\title{
EXPERIMENTAL INVESTIGATION OF THE NEAR WALL FLOW STRUCTURE OF A LOW REYNOLDS NUMBER 3-D TURBULENT BOUNDARY LAYER
}

\author{
By \\ Jonathan L. Fleming
}

A DisSERTATION SUBMITTED TO THE FACULTY OF

VIRGINIA POLYTECHNIC INSTITUTE AND STATE UNIVERSITY

IN PARTIAL FULFILLMENT OF THE REQUIREMENTS FOR THE DEGREE OF DOCTOR OF PHILOSOPHY

IN

Aerospace EngineEring

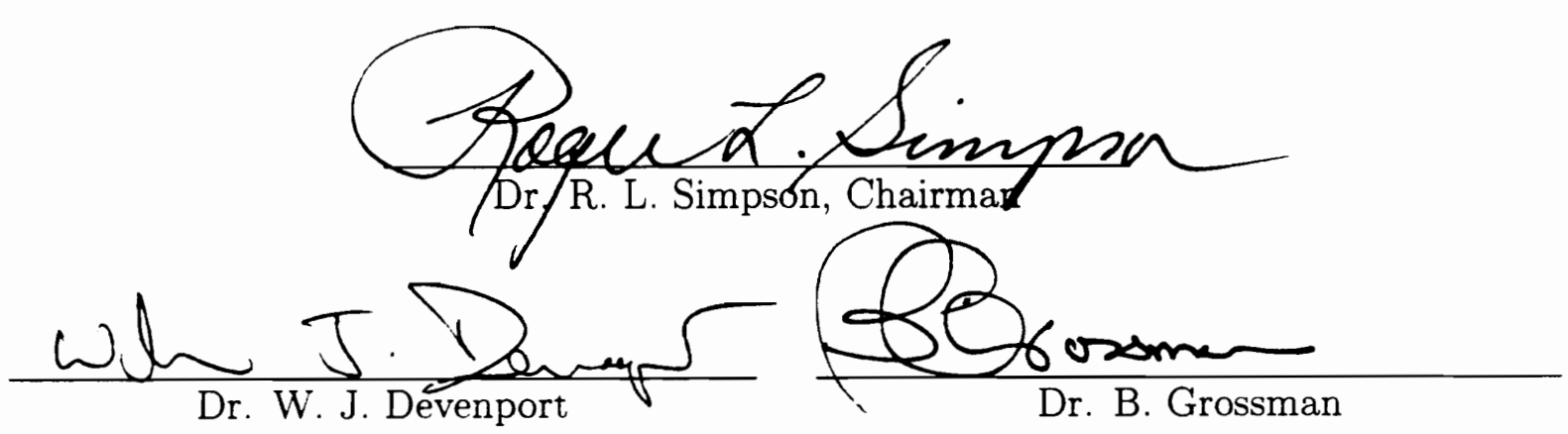

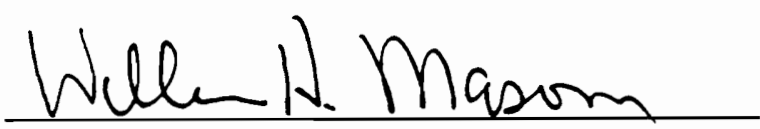

Dr. W. H. Mason

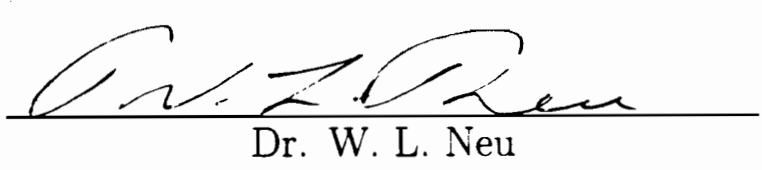

Dr. W. L. Neu

November 1996

Blacksburg, Virginia

Keywords: LDA, Coherent Structures, Vortices, Digital Image Analysis 
0.2

$$
\begin{aligned}
& L D \\
& 5655 \\
& V 856 \\
& 1996 \\
& F 546
\end{aligned}
$$

C. 2 


\title{
EXPERIMENTAL INVESTIGATION OF THE NEAR WALL FLOW STRUCTURE OF A LOW REYNOLDS NUMBER 3-D TURBULENT BOUNDARY LAYER
}

\author{
by \\ Jonathan L. Fleming \\ Dr. Roger L. Simpson, Chairman \\ Department of Aerospace and Ocean Engineering
}

(ABSTRACT)

Laser Doppler velocimetry (LDV) measurements and hydrogen-bubble flow-visualization techniques were used to examine the near-wall flow structure of 2-D and 3-D turbulent boundary layers (TBLs) over a range of low Reynolds numbers. The goals of this research were (1) an increased understanding of the flow physics in the near wall region of turbulent boundary layers, (2) to observe and quantify differences between 2-D and 3-D TBL flow structures, and (3) to document Reynolds number effects for 3-D TBLs. An ultimate application of this work would be to improve turbulence modeling for 3-D flows.

The LDV data have provided results detailing the turbulence structure of the 2-D and 3-D TBLs, as well as low uncertainty skin friction estimates. These results include mean Reynolds stress distributions, flow skewing results, and $U$ and $V$ spectra. Effects of Reynolds number for the 3-D flow were examined when possible. Comparison to results with the same 3-D flow geometry but at a significantly higher Reynolds number provided unique insight into the structure of 3-D TBLs. While the 3-D mean and fluctuating velocities were found to be highly dependent on Reynolds number, a previously defined shear stress parameter was discovered to be invariant with Reynolds number.

The hydrogen-bubble technique was used as a flow-visualization tool to examine the near-wall flow structure of 2-D and 3-D TBLs. Both the quantitative and qualitative results displayed larger turbulent fluctuations with more highly concentrated vorticity regions for the 2-D flow. The 2-D low-speed streaky structures experienced greater interaction with the outer region high-momentum fluid than observed for the 3-D flow. The near-wall 3-D flow structures were generally more quiescent. Numerical parameters quantified the observed differences, and characterized the low-speed streak and high-speed sweep events. All observations indicated a more stable near-wall flow structure with less turbulent interactions occurring between the inner and log regions for a 3-D TBL. 
The author very gratefully acknowledges the support of the Advanced Research Project Agency (Mr. Gary Jones) through ONR grant number N00014-90-J-1909, Dr. Edwin Rood, Program Manager. This work was also supported by NASA Langley grant NAG-1-1303, Dr. Stephen Robinson, Technical Monitor. Without the support of these agencies, this work would not have been possible.

I have been fortunate to have had the most patient advisor a graduate student could ask for. Thank you Dr. Simpson, for your guidance, suggestions, and encouragement. Someday we'll be amazed by how simple this turbulent flow business really is ... (and wondering why we couldn't have figured it out while we were alive).

Thanks also go to the other members of my commitee: Dr. Grossman, Dr. Mason, Dr. Neu, and Dr. Devenport. Even though our day-to-day interactions were limited, I feel that I have learned much about engineering and also life in general from each one of you during my years of roaming the halls of Randolph. Special thanks go to Dr. Devenport and Dr. Semih Ölçmen for being my wind tunnel "mentors" and helping me along when I was a beginning graduate student. Also, I am grateful for Dr. Ölçmen's permission to use his high Reynolds number LDV data in this dissertation. Many thanks go to Dr. Kevin Shinpaugh for assembling and aligning the LDV system described in chapter 2.

It would take several pages to properly thank everyone who has helped me over the years, so here is the short list. Thanks goes to the departmental secretaries (Betty Williams, Wanda Foushee, and Gay Stafford) who were always very quick to help me out. I think they were just trying to get me out of the office. I also (grudgingly) thank the guys in the shop ... I'm not going to name them for fear of ruining any future background checks. I am also grateful for fellow grad students like Todd Wetzel, Kraig Willet, Dan Hammerand, and the many others who have made this place too much fun to leave.

I have been blessed with a very supportive family. Thank you mom and dad (and mom-in-law) for all the encouragement and love. You'll be happy to find out that I now know the value of hard work and commitment (about $\$ 3.55$ an hour for a bumbling grad student, but hey, it's more than the art majors get). And of course very special thanks go to the most wonderful two ladies in my life - Karen and Jennifer. You two are the sweetest evidence of God's grace in my life. 
Page

List of Tables $\ldots \ldots \ldots \ldots \ldots \ldots \ldots \ldots \ldots \ldots \ldots \ldots \ldots \ldots \ldots \ldots \ldots \ldots$

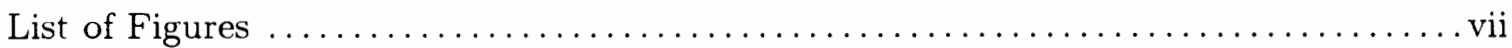

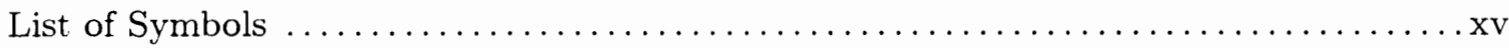

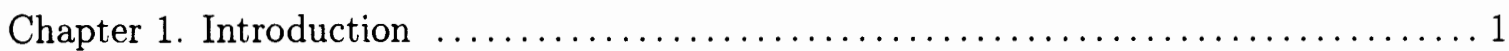

1.1 Current Research Motivations and Goals .......................... 2

1.2 Review of Wall-Bounded Turbulent Flow Research $\ldots \ldots \ldots \ldots \ldots \ldots \ldots \ldots$

1.2.1 2-D Turbulent Boundary Layers .......................... 5

1.2.2 3-D Turbulent Boundary Layers .......................... 8

1.3 Summary of Current Research Program ......................... 10

1.3.1 LDV Measurements .................................... 10

1.3.2 Hydrogen-Bubble Flow Visualizations $\ldots \ldots \ldots \ldots \ldots \ldots \ldots \ldots \ldots \ldots \ldots$

Chapter 2. Experimental Apparatus and Techniques $\ldots \ldots \ldots \ldots \ldots \ldots \ldots \ldots \ldots \ldots \ldots$

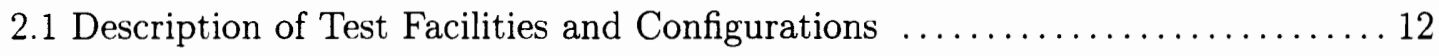

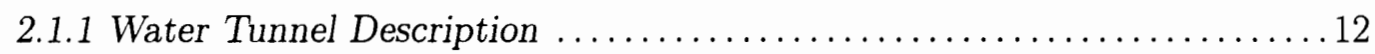

2.1.2 Determining Test Fluid Properties .......................... 13

2.1.3 Determining Freestream Flow Velocity ...................... 14

2.1.4 Potential Core and Approach Boundary Layer Documentation ......... 15

2.1.5 Model Description ....................................... 19

2.1.6 2-D and 3-D Test Configurations ................................. 19

2.2 Laser Doppler Velocimetry Apparatus and Setup ..................... 20

2.2.1 Basic Principles of LDV Operation ........................... 21

2.2.2 Current Implementation of the LDV Technique ................ 22

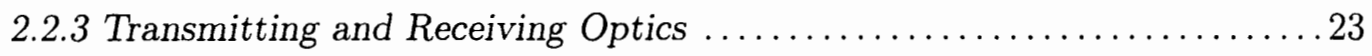

2.2.4 LDV Data Recording and Signal Analysis ................... 25

$2.2 .5 \mathrm{LDV}$ Data Reduction and Uncertainties ...................... 26

2.3 Hydrogen-Bubble Apparatus and Setup ............................ 31

2.3.1 Hydrogen-Bubble Technique Background ...................... 31

2.3.2 Current Implementation of the Hydrogen-Bubble Technique . . . . . . . 32

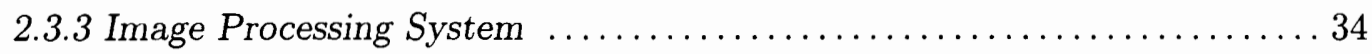


2.3.4 Analyzing Flow-Visualization Data ......................... 36

2.3.5 Flow-Visualization Uncertainty Analysis ................... 37

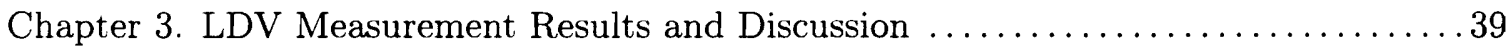

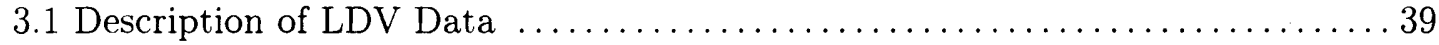

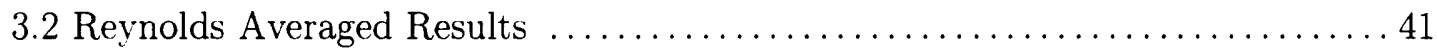

3.2.1 Measured Quantities .................................... 41

3.2.2 Derived Quantities .................................. 47

3.3 LDV Autocorrelation Results .................................. 52

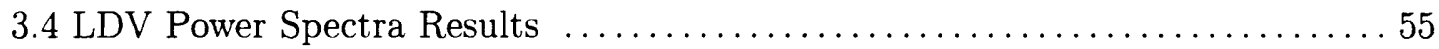

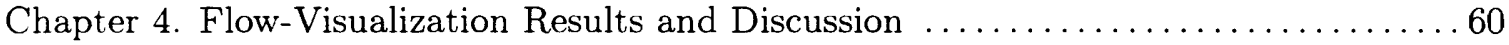

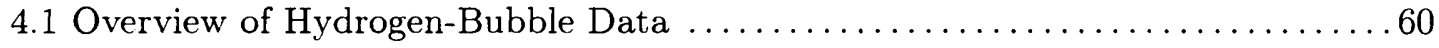

4.1.1 Hydrogen-Bubble Wire Coordinates ........................61

4.1.2 Quantitative Data Regions ............................61

4.1.3 Local Pressure Fields ................................... 61

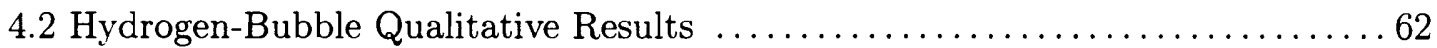

4.3 Hydrogen-Bubble Quantitative Results ..........................64

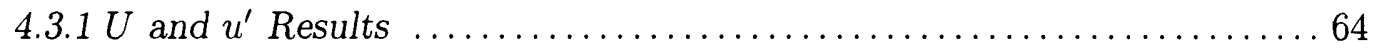

4.3.2 Low-Speed Streak and High-Speed Sweep Results ................ 65

4.3.3 Other Streak/Sweep Parameter Results .................... 68

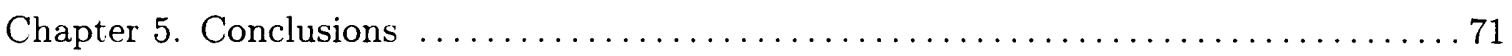

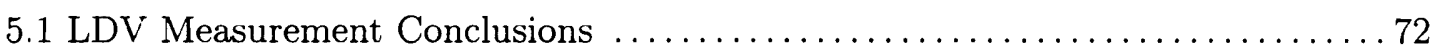

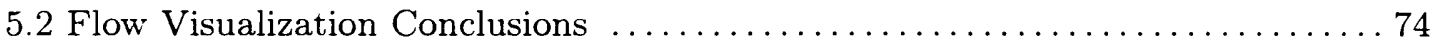

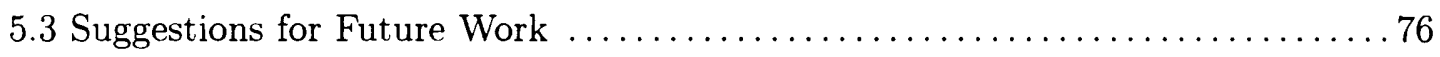

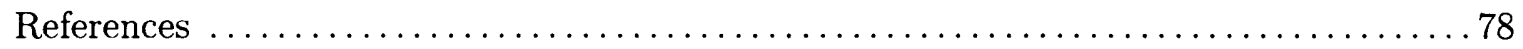

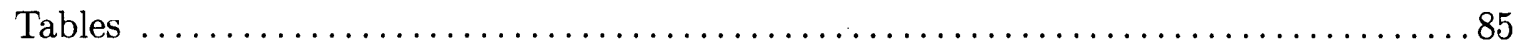

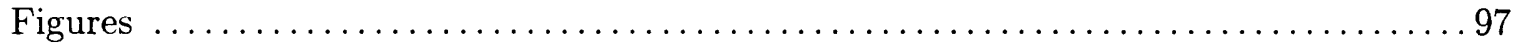

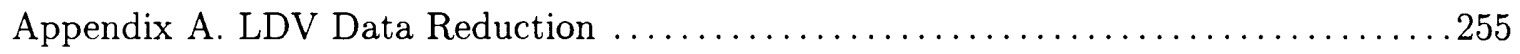

Appendix B. Data Reduction Programs . ............................... 259

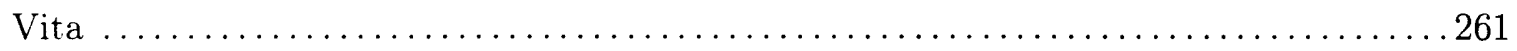




\section{LIST OF TABLES}

Table 1.1 Experimental test matrix used to set up the LDV measurements.

Table 1.2 Experimental test matrix used to set up the hydrogen-bubble flow-visualization experiments. 87

Table 2.1 Fluid properties for the different parts of the author's experimental work. . 88

Table 2.2 Freestream parameters for nominal tunnel speeds of $10,15,20$, and $30 \mathrm{~cm} / \mathrm{s} . \quad 89$

Table 2.3 Displacements for the water tunnel test section false side walls. .90

Table 2.4 Estimated uncertainties for quantities affecting the LDV experimental results. 91

Table 2.5 Nominal uncertainties for LDV data at 20:1 odds. .92

Table 3.1 Boundary layer parameters for 2-D and 3-D cases.

Table 3.2 Boundary layer shape parameters for the 3-D cases at station 5 .

Table 4.1 Actual experimental test matrix for the hydrogen-bubble flow-visualization experiments, separated into 2-D and 3-D sections. 95

Table 4.2 Near-wall structural parameter results from the 2-D and 3-D hydrogen-bubble flow visualization data, nominal reference velocity $=15 \mathrm{~cm} / \mathrm{s}$. .96 


\section{LIST OF FIGURES}

Fig. 1.1 3-D flow around a wing-body junction, from Ölçmen and Simpson (1997). . . 98

Fig. 1.2 3-D flow patterns around a slender body at angle of attack (from Wetzel and Simpson, 1997).

Fig. 1.3 The typical ranges of momentum thickness Reynolds numbers encountered for various types of flows.

Fig. 1.4 Comparisons of Spalart's (1988) DNS results and Ölçmen and Simpson's (1995) 2-D experimental results.

Fig. 1.5 Near-wall low-speed streak spacing results from Kim et al. (1971).

Fig. 1.6 Robinson's (1991) observed flow structures from DNS results. 104

Fig. 1.7 2-D computational results from Robinson, et al.(1991), using Spalart's Direct

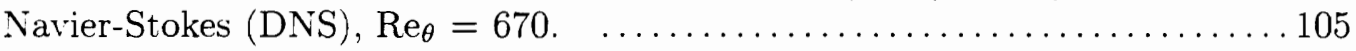

Fig. 1.8 Typical 3-D turbulent boundary layer mean flow distributions. 106

Fig. 1.9 Graphical representations of the quadrant and octant analysis schemes. $\ldots 107$

Fig. 1.10 Streamwise elevation view of a conceptual model for the near-wall sweep/ejection process for mean 2-D turbulent boundary layers.

Fig. 1.11 Streamwise elevation and plan views of a conceptual model for the near-wall sweep/ejection process for pressure-driven 3-D turbulent boundary layers. . 109

Fig. 1.12 Observed variations in fluid trajectories for ejection and sweep events in the presence of spanwise (3-D) flow.

Fig. 1.13 Surface oil flow results for current experimental wing-body junction geometry, approach $\operatorname{Re}_{\theta} \approx 7000$.

Fig. 1.14 A frame from a hydrogen-bubble flow-visualization video documenting the wingbody junction vortex structure (Kim, 1991).

Fig. 2.1 Top view of Virginia Tech Aerospace and Ocean Engineering water tunnel. 113

Fig. 2.2 Sketch of side view of water tunnel test section, showing contraction, false floor, and appendage location. 
Fig. 2.3 Diagram of appendage and tunnel coordinate system.

Fig. 2.4 Plot of current kinematic viscosity results compared to published data.

Fig. 2.5 Sketch of the special hydrogen-bubble probe used to check the accuracy of the electromagnetic water current meter.

Fig. 2.6 Freestream $U$ autocorrelation results for $U_{\text {ref }}=10,15,20$, and $30 \mathrm{~cm} / \mathrm{s}$. $\quad \ldots 118$

Fig. 2.7 Freestream $U$ autospectra results for $U_{r e f}=10,15,20$, and $30 \mathrm{~cm} / \mathrm{s} . \quad \ldots 119$

Fig. 2.8 2-D flow boundary layer profile data, documenting the test section boundary layer and lower portion of the test flow's "inviscid core." .................120

Fig. 2.9 Shear stress correlation results for the 2-D boundary layers.

Fig. 2.10 Diagram explaining the effects that a weak streamwise vortex would have on the distribution of $V$.

Fig. 2.11 Sketch showing typical open-channel mean velocity contours and secondary flow patterns (after Sellin, 1970).

Fig. 2.12 Appendage geometry used for 3-D flow test case (3:2 elliptic wing, NACA 0020 tail).

Fig. 2.13 Side wall displacement for 2-D flow case. .126

Fig. 2.14 Side wall displacement for 3-D flow case. 127

Fig. 2.15 Basic elements of a one-component LDV system (after Durst, et al., 1981) . 128

Fig. 2.16 Sketch revealing the underlying principles of how the LDV technique works. 129

Fig. 2.17 Photograph of LDV system configured for the 2-D case. 130

Fig. 2.18 Top view of LDV setup and component list for $20^{\circ}$ cases. 131

Fig. 2.19 Top view of LDV setup showing the 3 different optical configurations used to get all mean and fluctuating velocity components.

Fig. 2.20 Top view of LDV setup with $+45^{\circ}$ optical configuration (case C) detailed. $\quad .133$

Fig. 2.21 Side view of optical table mounted on milling machine base. 134

Fig. 2.22 Axis systems for the different LDV optical configurations. 135

Fig. 2.23 A summary of Snell's law of refraction. 136 
Fig. 2.24 Pictures of water cell used for $-45^{\circ}$ case (case B). $\ldots \ldots \ldots \ldots \ldots \ldots \ldots \ldots \ldots$

Fig. 2.25 Block diagram of equipment used to receive and process LDV signals. . . . 138

Fig. 2.26 Sample of velocity biasing effects inherent in LDV measurements. . . . . . 139

Fig. 2.27 Sample of gradient broadening corrections from Ölçmen and Simpson's (1995) LDV data. ........................................... 140

Fig. 2.28 Photographs of hydrogen-bubble probe, $\ldots \ldots \ldots \ldots \ldots \ldots \ldots \ldots \ldots \ldots \ldots \ldots \ldots \ldots$

Fig. 2.29 Camera and hydrogen-bubble probe position for $2-\mathrm{D}$ flow visualizations. . . 142

Fig. 2.30 Sketch of bubble wire position for the 3-D flow visualizations. $\ldots \ldots \ldots \ldots 143$

Fig. 2.31 Field of view diagrams for the hydrogen-bubble flow visualizations. $\ldots \ldots \ldots 144$

Fig. 2.32 Top view of light placement for the 2-D hydrogen-bubble flow visualizations. 145

Fig. 2.33 Top view of light placement for the 3-D hydrogen-bubble flow visualizations. 146

Fig. 2.34 Block diagram of equipment used to generate hydrogen bubbles. $\quad \ldots \ldots \ldots 147$

Fig. 2.35 Block diagram of equipment used to store and analyze hydrogen-bubble flow vi-

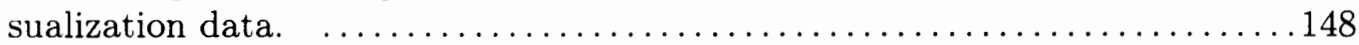

Fig. 2.36 Sample constant-sheet images of hydrogen-bubble flow-visualization results. 149

Fig. 2.37 Sample time-line images of hydrogen-bubble flow-visualization results. $\ldots 150$

Fig. 2.38 A sample calibration image used for the 3-D flow case. $\ldots \ldots \ldots \ldots \ldots \ldots 151$

Fig. 2.39 Example of digital image filtering and enhancement. $\ldots \ldots \ldots \ldots \ldots \ldots \ldots 2$

Fig. 2.40 Outline of the image processing procedure. $\ldots \ldots \ldots \ldots \ldots \ldots \ldots \ldots \ldots \ldots \ldots \ldots \ldots$

Fig. 2.41 Examples of the automated time-of-flight reduction technique (3-D flow). ..154

Fig. 2.42 More examples of the time-of-flight reduction technique (2-D flow). $\ldots \ldots 155$

Fig. 3.1 The different coordinate systems traditionally used for the presentation of 3-D boundary layer data.

Fig. 3.2 Skin friction coefficient and shape factor vs. Reynolds number for current 2-D and 3-D data. 157

Fig. 3.3 3-D lateral shape parameters vs. Reynolds number for the current 3-D data. 158 
Fig. 3.4 2-D flow boundary laver profiles, plotted to display the shape of the mean velocity distribution.

Fig. 3.5 3-D flow boundary layer profiles, plotted to display the shape of the mean velocity distribution. 160

Fig. 3.6 2-D mean velocity boundary layer profiles. 161

Fig. 3.7 The 2-D velocity defect profile results. 162

Fig. 3.8 3-D boundary layer profiles of $Q$. 163

Fig. 3.9 3-D mean velocity boundary layer profiles. 164

Fig. 3.10 Detail of the 2-D $V$ results. 165

Fig. 3.11 Detail of the 3-D $V$ results. 166

Fig. 3.12 Hodograph plot, $U$ and $W$ scaled using $U_{\boldsymbol{e}}$. 167

Fig. 3.13 2-D normal stress results. 168

Fig. 3.14 2-D normal stress results from Klebanoff (1955) and Ölçmen and Simpson, station 0 data (1995). 169

Fig. 3.15 3-D normal stress results. 170

Fig. 3.16 Fit results for the peak $u^{\prime}$ normal stress for the 3-D boundary layer cases. . 173

Fig. 3.17 2-D results for the turbulent kinetic energy, $k$. 174

Fig. 3.18 3-D results for the turbulent kinetic energy, $k$. 175

Fig. 3.19 Fit results for the peak turbulent kinetic energy levels for the 3-D boundary layer cases. 176

Fig. 3.20 2-D mean Reynolds shear stress results. 177

Fig. 3.21 3-D mean Reynolds shear stress results. 178

Fig. 3.22 3-D mean Reynolds shear stress $-\overline{u v}$ results. 179

Fig. 3.23 3-D mean Reynolds shear stress $-\overline{v w}$ results. 180

Fig. 3.24 3-D mean Reynolds shear stress $-\overline{u w}$ results. 181

Fig. 3.25 Mean Reynolds shear stress correlation results. 182 
Fig. 3.26 Profiles of the total shear stress magnitude for the 3-D boundary layers.

$\ldots 183$

Fig. 3.27 Typical streamwise velocity rms, skewness, and flatness results for a 2-D turbulent boundary layer.

Fig. 3.28 2-D skewness results for $u$ and $v$. 185

Fig. 3.29 2-D flatness results for $u$ and $v$. 186

Fig. 3.30 2-D flatness vs. skewness ${ }^{2}$ results for $u$ and $v$. 187

Fig. 3.31 Skewness results for the 3-D boundary layers. 188

Fig. 3.32 Triple products results for the 3-D boundary layers. 189

Fig. 3.33 2-D Townsend structural parameter results. 190

Fig. 3.34 3-D Townsend structural parameter results. 191

Fig. 3.35 2-D shear stress parameter, $1 / S$, results.

Fig. 3.36 3-D shear stress parameter, $1 / S$, results. 193

Fig. 3.37 $-1 / C$ results for the $3-\mathrm{D}$ flow.

Fig. 3.38 Freestream coordinate system results for $\beta_{\mathrm{FA}}, \beta_{\mathrm{FGA}}$, and $\beta_{\tau}$. 195

Fig. 3.39 $\beta_{\mathrm{FA}}$ freestream results. 197

Fig. 3.40 $\beta_{\mathrm{FGA}}$ freestream results. 198

Fig. 3.41 $\beta_{\tau}$ freestream results.

Fig. 3.42 Freestream coordinate system results for $\beta_{\mathrm{FA}}(\mathrm{FA}), \beta_{\mathrm{FGA}}(\mathrm{FGA})$, and $\beta_{\tau}$ (SSA) (reprinted from Ölçmen and Simpson, 1995).

Fig. 3.43 Shear stress lag angle $\left(\beta_{\mathrm{lag}}\right)$ freestream results 201

Fig. 3.44 Freestream results for $\beta_{\mathrm{FA}}, \beta_{\mathrm{FGA}}, \beta_{\tau}$, and $\beta_{\text {lag }}$ plotted vs. $y / T$. 202

Fig. 3.45 Freestream results for the anisotropy factor, $N$. 204

Fig. 3.46 2-D $U$ autocorrelation results, $U_{r e f}=10 \mathrm{~cm} / \mathrm{s}, \operatorname{Re}_{\theta}=450$. 205

Fig. 3.47 2-D $U$ autocorrelation results, $U_{\text {ref }}=15 \mathrm{~cm} / \mathrm{s}, \operatorname{Re}_{\theta}=300$. 206

Fig. $3.482-\mathrm{D} U$ autocorrelation results, $U_{r e f}=20 \mathrm{~cm} / \mathrm{s}, \operatorname{Re}_{\theta}=425$. 207 
Fig. 3.49 2-D $U$ autocorrelation results, $U_{r e f}=30 \mathrm{~cm} / \mathrm{s}, \operatorname{Re}_{\theta}=570$. .208

Fig. 3.50 3-D $U$ autocorrelation results, $U_{r e f}=10 \mathrm{~cm} / \mathrm{s}, \operatorname{Re}_{\theta}=500$. 209

Fig. 3.51 3-D $U$ autocorrelation results, $U_{r e f}=15 \mathrm{~cm} / \mathrm{s}, \operatorname{Re}_{\theta}=760$. 210

Fig. 3.52 3-D $U$ autocorrelation results, $U_{r e f}=20 \mathrm{~cm} / \mathrm{s}, \operatorname{Re}_{\theta}=890$. .211

Fig. 3.53 Autocorrelation length scale results for the 2-D and 3-D data. 212

Fig. 3.54 Autocorrelation length scale results vs. $\operatorname{Re}_{\theta}$ for $2-\mathrm{D}$ and $3-\mathrm{D}$ data. 213

Fig. 3.55 Noise subtraction and smoothing results for typical LDV raw spectral data. 214

Fig. 3.56 2-D $U$ and $V$ power spectral results, $U_{\text {ref }}=10 \mathrm{~cm} / \mathrm{s}, \operatorname{Re}_{\theta}=450$. 215

Fig. $3.572-\mathrm{D} U$ and $V$ power spectral results, $U_{r e f}=15 \mathrm{~cm} / \mathrm{s}, \operatorname{Re}_{\theta}=300$. 216

Fig. 3.58 2-D $U$ and $V$ power spectral results, $U_{r e f}=20 \mathrm{~cm} / \mathrm{s}, \operatorname{Re}_{\theta}=425$. 217

Fig. 3.59 2-D $U$ and $V$ power spectral results, $U_{r e f}=30 \mathrm{~cm} / \mathrm{s}, \operatorname{Re}_{\theta}=570$. 218

Fig. 3.60 2-D $U$ power spectra from Ahn and Simpson's (1987). 219

Fig. 3.61 2-D $V$ power spectra from Ölçmen (1997). 220

Fig. 3.62 3-D $U$ and $V$ power spectral results, $U_{\text {ref }}=10 \mathrm{~cm} / \mathrm{s}, \operatorname{Re}_{\theta}=500$. 221

Fig. 3.63 3-D $U$ and $V$ power spectral results, $U_{r e f}=15 \mathrm{~cm} / \mathrm{s}, \operatorname{Re}_{\theta}=760$. 222

Fig. 3.64 3-D $U$ and $V$ power spectral results, $U_{r e f}=20 \mathrm{~cm} / \mathrm{s}, \operatorname{Re}_{\theta}=890$.

Fig. 3.65 3-D $U$ power spectra from Ha and Simpson's (1993b). 224

Fig. 3.66 $U$ and $V$ power spectral results, $U_{\text {ref }}=15 \mathrm{~cm} / \mathrm{s}$, non-dimensionalized using outer scaling ( $\delta$ ). (a) $2-\mathrm{D}, \operatorname{Re}_{\theta}=300$, and (b) $3-\mathrm{D}, \operatorname{Re}_{\theta}=760$. 225

Fig. 3.67 2-D $U$ spectral results for a range of Reynolds numbers, $8.9<y^{+}<10$. $\ldots 226$

Fig. 3.68 2-D $V$ spectral results for a range of Reynolds numbers, $8.9<y^{+}<10$. $\ldots 227$

Fig. 3.69 2-D $U$ spectral results for a range of Reynolds numbers, $0.70<y / \delta<0.77$. 228

Fig. 3.70 2-D $V$ spectral results for a range of Reynolds numbers, $y / \delta=0.77$. 229

Fig. 3.71 3-D $U$ spectral results for a range of Reynolds numbers, $11.6<y^{+}<14.0$. .230 
Fig. 3.72 3-D $V$ spectral results for a range of Reynolds numbers, $11.6<y^{+}<14.0$. . 231

Fig. 3.73 3-D $U$ spectral results for a range of Reynolds numbers, $0.35<y / \delta<0.49$. 232

Fig. 3.74 3 -D effects on the $U$ power spectral results, $y^{+} \approx 10, U_{\text {ref }}=15 \mathrm{~cm} / \mathrm{s} . \quad \ldots 233$

Fig. 3.75 3 -D effects on the $U$ power spectral results, $y^{+} \approx 50, U_{\text {ref }}=15 \mathrm{~cm} / \mathrm{s} . \quad \ldots 234$

Fig. 3.76 3 -D effects on the $U$ power spectral results, $y^{\prime} \delta=0.77, U_{\text {ref }}=15 \mathrm{~cm} / \mathrm{s} . \quad \ldots 235$

Fig. 3.77 3-D effects on the $V$ power spectral results, $y^{+} \approx 10, U_{\text {ref }}=15 \mathrm{~cm} / \mathrm{s} . \quad \ldots 236$

Fig. 3.783 -D effects on the $V$ power spectral results, $y^{+} \approx 50, U_{\text {ref }}=15 \mathrm{~cm} / \mathrm{s} . \quad \ldots 237$

Fig. 3.79 3 -D effects on the $V$ power spectral results, $y / \delta=0.77,0.40, U_{r e f}=15 \mathrm{~cm} / \mathrm{s} . \quad 238$

Fig. 4.1 Fig. 2.31 with wire coordinate systems drawn. $\ldots \ldots \ldots \ldots \ldots \ldots \ldots \ldots 239$

Fig. 4.2 Sketch of the estimated separation line location relative to the bubble-producing portion of the hydrogen-bubble wire for the author's low Reynolds number 3-D

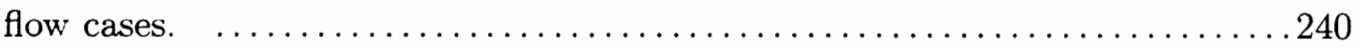

Fig. 4.3 Computed freestream pressure contours for the baseline appendage geometry. 241

Fig. 4.4 Computed freestream pressure contours overlayed by favorable pressure gradient unit direction vectors.

Fig. 4.5 Detail of the computed freestream $C_{p}$ along the 3-D bubble wire. 243

Fig. 4.6 Computed freestream flow direction along 3-D bubble wire. 244

Fig. 4.7 Detail of computed freestream flow directions along 3-D bubble wire. 245

Fig. 4.8 Constant-sheet hydrogen-bubble images for a nominal $U_{e}$ of $15 \mathrm{~cm} / \mathrm{s}$. $\ldots . .246$

Fig. 4.9 Time-line hydrogen-bubble images for a nominal $U_{e}$ of $15 \mathrm{~cm} / \mathrm{s}$. 247

Fig. 4.10 2-D flow-visualization results for $U$ and $u^{\prime}, U_{e}=15 \mathrm{~cm} / \mathrm{s}, \operatorname{Re}_{\theta}=300$. 248

Fig. 4.11 3-D quantitative flow-visualization results for $U$ and $u^{\prime}$, bubble wire at $20^{\circ}, U_{e}=$ $15 \mathrm{~cm} / \mathrm{s}, \operatorname{Re}_{\theta}=760$.

Fig. 4.12 Skin friction results determined for the $U_{e}=15 \mathrm{~cm} / \mathrm{s}$ cases.

Fig. 4.13 Sketch defining event geometry parameters used to calculate quantitative structural quantities. 251 
Fig. 4.14 $\lambda^{+}$probability distributions for the 2-D and 3-D TBLs.

.252

Fig. 4.15 The low-speed event momentum flux ratio $\left(E_{\mathrm{LS}}\right)$ probability distributions for 2-D and $3-\mathrm{D}$ turbulent boundary layers at $y^{+} \approx 10, U_{e}=15 \mathrm{~cm} / \mathrm{s} . \quad \ldots \ldots \ldots .253$

Fig. 4.16 Spatially-averaged vorticity $\left(\Omega^{+}\right)$probability distributions for the 2-D and 3-D turbulent boundary layers for the $U_{e}=15 \mathrm{~cm} / \mathrm{s}$ cases. 254 
Note: When a symbol is used which has multiple definitions, the author has taken care to make sure the symbol's usage in the text or equation is free from ambiguity.

$a . b \ldots \ldots \ldots \ldots \ldots$ Parameters used to define instantaneous turbulent event spatial bounds.

$a_{1} \ldots \ldots \ldots \ldots \ldots$ Townsend structural parameter, see equation 1.1 .

$C \ldots \ldots \ldots \ldots$ Chord length of appendage, or 3-D shear stress parameter (see equation 3.5).

$C_{f} \ldots \ldots \ldots \ldots \ldots$ Skin friction coefficient, $C_{f} \equiv \tau_{w} / \frac{1}{2} \rho U_{e}^{2}$.

$C_{p} \ldots \ldots \ldots \ldots \ldots$ Pressure coefficient, $C_{p} \equiv \frac{\left(p-p_{\infty}\right)}{\frac{1}{2} \rho_{\infty} U_{\infty}^{2}}$.

$d \ldots \ldots \ldots \ldots \ldots$ Distance between adjacent hydrogen bubble time lines.

$d_{f s} \ldots \ldots \ldots \ldots \ldots$ LDV measurement volume fringe spacing.

$d_{m} \ldots \ldots \ldots \ldots \ldots$ LDV measurement volume diameter.

$E \ldots \ldots \ldots \ldots \ldots$ Event momentum flux ratio, defined by equation 4.1 .

$f \ldots \ldots \ldots \ldots \ldots$ Frequency, $\mathrm{Hz}$.

$F(f) \ldots \ldots \ldots \ldots$. Power spectral density function, defined by equation 3.16.

$F_{u}, F_{v}, F_{w} \ldots \ldots \ldots$ Flatness of $U, V$, and $W$ velocity components, $F_{u}=\overline{u^{4}} / u^{\prime 4}$.

FST P .......... Freestream turbulence parameter, defined by equation 2.1. Subscript indicates length scale involved.

$H \ldots \ldots \ldots \ldots$ B....... Boundary layer shape factor, $H \equiv \delta^{*} / \theta$.

$k \ldots \ldots \ldots \ldots \ldots$ Turbulent kinetic energy, $k=\frac{1}{2}\left(u^{\prime 2}+{v^{\prime 2}}^{2}+{w^{\prime}}^{2}\right)$.

$k_{1} \ldots \ldots \ldots \ldots \ldots$ Wave number, $k_{1}=2 \pi f / U$.

$L_{e}^{u} \ldots \ldots \ldots \ldots \ldots$ Turbulence length scale, defined by equation 2.2 .

$L_{\Lambda} \ldots \ldots \ldots \ldots \ldots$ Autocorrelation length scale, $L_{\Lambda}=U T_{\Lambda}$.

$L_{\lambda} \ldots \ldots \ldots \ldots \ldots$ Autocorrelation "micro" length scale, $L_{\lambda}=U T_{\lambda}$.

$l_{m} \ldots \ldots \ldots \ldots \ldots$ LDV measurement volume length.

$n \ldots \ldots \ldots \ldots$ Index of refraction.

$N \ldots \ldots \ldots \ldots \ldots$ Eddy viscosity anisotropy factor, $N=$ transverse eddy viscosity/ streamwise eddy viscosity.

$N_{d} \ldots \ldots \ldots \ldots \ldots$ Data density parameter for LDV measurements, $N_{\boldsymbol{d}}=T_{\Lambda}$ (mean data rate).

$Q \ldots \ldots \ldots \ldots$ Component of mean velocity vector in $x z$ plane, $Q=\sqrt{U^{2}+W^{2}}$.

$R_{u u} \ldots \ldots \ldots$. Velocity autocorrelation coefficient, defined by equation 3.11.

$\operatorname{Re}_{\lambda} \ldots \ldots \ldots \ldots$ Turbulent Reynolds number, see equation 3.5.

$\operatorname{Re}_{\theta} \ldots \ldots \ldots \ldots$ Reynolds number based on momentum thickness, $\theta$. 
$S \ldots \ldots \ldots \ldots$ Shear stress parameter, $S \equiv v^{\prime 2} /|\tau / \rho|$.

$S_{u} . S_{v} . S_{w} \ldots \ldots \ldots$ Skewness of $U, V$, and $W$ velocity components, $S_{u}=\overline{u^{3}} / u^{\prime 3}$.

t............. Time.

$T \ldots \ldots \ldots \ldots \ldots$ Maximum thickness of appendage.

$T_{\Lambda} \ldots \ldots \ldots \ldots \ldots$ Autocorrelation integral time scale, defined by equation 3.13 .

$T_{\lambda} \ldots \ldots \ldots \ldots \ldots$ Autocorrelation "micro" time scale, defined by equation 3.14 .

TBL ............ Turbulent boundary layer (acronym).

$u_{\tau} \ldots \ldots \ldots \ldots \ldots$ Friction velocity, $u_{\tau} \equiv \sqrt{\tau_{w} / \rho}$.

$u^{\prime} . v^{\prime}, w^{\prime} \ldots \ldots \ldots \ldots$ Root mean square of $x, y$, and $z$ velocity fluctuations, $u^{\prime}=\sqrt{\overline{\overline{u^{\prime 2}}}}$.

$U, V, W \ldots \ldots \ldots \ldots$ Time mean velocity components in $x, y$, and $z$ directions. These symbols were also used to refer to the general velocity component directions for discussion purposes.

$U_{\perp} . V_{\perp} \ldots \ldots \ldots \ldots$ Instantaneous velocity components perpendicular to the LDV measurement volume fringe patterns.

$U_{e} \ldots \ldots \ldots \ldots \ldots$ Edge velocity, or the local boundary layer freestream velocity.

$U_{r e f} \ldots \ldots \ldots \ldots \ldots$ Test flow reference velocity, often a nominal value.

$-\overline{u v},-\overline{v w},-\overline{u w} \ldots .$. Mean Reynolds turbulent shear stresses.

$x . y . z \ldots \ldots \ldots \ldots$ Nominal coordinate directions. $x$ is in the streamwise direction, $y$ is perpendicular to the tunnel floor, and $z$ completes the right handed coordinate system.

$Z_{e} \ldots \ldots \ldots \ldots \ldots$ Transverse location of a turbulent streak or sweep event, usually defined a local maximum or minimum velocity.

Greek Symbols

$\beta \ldots \ldots \ldots \ldots \ldots$ (1) Intersecting beam angle for the LDV system, or (2) Instantaneous local flow angle.

$\beta_{e} \ldots \ldots \ldots \ldots$ Local freestream flow angle, $\beta_{e}=\tan ^{-1}\left(W_{e} / U_{e}\right)$.

$\beta_{\mathrm{FA}} \ldots \ldots \ldots \ldots$ Local mean flow angle, $\beta_{\mathrm{FA}}=\tan ^{-1}(W / U)$.

$\beta_{\mathrm{FGA}} \ldots \ldots \ldots \ldots$ Local flow gradient angle, see equation 3.7.

$\beta_{\text {lag }} \ldots \ldots \ldots \ldots$ Local shear stress lag angle, $\beta_{\text {lag }}=\beta_{\mathrm{FGA}}-\beta_{\tau}$.

$\beta_{w} \ldots \ldots \ldots \ldots$ Mean flow angle of the near-wall collateral flow.

$\beta_{\tau} \ldots \ldots \ldots \ldots$ Local shear stress angle, see equation 3.8 .

$\delta \ldots \ldots \ldots \ldots \ldots$ Boundary layer thickness, defined by $U / U_{e}=0.99$.

$\delta^{*} \ldots \ldots \ldots \ldots \ldots$ Boundary layer displacement thickness.

$\varepsilon \ldots \ldots \ldots \ldots \ldots$ Turbulent event velocity magnitude, $\varepsilon^{+}=u_{\text {event }} / u_{\tau}$.

$\theta \ldots \ldots \ldots \ldots$ Boundary layer momentum thickness.

$\kappa \ldots \ldots \ldots \ldots \ldots$ Turbulent event transverse extent, or width.

$\lambda \ldots \ldots \ldots \ldots$ (1) Wavelength of laser beam, or (2) Turbulent event transverse spacing. 


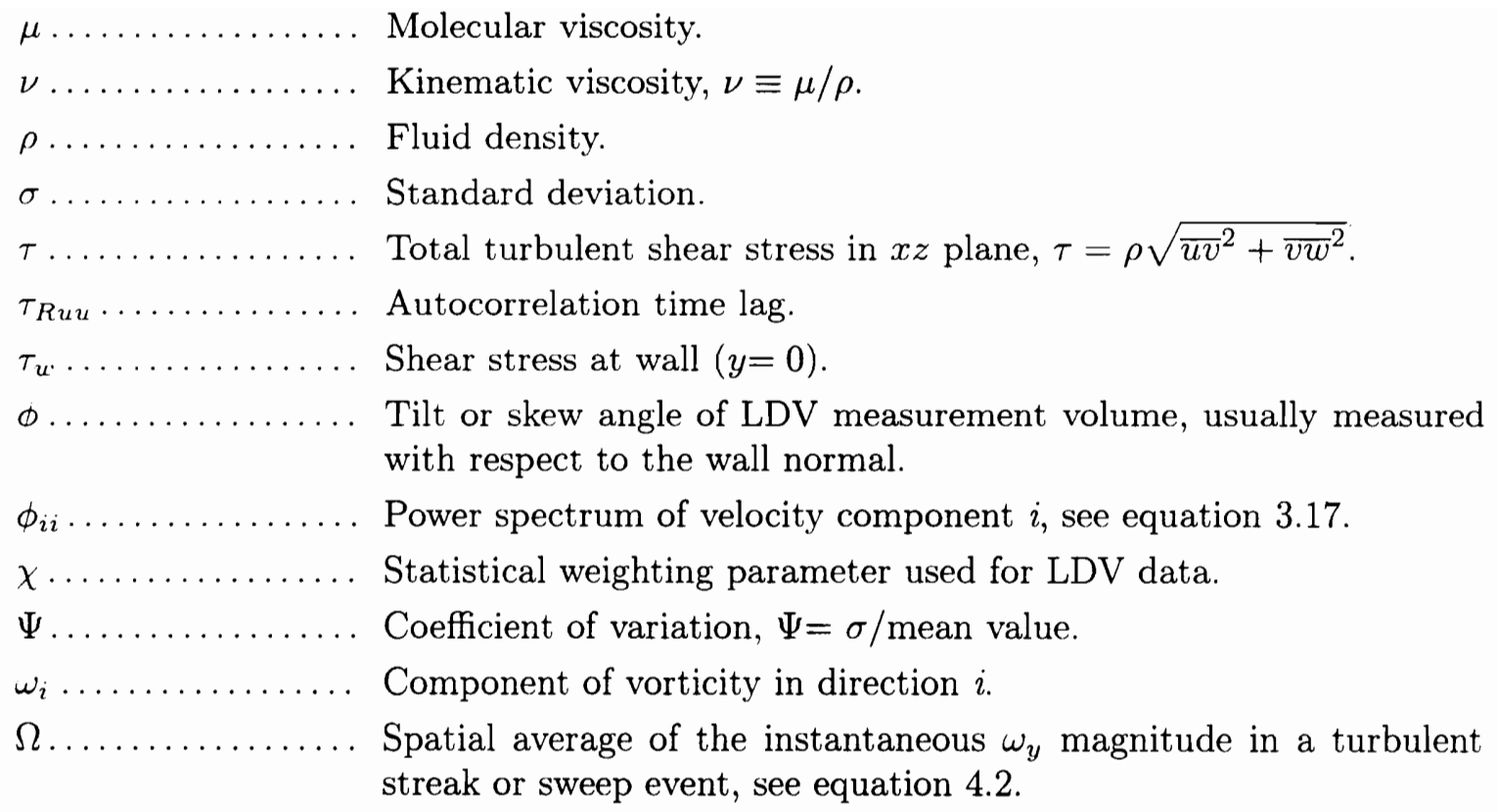

\section{Superscripts and Subscripts}

()$^{+} \ldots \ldots \ldots \ldots$ Quantity normalized using wall units. Velocities are non-dimensionalized using $1 / u_{\tau}$ and lengths are non-dimensionalized using $u_{\tau} / \nu$. More complex normalizations are defined in the symbol list or the text.

( $)_{F S} \ldots \ldots \ldots \ldots$ Quantity presented in the freestream coordinate system.

( $)_{\text {HS }} \ldots \ldots \ldots \ldots$..... Quantity describes high speed turbulent events (sweeps).

( $)_{\text {LS }} \ldots \ldots \ldots \ldots$. Quantity describes low speed turbulent events (streaks).

()$_{\mathrm{mp}} \ldots \ldots \ldots \ldots$...... Most probable value of () .

( $)_{\mathrm{TC}} \ldots \ldots \ldots \ldots$ Q Quantity presented in the tunnel coordinate system. 


\section{INTRODUCTION}

A wall-bounded fluid flow's "boundary layer" is the region where the flow transitions from zero velocity (relative to the wall) at the wall's surface to the "freestream" or "edge" velocity away from the wall. This region is typically very thin compared to the entire region of interest, such as the flow around an aircraft wing at high speed.

However, the boundary layer's small size belies its importance, and it is of great engineering interest for primarily two reasons. First, this viscous region is directly responsible for a flow's skin friction drag. Skin friction drag comprises $50 \%$ of a commercial airliner's drag, $90 \%$ of the drag on submersibles, and $100 \%$ of the drag in pipe flows (Gad-el-Hak and Bandyopadhyay, 1994). Second, when a boundary layer becomes detached, or separated, from its bounding surface, the entire flowfield is changed dramatically. The characteristics of an aircraft's boundary layer influence its speed, range, maneuverability, minimum landing speed, and many other aspects of its performance. If flow separation can be controlled, aircraft lift can be enhanced and form drag can be decreased.

The vast majority of boundary layers are turbulent. Turbulent flows are characterized by seemingly random swirls, or eddies of fluid, occurring in all shapes and sizes. Turbulence is a property of the flow, not the fluid. To understand boundary layers, one must also understand the physics of turbulent flows. Unfortunately, turbulent flows are very difficult to predict or model using modern engineering methods. One noted fluids researcher has called turbulence "the chief outstanding difficulty of our subject" (Bradshaw, 1992).

Turbulent flows occur in nature in the atmosphere and oceans, around buildings and bridge piers, and over sand dunes and ocean waves. From studying flows around vehicles, to analyzing sediment transport by wind or water, to studying the flow of blood through the human heart, turbulent flows are of constant practical interest.

The author has hopefully made a useful contribution to the large body of basic turbulence research with this work. The current experimental work examined the near-wall spanwise flow structure of a spatially developing, pressure-driven 3-D turbulent boundary layer, and compared it to a 2-D TBL using two complementary measurement techniques. This research was carried out in a low speed, low Reynolds number $\left(U_{r e f}<30 \mathrm{~cm} / \mathrm{s}, \operatorname{Re}_{\theta}<\right.$ 
900) water tunnel facility. The effects of Reynolds number on the 3-D turbulent boundary laver were examined by comparing to a higher Reynolds number boundary layer which had the same flow geometry (Ölçmen and Simpson, 1995). Data from the Ölçmen and Simpson flow case are shown in Fig. 1.1, along with a sketch of the wing-body junction geometry. At Ölçmen and Simpson's \#5 station, the Reynolds number was approximately 9500, and the near-wall region was very thin $\left(y^{+}=10\right.$ at $\left.y=0.14 \mathrm{~mm}\right)$. The current test flow's nearwall region was an order of magnitude thicker, which facilitated a detailed examination of the inner region. The current research program's motivations and objectives are described along with background information in section 1.1 .

\subsection{Current Research Motivations and Goals}

Why study 3-D boundary layers?

Three-dimensional pressure-driven turbulent boundary layers are encountered in over $95 \%$ of "real world" flows. Ironically, the vast majority of past turbulence research has focused on 2-D flows, without advancing the understanding of 3-D turbulent boundary layers (TBLs). Most results from 2-D TBLs cannot be directly applied to 3-D TBLs, as discussed extensively by Ölçmen and Simpson $(1991,1993,1995)$. The differences between 2-D and 3-D TBLs indicate fundamental changes in the flow physics, which is why existing 2-D turbulence models do not work well when adapted to general 3-D flows. Some of the most basic differences include the following (from Ölçmen and Simpson, 1993a; Johnston and Flack, 1996; Eaton, 1995):

- The eddy viscosity concept fails for general 3-D layers. The anisotropy factor, $N$, is not constant.

- The total Reynolds shear stress in the $x z$ plane is lower for a 3-D TBL than for a 2-D TBL. Also, the shear direction does not coincide with the mean velocity gradient direction (this is related to the non-homogeneous eddy viscosity).

- The Townsend structural parameter, $a_{1}$, defined as

$$
a_{1}=\frac{\sqrt{{\overline{u^{\prime} v^{\prime}}}^{2}+{\overline{v^{\prime} w^{\prime}}}^{2}}}{2 k}
$$

typically has a constant value of 0.15 outside of the buffer layer for 2-D TBLs. This parameter has been observed to decrease with increasing flow three-dimensionality.

The current turbulence models do not predict the observed reductions for 3-D Reynolds shearing stresses. This reduction must be due to a fundamental structural difference for 
3-D TBLs. One of the ultimate goals of turbulence research is to accurately predict the flow around general 3-D geometries. There is much to be learned before this goal is met. For example, not even the current state-of-the-art flow solvers can correctly predict the flow separation locations for the geometrically simple 3-D case shown in Fig. 1.2.

Why study Reynolds number effects?

First a review-what is the Reynolds number? It is the non-dimensional grouping formed by (length)(velocity)(fluid density)/(fluid viscosity). The Reynolds number characterizes the relative magnitudes of the inertial and viscous forces in a given flow. The importance of the Reynolds number is illustrated by the non-dimensional equation of motion for an incompressible fluid:

$$
\frac{\partial \vec{u}}{\partial t}+(\vec{u} \cdot \nabla) \vec{u}=-\nabla P+\frac{1}{\operatorname{Re}} \nabla^{2} \vec{u}
$$

This equation indicates the need for Reynolds number similarity as well as geometric similarity when performing fluids experiments. This condition is also referred to as "dynamic similarity."

This leads us to a primary reason for studying the effects of Reynolds number on turbulent boundary layers. Figure 1.3 displays the ranges of momentum thickness Reynolds numbers for different types of flows (from Gad-el-Hak and Bandyopadhyay, 1994). The gap between real-world flows and typical laboratory flows is clearly evident. Understanding the effects of changing Reynolds number becomes essential to the correct interpretation of test results.

The emerging success of direct numerical simulation (DNS) experiments has created another need to quantify Reynolds number effects for TBLs. These simulations integrate and obtain solutions to the full Navier-Stokes equation at each time step in the flow history. DNS results have provided unique insights into the characteristics of the time-evolving flow structures. However, the computations are so expensive in terms of computer memory and solution time that only low Reynolds number cases have been analyzed. Figure 1.4 compares Spalart's (1988) DNS results $\left(\operatorname{Re}_{\theta}=1410\right)$ to the 2-D approach boundary layer of Ölçmen and Simpson (1995) $\left(\operatorname{Re}_{\theta}=5700\right)$. The differences between these results are significant, but are they due to Reynolds number effects, experimental error, or computational error? A clear understanding of all issues concerning this type of comparison is needed to come to the correct conclusion. These computational "experiments" have provided a wealth of information, but the application of DNS results to practical engineering flows depends on understanding Reynolds number effects. 
Studies of "coherent motions" or flow structures in turbulent boundary layers have contributed to the understanding of the complex turbulence production process. A typical study of coherent motions in a boundary layer often involves flow-visualizations at low Reynolds numbers, usually below 2000. However, the geometry of the coherent structures has been observed to change significantly with increasing $\operatorname{Re}_{\theta}$. These structures, typically loosely wound vortical structures at low Reynolds numbers, become smaller, more contorted, and more tightly wound as $\mathrm{Re}_{\theta}$ increases (Head and Bandyopadhyay, 1981). While the basic turbulent flow mechanics can be studied at low to moderate values of $\operatorname{Re}_{\theta}$, an understanding of Reynolds number effects is crucial in order to extrapolate these results to larger scale. higher speed flows. Also, recent questions have arisen concerning scaling laws for these coherent motions, indicating that perhaps a more advanced understanding of Reynolds number behavior is required.

The changes in flow structures with increasing $\operatorname{Re}_{\theta}$ illustrates the basic fact that TBLs at larger Reynolds numbers contain a broader range of turbulent length scales. The largest scales are not strongly Reynolds number dependent, since they scale on the boundary layer thickness. The small structures that scale on viscosity continue to decrease in size with increasing $\operatorname{Re}_{\theta}$ (remember, the Reynolds number is the ratio of inertial to viscous forces in a flow). Turbulent spectra results directly show this effect, as the turbulent energy becomes distributed over a wider range of wavenumbers with increasing $\operatorname{Re}_{\theta}$ (Townsend, 1976).

\section{Why perform hydrogen-bubble flow visualizations?}

In most turbulent boundary layers, the majority of the turbulence production takes place in a thin, near-wall layer, making the study of these flows inherently difficult. Threedimensionality compounds these problems, since additional data are usually required. Information in the spanwise, or $z$ direction, is needed to fully describe 3-D TBLs. Multiple sensor techniques have proven useful for some spanwise measurements (Gupta et al., 1971, and $\mathrm{Ha}$ and Simpson, 1993a,b). However, using multiple sensors in the near-wall region of a turbulent boundary layer is usually very difficult, since the region of interest can be less than $0.2 \mathrm{~mm}$ thick at moderate Reynolds numbers.

Using the hydrogen-bubble technique in a water tunnel usually solves both these problems. The near-wall region is now much thicker, up to about $2 \mathrm{~mm}$ at $y^{+}=15$, depending on flow speed. The use of hydrogen-bubble flow visualization is also a relatively simple way of obtaining information in the spanwise direction. The usefulness of this technique has been time proven over of a period of more than 30 years. As we will see later, getting quantitative results was the most difficult part of using the hydrogen-bubble technique. 


\section{Research Goals}

All basic research boils down to observing nature and gathering facts about the world in which we live. The author's basic research program was no different. A 3-D turbulent boundary layer was investigated for the effects of Reynolds number and 3-dimensionality primarily to see what was there. But the author was also truly interested in obtaining results that would advance the current understanding of basic turbulent flow physics. In addition to examining the behavior of the flow with increasing $\operatorname{Re}_{\theta}$, turbulence modeling parameters were examined for the $3-\mathrm{D}$ cases and compared to $2-\mathrm{D}$ results when possible. Another research goal was to quantify the changes caused by a mean spanwise flow on the near-wall streak structure. The logical ultimate goal of this work would be to improve the turbulence modeling and prediction of 3-D flows.

\subsection{Review of Wall-Bounded Turbulent Flow Research}

The existing body of experimental results can be broadly classified into two groups. The first group examined boundary layers by gathering results at discrete flow locations and analyzing the data statistically, usually by applying Reynolds decomposition (i.e., the data are decomposed into mean and fluctuating quantities). The second group examined what are called "coherent motions" of these flows. Robinson (1991a) defines a coherent motion as: a three-dimensional region of the flow over which at least one fundamental flow variable (velocity component, density, temperature, etc.) exhibits significant correlation with itself or with another variable over a range of space and/or time that is significantly larger than the smallest local scales of the flow. Flow-visualization techniques are generally used to study the coherent motions or structures in a turbulent flow. Coherent motions can be studied using pointwise measurements and "conditional sampling." The latter techniques usually involve some type of triggering event to signal the passage of a coherent structure. The following sections discuss both types of experiments for 2-D and 3-D boundary layers.

\subsubsection{2-D Turbulent Boundary Layers}

Research on the basic wall-bounded 2-D turbulent boundary layer has been extensive, to say the least. The simplest form of this flow is sometimes called a "canonical" boundary layer. This term refers to a fully turbulent flow over a smooth, flat, stationary wall with: zero pressure gradient, 2-D steady mean flow, no freestream turbulence, constant density, single-phase, Newtonian fluid, and no force fields (Robinson, 1991a).

A considerable body of research has documented the effects of Reynolds number on the "canonical" boundary layer. These experiments have typically covered $\mathrm{Re}_{\theta}$ ranges from 
approximately 300 to 10,000 . Among these studies are Purtell et al. (1981) (700< $\operatorname{Re}_{\theta}$ $<4090)$ and Ching et al. (1994) $\left(400<\operatorname{Re}_{\theta}<1316\right)$. One interesting study examined the inner region of the atmospheric surface layer over the Great Salt Lake Desert salt flats (Klewicki and Metzger, 1996). The estimated value of $\operatorname{Re}_{\theta}$ was $4 \times 10^{6}$. These results have shown that the increasing inertial effects are "felt" far into the boundary layer (usually down to the inner portion of the log region), and that scaling on wall variables does not collapse the data over all ranges of $\operatorname{Re}_{\theta}$. Gad-el-Hak and Bandyopadhyay (1994) provide a current survey of Reynolds number effects in TBLs. No 3-dimensional results are mentioned in this survey. Gad-el-Hak and Bandyopadhyay present a long list of remarks at the end of their report. Their final remark states that Reynolds number effects cannot be extrapolated to non-canonical cases in a straightforward manner.

Many researchers have studied the near-wall region of 2-D TBLs using flowvisualization techniques, primarily in water (Schraub et al., 1964; Kim et al., 1968; Oldaker and Tiederman, 1977; Praturi and Brodkey, 1978; Nakagawa and Nezu, 1981; Smith and Metzler, 1983, and others). These investigations of the coherent motions in 2-D boundary lavers have found that the near-wall region of turbulent boundary layers $\left(y^{+}<30\right)$ is dominated by streamwise, low-speed streaky structures, and their counterparts, high-speed "sweeps." Sample near-wall flow-visualization results from the author's test cases are shown in Fig. 2.36. Van Dyke's (1982) superb volume of flow-visualization results contains many pictures which document the near-wall and outer-region structures of turbulent boundary lavers.

Runstadler et al. (1963) were the first of many researchers to quantify these streaks. Since then, a "universal" streak spacing of $\overline{\lambda^{+}}=100$ has been generally accepted. Figure 1.5 presents some early results for the streak spacing variation with flow speed and streamwise pressure gradient. The scatter shown in this figure is typical, but the results are usually concentrated near $\overline{\lambda^{+}}=100$. Smith and Metzler (1983) present a survey of the streak spacing results available at that time. The vast majority of the data for $\overline{\lambda^{+}}$was between 90 and 110 .

Other researchers, such as Gupta et al.(1971) and Nakagawa and Nezu (1981), have used multiple sensor techniques to examine characteristics of the near-wall flow structures. These types of measurements do not rely on flow-visualization techniques and can be performed at larger Reynolds numbers (Gupta et al. went up to $\operatorname{Re}_{\theta}=6500$ ). Additional details about the turbulence can be obtained using these techniques, but the streak spacing results appear to have more scatter compared to the flow-visualization results. However, the average spanwise streak spacing results usually match those obtained using flow-visualization. 
These low-speed streaks have generated so much interest because they appear to be involved in the turbulence production through a process referred to by Kim et al. (1971) as "bursting." This process occurs when the low speed streaks become unstable and begin to oscillate and rise away from the wall. Soon after this, the coherence of the streak structure is lost (the streak "bursts") because of its interaction with surrounding higher-speed fluid. Because of this relationship to the production of turbulence, these streaks have been thought to be a reflection of near-wall flow physics, and would therefore yield information about skin friction and heat transfer at the wall (Oldaker and Tiedermann, 1977).

More recent studies of 2-D turbulent boundary layer flow structures were conducted using direct numerical simulation (DNS) techniques (Robinson, 1990; Robinson et al., 1991; Spalart, 1988; Jiménez and Moin, 1991; Bernard et al., 1993, among others). These studies have been very useful, even groundbreaking, by providing insight into the type of structures which exist in 2-D turbulent boundary layers at low Reynolds numbers $\left(300<\operatorname{Re}_{\theta}<1410\right)$. The results of these simulations have agreed remarkably well with existing experimental results, and in some regards these results are considered more accurate (see section 3.2.2 for a brief discussion on this topic). While most of this work has concentrated on low $\operatorname{Re}_{\theta}$ 2-D TBLs, some recent DNS studies have examined 3-D flows as well (Senstad and Moin, 1992).

Robinson (1991a) determined that the near-wall low-speed streaks were closely related to near-wall longitudinal, or "quasi-streamwise" vortices. Figure 1.6 displays sketches of these structures and their observed interactions. The regenerative or cause-and-effect relationship between the low-speed streaks is apparent in these sketches. The near-wall vortices appear to have been formed from the trailing legs of expanding arch or horseshoe shaped structures. These near-wall vortices pump low momentum fluid away from the wall, creating the new low-speed streaks, which in turn create the new "baby" arches. Note the use of Wallace et al.'s (1972) and Willmarth and Lu's (1972) 2-D quadrant nomenclature (Fig. 1.9) by Robinson to describe the shear stresses generated by these coherent structures.

A very interesting video was created from the results of this DNS experiment (Robinson et al., 1991). The DNS results were visualized using a series of time-history animations of the flow structures. Low pressure contour surfaces were used to visualize the arches and quasi-streamwise vortices in space, while other results were presented using a series of planar slices through the flow's control volume. Figure 1.7 displays two sample images from this video. These images reveal how complex the flow structures are for even a very basic wall-bounded turbulent flow. 


\subsubsection{3-D Turbulent Boundary Layers}

The presence of a non-zero mean spanwise flow in a boundary layer can dramatically change its turbulence characteristics. An infinite variety of 3-D TBLs are possible by simply changing the spanwise flow distribution. Figure 1.8 illustrates two distinctly different types of crossflow distributions, the uni-directional and the bi-directional, or "S" shaped profile. The latter profile shape creates a rather complex boundary layer, and is usually produced by 3 -D flows with a transverse pressure gradients which changes direction as the flow moves downstream.

The results from 3-D turbulent boundary layer studies pale in comparison to the large body of 2-D turbulence research. The author found very little information regarding Reynolds number effects for 3-D TBLs. As mentioned in section 1.2.1, Gad-el-Hak and Bandyopadhyay's (1994) rather comprehensive survey of Reynolds number effects on wallbounded turbulent flows did not discuss any results from 3-D flows. Eaton (1995) briefly discusses a few observed effects of Reynolds number for 3-D flows (he does not reference the source of these observations). He states that the general consensus is that the nearwall vortices scale on viscous length scales, so that increasing the Reynolds number would reduce their size. Also, the mean crossflow velocity profiles seemed to scale on outer-layer variables. If these observations were accurate, then at large Reynolds numbers, the vortices would be quite small compared to the region of rapid skewing near the wall. Eaton then hypothesizes that the near wall vortices would be so small that the spanwise flow would not distort them, and that the structural changes would be negligible. Unfortunately no experimental data have been taken to test his hypothesis.

However, some recent experiments have begun to shed some light on the effects of 3-dimensionality on the near-wall flow structures for 3-D TBLs. Experimental hot-wire and hot-film results in a spatially developing 3-D TBL have shown that the boundary layer length scales are reduced as the flow becomes more 3-D, and that the mean motion of the coherent structures is skewed in a direction between the local turbulent shear stress and local mean velocity vectors (Ha and Simpson, 1993). Chiang and Eaton (1993), and Flack and Johnston (1993) examined the quasi-streamwise vortex interactions and associated ejection behavior for 3-D TBLs using hydrogen bubbles and dye injection. They found that the transverse flow appeared to allow for different types of ejection events and multiple vortex interactions that were not present in 2-D turbulent boundary layers. Johnston and Flack (1996) present a review of 3-D experimental results and study several different data sets. These researchers examined how $a_{1}$ changes with flow skewing and $\operatorname{Re}_{\theta}$. A modest reduction in $a_{1}$ appeared to exist with increasing Reynolds number. The consensus from 
the (somewhat slow) growing body of 3-D results is that it is the influence of the near-wall spanwise flow or pressure gradient that inhibits the turbulence production mechanisms in a 3-D TBL.

Figure 1.9 compares the probability distributions of instantaneous velocity fluctuations for a 2-D and 3-D boundary layer. For the 2-D layer, the quandrant analysis concept proposed by both Wallace et al. (1972) and Willmarth and Lu (1972) has proven very useful. Robinson (1991a) used this type of analysis extensively. An extension of this idea has been put forth by Simpson and Devenport (1990) to analyze 3-D fluctuations, and was referred to as an "octant" analysis (Fig. 1.9b). For the 2-D case, the $w^{\prime}$ fluctuations are assumed symmetric, and are ignored. For the 3-D case, the flow is no longer symmetric, and the probability distribution is allowed to become skewed by considering the $w^{\prime}$ fluctuations. These ideas may turn out to be quite useful, because if the fluctuation probabiltiy distributions could be modeled, all Reynolds stress tensor terms could be calculated directly.

Figure 1.10 diagrams a conceptual model for a typical sweep/ejection process in a 2-D TBL. A wallward "sweep" of high momentum fluid displaces near-wall low momentum fluid, either to one side or in front of the sweep fluid. Because of continuity requirements in the mean 2-D flow, this near wall fluid is displaced away from the wall and forms an ejection. Both ejections and sweeps have positive contributions to the Reynolds shear stress, and are sometimes referred to as "active" or stress-producing motions.

Simpson and Devenport (1990) proposed a new sweep-ejection behavior for a case in which a spanwise pressure gradient exists (Fig. 1.11). As a sweep approaches the wall, its direction is more parallel to the freestream direction than the near wall fluid. Since $u^{\prime}>0$ and $v^{\prime}<0$ for this sweep, it contributes to a positive Reynolds shear stress. The slow near-wall fluid is moved more in the spanwise direction by the non-zero transverse pressure gradient than the higher velocity incoming fluid. More of the displaced near wall fluid will move in the direction of lower spanwise pressure than in the direction of higher spanwise pressure. As seen in Fig. 1.11, the displaced near-wall fluid need not be ejected away from the wall, as in the 2-D case. Since the mean flow now has a "third degree of spatial freedom," the average near-wall spanwise displacement fluctuations are no longer constrained to zero. Therefore, not as much displaced fluid is ejected, and there will be a lower contribution of ejections to the streamwise Reynolds shear stress $-\overline{u v}$. The spanwise shear stress $-\overline{v w}$ would be largely due to ejections, since the incoming sweep typically has a much lower spanwise velocity component.

3-D DNS results from Senstad and Moin (1992) revealed changes in the turbulent sweep-ejection process that are in qualitative agreement with this postulated turbulence 
production mechanism. They report that the shear stress producing fluid motions between the near-wall and outer regions was decreased by the near-wall mean crossflow and resulting changes in fluid particle trajectories. These changes in particle trajectories are shown in Fig. 1.12 .

It is the author's opinion that using conceptual models or physical descriptions of the turbulent flow mechanisms offer the most promise for the development of accurate turbulence models for 3-D flows.

\subsection{Summary of Current Research Program}

The current experimental work examines the near-wall spanwise flow structure of a spatially developing, pressure-driven 3-D TBL, and compares it to a 2-D TBL. Reynolds number effects for a 3-D TBL were investigated by comparing to a higher Reynolds number boundary laver with the same flow geometry (Ölçmen and Simpson, 1995). The current research was performed using a low speed, low Reynolds number water tunnel facility $\left(U_{e}<30 \mathrm{~cm} / \mathrm{s}, R_{\theta}<900\right)$. Laser Doppler velocimetry (LDV) and hydrogen-bubble flow visualization were used to investigate the test flows.

The 3-D flow geometry was created using a wing-body junction flow. This geometry was unique because of its extensive use by other researchers at a variety of approach flow conditions (Ölçmen and Simpson, 1990, 1995; Ha and Simpson, 1993a, b; Fleming et al. 1991, 1993; and Kim, 1991). Figure 1.13 shows surface oil flow results from Ölçmen and Simpson (1995). Ölçmen's LDV measurement stations 0-7 are shown on the right hand side of the appendage. Kim (1991) performed hydrogen-bubble flow visualization in the nose region of this wing-body junction flow. These tests were performed in the same water tunnel facility used by the author for this work. Figure 1.14 displays a sample image from Kim's work.

\subsubsection{LDV Measurements}

A two-component LDV system was used to take boundary layer profile data for the 2-D TBL and the 3-D TBL at Ölçmen's station number 5. Table 1.1 lists the test matrix used to design the LDV measurement phase of the author's work. Note that the Reynolds numbers listed in this table were a priori estimates. Results from the LDV measurements were used to calculate the actual test values of $\operatorname{Re}_{\theta}$ (Table 3.1). The LDV data were used in support of the hydrogen-bubble flow visualization work, and also to provide a more detailed look at these low Reynolds number boundary layers. The LDV boundary layer profile results include mean velocities and the complete Reynolds stress tensor measured 
well into the boundary layer's laminar sublayer $\left(y^{+}<7\right)$. Accurate skin friction results could be obtained with this very near-wall data. Spectra and autocorrelation results were also obtained at various locations throughout the boundary layer and in the freestream (Table 1.1). All LDV results are presented in chapter 3.

\subsubsection{Hydrogen-Bubble Flow Visualizations}

To visualize and study the differences between 2-D and 3-D TBL near wall structures, a wire producing hydrogen bubbles was held parallel to the floor of the tunnel (in the $x z$ plane) and electronically pulsed to produce "time lines." Table 1.2 lists the test matrix for the flow-visualization phase of the author's research. Note that several different wire skew angles were used for the 3-D flow visualizations.

The hydrogen-bubble flow-visualization results were videotaped and studied to obtain qualitative results. Individual frames and image sequences of the video were studied using a PC-based image digitizing/processing system to obtain quantitative results. Instantaneous $U(z)$ distributions were obtained from the time line images, and the 2-D and 3-D lowspeed streak spacing could be statistically analyzed. New streak (and sweep) parameters are discussed and presented in chapter 4 . 
The author's experimental work was performed in the Virginia Tech Aerospace and Ocean Engineering Department's $2 \mathrm{ft} \times 2 \mathrm{ft}$ water tunnel. This tunnel was initially used during the summer of 1987 for researching the internal flow characteristics of the space shuttle solid rocket boosters (Waesche et al., 1989). It has also been used in the research of turbulent jets (Hsu, 1989) and wing/body junction flows (Kim, 1991).

The author's experimental work proceeded in two phases: the first was near-wall hydrogen-bubble flow visualization, and the second was LDV boundary layer measurements. The LDV measurements will be discussed first in chapter 3 , since these results enhance the interpretation of the flow-visualization results (chapter 4). The LDV apparatus and techniques are also discussed prior to the flow-visualization techniques. The ardent reader will note that describing both techniques has made for a lengthy chapter.

\subsection{Description of Test Facilities and Configurations}

\subsubsection{Water Tunnel Description}

The Virginia Tech Aerospace and Ocean Engineering water tunnel is a horizontal, closed loop design, as shown in Fig. 2.1. This tunnel was designed primarily for flow visualization experiments, and it features a large glass test section with upstream and downstream viewing windows. The water is circulated through $0.457 \mathrm{~m}$ diameter PVC pipe, except for the settling chamber and test sections, which are $0.71 \mathrm{~m}$ square and $0.61 \mathrm{~m}$ square, respectively. The test section is $2.44 \mathrm{~m}$ long, and the settling chamber is $1.54 \mathrm{~m}$ in length. The tunnel's centerline follows a rectangular path roughly $4.8 \times 7.3 \mathrm{~m}$, and holds approximately 3800 liters (1000 gallons) of water. The tunnel is supported and leveled $1.32 \mathrm{~m}$ above the laboratory floor using an aluminum channel frame. Plywood supports attached to the framework are used to cradle the PVC pipe sections.

The flow is driven by an Ingersoll-Rand $0.46 \mathrm{~m}$ diameter axial pump. The pump is powered by a 3 phase, $10 \mathrm{hp}$ induction motor, controlled by a Toshiba TOSVERT-130 $\mathrm{H} / \mathrm{H} 1$ low acoustic-noise transitorized inverter. The pump speed is reduced by a 6 to 1 belt 
drive assembly. A section of honeycomb (see following description of settling chamber) was installed at the suction side of the pump. This helps to isolate the test section from the pump (Hsu, 1989). Test section flow speeds up to $0.5 \mathrm{~m} / \mathrm{s}$ are possible using this water tunnel.

The settling chamber/test section components of the water tunnel are actually channels, so the test flow has a free surface. These sections were constructed of $1.2 \mathrm{~cm}$ plexiglas (settling chamber) and $1.2 \mathrm{~cm}$ plate glass (test section) held together by an aluminum frame and bulkheads. The settling chamber contains 2 honeycomb sections, 6 turbulence screens, and a 1.6:1 ratio contraction. The $7.62 \mathrm{~cm}$ thick honeycomb sections were coated with water-proof epoxy for longevity. The six-sided cells measure approximately $0.5 \mathrm{~cm}$ from side to side. The screens were constructed of a progressively finer mesh as the flow nears the contraction. The honeycomb and screen frames, as well as the contraction, were made of plexiglas. A $5 \mathrm{~mm}$ square boundary layer trip was positioned $5.7 \mathrm{~cm}$ prior to the contraction exit, where the remaining contraction ratio is approximately 1.1:1.

In the test section, a false floor and flexible side walls created the desired geometry for the different test cases. Figure 2.2 shows the $5.6 \mathrm{~mm}$ thick plexiglas false floor in place. Figures 2.13 and 2.14 show the different configurations for the plexiglas side walls, which are $2.4 \mathrm{~mm}$ thick. Threaded rods below the false floor and above the test section were used to position the flexible side walls. The positioning of these walls is discussed in section 2.1.6.

\subsubsection{Determining Test Fluid Properties}

For the hydrogen-bubble flow visualizations, the test fluid contained several additives: sodium sulfate $\left(\mathrm{Na}_{2} \mathrm{SO}_{4}\right)$, Kodak Photo-Flo 600 solution (concentrated), and normal household bleach. Approximately 8 liters of bleach $(\approx 5 \%$ sodium hypochlorite) was added to prevent algae growth (total test fluid bleach concentration $\approx 0.2 \%$ ). The Kodak Photo-Flo solution was used to eliminate small, extraneous air bubbles. The active ingredient in this solution was ethylene glycol. Since the solution was concentrated, very little was actually used ( $\approx 1$ liter, total test fluid concentration $\approx 0.026 \%$ ). The sodium sulfate was used as a catalyst for the creation of the hydrogen bubbles. Approximately 500 grams of granular sodium sulfate was added (total test fluid concentration $\approx 0.013 \%$ by weight).

Because of these chemical additives, a Brookfield Digital Viscometer (model LVTDVII) was used to determine the molecular viscosity, $\mu$. This device measured viscosity using concentric rotating cylinders. The manufacturer's specified accuracy was \pm 0.1 centipoise for the selected scale of 0 to $10 \mathrm{cps}$. Viscosity measurements were made at two different 
water temperatures, $17^{\circ} \mathrm{C}$ and $20^{\circ} \mathrm{C}$. At $17^{\circ} \mathrm{C}, 5$ realizations yielded an average $\mu$ of 1.046 centipoise, with a standard deviation of $0.008 \mathrm{cps}$ ( $95 \%$ confidence interval, or $\pm 2 \sigma=$ $\pm 0.016 \mathrm{cps}$ ). For $20^{\circ} \mathrm{C}, 6$ realizations yielded an average $\mu$ of $0.993 \mathrm{cps}$, with a standard deviation of $0.032 \mathrm{cps}( \pm 2 \sigma= \pm 0.064 \mathrm{cps})$. Since both uncertainty levels due to random effects were less than the total accuracy of the viscometer, the manufacturer's specified accuracy was used as the uncertainty for the author's viscosity measurements (see Fig. 2.4).

The difference in water temperatures was due to changing ambient laboratory temperatures. which flucuated with average outdoor temperatures. The $17^{\circ} \mathrm{C}$ temperature was measured during February, when the 2-D flow-visualizations were performed. The $20^{\circ} \mathrm{C}$ temperature was measured during April, when the 3-D work was done.

For the LDV measurements, the flow additives were seeding particles and bleach. The low concentrations of both of these additives were considered negligible, and $\mu$ for fresh water was used for the LDV work. The author performed the 2-D and 3-D LDV measurements from late February to early March, and the ambient laboratory temperature averaged out to $\approx 18^{\circ} \mathrm{C}$. At this temperature, $\mu=1.054 \mathrm{cps}$ for fresh water.

Table 2.1 displays the final results of the molecular and kinematic viscosities for the different experimental stages. Data from Fogiel (1983) was used as the source for determining the variation in water density at different temperatures. Note that no uncertainty was specified for the LDV test flow (fresh water) viscosity, but a review of several different sources indicate that a small uncertainty of $\approx \pm 0.02 \mathrm{cps}$ would not be inappropriate. The fresh water data presented in Fig. 2.4 was taken from Brown and Marco (1958) and the seawater data from Touloukian and Ho (1975). The test flow kinematic viscosity agreed closely with fresh water data, and also with Kim's data ( $\nu=0.0104$, from Kim, 1991). This latter result is particularly significant since this researcher used similiar additives to produce hydrogen bubbles in water.

\subsubsection{Determining Freestream Flow Velocity}

At low flow speeds, such as the speeds used for the current experiments, it becomes difficult to easily obtain low uncertainty readings for the freestream flow velocity, $U_{e}$. A Marsh-McBirney electromagnetic flow meter was used to set the nominal flow speeds. The flow meter uncertainty, as specified by the manufacturer, was $\pm 2 \%$ of the actual reading.

To check the accuracy of the electromagnetic flow meter, 2 tests were performed. The first involved timing styrofoam particles on the surface of the water over a distance of $\approx 1$ $\mathrm{m}$ through the test section. The second test used a specially constructed hydrogen bubble 
probe. Shown in Fig. 2.5, this device provided a convenient way to check the inviscid core flow speed. The results of these tests satisfied the author that the electromagnetic water current meter was working satisfactorily.

When making the 2-D LDV measurements, $U_{e}$ could be measured directly and compared to the electromagnetic flow meter reading. For the more complex 3-D LDV measurements, this was not true, and it became critical to keep the tunnel speed constant between different profile measurements (see section 3.1.1 for more details). In this case, the electromagnetic flow meter was used for test flow speed repeatability, not to set a particular flow speed. For the 3-D case, $U_{e}$ was measured directly at the 3-D location, and $U_{r e f}$ was estimated by using the potential flow calculation results (see sections 2.1.6 and 3.1.1).

For the hydrogen bubble flow visualizations, the electromagnetic flow meter was used to set and monitor the flow speed. No other indicators of the test flow velocity were used.

\subsubsection{Potential Core and Approach Boundary Layer Documentation}

The approach boundary layer and "potential core" (or freestream) in the water tunnel test section were verified using the 2-D LDV boundary layer profiles and special long time records made at $y=14 \mathrm{~cm}$. These long time records contained up to 30 minutes of $u(t)$ data.

The turbulence intensity and other freestream results are listed in Table 2.2. The turbulence intensities ranged from $1.6 \%$ to $1.9 \%$, which are reasonable levels considering the low speeds used in this facility. However, when evaluating the overall quality of the freestream flow, the turbulence length scales are also important. Hancock and Bradshaw (1983) presented a freestream turbulence parameter (FSTP) which they defined as

$$
F S T P_{L_{e}^{u}}=\frac{\left(\frac{u^{\prime}}{U_{e}}\right) \times 100}{\left(\frac{L_{e}^{u}}{\delta_{0.995}}+2.0\right)}
$$

The length scale $L_{e}^{u}$ is a dissipation length parameter, and was also defined by Hancock and Bradshaw (1983) as

$$
U_{e} \frac{d\left(u^{\prime 2}\right)_{e}}{d x}=\frac{-\left(u^{\prime 2}\right)_{e}^{3 / 2}}{L_{e}^{u}}
$$

Since no streamwise turbulence data were available for the current study, the autocorrelation integral time scale was used to form an integral length scale (equations 3.12 and 3.13 , repeated here):

$$
T_{\Lambda}=\int_{0}^{\infty} R_{u u}\left(\tau_{R u u}\right) d \tau_{R u u}
$$




$$
L_{\Lambda}=U T_{\Lambda}
$$

See section 3.3 for a description of the autocorrelation function $R_{u u}\left(\tau_{R u u}\right)$. The freestream results for $R_{u u}$ are shown in Fig. 2.6.

Results from several researchers have shown that the ratio of $L_{e}^{u} / L_{\Lambda}$ varies from 1.1 to 2.6 (Thole and Bogard, 1996). To arrive at a conservative value of FSTP, the author used a straight substitution of $L_{\Lambda}$ into eqn. 2.1. The results for $F S T P_{L_{\Lambda}}$ are listed in Table 2.2. $T_{\lambda}$ and $L_{\lambda}$ are time and length "microscales" and are related to the radius of curvature of $R_{u u}$ at $\tau_{R u u}=0$ (see section 3.4 ). Both length scales generally decrease with increasing $U_{r e f}$. The microscale information is listed in Table 2.2 primarily for comparison with the data presented in section 3.3 and Fig. 3.53. Note that the ratio $L_{\lambda} / L_{\Lambda}$ is closer to unity for the freestream data compared to the boundary layer data, revealing more uniformly sized disturbances for the freestream flow.

Hancock and Bradshaw (1983) correlated changes in skin friction and shape parameters with FSTP. For the current range of $F S T P_{L_{\Lambda}}$, the changes in $C_{f}$ and $H$ should be less than $5 \%$ and $2.5 \%$, respectively. Castro's (1984) results indicate that at low Reynolds numbers $\left(\operatorname{Re}_{\theta}<1000\right)$, the effects on $C_{f}$ and $H$ from freestream turbulence may be further suppressed, but no clear trend was found from that data set. The values of $C_{f}$ and $H$ listed in Table 2.2 compare very favorably with data from Purtell et al. (1981).

Hoffman and Mohammadi (1991) examined the decrease in Coles' wake parameter, $\Pi$, with increasing FSTP. $\Pi$ can be found from the relation (simplified from eqn. 3.1)

$$
\frac{U_{e}}{u_{\tau}}=\frac{1}{\kappa} \ln \frac{\delta u_{\tau}}{\nu}+C+\frac{2 \Pi}{\kappa}
$$

where $\kappa=0.40$ and $C=5.1$ (Coles (1956) constants). The current $2-D$ values for $\Pi$ are listed in Table 2.2. Note that for low freestream turbulence levels, $\Pi \approx 0.50$ to 0.55 . Coles (1956) recommended using $\Pi=0.55$. While the author's data do not show a uniform decrease in $\Pi$ with increasing $F S T P_{L_{\Lambda}}$, published results show large scatter for this correlation (Hoffman and Mohammadi, 1991, and Thole and Bogard, 1996). The author's data fell within the range shown for the published results.

Figure 2.7 displays the current freestream spectral results. These spectra were normalized using $L_{\Lambda}$ and $U_{\text {freestream }}$ in the following manner:

$$
\begin{aligned}
k_{1} L_{\Lambda} & =\frac{2 \pi f}{U_{\text {freestream }}} L_{\Lambda} \\
\phi_{u u}\left(k_{1} L_{\Lambda}\right) & =\frac{U_{\text {freestream }}}{2 \pi L_{\Lambda}} u^{\prime 2} F(f)
\end{aligned}
$$


The plot of the first moment of the spectra (Fig. 2.7b) shows the relative contributions to the total energy for the different wavenumbers. The largest energy contributions will always be in the region where $F(f) \propto\left(k_{1}\right)^{-1}$, also known as the -1 region. This region is centered about $k_{1} L_{\Lambda}=1$ for the current freestream results. $k_{1} L_{\Lambda}=1$ corresponds to dimensional frequencies between 0.5 and $2 \mathrm{~Hz}$ for the different flow speeds. The energy contribution peak is sharper than results obtained for $y<14 \mathrm{~cm}$ (see section 3.4), indicating that a relatively narrow range of frequencies is responsible for the elevated freestream turbulence levels.

At larger wavenumbers, the spectral results appear to obey Kolmogorov's $k_{1}^{-5 / 3}$ law, at least for the 20 and $30 \mathrm{~cm} / \mathrm{s}$ cases. Past this $k_{1}^{-5 / 3}$ region, the spectral data do not continue to fall off as expected. This is normal for spectra calculated using non-continuous data, such as LDV data (Adrian and Yao, 1987). Refer to section 3.4 for a detailed discussion of the current spectral results for the current experimental boundary layers.

The spectral results seem to suggest that a relatively low frequency, "surging" type of unsteadiness is the main contributor to the freestream turbulence. This type of unsteadiness was considered an "inactive" (and irrotational) motion, which does not significantly affect the boundary layer (Bradshaw, 1967). However, the 2-D boundary layer profile data, shown in Fig. 2.8. show what appears to be some type of flow anomaly from approximately $y=$ $3 \mathrm{~cm}$ to over $10 \mathrm{~cm}$. The data shown in Fig. 2.8 were plotted with $y$ on a linear scale to emphasize the transition from the outer region of the boundary layer to the "freestream" (which was measured at $y=14 \mathrm{~cm}$ ). Chapter 3 contains a more traditional presentation of the 2-D boundary layer data (Figs. 3.6, 3.7, 3.13, and 3.20).

The plot of $U / U_{e}$ (Fig. 2.8a) shows a wake-like defect region. Figure $2.8 \mathrm{~b}$ reveals that this region was associated with elevated $V / U_{e}$ values. There was no obstruction upstream of the test section which would cause a conventional wake, such as a strut or rod. From $U_{\text {ref }}=10 \mathrm{~cm} / \mathrm{s}$ to $U_{\text {ref }}=15 \mathrm{~cm} / \mathrm{s}$, it appears that the flow underwent a major change in structure from a sub-critical to critical flow state. The sub-critical state was characterized by a much thicker boundary layer and large $V / U_{e}$ values at the the edge of the boundary layer. This structural change was likely associated with some discrete flow anomaly or Reynolds number dependent stall occurring in the settling chamber/contraction section. Other characteristics of the outer region flow structure are elevated normal and shear stress levels (Figs. 2.8c and 2.8d). Note that while the normal stresses may not go to zero at $y=\delta$, the shear stress levels generally should for turbulent boundary layers (Ölçmen and Simpson, 1995).

The distribution of $V$ for the $U_{\text {ref }}=10 \mathrm{~cm} / \mathrm{s}$ case suggests that a weak longitudinal vortex $\left(\omega_{x}>0\right)$ existed for this 2-D flow case (Fig. 2.10). This vortex could have been 
created by a separation off a sharp edge upstream of the contraction section or a stalled portion of the test flow. Based on Fig. 2.8b, this vortex would have had to grow weaker for the higher Reynolds number cases, which suggests that this problem was associated with some type of Reynolds number dependent stall.

Consideration of the author's current data for the 3-D flow case seems to support this hypothesized description of the outer flow structure. The 3-D data did not display any flow abnormalities or anomalies outside of the boundary layer (see Figs. 3.5, 3.15, and 3.21). This suggests that a streamwise vortex existed and that the 3-D flow's non-zero streamwise pressure gradient eliminated the vortex, or that the vortex passed on the other side (port side, $z>0$ ) of the appendage.

LDV $V$ measurement volume misalignment could have easily contributed to this elevated freestream values of $V$. If the beams were skewed into the streamwise direction by even $1^{\circ}$, the $V$ readings would be contaminated by $2 \%$ of $U$. Even if the LDV optics were properly aligned, a non-zero pitch angle for the freestream flow would have the same affect.

Regardless of what caused the outer region flow structure for the 2-D flow case, the structure itself does not appear to adversely affect the near-wall portion of the boundary layer. As discussed in chapter 3, the data agree well with previously published low Reynolds number data. The shear stress correlation results shown in Fig. 2.9 are a good indicator of a "nominal" boundary layer. Maximum shear stress correlation values of approximately 0.45 are typical for a 2-D TBL. The author's peak $-\overline{u v} /\left(u^{\prime}\right)\left(v^{\prime}\right)$ results are between 0.35 and 0.4, which agrees with data presented by Thole and Bogard (1996) for FSTP $=1.0$. The reduction in maximum correlation levels (slightly below 0.4 ) are because of elevated freestream turbulence levels, and are not the result of some flow anomaly occurring between the boundary layer edge and the freestream potential core.

Finally, it is worth briefly discussing secondary flows which occur in open and closed channels. These secondary flow structures are well documented (Nezu and Nakagawa, 1993, Sellin, 1970, and many others), and are caused by the flow's mean shear stress distribution (Prandtl's secondary flow of the "second kind," see Gessner, 1973). The water tunnel test section was configured as an open channel for the author's testing. Figure 2.11 shows typical mean flow contours and secondary flow patterns for a well-developed open channel flow (2:1 aspect ratio). The current channel test flow, however, could not be considered well-developed $\left(x / h_{\text {channel }} \approx 2\right)$. Additionally, the flow pattern shown in Fig. 2.11 would result in negative $V$ velocities near the center of the channel, not positive. Therefore, it is highly unlikely that any shear-stress-driven secondary flow typical for open channel flows is the cause for the observed outer region flow structure. 


\subsubsection{Model Description}

The wing-body junction geometry, used to create the 3-D turbulent boundary layer, consisted of a streamlined appendage mounted perpendicular to the test section false floor. The wing leading edge was mounted $1.19 \mathrm{~m}$ downstream of the contraction exit. The wing leading edge location also determined the $x=0, y=0, z=0$ position for the tunnel coordinate system (Fig. 2.3).

The wing's geometry was created by joining a 3:2 elliptic nose to a NACA 0020 tail section at their respective maximum thickness locations (Fig. 2.12). This formed a relatively blunt appendage with a maximum thickness to chord ratio of $T / C=0.235$. As mentioned in section 1.2, the flow around this geometry is documented over a range of approach boundary layer Reynolds numbers (Devenport and Simpson, 1990, Ölçmen and Simpson, 1990, Fleming et al., 1993, Kim, 1991, among many others).

The appendage model used by the author was the same model as used by Kim (1991). It was constructed of $3 \mathrm{~mm}$ plexiglas sheet for the wing skin and $17 \mathrm{~mm}$ plexiglas ribs. The appendage chord length $C=61.0 \mathrm{~cm}$, thickness $T=14.4 \mathrm{~cm}$, and height $=58.4$ $\mathrm{cm}$. Since the model thickness was $24 \%$ of the test section width, the false side walls were used to approximate streamlines in order to minimize blockage effects. The 3 -D side wall configuration is discussed in section 2.1.6.

Figure 1.13 shows an oil flow around the nose of this geometry in a wind tunnel at $\operatorname{Re}_{\theta}=6700$. Figure 1.14 is a frame from a hydrogen-bubble flow-visualization video showing the horseshow junction vortex in the nose region.

\subsubsection{2-D and 3-D Test Configurations}

Two test configurations existed for the LDV measurements and the hydrogen bubble flow visualizations, a 2-D flow case, and a 3-D flow case. In each case, the test-section side walls were adjusted to relieve flow blockage (for the 3-D case) and to allow for the growing side-wall boundary layers (both 2-D and 3-D cases).

The 2-D flow configuration is shown in Fig. 2.13. Power law calculations were used to estimate the growth of the side-wall boundary layer displacement thickness. The false side walls were adjusted using these calculations in an attempt to produce a test flow with zero streamwise pressure gradient. Typical power law relations used to estimate boundary layer parameters throughout the author's experimental work are

$$
\begin{aligned}
\delta / X & \approx 0.37\left(\operatorname{Re}_{X}\right)^{-0.2} \\
\theta / X & \approx 0.036\left(\operatorname{Re}_{X}\right)^{-0.2} \\
C_{f} & \approx 0.0592\left(\operatorname{Re}_{X}\right)^{-0.2}
\end{aligned}
$$


where $X$ is measured from some virtual origin (White, 1974). The shape factor, $H$, was assumed constant $(H=1.5)$ and was used to calculate $\delta^{*}$. A nominal flow speed of $U_{r e f}$ $=15 \mathrm{~cm} / \mathrm{s}$ was used for these calculations. The final side-wall displacements are listed in Table 2.3 .

Figure 2.13 shows that the actual 2-D side-wall positions were slightly wider than the calculated positions. The total increase in width is $0.93 \mathrm{~cm}$. The estimated adverse streamwise pressure gradient created by the diverging side walls is very small $\left(\approx 0.00018 \mathrm{~cm}^{-1}\right)$. The design of the aft portion of the water tunnel test section may have also created a slight adverse pressure gradient (Fig. 2.1).

Figure 2.14 shows the actual side-wall positions for the 3 -D configuration. The positioning was determined using a potential flow solution and adding the side-wall boundary layer displacement thickness to the streamline displacements. The potential flow solver accuracy was verified using data from Abbott and Von Doenhoff (1959), and was reported by Fleming (1991). A relation for the growth of turbulent boundary layers in the presence of non-zero pressure gradients was used to estimate $\delta^{*}$ for the final 3-D configuration. This relation (from Kays, 1966), is

$$
\delta^{*}=\frac{0.048 \nu^{0.2}}{R U_{e}^{3.29}}\left(\int_{0}^{\infty} R^{5 / 4} U_{e}^{3.86} d x\right)^{0.8}
$$

Here, $R$ is the surface radius of curvature. Eqn. 2.5 was derived using a constant shape factor of $H=1.29$. This value is within $10 \%$ of the values calculated for the $3-\mathrm{D}$ cases (Table 3.1).

The actual 3-D side-wall positions closely matched the desired positions, except at the wing maximum thickness location. At this location, the side walls were at their maximum deflection, which was approximately $0.5 \mathrm{~cm}$ less than the required deflection. However, the side-wall displacement calculations are at best only approximations, and in light of this fact, the final side-wall displacements were considered satisfactory.

\subsection{Laser Doppler Velocimetry Apparatus and Setup}

To facilitate a detailed examination of boundary layer parameters and turbulence characteristics, a two-component laser Doppler velocimetry (LDV) system was used to measure simultaneous $U$ and $V$ velocity components. This section briefly introduces basic LDV operation, and then goes on to detail the author's implementation of this technique. 


\subsubsection{Basic Principles of LDV Operation}

The LDV technique relies on the interference fringe pattern created by intersecting beams of coherent light. This is a relatively modern technique, first being used by Yeh and Cummins (1964) to measure the fully developed laminar pipe flow of water. One of the main advantages of LDV is that it is a "non-intrusive" method; no flow disturbances are typically required to use this technique. With a good LDV system design, low uncertainty data can be measured very near a wall, which makes this a good technique for detailing boundary laver features and characteristics. Some of the disadvantages of using this technique are higher equipment costs, more difficulty in setup when compared to other methods, such as hot-wire anemometry, and typically slower and non-uniform data rates. To learn the many details and variations of this technique, Durst et al. (1981) is a good place to begin.

Figure 2.15 diagrams the components of a very basic LDV system. A laser light source is split into multiple beams, and transmitting optics focus these beams into a very small region, known as the "measurement volume" or "probe volume." This region is shaped like an oblong spheroid. In the probe volume, planar interference fringes are created by the intersecting coherent wave fronts of beams (Fig. 2.16). The spacing of the interference fringes is

$$
d_{f s}=\frac{\lambda}{2 \sin \left(\frac{\beta}{2}\right)}
$$

where $\lambda$ is the light wave length and $\beta$ is the beam intersection angle.

Small seeding particles, usually with diameters less than the light fringe spacing, scatter the light as they move through the probe volume. Receiving optics gather the scattered light and a photomultiplier converts this information into a voltage signal, sometimes referred to as a "Doppler burst." The frequency of the Doppler burst is directly related to the velocity of the seed particle, and can be expressed as

$$
U_{\perp}=(f)\left(d_{f s}\right)
$$

Here $U_{\perp}$ is the seed particle's velocity component perpendicular to the interference fringes, a direction which is determined by the transmitting optics' geometry.

If both laser beams have the same frequency, the interference fringe pattern is stationary. However, If one of the laser beams is frequency shifted a relatively small amount $\left(f_{s} \ll f_{\text {light }}\right)$, the interference fringes will translate, enabling the correct measurement of zero or reverse flow. Shifting the laser beam frequency can be accomplished using several different methods. For the current experimental work, commercially available acousto-optic cells (Bragg cells) were used to perform the desired beam frequency shifts. 
If the interference fringes are moving in the positive velocity direction, equation $2.7 a$ becomes

$$
U_{\perp}=\left(f-f_{s}\right)\left(d_{f s}\right)
$$

where $f_{s}$ is the beam shift frequency. This equation is the essence of the LDV technique. In the following sections, the author's implementation of this technique is detailed.

\subsubsection{Current Implementation of the LDV Technique}

Figure 2.17 shows a photograph of the current LDV system used to take detailed measurements of the test boundary layer. Figure 2.18 is a schematic of the LDV system as it was set up for the 2-D case. This is a two color system, capable of measuring two simultaneous velocity components (nominally $U$ and $V$ ). To get all velocity components for the 3-D flow case, 3 profiles were made at different beam orientations, as shown in Fig. 2.19. The optical configuration used for the $3-\mathrm{D} 0^{\circ}$ case was very similar to the $2-\mathrm{D}$ case. A mroe detailed diagram of case $\mathrm{C}\left(-45^{\circ}\right)$ for the 3-D flow is shown in Fig. 2.20. Further details of the transmitting and recieving optics are discussed in section 2.2.3.

For both 2-D and 3-D flow cases, profile measurements were performed at one $x, z$ location for varying Reynolds numbers. The 2-D $x, z$ measurement location matches that of the 2-D hydrogen-bubble flow visualizations $\left(x_{T C} \approx-4.7 \mathrm{~cm}, z_{T C} \approx-3.65 \mathrm{~cm}\right)$. The 3-D measurement location matched Ölçmen's \#5 station and the 3-D hydrogen-bubble flow visualizations $(x / C=0.0215, z / C=-0.245)$. For the 3 -D cases, boundary layer profiles were measured for nominal velocities of 10,15 , and $20 \mathrm{~cm} / \mathrm{s}$. For the 2-D case, profiles were measured for 15,20 , and $30 \mathrm{~cm} / \mathrm{s}$. The resulting boundary layer parameters for these cases are shown in Table 3.1.

The LDV system components were arranged on a 4' $\times 6^{\prime}$ optical table, which was mounted on a large milling machine base (Figs. 2.19 and 2.21). The base was moved on casters for large changes in $x$ and $z$. Once the base was stabilized on blocks, small changes in $x, y$, and $z$ were possible using the milling machine's compound traverse. The traverse was used to move the optical table from $y=0$ to over $15 \mathrm{~cm}$ above the test section's false floor. As shown in Fig. 2.21, the optical table was mounted at an angle on the milling machine base, so that the beam crossing point (the measurement volume) could be brought down to the wall with no interference with the lower $V$ component beam. For the $0^{\circ}$ cases, this angle was $5.6^{\circ}$. For the $\pm 45^{\circ}$ cases, the angle was $5.6^{\circ}$.

Figure 2.22 shows the velocity component directions for the different flow cases. For the 2-D flow case, the $U$ component measured was aligned with $x_{\mathrm{TC}}$, and $V$ was measured in a direction $5.2^{\circ}$ from the wall normal. For the 3-D flow case, 3 pairs of $U$ and $V$ velocity 
measurements were performed at each flow speed, and are referred to as cases A, B, and C. Figure 2.19 shows the laser beam orientation for each case (case A - $0^{\circ}$, case $\mathrm{B}--45^{\circ}$, case $\left.\mathrm{C}-+45^{\circ}\right)$. These 3 orientations enabled all velocity components and Reynolds stress components to be measured for the 3-D boundary layer. Note that not all orientations were used simultaneously. The standard procedure was to configure the LDV system for a given case, measure all profiles for that case over the selected range in $\operatorname{Re}_{\theta}$, and then reconfigure the LDV for the next case. The final order was: 1) all 2-D cases, 2) 3-D case $\left.\mathrm{A}\left(0^{\circ}\right), 3\right)$ 3 -D case B $\left(-45^{\circ}\right)$, and 4) 3-D case C $\left(+45^{\circ}\right)$. Section 2.2 .5 and Appendix A discuss the data reduction methods used to extract the final results from the raw data.

\subsubsection{Transmitting and Receiving Optics}

A Spectra Physics argon-ion laser (laser model 164-03, exciter model 265), with a total power $\approx 1.5 \mathrm{~W}$ was used to produce 2 blue beams $(\lambda=488.0 \mathrm{~nm})$ and 2 green beams $(\lambda=$ $514.5 \mathrm{~nm})$. The four beams used to make the measurements were nominally $100 \mathrm{~mW}$ each. The blue beam pair was used to measure velocity components nominally parallel to the floor, and the green beam pair was used to measure components nominally perpendicular to the floor. All four beams crossed at the same point, creating two overlapping measurement volumes, and simultaneous $U$ and $V$ measurements were possible. The beam pairs were spaced at $50 \mathrm{~mm}$, and TSI 10966 alignment blocks were used to align the beams parallel to each other and the optical table.

Figure 2.18 details the equipment used to transmit the laser beams. After the main beam leaves the laser, it is split into two colors using a dispersion prism (part d). These two beams are then guided into the "optics train" - a row of optical components which splits each beam to create two beam pairs, and then frequency shifts one beam from each pair using TSI 9182 Bragg cells (parts $m$ and $n$ ). Two assemblies of four small mirrors (parts o) directed the laser beams back toward the water tunnel. These mirrors enabled adjustments to be made for slight beam misalignment, usually caused by refraction problems.

The effects of the changing index of refraction as the laser beams entered the water tunnel test section were accounted for during all phases of the LDV measurements. Figure 2.23 offers a summary of Snell's law of refraction. The main refraction problems were encountered for the $\pm 45^{\circ} 3$-D cases. As discussed later in this section, custom built "water cells" solved these problems.

For the $2-\mathrm{D}$ and $0^{\circ} 3-\mathrm{D}$ cases, a $250 \mathrm{~mm}$ focal length transmitting lens was used. The beam intersection angle, $\beta$, and the measurement fringe spacing, $d_{f s}$, were $8.5^{\circ}$ and 2.5 $\mu \mathrm{m}$. respectively, for these cases. A beam expander was not used, and the diameter of the 
beams defined by the $e^{-2}$ intensity points was $\approx 1.5 \mathrm{~mm}$. From these dimensions, the beam waist diameter was estimated at $0.11 \mathrm{~mm}$ and the corresponding beam crossing length was $1.5 \mathrm{~mm}$. The actual measurement volume diameter and length $\left(d_{m}\right.$ and $\left.l_{m}\right)$ also depends on the receiving optics, as discussed later in this section.

For the $\pm 45^{\circ}$ cases (Fig. 2.20), a $400 \mathrm{~mm}$ focal length transmitting lens was used, and the beam angle, $\beta$, and fringe spacings, $d_{f s}$, were $5.4^{\circ}$ and $3.8 \mu \mathrm{m}$, respectively. The beam crossing dimensions were: diameter $=0.175 \mathrm{~mm}$, length $=3.75 \mathrm{~mm}$.

One great advantage of using a water tunnel for LDV measurements is that flow seeding is very easy, and data rates are generally higher than data rates in air. Optimal scattered light signals are realized when the seed particle diameter is approximately $0.5\left(d_{f s}\right)$, and when no more than one seed particle is present in the measurement volume at any given time (Durst et al., 1981). It is also desired that these particles are of uniform size or "monodispersed." Because of the dirt and even rust particles present in the current facility, it was impossible to obtain monodispersed seeding. However, good results were obtained with a combination of $2.0 \mu \mathrm{m}$ polystyrene latex spheres (PSLs) and $1.5 \mu \mathrm{m}$ silicon carbide particles. The mean data rates achieved using this seeding were approximately $1 / 8$ to $1 / 5$ of the maximum possible data rate (see section 2.2.4).

A disadvantage to performing LDV measurements in a water tunnel is that refraction becomes a problem when the laser beam pair bisector does not enter normal to the test section's outer glass wall. For the $0^{\circ}$ cases, the beams' entrance angles were small (near $90^{\circ}$ ), so the refraction problem was minimal and misalignments could be corrected using the independent mirror assemblies (parts o in Fig. 2.18).

For the $\pm 45^{\circ}$ cases, plexiglas cells filled with water were used to minimize the refraction problem (Figs. 2.19 and 2.24). Without these water cells, it would have been virtually impossible to have both the green and blue measurement volumes coincide. The water cells were designed such that their outer face was perpendicular to the central axis of the incoming laser beams. The cells rested on a support on the optical table, sliding along the test section wall as the table was traversed. A narrow air gap between the water cell and the tunnel wall caused the laser beams at the largest incident angles to be almost totally internally reflected. This problem was solved by injecting water into the thin gap between the water cell and the tunnel wall. Since the surface of the water cell was very flat, water could be suspended in this gap for several hours using the capillary effect. The independently mounted steering mirrors were essential for achieving beam alignment for these highly skewed cases.

The receiving optics were mounted at approximately $22^{\circ}$ backscatter for the $0^{\circ}$ cases and $45^{\circ}$ backscatter for the $\pm 45^{\circ}$ cases. The optics consisted of a $400 \mathrm{~mm}$ lens ( $0^{\circ}$ cases) or a 
$250 \mathrm{~mm}$ lens $\left( \pm 45^{\circ}\right.$ cases $)$ mounted to a TSI color separator module, with a photomultiplier for each color channel. Each photomultiplier was equipped with a $200 \mu \mathrm{m}$ diameter pinhole. The estimated measurement volume diameters $\left(d_{m}\right)$ were $105 \mu \mathrm{m}$ and $50 \mu \mathrm{m}$ for the $0^{\circ}$ 2-D and 3-D cases, and $90 \mu \mathrm{m}$ for the $\pm 45^{\circ} 3$-D cases. In each case, these measurements are smaller than the beam crossing diameters, which means that the receiving optics for this setup determined the final measurement volume diameter. The measurement volume lengths were shortened by using off-axis backscattering. For the 2-D case, the transverse length of the measurement volume $\left(l_{m}\right)$ was $410 \mu \mathrm{m}$. For the $3-\mathrm{D} 0^{\circ}$ case, $l_{m}=390 \mu \mathrm{m}$, and for cases $\mathrm{B}$ and $\mathrm{C}, l_{m}=250 \mu \mathrm{m}$. In terms of viscous units, the probe volume diameter ranged from $\Delta y^{+} \approx 0.37$ to 1.5 . The probe volume length ranged from $\Delta z^{+} \approx 2$ to 6.5 . The 3-D measurement volume sizes were generally smaller compared to the 2-D case, since the receiving optics were closer to the beam crossing location. Measurement volume size is not only important when considering the maximum spatial resolution of the flowfield, but also when attempting to minimize the effects of gradient broadening, as discussed in section 2.2.5.

\subsubsection{LDV Data Recording and Signal Analysis}

Figure 2.25 displays a block diagram of the equipment used to record and save the raw LDV frequency data. The photomultiplier signals were downmixed using the TSI LV frequency shifters. The resulting effective frequency shifts ranged from $-50 \mathrm{kHz}$ to $+50 \mathrm{kHz}$. The blue signal ( $U$ and $W$ velocity components) was low pass filtered using a Kron-Hite filter with the $-3 \mathrm{~dB}$ point at $\approx 100 \mathrm{kHz}$. Two Macrodyne frequency domain processors converted the raw PM signals to digital frequency output. The selected bandwidth for the Macrodyne processors was $0-0.2 \mathrm{mHz}$. At this bandwidth, the point sampling rate was $0.5 \mathrm{mHz}$, and 256 points were used to record each Doppler burst. For this setup, each burst record length was $512 \mu \mathrm{s}$, and the output frequency resolution (or "bit noise") of the Macrodynes was $160 \mathrm{~Hz}$.

The frequency domain processors were operated in coincidence mode to gather simultaneous $U, V$ velocity data pairs. The coincidence window times used were approximately $1 / 2$ of the Doppler burst record length (300 to $160 \mu \mathrm{s}$ ). A reduction in the effective measurement volume size is an added benefit of using the Macrodyne processors in coincidence mode. While using coincidence mode, nominal average data rates were on the order of 100 $\mathrm{Hz}$. The total measurement time was approximately 4 minutes at each point $(24,000$ to 30.000 samples). 
The $\Delta t$ between each Doppler burst is known as the particle interarrival time, and constantly changing throughout each data record. The interarrival time probability distribution should obey a Poisson distribution, since we are dealing with a "Poisson experiment" (defined as an experiment where the random variable is the number of successes for a given time interval). Checks of the interarrival time probability distribution showed that the flow of particles through the LDV measurement volume did indeed obey a Poisson distribution.

The digital output of the Macrodynes was recorded and stored using a Dostek 1400A LV interface and a 486 PC-compatible computer. The raw frequencies and interarrival times for the $U, V$ data pairs were saved to disk using the Dostek software. Including data taken for calculating spectra (discussed below), about 400 megabytes of LDV data were taken. These data were archived in binary format using a Panasonic LF-5010 optical WORM drive.

To determine the feasibility of measuring power spectra using LDV, a simple analysis to estimate the largest expected frequencies was performed. Using data presented in Bogard and Tiederman (1986), the boundary layer ejection (or burst) frequencies were estimated at approximately 8 to $10 \mathrm{~Hz}$. Knowing that the smallest length scales have frequencies about 1 order of magnitude greater than the bursting frequencies, one determines that spectral measurements should include frequencies up to $100 \mathrm{~Hz}$. Therefore, to satisfy the Nyquist anti-aliasing criteria, the sampling frequency for data used to calculate spectra should be at least $200 \mathrm{~Hz}$.

To maximize the data rate of the Macrodyne processors, the Doppler burst record length was reduced to 128 points, and the $U, V$ coincidence mode was turned off. Turning the coincidence off meant that $U$ and $V$ spectra data had to be aquired separately, but data rates above $500 \mathrm{~Hz}$ became possible using these processor settings. These data rates easily met the Nyquist requirement. Note that about $1 \mathrm{kHz}$ would be a continuous signal for this flow (a particle in the measurement volume at all time). The spectral data records were recorded in 45,000 point data blocks. Usually 15 data blocks were recorded. At the highest sampling rates, these data blocks were about 60 seconds long. $U$ and $V$ spectral data were taken at $y^{+} \approx 10$ and 50 , and $y / \delta \approx 0.5,0.8$ and $>1.0$ for both the 2-D and 3-D locations.

\subsubsection{LDV Data Reduction and Uncertainties}

In this section, the LDV data reduction and uncertainty estimates are described. Reducing the raw LDV data into their final form involved many steps, each adding to the total uncertainty level. Briefly, the steps involved were: 
1. Reducing the raw frequency data to individual velocity realizations.

2. Obtaining mean and fluctuating velocity statistics, including higher order statistics. Usually the probability distribution of the data was used to reject spurious data points.

3. The LDV data must eventually be transformed into the coordinate system of interest. For the 2-D case, one profile was taken, but the $V$ component was measured at a slight tilt angle. For the 3-D case, data from 3 profiles were combined to obtain one set of profile data, including all Reynolds stresses. Once the data were reduced into an orthogonal coordinate system, a tensor transformation could be used to calculate results for different coordinate systems. The transformation equations used are described in appendix A.

4. Many more experimental quantities can be derived from the basic LDV profile data, such as skin friction, local mean flow angles, shear stress angles, Townsend's structural parameter, etc. Some uncertainties were estimated for the most basic quantities, but a complete discussion is beyond the scope of this research.

Kline and McClintock's (1953) single sample uncertainty analysis method was used extensively to estimate the LDV data uncertainties. This method assumes that each uncertainty source is uncorrelated with the other uncertainty sources, and that the total uncertainty is the square root of the sum of the squares of all uncertainty contributions. Uncertainty results using this method will always be dominated by the largest contributions to the total uncertainty, so some smaller (and usually more obscure) sources of uncertainty can often be neglected in the final analysis. This is not to suggest that these sources should be ignored. All uncertainties given are for 20:1 odds $( \pm 2 \sigma)$ unless otherwise stated.

\section{Step 1. Reducing raw frequency data}

Equation $2.7 \mathrm{~b}$ was used to obtain $U_{\perp}$ and $V_{\perp}$. The two sources of uncertainty were the fringe spacing, $d_{f s}$, and the frequency difference, $f-f_{s}$. For the standard Macrodyne settings, the frequency resolution was $\pm 160 \mathrm{~Hz}$, which corresponded to a velocity resolution of $\approx \pm 0.04 \mathrm{~cm} / \mathrm{s}$. This uncertainty source is a random uncertainty, and was minimized by acquiring statistics for large numbers of samples. A detailed analysis of the uncertainty for $d_{f s}$ revealed an uncertainty of only about $\pm 0.7 \%$. This uncertainty source is considered a bias uncertainty, and could be minimized by normalizing the final results. In this case, one could normalize by $U_{e}$ to remove the dependence on $d_{f s}$. The raw uncertainties for individual realizations of $U_{\perp}$ and $V_{\perp}$ were less than $2 \%$ for velocities above $5 \mathrm{~cm} / \mathrm{s}$. These uncertainties for the LDV "raw" results are listed in Table 2.4.

The positioning uncertainties are listed in Table 2.4 as well. The $x$ and $z$ uncertainties were only critical for the 3 -D case. For this case, 3 profiles measured with different LDV 
beam geometries were used to acquire all mean and fluctuating velocity components at a singe point. The uncertainty for $x$ and $z$ was about $0.7 \%$ of the wing thickness. The estimated maximum change in $C_{p}$ for this positional uncertainty was $2 \%$. This figure is based on the inviscid $C_{p}$ calculations discussed in chapter 4 .

The uncertainty in $y$ was important for determining $u_{\tau}$, since it was found using the relation

$$
u_{\tau}^{2}=\frac{\tau_{w}}{\rho}=\frac{\mu}{\rho}\left(\frac{\partial U}{\partial y}\right)_{y=0}
$$

When reducing the LDV data, the $y$ datum was shifted slightly to obtain an optimum fit with equation 2.8. The resulting $y$ shifts were always quite small, and were used to estimate the uncertainty on $y$ (Table 2.4). Djenidi and Antonia (1993) examined the reliablity of using LDV data to calculate $u_{\tau}$. For their low Reynolds number 2-D data, they report $u_{\tau}$ uncertainies of $3 \%$. The author's uncertainty was $5 \%$ for the 2 -D case because of the existing uncertainties for $y$ and $U$, and rose to $7 \%$ for the $3-\mathrm{D}$ case because of the additional velocity component involved.

\section{Step 2a. Obtaining mean and fluctuating statistics}

The large sample sizes (20,000 to 30,000 samples/point) effectively removed any uncertainties due to the finite Macrodyne frequency resolution. To remove noisy or bad data points from the data records, a technique similar to that used by Chesnakas and Simpson (1994), and Ölçmen and Simpson (1990) was employed. This technique involved rejecting data by examining the probability distribution or histogram of the entire data record. A data record from a turbulent boundary layer usually approximates a random process with a Gaussian distribution. When plotted on a log scale, a Gaussian histogram has a parabolic shape, so one would expect a similarly shaped histogram from data from a TBL. Data that fall outside the "skirts" of these histogram can be confidently discarded as spurious data.

Ölçmen examined at several hundred of these histograms to determine how this sometimes subjective process affected the final statistics (Ölçmen and Simpson, 1990). He found that mean quantities could vary up to $\pm 0.5 \%$, and that second-order turbulence quantities could vary up to $\pm 4 \%$. Third- and fourth-order terms, however, were very sensitive to any modifications to the histograms. Ölçmen did not quantify these uncertainties, but it is the author's experience that these effects can vary the higher order results by over $\pm 20 \%$.

Their are several inherent sources in LDV measurement systems which bias or "broaden" the uncertainty of the LDV statistical results. These bias errors are systematic, and are functions of the flow velocity, flow gradients, and turbulence intensity. Two of these sources will be discussed here: velocity biasing and flow gradient broadening. A 
discussion of all sources of statistical biasing is beyond the scope of this work, and the interested reader is referred to Durst et al. (1981) for more information.

Velocity biasing occurs because the instantaneous data rate is correlated to the velocity of the seed particles. One method to correct for this biasing is the known as the McLaughlin and Tiedermann (1973) correction or inverse velocity weighting. Using this method, weighted averages are used to calculate the final statistics, where the weighting term is the inverse of the total instantaneous velocity, or $\chi_{i}=1 / \sqrt{U_{i}^{2}+V_{i}^{2}+W_{i}^{2}}$. The author used a modified inverse velocity weighting scheme recommended by Tummers et al. (1992) to correct for the biasing for the 2-D flow case. This weighting incorporates an estimate of $w$ for the inverse velocity weighting, and is referred to by Tummers et al. as the " $2 \mathrm{D}+$ " weighting. Figure 2.26 shows the effects of applying this inverse velocity weighting scheme to a sample of the current data.

A modified version of this scheme was used for the 3-D data. For these cases where the mean spanwise flow was non-zero, an a priori estimate of the local mean flow angle was used to estimate the mean $W$ for use in the velocity weighting.

Fuchs et al. (1994) also studied the problem of statistical biasing. of LDV data. They found that using transit time weighting (similar to inverse velocity weighting) provided the most accurate corrections to the data regardless of the LDV "data density." The data density is often defined as $N_{d}=\left(T_{\Lambda}\right)$ (mean data rate), where $T_{\Lambda}$ is the integral time scale, defined by equation 3.13. For the author's LDV data, the data density was quite high, normally above 10. According to Fuchs et al., using a weighting scheme based on the particle interarrival time should give similar results for $N_{d}>10$ for a wide range of turbulence intensities. Winter et al. (1991) report good agreement between inverse velocity weighting results and sample-and-hold algorithms for turbulence intensities of up to $30 \%$. Sample-and-hold algorithms remove the statistical bias by reconstructing the data record as a series of data points with uniform $\delta t$.

Another source of statistical biasing, known as gradient broadening, is dependent on the measurement volume size and flow gradient magnitudes. Gradient broadening occurs when volume-averaged measurements are taken in a flow-field with non-zero mean velocity gradients. In the presence of linear gradients, mean velocities results will not be affected by gradient broadening, but measured fluctuating quantities will be larger than the true quantities. In a boundary layer, the near-wall $\partial() / \partial y$ gradients are the greatest concern. Ölçmen and Simpson (1995) report that gradient broadening effects were most significant in the near-wall regions for the $u^{\prime}$ and $-\overline{u w}$ results. Figure 2.27 presents Ölçmen and Simpson's corrected data. For $y^{+}>10$, the corrections were negligible for this data. Since 
the $\Delta y^{+}$sizes of the author's measurement volumes were smaller (often much smaller) than that of Ölçmen and Simpson (0.37 to 1.5 compared to 2), gradient broadening corrections were not made.

\section{Step $2 b$. Obtaining velocity vs. time data records}

The data used to calculate spectra and autocorrelations were processed differently. The raw data were processed into records with uniform $\Delta t$ 's between samples by applying a sample-and-hold type of interpolation scheme to the original time series data. The success of this type of interpolation, or any other type, depends on an experimental parameter referred to previously as the "data density," or $N_{d}$, where $N_{d}=$ (time scale)(mean data rate). This parameter is simply an indicator of how fast the data rate was relative to the important time scales in the flow. When using the integral time scale $T_{\Lambda}$ (from equation 3.10 ), the data density for the time series data was much greater than 10. Lee and Sung (1994) found that using sample-and-hold interpolation schemes to estimate spectra worked well for data densities as low as 1.0 .

Some noise was present in the time-series data, and was eliminated using a maximum "data jump" criteria. The "data jump" was defined as the difference between adjacent data points. If this difference exceeded a predefined amount, typically $\pm 3 \sigma$, it was discarded. Usually only $0.2 \%$ to $0.5 \%$ of the data were rejected. To get better results near $\tau=0$ for the autocorrelation results, a low pass digital filter was applied to the time-series data (third order Butterworth filter, design from Peled and Liu, 1976). A typical cutoff frequency was $30 \mathrm{~Hz}$. Only the data used to calculate autocorrelations were filtered, none of the data used to calculate spectra were filtered. Approximately 100 block averages were used to obtain the final spectra results, so the estimated relative error for $\Phi(f)$ was $10 \%$ (Bendat and Piersol, 1986).

\section{Transforming the data to different coordinate systems}

Appendix A details the equations used to reduce the results from the LDV coordinate system (Fig. 2.22) to the desired 2-D or 3-D coordinate system. This process for the 2-D case simply involved rotating the $y$ axis about the LDV $x$ axis (aligned with the $x_{\mathrm{TC}}$ axis) by $5.2^{\circ}$. The process for the $3-\mathrm{D}$ case was much more complex, and involved 2 coordinate transformations to obtain the data in tunnel coordinates. A degree of redundancy existed for the 3-D data since 3 profiles were measured at the same point for each flow condition. This redundancy was used to correct the data for small differences in flow speed between the profile measurements, and to correct for Macrodyne signal processing errors (operator error for the $\pm 45^{\circ}$ cases). The estimated uncertainties for the 2-D and 3-D data are listed in Table 2.5. 


\section{Quantities calculated from experimental data}

After the mean and fluctuating velocity components have been determined, other quantities can be found, such as friction velocity $\left(u_{\tau}\right)$, Townsend's structural parameter, $\left(a_{1}\right)$, various flow angles $\left(\beta_{\mathrm{FA}}, \beta_{\mathrm{FGA}}, \beta_{\tau}\right.$, etc. $)$, and boundary layer shape parameters $\left(\delta, \delta^{*}, \theta\right.$, etc.). Uncertainties in the most basic experimental results affect these secondary results, some of which have been listed in Table 2.5. The derived quantities and results are discussed in chapter 3.

No attempt was made to determine the uncertainties on all of the secondary or derived quantities, but the following guidelines generally apply. Uncertainties tend to be largest in the near-wall regions, and here the relative uncertainties can become very large. A quantity with a nominal uncertainty of $7 \%$ might increase to over $30 \%$ uncertainty in these regions. Results involving derivatives and/or fluctuating quantities generally have the highest uncertainties, while results involving integrals will have lower uncertainties. It is the author's opinion that the inclusion of uncertainties for every data point would tend to trivialize the data. A responsible researcher or reader should know and understand that these uncertainties exist, but should not cripple one's ability to interpret the existing trends in the data or to make comparisons between data sets. One should always use sound and reasonable judgment.

\subsection{Hydrogen Bubble Apparatus and Setup}

The hydrogen-bubble technique was used to visualize the near-wall region of the test boundary layers. It was used to produce continuous bubble sheets as well as bubble "timelines." This section gives a brief history of the technique and describes the author's implementation of this method in detail.

\subsubsection{Hydrogen-Bubble Technique Background}

The hydrogen-bubble technique employs the electrolysis of water to create bubbles which function as fluid markers to visualize the flow. Electrolysis simply involves applying a voltage difference across two electrodes in water, the anode (positive voltage) produces oxygen, and the cathode (negative voltage) produces hydrogen as the water molecules $\left(\mathrm{H}_{2} \mathrm{O}\right)$ are decomposed. Since hydrogen molecules are produced at twice the rate of oxygen molecules, it is most logical to use the hydrogen bubbles produced at the cathode as the fluid markers.

Accounts vary as to who was first to discover or apply this technique. Kollin (1953) used a copper wire to study laminar pipe flow. Wortmann (also in 1953) used the electrical 
discharge of tellurium to mark the fluid, not an ideal method since tellurium is toxic. (The description of Wortmann's work is from Schraub et al.(1964), as the author does not read German!) Geller (1955) was probably the first to use this technique in its modern form. He was the first to use a thin platinum wire (0.006" in diameter) as the cathode. Geller even pulsed the bubble wire to create timelines to visualize a boundary layer in a duct flow. Because of its ease of use, this technique was quickly adopted and used within the aerospace industry. as described by Clutter and Smith (1961).

Schraub et al.(1964) presents possibly the most detailed early description of this technique, complete with a very thorough uncertainty analysis. This paper describes two methods for analyzing hydrogen-bubble images: the multiple-frame (or "pathline") method, and the single-frame (or "streakline") method. The former is used for determining velocities on a global scale, the latter is simpler but can only be used to determine velocities near the bubble-producing wire. For the current study, the single-frame method was employed to obtain instantaneous $U(z)$ distributions across the 2-D and 3-D boundary layers. From these results, the near-wall streak/sweep structure could be investigated quantitatively.

\subsubsection{Current Implementation of the Hydrogen-Bubble Technique}

The author's implementation of the hydrogen-bubble technique was very straight forward. For both flow cases, a single bubble-generating wire was held parallel to the test section floor at various $y$ locations in the boundary layer. These $y$ locations were determined by using an estimate of $u_{\tau}$ to match the $y^{+}$locations listed Table 1.2. More accurate $y^{+}$locations were determined using the LDV results, and are listed in Table 4.1. (Note: the hydrogen bubble testing was done prior to the LDV work.)

A $64 \mathrm{~cm}$ long forked probe was used to hold the bubble-generating wire in place (Fig. 2.28 ). The probe was constructed of streamlined tubing to minimize flow interference. The wire mounting points were $16 \mathrm{~cm}$ apart and were designed to tension and hold the wire horizontally at precise $y$ locations. Repeatable positioning of the wire in the $x z$ plane was accomplished using small guide pins attached to the probe ends and locating holes drilled in the false floor.

To obtain the optimum bubble size and keep the wire wake size to a minimum, very thin wire was used to produce the hydrogen bubbles. Platinum wire with a diameter of 25 $\mu \mathrm{m}$ (0.001 inches) was used for the 2-D flow-visualization work. This diameter was found to be a bit too fragile, and a larger diameter of $50 \mu \mathrm{m}$ (0.002 inches) was used for the 3-D flow case. The Reynolds number based on wire diameter determined the type of wake created by the wire. The largest wire Reynolds numbers encountered while performing the flow 
visualizations was about 7 . The flow around circular cylinders separates at approximately $\operatorname{Re}_{\mathrm{D}}=10$, and remains steady up to $\mathrm{Re}_{\mathrm{D}}=40$ (Van Dyke, 1982) The local turbulence increased the effective wire Reynolds number, but close observations revealed that the flow remained steady around the wire.

The wire size is also related to the bubble size. The bubbles which are produced and swept off the wire are about $1 / 2$ the wire radius. If the wire is too large, the bubbles will become buovant and will no longer be accurate fluid markers (Schraub et al., 1964). No buoyancy effects were noticed by the author for either size wire. The estimated size of the bubbles in viscous units ranged from 0.1 to 0.5 , depending on flow conditions and wire size.

Figures 2.29 and 2.30 show the probe positioned for the 2-D and 3-D experimental setups, respectively. The $2-\mathrm{D}$ location $\left(x_{T C}=-4.7 \mathrm{~cm}, z_{T C}=0.0 \mathrm{~cm}\right)$ was selected so that $\operatorname{Re}_{\theta}$ would be approximately equal to the 3 -D flow case at the same flow speeds. For this flow case, the wire was held perpendicular to the oncoming flow (parallel to the $z_{\mathrm{TC}}$ axis). Note that when $x, z$ coordinates are given for a wire position, it is implied that this was the location of the midpoint of the wire.

Figure 2.30 shows the wire positioned at Ölçmen's \#5 station $(x / C=0.0215, z / C=$ $0.245)$. The wire could be rotated to different angles in the $x z$ plane at this location. Note that the wire was positioned on the port side of the appendage, and the video was filmed looking up through the floor. An observer looking down at a bubble wire in the flow on the starboard side of the wing would have a similar view, and throughout this work, data are visually presented in this fashion. Figure 2.31 shows sketches of the field of view used for the flow-visualization work. Note that only a portion of the wire was used to produce bubbles. The ends of the wire near the supports were masked using thinned liquid tape (GC Electronics catalog no. 10-1762), as suggested by Schraub et al.(1964). This mixture was applied using an airbrush. The bubble producing portion of the wire was about $14 \mathrm{~cm}$ for the 2-D case, and $10 \mathrm{~cm}$ for the 3-D case. By using this masking technique, regions of the flow which may experience interference from the bubble wire probe legs were not visualized. Sample constant-sheet and time-line images do not display any noticeable interference at the bubble field edges (Figs. 2.36and 2.37).

The lighting and camera positions are crucial for obtaining good results using the hydrogen-bubble technique. For the current work, the camera was placed underneath the water tunnel, and all video was taken viewing through the glass test-section floor. A combination of $500 \mathrm{~W}$ halogen and $300 \mathrm{~W}$ tungsten lamps were used to backlight the bubbles at an oblique angle $\left(\approx 45^{\circ}\right.$ to $\left.60^{\circ}\right)$. Figures 2.32 and 2.33 diagram the 2-D and 3-D lighting setups, respectively. Since the bubble wire was near the side of the tunnel for the 
3-D flow case, the lights could be placed closer to the wire, and better flow-visualization results were obtained for this case. Acrylic Fresnel lenses were used to help focus the halogen lamps. Cooling fans were necessary to prevent these lenses from becoming heat damaged.

A block diagram of the bubble generating equipment used for this work is shown in Fig. 2.34. The bubble generator was previously used and documented by Kim (1991). A toggle was added to the generator's cathode output to enable switching between constant sheet and time line modes of operation. For producing the time lines, a function generator with variable duty cycle was used to pulse the wire at the desired frequency. A counter and oscilloscope were used to monitor the pulsing frequency. The pulsing frequency was determined as an average of these two readings. The difference in frequency readings was usually $\approx 2 \%$. An isolation transformer prevented ground loops from occurring in the bubble-generating electronics. The variac made it possible to vary the wire voltage level $(70$ to $140 \mathrm{Vdc}$ ) to produce bubbles of the desired size at different flow speeds. Note that the bubble generator was equipped with a polarity switch, which enabled the bubble wire to be periodically "cleaned." In practice, this switch was used often, as bubble degradation would occur after only a few minutes of continuous operation. The author will concur with Schraub et al.'s observation that this technique can be "finicky."

Sodium sulfate and Kodak Photo-Flo 600 solution were added to the water for this phase of the experimental work. The sodium sulfate, an electrolyte, acted as a catalyst to enhance the production of hydrogen bubbles (suggested by Schraub et al., 1964). The Kodak Photo-Flo solution helped to eliminate extraneous bubbles in the flow, which would usually be present in the worst possible place, such as the test section floor.

\subsubsection{Image Processing System}

The image processing system used to record and analyze the flow-visualization data is diagrammed in Fig. 2.35, and Figs. 2.36 and 2.37 show samples of the hydrogen-bubble constant sheet and time line images. A Sony DXC-151 camera paired with a D.O. Industries Navitron TV zoom lens (12.5 to $70 \mathrm{~mm}$ ) was used to film the hydrogen-bubble flow visualization. This camera featured a $768 \times 493$ pixel color CCD sensor, and could output three different types of video signals: RGB, Y/C (same as S-VHS), or standard NTSC. The camera also had variable gain and electronic shutter settings, producing single frame images that were free of blurring. The camera framing rate was the standard $30 \mathrm{frames} / \mathrm{sec}$. The video output horizontal resolution was $460 \mathrm{TV}$ lines.

The high resolution S-VHS output of the camera was recorded using an S-VHS VCR (Panasonic model AG-1960). The recorded data were then played back through a digital 
time-base corrector/frame synchronizer (Hotronic model AP41-SP), which stabilized the video signal. The time-base corrector was mainly used to digitally freeze the video signal. At this point let us examine what is happening when attempting to digitize a video signal. Each "frame" of a video signal are made up of 2 fields, referred to as "odd" and "even" fields. These field names come from the fact that each field is made up of alternating horizontal TV lines. So for the author's equipment, which produces and stores 460 lines of TV resolution, each field consists of 230 lines of resolution. This is important because when freezing a video "frame", it is actually 2 fields that get frozen, and it is this image that gets digitized by the computer. If one were to try to freeze a conventional NTSC or $\mathrm{Y} / \mathrm{C}$ video signal frame, the image would look blurred or jittery if the image on the tape is in motion. For freezing still images, this isn't a problem, and one can freeze an entire frame and get the highest resolution possible. In the author's case, however, fields had to be used, so the ultimate video resolution was only half of the camera's maximum. The time-base corrector could be selected to freeze fields as well as entire frames, and one could even select the "odd" or "even" field to display.

As an additional side note, it is worth mentioning a feature of the time-base corrector that the author came to rely on heavily. The Hotronic AP41 unit came equipped with a "strobe" setting, which continuously froze either frames or fields at a set rate (16 strobe speeds were available). This strobe feature enabled the author to see the hydrogen-bubble sheets or timelines very clearly, and it was very evident when the bubble quality was poor or the time lines were not set to the optimum frequency or pulse length. Using the strobe setting saved time and video tape.

As seen in Fig. 2.35, the image data were digitized using a 486-based PC equipped with a Truevision TARGA + 16/32 frame grabber. This ISA bus frame grabber had 1 Mbyte of onboard memory, and could capture images in 24-bit color. Different capture resolutions were offered, and 3 signal input options were available (NTSC, S-VHS, or RGB). For the current experiment, software from Jandel Scientific was used to control the frame grabber and store 8-bit grey-scale images at resolutions of $640 \times 480$ pixels, which is a standard VGA resolution. Images captured using this resolution had "square" pixels, which means that the spatial resolution of each pixel was equal in each direction. Calibration images, similar to the one shown in Fig. 2.38 were used to determine the spatial resolution and the bubble wire location in screen units. The actual spatial resolution of the digitized images ranged from $0.034 \mathrm{~cm} /$ pixel $(2-\mathrm{D})$ to $0.025 \mathrm{~cm} /$ pixel $(3-\mathrm{D})$. The images were saved using the standard Windows 3.1 bitmap format (*.bmp), and were archived using a Panasonic LF-5010 optical drive. 


\subsubsection{Analyzing Flow-Visualization Data}

To get quantitative results from the flow-visualization images, a robust algorithm was needed to convert the time-line geometry information to velocity information. A highly automated process was desired to minimize subjective errors. In general, two types of schemes are possible, single-frame and multiple-frame (Schraub et al., 1964). The singleframe technique is also referred to as a "time-of-flight" estimate (Bruneau et al., 1992) This method was chosen because of the low framing rate for standard S-VHS video $(1 / 60 \mathrm{sec}$.), and the inherent difficulty in automating a multi-frame technique (essentially a particle tracking problem) using the available equipment. The single-frame technique has been used in the past by Davis and Fox (1967) and Kim et al. (1968). More recent researchers have used digital image processing with this technique (Lu and Smith, 1985, and Bruneau et al., 1992)

Before analyzing the time-line flow-visualization data, low-pass filtering techniques were used to decrease the image noise levels. Usually, the image was first filtered using a median or averaging filter. After that, the image contrast was enhanced by stretching the image histogram. Figure 2.39 shows before and after samples of a filtered and enhanced image. Lindley (1991) describes these digital image processing techniques.

An outline of the digital image reduction procedure is shown in Fig. 2.40. User input to the reduction process is indicated in this figure by the gray-shaded boxes. A dark gray box signifies user decisions which can effect the final results. The image reduction process should be allowed to proceed with as few adjustments as possible. By applying uniform analysis procedures and streak/sweep selection criteria, much of the subjective nature of the data reduction was removed. The streak/sweep selection criteria were based on suggestions by Smith and Metzler (1983), and are discussed in section 4.3. These criteria can be thought of as a type of "triggering" which tells the computer to analyze a particular flow structure as a low-speed streak or high-speed sweep.

Note that an "interactive shift" was used during the program runs to correct for frameto-frame jitter. This jitter, caused by the instability of the VCR output, meant that the spatial calibration could be off by as much as 4 pixels in either the $x$ or $z$ directions. To correct for this random shifting, a constant point of reference in each series of images was selected. For each frame the program user would interactively move the "reference pixel" to match this reference point, and the program would translate the calibration accordingly. The jitter problem did not affect the image rotation, and was not a source of uncertainty since it could be very accurately corrected. 
Figures 2.41 and 2.42 show sample display screens from the image processing program "REDFV32." This program essentially calculated the image intensities along a line perpendicular to the bubble wire. Peaks in the intensity distribution were used to determine the distance between adjacent timelines. The two most recently shed timelines from the wire were used because of the uncertainty in time associated with the bubbles still on the wire. This method worked well for high contrast, low noise images, but was difficult to implement for poor quality image data. The 2-D images were particularly difficult to analyze. Employing image enhancing techniques helped but not all the problems were solved. As seen in Fig. 2.41a, spurious $U(z)$ data points often occurred. To eliminate these data, local rejection limits of $\pm 3 \sigma$ were usually implemented. After much trial and error, an algorithm that was robust enough to deal with all but the most troublesome images was finally developed (could handle about $97 \%$ of the images).

Using the "time-of-flight" algorithm, the average $U$ (normal to the bubble wire) over the pulsing period was calculated using the simple relation

$$
U=(d)(\text { frequency })
$$

If the pulsing period is small enough compared to the time scale of the flow structures of interest, the velocity determined using equation 2.9 could be considered an instantaneous velocity. For the author's work, typically 4 to 10 wire pulses occurred within one integral time scale, so this approximation was good for this particular flow.

By using this technique, instantaneous $U(z)$ distributions were found with high spatial resolution, data not easily obtained using conventional experimental methods. Although this method revealed only the velocity component normal to the bubble wire (of concern mainly for the 3-D flow), the flow-visualization results show the mean flow to be within approximately $10^{\circ}$ of the wire at all $z$ locations. Even at a $10^{\circ}$ "worst case" limit, $98 \%$ of the streamwise mean velocity is measured.

\subsubsection{Flow-Visualization Uncertainty Analysis}

Schraub et al. (1964) presents a thorough uncertainty investigation of both single and multiple frame techniques. For the specific test flow conditions of their analysis, Schraub et al. found an experimental uncertainty of $6 \%$ for the single frame technique. The current authors applied the uncertainty estimating technique of Kline and McClintock (1953) to equation 2.9 to estimate the $\pm 2 \sigma$ bounds of the velocity data. For the current data, the estimated uncertainties in $U$ ranged from $3.5 \%$ to $8.5 \%$ of $U_{r e f}$, depending on the local 
flow velocity, the wire pulsing frequency, and the image spatial resolution. The estimated uncertainties for $u^{\prime}$ are $5 \%$ to $10 \%$.

Recent work by Bruneau et al. (1992) showed that results from this type of analysis compared very well to LDV results in an experiment which examined turbulent spots in laminar boundary layers. They report that the largest source of error was the limited pixel resolution of the video camera, and that the highest uncertainties occurred during the passage of structures which created large velocities in the direction parallel to the bubble wire. See chapter 4 for a comparison of the current flow-visualization and LDV results.

Many of the image data uncertainties could be described as resulting from "random biases", with the results from each image being subjected to the same biases. So even though the absolute experimental uncertainties were relatively large, the data could be reliably used in a comparative fashion. These results were more than adequate for examining the the near-wall flow structure geometry. 


\section{LDV MEASUREMENT RESULTS AND DISCUSSION}

This chapter discusses the LDV results, making comparisons between the 2-D and 3-D flow cases and Reynolds numbers when possible. Higher Reynolds number data for 2-D boundary layers were examined and Ölçmen and Simpson $(1990,1995)$ have taken data at higher Reynolds numbers using the same 3-D geometry as the author. This 3-D data are plotted with the author's current data for comparison purposes.

\subsection{Description of LDV Data}

To get the LDV results, one profile was measured at three different Reynolds numbers for the 2-D case, while three profiles were measured for three different Reynolds numbers (9 total profiles) for the 3-D case. Tables 3.1 and 3.2 list the boundary layer and shape parameters for the 3 profiles measured. Note that Ölçmen and Simpson's (1995) high Reynolds number case is included, and will usually be referred simply as the Ölçmen data. Table 3.2 lists the additional spanwise shape parameters, and also lists the parameter definitions.

The Reynolds number ranges for the 2-D and 3-D cases differed, being 300 to 560 for the 2-D case, and 500 to 880 for the 3-D TBL. These ranges were somewhat narrow for examining $\operatorname{Re}_{\theta}$ effects, but the $2-\mathrm{D}$ results could be compared to the large body of existing 2-D TBL results. The current 3-D results were compared to and plotted against Ölçmen's data, which had the same flow geometry but with a local Reynolds number of $\operatorname{Re}_{\theta}=9520$.

The skin friction and shape factors for the 2-D flow agreed well with other low Reynolds number data (Purtell et al., 1981, Hama, 1954). For the 3-D case, $C_{f}$ was approximately $20 \%$ higher, and $H$ was about $10 \%$ lower than comparable 2-D data. The 3 -D boundary layer thicknesses were about twice the size of the 2-D cases for similar freestream velocities. Ölçmen's high Reynolds number flow has very similar boundary layer thicknesses compared to the current 3-D data, but the skin friction is approximately 100 times greater. The author's skin friction results were determined directly from sublayer data $\left(y^{+}<7\right)$.

The current LDV measurement techniques are described in section 2.2. Table 1.1 lists the locations and reference velocities used to design the LDV measurements. The 3 different 
LDV optical configurations were used for the 3-D flow case to obtain all mean, fluctuating, and Reynolds shear stress components. Only the $U$ and $V$ components were measured for the 2-D flow.

While Table 3.1 lists the local flow conditions for the measured boundary layers, one flow parameter has not been discussed - the local pressure gradients. A very slight adverse pressure gradient may have been present for the 2 -D flow cases $\left(\partial C_{p} / \partial x \approx 0.00018 \mathrm{~cm}^{-1}\right.$, see sections 2.1.6 and 4.1.3). The pressure field for the 3-D case was much more complex, but the local pressure gradients given by Ölçmen and Simpson (1990) are $\partial C_{p} / \partial x \approx$ $-0.058 \mathrm{~cm}^{-1}$ and $\partial C_{p} / \partial z \approx 0.03 \mathrm{~cm}^{-1}$ at station \#5. These 3-D pressure gradients should be similar for the author's lower Reynolds number flow. Section 4.1.3 contains a discussion of the 3-D pressure field as it pertains to the hydrogen-bubble flow visualization results.

When presenting 2-D data, the biggest decision that must be made is what to nondimensionalize the velocity and lengths by - should one use inner scaling variables $\left(u_{\tau}\right.$, $\left.u_{\tau} / \nu\right)$, or outer scaling variables $\left(U_{e}, \delta\right)$ ? In this chapter, almost all the data $(2-\mathrm{D}$ and 3-D) are presented using "companion" plots, which display the results using both sets of scaling parameters. For some of the data, this probably was not necessary, but was done for completeness.

When considering 3-D data presentation, another question arises - what coordinate system should be used to present the data? Figure 3.1 shows several standard coordinate systems. Tunnel coordinates (TC) are defined as being aligned with the test section coordinate system. Usually this means that $x_{\mathrm{TC}}$ is pointing downstream, $y_{\mathrm{TC}}$ is perpendicular to the floor, and $z_{\mathrm{TC}}$ completes the right-handed coordinate system (see Fig. 2.3). Most of the other coordinates systems vary by chosing different references for the $x$ direction. Freestream coordinates (FS) align the $x$ axis with the local freestream direction, and wall coordinates (WC) align the $x$ axis with the wall shear stress direction.

For the current 3-D data, the freesteam coordinate system was chosen to present the majority of the data. At station \#5, the near wall and freestream flow angles differ by $7^{\circ}$ at most, so differences between results presented using FS or WC systems are small. For Ölçmen's higher Reynolds number data, the near-wall and freestream flow angle difference increases to $12^{\circ}$, which is large enough to see some differences depending on how the data are presented.

For descriptions of the data reduction techniques and data uncertainties, refer to Table 2.5 and sections 2.2 .4 and 2.2.5. 


\subsection{Reynolds Averaged Results}

In this section, the Reynolds averaged mean and fluctuating results are presented. These results also include the complete Reynolds stress tensor for the 3-D flow cases, along with the skewness and flatness results for the 2-D TBLs. The skewness and $\overline{u^{2} v}, \overline{u v^{2}}$ triple products are also presented for the 3-D profiles. The data are non-dimensionalized using both inner and outer scaling, and the 3-D data are presented using the freestream coordinate system (except for the skewness and triple products results, which are presented using tunnel coordinates).

\subsubsection{Measured Quantities}

Measured quantities refer to the most fundamental or basic LDV results - the mean and fluctuating velocity results. The results discussed in this section include up to fourth order fluctuating velocity statistics.

\section{Boundary Layer Skin Friction and Shape Parameters}

As mentioned in section 3.1, the current 2-D TBL $C_{f}$ and shape factor results for the agreed well with other low Reynolds number data. Figure 3.2 displays the current 2-D and 3-D data along with data from Purtell et al.(1981) and Hama (1954). For the 3-D case, $C_{f}$ was approximately $20 \%$ higher, and $H$ was about $10 \%$ lower than comparable $2-\mathrm{D}$ data.

The 3-D boundary layer shape parameter results were calculated for the freestream coordinates system and are listed in Table 3.2. The shape parameters containing crossflow terms were examined and plotted against $\operatorname{Re}_{\theta}$ in Fig. 3.3. The lateral shape parameter, $\delta_{4} / \delta_{5}$, does not vary uniformly for the 3-D TBLs examined, possibly since the lateral momentum thickness still contains a $U / U_{e}$ term. The cross-product and cross-flow momentum thicknesses did vary with Reynolds number, as shown in Fig. 3.3 b. Of all the shape parameters calculated, the cross-flow momentum thickness had the highest correlation with $\log \left(\operatorname{Re}_{\theta}\right)$.

\section{Mean Velocities}

Figures 3.4 and 3.5 display the 2-D and 3-D boundary layer profiles using linear axii for plotting. The 2-D profiles appeared to be under the influence of some flow anomaly which was not detected for the 3-D flow case. This influence was observed primarily in the $V$ and $-\overline{u v}$ results. A detailed look at the test section freestream flow characteristics and 2-D data profiles revealed that the influence of this flow anomaly was limited to the region outside of the boundary layer. For complete details, refer to section 2.1.4. 
However, the 3-D boundary layer mean profiles (Fig. 3.5) show a much more uniform freestream flow, and $\delta$ for all three Reynolds numbers were very similar. The profile shapes get slightly more "full" with increasing $\mathrm{Re}_{\theta}$, a fact quantified by the decreasing values of $\delta^{*}$ and $\theta$ for these flow cases (see Table 3.1).

A general observation can be made here before discussing the LDV data. Using inner scaling to present the results works well near the wall, and using outer scaling tends to work best away from the wall. For the results presented here, inner scaling seemed to work best when looking at features over the entire profile range, except near the boundary layer edge. Scaling velocities on $u_{\tau}$ seemed to capture the variations due to Reynolds number effects, and since many of the interesting profile features are found in the buffer layer, it is obvious that using $y^{+}$scaling would be the best choice.

Figures 3.6, 3.8, and 3.9 display the mean flow results. The sublayer relation, $u^{+}=y^{+}$, and the law-of-the-wall for the log regions are shown in Figs. 3.6 and 3.8. Coles constants were used for the law-of-the-wall $(\kappa=0.40, C=5.1)$, for each case. In general, turbulent boundary layer profiles over a range of Reynolds numbers do not collapse when using inner flow scaling (Purtell et al., 1981 and Ching et al., 1994), but the current 2-D mean results collapsed well for the limited range in $\mathrm{Re}_{\theta}$ using wall variables.

The velocity defect plot results are shown in Fig. 3.7 for the 2-D boundary layers. The curve fit shown is the "law of the wake" function, expressed as

$$
\frac{U_{e}-\bar{U}}{u_{\tau}}=\frac{U_{e}}{u_{\tau}}-\frac{1}{\kappa} \ln y^{+}+C+\frac{2 \Pi}{\kappa} \sin ^{2}\left(\frac{\pi}{2} \frac{y}{\delta}\right)
$$

(Coles, 1956). The law of the wake fit results shown in Fig. 3.7 were calculated for the $U_{r e f}=15 \mathrm{~cm} / \mathrm{s}$ case. As shown in Fig. 3.6, the wake regions of these low Reynolds number TBLs were very weak. The wake parameter, $\Pi$, which quantifies the wake strength, generally decreases with elevated freestream turbulence (Thole and Bogard, 1996, Hoffman and Mohammadi, 1991), and is also Reynolds number dependent, particularly at low Reynolds numbers. Purtell et al. (1981) report a rapid increase in $\Pi$ with $\operatorname{Re}_{\theta}$ up to $\operatorname{Re}_{\theta} \approx 2000$. Data presented by Gad-el-Hak and Bandyopadhyay (1994) shows similar behavior for $\Pi$ vs. $\operatorname{Re}_{\theta}$. The author's current results for $\Pi$, shown in Table 2.2, are in good agreement with these previous results. Refer to section 2.1.4 for more details.

The log region fit using Johnston's (1960) formulation for $Q,\left(Q=\bar{U}_{\mathrm{FS}} / \cos \left(\beta_{w}\right)_{\mathrm{FS}}\right)$, did not work well for the 3-D data. Ölçmen and Simpson (1990) showed that no law-ofthe-wall formulation works well for general 3-D boundary layers. The Johnston log region fit does improve with increasing $\operatorname{Re}_{\theta}$. The shallow log region slope was characteristic of all 
mean profiles in the wing-junction flow. The inner region curve fit, $Q^{+}=y^{+}$worked well up to about $y^{+}=6$.

Figures 3.6 and 3.9 reveal that the $V$ magnitudes were small compared to $U$. Figure 3.9 shows that the mean spanwise velocity, $W$, increased with $\operatorname{Re}_{\theta}$. The $3-\mathrm{D}$ flow skewing is discussed in detail in section 3.2.2.

$V$ results are plotted separately in Figs. 3.10 and 3.11. The maximum levels of $V$ occurred near the edge of the boundary layer for both the 2-D and 3-D flows, and therefore $V$ features scaled best using outer variables. Note that these maximum levels for the 3$\mathrm{D}$ cases were negative, which would indicate that the displacement thickness is growing thinner at this location as the flow moves downstream. No consistent pattern for Reynolds number effects were evident from these results.

Figure 3.12 shows the Gruschwitz-Johnston "polar plot" or "hodograph" plot (Johnston, 1960) for the 3-D flow cases. This plot displays the locus of points formed by the mean velocity vector in the $x z$ plane (freestream coordinate system data). The much larger flow skewing as $U / U_{e}$ decreased is an obvious high Reynolds number effect. Near the wall, a collateral mean flow region should exist, and would be shown in this plot by curves with constant slopes and zero intercepts at low values of $U / U_{e}$. This behavior was seen for all the data except for the $10 \mathrm{~cm} / \mathrm{s}$ case, which appears to be subject to a bias error near the wall.

The slope of $d W / d U$ in the outer region of the boundary layer can be approximated from the Squire-Winter-Hawthorne inviscid secondary flow formula. This approximation assumes that all viscous and Reynolds stresses are negligible, and that the only non-zero vorticity component is $\omega_{z}$. Eventually, the relation

$$
\frac{d W}{d U} \approx-2 \beta_{e}
$$

can be derived for a 3-D TBL's outer region at small flow deflections (Bradshaw, 1986). $\beta_{e}$ is the freestream turning angle relative to the tunnel coordinate system.

One might not expect this relation to hold true for a relatively complex 3-D flow, such as at station 5 for the current wing-body junction flow. Before station 5 was reached, the spanwise pressure gradient has reversed sign, and $\beta_{e}$, having reached a local maximum upstream of station 5 , is starting to relax back to $0^{\circ}$. However, equation 3.2 still approximates portions of the outer region $d W / d U$ slopes quite well, even for the lower Reynolds number cases which have some slight bi-directional skewing (Fig. 3.12). 


\section{Mean Normal Stresses}

Figures 3.13 and 3.15 display the current normal stress results. The 2-D results collapsed well when non-dimensionalized by $u_{\tau}$. There was only a hint of the typical $u^{\prime}$ outer region plateau for these low Reynolds numbers. Ölçmen and Simpson's (1995) data at station 0 and local $\operatorname{Re}_{\theta}=7800$ are shown in Fig. 3.14, along with Klebanoff's 1955 data. Using a scaling factor of $U_{e} / u_{\tau}=30.34$ for Ölçmen and Simpson's data, peak turbulence levels of 3.34 and 1.2 were found for $u^{\prime} / u_{\tau}$ and $v^{\prime} / u_{\tau}$, respectively. The skin friction velocity seemed to be more effective compared to $U_{e}$ for collapsing the peak 2-D turbulence levels.

The 3-D near-wall maximum $u^{\prime}$ turbulence levels (scaled on $u_{\tau}$ ) increased substantially with $\operatorname{Re}_{\theta} . v^{\prime} / u_{\tau}$ increased by over $30 \%$ for the high Reynolds number case for $y^{+}<50$. $w^{\prime} / u_{\tau}$ increased by almost $50 \%$ for the high Reynolds number data. The plateau regions for $u^{\prime}$ and $v^{\prime}$ in the outer region scaled on $U_{e}$ for this data. Interestingly, comparison of Fig. 3.15a and 3.15b seemed to indicate that one should scale the turbulence levels using outer scaling $\left(U_{e}\right)$ and inner scaling for $y\left(y^{+}\right)$for the best overall data collapse. However, to preserve the 3-D Reynolds number dependence, one should scale the turbulence levels using $u_{\tau}$. Remember that $u_{\tau}$ appeared to best correlate the 2-D normal stress data as well.

Klewicki and Metzger (1996) present a correlation for the near-wall $u^{\prime} / u_{\tau}$ peak levels vs. $\operatorname{Re}_{\theta}$ for $2-\mathrm{D}$ TBLs. The given correlation is

$$
\left(\frac{u^{\prime}}{u_{\tau}}\right)_{\text {peak }}=1.65+0.37 \log \left(\operatorname{Re}_{\theta}\right)
$$

and was shown to agree with 2 -D boundary layer data from $\operatorname{Re}_{\theta} \approx 200 \sim 4 \times 10^{6}$. For $\operatorname{Re}_{\theta}=500$, the predicted peak turbulence level from equation 3.3 was 2.65 , which agrees to within $5 \%$ of the current $2-\mathrm{D}$ data $\left(\left(u^{\prime} / u_{\tau}\right)_{\text {peak }} \approx 2.75\right.$ ). For $\operatorname{Re}_{\theta}=7800$ (Ölçmen and Simpson's data from Fig. 3.14), equation 3.3 under predicts $\left(u^{\prime} / u_{\tau}\right)_{\text {peak }}$ by only $7.5 \%$.

Although the overall turbulence levels were lower for the 3-D case, the 3-D data displayed a larger variation in the peak $u^{\prime} / u_{\tau}$ values with $\operatorname{Re}_{\theta}$. The results from a curve fit using the same form of equation 3.3 are shown in Fig. 3.16. This equation has a slope twice as large as the $2-\mathrm{D}$ data $(0.72)$, but these results are for a much smaller range in Reynolds number. One would also expect to find any such curve fit to vary for different flow geometries.

The 2-D $u^{\prime}$ and $v^{\prime}$ results were approximately $25 \%$ larger than their 3-D counterparts. The near-wall $u^{\prime}$ peaks were located at $y^{+} \approx 12-15$ for both the 2-D and 3-D data. The high turbulence levels at the boundary layer edge for the 2-D case were a result of freestream unsteadiness, as discussed in section 2.1.3. In the outer region of the 3-D flow, 
the $u^{\prime}$ and $w^{\prime}$ turbulence levels were about equal. This result was also seen by Ölçmen and Simpson (1995) as the three-dimensionality of their flow increased.

Results for the turbulent kinetic energy, $k$, roughly paralleled those for $u^{\prime}$ (see Figs. 3.17 and 3.18). The 2-D data collapsed well based on inner scaling, but the 3-D data displayed a strong Reynolds number dependence for the near wall turbulence peak. Another curve fit using the same form as equation 3.3 was tried for the 3-D $\left(k / u_{\tau}^{2}\right)_{\text {peak }}$ data (Fig. 3.19). The fit results are shown in the figure. Note that the correlation was better for these data than for the 3-D $\left(u^{\prime} / u_{\tau}\right)_{\text {peak }}$ vs. $\operatorname{Re}_{\theta}$ data.

\section{Mean Reynolds Shear Stresses}

The mean Reynolds stresses are shown in Figs. 3.20 - 3.24. Again, results from Ölçmen and Simpson (1995) are included for comparison. Figure 3.20 reveals that the total 2-D shear stress (viscous plus turbulent stresses) was comprised mainly of turbulent stresses in the $\log$ region of the boundary layer, since $-\overline{u v} / u_{\tau}^{2}$ was almost 1 .

For all 3-D cases, $|\tau / \rho| / u_{\tau}^{2}$ was only half of the 2 -D value (Figs. 3.21 and 3.22). This reduction in $-\overline{u v}$ occurred even though the 3-D flow's shear stress correlation, $-\overline{u v} /\left(u^{\prime}\right)\left(v^{\prime}\right)$, was approximately 0.45 above $y^{+}=10$ (Fig. 3.25). This is a large enough correlation to indicate that the low turbulent shear stress levels for the 3-D case was not a result of measurement error (Schlichting, 1955). The total 3-D shear stress, including the viscous stress, was calculated and plotted in Fig. 3.26. Reduced levels of total shear stress were observed for the 3-D flow case at all Reynolds numbers.

The variation in $\operatorname{Re}_{\theta}$ for the $2-\mathrm{D}$ TBL was too limited to see the same Reynolds number effects as did Ching et al., (1994). The 2-D TBL $-\overline{u v}$ data seemed to collapse well for this range of $\operatorname{Re}_{\theta}$, but the maximum values of $-\overline{u v}$ were about $10 \%$ higher than those observed by Ching et al. for similar Reynolds numbers.

Figure 3.22 shows the increase in $-\overline{u v}$ for the $3-\mathrm{D}$ data with $\operatorname{Re}_{\theta}$. The $-\overline{u v}$ results for the 3 lowest Reynolds number resembled nominal 2-D data, but at only about $50 \%$ of the turbulence intensities. For Ölçmen's high $\operatorname{Re}_{\theta}$ case, the distribution of $-\overline{u v}$ was double peaked, with the local minima occurring near the location of maximum crossflow and minimum $-\overline{u w}$ (see Figs. 3.9 and 3.21).

A major 3-D Reynolds number effect was observed by comparing the distribution of $-\overline{u w}$ in Fig. 3.21 or 3.24 . The near wall peaks in $-\overline{u w}$ increased rapidly with $\operatorname{Re}_{\theta}$. At the largest 3-D Reynolds number (Ölçmen's data, Fig. 3.21), the $-\overline{u w}$ shear stress peak was 3 times greater than the $-\overline{u v}$ stress. This large difference was not seen for the author's low $\operatorname{Re}_{\theta}$ data. 
Figure 3.23 shows that the near-wall values of $-\overline{v w}$ also increase with Reynolds number (increasing toward larger negative values). These near-wall increases in $-\overline{u w}$ and $-\overline{v w}$ were likely due to an increase in $\partial \bar{W} / \partial y$ with $\operatorname{Re}_{\theta}$ (see Fig. 3.9), since this quantity contributes to the production of these stresses. Explanations for the variations observed in the outer region distribution of $-\overline{u w}$ for the $\operatorname{Re}_{\theta}=760$ and 880 data are unknown at this time (Fig. $3.24)$.

\section{Higher Order Fluctuating Terms}

The skewness and flatness results for the 2-D flow are shown in Figs. 3.28-3.30. The definitions of skewness and flatness are

$$
S_{u}=\frac{\overline{u^{\prime 3}}}{{\overline{u^{\prime}}}^{3}}, \quad F_{u}=\frac{\overline{u^{\prime 4}}}{\overline{u^{\prime}}}
$$

$S_{v}$ and $F_{v}$ are defined similarly. For reference, a Gaussian probability distribution has a skewness of 0.0 and a flatness of 3.0. A square or "flattop" probability distribution has a flatness of 1.0. The flatness parameter is sometimes referred to as the kurtosis.

Figure 3.27 shows typical skewness and flatness results for the streamwise velocity component in a 2-D TBL (from Durst et al., 1992). Skewness results near the wall are positive because of the presence of much higher speed fluid being swept near the wall. Near the edge of the boundary layer, the skewness is negative for the opposite reason, with much slower speed fluid being ejected and brought out of the near-wall regions of the TBL. At or near the peak $u^{\prime}$ position, the skewness becomes zero (the probability distribution is symmetric) and the flatness reaches a local minimum.

The current skewness and flatness results are plotted in Figs. 3.28-3.29. These data were similar to the typical results shown in Fig. 3.27. No trends based on the variation in $\operatorname{Re}_{\theta}$ are apparent from this data. The only real variation in any of these quantities is the near-wall values of $F_{v}$. Klewicki and Metzger (1996) present data which suggest that $S_{u}$ and $F_{u}$ values increase with $\mathrm{Re}_{\theta}$, at least in the near-wall region. The small $\operatorname{Re}_{\theta}$ range for the current 2-D data probably would not reveal any existing trends.

Figure 3.30 presents results using a curve fit from Durst et al., (1991) for $F_{u}$ vs. $S_{u}^{2}$ and $F_{v}$ vs. $S_{v}^{2}$. The curve fit equations are given in the figure captions. These equations fit the author's current 2-D data well.

The 3-D skewing results for $u^{\prime}$ and $v^{\prime}$ are shown in Fig. 3.31. These results, presented in tunnel coordinates, did not display any distinct trends for changing $\mathrm{Re}_{\theta}$. Differences were observed between the author's and Ölçmen's $V$ skewness data near the wall. The 
uncertainties in this region for higher order $V$ terms are very high, however, so no reliable conclusions can be drawn from this near-wall variation in the $V$ skewness results.

The $\overline{u^{2} v}$ and $\overline{u v^{2}}$ triple products are presented in Fig. 3.32 using inner flow scaling in tunnel coordinates. The local maxima and minima peaks increased substantially with increasing $\operatorname{Re}_{\theta}$, and the locations of these peaks were correlated using $y^{+}$.

\subsubsection{Derived Quantities}

Derived quantities refer to results obtained through calculations performed using the basic LDV results. These quantities include flow angles, shear stress parameters, and structural parameters such as Townsend's structural parameter, $a_{1}$.

\section{Townsend's structural parameter, $a_{1}$}

Townsend's structural parameter, $a_{1}$, has become a commonly calculated turbulence parameter. It is simply the ratio of the turbulent shear stress in the $x z$ plane to twice the turbulent kinetic energy, or in equation form (repeated here from chapter 1):

$$
a_{1}=\frac{\sqrt{{\overline{u^{\prime} v^{\prime}}}^{2}+{\overline{v^{\prime} w^{\prime}}}^{2}}}{2 k}
$$

This quantity can be considered a type of turbulence "efficiency factor," which relates the ability of the random turbulence energy $(k)$ to create and sustain correlated shear stresses (Johnston and Flack, 1996). This parameter has been found to be $\approx 0.15$ for $2-\mathrm{D}$ turbulent boundary layers, but somewhat less for 3-D TBLs. Johnston and Flack (1996) present an in depth review and study of the changes in $a_{1}$ for different 3-D flows. The relative decrease in $a_{1}$ for these flows ranges from roughly $6 \%$ to $33 \%$.

The author's current results for $a_{1}$ are shown in Figs. 3.33 and 3.34. Note that for the

$2-\mathrm{D}$ data, $\overline{w^{\prime}}=\frac{1}{2}\left(\overline{u^{\prime}}+\overline{v^{\prime}}\right)$ was used to calculate $k$. The $2-\mathrm{D}$ results agreed well with the low $\operatorname{Re}_{\theta}$ results of Flack and Johnston (1993). Note that the low Reynolds number data do not peak at the "typical" value of 0.15 , but are about $25 \%$ lower at $a_{1}=0.11$. The author's data show a slight increase in $a_{1}$ with $\operatorname{Re}_{\theta}$. Johnston and Flack (1996) present results showing that $a_{1}$ does increase with $\operatorname{Re}_{\theta}$.

Calculations of $a_{1}$ for the 3-D data agreed with previous 3-D TBL results, as the peak in $a_{1}$ did not quite reach 0.15 . A region where $a_{1}$ was constant with values $\approx 0.13$ to 0.14 existed at all Reynolds numbers tested for the 3-D case. Note that these values of $a_{1}$ were larger than the author's 2-D $a_{1}$ data. Ölçmen's data display a unique profile for $a_{1}$. This type of "kink" has been noted by Johnston and Flack (1996). They report that for higher 
Reynolds number cases, some of the upstream, 2-D turbulence structure was preserved in the outer layer of the TBL, making $a_{1}$ larger in the outer part of the boundary layer. Again, for the 3-D data, the author's data suggest a slight increase in $a_{1}$ with $\operatorname{Re}_{\theta}$ in the outer region

\section{$1 / S$ and $-1 / C$}

The parameter $S$ is the ratio of $v^{\prime 2}$ to the total turbulent shear stress in the $x z$ plane, or in equation form:

$$
S=\frac{\overline{v^{\prime 2}}}{|\tau| / \rho}
$$

For a 3-D flow, $|\tau / \rho|=\sqrt{{\overline{u^{\prime} v^{\prime}}}^{2}+{\overline{v^{\prime} w^{\prime}}}^{2}}$. For a 2-D flow, $|\tau / \rho|$ reduces to simply the mean Reynolds shear stress, $-\overline{u v}$. Like $a_{1}$, this parameter is invariant to changes in coordinate systems. Instead of relating the shear stress to $k, S$ involves only $v^{\prime}$, the normal stress component largely responsible for the shear stress production. For a $2-\mathrm{D}$ flow, $1 / S$ is the ratio of $-\overline{u w}$ production to $-\overline{v w}$ production (Ölçmen and Simpson, 1995). For a 3-D flow, $1 / S$ is a good approximation of this ratio, especially in the outer region.

Figures 3.35 and 3.36 display the current results for $1 / S$. Both flow cases displayed a region of constant $1 / S$ away from the wall. This region was smaller for the 2 -D TBL due to the low $\operatorname{Re}_{\theta}$ values for this case. The current results for $1 / S$ show no Reynolds number dependence away from the wall, and the outer region values appear approximately the same for the $2-\mathrm{D}$ and $3-\mathrm{D}$ data $(\approx 0.7)$. These results are consistent with Ching et al. (1994). Ölçmen and Simpson (1995) show slightly lower values of $1 / S(\approx 0.5$ to 0.6$)$ in the outer layer as their flow progresses from stations 1 to 7 (Fig. 1.13). This small decrease may be a Reynolds number effect similar to that noted earlier for $a_{1}$, or it may be due to the changing local flow geometry.

As $y$ decreases $1 / S$ begins to rise sharply for both the 2-D and 3-D cases. This rise can be predicted by using the continuity equation to determine how $\tau / \rho$ and $\overline{v^{\prime 2}}$ vary as $y$ approaches zero. One can show that $1 / S$ is proportional to $1 / y$ as $y$ goes to zero, so $1 / S$ must go to infinity at the wall. Current DNS results show this trend correctly, but the experimental data do not (Spalart, 1988). It is most likely that this disagreement was caused by elevated $v^{\prime}$ measurements very near the wall. This appears to be a universal problem with near-wall LDV data (Plesniak, 1995), and may be caused by the behavior of the flow seeding particles near the wall.

These apparent measurement problems do not change the fact that $S$ still correlates $v^{\prime}$ and $|\tau|$ very well. This parameter was apparently very effective at correlating active 
motions which contribute to both $v^{\prime}$ and $-\overline{u v}$. This correlation appears to hold over a large range of Reynolds numbers and also between 2-D and 3-D flow geometries. Ching et al. also noted that this parameter seemed to be relatively invariant with $\operatorname{Re}_{\theta}$ for their $2-\mathrm{D}$ boundary laver data.

For a 3-D TBL, the ratio of $-\overline{v w}$ production to $-\overline{u w}$ production can be calculated more accurately (but still after making a few assumptions) as

$$
C=\frac{-{\overline{v^{\prime} w^{\prime}}}_{\text {prod }}}{-\overline{u^{\prime} w^{\prime}}{ }_{\text {prod }}}=\frac{{\overline{v^{\prime}}}^{2} \tan \left(\beta_{\mathrm{FGA}}\right)}{\overline{u^{\prime} v^{\prime}} \tan \left(\beta_{\mathrm{FGA}}\right)+\overline{v^{\prime} w^{\prime}}}
$$

(from Ölçmen and Simpson, 1995). Note that if equation 3.5 is applied to a 2-D flow, one finds that $-1 / C=1 / S$. Figure 3.37 shows the results of calculating $-1 / C$ for the available $3-\mathrm{D}$ data. The distribution of this parameter is similar to $1 / S$ through the boundary layer, with near-wall peaks present, and a constant region away from the wall. It appears that $-1 / C$ may slightly correlate the data better compared to $1 / S$, even though the results for this parameter show more scatter for $y^{+}<50$. This scatter is due to the large relative uncertainties encountered when evaluating equation 3.5 near the wall.

\section{3-D Flow Angles}

The three-dimensionality of a boundary layer can be characterized by the local mean flow angles $\left(\beta_{\mathrm{FA}}\right)$, flow gradient angles $\left(\beta_{\mathrm{FGA}}\right)$, and shear stress angles $\left(\beta_{\tau}\right)$. These angles are defined as

$$
\begin{gathered}
\beta_{\mathrm{FA}}=\tan ^{-1}\left(\frac{\bar{W}}{\bar{U}}\right) \\
\beta_{\mathrm{FGA}}=\tan ^{-1}\left(\frac{\partial \bar{W} / \partial y}{\partial \bar{U} / \partial y}\right) \\
\beta_{\tau}=\tan ^{-1}\left(\frac{-\overline{v^{\prime} w^{\prime}}}{-\overline{u^{\prime} v^{\prime}}}\right)
\end{gathered}
$$

and are relative to the coordinate system used to define $U, W,-\overline{u v}$, and $-\overline{v w}$. Figure 3.38 displays profiles of the local mean flow angles for the current data in freestream coordinates. Flow angle data presented in this coordinate system will go to $0^{\circ}$ at the layer edge by definition of the freestream coordinate system.

To determine $\beta_{w}$ accurately, one would like to observe a near-wall region of constant $\beta_{\text {FA }}$ (see Fig. 3.39), which indicates that a near-wall collateral flow region had been found. No near-wall collateral region was observed for any of the current data, and $\beta_{\mathrm{FA}}$ was estimated from the near-wall data point for each Reynolds number. Note that the angles 
presented in Fig. 3.39 are for the freestream coordinate system, so $\beta_{w}$ determined using this data would be offset by $\beta_{e}$.

Table 3.1 compares the freestream and wall flow angles for the 4 cases shown in Fig. 3.38. $\beta_{e}$ did not show any significant variation with increasing Reynolds number. The estimated values for $\beta_{w}$ did change with changing $\operatorname{Re}_{\theta}$, increasing to almost $-20^{\circ}$ for the $\operatorname{Re}_{\theta}=9520$ case. This is evidence of the inertial forces created by the flow geometry being "felt" farther into the viscous inner region of the high Reynolds number boundary layer.

Examining Figs. 3.39 and 3.40 more closely, one sees that the $y^{+}$locations of the near wall $\beta_{\mathrm{FA}}$ and $\beta_{\mathrm{FGA}}$ minima did not change significantly for the 3 lowest Reynolds numbers $\left(y^{+} \approx 20\right.$ to 25$)$. At the highest Reynolds number this position increased to $\left(y^{+} \approx 40\right.$ to 60$)$, which was still within the log layer for this flow. The maximum flow skewing for the current data was $-10^{\circ}$ (with respect to the freestream flow angle), but increased to $-18^{\circ}$ for the highest Reynolds number. The relative near-wall increase in $\beta_{\text {FGA }}$ was slightly less, from the $-15^{\circ}$ to $-20^{\circ}$ range (low $\operatorname{Re}_{\theta}$ ) to $-25^{\circ}$ (high $\operatorname{Re}_{\theta}$ ). The peak values for $\beta_{\mathrm{FGA}}$ near the edge of the boundary layer were nearly constant $\left(\approx 40^{\circ}\right)$ for all but the $\operatorname{Re}_{\theta}=760$ case. In general, the Reynolds number dependence was more visible for $\beta_{\mathrm{FA}}, \beta_{\mathrm{FGA}}$, and $\beta_{\tau}$ in the near-wall regions. Note that neither inner nor outer flow scaling appeared to correlate the near wall and outer region peaks for $\beta_{\mathrm{FGA}}$ and $\beta_{\tau}$. This topic will be revisited at the end of this section.

The local shear stress angles are shown in Figs. 3.38 and 3.41If $\beta_{\tau}$ and $\beta_{\mathrm{FGA}}$ were equal, it would indicate that the streamwise and spanwise eddy viscosities were equal. This is not true for a generalized 3-D TBL (Ölçmen and Simpson, 1993a). By definition, in a 3-D TBL the crossflow skewing changes as the flow moves downstream. As the flow gradient angle changes, the shear stress vector seeks realignment with $\beta_{\mathrm{FGA}}$. The change is not instantaneous, and $\beta_{\tau}$ usually lags $\beta_{\mathrm{FGA}}$. It is necessary to examine the particular flow's history to determine the exact lead/lag relationship between $\beta_{\tau}$ and $\beta_{\mathrm{FGA}}$. Figure 3.42 , from Ölçmen and Simpson (1995), shows the history of these angles for stations 1 thru 7 for the current 3-D flow geometry. These data clearly show that $\beta_{\tau}$ lags $\beta_{\mathrm{FGA}}$ for the current 3-D junction flow geometry. This is most easily seen in the outer region of the boundary layer. For the locations of stations 1 thru 7, refer to Fig. 1.13.

To directly examine the shear stress lag angle, $\beta_{\text {lag }}$ was calculated and plotted (Fig. 3.43). $\beta_{\text {lag }}$ was simply defined as

$$
\beta_{\mathrm{lag}}=\beta_{\mathrm{FGA}}-\beta_{\tau}
$$

The results shown in Figs. 3.43 indicate that $\left|\beta_{\tau}\right|$ increases near the wall for decreasing $\operatorname{Re}_{\theta}$. This is what one might expect to find as viscous forces become larger relative to the inertial 
forces in the inner region. Examination of Fig. 3.41 revealed that most of the change in 3.43 appeared in the distribution of $\beta_{\tau}$. The inner region shear stress angles varied by over $20^{\circ}$ with increasing $\operatorname{Re}_{\theta}$. No obvious trends appeared in the outer region. The local peak values in the outer region were approximately equal for all but the $\operatorname{Re}_{\theta}=890$ case.

As a final note on the 3-D boundary layer angles, an interesting feature of this particular data set was discovered. The astute observer will have noticed that the 3-D TBL angles results do not scale well on $y^{+}$or $y / \delta$ (see Figs. 3.39-3.41). Fleming et al. (1993) noted that for the same wing-body junction geometries, but with different approach boundary layer conditions, most boundary layer profile features scaled on the maximum wing thickness $(y / T)$, and not on $y^{+}$or $y / \delta$. Figure 3.44 shows the current flow angle results plotted vs. $y / T$. These results suggest (for this flow at least) that $T$ is still the most appropriate scaling parameter even when comparing flows with order of magnitude differences in $\operatorname{Re}_{\theta}$. This seems logical, since the pressure field scales on the appendage geometry. The depth into the boundary layer that the inertial forces are "felt" would then scale on $T$, which is why the $y$ location of the peaks in $\beta_{\mathrm{FA}}, \beta_{\mathrm{FGA}}, \beta_{\tau}$, and $\beta_{\text {lag }}$ are correlated using $y / T$.

\section{Anisotropy Factor, $N$}

Many turbulence models are based on the eddy viscosity concept, that is the shear stresses are related by a constant to the mean velocity gradients. This constant is often called the eddy viscosity. While this concept has proven useful for 2-D flows, the extension of this idea to 3-D flows has been fraught with difficulty (Ölçmen and Simpson, 1993). The fundamental problem lies in the fact that the transverse eddy viscosity varies greatly depending on the local 3-D crossflow and also the flow history.

The eddy viscosity anisotropy factor, $N$ was calculated for the current 3-D data to investigate any Reynolds number effects. $N$ is the ratio of the transverse to streamwise eddy viscosities, and was calculated as

$$
N=\frac{-\overline{v^{\prime} w^{\prime}} /(\partial \bar{W} / \partial y)}{-\overline{u^{\prime} v^{\prime}} /(\partial \bar{U} / \partial y)}
$$

Figure 3.45 displays the results for $N$. While it is hard to discern any trends because of the scatter present for the author's data, there does not appear to be any significant change in $N$ with increasing $\operatorname{Re}_{\theta}$. This result was based primarily on the results for the outer region of the boundary layers $\left(y^{+}>40\right)$. 


\subsection{LDV Autocorrelation Results}

As listed in the LDV measurement test matrix, long-time-record data were obtained at various $y$ locations for both the $2-\mathrm{D}$ and $3-\mathrm{D}$ cases. These data were used to calculate autocorrelation results and autospectra (see section 3.4).

To obtain more information about a fluctuating signal, an autocorrelation function is often useful. These functions show how the values of $U$ over time are related by forming an autocorrelation coefficient, $R_{u u}$, defined by

$$
R_{u u}\left(\tau_{R_{u u}}\right)=\frac{\overline{u^{\prime}(t) u^{\prime}\left(t+\tau_{R_{u u}}\right)}}{\overline{u^{\prime 2}}}
$$

Note that $R_{u u}\left(\tau_{R u u}\right)=R_{u u}\left(-\tau_{R u u}\right)$, and that $R_{u u}$ depends only on the time difference $\tau_{R u u}$, and not the time origin (Tennekes and Lumley, 1972). The autocorrelation time lag was designated $\tau_{R u u}$ to avoid confusion with the shear stress $\tau$.

Current autocorrelation results are shown in Figs. 3.46 - 3.52. These plots were obtained by ensemble averaging the autocorrelation results from 90 ten second data blocks for each $y$ location. Because of the measurement techniques used, only tunnel coordinate system results were available for the 3-D data. The results are presented in two plots for each flow case. The first plot shows the data for all time lags $\tau_{R u u}$ calculated (up to 5 seconds). The second plot shows the detail of the autocorrelations from $\tau_{R u u}=0.0$ to 0.5 seconds.

The autocorrelation results were used to examine the streamwise flow structure by calculating integral length scales $\left(L_{\Lambda}\right)$ and autocorrelation microscales $\left(L_{\lambda}\right)$. These length scales were found using the local mean velocities and time scales calculated from $R_{u u}$ :

$$
L_{\Lambda}=U T_{\Lambda}, \quad L_{\lambda}=U T_{\lambda}
$$

The integral time scale is defined as

$$
T_{\Lambda}=\int_{0}^{\infty} R_{u u}\left(\tau_{R u u}\right) d \tau_{R u u}
$$

$T_{\Lambda}$ gives an approximate measure of how long $U$ remains correlated, and $L_{\Lambda}$ gives an approximate mean streamwise dimension for the passing coherent structures.

A data processing note is in order at this point. Because of small tunnel speed variations over relatively long periods of time (over 15 minutes), the data's block mean velocities varied slightly - the data were not "stationary." For stationary data, the contribution to the integral in equation 3.10 should be almost zero after $R_{u u}$ crosses the zero axis for the first 
time (the idealized $R_{u u}$ function resembles a severely damped cosine function). Therefore, to avoid bias errors created by integrating non-stationary data out to $\tau_{R u u}=5$ seconds, the current $T_{\Lambda}$ results were determined by integrating $R_{u u}$ up to its first zero crossing. This same procedure was used to calculate $T_{\Lambda}$ for the freestream data discussed in section 2.1.4. Close examination of the results in Figs. 3.46 - 3.52 reveals that this difference would not have significantly changed the results for the majority of the autocorrelation data.

The microscale. $T_{\lambda}$, is related to the curvature of $R_{u u}$ at zero time lag using

$$
\left.\frac{d^{2}\left(R_{u u}\right)}{d \tau_{R_{u u}}{ }^{2}}\right|_{\tau_{R_{u u}=0}}=\frac{-2}{T_{\lambda}{ }^{2}}
$$

(Tennekes and Lumley, 1972). Uncertainties for the $T_{\lambda}$ results were approximately $5 \%$ (20:1 odds). This time scale is comparable to Taylor's microscale, found similarly from spatial correlation results. The two length scales, $L_{\Lambda}$ and $L_{\lambda}$, give a rough estimate of the size range of the coherent structures.

Converting time scales to length scales using the local mean velocity is an accurate measure of the actual length scales only if the flow structures do not substantially change during their time of passage, and if the convective velocities are equal to the local mean velocities. The first condition is met if Taylor's "frozen flow" hypothesis holds true. This hypothesis becomes a good approximation if $u^{\prime} / U \ll 1$ (Landahl and Mollo-Christensen, 1992; and Lumley, 1965). Ahn and Simpson (1987) have shown that for a 2-D turbulent boundary layer, the convective velocities vary depending on structure size, but become most uniform across wavenumber space for $0.08<y / \delta<0.5\left(\operatorname{Re}_{\theta}=3270\right)$. The ratio of $U_{\text {convective }} / U$ generally increases with decreasing $y / \delta$, particularly near the wall. These are also important considerations to remember when interpreting the spectral results (section $3.4)$.

The 2-D and 3-D length scale results are compared in Fig. 3.53. Figure 3.53a shows that the 3-D length scales, non-dimensionalized by $\delta$, were usually much less than the $2-\mathrm{D}$ length scales, especially for $0.1<y / \delta<1.0$. Results from $\mathrm{Ha}$ and Simpson (1993b) for a wind tunnel flow around the same wing-body junction geometry at higher $\operatorname{Re}_{\theta}$ revealed a $50 \%$ decrease for length scales in the $y$ direction with increasing three-dimensionality. The 3 -D length scale estimates (based on $U$ ) are likely underestimated slightly ( $\approx 3 \%$ to $8 \%$ ), since they were found using tunnel coordinate system data, and the mean flow direction was skewed up to $20^{\circ}$ from the $x$ axis. Note that the 2 -D freestream results for $L_{\Lambda} / \delta$ range between 0.69 and 1.65 (see section 2.1.4 and Table 2.2).

The comparison of the 2-D and 3-D length scale results is valid if the convective velocity variations are comparable between the two flow cases. Ha and Simpson (1993b) 
have convective velocity results for the current 3-D geometry at a larger Reynolds number (Ölçmen and Simpson's (1995) flow case). Their results were measured in the direction of maximum coherency for each location. At station 5 , these angles approximated the mean flow angles. While $\mathrm{Ha}$ and Simpson's convective velocity results varied greatly for stations 0 thru 6, their results for station 5 are comparable to Ahn and Simpson's 2-D results, particularly for the smaller scale structures (larger wavenumbers).

Results for the length scale ratio, $L_{\lambda} / L_{\Lambda}$, are shown in Fig. 3.53b. This ratio was $25 \%$ to $35 \%$ greater for the $3-\mathrm{D}$ flow case, a result which suggests that a smaller overall range of length scales was present for the 3-D flow. The increase in $L_{\lambda} / L_{\Lambda}$, coupled with the decrease in $L_{\Lambda} / \delta$, suggests that the three-dimensionality of the wing-body junction flow affected the larger outer region structures or eddies without decreasing the length scales associated with smaller structures.

When considering how pressure-induced flow skewing might affect the coherent boundary layer structures, the results shown in Fig. 3.53 can be explained by considering the effects of the mean flow skewing. Near the wall, the coherent structures' $y$ dimensions are relatively small, and the length scale reductions due to skewing effects are minimized in this region. Away from the wall, the $\Delta y$ extent of the structures grow much larger (Fig. 1.6), and any flow skewing would tend to break these structures down more effectively. The same is true when considering the relative sizes of the the smallest to largest coherent structures for the 3-D flow case. The smaller structures would be more resistant to changes caused by the $3-\mathrm{D}$ flow skewing simply because of their size. This idea is similar to the "toppling" mechanism hypothesized by Bradshaw and Pontikos (1985) which affects the large scale structures in a pressure-skewed TBL.

The effects of Reynolds number on length scale were also examined. Figure 3.54a shows that $L_{\Lambda} / \delta$ tends to decrease with $\operatorname{Re}_{\theta}$. Note that the $y$ ranges for the data are shown in the legend. This trend was expected since it has been observed that the coherent structures are smaller at higher Reynolds numbers (Head and Bandyopadhyay, 1981). The results for $L_{\lambda} / L_{\Lambda}$ vs. $\operatorname{Re}_{\theta}$ are shown in Fig. 3.54b. Since it has been observed that the coherent structures are less "chopped up" for low Reynolds numbers, one might expect $L_{\lambda} / L_{\Lambda}$ to approach unity with decreasing $\operatorname{Re}_{\theta}$. The 2-D freestream results for $L_{\lambda} / L_{\Lambda}$ are listed in Table 2.2 (also see section 2.1.4). These results ranged from 0.97 to 0.55 , and do show a definite trend as the length scale ratio increases with decreasing $\operatorname{Re}_{\theta}$. However, the current 2-D and 3-D results for $y / \delta<1$ do not show any general trends and are inconclusive. 


\subsection{LDV Power Spectra Results}

Because of the relatively high data rates achieved with the current LDV system, $U$ and $V$ power spectra could be measured. The Nyquist requirement was easily met for the frequencies of interest, and the data densities typically exceeded 10. The techniques used for taking the current spectral measurements using LDV were discussed in sections 2.2.4 and 2.2.5.

Figure 3.55 shows sample raw spectra data for the 2-D and 3-D cases. Each plot is the result of ensemble averaging the spectral results from approximately 90 ten second blocks for each $y$ location. The noise present at higher frequencies is typical for spectra calculated from LDV data, such as shown by Srikantaiah and Coleman (1985) and Adrian and Yao (1987). The frequency at which this noise becomes significant can be related to the LDV's maximum data rate (Høst-Madsen and Caspersen, 1994). Adrian and Yao (1987) suggested sampling at a data rate 20 times larger than the maximum frequency to be calculated to avoid problems with noise.

To improve the current spectral results, an attempt was made to reduce the high frequency spectral noise level. The noise distribution was assumed white, and was estimated using the spectral results at the highest available frequencies. This noise level was then subtracted from the raw spectra results at all frequencies, and the final spectra were smoothed using bin averaging (Fig. 3.55). As seen in Fig. 3.55, the spectra results in the -1 and $-5 / 3$ regions were not affected by the noise subtraction. However, the overall success of the noise subtraction at higher frequencies was only marginal.

The "- 1 " and " $-5 / 3$ " regions are shown in Fig. 3.55 and in the majority of the other spectral plots. These labels refer to regions of the spectral data that are proportional to $f^{-1}$ and $f^{-5 / 3}$ (or $k_{1}^{-1}$ and $k_{1}^{-5 / 3}$ ) respectively. The -1 region is where the maximum spectral energy content is contained, which can be seen most easily in a plot of the first moment of the spectra $(f F(f))$. The first moment peak or plateau defines the -1 region (Fig. 3.56).

The $-5 / 3$ region is known as the "inertial subrange," and was hypothesized by Kolmogorov in 1941. The inertial subrange is an overlap region between higher frequency scales influenced by viscosity and lower frequencies influenced by inertial effects. It can be shown that this $-5 / 3$ overlap region must exist if the turbulent Reynolds number is sufficiently large (Tennekes and Lumley, 1972; Townsend, 1976; and Landahl and Mollo-Christensen, 1992). The turbulent Reynolds number formed using the micro length scale is

$$
\operatorname{Re}_{\lambda}=\frac{u^{\prime} L_{\lambda}}{\nu}
$$


where $L_{\Lambda}$ is related to the curvature of the autocorrelation function at zero time lag (see section 3.3). $\mathrm{Re}_{\lambda}$ can be related to the ratio of the large-eddy time scales and the time scales of the strain-rate fluctuations (Corrsin, 1959). For the current data, $\operatorname{Re}_{\lambda}$ was relatively small, ranging from approximately 50 to 150 . Therefore, the extent of the inertial subrange for the current spectra results was reduced compared to higher Reynolds number results (Figs. $3.56-3.64)$.

\section{Present Spectral Results}

The present power spectrum results for $U$ and $V$ are shown in Figs. $3.56-3.66$. The 3 -D spectral data were obtained using the tunnel coordinate system. The current results are presented using the inner flow scaling proposed by Perry et al. (1985). These scaling parameters were defined as

$$
\begin{gathered}
u^{\prime 2}=\int_{0}^{\infty} u^{\prime 2} F_{u u}(f) d f \\
\phi_{u u}\left(k_{1} y\right)=\frac{U}{2 \pi y} u^{\prime 2} F_{u u}(f)
\end{gathered}
$$

To obtain Perry et al.'s outer flow scaling, substitute $\delta$ for $y$. The scaling parameters for $\checkmark$ were

$$
\begin{gathered}
v^{\prime 2}=\int_{0}^{\infty} v^{\prime 2} F_{v v}(f) d f \\
\phi_{v v}\left(k_{1} y\right)=\frac{U}{2 \pi y} v^{\prime 2} F_{v v}(f)
\end{gathered}
$$

Note that the definition of $k_{1}$ did not change for $\phi_{v v}$. Perry et al.'s inner flow scaling was also used by Ahn and Simpson (1987) and Ha and Simpson (1993b).

Inner scaling was preferred to outer scaling because it collapses the data better in the $-1,-5 / 3$, and higher wavenumber regions. However, the inner scaling parameters do not collapse the spectra for near-wall data. This is true for $y^{+}$values up to the outer edge of the buffer layer (Ha and Simpson, 1993b). Compare Fig. 3.57a to Fig. 3.66a, and Fig. 3.63a to Fig. $3.66 \mathrm{~b}$ to observe the effects of the different types of scaling. Ha and Simpson found that the outer flow scaling collapsed the data into two groups, one group for $y^{+}<500$, and another for $y^{+}>500$ (local $\operatorname{Re}_{\theta} \approx 9500$ ).

Both $U$ and $V$ spectra display -1 and $-5 / 3$ regions. For the $U$ data, the width of these regions each extended about $1 / 2$ to $3 / 4$ of a decade in wavenumber. For the $V$ data, the -1 region was often narrower than the inertial subrange, extending only about $1 / 3$ to $1 / 2$ of a decade. The highest measured wavenumbers not affected by noise had a -3 slope. For large $\operatorname{Re}_{\theta}$, spectra at the highest wavenumbers (responsible for the viscous dissipation) typically have a -7 slope (Townsend, 1976). 
The $U$ power spectra vary with $y$ similar to by Perry et, al.'s (1985) results. The present 2-D and 3-D results show the $U$ power spectra increasing with decreasing $y$ for low wavenumbers, and the $-5 / 3$ region extending to larger wavenumbers with increasing $y$. Note that the wavenumbers associated with maximum spectral peaks also decreases with decreasing $y$. Ahn and Simpson's (1987) results show that spectral levels reach maximum values at the $y$ locations where the most intense turbulent mixing occurs (Fig. 3.60).

The $V$ spectral results were quite different compared to the $U$ results. Variations in the maximum spectral values with $y$ were small compared to the $U$ results. In fact, the 3-D maximum spectral values for $y^{+}=11.6$ and 60 were almost equal (Fig. 3.63). The most intense 2-D $V$ fluctuations were found near $y^{+}=50$, which was also the $y$ location which shows the largest spectral values (Figs. 3.57and 3.58). Also, the $V$ results displayed a distinct "plateau" region at low wavenumbers, where $\phi_{v v}$ was constant for up to a decade in wavenumber space (Figs. 3.56-3.59). Higher Reynolds number $V$ spectral results from Ölçmen (1997) show a region of constant $F_{v v}$ which persists for approximately 3 decades in wavenumber space. Note that this data were plotted using a different type of inner scaling $\left(\nu / u_{\tau}\right.$ was used in place of $\left.y\right)$. Ölçmen's data display increasing spectral levels with increasing $y$, up to $y^{+}=325$. Above this value, the maximum spectral levels changed very little. This was also near the $y^{+}$location where Ölçmen's 2-D $v^{\prime}$ results reach a maximum (Fig. 1.4). These trends match the author's current data.

\section{Reynolds Number Effects}

Figures 3.67 - 3.73 compare spectral results over the current range of Reynolds numbers. $U$ and $V$ results are presented for $y^{+} \approx 10$ and $y / \delta=0.70 \sim 0.77$ (2-D) and $y / \delta=0.35 \sim 0.49$ (3-D). No significant variations in spectral distribution could be found over the author's limited Reynolds number range. However, comparison with the higher Reynolds number data of Ahn and Simpson (1987) (2-D data) and Ha and Simpson (1993b) (3-D data) was possible. These data are shown in Figs. 3.60 and 3.65, respectively.

As discussed by Tennekes and Lumley (1972), as the turbulent Reynolds number increases, the width of the $-5 / 3$ region should grow. Also, when using inner scaling parameters, the maximum spectral peaks at low wavenumbers increase with increasing $\operatorname{Re}_{\theta}$. Ahn and Simpson (1987) observed both trends when comparing 2-D spectra.

When the current 2-D data are compared to Ahn and Simpson's data, the only effect observed is the increase in the $-5 / 3$ region width. The low wavenumber spectral peaks are about equal near the wall (Figs. 3.60 and 3.67). Away from the wall, the low $\operatorname{Re}_{\theta}$ data spectral peaks are larger, not smaller (Figs. 3.60 and 3.69). 
For the 3-D data, the higher $\operatorname{Re}_{\theta}$ data of Ha and Simpson (1993b) show $U$ spectral peaks approximately half a decade larger at both $y$ locations (Figs. 3.65, 3.71, and 3.73). The $-5 / 3$ region is again larger for the higher Reynolds number case, especially for the data above $y / \delta=0.35$.

\section{3-D vs. 2-D Spectra Results}

Comparisons of the 2-D and 3-D $U$ and $V$ spectral results for the $U_{r e f}=15 \mathrm{~cm} / \mathrm{s}$ cases are shown in Figs. 3.74 - 3.79. These plots reveal the wavenumbers responsible for the decrease in turbulence levels for the 3-D boundary layer. The $U$ results show that a decrease in energy at the lower wavenumbers caused the lower turbulence levels. The opposite was seen from the $V$ results - the energy increase was due to differences in the moderate to high wavenumber range. Note that these comparisons were made assuming the convective velocities scale on $U$ in a consistent manner for both flow cases, which may not be a particularly good assumption. Comparison of convective velocity data from Ahn and Simpson (1987) and $\mathrm{Ha}$ and Simpson (1993b) show that for the current data, this assumption may be valid, at least for station 5 . See section 3.3 for more discussion on this topic. Perry et al. (1986) also discusses possible biasing errors present in wavenumber spectra caused by variations in convective velocity with wavenumber.

The 2-D vs. 3-D comparison results for the $U$ power spectra show that the decrease in energy occurred mainly at the smallest wavenumbers, with this difference increasing with increasing $y$. At $y^{+} \approx 10$, there was a roughly uniform spectral decrease across all wavenumbers for the $3-\mathrm{D}$ data (Fig. 3.74). For $y^{+} \approx 50$, the energy contribution at the lower wavenumbers begins to drop slightly, with a uniform difference across the -1 region (Fig. 3.75). At both these $y$ locations, the -1 and $-5 / 3$ regions were about equal in width.

At $y / \delta=0.77$, the $3-\mathrm{D}$ drop in energy contribution was more pronounced at the lower wavenumbers, and the 3-D - 1 region spanned about $1 / 2$ of a decade compared to an entire decade for the $2-\mathrm{D}$ data (Fig. 3.76). The width of the $-5 / 3$ region appeared about the same for the two cases. Note that the high wavenumber $U$ spectral data match more closely as $y$ increases.

The $V$ spectral results were quite different. The data tended to match closely at small wavenumbers, but the $3-\mathrm{D}-1$ region occurs at lower wavenumbers compared to the $2-\mathrm{D}$ data. The $3-\mathrm{D}$ data diverged from the $2-\mathrm{D}$ data near the beginning of the -1 region. This behavior was most evident at $y^{+} \approx 10$. The width of the -1 region was narrower for the 3 -D case, but the width of the $-5 / 3$ region appeared unchanged.

The comparison results for the $U$ spectra appear to be easily explained - the 3-D skewing has the greatest effect on the large scale, low wavenumber structures. This would 
be especially true in the outer layer, where the large coherent structures are oriented more vertically, and would be affected more by changes in the mean flow direction. Also note that the 3-D $U$ and $V-1$ regions were close together in wavenumber space, while these same regions for the $2-\mathrm{D}$ case were about $1 / 2$ of a decade apart. This indicates that the structures responsible for most of the 3-D $u$ and $v$ turbulent energy were more closely related in size than for the 2-D case. 


\section{FLOW-VISUALIZATION RESULTS AND DISCUSSION}

The hydrogen-bubble flow-visualization results are presented in this chapter. These results can be broadly classified into two categories; qualitative or quantitative. The former were gleaned from simple observation of the flow-visualization video, while the latter were the product of digital image analysis. These different Results from these two different methods point to a common conclusion - that the 2-D boundary layer experiences a more energetic momentum exchange between its inner and outer regions, and that the 3-D boundary layer possesses a more orderly or quiescent flow structure with much lower instantaneous vorticity levels.

\subsection{Overview of Flow-Visualization Data}

Table 1.2 lists the test matrix used to setup the hydrogen-bubble flow-visualization experiments. Refer to section 2.3 .2 for the exact placement of the hydrogen-bubble wire for the 2-D and 3-D flow cases. Table 4.1 lists the actual test matrix, with $U_{e}$ and $y^{+}$ determined using the LDV results. Note that 3 different bubble-wire angles were used for the $3-\mathrm{D}$ case, but the $3-\mathrm{D}$ results were obtained primarily using the $20^{\circ}$ wire angle. This angle was chosen because data from Ölçmen and Simpson $(1990,1995)$ show that the nearwall flow angle at station \#5 was about $19^{\circ}$. Also, the angle of a line normal to the local appendage surface extending through Ölçmen's \#5 station is almost exactly $20^{\circ}$. Since the local flow angles were changing along the bubble wire, sample flow visualizations were also performed with the wire at $10^{\circ}$ and $25^{\circ}$. The $20^{\circ}$ angle seemed to give the best results for the various flow speeds and $y$ locations, and was the primary angle used for the current study.

Two types of bubbles were produced for each test configuration, continuous sheet and pulsed time-line bubbles. Results from both types were used for the qualitative analysis, while the time-line images were used for the quantitative results. Low-speed streak and high-speed sweep behavior were studied using each type of analysis. 


\subsubsection{Hydrogen-Bubble Wire Coordinates}

Special coordinate systems were used to simplify the presentation of the flowvisualization data. These coordinate systems, shown in Fig. 4.1, have their $z$ axii aligned with the hydrogen-bubble wire for their respective configurations. $z=0.0$ is centered at the midpoint of the wire for the 2-D case, and at Ölçmen's station \#5 for the 3-D case. The quantitative results are presented using this coordinate system with no special designations. For the $3-\mathrm{D}$ case, all results presented are with the bubble wire rotated $20^{\circ}$.

\subsubsection{Quantitative Data Regions}

For both flow cases, only part of the bubble-producing portions of the wires were used to obtain quantitative data. For the 2-D case, this region was $z=0.0$ to $4.5 \mathrm{~cm}$. The hydrogen-bubble timelines in the $z<0.0$ region were more difficult to analyze. For some reason, timeline gaps frequently occurred in this region, possibly due to higher local turbulence levels. This problem may have been related to the odd behavior of the freestream flow for the 2-D LDV measurements (see section 2.1.4).

In order to minimize variations in local velocity and skin friction for the 3-D flow case, only the region $-1.5<z<3.0 \mathrm{~cm}$ was used for the quantitative analysis of the near-wall streaky structures. Note that the 2-D and 3-D regions examined are equal in width, $\Delta z=4.5 \mathrm{~cm}$, which should make the examination of the 2-D and 3-D flow structure spacing more uniform. For the $2-\mathrm{D}, U_{e}=15 \mathrm{~cm} / \mathrm{s}$ case, $\delta z^{+} \approx 320$, and for the $3-\mathrm{D}, U_{e}=$ $15 \mathrm{~cm} / \mathrm{s}$ case, $\delta z^{+} \approx 420$.

There was some concern about the results being influenced by the separation region created by the horseshoe junction vortex. Results from Kim (1991) and Ölçmen and Simpson (1995) were used to estimate the position of the separation line for $\operatorname{Re}_{\theta}<1000$. Figure 4.2 shows these results. The estimated separation line location was approximately $3 \mathrm{~cm}$ inboard of the bubble wire, and was not influencing the locations of the near wall streak structures revealed by the hydrogen bubbles. This result was supported by the $3-\mathrm{D}$ flowvisualization video, since the streaks were observed to change randomly in space and time.

\subsubsection{Local Pressure Fields}

Unlike more conventional measurement techniques, results from any type of flow visualization cannot be confined to a point. Once the fluid marker leaves its point of origin, it is subject to the time history of the fluid it is traveling with (Eulerian reference frame). For the quantitative results, the time-line bubbles are examined near the wire in order to 
get accurate results, but these results are still integrated or averaged over $\Delta t=1 / f$. This concern is also discussed in section 2.3.1.

On a broader scope, this means that the global flow-field conditions are very important to interpreting any flow-visualization result. The pressure field is a primary concern. For the 2 -D case. the pressure gradients should have been zero in all directions. For the current test case, a slight negative pressure gradient may have been present $\left(\partial C_{p} / \partial x \approx 0.00018 \mathrm{~cm}^{-1}\right.$, see section 2.1.6). For the 3-D case, the pressure field was much more complex.

An inviscid vortex panel flow solver was used to estimate and characterize the local flow conditions around Ölçmen's \#5 station. The same flow solver was used with success by Fleming (1991). The inviscid pressure and flow direction results are shown in Figs. 4.3 - 4.7. Figures 4.3 and 4.4 display the freestream pressure contours along the appendage. Ölçmen and Simpson (1995) show (for their higher Reynolds number case) that the while the static pressure contours look similar at the test wall, the $C_{p}$ magnitudes are roughly $50 \%$ of the inviscid results. Ölçmen and Simpson's (1990) experimental results show pressure gradients of $\partial C_{p} / \partial x=-0.058 \mathrm{~cm}^{-1}$ and $\partial C_{p} / \partial z=0.03 \mathrm{~cm}^{-1}$ at station $\# 5, \operatorname{Re}_{\theta}=6000$. These results indicate favorable pressure gradients in the downstream direction and toward the appendage. When these results are transformed to a coordinate system rotated $-20^{\circ}$ (i.e., aligned with the bubble wire direction), the pressure gradient is $\partial C_{p} / \partial z=0.008 \mathrm{~cm}^{-1}$.

The computed freestream $C_{p}$ contour results are useful for showing how the pressure field is changing from primarily a favorable streamwise pressure gradient, to a transverse pressure gradient (favorable toward the appendage), to an adverse pressure gradient downstream of the appendage maximum thickness. Figure 4.5 details the estimated $C_{p}$ distribution along the bubble wire. The calculated freestream $C_{p}$ at station \#5 was -0.17 . The computed pressure gradient along the wire at station $\# 5$ is $\partial C_{p} / \partial z=0.019 \mathrm{~cm}^{-1}$. The experimental result from Ölçmen and Simpson (discussed above) is $42 \%$ of this computed result (again, roughly half).

The local freestream flow angles, $\beta_{e}$, along the bubble wire for the 3-D case are shown in Figs. 4.6 and 4.7. At station \#5, the computed flow angle was $-9.8^{\circ}$ (negative angle mean the flow is directed away from appendage). For the 3 flow speeds measured for the 3 -D case using LDV, $\beta_{e}$ ranged from $-7.5^{\circ}$ to $-9.2^{\circ}$, with the agreement improving with flow speed (see Table 3.1).

\subsection{Hydrogen-Bubble Qualitative Results}

Constant-sheet flow visualization enabled comparison of the 2-D and 3-D low speed streaky structures at $y^{+} \approx 5,10$, and 30 for a nominal flow speed of $15 \mathrm{~cm} / \mathrm{s}$ (Fig. 4.8) The 
local Reynolds numbers were 300 (2-D) and 760 (3-D). The observed 2-D structures were very similar to the earlier results of Schraub et al. (1964), Kim et al. (1968), Robinson et al. (1991), and many others. Near the wall, the low speed streaks stretched up to 1000 viscous units in the streamwise direction, and broke up (or burst) when they began to oscillate. The oscillations were possibly accompanied by the streak rising through the boundary layer. Although rare, sometimes a streak could be seen wound in a downstream helical pattern, suggesting an interaction of the streaks with near-wall quasi-streamwise vortical structures The largest, most active oscillations occurred at $y^{+} \approx 10$, as the transverse fluctuations grew with increasing $y$. For the 2-D TBL, the high speed regions interacted strongly with the low speed streaks, often further dividing or separating the low speed structures with quick inrushes of high momentum fluid. The oscillations appeared to be caused in part by the rapid dividing and reformation (or coalescence) of a singular streak. Nakagawa and Nezu (1981) observed that these divisions caused by the high speed sweeps are part of the streak break-up mechanism.

Pockets of unmarked fluid near the wire are more often visible for the 2-D TBL (Fig. $4.8 \mathrm{a} \sim \mathrm{c})$. The more rapid formation of these pockets suggests stronger $v$ fluctuations for the 2-D case, a result supported by the LDV data results (see Figs. 3.13 and 3.15). In general, when compared to the 2-D case, the 3-D TBL did not experience as much mixing (the flow seemed more "orderly"), and the streaks were not as prone to the same oscillations or interactions. The 3-D flow was observed to be more quiescent overall, and was not dominated by the strong inrushes of high speed fluid common in the 2-D TBL. When the 3-D streaks experienced oscillations, adjacent streaks often oscillated in unison, keeping the total number of streaks constant. This was different from the 2-D case, where the 2-D oscillations led to streak coalescence and division. The 3-D streak locations did not change as rapidly when compared to the $2-\mathrm{D}$ streaks. The local acceleration around the side of the appendage may have been partially responsible for the increased stability of the 3-D TBL structure. This issue is discussed later in this chapter.

Above $y^{+}=15$, transverse structures began to dominate both flow cases. Figures $4.8 \mathrm{c}$ and $4.8 \mathrm{f}$, at $y^{+}=30$, start to display the larger transverse structures in the flow. These relatively near-wall transverse structures may be what Robinson (1991) referred to as "secondary arches" (Fig. 1.6). The flow-visualization video revealed that these structures are not a primary source of the turbulence production, as they seemed to be convected downstream while not experiencing much turbulent mixing. Note that the 2-D streaky structures were severely kinked at $y^{+}=30$, in contrast to the smaller transverse fluctuations for the 3-D flow. 
Comparison of the time lines (Fig. 4.9) revealed another fundamental difference between the flows. The 2-D TBL displays very narrow low speed peaks in the spanwise velocity distribution, often separated by regions of uniformly distributed high-speed fluid. The time lines in these regions were plateau shaped. The large spanwise velocity gradients at the edges of these "plateaus" created regions of highly concentrated vorticity, $\omega_{y}$. The 3-D flow possessed lower spanwise velocity gradients, indicating that these regions of concentrated vorticity were more diffused. One hypothesis is that the $\partial \bar{W} / \partial y$ velocity gradient and spanwise pressure gradient influenced the longitudinal vortex structures in the 3-D TBL, created a preferred direction of rotation, and ultimately decreased the near-wall concentrated vorticity regions. The instantaneous $\omega_{y}$ vorticity present in the low and high speed structures is discussed in the quantitative results section.

\subsection{Hydrogen-Bubble Quantitative Results}

This section discusses results from a quantitative analysis performed on digitized hydrogen-bubble flow-visualization images. The method used is very briefly discussed here. Details of the techniques and computer software used are in section 2.3.

To get quantitative results from the flow visualization results, automated computer processing was used to converted the time line geometry information to velocity information. A "single-frame" technique was used because of the low framing rate for standard S-VHS video ( 60 frames/sec, or 30 fields/sec), and the inherent difficulty in automating a multipleframe technique. Refer to Figs. 2.41 and 2.42 for sample display screens of the reduction technique used.

The images examined by the author using this technique were from the $y^{+} \approx 5$ and $10, U_{r e f}=15 \mathrm{~cm} / \mathrm{s}$ cases for the 2-D and 3-D flow geometries. Examining these four sets of images revealed several quantifiable differences between the 2-D and 3-D cases.

As mention in section 4.1.2, the complete transverse $(z)$ range was not used for the quantitative results. For the 2-D case, only the $z=0.0$ to $4.5 \mathrm{~cm}$ results are presented. For the 3-D case, $z=-4.5$ to $3 \mathrm{~cm} U$ and $u^{\prime}$ data are presented, but only the region from $z=-1.5$ to 3.0 was used for quantifying the near-wall streaky structures.

\subsection{1 $U$ and $u^{\prime}$ Results}

Figures 4.10 and 4.11 show the time mean results for the 2-D and 3-D turbulent boundary layer data. Note that the positive $z$ direction for the 3-D TBL is pointing away from the wing. The data were obtained by sampling the video at a rate of 1 frame/sec. This corresponds to a dimensionless time of $t U_{e} / \delta \approx 3$, long enough that each instantaneous 
$U(z)$ distribution could be assumed independent, yet short enough to obtain sufficient data from 4 to 5 minute video clips. For each case, roughly 240 images were analyzed to obtain the time mean results. This might seem like a small sample set compared to the LDV data. but is large compared to past researchers using non-automated data analysis. For comparison, Smith and Metzler (1983) typically analyzed 45 to 60 frames per flow case.

Figures $4.10 \mathrm{a}$ and $4.11 \mathrm{a}$ display the mean flow distributions, $U(z)$, for the 2-D and 3-D TBLs for $U_{r e f}=15 \mathrm{~cm} / \mathrm{s}$. The small peaks at $z=0$ for both cases are the result of locating marks on the plexiglas false floor. These marks hindered the image data reduction at $z=$ 0 , and the attempted correction at these locations was not $100 \%$ effective. This problem is most visible in Fig. 4.12, the friction velocity results. Note the spanwise change in $U$ for the 3 -D case. The $2-\mathrm{D}$ case also displayed a small $(\approx 10 \%)$ change in $U$ over a span of 3 $\mathrm{cm}$. If the bubble wire was not parallel to the floor, results like this will occur. But since the results at $y^{+}=5$ and 10 are similar, it was likely that $U$ varied slightly in the spanwise direction. The scatter in both plots is an indication of the typical uncertainty levels for this type of data.

The rms velocity fluctuations, $u^{\prime}$, are displayed in Figs. $4.10 \mathrm{~b}$ and $4.11 \mathrm{~b}$. The increased uncertainty for this quantity is apparent by the increased scatter in the results. The 3-D $u^{\prime}(z)$ distribution does not vary as much as one might expect for the given pressure field. The 3 -D turbulence intensities are $\approx 25 \%$ lower than for the 2 -D case at both $y^{+}$locations. Note that the 3-D results are normalized by $U_{r e f}$, not an edge velocity. The spanwise variation in $U$ and $u^{\prime}$ would not be as apparent if the data were normalized by the edge velocity at each $z$ location plotted.

These quantitative results are "in the same ballpark" as the LDV data, with relative differences ranging from $3 \%$ to $20 \%$. However, it is difficult to quantify the uncertainty of the hydrogen-bubble analysis based on a comparison to the LDV results, since small changes in $y$ lead to large differences in the final results. For example, for $y^{+}=5, U_{r e f}=$ $15 \mathrm{~cm} / \mathrm{s}$, a $0.7 \mathrm{~mm}$ change in the wire height will cause a $20 \%$ relative change in $U / U_{\text {ref }}$.

An attempt was made to estimate $u_{\tau}(z)$ using the limited amount of data. Figure 4.12 shows these results. For the $2-\mathrm{D}$ case, the agreement was within $8 \%$ of the LDV data (see Table 3.1) . The agreement was considerably worse for the 3 -D data (22\%), but still within the uncertainty bounds calculated for a $u_{\tau}$ estimate found using the flow-visualization data.

\subsubsection{Low-Speed Streak and High-Speed Sweep Results}

Since ordinary statistical quantities of the instantaneous $U(z)$ distributions are of limited interest, what else can be done with this data? One thing that has been done in the past is to look at the near-wall low-speed streaky structure. 
The spacing of these structures has been documented by many researchers for 2-D TBLs (Gupta et al., 1971. Nagawa and Nezu, 1981, Smith and Metzler, 1983, and others), but only a fraction of TBL studies have examined 3-D flows. For 2-D layers, the streak spacing, $\lambda^{+}$, is usually found to be $\approx 100$. Ha and Simpson (1993) show that the streak spacing may be decreased slightly for a 3-D boundary layer. Flack and Johnston (1993) report that $\lambda^{+}$for 2 different 3-D TBLs remained near 100 .

Since these streaky structures indicate the positioning of the near-wall quasi-streamwise vortices. their spacing and other characteristics were studied using the current flowvisualization data. Not only were the low-speed structures studied, but the high-speed events were examined as well. These events are usually referred to as high-speed sweeps. Other streak/sweep parameters were examined and are discussed in section 4.3.3.

The $U(z)$ velocity distributions were examined for sweep and streak events using a thresholding technique to identify the events. Figure 4.13 shows a sketch describing this technique graphically. Note that the local mean velocity is subtracted from the instantaneous velocity distributions, so one ends up analyzing $(U-\bar{U})$ distributions. The current thresholding level to indentify the event bounds (points $a$ and $b$ in Fig. 4.13) was $|U-\bar{U}| / \overline{u^{\prime}}=0.2$.

This thresholding technique was used as a "first cut" to identify possible low or high speed events. Smith and Metzler's (1983) criterion for counting an event as a low speed streak was used in the present study for both low and high speed events as a "second cut" criterion. This criterion required the momentum flux ratio between adjacent low and high speed regions must exceed a set value (Smith and Metzler used a 2:1 ratio). This value can be approximated using adjacent maximum and minimum values of $U$, and then calculating $\left(U_{\text {high }} / U_{\text {low }}\right)^{2}$. To increase the statistical significance of the results (i.e., to look at more events), a 1.5:1 momentum flux ratio was used by the author as the acceptance criterion for the streak or sweep events. It was found that decreasing the momentum flux ratio to 1.5 did not greatly affect the average low-speed streak spacings (less than a $4 \%$ decrease in $\overline{\lambda^{+}}$for the current data). Smith and Metzler report the same findings (3\% decrease in $\left.\overline{\lambda^{+}}\right)$. The streak location probability distributions are not presented here, but these results show no preferred streak positions in the regions of interest for both flow cases. This was an issue for the 3-D case, where the streak locations may have been influenced by the horseshoe vortex mean flow structure. Overall, approximately 600 events were identified and examined for each flow case.

Another factor affecting the streak spacing results was the limited $\Delta z$ range of the current data. For each case, this range was about $\Delta z^{+} \approx 350$, while for other researcher's 
studies. $\Delta z$ was usually closer to $1000 . \lambda$ histograms for large $\Delta z$ ranges show spacings in excess of 300 for some events, while the current study limited the maximum spacings accepted to $\approx 200$, due to spatial variations in the mean flow. However, the lower order statistics and probability distributions $\left(\lambda^{+}<150\right)$ compare well with the results of other researchers. The statistical coefficient of variation ( $\Psi=\sigma /$ meanvalue $)$ is in the 0.30 to 0.40 range. and the ratio $\lambda_{\mathrm{mp}} / \bar{\lambda}$ varies from 0.80 to 0.90 (see Table 4.2 ). Both results are in good agreement with other researchers' findings (Smith and Metzler, 1983, Gupta et al., 1971).

Table 4.2 lists the streak spacing statistics, and Fig. 4.14 shows the $\lambda^{+}$histograms for the current data. For the current test cases, the mean low-speed streak spacing was increased by $23 \%$ for the $3-\mathrm{D}$ data. The streak variance increased as well for the 3-D data, being $30 \%$ larger.

At $y^{+}=5, \lambda^{+}$mp was $30 \%$ larger for the 3 -D case compared to the 2 -D case. At $y^{+}=$ 10 , the peak in the 2-D probability distribution was much broader, and $\lambda^{+}{ }_{\mathrm{mp}}(\approx 108)$ was not well defined. $\lambda^{+}{ }_{\mathrm{mp}}$ for the 3-D case did not change farther away from the wall. For both cases, $\overline{\lambda^{+}}$increased slightly with $y$. Smith and Metzler's (1983) results show about a $5 \%$ to $10 \%$ increase in $\overline{\lambda^{+}}$from $y^{+}=5$ to 10 . Nakagawa and Nezu (1981) suggest that the streak spacing increases with $y$ because streak divisions diminish as $y$ increases, while streak coalescing still occurs, thus creating less total streaks. The streak spacing variance did not change with $y$.

Nakagawa and Naga (1981) have indicated that the streak spacing histograms may be represented using log-normal distributions. The log-normal distribution curves for the current data are shown in Fig. 4.14, and appear to fit the 3-D data very well. The fit results for the 2-D data were almost as good.

As mentioned above, a possibly significant result from examining the low speed streak spacing was that $\overline{\lambda^{+}}$is $\approx 23 \%$ higher for the 3-D case compared to the 2-D (Table 4.2). It should be noted that the mean dimensional spacing of the low speed streaks was roughly equal for the two boundary layers at both $y^{+}$locations. The factor that increases $\lambda^{+}$for the $3-\mathrm{D}$ case is the increased skin friction velocity, $u_{\tau}$. This increase in $\overline{\lambda^{+}}$for the $3-\mathrm{D}$ case is not consistent with previous results, and should be examined more closely.

One parameter that changes between the two flows is the Reynolds number. $\mathrm{Re}_{\theta}$ for the 3-D flow was 760, compared to 300 for the 2-D flow. But Smith and Metzler's compilation of past 2-D results do not show any significant dependence on Reynolds number, so we must look for other sources of discrepanies. The most obvious reason for the increase in $\overline{\lambda^{+}}$is the increasing $u_{\tau}$ for the $3-\mathrm{D}$ case, since the physical or dimensional streak spacing is roughly 
same for both flow cases. The current test flow travels approximately $2000 x^{+}$units while transitioning from a 2-D to $3-\mathrm{D}$ TBL. This distance is only about 2 streak lifetimes. It is quite likely that the near-wall turbulence structure cannot respond that quickly to changes in the local mean flow conditions, and that the turbulence structure is "frozen" compared to the more rapidly changing mean flow. Utilizing turbulence distortion theory, where one studies the effects of flow distortion on various sizes of turbulent eddies may be one way to examine this issue. If the increase in $\overline{\lambda^{+}}$is due to the rapidly changing mean flow, it would be interesting to determine when the near-wall flow structure "relaxed" back to its nominal spacing of $\overline{\lambda^{+}}=100$.

Regardless of how the increase in $\overline{\lambda^{+}}$for the 3-D case came about, this finding seems to make the current flow-visualization results more cohesive or internally consistent. Qualitatively, the 2-D flow revealed a more active inner region. The 2-D flow case displayed more energetic streak oscillations, with the vigorous low speed streak/high speed sweep interactions resulting in rapid streak coalescence and division. The greater streak activity would tend to result in a more closely spaced streak structure, which the current quantitative results show. Other research has shown that the 3-D turbulent boundary layer's mean crossflow creates variations in the near-wall ejection/sweep activity which are not seen in the 2-D case (Senstad and Moin, 1992, Flack and Johnston, 1993). These variations may be responsible for the apparent increase of $\overline{\lambda^{+}}$for the 3-D layer. Also, the mean crossflow present in a 3-D TBL typically creates a region of concentrated $\omega_{x}$ vorticity near the wall. It is possible that this vorticity influences or inhibits the creation of near-wall vortices of a particular rotational direction, which would precipitate an increase in streak spacing for the 3-D flow.

The next two parameters listed in Table 4.2 are the streak/sweep width, $\kappa$ (nondimensionalized by $u_{\tau} / \nu$ ), and velocity magnitude, $\varepsilon$ (non-dimensionalized by $u_{\tau}$ ). The results for $\kappa^{+}$revealed that the average 3 -D streak or sweep was about $20 \%$ wider than the average 2-D event. The near-wall event velocity magnitudes for the 2-D TBL were double those for the 3-D case. These parameters indicate narrow, highly energetic events for the 2-D case, compared to broader, more sedate events for the 3-D case. There was more inner region activity for the 2-D TBL, where greater quantities of low speed fluid were involved in the ejection/burst process. These results are all consistent with the observations of the flow-visualization video.

\subsubsection{Other Streak/Sweep Parameter Results}

In an attempt to better quantify the streak/sweep characteristics, two additional parameters were derived. The first parameter was termed the "event momentum flux ratio," 
and was designated using the symbol $E$. This parameter is the ratio of the low-speed streak (or high-speed sweep) momentum to the mean flow momentum over the same $\Delta z$ range. In equation form, this ratio was defined as

$$
E=\frac{\int_{a}^{b} U(z)^{2} d z}{\int_{a}^{b} \bar{U}(z)^{2} d z}
$$

where $a$ and $b$ are the locations where $U-U_{\text {threshold }}=0$ (Fig. 4.13). This ratio quantifies the strength of a low or high speed perturbation in the boundary layer. $E$ values near 1.0 reveal that the low or high speed events do not possess large changes in momentum. The results shown in Table 4.2 indicate that the creation of low speed streaks and high speed sweeps with large momentum changes was inhibited for the 3-D flow compared to the 2-D TBL. $E_{\mathrm{LS}}$ results for the $2-\mathrm{D}$ case were about $8.5 \%$ lower at both $y^{+}$locations, showing that the relative streak momentum loss is greater for the 2-D boundary layer.

$E$ for the high speed sweep events was also calculated. These results revealed that $E_{\mathrm{HS}}$ was $7 \%$ to $10 \%$ higher for the $2-\mathrm{D}$ case, again showing a greater exchange of momentum between the inner and outer regions for the 2-D TBL.

Figure 4.15 shows the probability distribution for the $E_{\mathrm{LS}}$ results at $y^{+}=10$. The 3 -D TBL $E_{\mathrm{LS}}$ results have $30 \%$ less variance and are closer to unity, compared to the 2-D values. These findings corroborate the earlier observation that the 3 -D flow appears to be more organized, with less momentum exchange between the inner and outer regions.

The last parameter examined was the instantaneous spatial average of $\left|\omega_{y}\right|$ created by the low and high speed events. As mentioned previously, recent DNS results have shown that the longitudinal (or quasi-streamwise) vortices play a key role in the inner region bursting process. The $y$ component of vorticity is related to these longitudinal vortices in two ways. The low-speed streaks and high-speed sweeps are influenced by and interact with these near-wall vortices. The quasi-streamwise vortices can create large $\partial U / \partial z$ velocity gradients, therefore increasing $\omega_{y}$. Likewise, an increase in $\omega_{y}$ can enhance the strength of the longitudinal vortices when it becomes skewed into the $x$ direction by the $\partial U / \partial y$ velocity gradient. This interaction between the different vorticity components is part of a turbulent boundary layer's regenerative process. If one or more of the vorticity components were decreased or diffused, then the turbulence production would be suppressed.

To compare the relative vorticity levels present in the 2-D and 3-D boundary layers, the average $\omega_{y}$ vorticity was calculated for each case. The instantaneous spatial average for $\left|\omega_{y}\right|$ due only to the low or high speed events is

$$
\Omega=\frac{1}{z_{m}-a} \int_{a}^{z_{m}}\left|\omega_{y}-\overline{\omega_{y}}\right| d z+\frac{1}{b-z_{m}} \int_{z_{m}}^{b}\left|\omega_{y}-\overline{\omega_{y}}\right| d z
$$


If the following assumptions are made:

- $|\partial U / \partial z| \gg|\partial W / \partial x|$ (such that $\left.\omega_{y} \approx \partial U / \partial z\right)$

- event symmetry $\left(z_{m}-a \approx b-z_{m}\right)$

- $U(z)$ is linear

then the above equation simplifies to

$$
\Omega_{\mathrm{LS}}^{+}=\frac{4}{\kappa^{+}} \frac{\left(U_{\mathrm{threshold}}-U\left(z_{m}\right)\right)}{u_{\tau}}
$$

and

$$
\Omega_{\mathrm{HS}}^{+}=\frac{4}{\kappa^{+}} \frac{\left(U\left(z_{m}\right)-U_{\text {threshold }}\right)}{u_{\tau}}
$$

which can be rewritten as

$$
\Omega_{\mathrm{LS}, \mathrm{HS}}^{+}=\frac{4\left|\varepsilon_{\mathrm{LS}, \mathrm{HS}}\right|}{\kappa_{\mathrm{LS}, \mathrm{HS}}^{+}}
$$

Note that equation 4.2 is dimensionless.

The $\Omega^{+}$results for both low speed and high speed events were over $100 \%$ greater for the 2-D case compared to the 3-D case (Table 4.2). The much higher levels of $\omega_{y}$ for the 2-D laver indicate more dynamic turbulence producing flow structures with higher concentrations of vorticity in the near-wall region.

The probability distributions for $\Omega_{\mathrm{LS}}^{+}\left(y^{+}=10\right)$ and $\Omega_{\mathrm{HS}}^{+}\left(y^{+}=5\right)$ are shown in Fig. 4.16. These distributions show that the variance in $\Omega^{+}$for both streak and sweeps was $50 \%$ less for the 3-D data. The 2-D TBL displays more event occurrences at higher vorticity levels. This is somewhat similar to the $E_{\mathrm{LS}}$ results (Fig. 4.15), where events occurring at higher "activity" levels for the 2-D TBL skew the probability distributions, even though the most probable values for the two cases were not that far apart. 


\section{CONCLUSIONS}

This research involved examining and comparing a variety of experimental results from wall-bounded 2-D and 3-D turbulent boundary layers. Reynolds number effects were investigated for the 3-D boundary layer by comparing to previous results using the same flow geometry but at a significantly larger Reynolds number. Unique parameters were defined to help quantify the structure of the near-wall turbulence and its dependence on different flow geometries.

By meeting the goals of this basic research program, a gap in the existing body of wall-bounded turbulent flow research has begun to be filled. A 3-D TBL was examined for effects of Reynolds number and effects of the 3-D flow geometry, and the flow variations were recorded and quantified. As with most basic research, it usually depends on others to apply the new information. Hopefully this research will be applied, whether it be to assist interpretation of wind tunnel results, to hypothesize new turbulence models based on observed flow physics, to realize practical boundary layer flow control, or to design future experiments for basic turbulence research.

To accomplish the research goals, the experimental work proceeded in two phases: LDV boundary layer measurements and hydrogen-bubble flow visualization. Both experiments were carried out in the Virginia Tech Aerospace and Ocean Engineering water tunnel facility. The major findings from each research phase are presented in this chapter.

Examining the effects of Reynolds number on a 3-D turbulent boundary layer has been a major part of this research's contribution to the general understanding of turbulent flows. The boundary layer of a complex three-dimensional flow was examined at low Reynolds numbers $\left(\operatorname{Re}_{\theta}=500,760\right.$, and 890$)$ and compared to a higher Reynolds number boundary laver with the identical flow geometry $\left(\operatorname{Re}_{\theta}=9520\right)$. All mean velocity components and the complete Reynolds stress tensor were measured for this flow. Several derived quantities were calculated and compared. As discussed in chapter 1, Reynolds number effects for 3-D TBLs have been virtually undocumented, which is surprising considering the importance of dynamic similarity in laboratory research. 
The author's hydrogen-bubble results have helped to quantify changes in the near-wall flow structure of 3-D TBLs. Similar to data documenting 3-D Reynolds number effects, flow-visualization results for 3-D TBLs are also relatively rare. The current study was even more unique in that this particular 3-D flow has been so well documented by past researchers. Most flow-visualization experiments are almost exclusively qualitative. This study extracted instantaneous velocity distributions and quantitatively analyzed the nearwall flow structures using digital image processing.

\subsection{LDV Measurement Conclusions}

The LDV measurement results were presented in chapter 3. Companion plots utilizing both inner- and outer-variable flow scaling were used to examine and present the results. The 2-D boundary layer results consistently agreed with previous low Reynolds number studies of TBLs. The current 2-D and 3-D results were compared to examine the effects of 3-dimensionality on TBLs at low Reynolds numbers. The major differences observed were:

- The 2-D normal stress levels were approximately $25 \%$ greater compared to the $3-\mathrm{D}$ case. However, an empirical relationship between Reynolds number and the peak value of $u^{\prime}$ near $y^{+}=15$ for the 3-D flow had a $100 \%$ larger slope. An empirical fit to the near-wall peak value in turbulent kinetic energy vs. Reynolds number for the 3-D TBL had an even larger slope with a significantly improved correlation coefficient.

- The mean Reynolds stress $-\overline{u v} / u_{\tau}^{2}$ was almost doubled for the 2-D case. The shear stress correlation coefficient $-\overline{u v} / u^{\prime} v^{\prime}$ for the 3 -D case was roughly the same compared to the 2-D flow.

- Townsend's structural parameter, $a_{1}$, was lower for the 2-D flow compared to the 3-D flow, which was a surprising result. This may have been a Reynolds number effect, since recent studies have shown that $a_{1}$ decreases with $\operatorname{Re}_{\theta}$. $\operatorname{Re}_{\theta}$ for the 2-D flow cases was up to $50 \%$ lower compared to the 3 -D cases.

- A better choice for a turbulent structural parameter would be the parameter referred to in this study as $S\left(S \equiv v^{\prime 2} /|\tau / \rho|\right)$. $1 / S$ results were approximately equal relatively constant in the outer regions of the 2-D and 3-D boundary layers. $S$ appears to have potential as a useful flow structure parameter, or at least more useful than $a_{1}$. The similarity of $1 / S$ between flow cases indicates that the same level of correlation is found between $v^{\prime}$ and the total $x z$ shear stress for both the 2-D and 3-D flow cases.

- Length scale results from the autocorrelation results revealed $50 \%$ shorter integral length scales for the 3-D flow. This result was consistent with previous results found using the same 3-D flow geometry (Ha and Simpson, 1993a,b). 
- $U$ spectral results revealed that the majority of the 2-D TBL's increase in $u^{\prime}$ energy occurred at low wavenumbers $\left(k_{1} y<1\right)$, which indicates that changes in the large-scale structures led to lower normal stress levels for the 3-D TBL. However, the $V$ spectral results revealed that the primary increase in $v^{\prime}$ occurred due to energy contributions at higher wavenumbers $\left(k_{1} y>1\right)$.

The current 3-D TBL was examined for Reynolds number effects in much more detail than most 2-D TBLs have been examined. Some characteristics of the 3-D boundary layer varied strongly with Reynolds number, while others appeared independent of Reynolds number. The main conclusions from the investigation of Reynolds number effects for a 3-D TBL were:

- The mean crossflow velocity increased significantly with Reynolds number. This was the most fundamental change due to Reynolds number effects. The relative increase in viscous forces in the near-wall region at low Reynolds numbers tended to keep the boundary layer's mean flow more closely aligned with the freestream direction. Neither inner or outer flow scaling successfully correlated the peak $W$ magnitudes or the $y$ peak locations for all Reynolds numbers. $W$ did appear to scale on $U_{e}$ in the outer layer, down to a minimum $y / \delta$ of 0.1 .

- As observed for 2-D TBLs, inner flow viscous scaling did not correlate the normal stress or tke results. The peak magnitudes of $u^{\prime}$ and $k$ could be correlated with $\operatorname{Re}_{\theta}$ using a semi-log curve fit.

- The higher-order fluctuating velocity terms were more sensitive to Reynolds number effects. The near-wall - $\overline{u w}$ shear stress increased significantly with Reynolds number due the increased turbulence production of the larger spanwise flow gradients. The $u^{\prime}$ and $v^{\prime}$ normal stress distributions were correlated to some degree using outer flow scaling in the outer region, down to a minimum $y / \delta$ of 0.15 . This location was approximately the inner limit for successful $W / U_{e}$ data scaling.

- The 3-D skewing results for $u^{\prime}$ and $v^{\prime}$ appeared to be only weakly Reynolds number dependent, if at all. The $\overline{u^{2} v}$ and $\overline{u v^{2}}$ triple products were Reynolds number dependent. The local maxima and minima peaks increased with increasing $\operatorname{Re}_{\boldsymbol{\theta}}$, and the locations of these peaks were correlated using $y^{+}$.

- The structural parameter $S\left(S \equiv v^{\prime 2} /|\tau / \rho|\right)$ was independent of Reynolds number near the wall for the 3-D boundary layer. This parameter was also found to vary little between the 2-D and 3-D TBLs. These findings indicate a direct relationship between $v^{\prime}$ and the total shear stress in the $x z$ plane, which appears to be invariant over a range of Reynolds numbers and with increasing 3-dimensionality. Note that this parameter is also independent of coordinate system rotation about the $y_{\mathrm{TC}}$ axis. 
- Another structural parameter, $-1 / C$, was found to correlate the $3-\mathrm{D}$ data above $y^{+} \approx$ 40 for all observed Reynolds numbers. This parameter is directly related to the ratio of the $-\overline{u w}$ production and $-\overline{v w}$ production terms. By finding parameters such as $S$ and $C$. which appear invariant for different Reynolds numbers and flow geometries, one is encouraged that a conceptually simple turbulence model will be found which accurately models the flow physics and turbulence production mechanisms.

- There was a significant change in the distribution of Townsend's structural parameter through the boundary layer with increasing $\operatorname{Re}_{\theta}$. This change was created by decreasing $-\overline{u v}$ and $-\overline{v w}$ shear stresses in the log region for the high Reynolds number case. These shear stress reductions were not correlated to the local turbulent kinetic energy. In light of these findings, the author does not recommend using $a_{1}$ for the modeling of turbulent boundary layers in general.

- For the 3-D case, the near-wall lag between $\beta_{\tau}$ and $\beta_{\mathrm{FGA}}$ increases for lower $\operatorname{Re}_{\theta}$ values. This is what one might expect to find as viscous forces become larger relative to the inertial forces in the inner region. No obvious Reynolds number trends appeared in the outer region for the shear stress lag angle.

- The most appropriate or useful scaling was usually found to be the inner scaling variables, $u_{\tau}$ and $u_{\tau} / \nu$. For quantities directly affected by the spanwise flow, evidence was found which indicated that a more appropriate scaling for $y$ was the flow geometry. By scaling $y$ on the maximum wing thickness $T$, the $y$ locations of the local maxima and minima for $\beta_{\mathrm{FA}}, \beta_{\mathrm{FGA}}, \beta_{\tau}$, and $\beta_{\text {lag }}$ were correlated for all $\operatorname{Re}_{\theta}$. This indicates that the minimum $y$ that the Reynolds number effects are significant scales on $T$ for this flow geometry.

\subsection{Flow Visualization Conclusions}

The hydrogen-bubble flow-visualization results were presented in chapter 4 . The LDV measurements provided detailed information about the boundary layers which was used to support the analysis of the flow-visualization data. The resulting data were examined qualitatively using results stored on videotape, and also quantitatively using digital image analysis.

Past research has indicated that near-wall longitudinal vortical structures play an important role in the production of turbulence in boundary layers. Past research has also suggested that increasing 3-dimensionality in a turbulent boundary layer greatly affects the behavior of any embedded streamwise vortical structures. For the present research, the 3-D effects on the interactions between the near-wall vortical structures and low-speed streaky 
structures could not be examined directly, but the results of these changing turbulent flow mechanisms were examined.

Both the qualitative and quantitative results indicated that the presence of a pressureinduced. transverse, mean velocity component led to more stable (or orderly) behavior of the inner-region flow structures. The increased stability of these near-wall flow structures led to less interaction between the inner and outer regions with a decrease in turbulence production.

Observation of both the constant-sheet and time-line flow-visualization results at nominal $y^{+}$locations of 5, 10 and 30 revealed several differences between the 2-D and 3-D TBL flow cases. The 2-D flow displayed more energetic streak oscillations. The vigorous low speed streak/high speed sweep interactions resulted in rapid streak coalescence and division. The strong sweeping motions helped to create large transverse velocity gradients and areas of concentrated vorticity. The 3 -D TBL results revealed a more orderly or quiescent flow structure. The streaky structures were not as prone to interactions with high momentum fluid. The smaller streak oscillations appeared to often occur in unison with other nearby streaks. The low speed streak positions were more stable, since the streaks did not experience the same coalescence/division behavior. The incoming high speed sweeps were not as energetic, and the transverse velocity gradients $(d U / d z)$ were not as great for the 3-D TBL.

Analysis of digitized hydrogen bubble time line flow-visualization data yielded instantaneous $U(z)$ velocity distributions. Unique parameters were used to help quantify the observed differences between the 2-D and 3-D flow structures. The quantitative results support the visual observations and comparisons:

- The streamwise turbulence intensities were approximately $25 \%$ lower for the 3-D case, an indication of the weaker streak/sweep interactions and decreased concentrations of turbulence producing vorticity.

- The low-speed streak spacings for the 3-D TBL were $23 \%$ larger than for the 2-D case. This appears to have been the result of the increased stability of the near-wall flow activity, with less streak coalescence and division. However, the increase in $\overline{\lambda^{+}}$ may have been solely due to the increase in the local skin friction for the 3-D TBL. While more study may be needed in this area, it was clearly obvious that the near-wall streaky structures behaved differently for the 3-D flow.

- The event momentum flux ratio $(E)$, calculated for both low-speed streak and highspeed sweep structures, shows that the 2-D TBL experiences a greater momentum exchange between the inner and outer regions of the boundary layer. The nondimensionalization of this parameter is not affected by changing skin friction values. 
- The instantaneous spatial average of $\omega_{y}(\Omega)$ present in a streak or sweep event was calculated. $\Omega^{+}$for the 2-D turbulent boundary layer was double that of the 3-D layer, illustrating the 2-D layer's increased turbulence production activity.

\subsection{Suggestions for Future Work}

In light of the vast amount of data available for zero-pressure gradient, 2-D wallbounded TBLs. the author suggests that future research of Reynolds number effects or nearwall turbulent flow structures should examine more "non-canonical" flow cases. However, these flows should still have practical applications.

The author's investigation of 3-D Reynolds number affects involved data from 2 different experiments utilizing the same wing-body junction geometry. An improved, more comprehensive experiment would involve using the same measurement apparatus in the same test facility. Although time consuming, obtaining enough data to examine "global" changes in the secondary flow structure with changing Reynolds number would be ideal. The most useful data would extend well into the boundary layer, to at least $y^{+}=10$, and preferably below $y^{+}=5$.

In light of this last test requirement for near-wall flow measurements, and also because higher-order terms are becoming more important in the evolving study of turbulent flow processes, the author recommends 3-component LDV measurements for obtaining the most useful data. Also, past research has shown that the higher-order terms are more sensitive to Reynolds number changes. An extension of the 2-D "quadrant analysis" to a more detailed "octant analysis" for 3-D TBLs would produce useful information.

Future examination of the near-wall turbulence structure using flow-visualization should not only look at the streak or sweep characteristics, but should also examine the quasi-streamwise vortical structures. Emphasis should be placed on how these structures are affected by the transverse pressure gradients and mean flow components. Also, the near-wall region should be examined most closely - the region visualized should be below $y^{+}=30$. Several researchers have examined embedded vortices in 2-D and 3-D TBLs (see chapter 1), but the size of these vortices was on the order of the boundary layer thickness. Future research should concentrate on the small near-wall intermittent structures if we hope to understand the physics of the turbulence production process.

One last suggestion regarding the design of future experiments. In addition to the transverse pressure gradients, the current 3-D flow possessed large streamwise pressure gradients. As noted by the discussion of the results in chapter 4, some ambiguity arose because of these streamwise gradients. An ideal study would impose a transverse pressure 
gradient while minimizing the streamwise pressure gradient. Some design compromises would probably have to be made to get a strong spanwise flow. An extension of the study of 3-D TBLs would involve "cross-over" flows, or flows were the transverse pressure gradient switches directions. Although not shown at the author's measurement location, wing-body junction flows will generate cross-over mean flow profiles. Because we do not yet fully understand the underlying turbulent flow physics, these types of 3-D flow fields have proven to be very difficult to calculate accurately. 
Abbott, I. H. and Von Doenhoff, A. E. (1959) Theory of Wing Sections, Dover Publications, Inc., New York, NY.

Adrian. R. J. and Yao, C. S. (1987) "Power Spectra of Fluid Velocities Measured by Laser Doppler Velocimetry," Experiments in Fluids, Vol. 5, pp. 17-28.

Ahn, S. and Simpson, R. L. (1987) "Convective Wave Speed and Spectral Features of Turbulent Boundary Layers," Paper No. AIAA-87-0198, 25th Aerospace Sciences Meeting and Exhibit, Reno, NV, Jan. 12-15.

Bendat, J. S. and Piersol, A. G. (1986) Random Data, Analysis and Measurement Procedures, John Wiley \& Sons, New York, NY.

Bernard, P. S., Thomas, J. M., and Handler, R. A. (1993) "Vortex Dynamics and the Production of Reynolds Stress," J. of Fluid Mech., Vol. 253, pp. 385-419.

Bogard. D. G. and Tiederman, W. G. (1986) "Burst Detection with Single-Point Velocity Measurements," J. of Fluid Mech., Vol. 162, pp. 389-413.

Bradshaw, P. (1967) "Inactive' Motion and Pressure Fluctuations in Turbulent Boundary Layers," J. of Fluid Mech., Vol. 30, part 2, pp. 241-258.

Bradshaw, P. (1986) "Physics and Modeling of Three-Dimensional Boundary Layers," Computation of Three-Dimensional Boundary Layers Including Separation, AGARD Report No. 741, Neuilly Sur Seine, France.

Bradshaw, P. (1992) "Turbulence: The Chief Outstanding Difficulty of Our Subject," Third Stewartson Memorial Lecture, presented at the Symposium on Numerical and Physical Aspects of Aerodynamic Flows, Long Beach, CA

Bradshaw, P. and Pontikos, N. S. (1985) "Measurements in the Turbulent Boundary Layer on an Infinite Swept Wing," J. of Fluid Mech., Vol. 159, pp. 105-130.

Brown, A. I. and Marco, S. M. (1958) Introduction to Heat Transfer, 3rd ed. McGraw-Hill Book Company, New York.

Bruneau, S. D., Krane, M. H., and Pauley, W. R. (1992) "Unsteady Boundary Layer Velocity Measurements Using Digital Image Processing of Hydrogen Bubble Timelines," Fluids Eng., Vol. 133.

Castro, I. P. (1984) "Effects of Free Stream Turbulence on Low Reynolds Number Boundary Layers," J. of Fluids Eng., Vol. 106, pp. 298-306.

Chesnakas, C. J. and Simpson, R. L. (1994) "An Investigation of the Three-Dimensional Turbulent Flow in the Cross-Flow Separation Region of a 6:1 Prolate Spheroid," Exp. in Fluids, Vol. 17, pp. 68-74.

Chesnakas, C. J. and Simpson, R. L. (1996) "A Detailed Investigation of the 3-D Separation about a 6:1 Prolate Spheroid at Angle of Attack," 34th Aerospace Sciences Meeting and Exhibit, Paper No. AIAA-96-0320, Reno, NV. 
Chiang. C. and Eaton, J. K. (1993) An Experimental Investigation of Corotating Disks and Single Disk Flow Structures, Report MD-62, Thermosciences Division, Dept. of Mech. Eng., Stanford University, Stanford, CA.

Ching, C. Y., Djenidi, L., and Antonia, R. A. (1994) "Low-Reynolds-Number Effects in a Turbulent Boundary Layer," Proceedings of Seventh Int. Sym. on App. of Laser Techniques to Fluid Mechanics, Lisbon, Portugal, July 11-14.

Clutter. D. O. and Smith, A. M. O. (1961) "Flow Visualization by Electrolysis of Water," Aerospace Engineering, Jan., pp. 24-27, 74-76.

Coles, D. (1956) "The Law of the Wake in the Turbulent Boundary Layer," J. of Fluid Mech., Vol. 1, pp. 191-226.

Corrsin, S. (1959) "Outline of Some Topics in Homogeneous Turbulent Flow," J. of Geophysical Research, Vol. 64, p. 2134.

Davis, W. and Fox, R. W. (1967) "An Evaluation of the Hydrogen Bubble Technique for the Quantitative Determination of Fluid Velocities Within Clear Tubes," ASME J. of Basic Eng., Vol. 89, pp. 771-781.

Devenport. W. J. and Simpson, R. L. (1990) "Time-Dependent and Time-Averaged Turbulence Structure Near the Nose of a Wing-Body Junction," J. of Fluid Mech., Vol. 210, pp. 23-55.

Djenidi, L. and Antonia, R. A. (1993) "LDA Measurements in Low Reynolds Number Turbulent Boundary Layer," Experiments in Fluids, (technical note), Vol. 14, pp. 280283.

Durst, F., Jovanovic, J., Kanevce, L. J. (1992) "Probability Density Distribution in Turbulent Wall Boundary Layer Flows," Turbulent Shear Flows, Springer-Verlag, pp. 197-220.

Durst, F., Melling, A., and Whitelaw, J. H. (1981) Principles and Practices of Laser-Doppler Anemometry, 2nd ed., Academic Press, New York, NY.

Eaton, J. K. (1995) "Effects of Mean Flow Three Dimensionality on Turbulent BoundaryLayer Structure," AIAA J., Vol. 33, No. 11, pp. 2020-2025.

Flack, K. A., and Johnston, J. P. (1993) Near-Wall Investigation of Three-Dimensional Turbulent Boundary Layers, Report MD-63, Thermosciences Division, Dept. of Mech. Eng., Stanford University, Stanford, CA.

Fleming, J. L. (1991) An Experimental Study of a Turbulent Wing-Body Junction and Wake Flow, M.S. Thesis, Virginia Polytechnic Institute and State University, Blacksburg, VA.

Fleming, J. L., Simpson, R. L., and Devenport, W. J. (1993) "An Experimental Study of a Turbulent Wing-Body Junction and Wake Flow," Experiments in Fluids, Vol. 14, pp. 366-378.

Fleming, J. L. and Simpson, R. L. (1994) "Experimental Investigation of the Near Wall Flow Structure of a Low Reynolds Number 3-D Turbulent Boundary Layer," 32nd Aerospace Sciences Meeting and Exhibit, Paper No. AIAA-94-0649, Reno, NV, Jan. 10-13. 
Fleming. J. L., Simpson, R. L, and Shinpaugh, K. A. (1995) "Further Investigation of the Near Wall Flow Structure of a Low Reynolds Number 3-D Turbulent Boundary Layer," 33nd Aerospace Sciences Meeting and Exhibit, Paper No. AIAA-95-0788, Reno, NV, Jan. 9-12.

Fogiel, M. (1983) The Fluid Mechanics and Dynamics Problem Solver, Staff of Research and Education Association, Dr. M. Fogiel, Director, Research and Education Association, Piscataway, New Jersey, p. 925.

French, R. H. (1985) Open-Channel Hydraulics, McGraw-Hill Book Company, New York.

Fuchs. W., Nobach, H., and Tropea, C. (1994) "Laser Doppler Anemometry Data Simulation: Application to Investigate the Accuracy of Statistical Estimators," AIAA J., Vol. 32, No. 9, pp. 1883-1889.

Gad-el-Hak, M. and Bandyopadhyay, P. R. (1994) "Reynolds Number Effects in WallBounded Turbulent Flows," Applied Mech. Rev., Vol. 47, No. 88.

Gessner, F. B. (1973) "The Origin of Secondary Flow in Turbulent Flow Along a Corner," J. of Fluid Mech., Vol. 58, pp. 1-25.

Geller, E. W. (1955) An Electrochemical Method of Visualizing the Boundary Layer, J. of Aero. Sciences, Vol. 22, No. 12, pp. 869-870.

Gupta, A. K., Laufer, J., and Kaplan, R. E. (1971) "Spatial Structure in the Viscous Sublayer," J. of Fluid Mech., Vol. 50, part 3, pp. 493-512.

Ha, S. and Simpson, R. L. (1993a) "An Experimental Investigation of a Three-Dimensional Turbulent Boundary Layer Using Multiple-Sensor Probes," Paper 2-3, Ninth Symposium on Turbulent Shear Flows, Kyoto, Japan, Aug. 16-18.

Ha, S. and Simpson, R. L. (1993b) An Experimental Study of Coherent Structures in a Three-Dimensional Turbulent Boundary Layer, VPI Technical Report No. VPI-AOE205, Virginia Polytechnic Institute and State University, Blacksburg, VA.

Hama, F. R. (1954) Trans. Soc. Nav. Arch. Mar. Eng., Vol. 62.

Hancock, P. E. and Bradshaw, P. (1983) "The Effect of Free-Stream Turbulence on Turbulent Boundary Layers," J. of Fluids Eng., Vol. 105, pp. 284-289.

Head, M. R. and Bandyopadhyay, P. (1981) "New Aspects of Turbulent Boundary Layer Structure," J. of Fluid Mech., V. 107, pp. 297-338.

Hoffmann, J. A. and Mohammadi, K. (1991) "Velocity Profiles for Turbulent Boundary Layers Under Freestream Turbulence," J. of Fluids Eng., Vol. 113, pp. 399-404.

Høst-Madsen, A. and Caspersen, C. (1994) "The Limitations in High Frequency Turbulence Spectrum Estimation using the Laser Doppler Anemometer," Proceedings of Seventh Int. Sym. on App. of Laser Techniques to Fluid Mechanics, Lisbon, Portugal, July 11-14.

Hsu, Cheng-Hsing (1989) Laser Doppler Anemometry Measurements of a Confined Turbulent Water Jet with a Uniform Background Flow, Ph.D. Dissertation, Virginia Polytechnic Institute and State University, Blacksburg, VA.

Jiménez, J. and Moin, P. (1991) "The Minimal Flow Unit in Near-Wall Turbulence," J. of Fluid Mech., Vol. 225, pp. 213-240. 
Johnston, J. P. (1960) "On the Three-Dimensional Turbulent Boundary Layer Generated by Secondary Flow," ASME J. Basic Eng., Vol. 92, pp. 233-248.

Johnston, J. P. and Flack, K. A. (1996) "Review - Advances in Three-Dimensional Turbulent Boundary Layers With Emphasis on the Wall-Layer Regions," ASME J. of Fluids Eng., Vol. 118, pp. 219-232.

Kays, W. M. (1966) Convective Heat and Mass Transfer, McGraw-Hill Book Company, United States.

Kim, H. T., Kline, S. J., and Reynolds, W. C. (1968) An Experimental Study of Turbulence Production near a Smooth Wall in a Turbulent Boundary Layer With Zero PressureGradient, Report MD-20, Thermosciences Division, Dept. of Mech. Eng., Stanford University, Stanford, CA.

Kim. H. T., Kline, S. J., and Reynolds, W. C. (1971) "The Production of Turbulence Near a Smooth Wall in a Turbulent Boundary Layer," J. of Fluid Mech., Vol. 50, pp. 133-160.

Kim, S. (1991) Observation and Measurements of Flow Structures in the Stagnation Region of a Wing-Body Junction, Ph.D. Dissertation, Virginia Polytechnic Institute and State University, Blacksburg, VA.

Klebanoff, P. S. (1955) "Characteristics of Turbulence in a Boundary Layer with Zero Pressure Gradient," NACA Rep. 1247.

Klewicki, J. C. and Metzger, M. M. (1996) "Viscous Wall Region Structure in High and Low Reynolds Number Turbulent Boundary Layers," 27th AIAA Fluid Dynamics Conference, Paper No. AIAA-96-2009, New Orleans, LA, June 17-20.

Kline, S. J. and McClintock (1953) "The Description of Uncertainties in Single Sample Experiments," Mech. Eng.

Kollin, A. (1953) "Demostration of Parabolic Velocity Distribution in Laminar Flow," American J. of Physics, Vol. 21, pp. 619-620.

Landahl, M. T. and Mollo-Christensen, E. (1992) Turbulence and Random Processes in Fluid Mechanics, second edition, Cambridge University Press, Melbourne, Australia.

Lee, D. H. and Sung, H. J. (1994) "Three-Dimensional Assesment of Spectral Bias in LDV," Proceedings of Seventh Int. Symposium on Applications of Laser Techniques to Fluid Mechanics, Lisbon, Portugal, July 11-14.

Lindley, Craig A. (1991) Practical Image Processing in C, John Wiley \& Sons, Inc., New York, NY.

Lu, L. J. and Smith, C. R. (1985), "Image Processing of Hydrogen Bubble Flow Visualization for Determination of Turbulence Statistics and Bursting Characteristics," Experiments in Fluids, Vol. 3, pp. 349-356.

Lumley, J. L. (1965) "On the Interpretation of Time Spectra Measured in High Intensity Shear Flows," Physics of Fluids, Vol. 8, p. 1056.

Luse, M. (1993) Bitmapped Graphics Programming in $C++$, Addison-Wesley Publishing Company, Reading, MA. 
McLaughlin, D. K. and Tiedermann, W. G., Jr. (1973) "Biasing Correction for Individual Realization of Laser Anemometer Measurements in Turbulent Flow," The Physics of Fluids. Vol. 16, No. 2, pp. 2082-2088.

Nakagawa, H. and Nezu, I. (1981) "Structure of Space-Time Correlations of Bursting Phenomena in an Open-Channel Flow," J. of Fluid Mech., Vol. 104, pp. 1-43.

Nakayama, A. (1983) "Measurements of Flows in the Trailing-Edge Region of Airfoils," Numerical and Physical Aspects of Aerodynamic Flows, Springer Verlag, pp. 233-255.

Nezu, I. and Nakagawa, H. (1993) Turbulence in Open-Channel Flows, IAHR Monograph Series, A. A. Balkema Publishers, Brookfield, VT.

Ölçmen, S., and Simpson, R. L. (1990) An Experimental Study of a Three-Dimensional Pressure-Driven Turbulent Boundary Layer, VPI Technical Report No. VPI-AOE-178, Virginia Polytechnic Institute and State University, Blacksburg, VA (available from DTIC).

Ölçmen, S., and Simpson, R. L. (1993a) "Evaluation of Algebraic Eddy-Viscosity Models in Three-Dimensional Turbulent Boundary-Layer Flows," AIAA J., Vol. 31, No. 9, pp. $1545-1554$.

Ölçmen, S., and Simpson, R. L. (1993b) "Perspective: On the Near Wall Similarity of ThreeDimensional Turbulent Boundary Layers," J. of Fluids Eng., Vol. 114, pp. 487-495.

Ölçmen, S., and Simpson, R. L. (1995) "An Experimental Study of a Three-Dimensional Pressure-Driven Turbulent Boundary Layer," J. of Fluid Mech., Vol. 290, pp. 225-262.

Ölçmen, S. (1997) personal communication.

Ölçmen, S., and Simpson, R. L. (1997) "Some Features of a Turbulent Wing-Body Junction Vortical Flow," 35th Aerospace Sciences Meeting and Exhibit, Paper No. AIAA-97-0651, Reno, NV, Jan. 6-10.

Oldaker, D. K. and Tiederman, W. G. (1977) "Spatial Structure of the viscous Sublayer in Drag-Reducing Channel Flows," The Physics of Fluids, Vol. 20, No. 10, Part II, pp. S133-S144.

Peled, Abraham and Liu, Bede (1976) Digital Signal Processing (Theory, Design, and Implementation), John Wiley \& Sons, Inc., New York, NY.

Perry, A. E., Henbest, S., and Chong, M. S. (1986) "A Theoretical and Experimental Study of Wall Turbulence," J. of Fluid Mech., Vol. 165, pp. 163-199.

Perry, A. E., Lim, K. L., and Henbest, S. M. (1985) "A Spectral Analysis of Smooth FlatPlate Boundary Layers," 5th Symposium on Turbulent Shear Flows, Ithaca, New York.

Plesniak, M. (1995) Private communication.

Praturi, A. K. and Brodkey, R. S. (1978) "A Stereoscopic Visual Study of Coherent Structures in Turbulent Shear Flow," J. of Fluid Mech., Vol. 89, pp. 251-272.

Purtell, L. P., Klebanoff, P. S., and Buckley, F. T. (1981) "Turbulent Boundary Layer at Low Reynolds Number," Phys. Fluids, Vol. 24, No. 5. 
Robinson. S. K. (1991a) The Kinematics of Turbulent Boundary Layer Structure, NASA TM 103859.

Robinson, S. K. (1991b) "Coherent Motions in the Turbulent Boundary Layer," Annual Rev. of Fluid Mech., Vol. 23, pp. 601-639.

Robinson, S. K., Kline, S. J., and Spalart, P. R. (1991) Turbulence Physics of a Low Reynolds Number Boundary Layer, NASA TM 103859 (video).

Runstadler, P. W., Kline, S. J., and Reynolds, W. C. (1963) Report MD-8, Dept. of Mech. Eng., Stanford University, Stanford, CA.

Schlichting, H. (1955) Boundary-Layer Theory, McGraw-Hill Book Company, New York, NY.

Schraub, F. A., Kline, S. J., Henry J., Runstadler, P. W. Jr., and Littell, A. (1964) "Use of Hydrogen Bubbles for Quantitative Determination of Time-Dependent Velocity Fields in Low-Speed Water Flows," Paper No. 64-WA/FE-20, ASME Winter Annual Meeting, New York, NY, Nov. 29-Dec. 3.

Sellin, R. H. J. (1970) Flow in Channels, Gordon and Breach Science Publishers, New York.

Senstad, O. and Moin, P. (1992) The Near Wall Mechanics of Three-Dimensional Turbulent Boundary Layers, Report TF-57, Thermosciences Division, Dept. of Mech. Eng., Stanford University, Stanford, CA.

Simpson, R. L. and Devenport, W. J. (1990) "A Conceptual Model for the Near Wall Region of Three-Dimensional Turbulent Boundary Layers," Boundary-Layer Structural Workshop, Langley Research Center, Hampton, VA, August 28-30.

Smith, C. R., and Metzler, S. P. (1983) "The Characteristics of Low-Speed Streaks in the Near-Wall Region of a Turbulent Boundary Layer," J. of Fluid Mech., Vol. 129, pp. $27-54$.

Spalart, P. R. (1988) "Direct Simulation of a Turbulent Boundary Layer up to $\operatorname{Re}_{\theta}=1410$," J. of Fluid Mech., Vol. 187, pp. 61-98.

Srikantaiah, D. V. and Coleman, H. W. (1985) "Turbulence Spectra from Individual Realization Laser Velocimetry Data," Experiments in Fluids, Vol. 3, pp. 35-44.

Tennekes, H. and Lumley, J. L. (1972) A First Course in Turbulence, The MIT Press, Cambridge, MA.

Thole, K. A. and Bogard, D. G. (1996) "High Freestream Turbulence Effects on Turbulent Boundary Layers," J. of Fluids Eng., Vol. 118, pp. 276-284.

Touloukian, Y. S. and Ho, C. Y. (1975) Thermophysical Properties of Matter - The TPRC Data Series, Y. S. Touloukian and C. Y. Ho (eds.), IFI/Plenum Data Co., New York, Vol. 11, p. 94 (as presented by Schetz, 1984).

Townsend, A. A. (1976) The Sturcture of Turbulent Shear Flow, (second edition), Cambridge University Press, New York, NY. 
Tummers, M. J., Absil L. H. J., and Passchier, D. M. (1992) "An Experimental Investigation of Velocity Bias in a Turbulent Flow," Proceedings of Sixth Int. Symposium on Applications of Laser Techniques to Fluid Mechanics and Workshop on Computers in Flow Measurements, Lisbon, Portugal, July 20-23.

Van Dyke, M. (1982) An Album of Fluid Motion, The Parabolic Press, Stanford, CA.

Waesche, R. H. W., Sargent, W. H., and Marchman, J. F. III (1989) "Space Shuttle Solid Rocket Motor Aft-End Internal Flows," J. of Propulsion and Power, Vol. 5, No. 6, pp. 650-656.

Wallace, J. M., Eckelmann, H., and Brodkey, R. (1972) "The Wall Region in Turbulent Shear Flow," J. of Fluid Mech., Vol. 54, pp. 39-48.

Wetzel, T. G. and Simpson, R. L. (1997) "Unsteady Three-Dimensional Crossflow Separation Measurements on a Prolate Spheroid Undergoing Time-Dependent Maneuvers," 35th Aerospace Sciences Meeting and Exhibit, Paper No. AIAA-97-0618, Reno, NV, Jan. 6-10.

Willmarth, W. W. and Lu, S. S. (1972) "Structure of the Reynolds Stress Near the Wall," J. of Fluid Mech., Vol. 55, pp. 65-92.

Winter, A. R., Graham, L. J. W., and Bremhorst, K. (1991) "Effects of Time Scales on Velocity Bias in LDA Measurements Using Sample and Hold Processing," Exp. in Fluids, Vol. 11, pp. 147-152.

White, F. M. (1974) Viscous Fluid Flow, McGraw-Hill Inc, United States.

Wortmann, F. X. (1953) "Eine Method zur Beobachtung ung Messung von Wasserstromungen mit Tellur," Zeitschrift für Angewandte Physik, 5 band, heft 6 .

Yeh. Y. and Cummins, H. Z. (1964) "Localized Flow Measurements with an He-Ne Laser Spectrometer," Appl. Phys. Letters, Vol. 4, p. 176. 
TABLES 
Table 1.1 Experimental test matrix for the laser Doppler velocimetry measurements. Listed $U_{\text {ref }}$ values are nominal, and $\operatorname{Re}_{\theta}$ values were estimated a priori using Kim's LDV data (1991). Actual test values for $U_{e}$ and $\operatorname{Re}_{\theta}$ are shown in Table 3.1. Locations are referenced to the tunnel coordinate system shown in Fig. 2.3 .

\begin{tabular}{|c|c|c|c|c|c|}
\hline $\begin{array}{l}\text { Meas. } \\
\text { Type }\end{array}$ & $\begin{array}{l}\text { Location } \\
(x, z)(\mathrm{cm})\end{array}$ & $\begin{array}{c}U_{\text {ref }} \\
(\mathrm{cm} / \mathrm{s}) \\
\end{array}$ & $\begin{array}{l}\operatorname{Re}_{\theta} \\
\text { (est.) } \\
\end{array}$ & $\begin{array}{l}\text { LDV system } \\
\text { orientation }(\mathrm{s})\end{array}$ & $\begin{array}{l}\text { Estimated } y \text { locations } \\
\text { for long data records }\end{array}$ \\
\hline $2-\mathrm{D}$ & $(-4.7,-3.0)$ & 10 & 330 & $0^{\circ}$ & $\begin{array}{l}y^{+} \approx 10 \\
y / \delta \approx .5\end{array}$ \\
\hline $2-\mathrm{D}$ & $(-4.7,-3.0)$ & 15 & 450 & $0^{\circ}$ & $\begin{array}{c}y^{+} \approx 10,50 \\
y / \delta \approx .5, .8,>1\end{array}$ \\
\hline $2-\mathrm{D}$ & $(-4.7,-3.0)$ & 20 & 575 & $0^{\circ}$ & $\begin{array}{c}y^{+} \approx 10,50 \\
y / \delta \approx .5, .8,>1\end{array}$ \\
\hline $2-\mathrm{D}$ & $(-4.7,-3.0)$ & 30 & 790 & $0^{\circ}$ & $\begin{array}{l}y^{+} \approx 10 \\
y / \delta \approx .5\end{array}$ \\
\hline $3-\mathrm{D}$ & $(1.31,-14.9)^{*}$ & 10 & 330 & $0^{\circ},-45^{\circ},+45^{\circ}$ & $\begin{array}{l}y^{+} \approx 10 \\
y / \delta \approx .5\end{array}$ \\
\hline $3-\mathrm{D}$ & $(1.31,-14.9)^{*}$ & 15 & 450 & $0^{\circ},-45^{\circ},+45^{\circ}$ & $\begin{array}{c}y^{+} \approx 10,50 \\
y / \delta \approx .5, .8,>1\end{array}$ \\
\hline $3-\mathrm{D}$ & $(1.31,-14.9)^{*}$ & 20 & 575 & $0^{\circ},-45^{\circ},+45^{\circ}$ & $\begin{array}{l}y^{+} \approx 10 \\
y / \delta \approx .5\end{array}$ \\
\hline
\end{tabular}

Notes:

*Ölçmen and Simpson's station \#5 location (1990, 1995). The negative values of $z$ indicate that the data was taken on the starboard side of the appendage.

Approximately 22 to 25 points were measured for each boundary layer profile, with $\approx 30,000 U, V$ data pairs per point.

${ }^{\dagger}$ Long time record data was used to calculate spectra and autocorrelations at the locations shown for the LDV data. These data records were typically over 15 minutes in length (non-contiguous). 
Table 1.2 Test matrix used for the hydrogen-bubble flow-visualization experiment. Listed $U_{r e f}$ values are nominal, and $\operatorname{Re}_{\theta}$ values were estimated a priori using Kim's LDV data (1991). The 3-D cases are further differentiated by the bubble wire angle (wrt the tunnel $z$-axis). Test $U_{e}$ and $\operatorname{Re}_{\theta}$ values for this experiment are shown in Table 4.1.

\begin{tabular}{|c|c|c|c|c|c|}
\hline \multirow{2}{*}{$\begin{array}{c}U_{\text {ref }} \\
(\mathrm{cm} / \mathrm{s})\end{array}$} & \multirow{2}{*}{$\operatorname{Re}_{\theta}$} & \multicolumn{4}{|c|}{ approximate $y^{+}$locations for: } \\
\cline { 3 - 6 } & & $2-\mathrm{D}$ & $3-\mathrm{D}\left(10^{\circ}\right)$ & $3-\mathrm{D}\left(20^{\circ}\right)$ & $3-\mathrm{D}\left(25^{\circ}\right)$ \\
\hline 10 & 330 & $3,5,10,30$ & $5,10,15,30$ & $\begin{array}{c}5,10,15, \\
20,25,30\end{array}$ & $5,10,15,30$ \\
& & & & $5,10,15$, & $5,10,15,30$ \\
& 450 & $5,10,30$ & $5,10,15,30$ & $\begin{array}{c}5,25,30 \\
20,30\end{array}$ \\
\hline 15 & & & & $5,10,15$, & $5,10,15,30$ \\
& 575 & $5,10,30$ & $5,10,15,30$ & $5,25,30$ & \\
\hline 30 & 790 & $5,10,30$ & - & - & - \\
\hline
\end{tabular}


Table 2.1 Fluid properties for the different parts of the author's experimental work.

\begin{tabular}{|c|c|c|c|}
\hline Measurement & Temperature & $\mu$ & $\nu$ \\
\hline 2-D flow vis. & $17^{\circ} \mathrm{C}$ & $1.046 \pm 0.1 \mathrm{cps}$ & $0.0105 \pm 0.001 \mathrm{~cm}^{2} / \mathrm{sec}$ \\
\hline 3-D flow vis. & $20^{\circ} \mathrm{C}$ & $0.993 \pm 0.1 \mathrm{cps}$ & $0.0099 \pm 0.001 \mathrm{~cm}^{2} / \mathrm{sec}$ \\
\hline LDV (2-D and 3-D) & $18^{\circ} \mathrm{C}$ & $1.054 \pm 0.1 \mathrm{cps}$ & $0.0106 \pm 0.001 \mathrm{~cm}^{2} / \mathrm{sec}$ \\
\hline
\end{tabular}

Note:

*Author's estimate based on published data. 
Table 2.2 Freestream parameters for nominal tunnel speeds of $10,15,20$, and $30 \mathrm{~cm} / \mathrm{s}$. All data points were taken $14 \mathrm{~cm}$ above the test section floor at the $2-\mathrm{D}$ boundary layer profile location $(x=-4.7, z=-3.0)$. Values of $\delta, C_{f}$, and $H$ are reprinted from Table 3.1 .

\begin{tabular}{|c|c|c|c|c|}
\hline Quantity & $U_{r e f}=10$ & $U_{\text {ref }}=15$ & $U_{\text {ref }}=20$ & $U_{\text {ref }}=30$ \\
\hline $\operatorname{Re}_{\theta}$ & 450 & 300 & 425 & 570 \\
\hline$\delta(\mathrm{cm})$ & 5.4 & 2.2 & 2.2 & 1.9 \\
\hline$u^{\prime} / U_{e}(\times 100)$ & 1.7 & 1.6 & 1.8 & 1.9 \\
\hline$T_{\Lambda}(\mathrm{sec})$ & 0.39 & 0.26 & 0.17 & 0.093 \\
\hline$L_{\Lambda}(\mathrm{cm})$ & 3.75 & 3.63 & 3.07 & 2.51 \\
\hline$T_{\lambda}(\mathrm{sec})$ & 0.30 & 0.25 & 0.14 & 0.051 \\
\hline$L_{\lambda}(\mathrm{cm})$ & 2.92 & 3.53 & 2.59 & 1.38 \\
\hline$L_{\Lambda} / \delta$ & 0.69 & 1.65 & 1.40 & 1.32 \\
\hline$L_{\lambda} / L_{\Lambda}$ & 0.78 & 0.97 & 0.84 & 0.55 \\
\hline$\Pi$ & 0.137 & 0.184 & 0.126 & 0.236 \\
\hline$F S T P_{L_{\Lambda}}$ & 0.63 & 0.44 & 0.53 & 0.57 \\
\hline$C_{f}\left(\times 10^{3}\right)$ & 5.1 & 5.8 & 5.5 & 4.9 \\
\hline$H$ & 1.55 & 1.61 & 1.54 & 1.50 \\
\hline
\end{tabular}


Table 2.3 Displacements for the water tunnel test section false side walls. See Figs. 2.13 and 2.14 and section 2.1.6 for more details. Measurements are given with respect to the tunnel coordinate svstem, as shown in Fig. 2.3.

\begin{tabular}{|c|c|}
\hline \multicolumn{2}{|c|}{ 2-D flow case } \\
\hline$x(\mathrm{~cm})$ & $z(\mathrm{~cm})$ \\
\hline-117.9 & 21.8 \\
\hline-86.4 & 22.1 \\
\hline-43.2 & 22.5 \\
\hline 12.7 & 22.9 \\
\hline 43.2 & 23.1 \\
\hline 73.7 & 23.3 \\
\hline 115.3 & 23.5 \\
\hline
\end{tabular}

\begin{tabular}{|c|c|}
\hline \multicolumn{2}{|c|}{ 3-D flow case } \\
\hline$x(\mathrm{~cm})$ & $z(\mathrm{~cm})$ \\
\hline-117.9 & 21.5 \\
\hline-86.4 & 21.7 \\
\hline-43.2 & 22.4 \\
\hline 12.7 & 25.1 \\
\hline 43.2 & 24.5 \\
\hline 73.7 & 22.8 \\
\hline 106.7 & 22.4 \\
\hline
\end{tabular}


Table 2.4 Estimated uncertainties for various quantities affecting the LDV experimental results. Uncertainties are given for 20:1 odds.

\begin{tabular}{|c|c|}
\hline Quantity & Uncertainty \\
\hline$d_{f s}$ & $\pm 0.7 \%$ \\
\hline freq (nominal data rate) & $\pm 160 \mathrm{~Hz}$ \\
\hline freq $^{(\text {fast data rate) }}{ }^{*}$ & $\pm 320 \mathrm{~Hz}$ \\
\hline$U_{\perp}, V_{\perp}$ & $\pm 0.1 \mathrm{~cm} / \mathrm{s}$ \\
\hline$x, z$ & $\pm 0.1 \mathrm{~cm}$ \\
\hline$y$ & $\pm 0.008 \mathrm{~cm}$ \\
\hline$\Delta y$ & $\pm 0.0015 \mathrm{~cm}$ \\
\hline
\end{tabular}

Note:

*Increase in the frequency uncertainty is due to the reduction in points used for FFT calculations by the Macrodyne LDV processors. The fast data rate was used for the autocorrelation and spectral data. 
Table 2.5 Nominal uncertainties for LDV data at 20:1 odds. The uncertainties are given as a fraction of the maximum profile values for that quantity, except for $V$ and $v^{\prime}$, which are based on the $U$ and $u^{\prime}$ maximum profile values. Uncertainties for 3-D data are for tunnel coordinate data, except as noted.

\begin{tabular}{|c|c|}
\hline \multicolumn{2}{|c|}{ 2-D flow case } \\
\hline Quantity & Uncertainty \\
\hline$U$ & $\pm 2.5 \%$ \\
\hline$V$ & $\pm 4 \%$ \\
\hline$u^{\prime}$ & $\pm 4 \%$ \\
\hline$v^{\prime}$ & $\pm 7 \%$ \\
\hline$-\overline{u v}$ & $\pm 7 \%$ \\
\hline$u_{\tau}$ & $\pm 5 \%$ \\
\hline
\end{tabular}

\begin{tabular}{|c|c|}
\hline \multicolumn{2}{|c|}{3 -D flow case } \\
\hline Quantity & Uncertainty \\
\hline$U, V$ & $\pm 2.5 \%$ \\
\hline$u^{\prime}, v^{\prime}$ & $\pm 4 \%$ \\
\hline$-\overline{u v}$ & $\pm 7 \%$ \\
\hline$W$ & $\pm 3.5 \%$ \\
\hline$w^{\prime}$ & $\pm 5 \%$ \\
\hline$-\overline{v w},-\overline{u w}$ & $\pm 8.5 \%$ \\
\hline$u_{\tau}{ }^{*}$ & $\pm 7 \%$ \\
\hline$\beta_{\mathrm{FA}}{ }^{*}$ & $\pm 4 \%$ \\
\hline$\beta_{\mathrm{FGA}}{ }^{*}$ & $\pm 6 \%$ \\
\hline$\beta_{\tau}{ }^{*}$ & $\pm 11 \%$ \\
\hline
\end{tabular}

Note:

${ }^{*}$ Uncertainties on derived quantities in freestream coordinates. 
Table 3.1 Boundary layer parameters for 2-D and 3-D cases. 3-D results are in freestream coordinates. Ölçmen data is from Ölçmen and Simpson (1995).

\begin{tabular}{|c|c|c|c|c|c|c|c|c|}
\hline Quantity & \multicolumn{4}{|c|}{ 2-D cases } & \multicolumn{3}{c|}{ 3-D cases } & Ölçmen \\
\hline$U_{\text {ref }}(\mathrm{cm} / \mathrm{s}$. nom. $)$ & 10 & 15 & 20 & 30 & 10 & 15 & 20 & $27.5 \mathrm{~m} / \mathrm{s}$ \\
\hline$U_{e}(\mathrm{~cm} / \mathrm{s})$ & 9.4 & 13.8 & 18.9 & 29.1 & 11.7 & 17.1 & 23.3 & $29.5 \mathrm{~m} / \mathrm{s}$ \\
\hline $\operatorname{Re}_{\theta}$ & 450 & 300 & 425 & 570 & 500 & 760 & 890 & 9520 \\
\hline$u_{\tau}(\mathrm{cm} / \mathrm{s})$ & 0.48 & 0.74 & 0.99 & 1.44 & 0.73 & 0.97 & 1.29 & $1.15 \mathrm{~m} / \mathrm{s}$ \\
\hline$C_{f} \times 10^{3}$ & 5.1 & 5.8 & 5.5 & 4.9 & 7.8 & 6.4 & 6.1 & 3.0 \\
\hline$\delta(\mathrm{cm})$ & 5.4 & 2.2 & 2.2 & 1.9 & 4.2 & 4.3 & 4.2 & 4.0 \\
\hline$\delta^{*}(\mathrm{~cm})$ & 0.78 & 0.37 & 0.36 & 0.30 & 0.68 & 0.63 & 0.52 & 0.54 \\
\hline$\theta(\mathrm{cm})$ & 0.50 & 0.23 & 0.23 & 0.20 & 0.48 & 0.46 & 0.40 & 0.41 \\
\hline$H$ & 1.55 & 1.61 & 1.54 & 1.50 & 1.43 & 1.36 & 1.32 & 1.32 \\
\hline$\beta_{e \mathrm{TC}}$ & - & - & - & - & $-7.5^{\circ}$ & $-8.1^{\circ}$ & $-9.2^{\circ}$ & $-7.7^{\circ}$ \\
\hline$\beta_{w \mathrm{TC}}$ & - & - & - & - & $-5.5^{\circ}$ & $-15.3^{\circ}$ & $-13.8^{\circ}$ & $-19.7^{\circ}$ \\
\hline
\end{tabular}


Table 3.2 Boundary layer shape parameters for the 3-D cases at station 5 . These results are presented in free-stream coordinates. Ölçmen data is from Ölçmen and Simpson (1995). Definitions for the different shape parameters are shown below the table, and are the same as those used by Ölçmen and Simpson.

\begin{tabular}{|c|c|c|c|c|}
\hline Quantity & \multicolumn{2}{|c|}{ current 3-D cases } & Ölçmen \\
\hline $\operatorname{Re}_{\theta}$ & 500 & 760 & 890 & 9520 \\
\hline$\delta(\mathrm{mm})$ & 42.4 & 42.5 & 42.0 & 39.6 \\
\hline$\delta_{1}(\mathrm{~mm})$ & 6.80 & 6.32 & 5.21 & 5.37 \\
\hline$\delta_{2}(\mathrm{~mm})$ & 6.74 & 6.24 & 5.12 & 5.23 \\
\hline$\delta_{3}(\mathrm{~mm})$ & 4.76 & 4.63 & 3.95 & 4.14 \\
\hline$\delta_{4}(\mathrm{~mm})$ & 0.99 & 1.27 & 1.62 & 1.90 \\
\hline$\delta_{5}(\mathrm{~mm})$ & -0.30 & -0.34 & -0.32 & -0.47 \\
\hline$\delta_{6}(\mathrm{~mm})$ & 0.70 & 0.92 & 1.30 & 1.43 \\
\hline$\delta_{7}(\mathrm{~mm})$ & -0.087 & -0.12 & -0.13 & -0.20 \\
\hline
\end{tabular}

$\delta=y$ where $U / U_{e}=0.995=$ boundary layer thickness

$\delta_{1}=\int_{0}^{\infty}\left(1-\frac{U}{U_{e}}\right) d y=$ streamwise displacement thickness

$\delta_{2}=\int_{0}^{\infty}\left(1-\frac{\left(U^{2}+W^{2}\right)^{1 / 2}}{U_{e}}\right) d y=$ magnitude displacement thickness

$\delta_{3}=\int_{0}^{\infty} \frac{U}{U_{e}}\left(1-\frac{U}{U_{e}}\right) d y=$ streamwise momentum thickness

$\delta_{4}=\int_{0}^{\infty}\left(-\frac{W}{U_{e}}\right) d y=$ lateral displacement thickness

$\delta_{5}=\int_{0}^{\infty} \frac{W}{U_{e}}\left(1-\frac{U}{U_{e}}\right) d y=$ lateral momentum thickness

$\delta_{6}=\int_{0}^{\infty}\left(-\frac{W U}{U_{e}^{2}}\right) d y=$ cross-product momentum thickness

$\delta_{7}=\int_{0}^{\infty}\left(-\frac{W^{2}}{U_{e}^{2}}\right) d y=$ cross-flow momentum thickness 
Table 4.1 Actual experimental test matrix for the hydrogen-bubble flow-visualization experiments, separated into 2-D and 3-D sections. Data listed here were calculated using LDV boundary layer profile results. This table can be directly compared to Table 1.1.

\begin{tabular}{|c|c|c|c|}
\hline \multicolumn{3}{|c|}{ 2-D cases, wire at $0^{\circ}, \nu=0.0105 \mathrm{~cm}^{2} / \mathrm{sec}$} \\
\hline$U_{e}(\mathrm{~cm} / \mathrm{s})$ & $\operatorname{Re}_{\theta}$ & $u_{\tau}(\mathrm{cm} / \mathrm{s})$ & $y^{+}$locations \\
\hline 9.4 & 450 & 0.48 & $2.6,4.4,8.8,26.3$ \\
\hline 13.8 & 300 & 0.74 & $4.7,9.4,28.3$ \\
\hline 18.9 & 425 & 0.99 & $4.9,9.7,29.2$ \\
\hline 29.1 & 570 & 1.44 & $4.9,9.8,29.6$ \\
\hline
\end{tabular}

\begin{tabular}{|c|c|c|c|}
\hline \multicolumn{4}{|c|}{$3-\mathrm{D}$ cases, wire at $20^{\circ}, \nu=0.0099 \mathrm{~cm}^{2} / \mathrm{sec}$} \\
\hline$U_{e}(\mathrm{~cm} / \mathrm{s})$ & $\operatorname{Re}_{\theta}$ & $u_{\tau}(\mathrm{cm} / \mathrm{s})$ & $y^{+}$locations \\
\hline 11.7 & 500 & 0.73 & $7.1,14.2,21.4,28.5,35.6,42.7$ \\
\hline 17.1 & 760 & 0.97 & $6.6,13.1,19.7,26.3,32.8,39.4$ \\
\hline 23.3 & 890 & 1.29 & $6.8,13.4,20.2,27.0,33.7,40.4$ \\
\hline
\end{tabular}

Notes:

3-D $U_{e}, \operatorname{Re}_{\theta}$, and $u_{\tau}$ results are expressed in freestream coordinates.

Only the $20^{\circ}$ data is shown for the $3-\mathrm{D}$ case, use Table 1.1 to determine $y^{+}$locations for other wire angles.

Comparison with Table 1.1 will show that the 2-D a priori $y^{+}$estimates were quite accurate, but the 3-D estimates were not. There is a 1:1 correspondence between the $y^{+}$locations listed in this table and the estimates listed in Table 1.1. 
Table 4.2 Near-wall structural parameter results from the 2-D and 3-D hydrogen-bubble flow visualization data, nominal reference velocity $=15 \mathrm{~cm} / \mathrm{s}$. These are quantitative results from the time-line data.

\begin{tabular}{|c|c|c|c|c|}
\hline \multirow{2}{*}{$\begin{array}{c}\text { Event } \\
\text { Parameter }\end{array}$} & \multicolumn{2}{|c|}{$2-\mathrm{D}\left(U_{\text {ref }}=15 \mathrm{~cm} / \mathrm{s}\right)$} & \multicolumn{2}{c|}{$3-\mathrm{D}\left(U_{\text {ref }}=15 \mathrm{~cm} / \mathrm{s}\right)$} \\
\cline { 2 - 5 } & $y^{+}=4.7$ & $y^{+}=9.4$ & $y^{+}=6.6$ & $y^{+}=13.1$ \\
\hline$\overline{\lambda^{+}}$ & 87 & 89 & 109 & 113 \\
\hline$\sigma_{\lambda^{+}}$ & 29.6 & 28.5 & 38.1 & 39.6 \\
\hline$\Psi_{\lambda}$ & 0.34 & 0.32 & 0.35 & 0.35 \\
\hline$\lambda_{\mathrm{mp}}^{+} / \overline{\lambda^{+}}$ & 0.8 & $1.08^{*}$ & 0.9 & 0.87 \\
\hline $\bar{\kappa}_{\mathrm{LS}}$ & 44 & 41 & 52 & 52 \\
\hline $\bar{\varepsilon}_{\mathrm{LS}}$ & -1.7 & -3.4 & -0.9 & -1.9 \\
\hline $\bar{\kappa}^{+}{ }_{\mathrm{HS}}$ & 40 & 42 & 52 & 53 \\
\hline $\bar{\varepsilon}_{\mathrm{HS}}$ & 2.1 & 3.5 & 1.0 & 2.0 \\
\hline $\bar{E}_{\mathrm{LS}}$ & 0.68 & 0.67 & 0.75 & 0.74 \\
\hline $\bar{\Omega}_{\mathrm{LS}}$ & 0.14 & 0.32 & 0.064 & 0.14 \\
\hline $\bar{E}_{\mathrm{HS}}$ & 1.52 & 1.46 & 1.38 & 1.36 \\
\hline $\bar{\Omega}_{\mathrm{HS}}$ & 0.18 & 0.30 & 0.073 & 0.14 \\
\hline
\end{tabular}

Note:

${ }^{*} \lambda_{\mathrm{mp}}^{+}$is not well defined for this flow case. 
FIGURES 
(a)
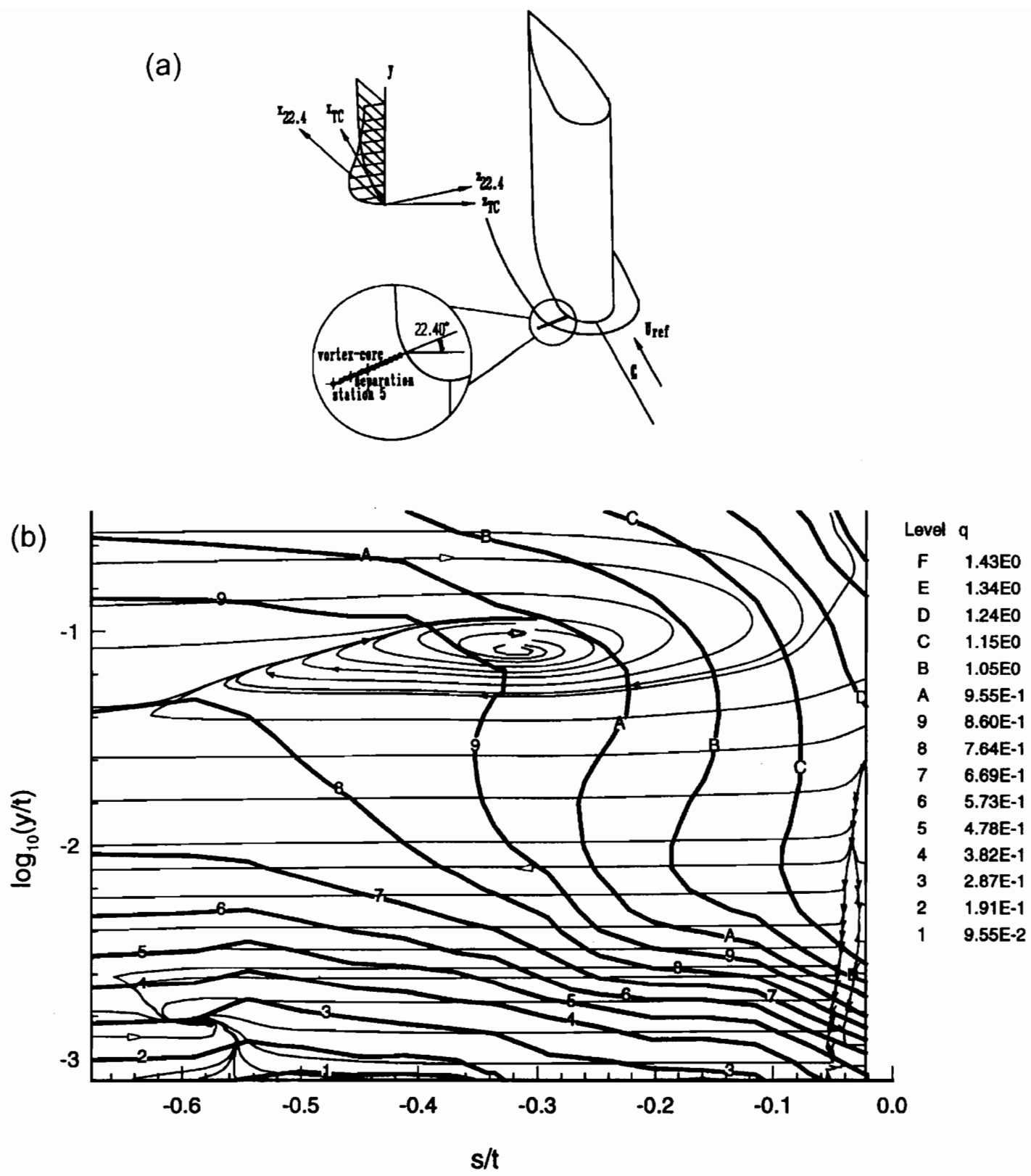

Fig. 1.1 3-D flow around a wing-body junction, from Ölçmen and Simpson (1997). Sketch (a) shows the approximate profile shape of the mean flow in the boundary layer and shows the location of the $y z$ plane containing Ölçmen's station \#5. Plot (b) shows the mean velocity vector magnitudes superimposed over the horseshoe vortex's secondary flow streamlines. Note that the $y$ axis is presented on a log scale, so the primary vortex appears flattened. $\operatorname{Re}_{\theta}$ for this flow was about 9500 at station $\# 5$. 

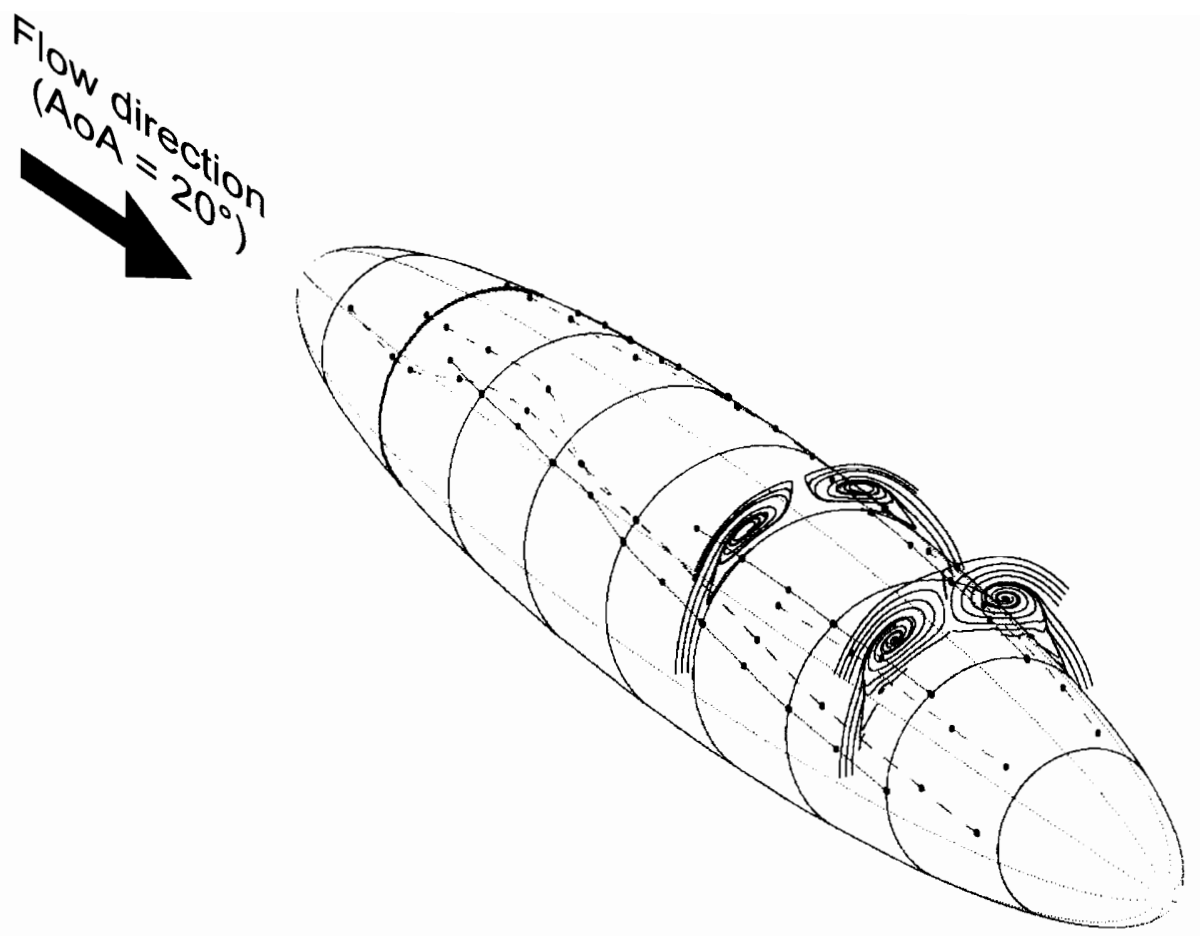

Fig. 1.2 3-D flow patterns around a slender body at angle of attack (from Wetzel and Simpson, 1997). Lines on body show the locations of primary flow separations and reattachment points. The mean secondary flow data are from Chesnakas and Simpson (1996). 


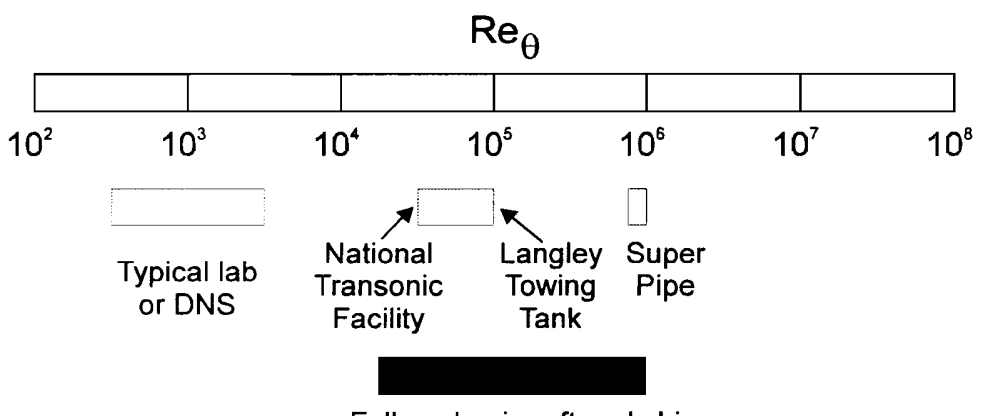

Full-scale aircraft and ships

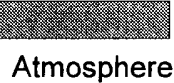

Fig. 1.3 The typical ranges of momentum thickness Reynolds numbers encountered for various types of flows (after Gad-el-Hak and Bandyopadhyay, 1994). 

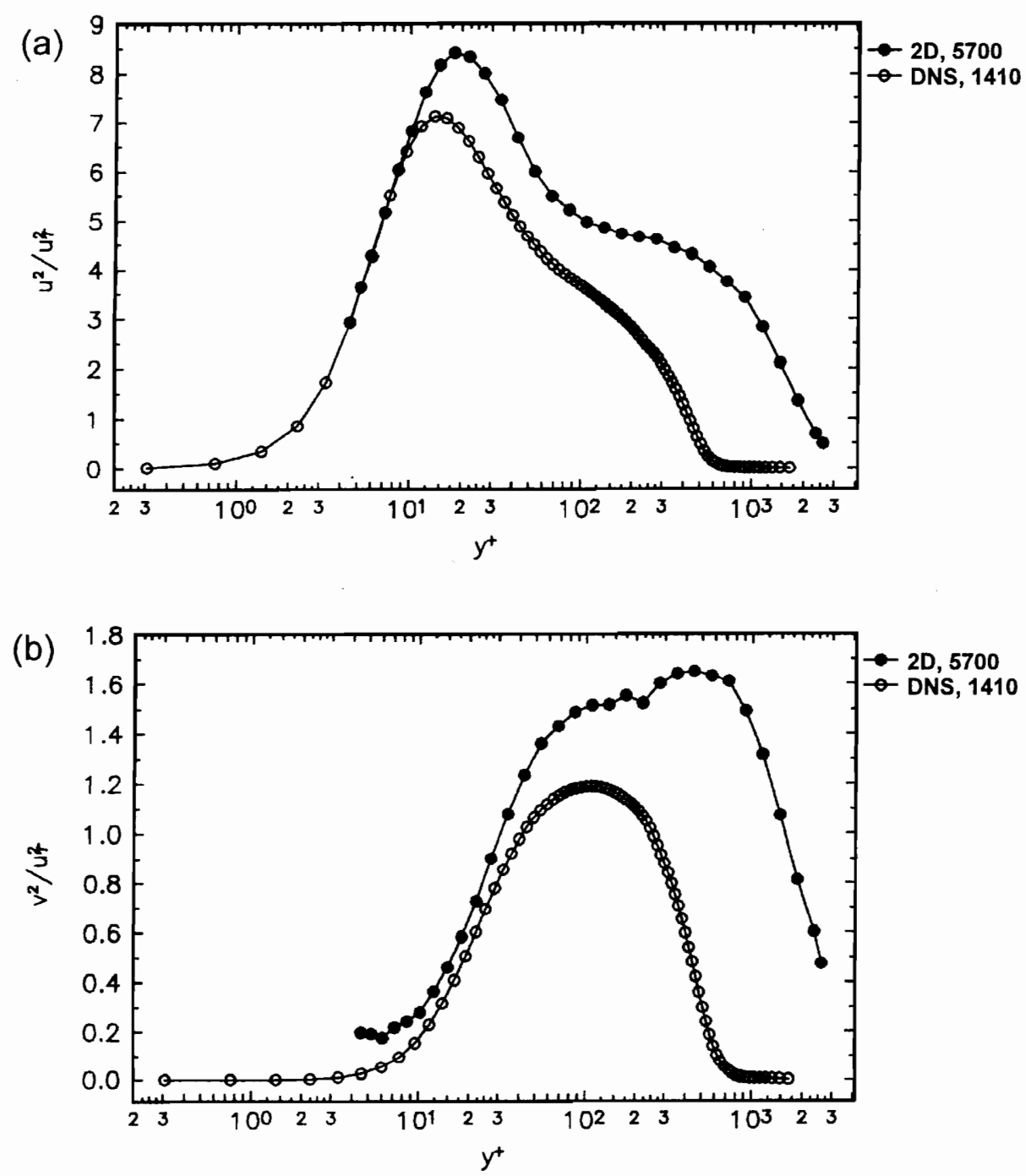

Fig. 1.4 Comparisons of Spalart's (1988) DNS results and Ölçmen and Simpson's (1995) 2-D experimental results. Spalart's simulation was for a Reynolds number of 1410, while Ölçmen and Simpson's Reynolds number was 5700. The $u^{\prime 2}$ results are shown in (a), and the $v^{2}$ results are shown in (b). Both plots use inner flow scaling. Plots are courtesy of Dr. S. Ölçmen (1997). 

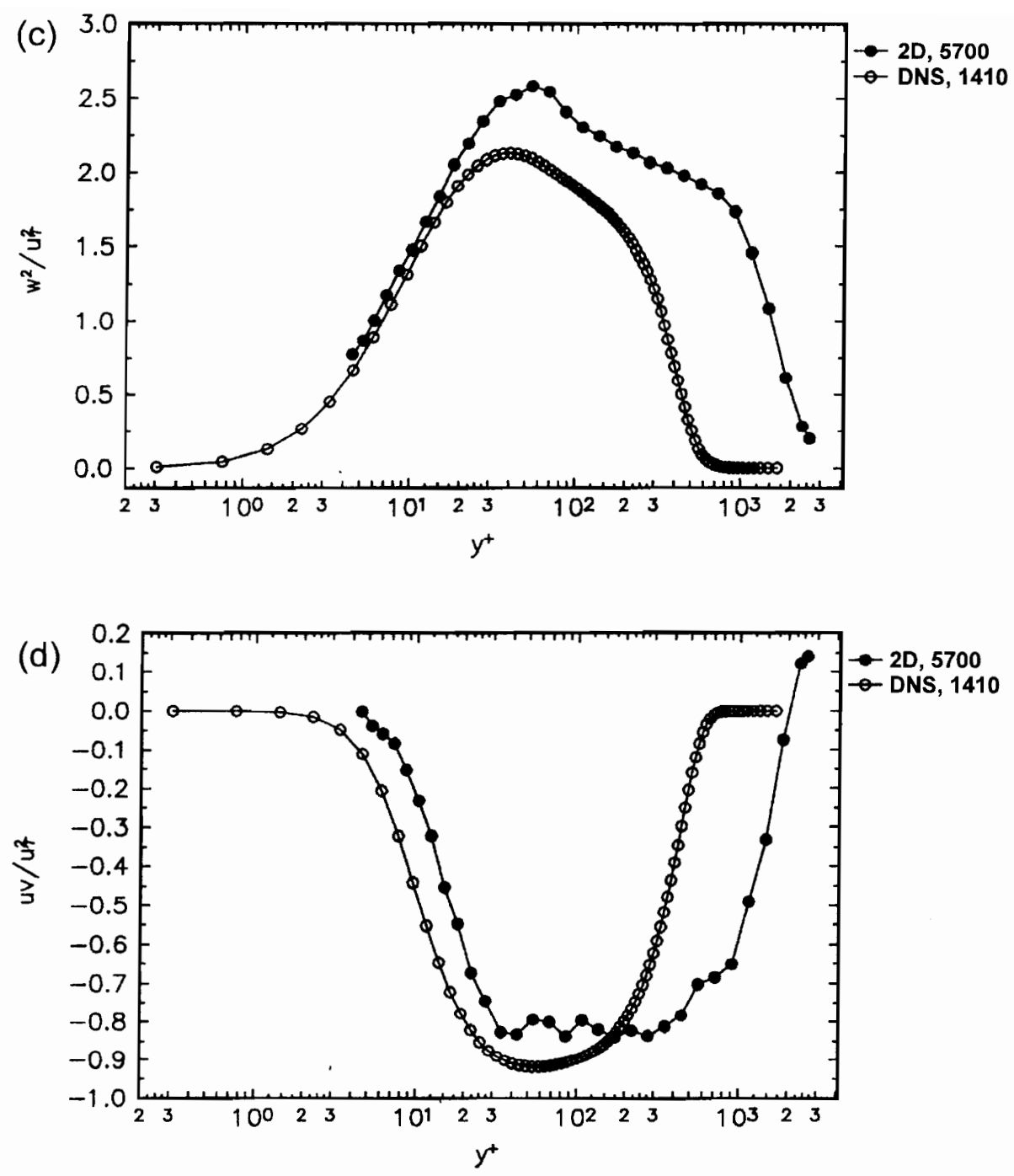

Fig. 1.4 (cont.) More comparisons of Spalart's (1988) DNS results and Ölçmen and Simpson's (1995) 2-D experimental results. Results for $w^{2}$ are shown in (c), and the $u^{\prime} v^{\prime}$ results are shown in (d). Again, both plots use inner flow scaling. Plots are courtesy of Dr. S. Ölçmen (1997). 

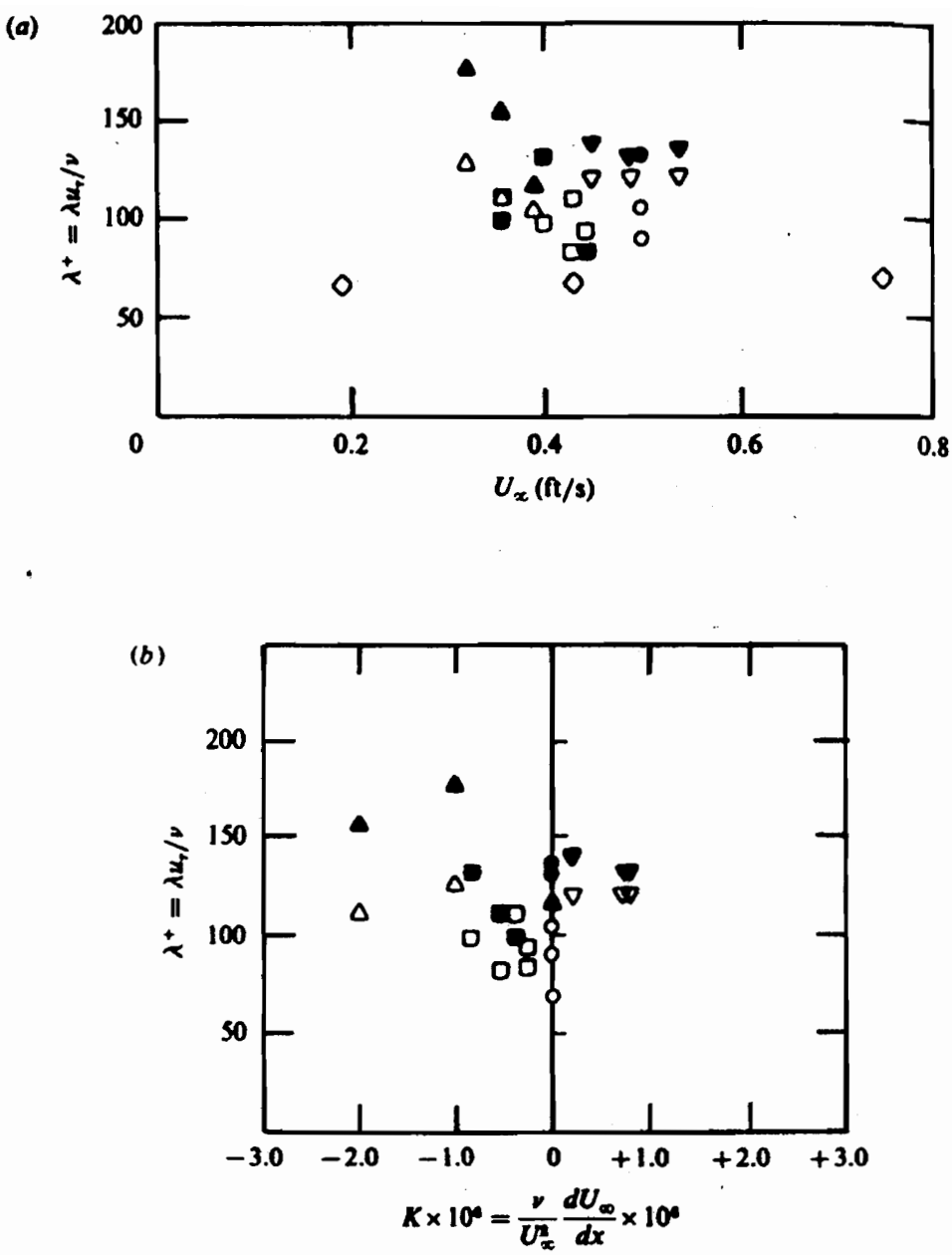

Fig. 1.5 Near-wall low-speed streak spacing results reprinted from Kim et al. (1971). Top figure (a) shows the effects of $U_{e}$, while (b) shows the influence of the streamwise pressure gradient. 


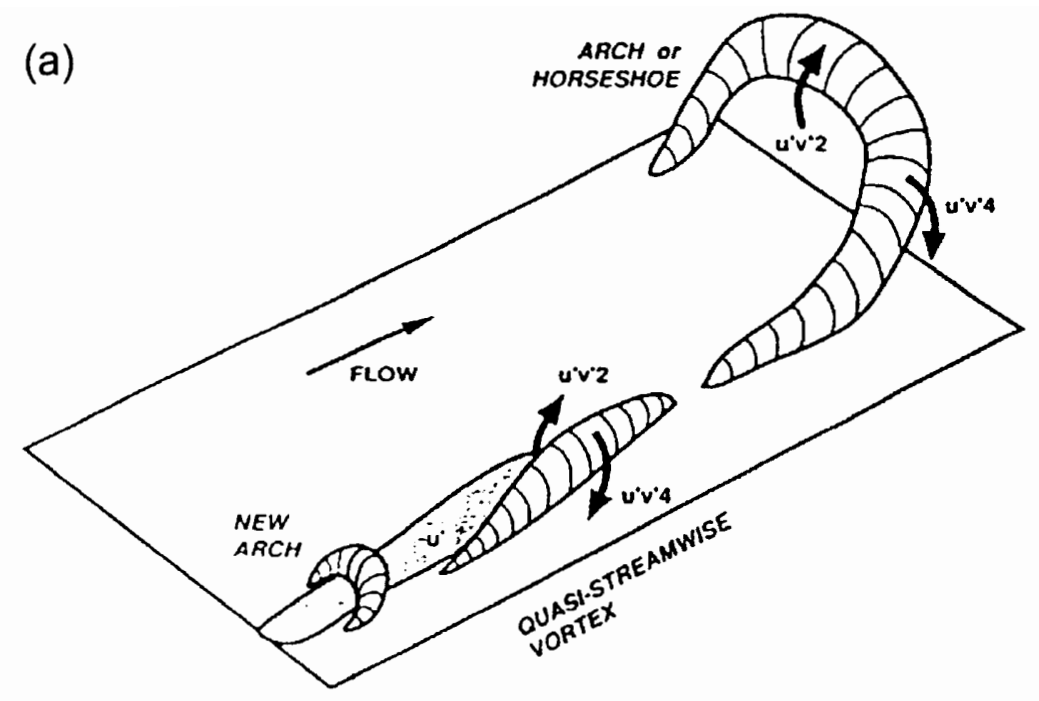

(b)
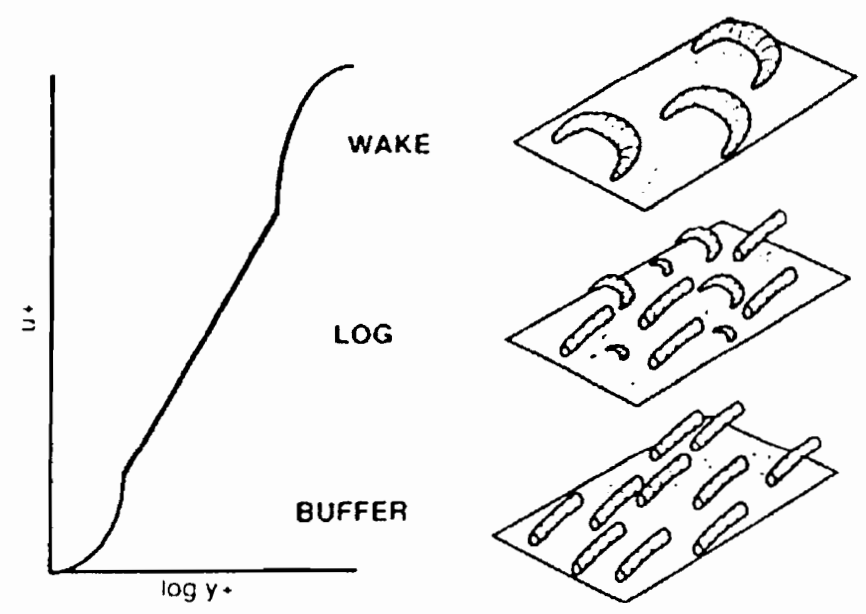

Fig. 1.6 Robinson's observed flow structures from DNS results. Sketch (a) is a simplified arch/trailing leg structure. Sketch (b) shows the flow structures observed in each part of the computed boundary layer. Both drawings are from Robinson (1991). 
(a)

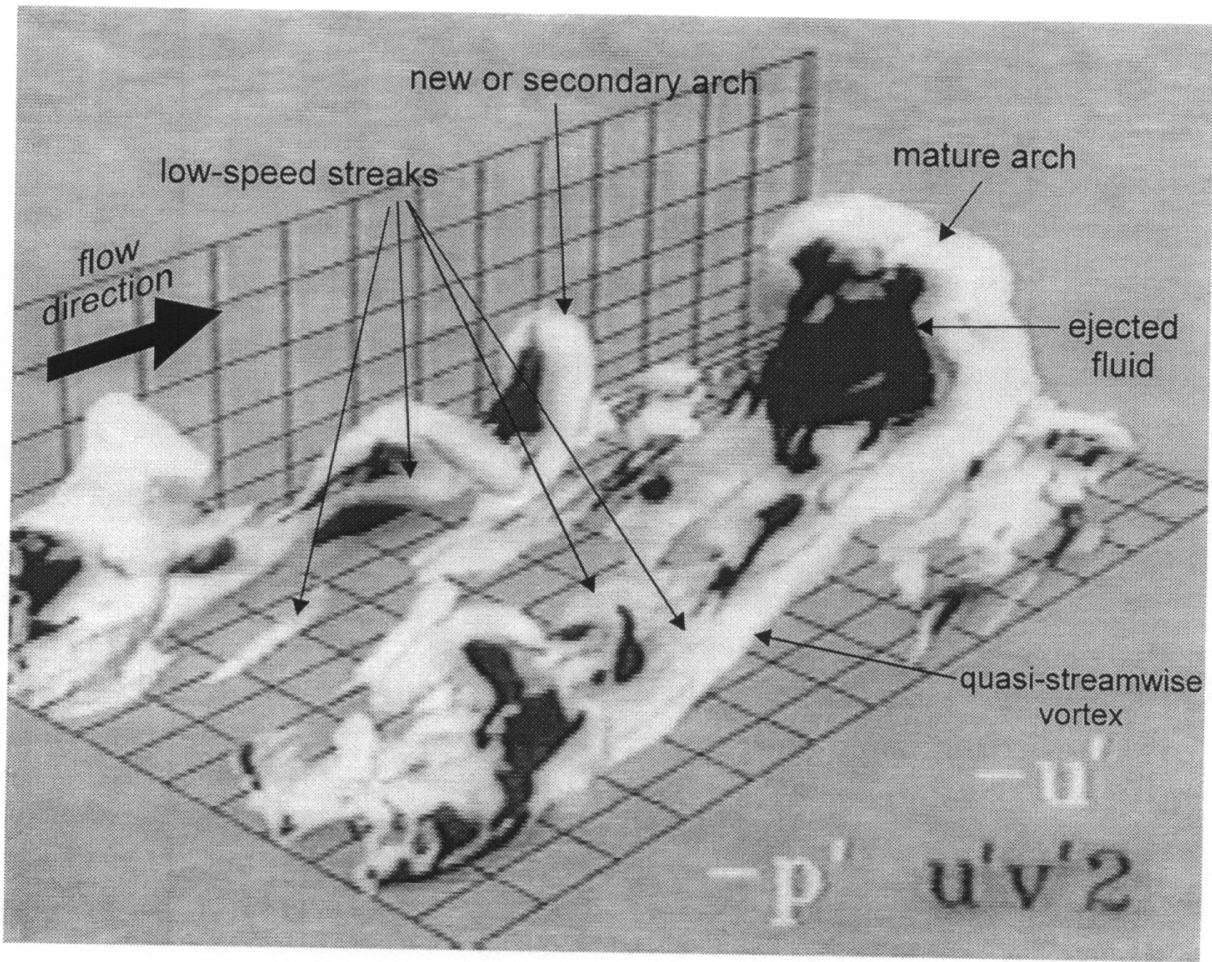

(b)

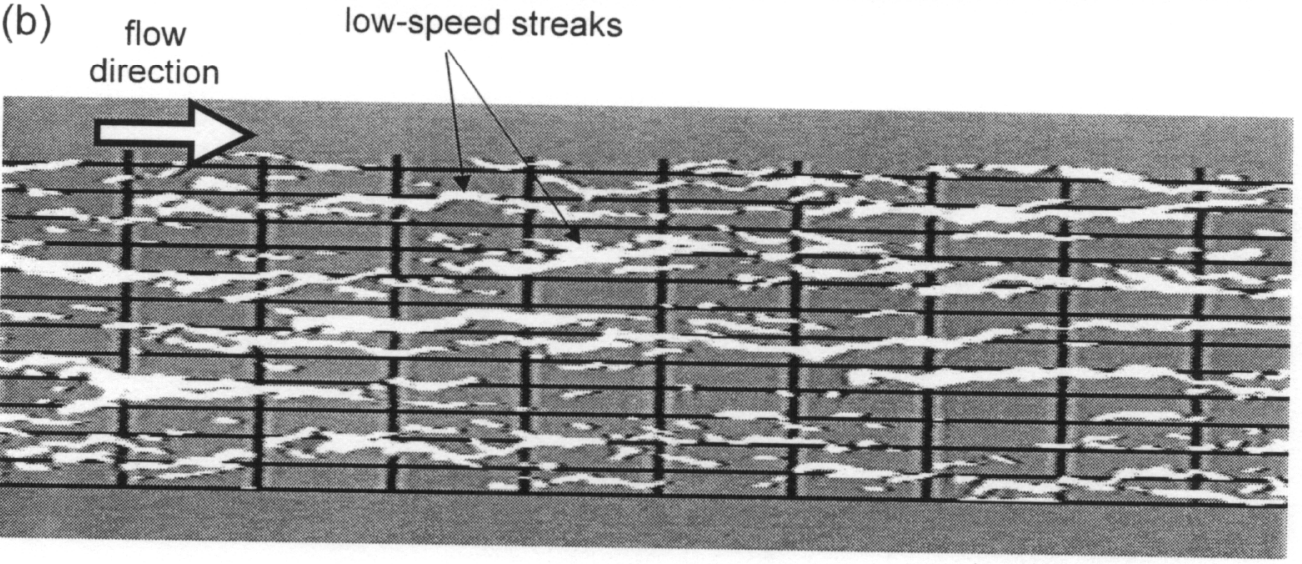

Fig. 1.7 2-D computational results from Robinson et al. (1991), using Spalart's Direct Navier-Stokes (DNS), $\operatorname{Re}_{\theta}=670$. Flow is from left to right for both video stills, Video still (a) shows 3 types of flow structures simultaneously; low speed $(-u)$, low pressure $\left(-p^{\prime}\right)$, and ejections $\left(u^{\prime} v^{\prime} 2\right)$. Frame (b) shows the low-speed streak structure in an $x z$ planar cut at $y^{+}=15$. Highlighted regions are fluid with $u^{\prime+}<-3.0$. Grid spacing is $\Delta x^{+} \approx 80, \Delta z^{+} \approx 35$. 
(a)

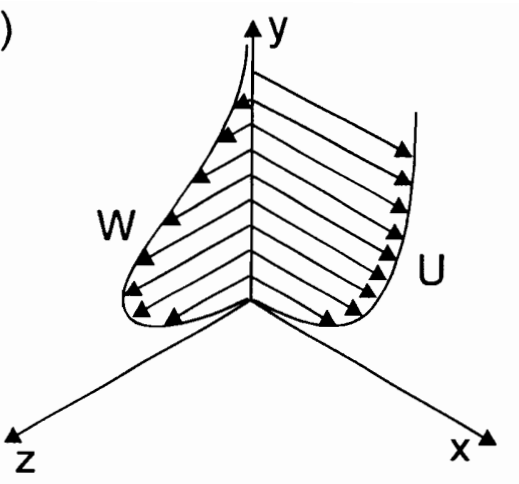

(b)

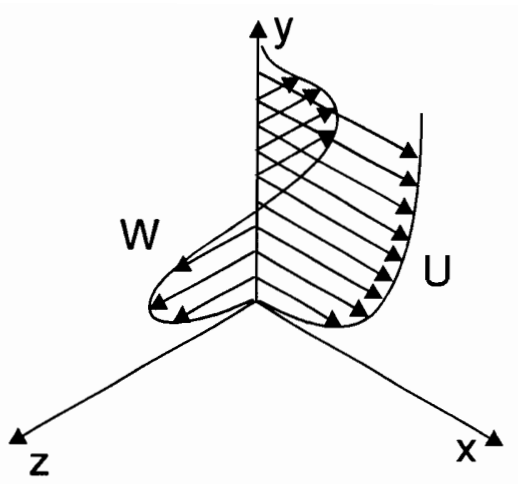

Fig. 1.8 Typical 3-D turbulent boundary layer mean flow distributions. (a) Uni-directional spanwise flow and (b) the more complex bi-directional spanwise flow case (after White, 1974). 
(a)

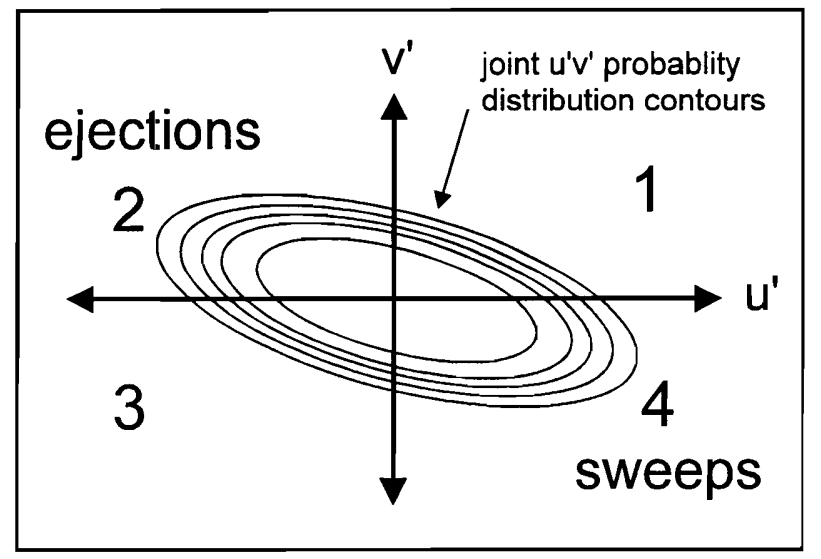

(b)

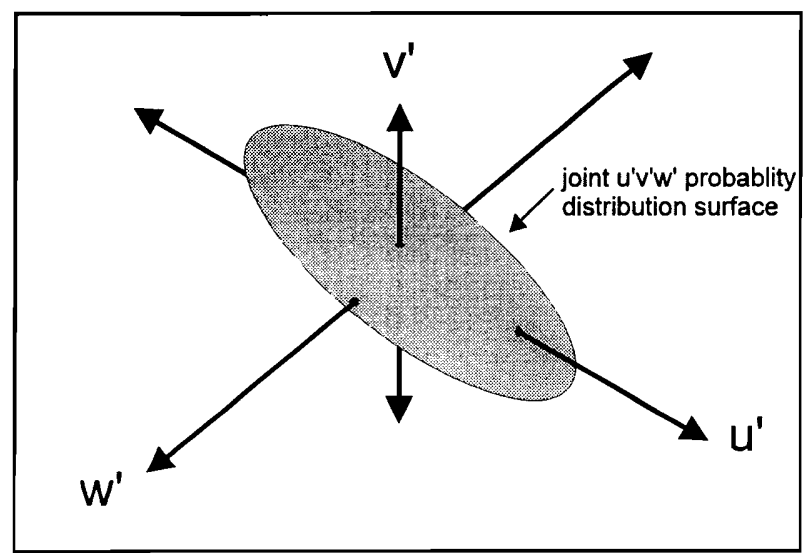

Fig. 1.9 Graphical representations of (a) the 2-D quadrant analysis of Wallace et al. (1972) and Willmarth and Lu (1972), and (b) the 3-D octant analysis proposed by Simpson and Devenport (1990). 


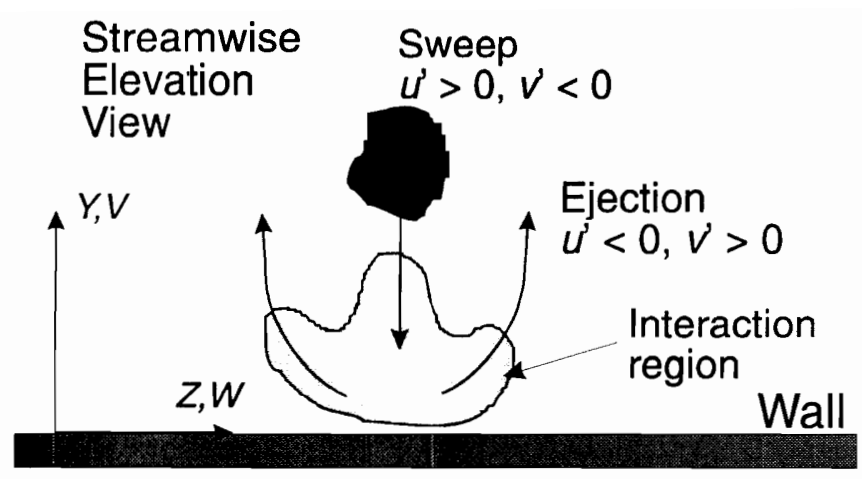

Fig. 1.10 Streamwise elevation view of a conceptual model for the near-wall sweep/ejection process for mean 2-D turbulent boundary layers. 


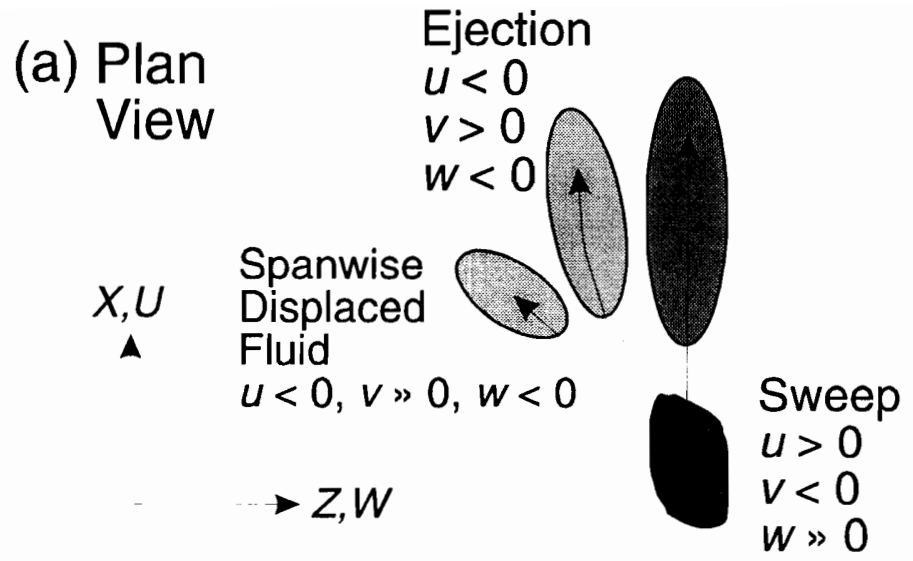

(b) Streamwise Elevation View

Fig. 1.11 Streamwise elevation (b) and plan views (a) of a conceptual model for the nearwall sweep/ejection process for pressure-driven 3-D turbulent boundary layers. 

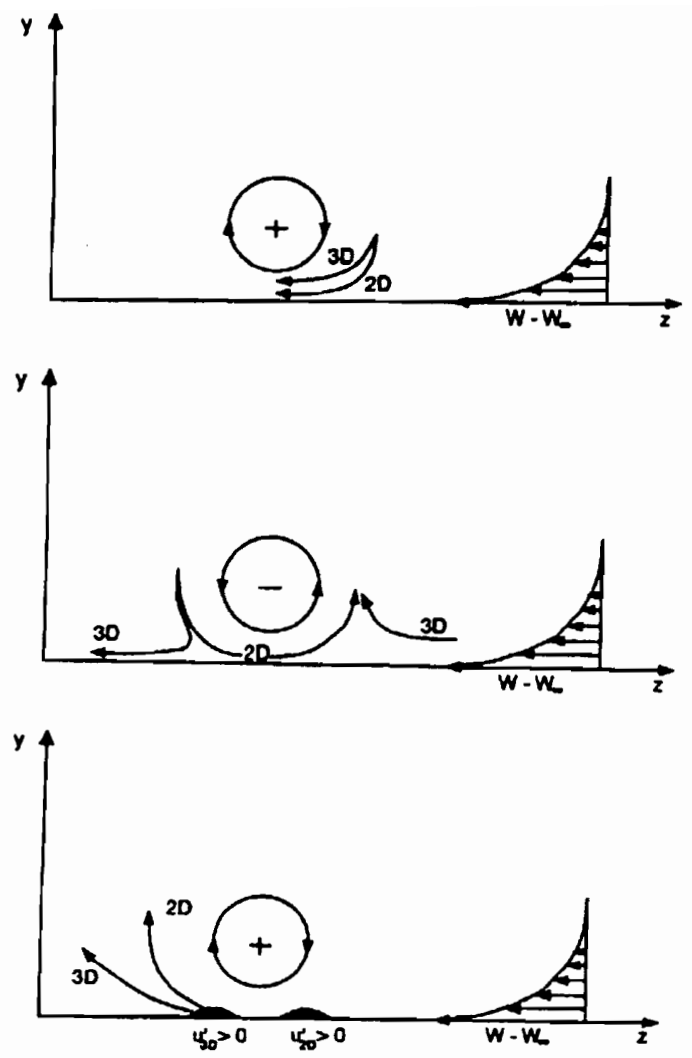

III

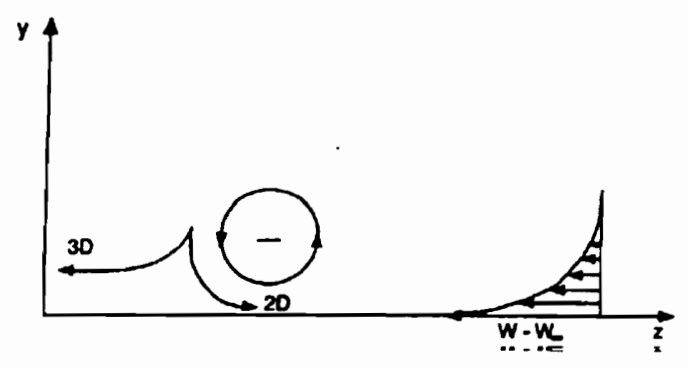

IV

Fig. 1.12 Observed variations in fluid trajectories for ejection and sweep events in the presence of spanwise (3-D) flow. Figures are from Senstad and Moin's (1992) DNS results. 


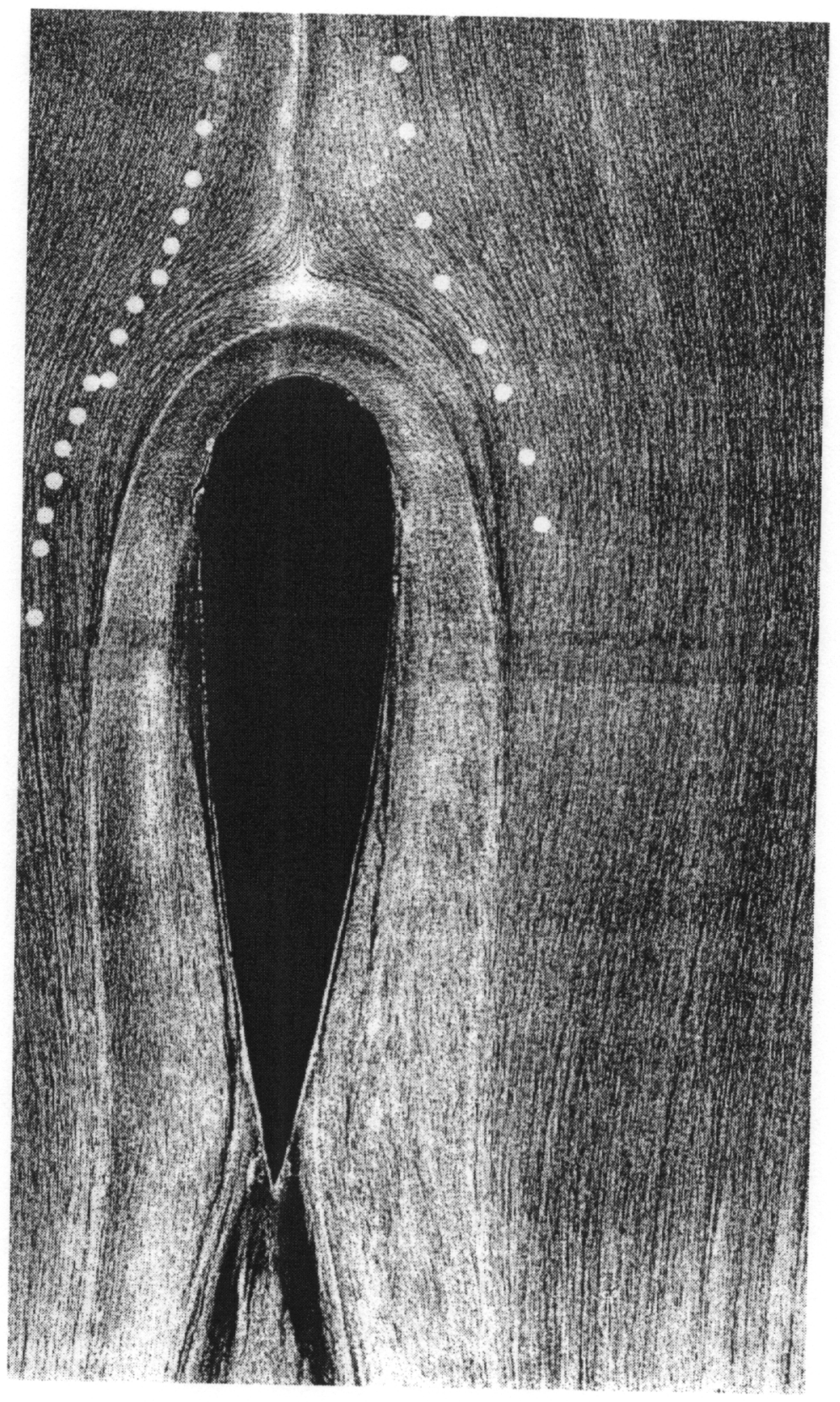

Fig. 1.13 Surface oil flow results for current experimental wing-body junction geometry, approach $\operatorname{Re}_{\theta} \approx 7000$ (from Ölçmen and Simpson, 1995). Ölçmen's LDV stations 0-7 are marked on the right side of the appendage location. Hotwire measurements were taken at the locations indicated on the left hand side of the appendage. 


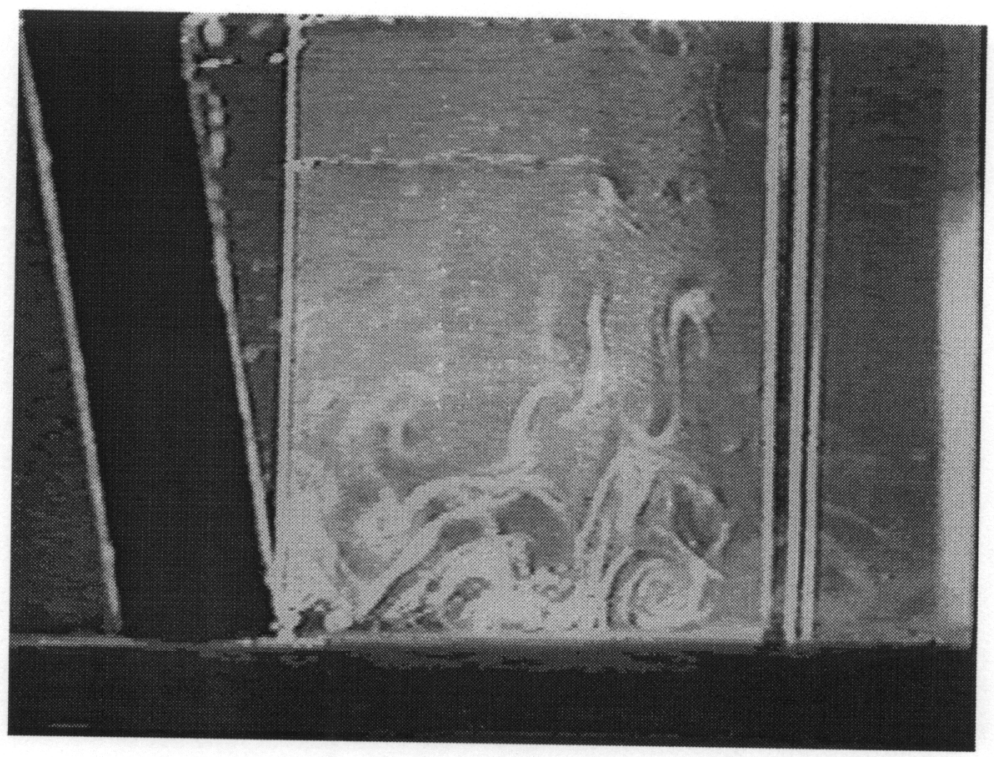

Fig. 1.14 A frame from a hydrogen-bubble flow-visualization video documenting the wingbody junction vortex structure (Kim, 1991). The printed image is $80 \%$ of full scale. The flow direction was from left to right. The approach flow Reynolds number was approximately 350 . 


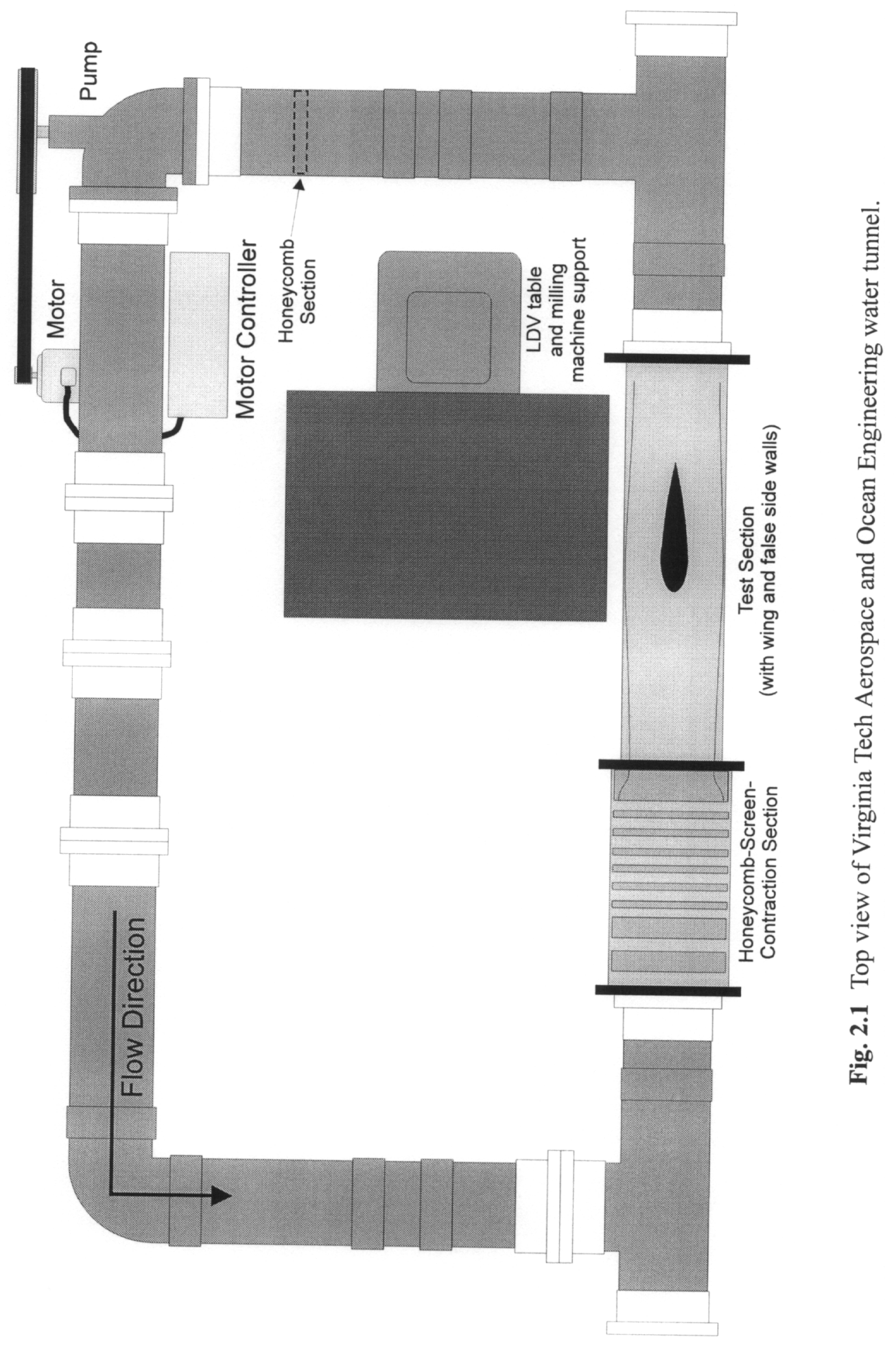




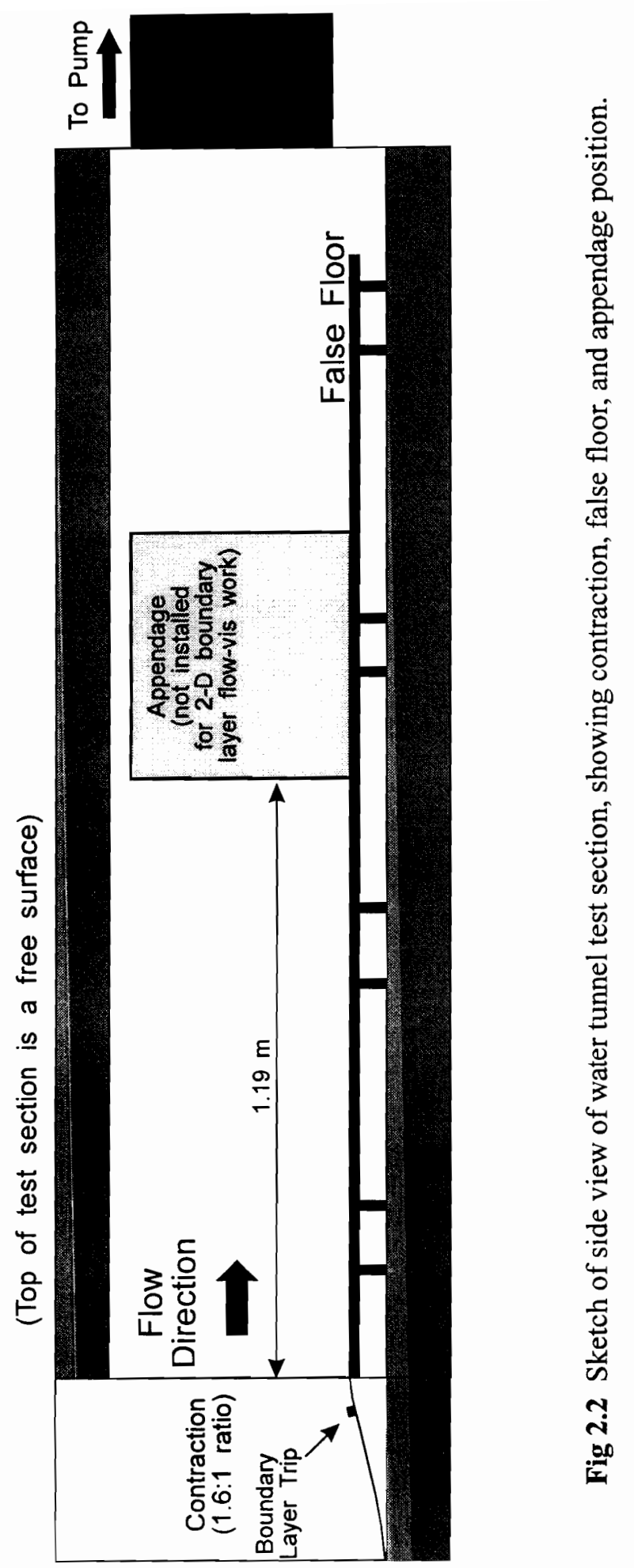




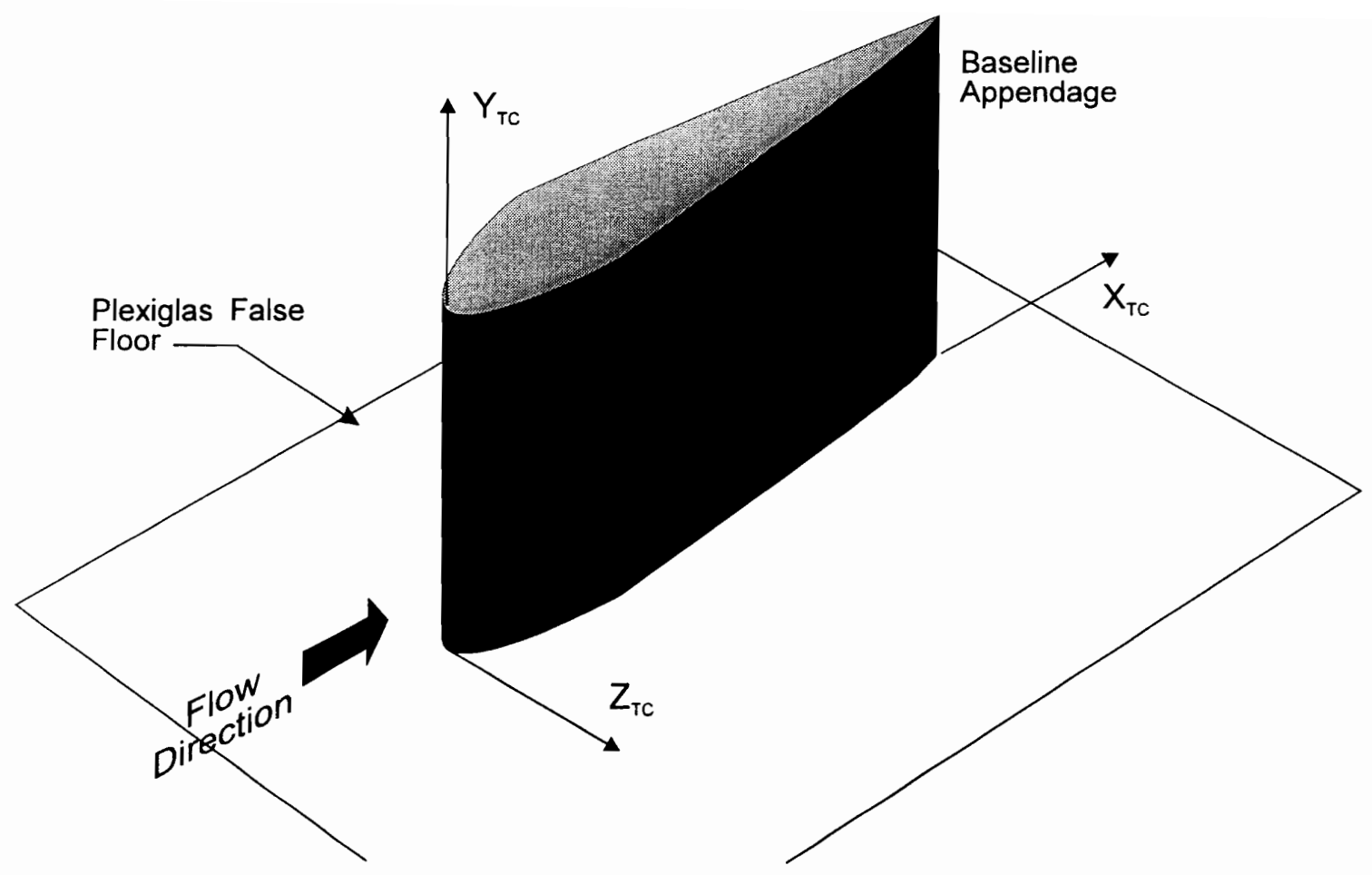

Fig. 2.3 Diagram of appendage and tunnel coordinate system. See Fig. 2.12 for the actual appendage shape. 


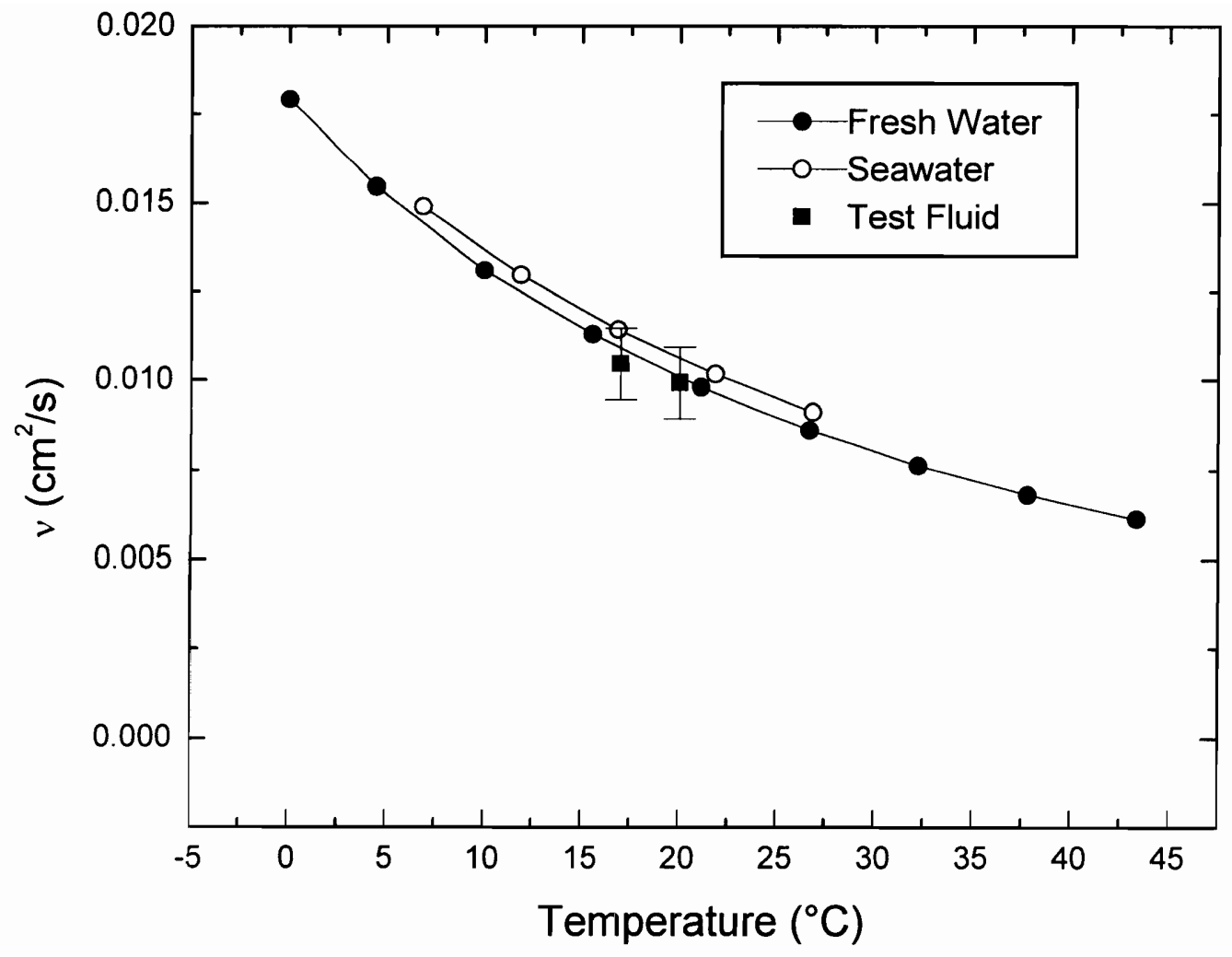

Fig. 2.4 Plot of current kinematic viscosity results compared to published data. Fresh water results are from Brown and Marco (1958), and seawater results are from Touloukian and Ho (1975). 


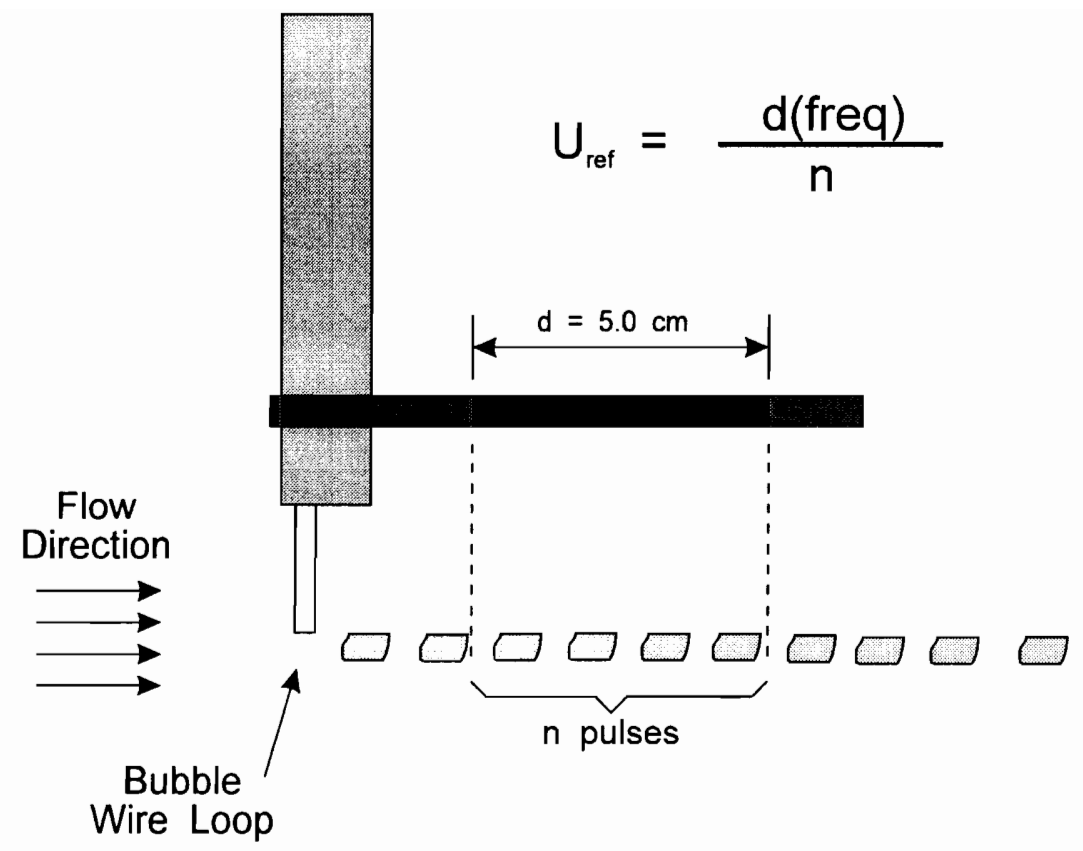

Fig. 2.5 Sketch of the special hydrogen-bubble probe used to check the accuracy of the electromagnetic water current meter. 

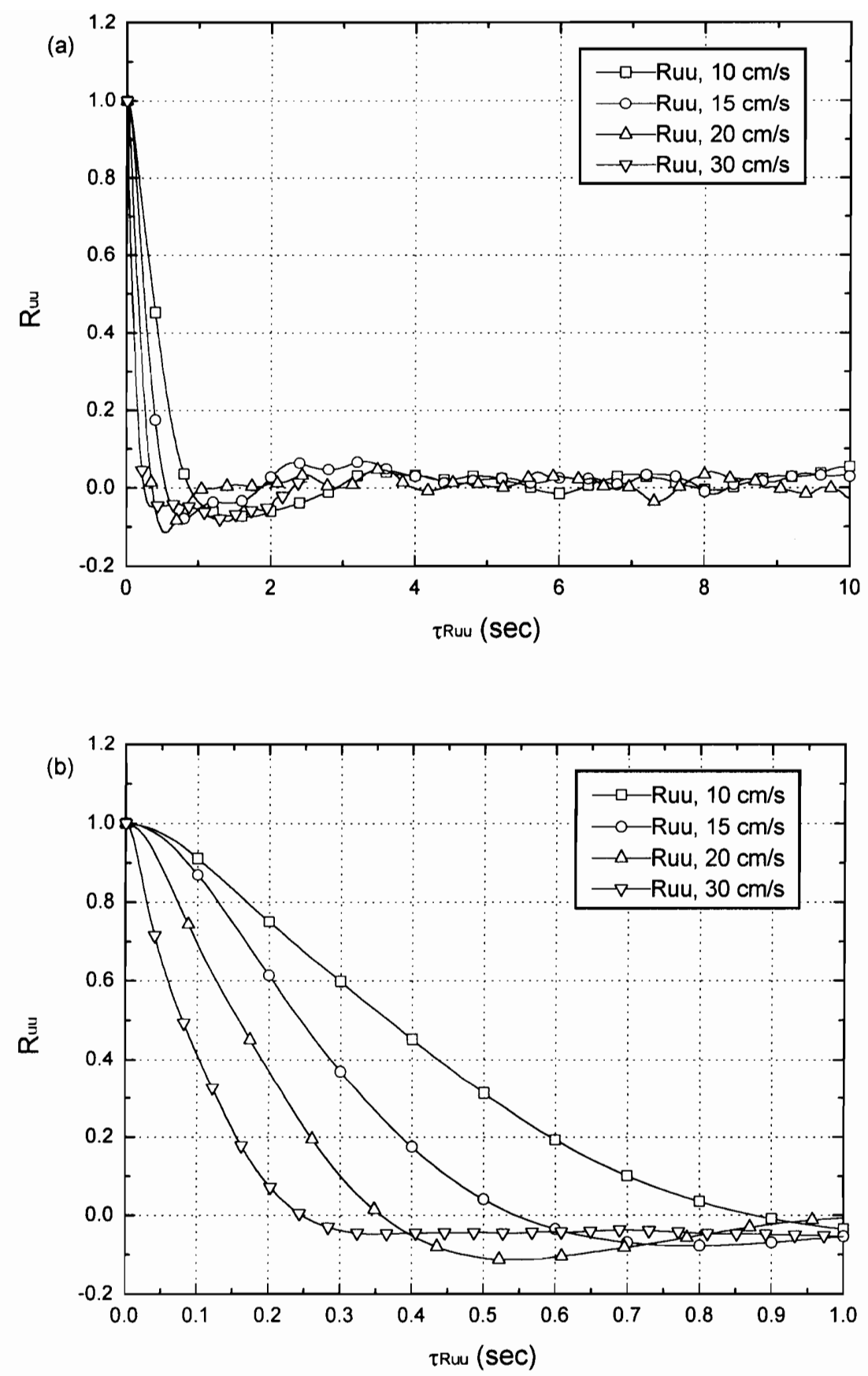

Fig. 2.6 Freestream $U$ autocorrelation results for nominal velocities of $10,15,20$, and 30 $\mathrm{cm} / \mathrm{s}$. (a) $R_{u u}$ results up to $\tau_{R u u}=10$ seconds, and (b) detail of $\tau_{R u u}=0$ to 1 second. See section 3.3 for autocorrelation details. (Note: not all data points are marked with symbols) 

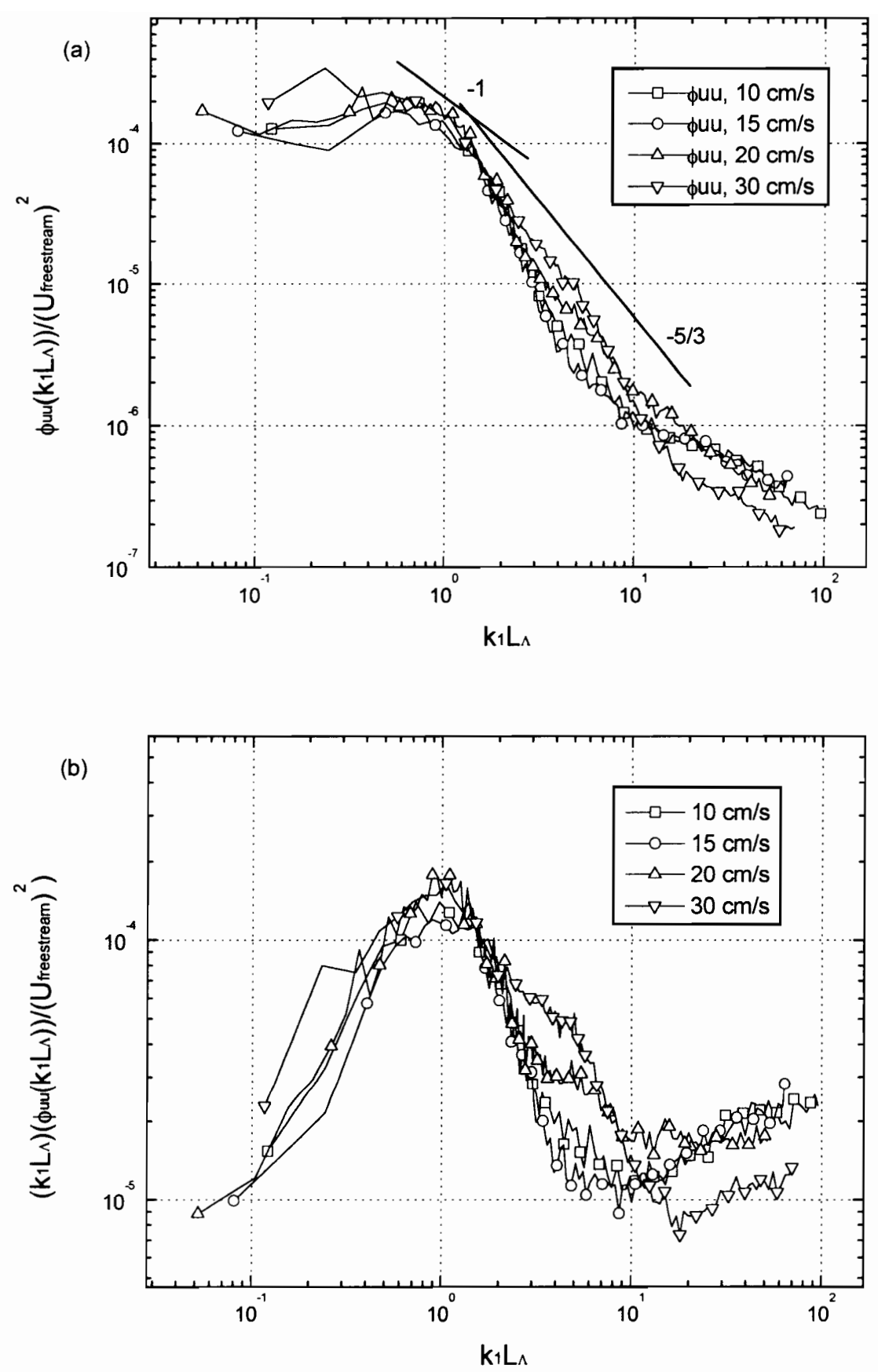

Fig. 2.7 Freestream $U$ autospectra results for nominal velocities of 10, 15, 20, and 30 $\mathrm{cm} / \mathrm{s}$. (a) Spectral results scaled using the autocorrelation integral length scales, $L_{\Lambda}$, and (b) first moment results. See section 3.4 for more spectral results and details. (Note: not all data points are marked with symbols) 

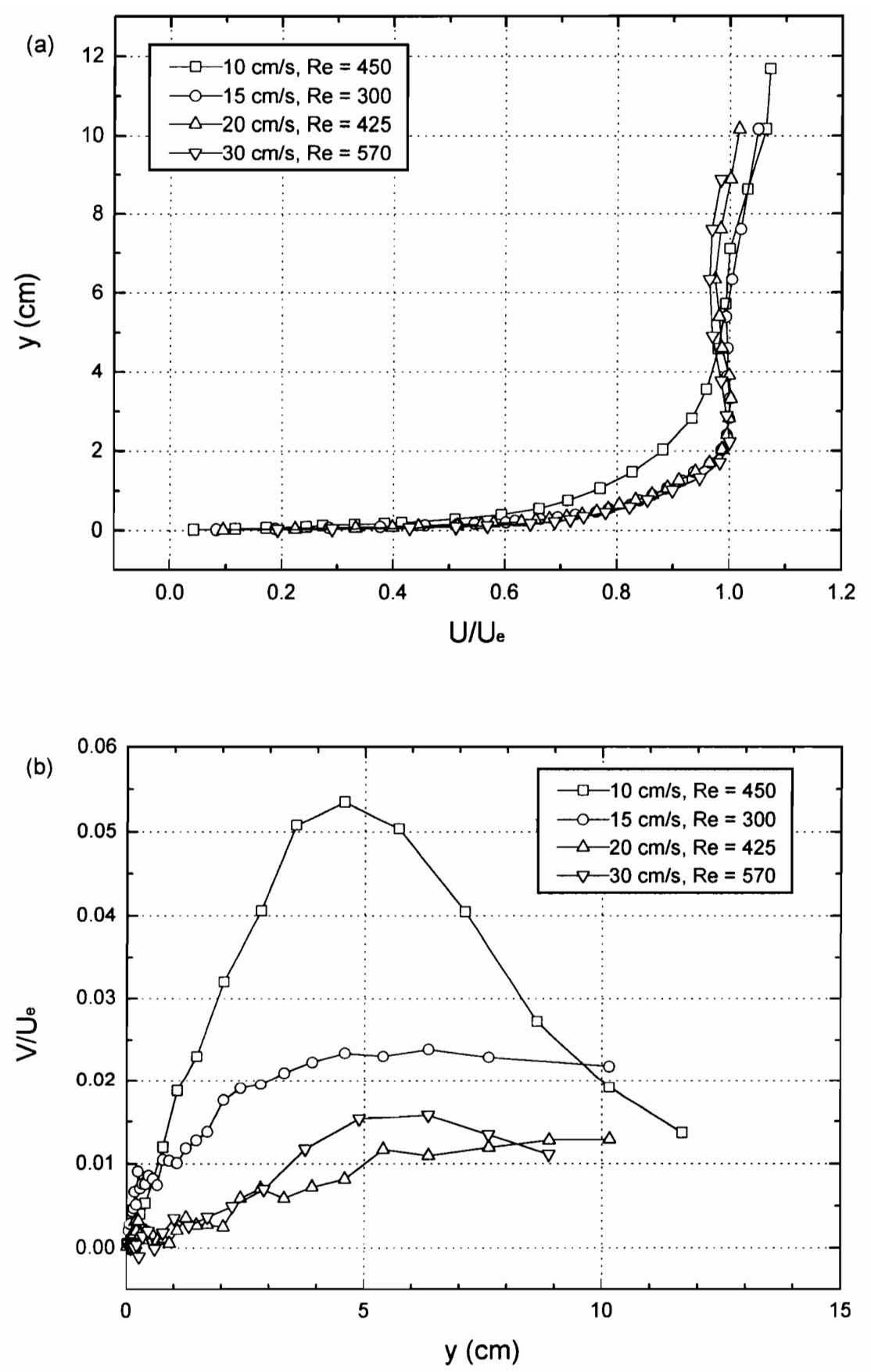

Fig. 2.8 2-D flow boundary layer profile data, documenting the test section boundary layer and lower portion of the test flow's "inviscid core." See Figs. 3.6 and 3.10 for the 2-D boundary layer data plotted using a $\log$ scale. (a) $U / U_{e}$ vs. $y$, note that $y$ is dimensional. This type of plot graphically displays the shape of the mean $U$ profile. (b) $V / U_{e}$ vs. $y$ (dimensional). 

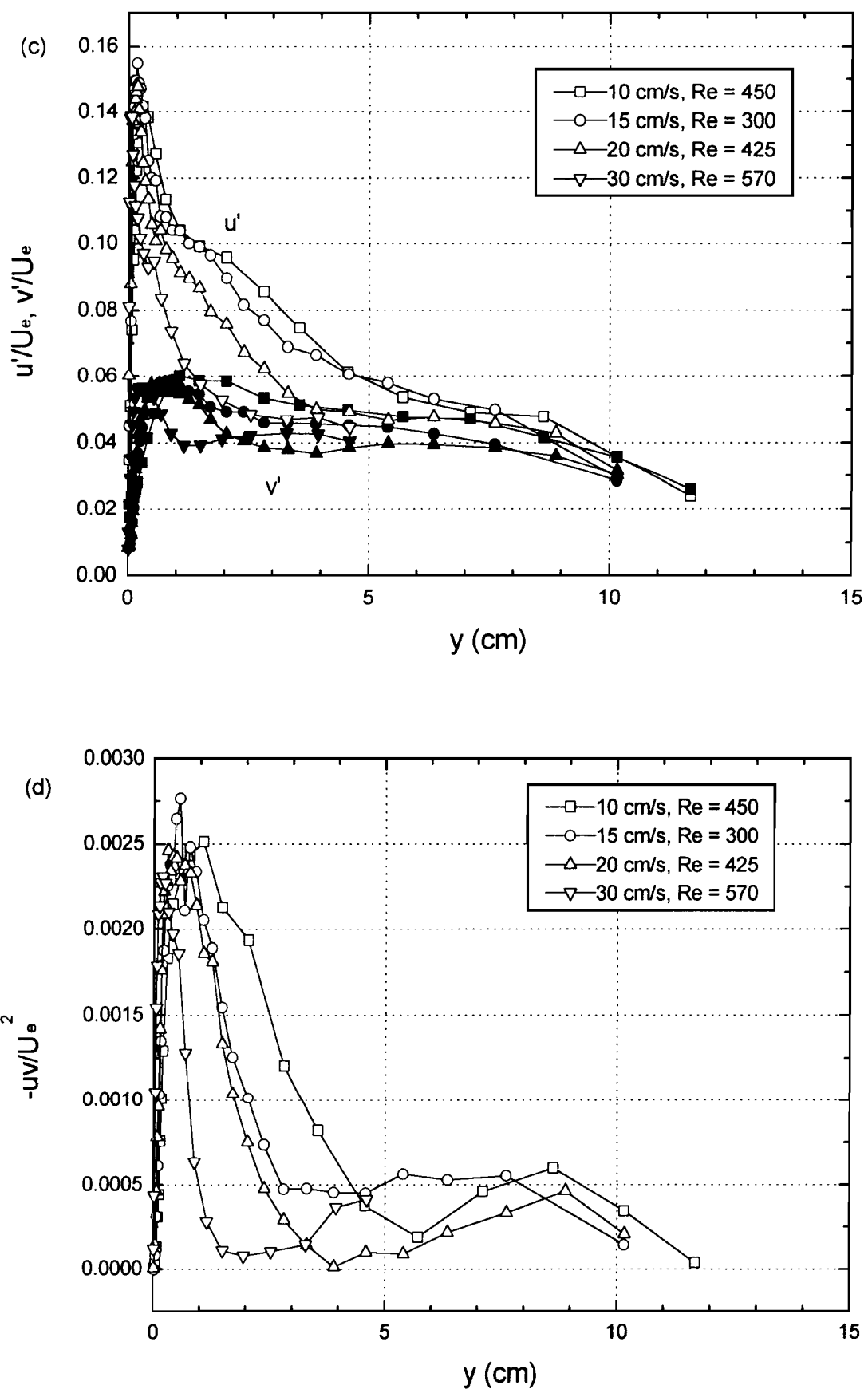

Fig. 2.8 (cont.) More 2-D flow boundary layer profile data. (c) Normal stress quantities, $u^{\prime}$ (open symbols) and $v^{\prime}$ (solid symbols), and (d) Reynolds shear stress, $-\overline{u v}$, both vs. $y$. 


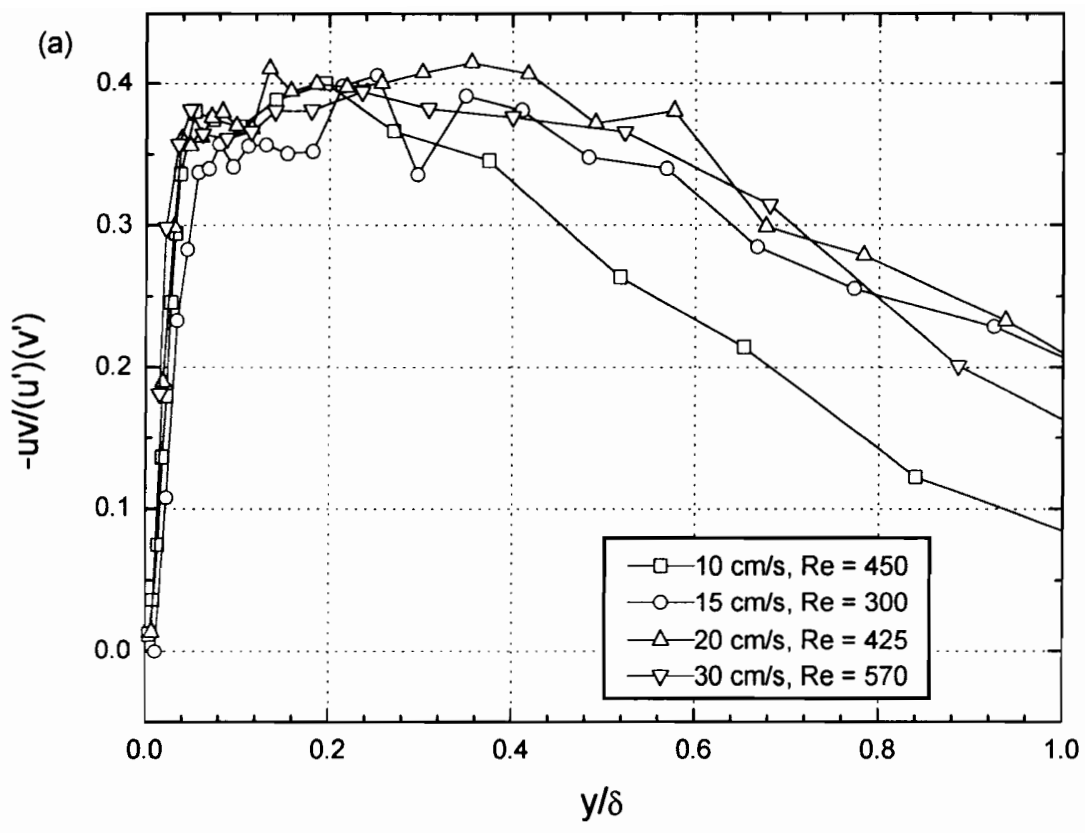

Fig. 2.9 Shear stress correlation results for the 2-D boundary layers. 


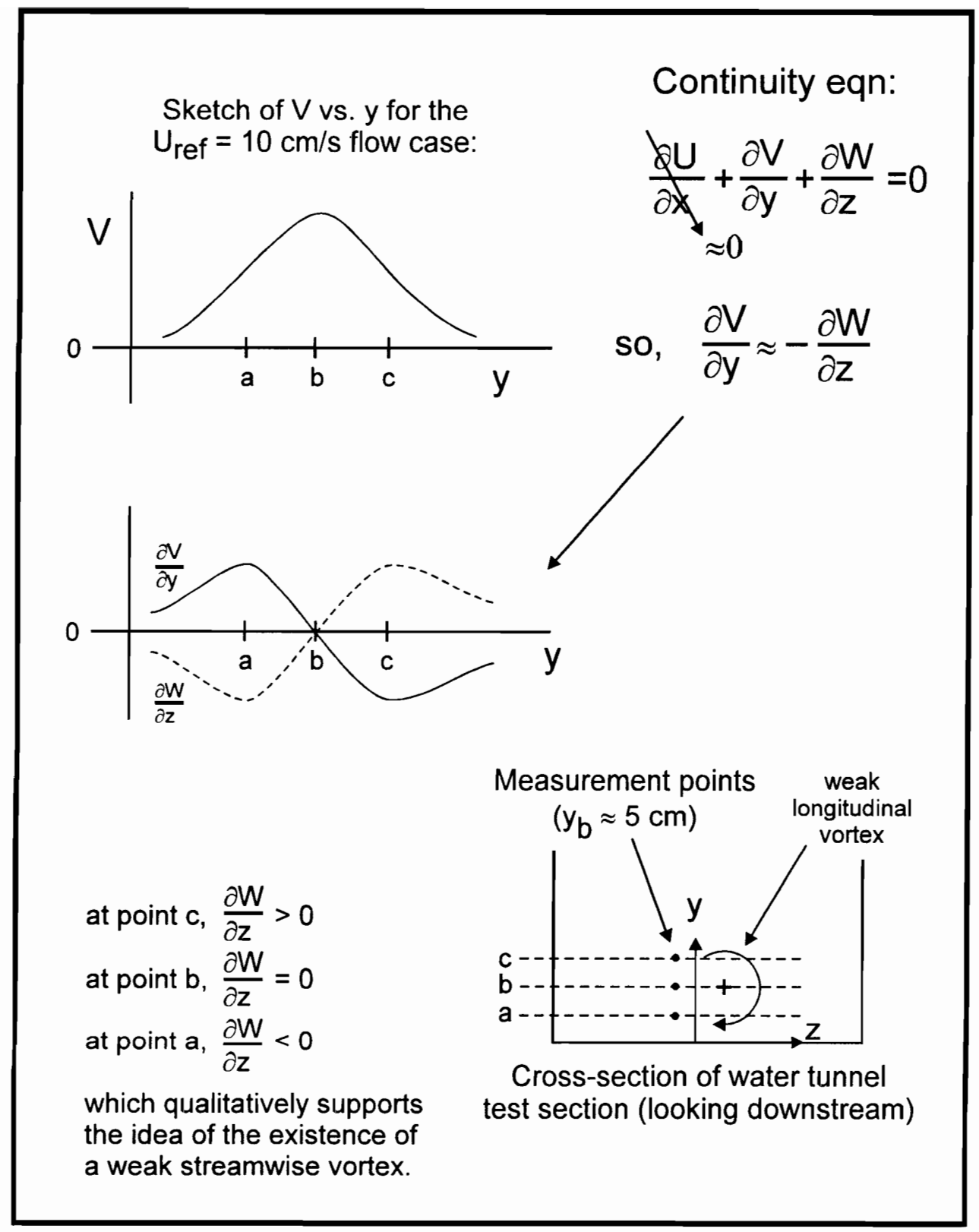

Fig. 2.10 Diagram explaining the effects that a weak streamwise vortex would have on the distribution of $V$. Based on Fig. 2.8(b), point $b$ would be located at approximately $y=5 \mathrm{~cm}$. 


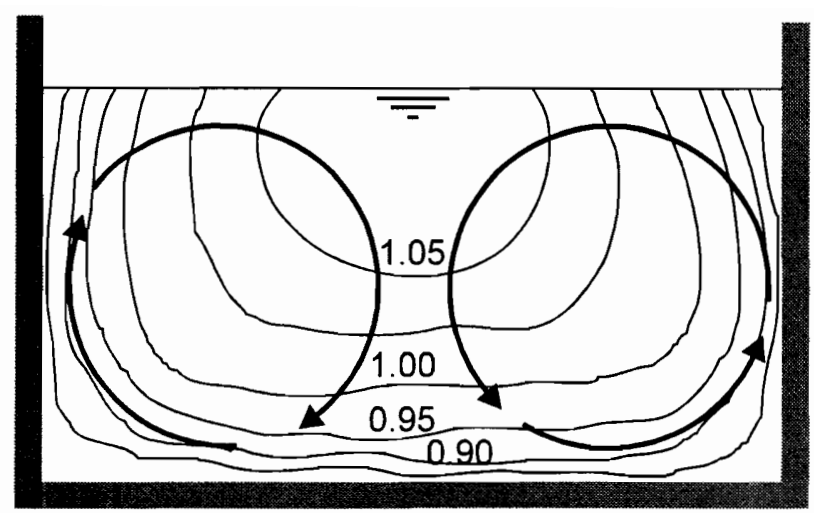

Fig. 2.11 Sketch showing typical open-channel mean velocity contours and secondary flow patterns (after Sellin, 1970). 


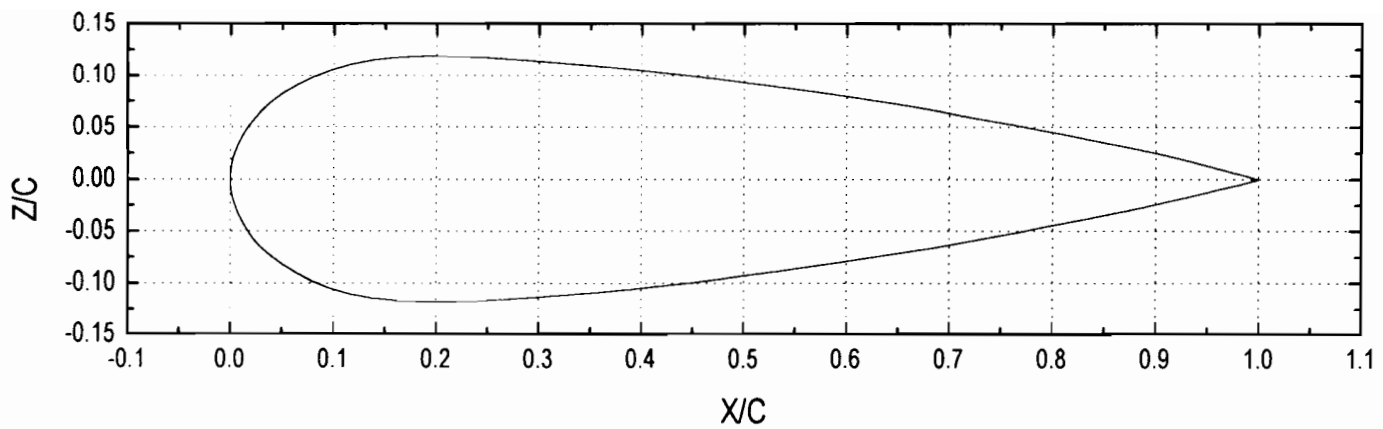

Fig. 2.12 Appendage geometry used for 3-D flow test case (3:2 elliptic wing, NACA 0020 tail). 


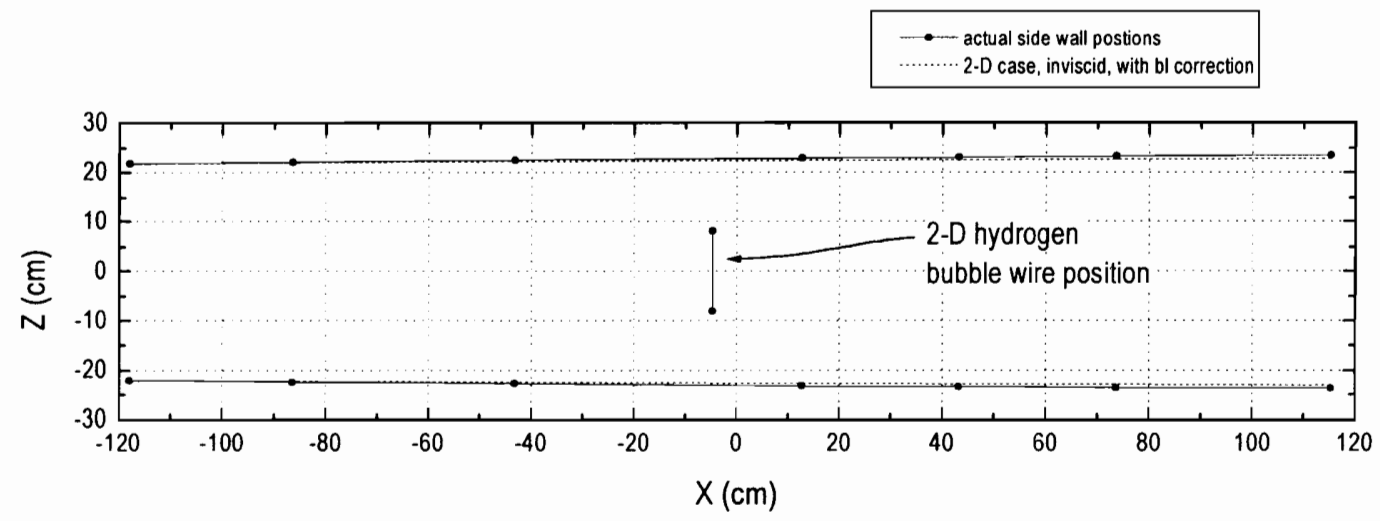

Fig. 2.13 Side wall displacement for 2-D flow case. See Table 2.3 for actual displaecment coordinates. 


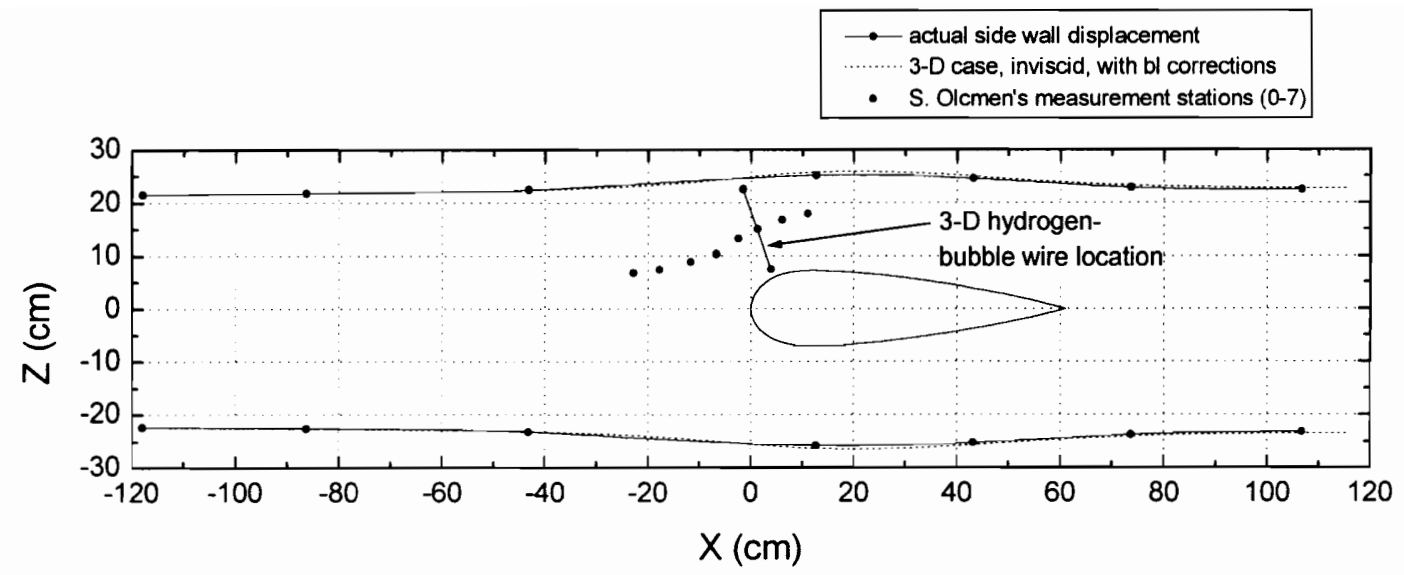

Fig. 2.14 Side wall displacement for 3-D flow case. Orientation of figure is the same as the bottom view of the test section. See Table 2.3 for actual displaecment coordinates. 

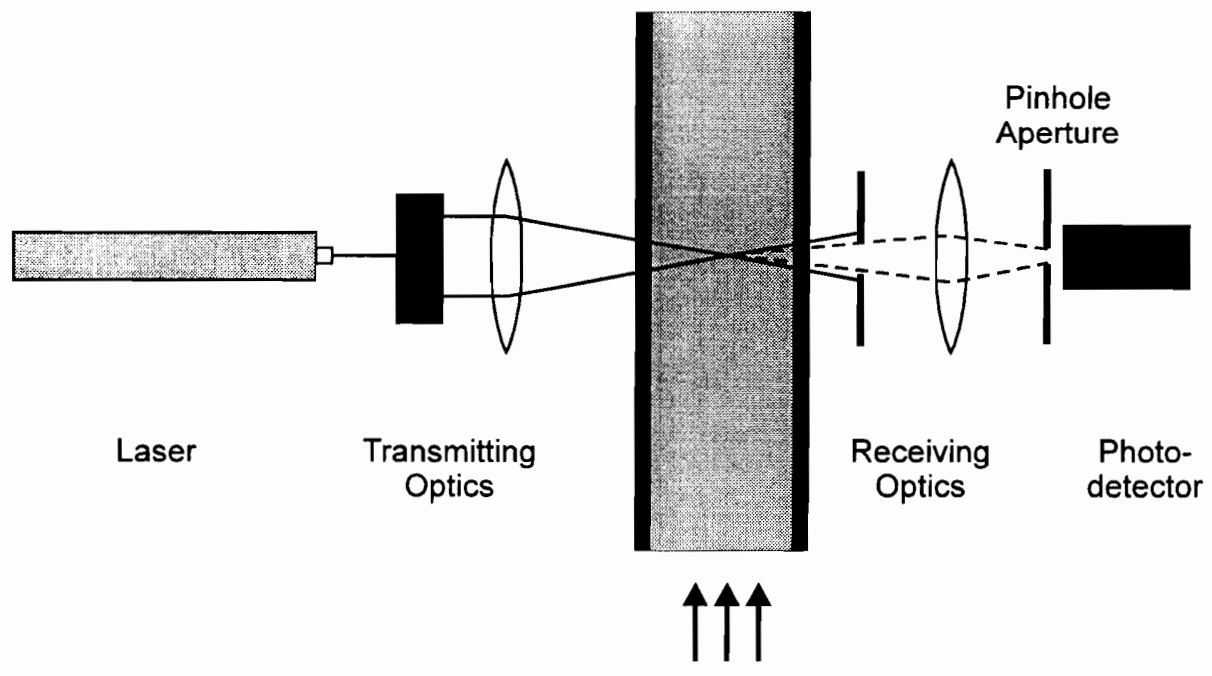

Flow

Direction

Fig. 2.15 Basic elements of a one-component LDV system (after Durst et al., 1981). 
(a)

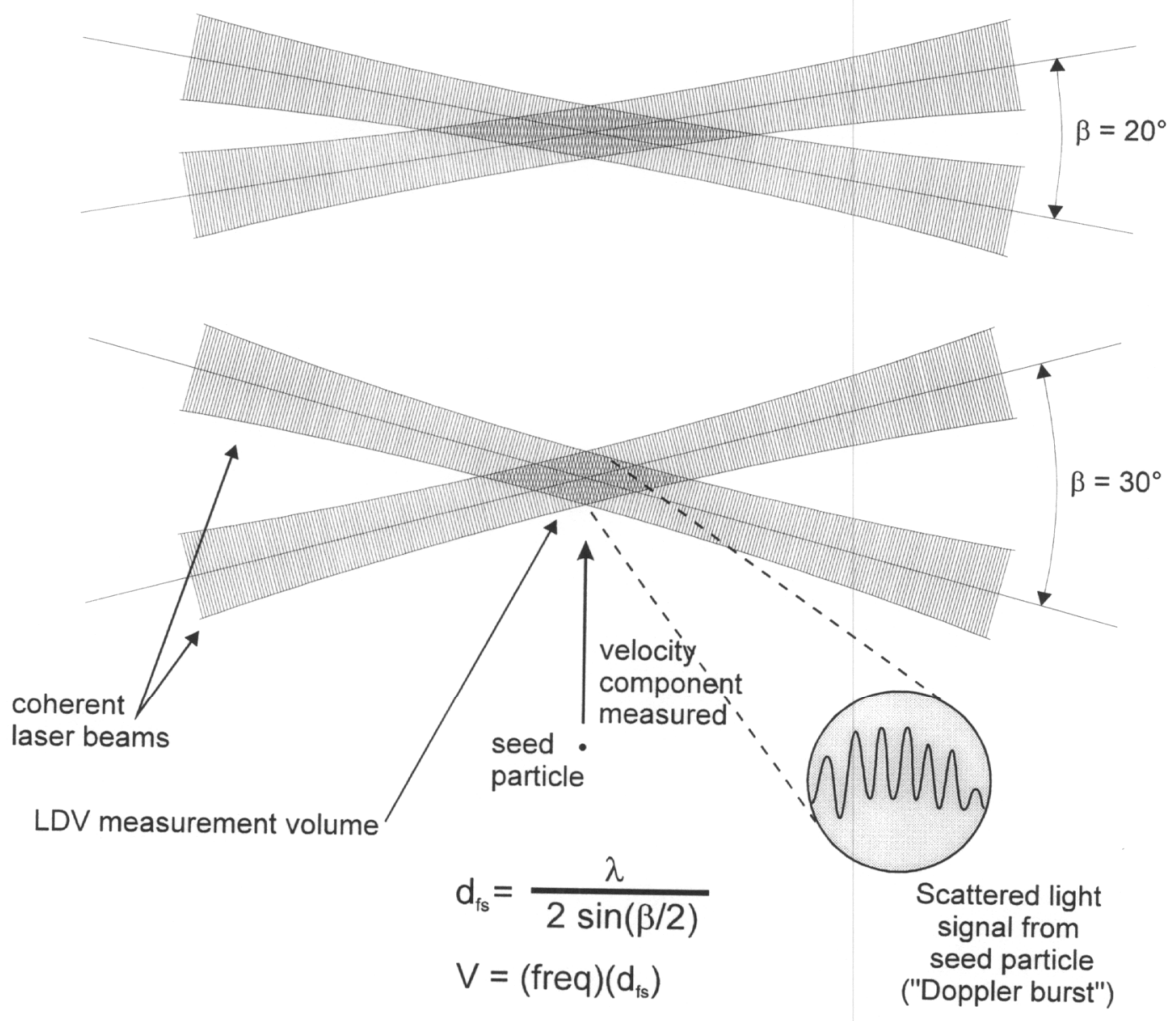

Fig. 2.16 Sketch revealing the underlying principles of how the LDV technique works. Comparison of (a) to (b) shows how the fringe spacing, $d_{f s}$, decreases with increasing intersection angle, $\beta$. 


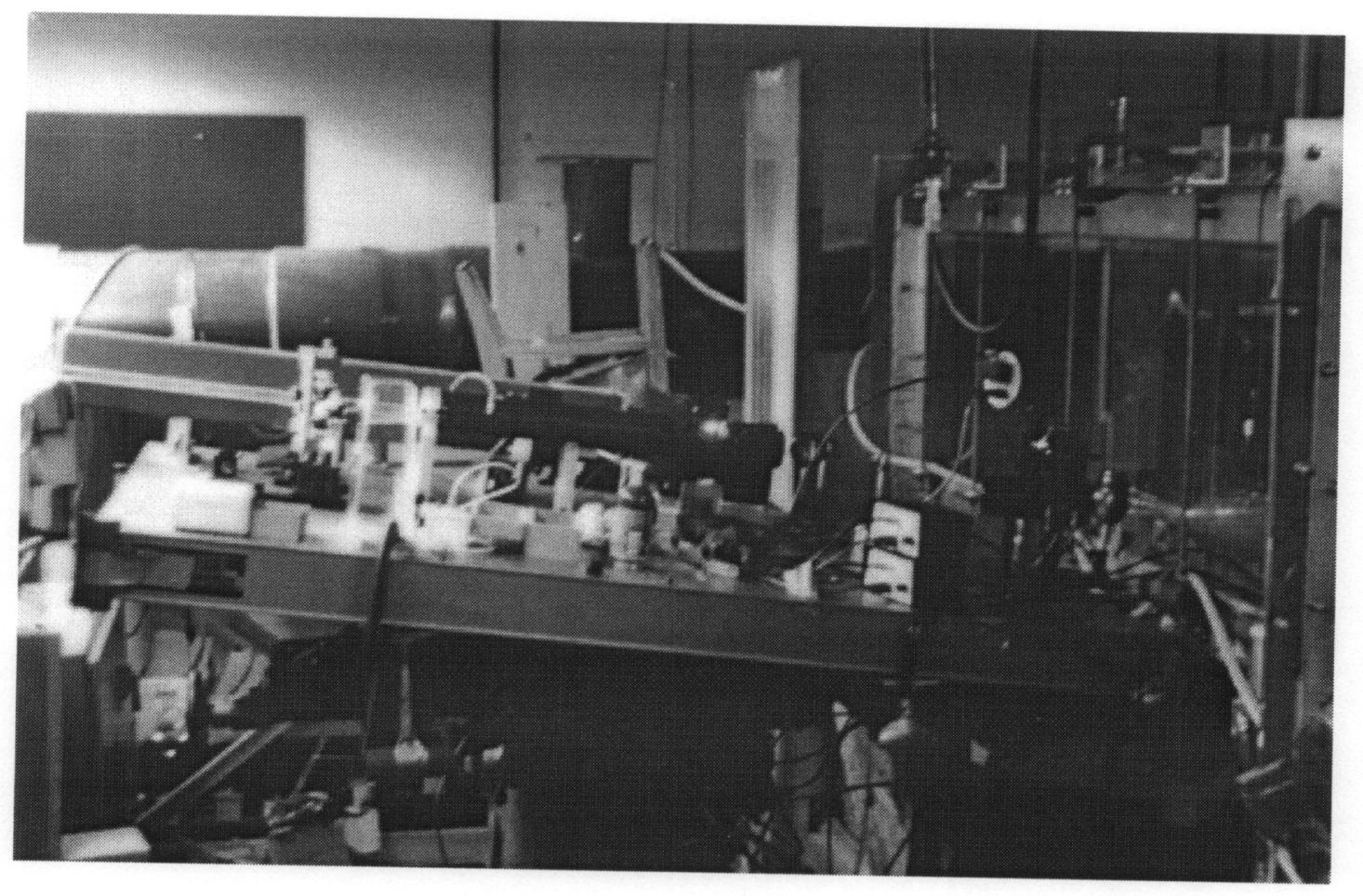

Fig. 2.17 Photograph of LDV system configured for the 2-D case. The water tunnel test section is on the right, and part of the water return line is visible behind the LDV system. 


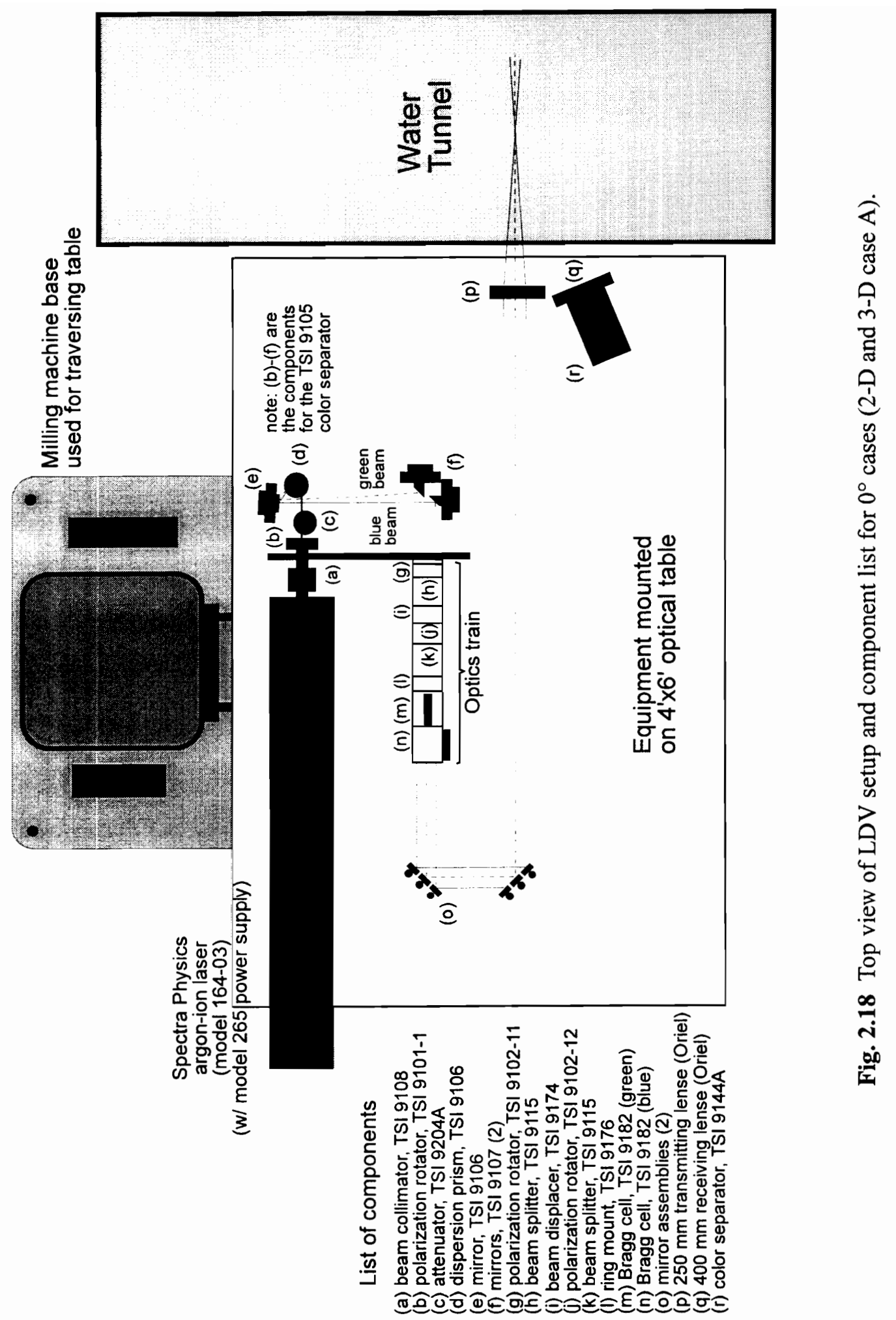




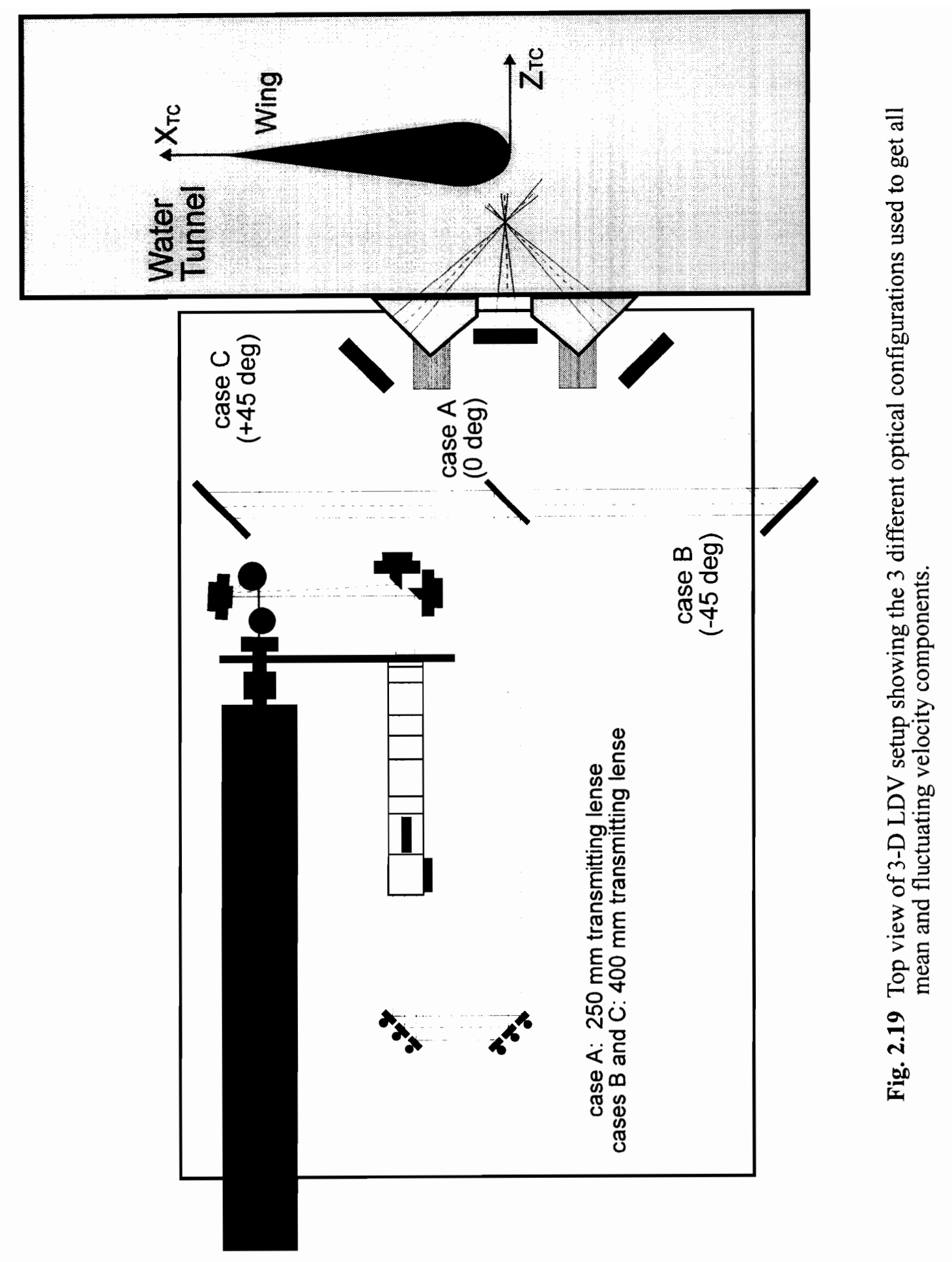




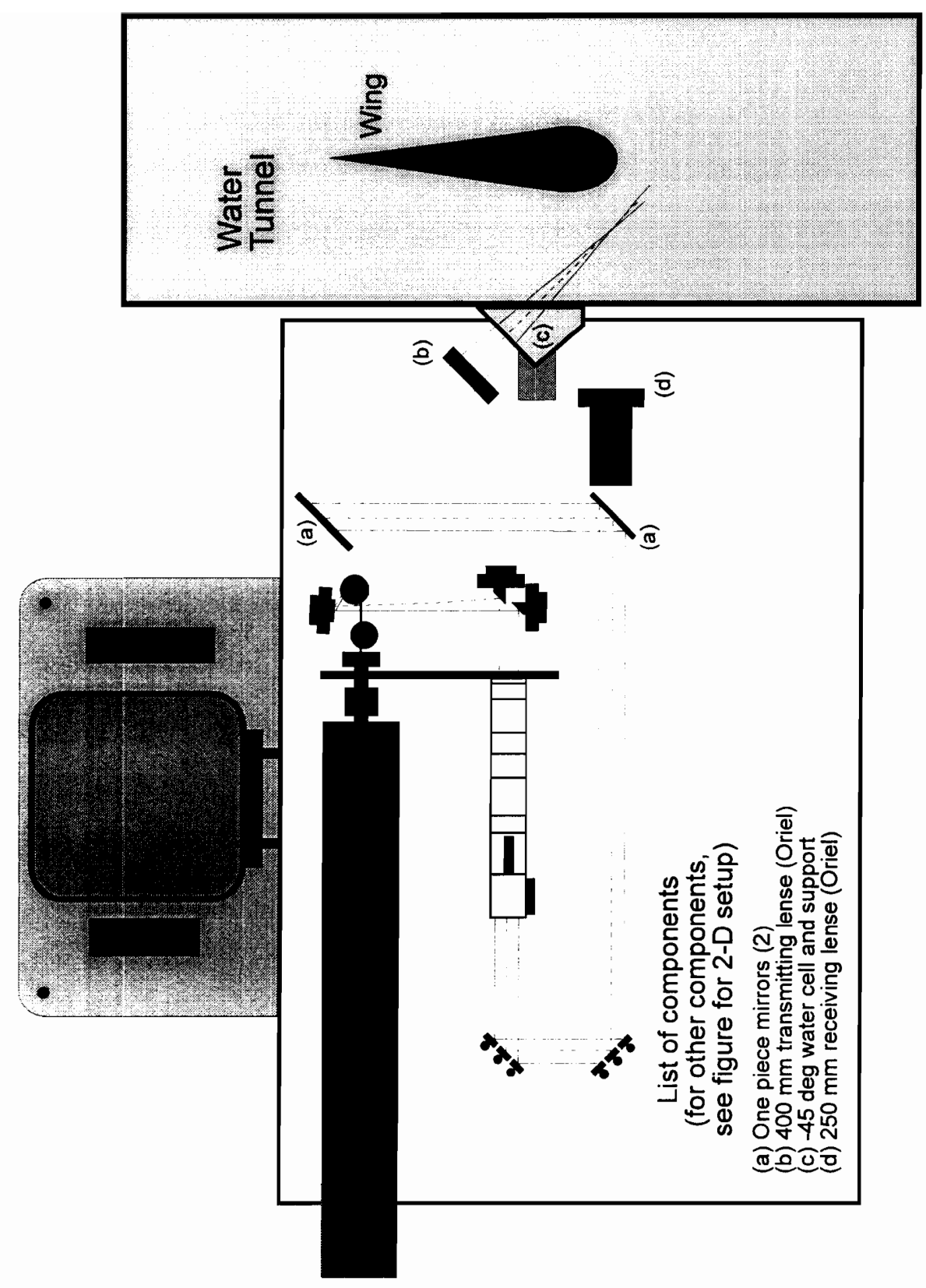

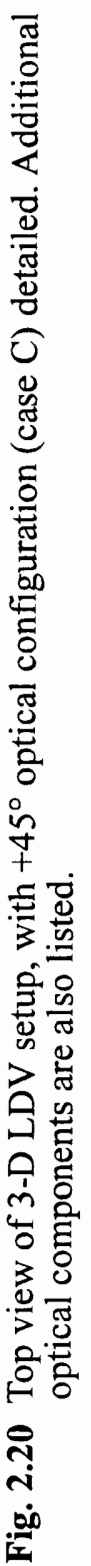




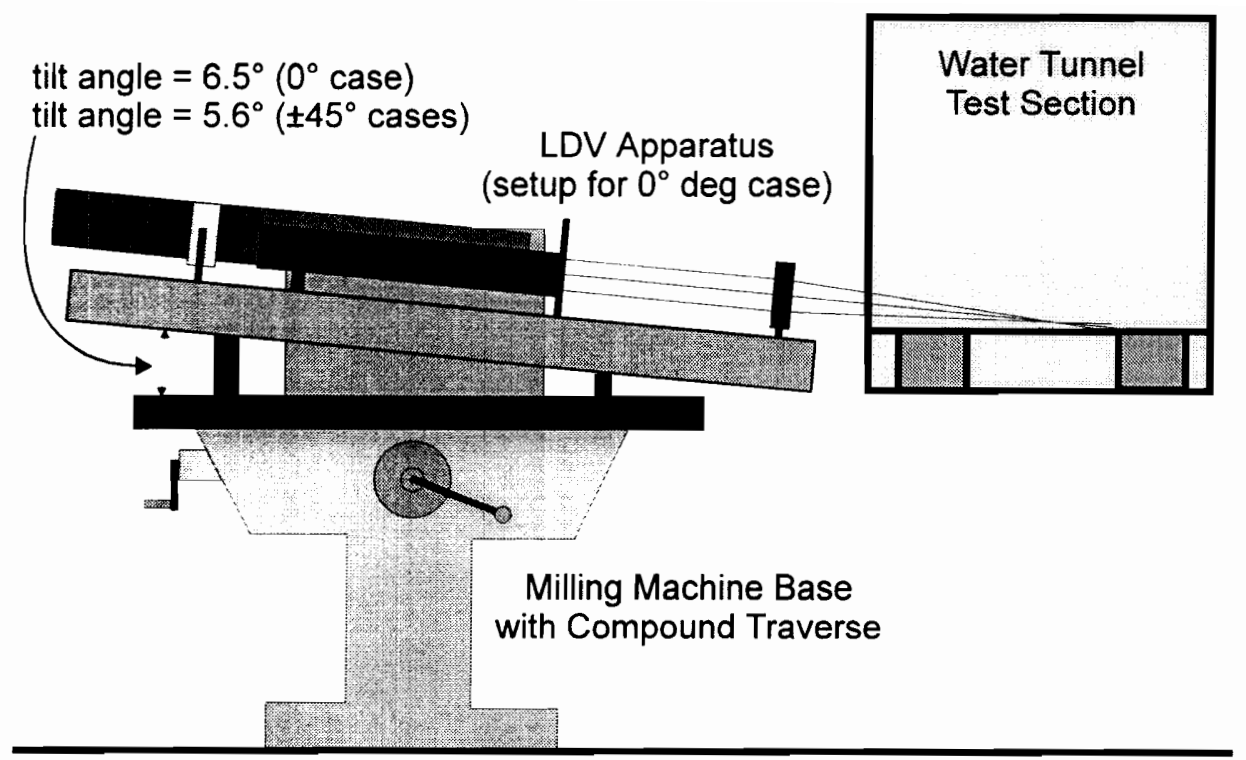

Fig. 2.21 Side view of optical table mounted on milling machine base, showing how the LDV system was tilted to enable near-wall measurements. 
(a)

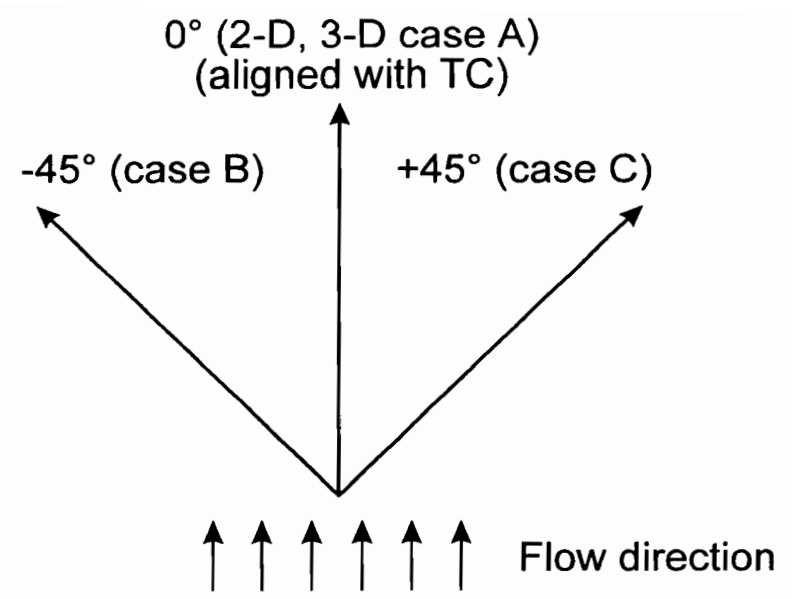

(b)

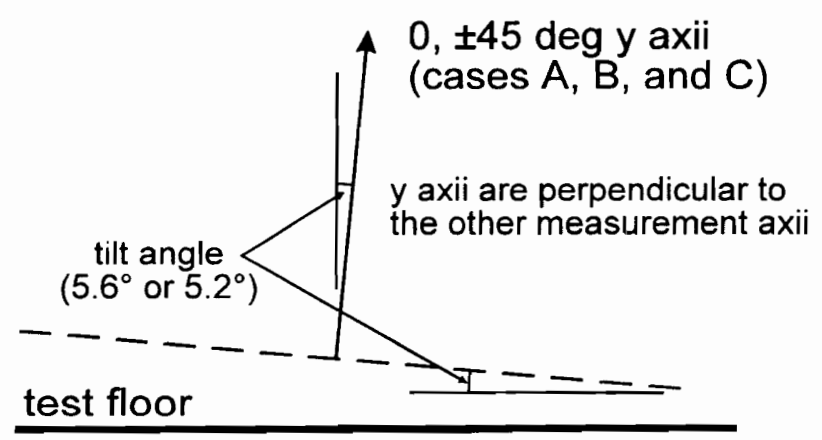

Fig. 2.22 Axis systems for the different LDV optical configurations. (a) shows the top view, while (b) shows the downstream view (looking along $x_{\mathrm{TC}}$ ). The tilt angle for cases $\mathrm{B}$ and $\mathrm{C}$ was $5.2^{\circ}$, and was $5.6^{\circ}$ for the 2-D case and case $\mathrm{A}$. 
Snell's law of refraction

$$
\begin{aligned}
\left(\mathrm{n}_{1}\right)\left(\sin \theta_{1}\right) & =\left(\mathrm{n}_{2}\right)\left(\sin \theta_{2}\right) \\
\lambda_{1} n_{1} & =\lambda_{2} \mathrm{n}_{2}
\end{aligned}
$$

$$
\mathrm{n}_{1}=1
$$

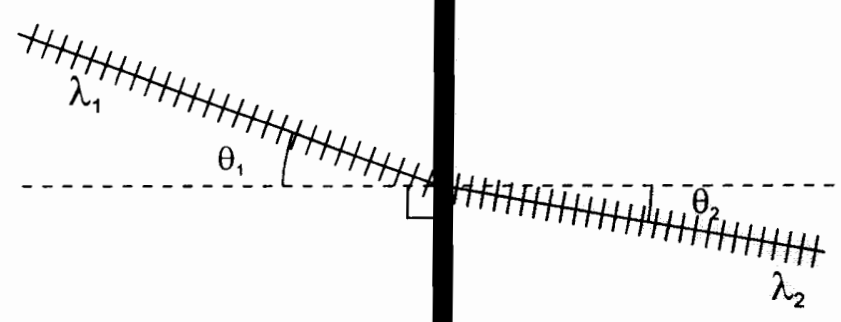

Fig. 2.23 A summary of Snell's law of refraction. 
(a)

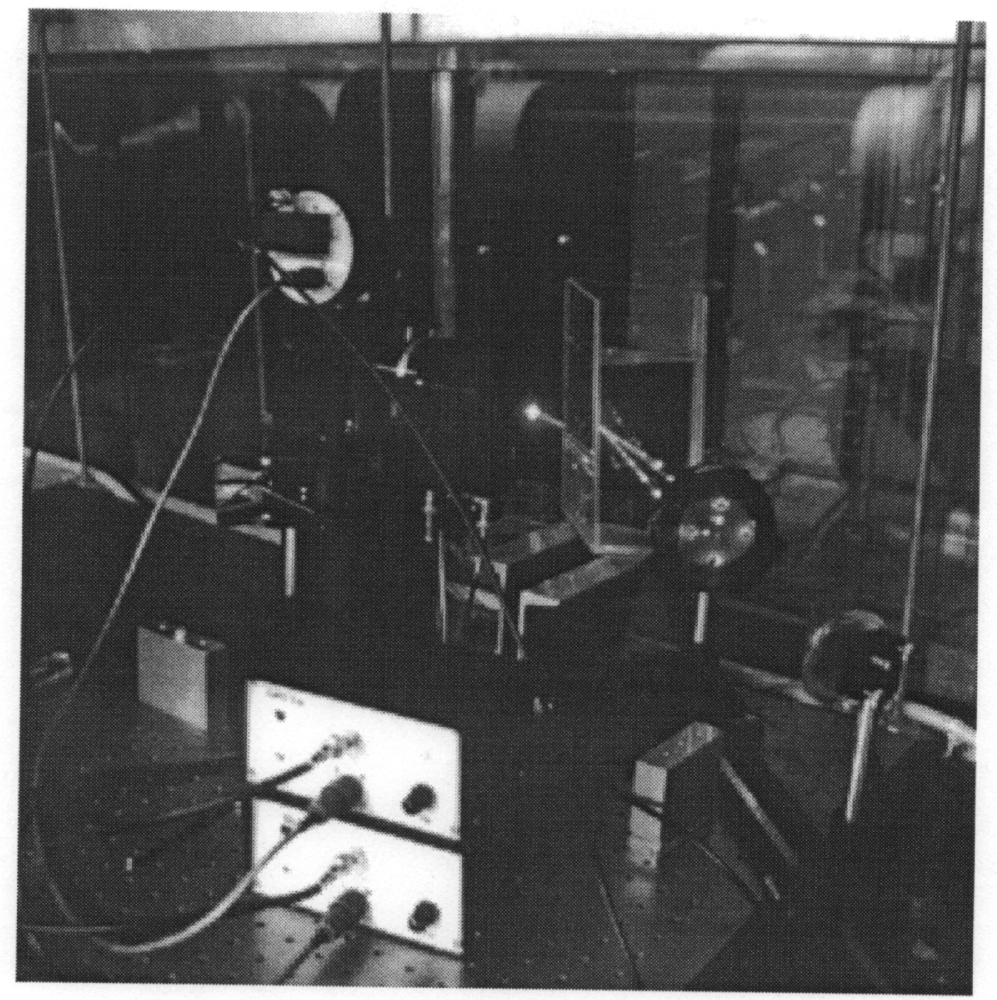

(b)

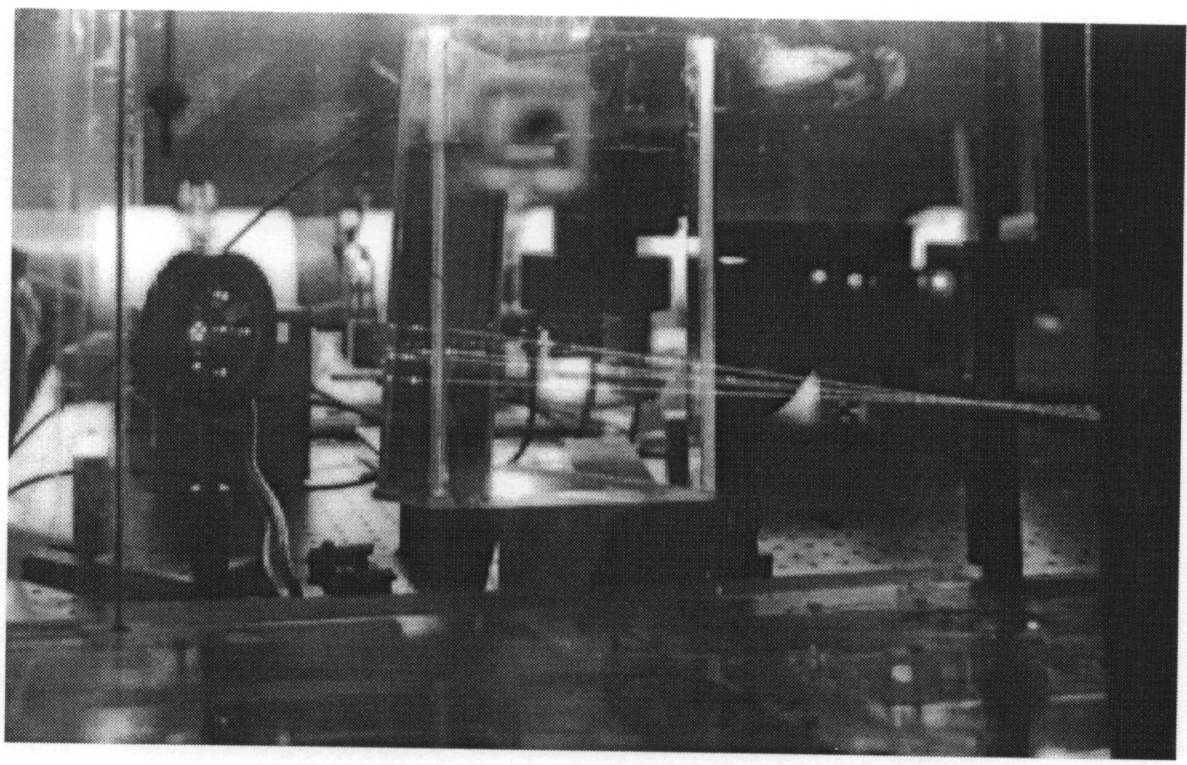

Fig. 2.24 Pictures of water cell used for $-45^{\circ}$ case (case B). These plexiglas cells minimized alignment problems caused by refraction. Views (a) and (b) are from opposite sides of the test section. 


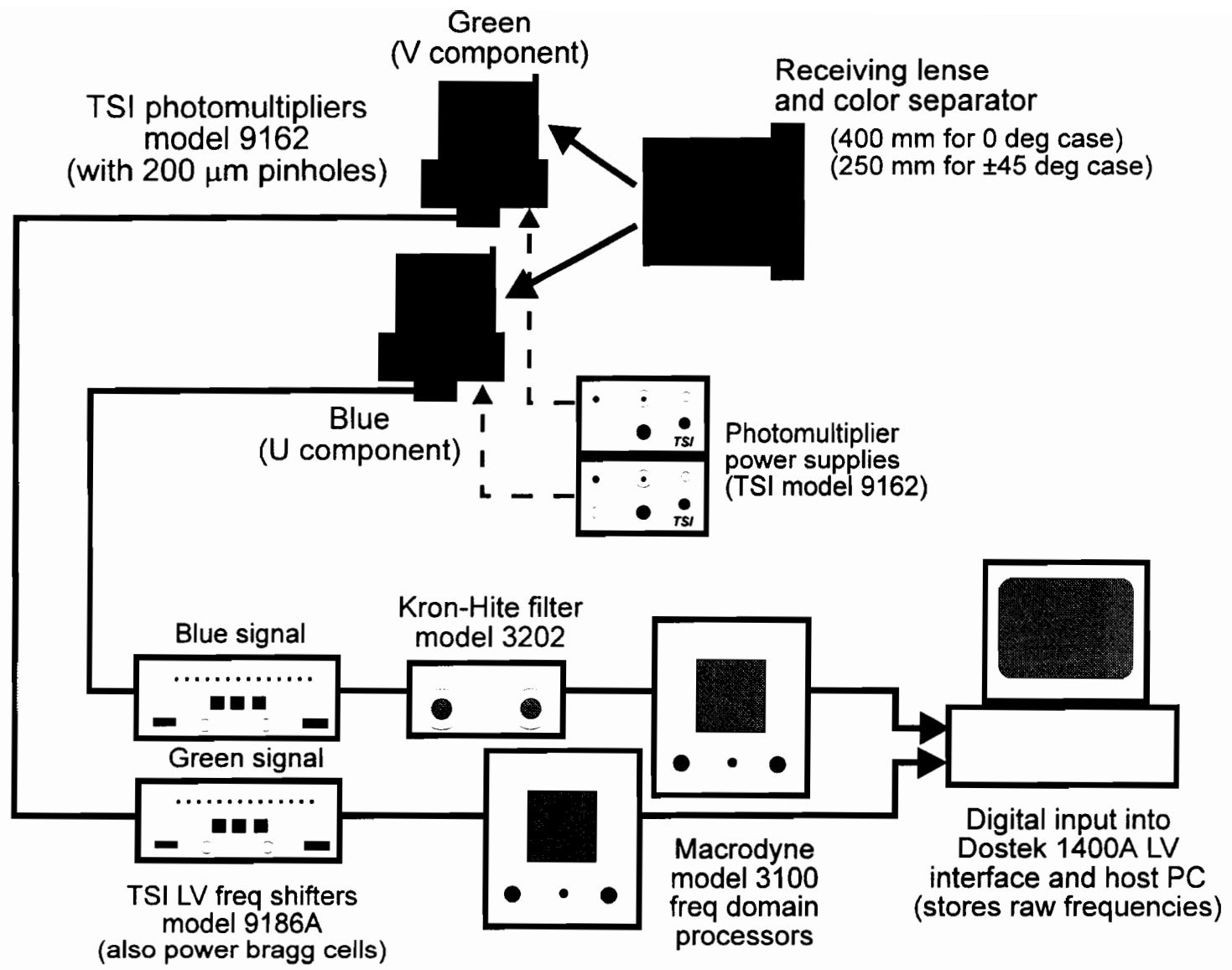

Fig. 2.25 Block diagram of equipment used to receive and process LDV signals. 

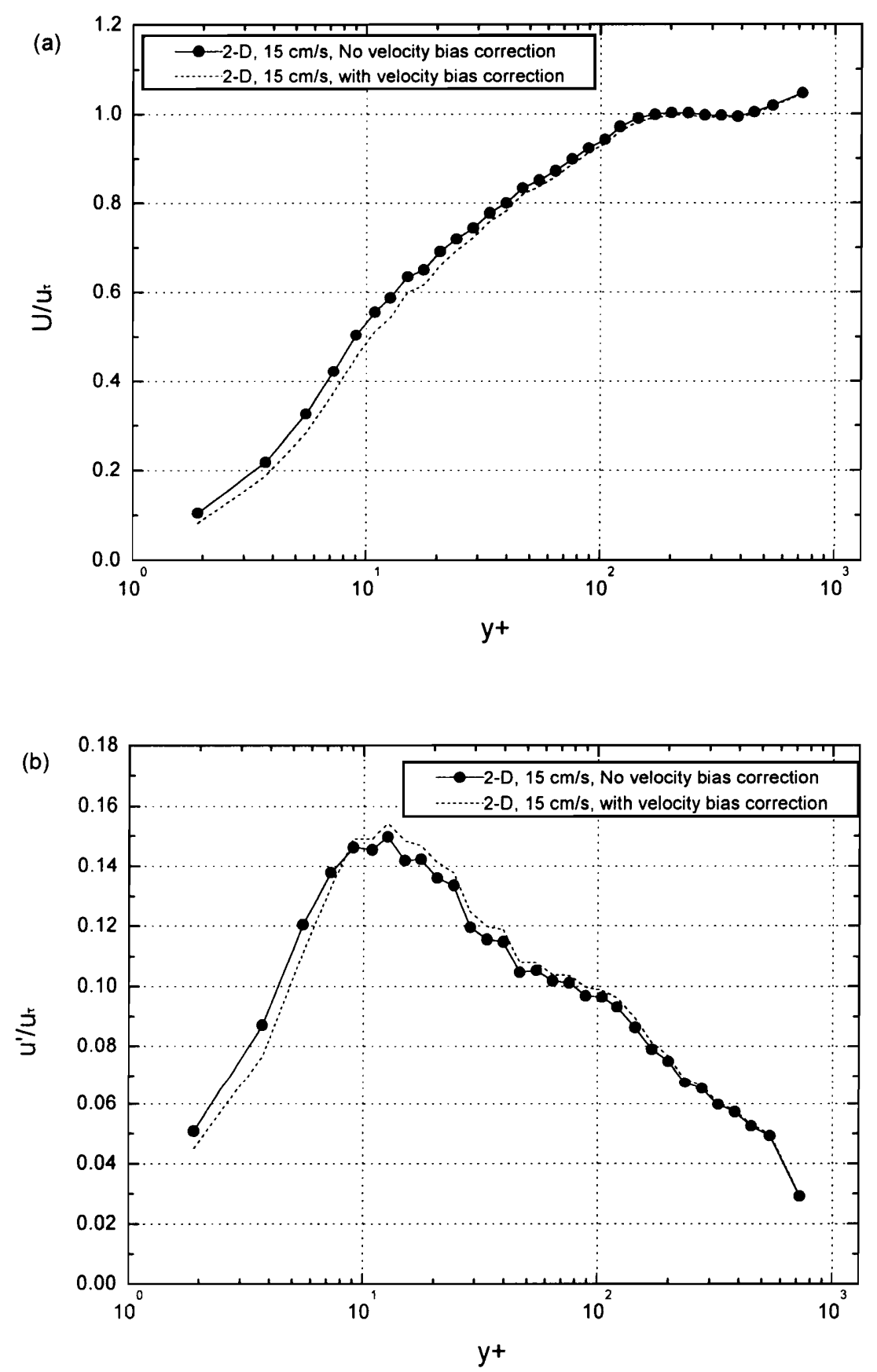

Fig. 2.26 Sample of velocity biasing effects inherent in LDV measurements. (a) shows results for $U$, and (b) shows results for $u^{\prime}$. Both plots from $2-\mathrm{D}, U_{e}=15 \mathrm{~cm} / \mathrm{s}$ data. The dashed line shows corrected results using $2 \mathrm{D}+$ weighting. 

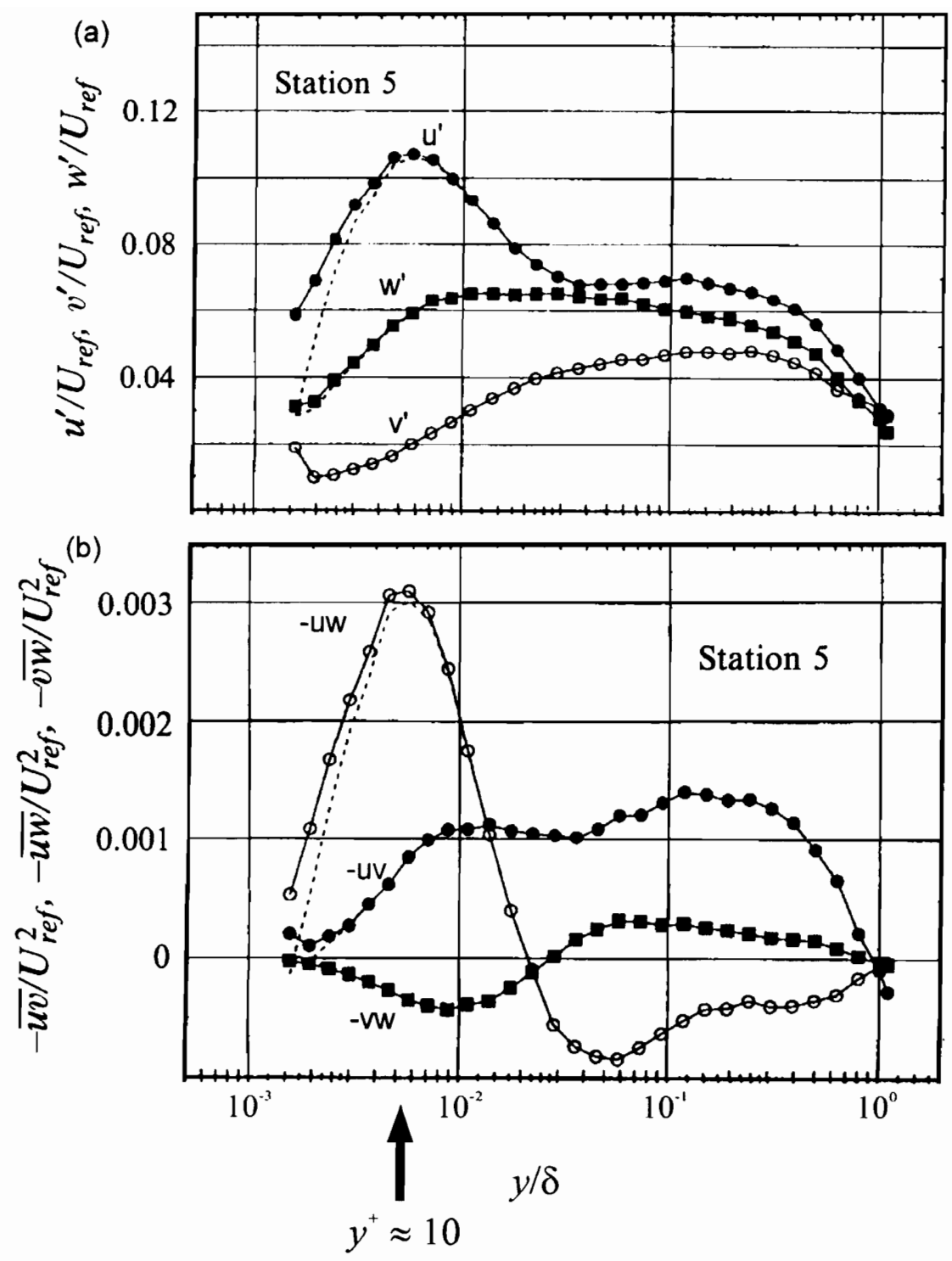

Fig. 2.27 Sample of gradient broadening corrections from Ölçmen and Simpson's (1995) LDV data. Dashed lines show the corrected data for (a) normal stress data, and (b) shear stress data. Only the $u^{\prime}$ and $-\overline{u w}$ near-wall results are significantly affected. The probe volume diameter for these measurements was about 2 viscous units. 
(a)

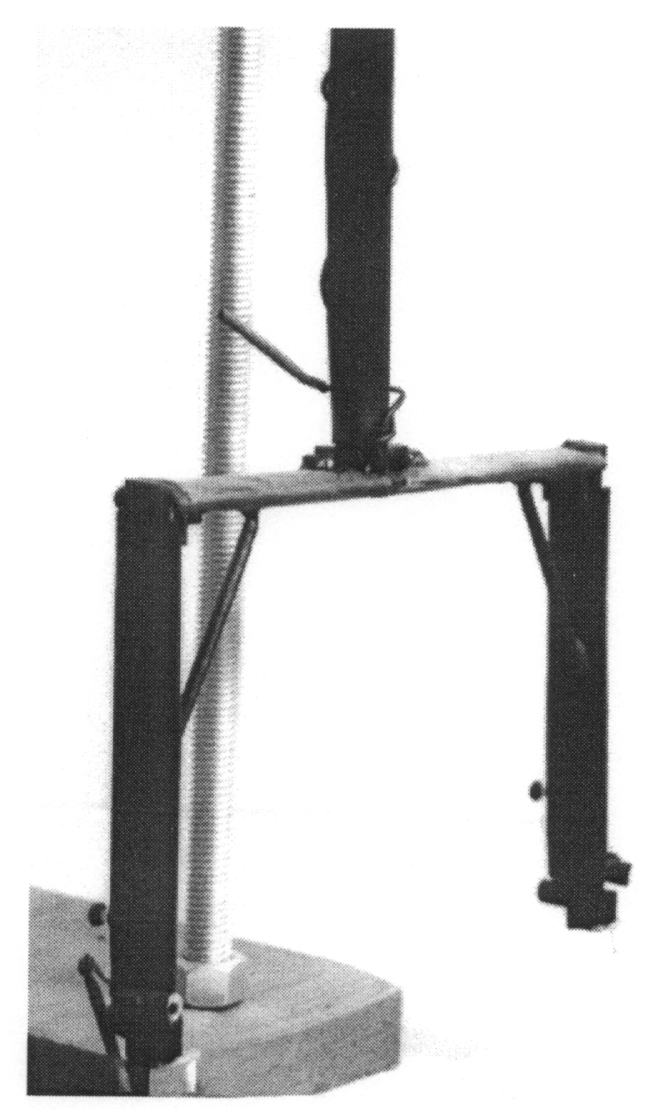

(b)

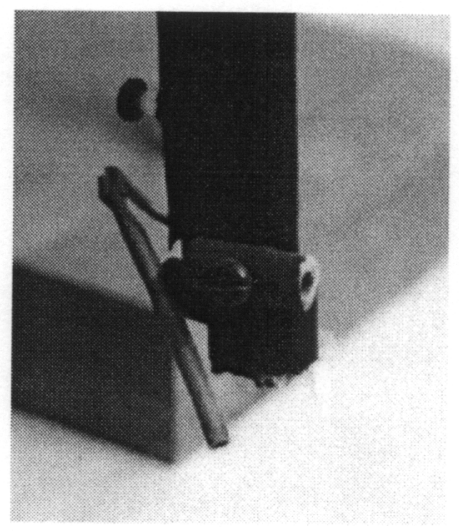

(c)

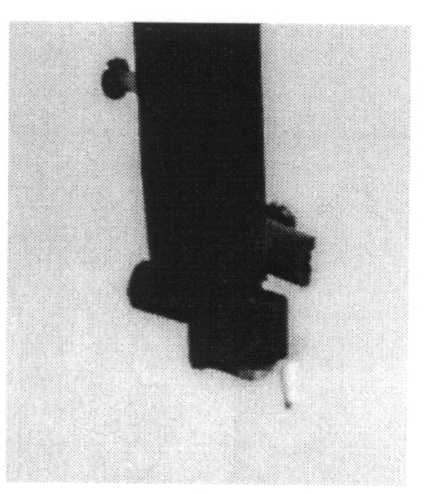

Fig. 2.28 Photographs of hydrogen-bubble probe, the device used to hold the bubble generating wire parallel to the test floor at various $y$ locations. Photo (a) shows the lower portion of the probe, while (b) and (c) are details of the wire attachment points. The wire was tensioned using a technique similar to tightening a string on a musical instrument. 


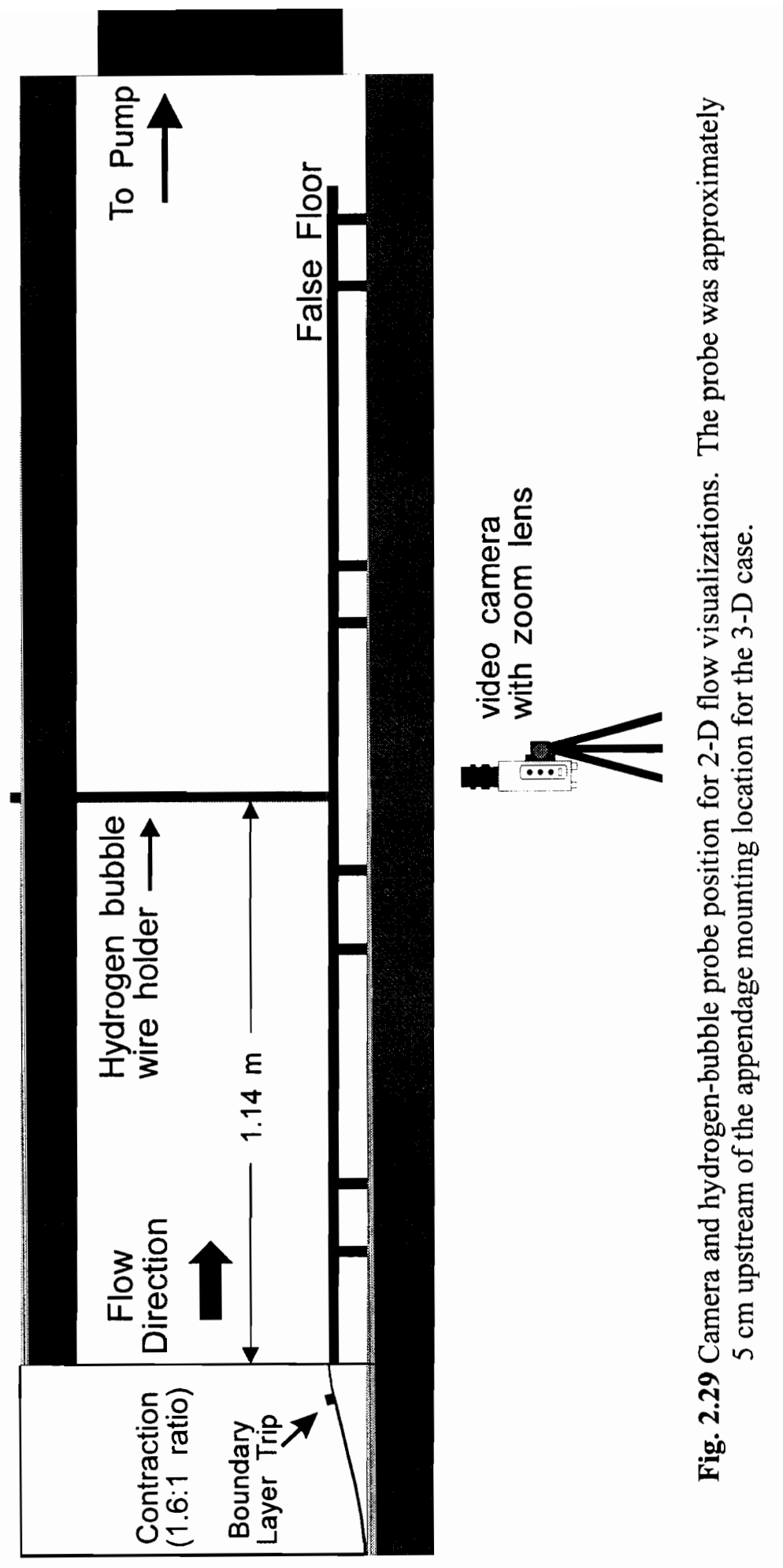




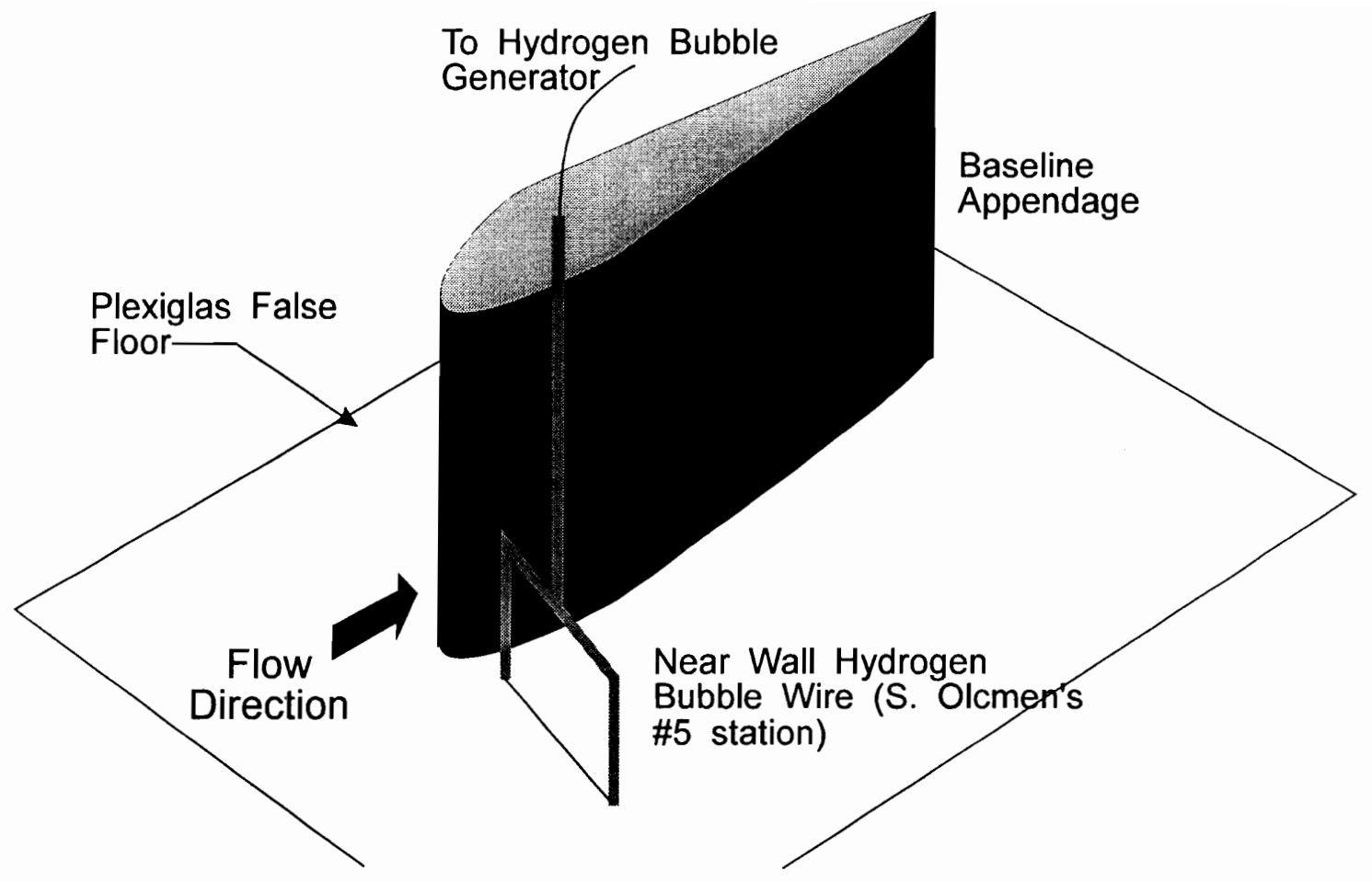

Fig. 2.30 Sketch of bubble wire position for the 3-D hydrogen-bubble flow visualizations. 
Flow Direction

(a)
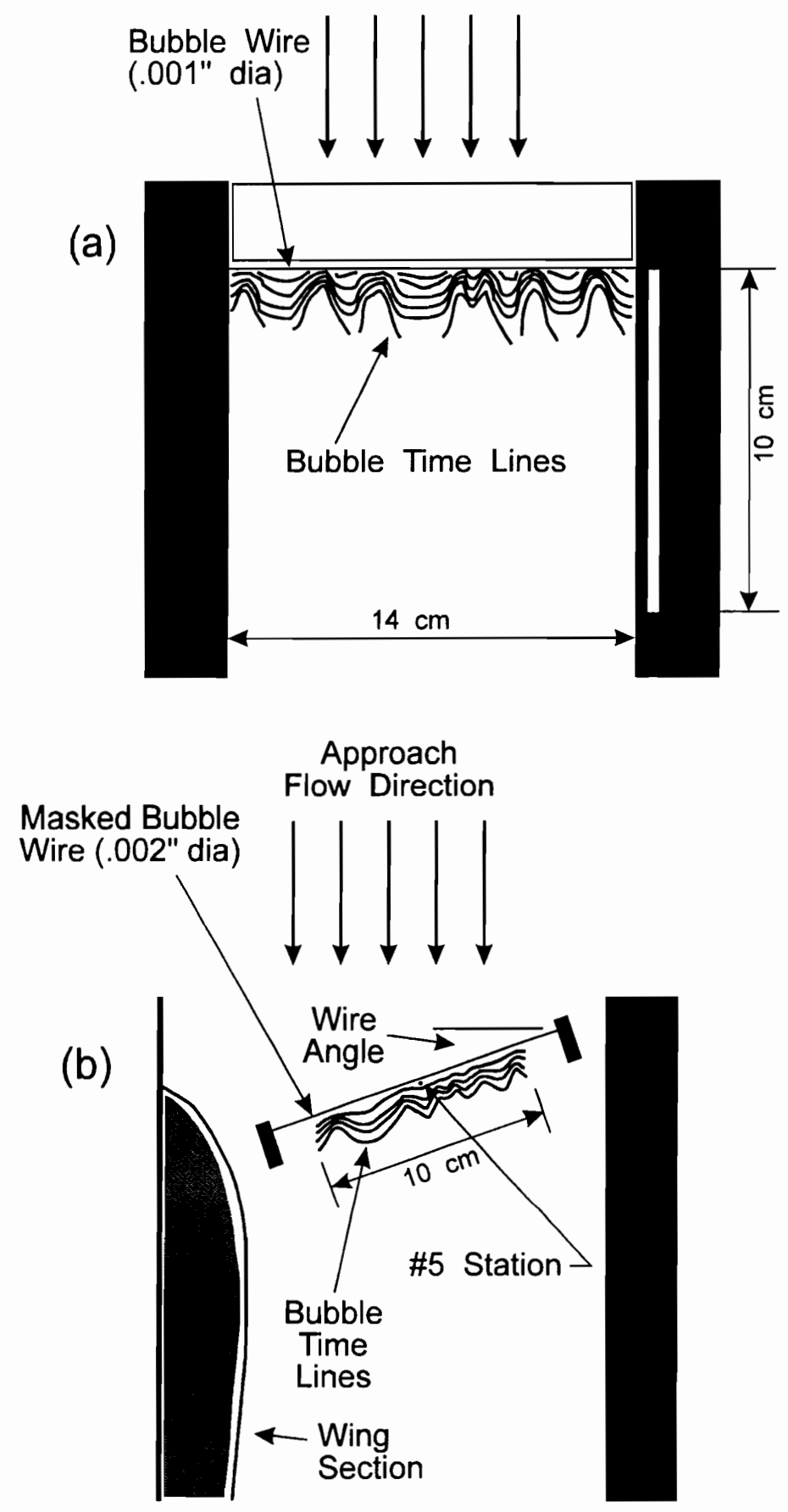

Fig. 2.31 Field of view diagrams for the hydrogen-bubble flow visualizations. The viewpoint for both sketches is looking up through the test section floor. Sketch (a) is the $2-\mathrm{D}$ case, (b) is the 3-D case. Note that the wire ends are masked for the 3-D case. 


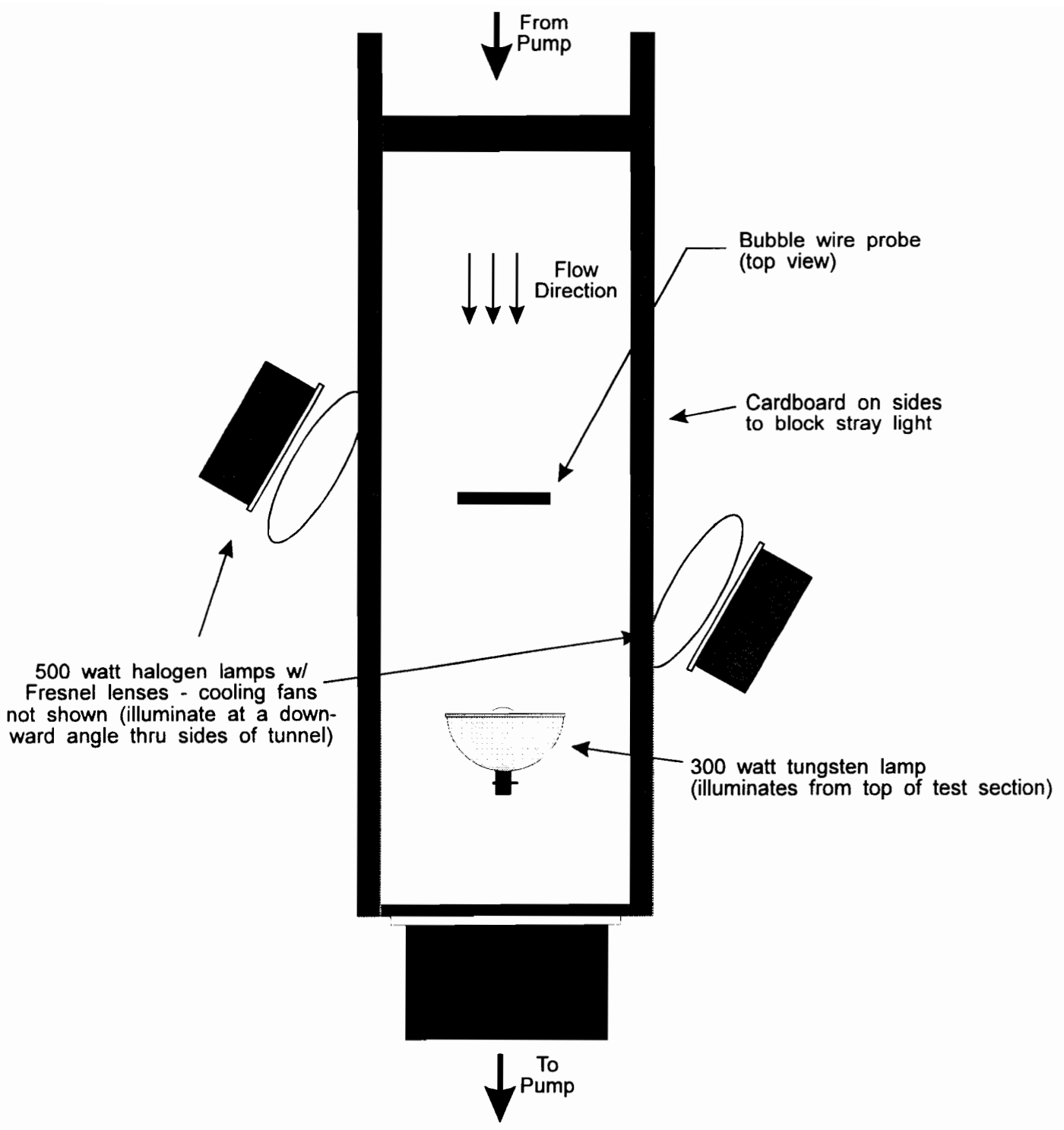

Fig. 2.32 Top view of light placement for the 2-D hydrogen-bubble flow visualizations. 


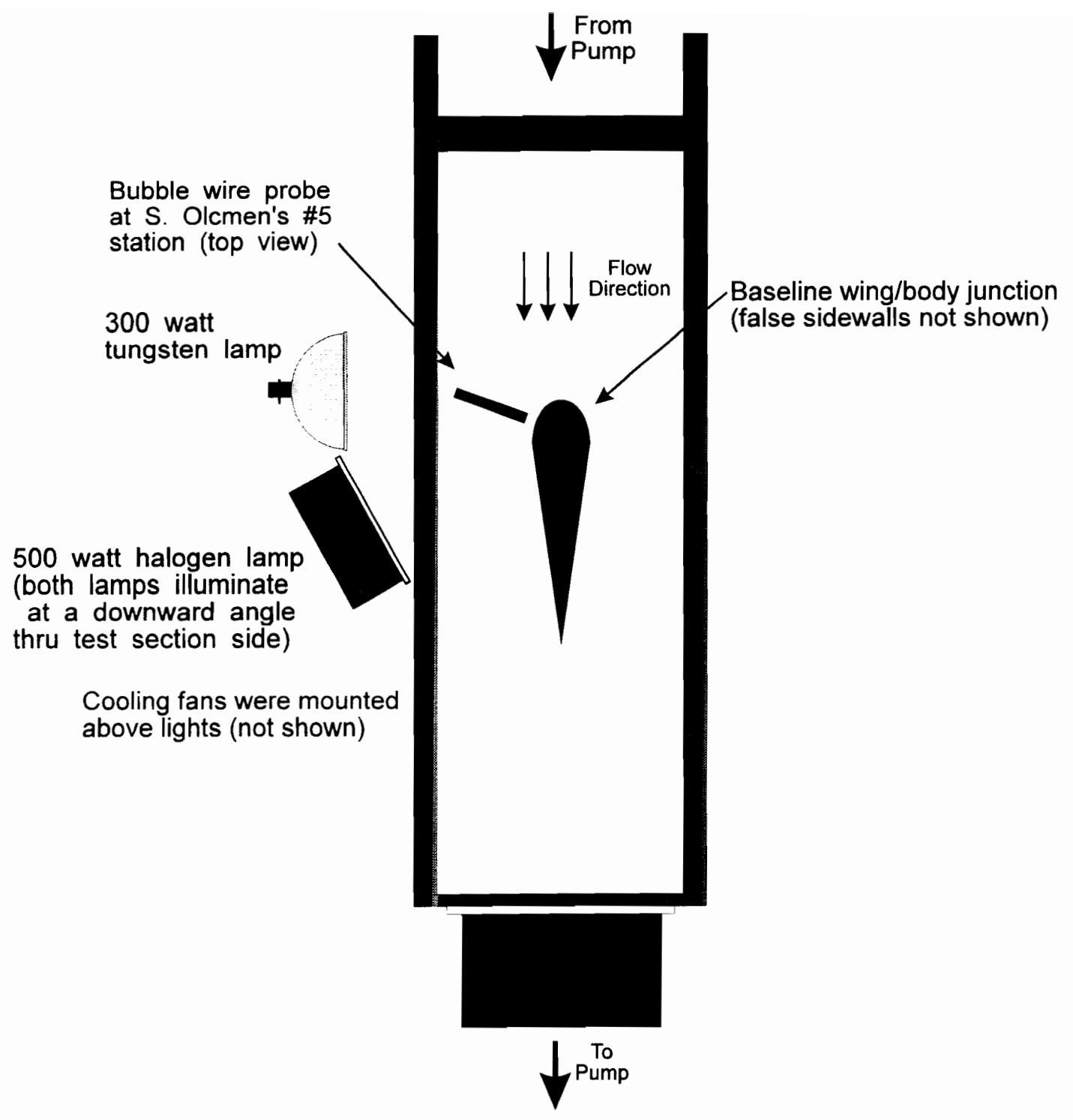

Fig. 2.33 Top view of light placement for the 3-D hydrogen-bubble flow visualizations. 


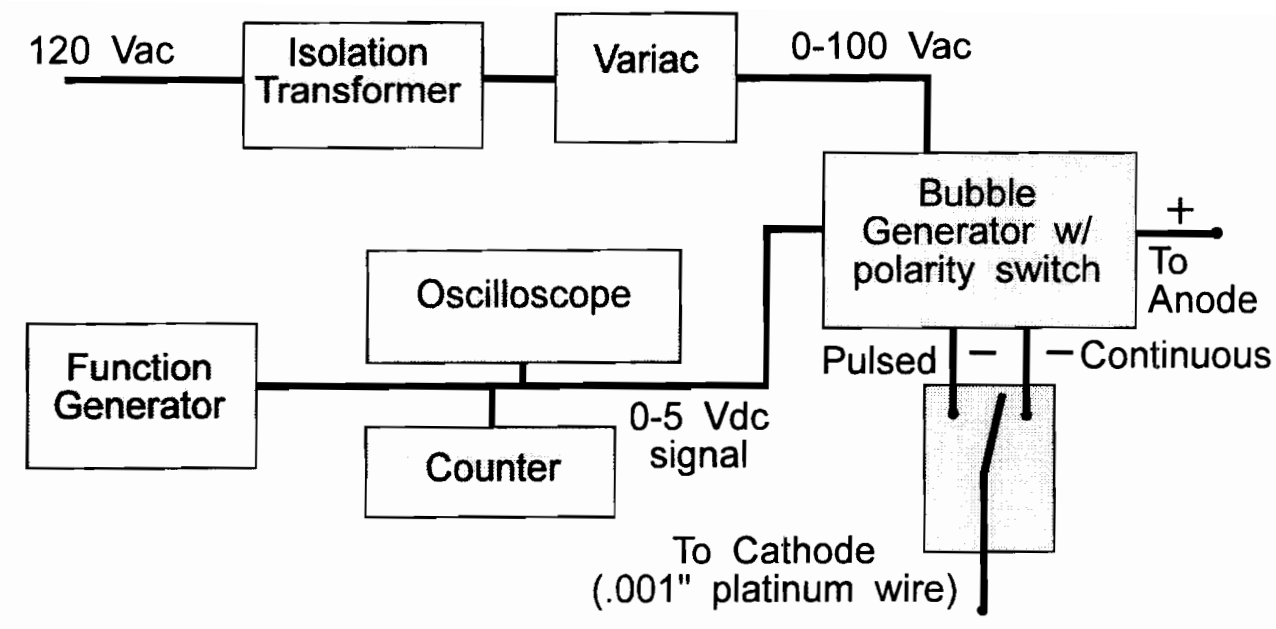

Fig. 2.34 Block diagram of equipment used to generate constant sheet and pulsed time-line hydrogen bubbles. 


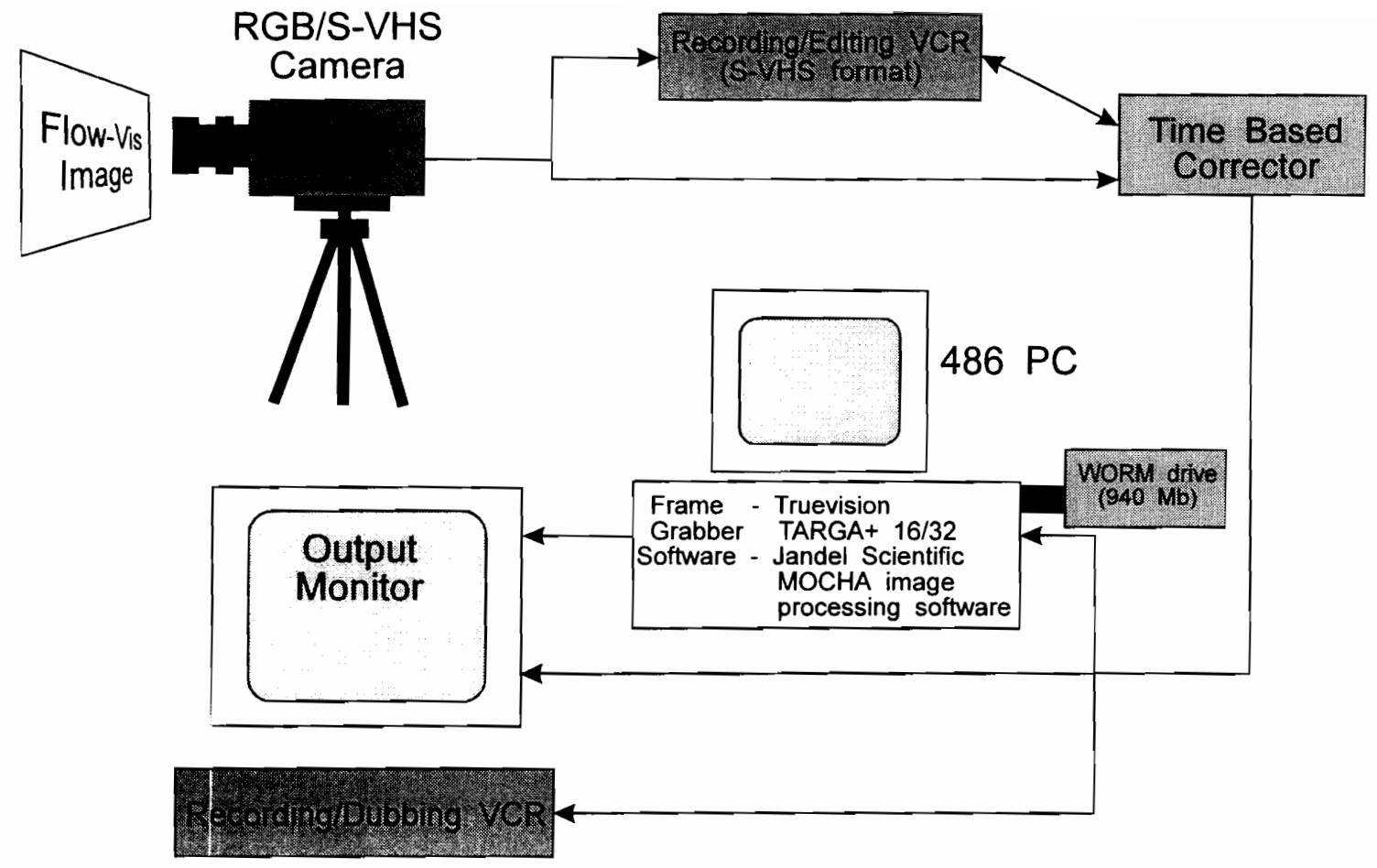

Fig. 2.35 Block diagram of equipment used to store and analyze hydrogen-bubble flow visualization data. 
(a)

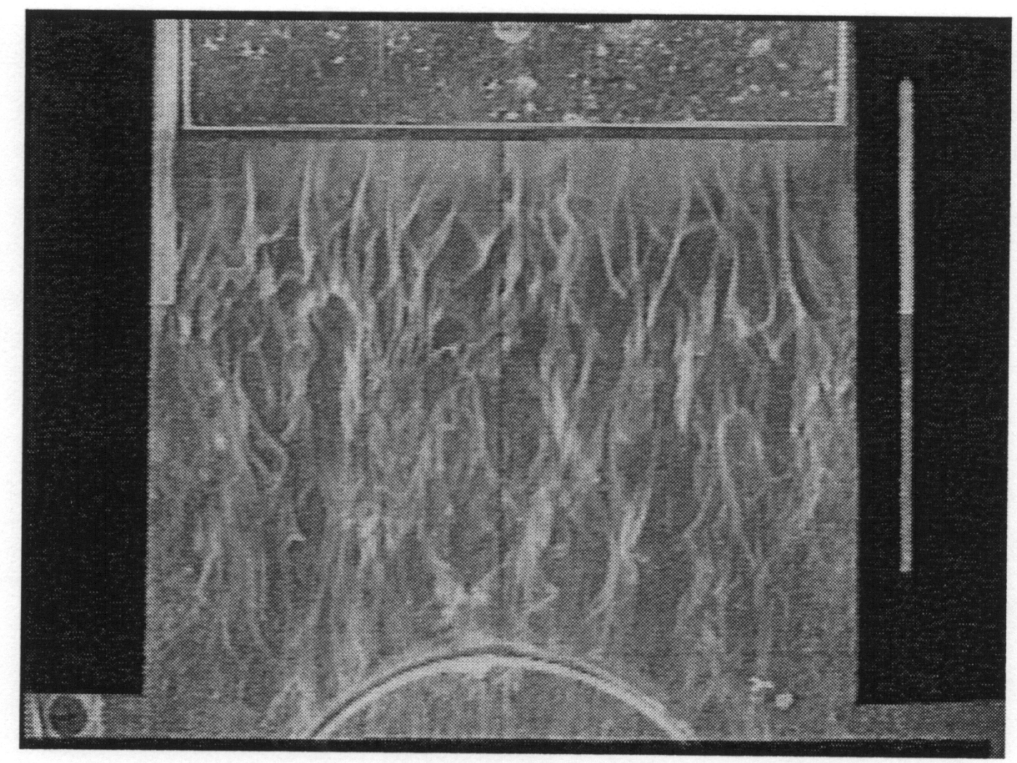

(b)

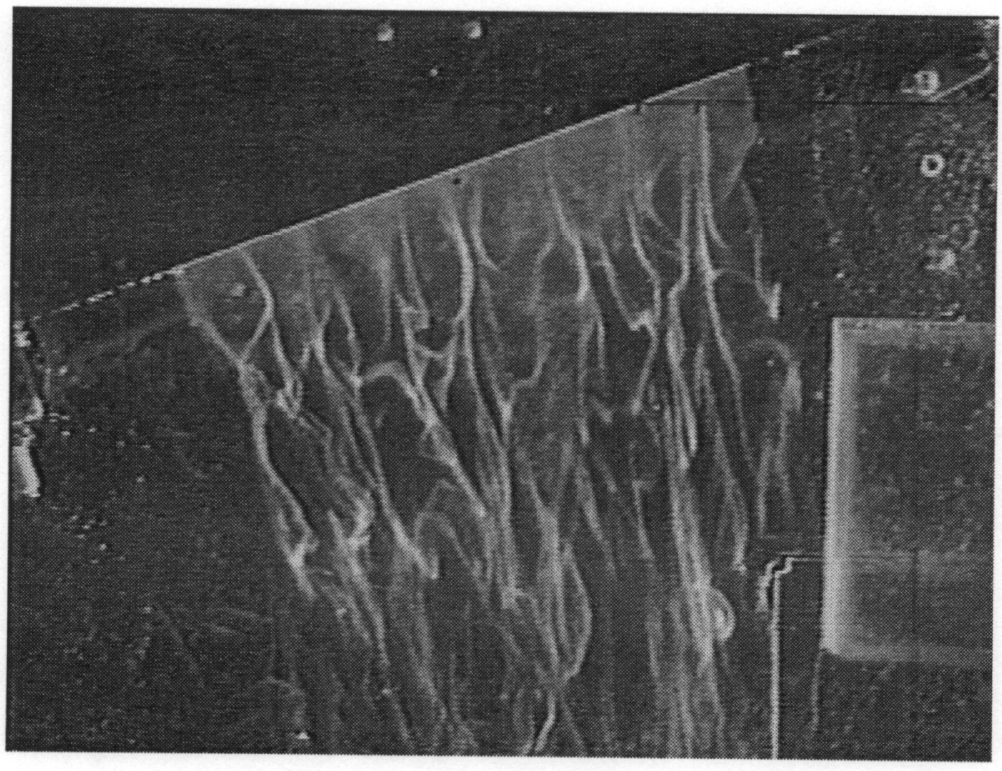

Fig. 2.36 Sample constant-sheet images of hydrogen-bubble flow-visualization results, images captured from video. (a) $2-\mathrm{D}, y^{+} \approx 10, U_{e} \approx 15 \mathrm{~cm} / \mathrm{s}$, and (b) 3 -D, $y^{+} \approx 10$, $U_{e} \approx 15 \mathrm{~cm} / \mathrm{s}$. 
(a)

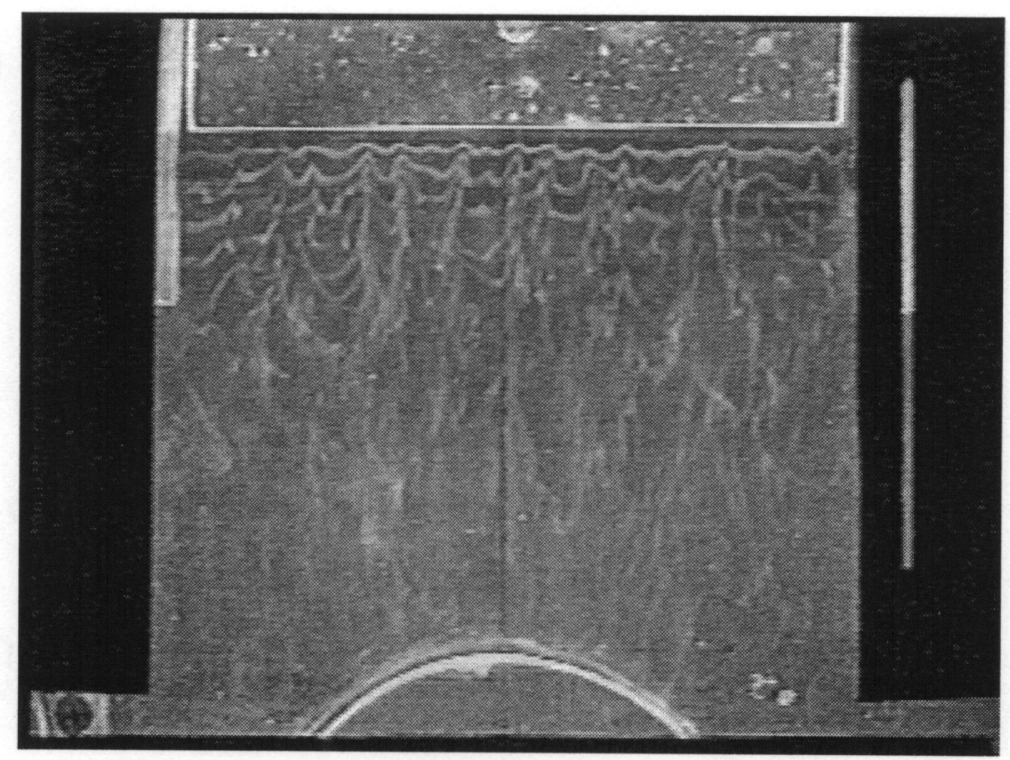

(b)

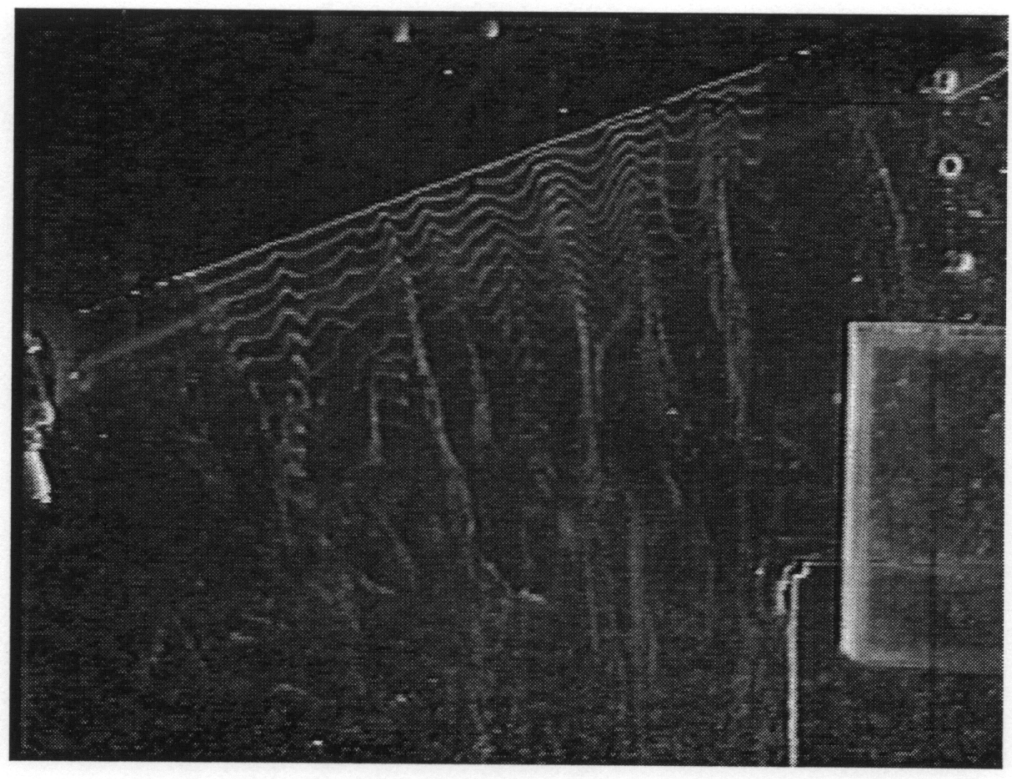

Fig. 2.37 Sample time-line images of hydrogen-bubble flow-visualization results, images captured from video. (a) $2-\mathrm{D}, y^{+} \approx 10, U_{e} \approx 15 \mathrm{~cm} / \mathrm{s}$, and (b) $3-\mathrm{D}, y^{+} \approx 10, U_{e} \approx 15$ $\mathrm{cm} / \mathrm{s}$. 


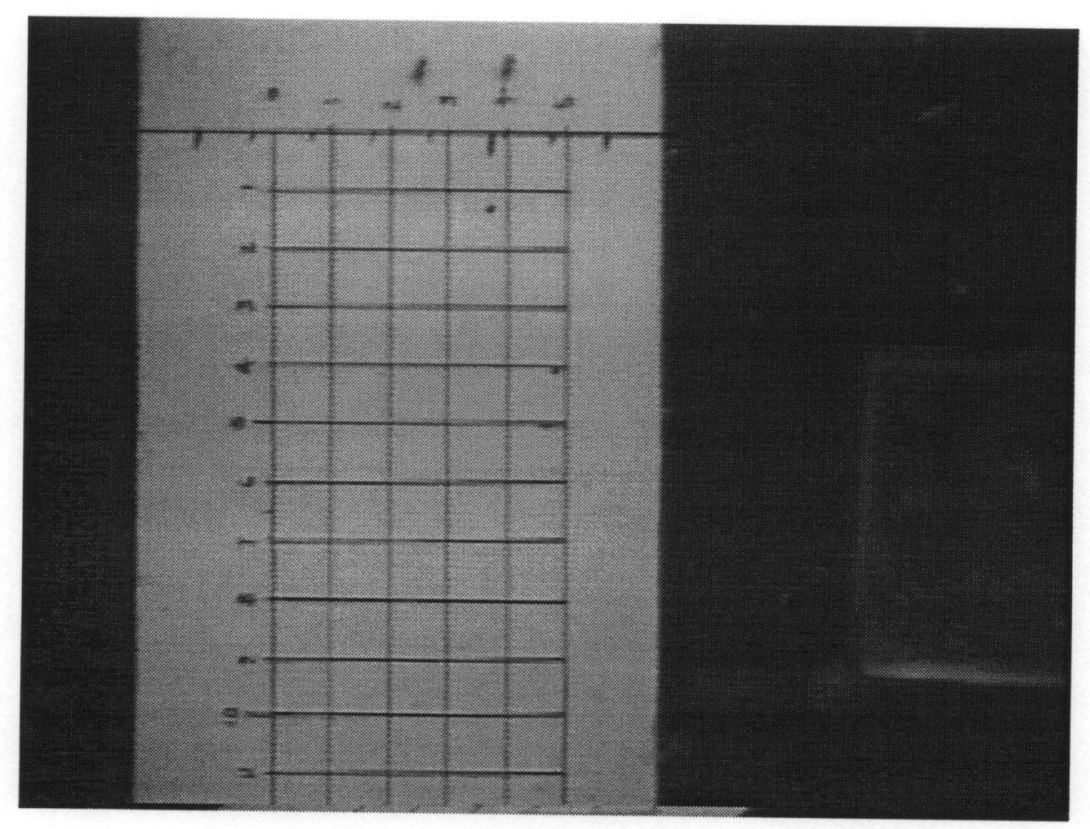

Fig. 2.38 A sample calibration image used for the 3-D flow case. A $1 \times 1 \mathrm{~cm}$ grid is shown. The point marking Ölçmen's \#5 station is visible in the upper part of the image, in grid cell $(4,2)$. 


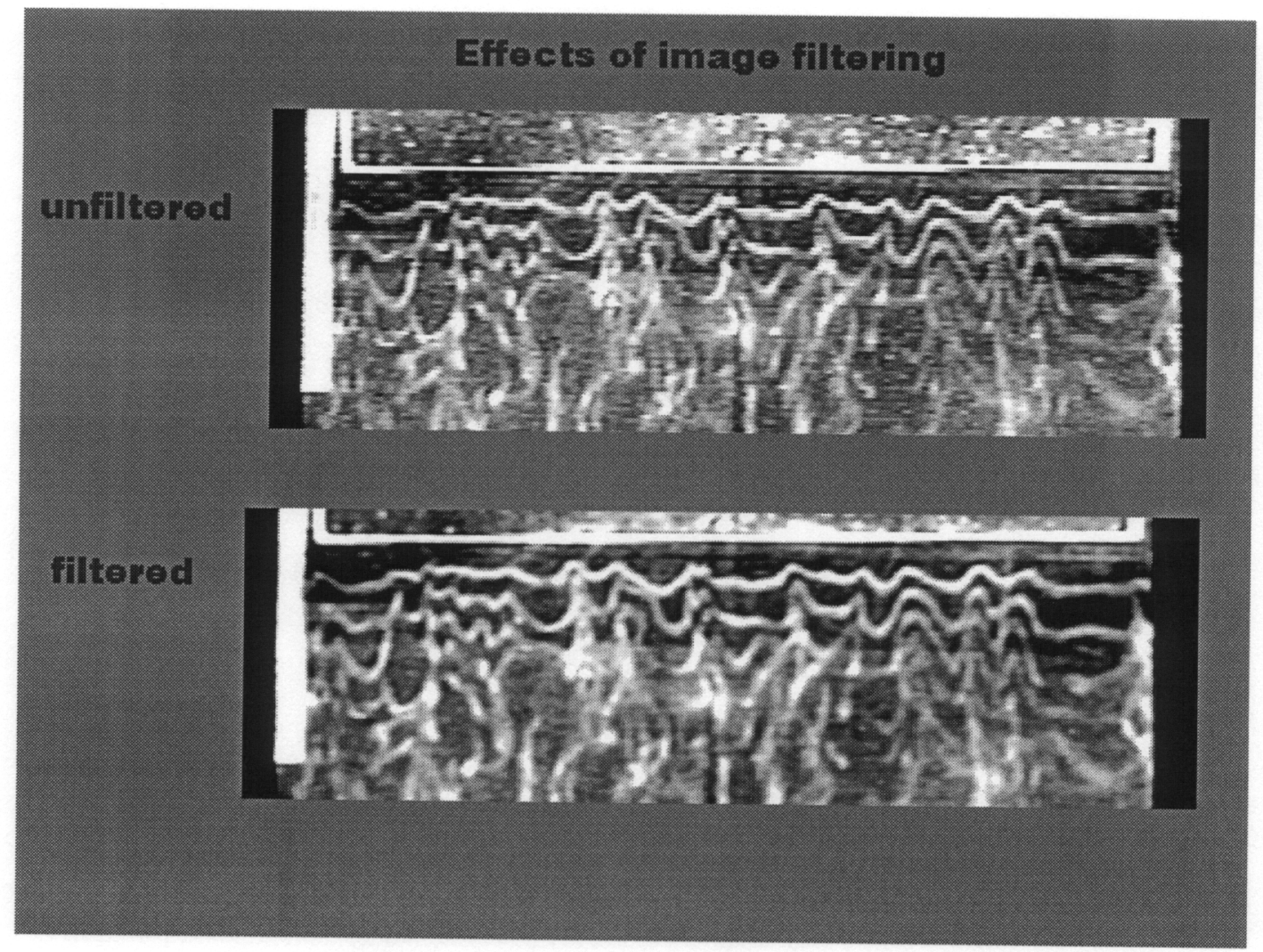

Fig. 2.39 Example of how filtering and enhancing the digitized flow-visualization results can decrease image noise and enable automated quantitative reduction of the data. This example shows 2-D data, which was usually of poorer quality compared to the 3-D data. 


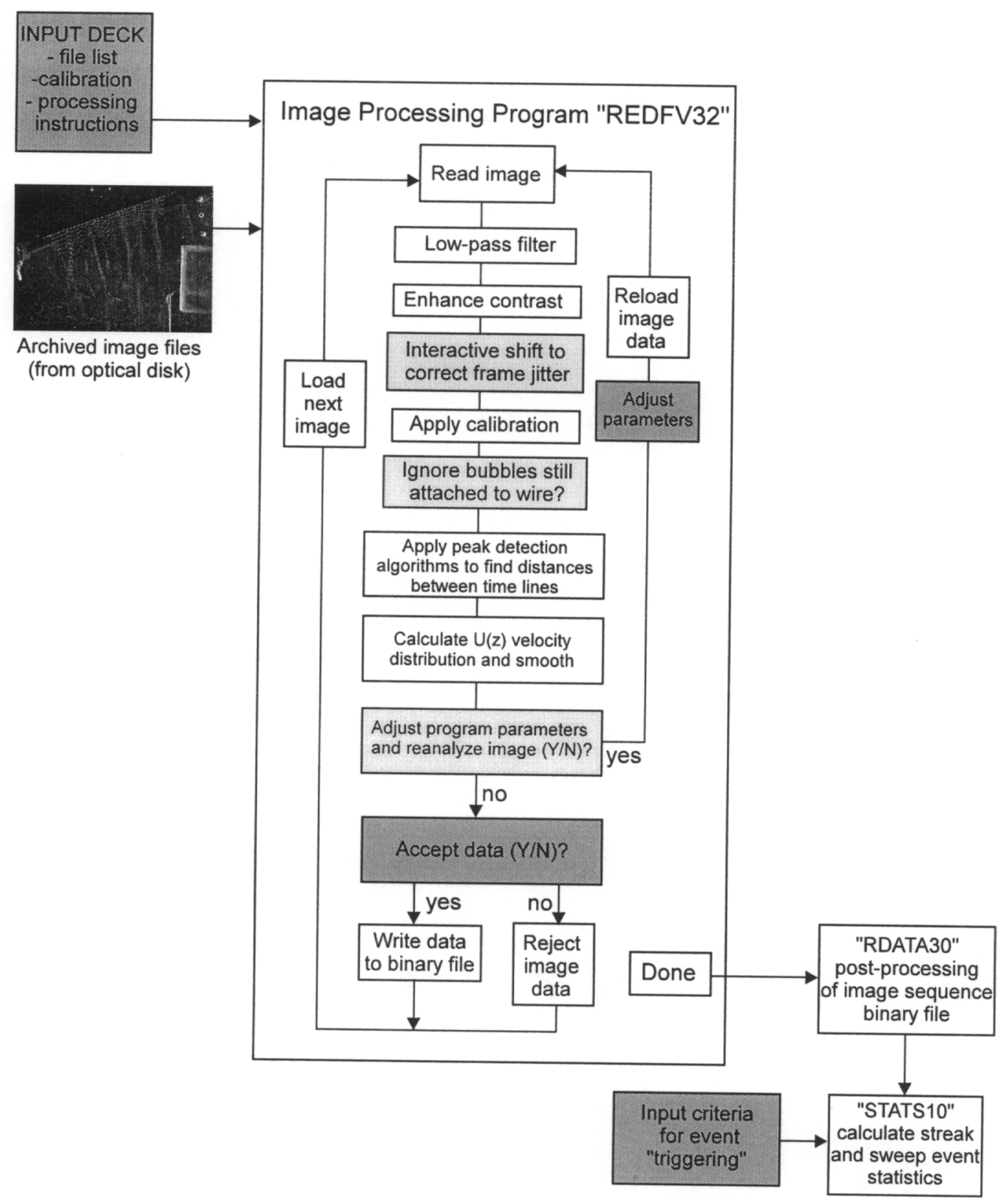

Fig. 2.40 Outline of the image processing procedure used to obtain quantitative results from the time-line flow-visualization images. Lightly shaded boxes indicate when user input was needed as part of the data analysis. Dark shaded boxes indicate subjective inputs or decisions. 
(a)

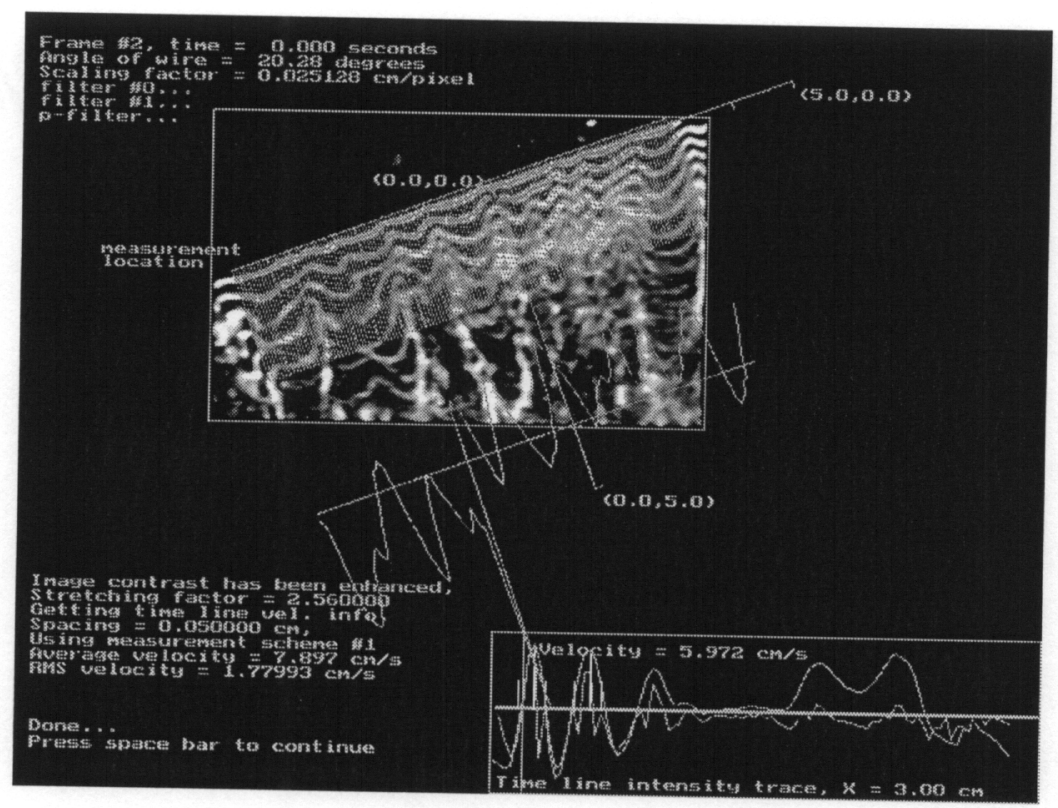

(b)

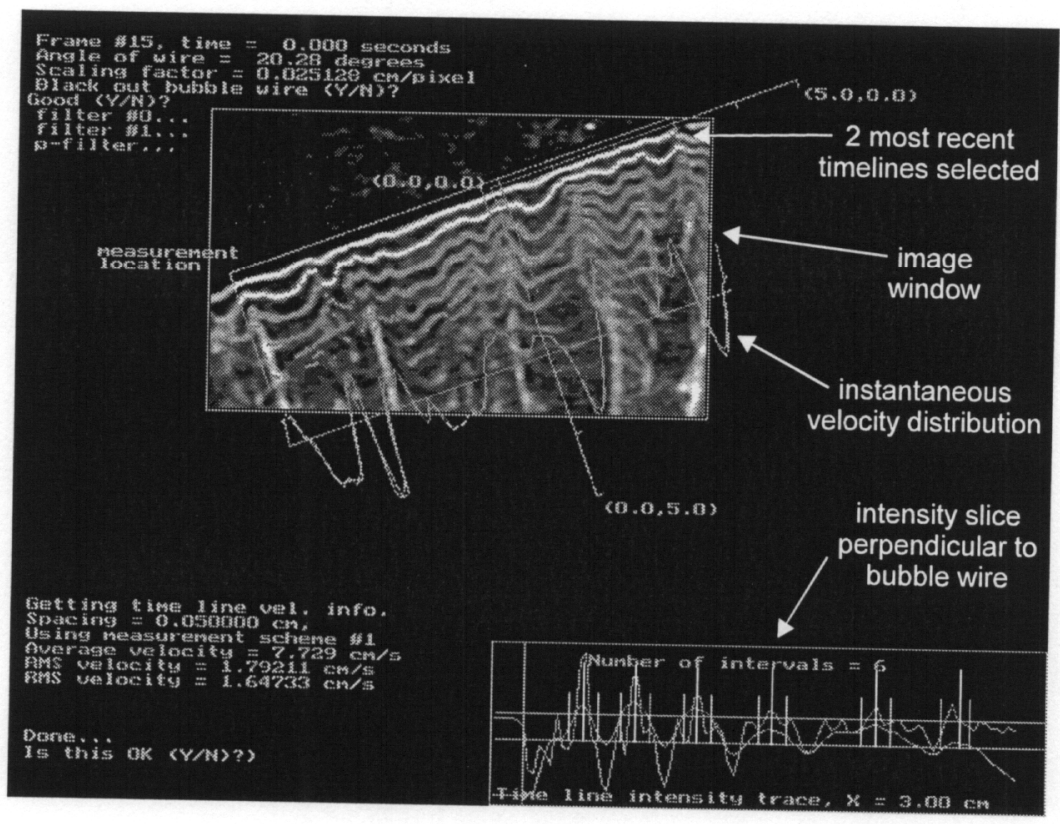

Fig. 2.41 Examples of the automated time-of-flight reduction technique used to obtain quantitative data from the hydrogen-bubble flow visualization video. These results are from the $3-\mathrm{D}$ case. Note that only a portion of the image is shown on these sample screens. The $U(z)$ velocity distribution is plotted immediately below the time lines. The plot in the lower right corner of the screen is one intensity "slice" perpendicular to the time lines. 
(a)

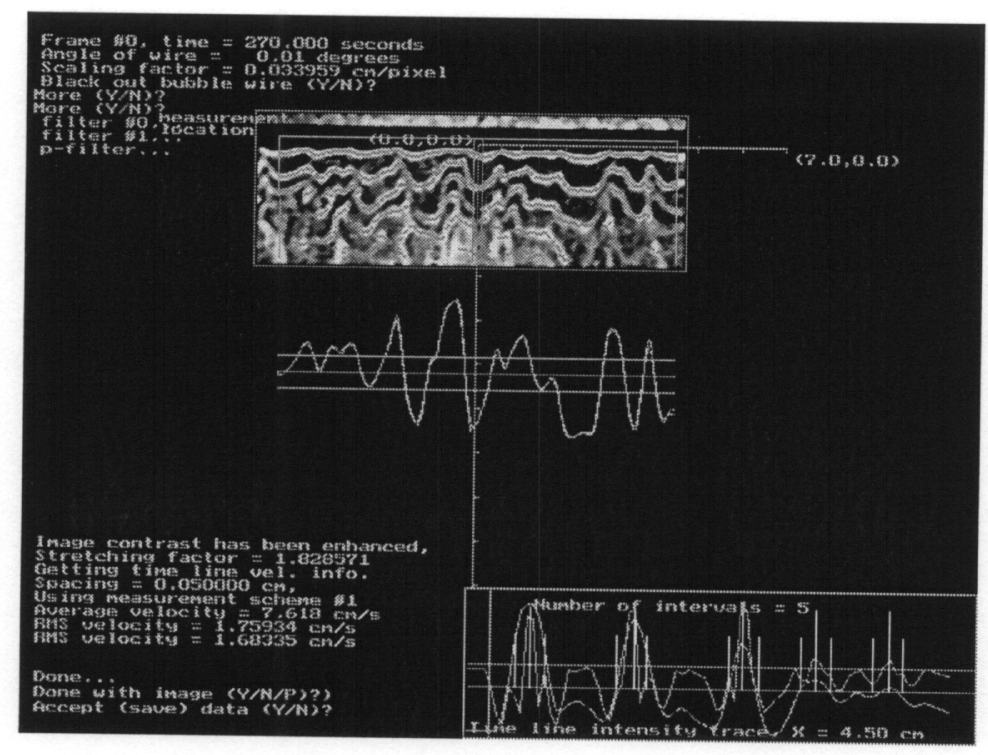

(b)

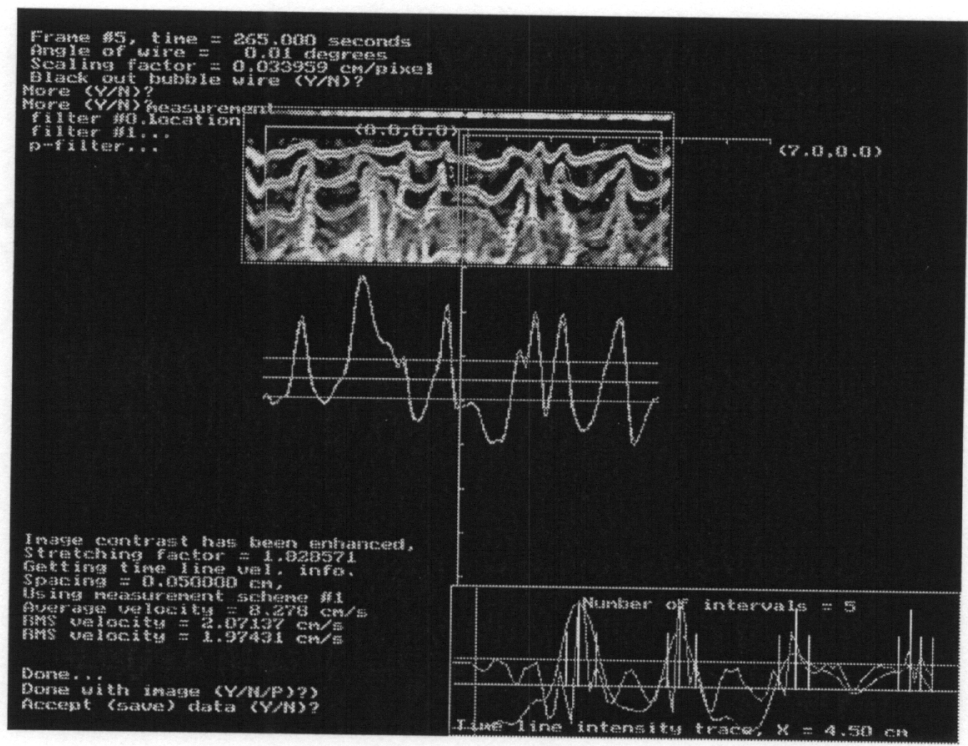

Fig. 2.42 More examples of the time-of-flight reduction technique used to obtain quantitative data from the hydrogen-bubble time lines. These results are from the 2-D case, which tended to be the more difficult case. 


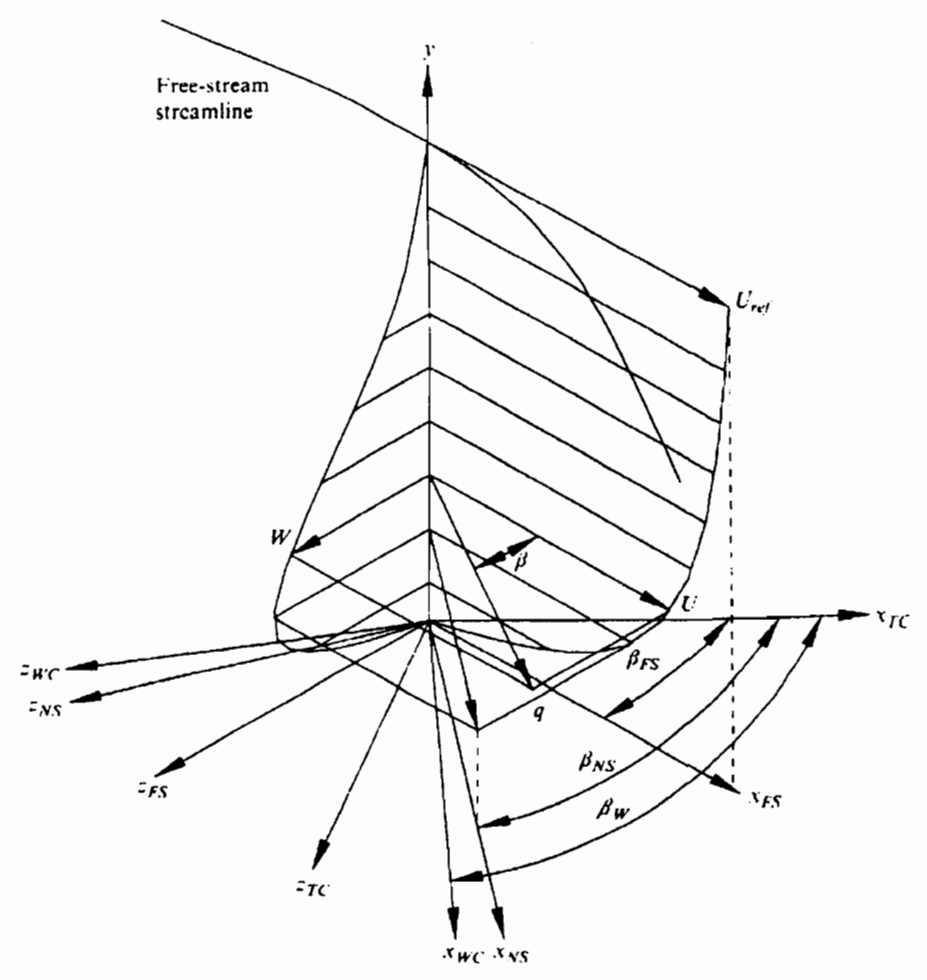

Fig. 3.1 The different coordinate systems traditionally used for the presentation of 3-D boundary layer data (from Ölçmen and Simpson, 1995). 

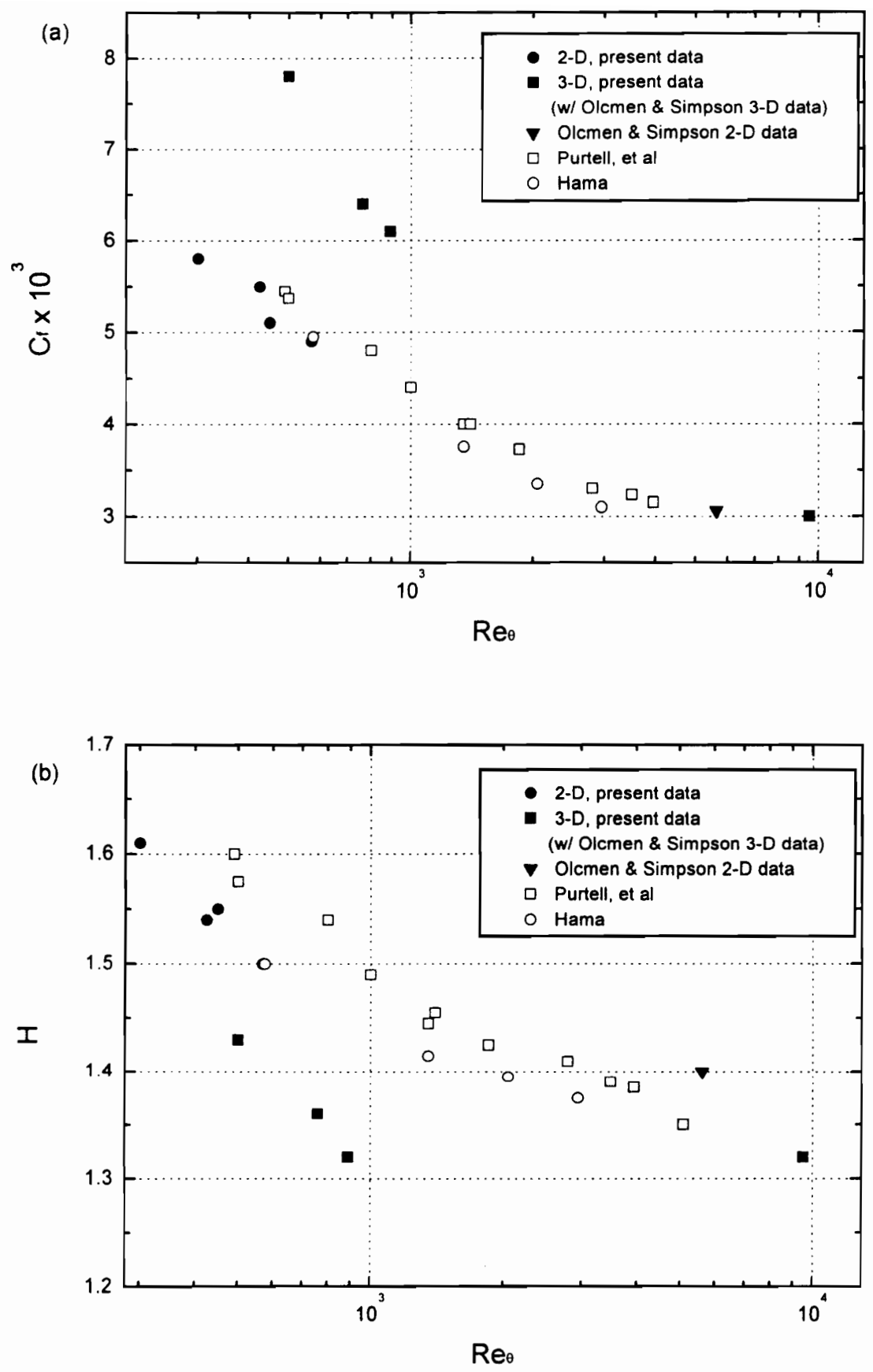

Fig. 3.2 Skin friction coefficient and shape factor vs. Reynolds number for current 2-D and 3-D data. Data from Purtell, et al. (1981) and Hama (1954) are also plotted. For figure (a) the total $C_{f}$ magnitude was plotted for the 3-D data. For figure (b) the 3 -D shape factor was calculated using freestream coordinate system results. 

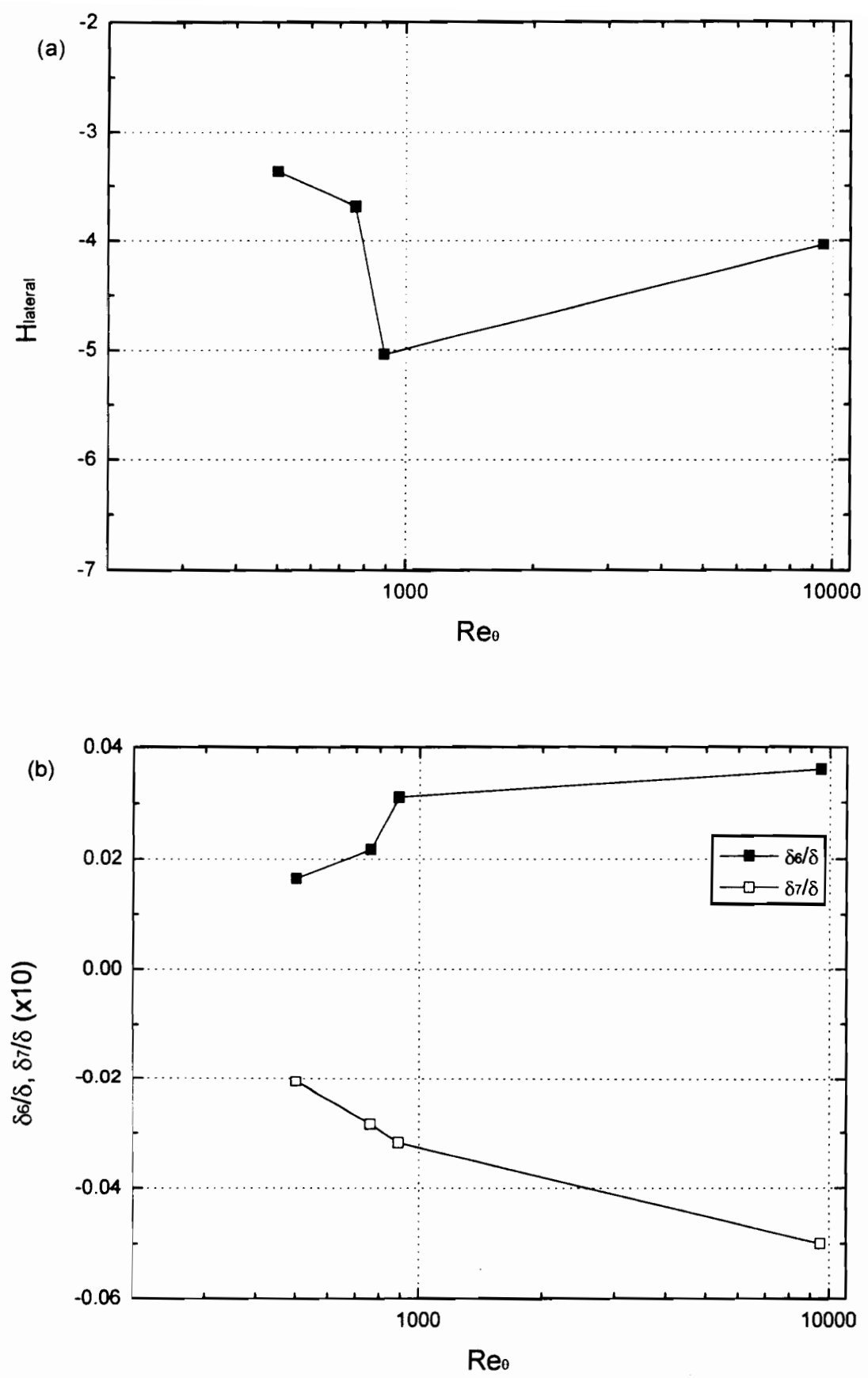

Fig. 3.3 3-D lateral shape parameters vs. Reynolds number for the current 3-D data and Ölçmen and Simpson's (1995) data. The lateral shape factor, $\left(\delta_{4} / \delta_{5}\right)$ is shown for plot (a), and plot (b) displays the cross-product momentum thickness $\left(\delta_{6}\right)$ and the cross-flow momentum thickness $\left(\delta_{7}\right)$, both non-dimensionalized by the boundary layer thickness. See Table 3.2 for shape parameter equations. 

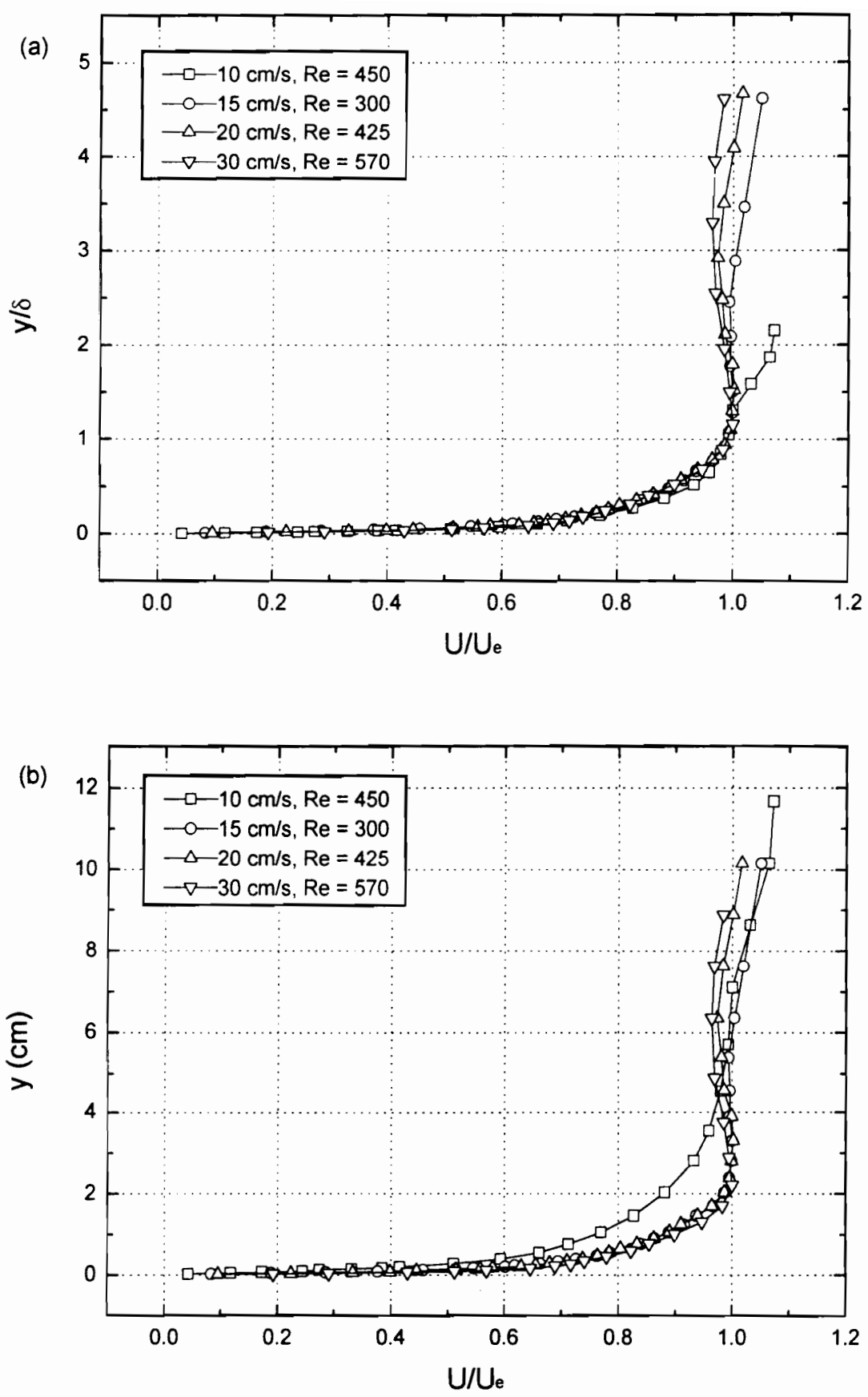

Fig. 3.4 2-D flow boundary layer profiles, plotted to display the shape of the mean velocity distribution. For (a), $y$ is non-dimensionalized by $\delta$, while for (b), the $y$ axis is not non-dimensionalized. 

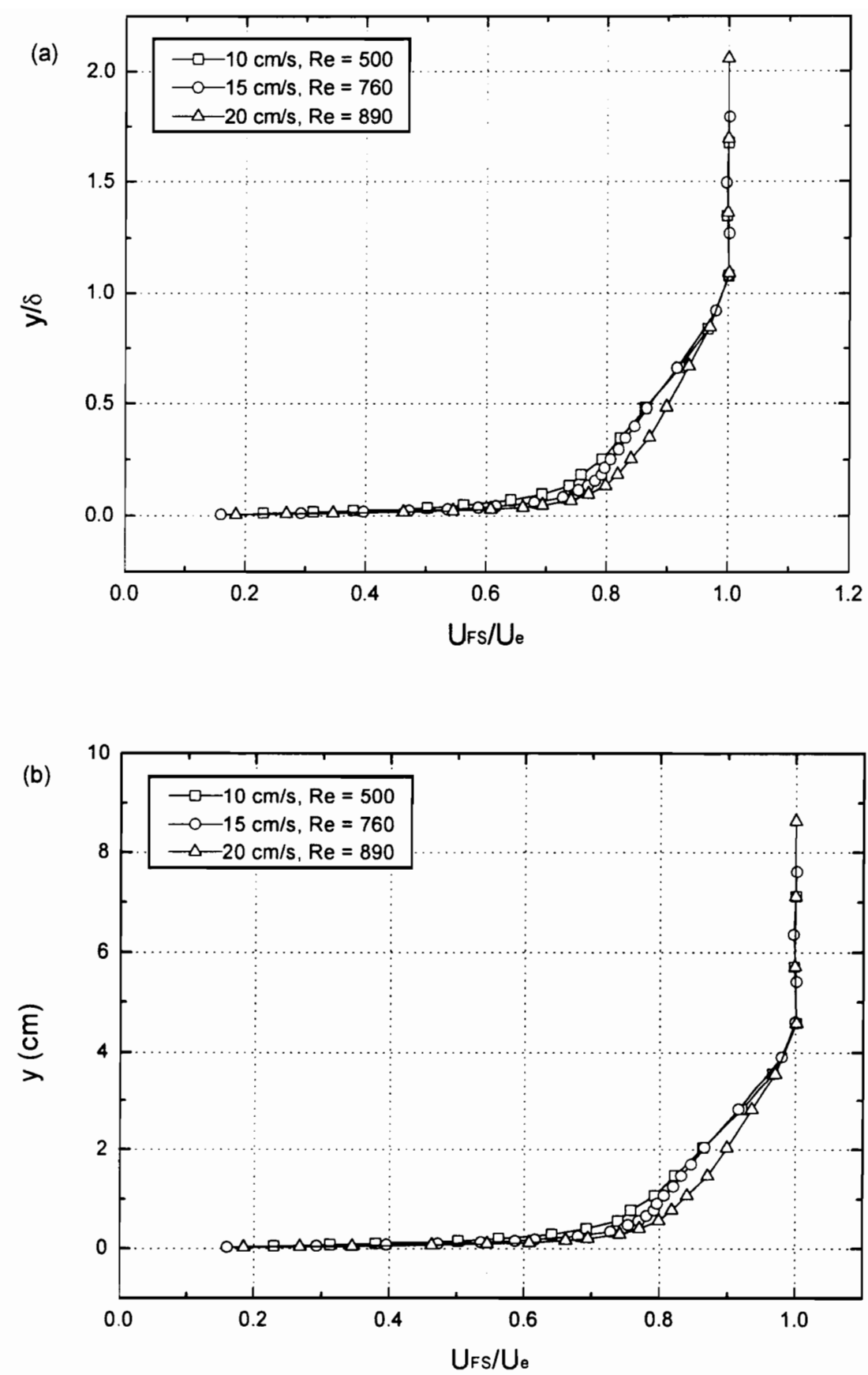

Fig. 3.5 3-D flow boundary layer profiles, plotted to display the shape of the mean velocity distribution. For (a), $y$ is non-dimensionalized by $\delta$, while for (b), $y$ is not nondimensionalized. Note the linear shape of the profiles from $\approx 0.15 \delta$ to the edge of the boundary layer. 

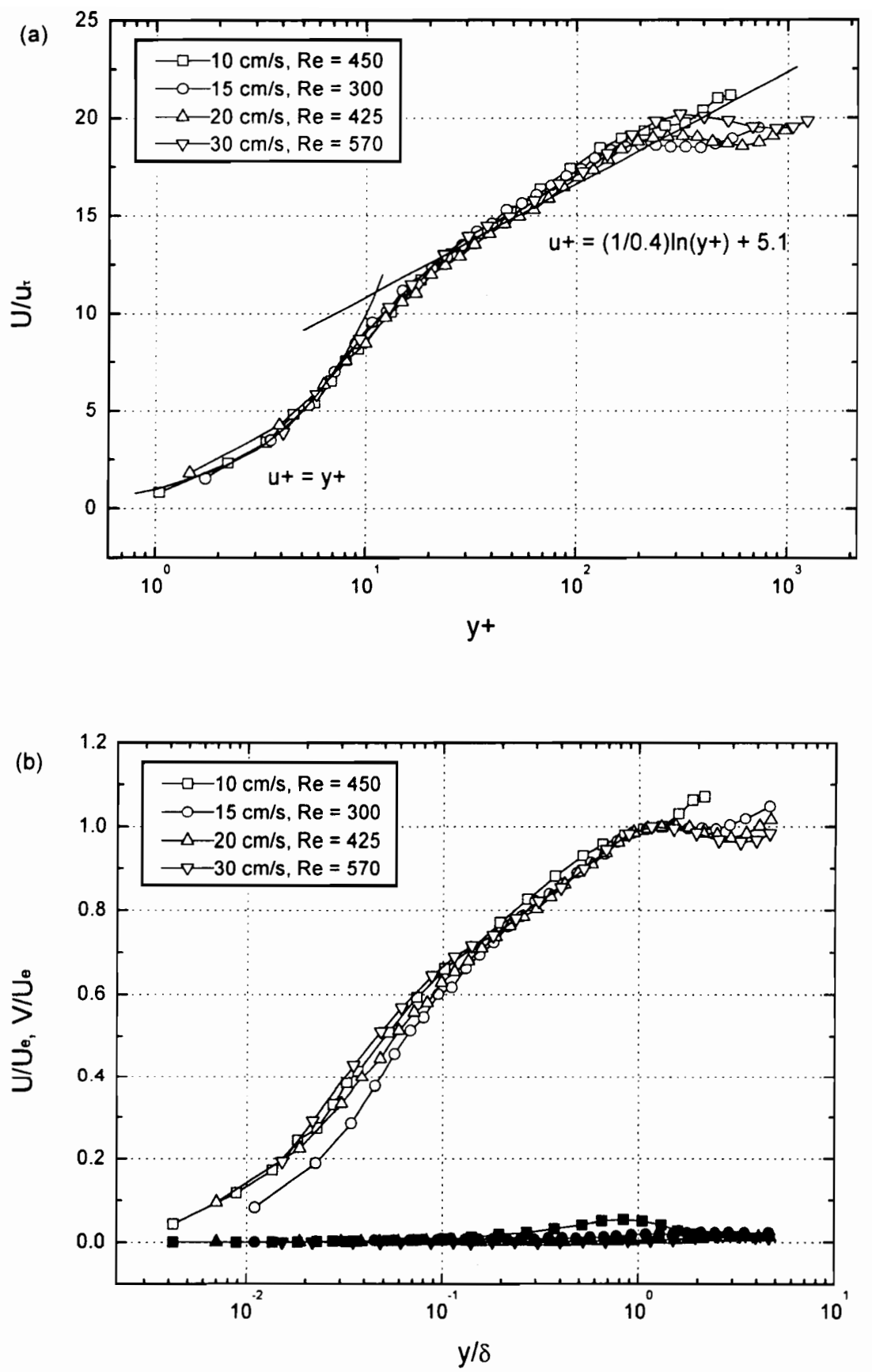

Fig. 3.6 2-D mean velocity boundary layer profiles. (a) is non-dimensionalized using inner scaling, with the inner and log region curve fits shown. Coles' wall-law constants are used for the log region. (b) shows the $U$ (open symbols) and $V$ (solid symbols) results non-dimensionalized using outer scaling. 


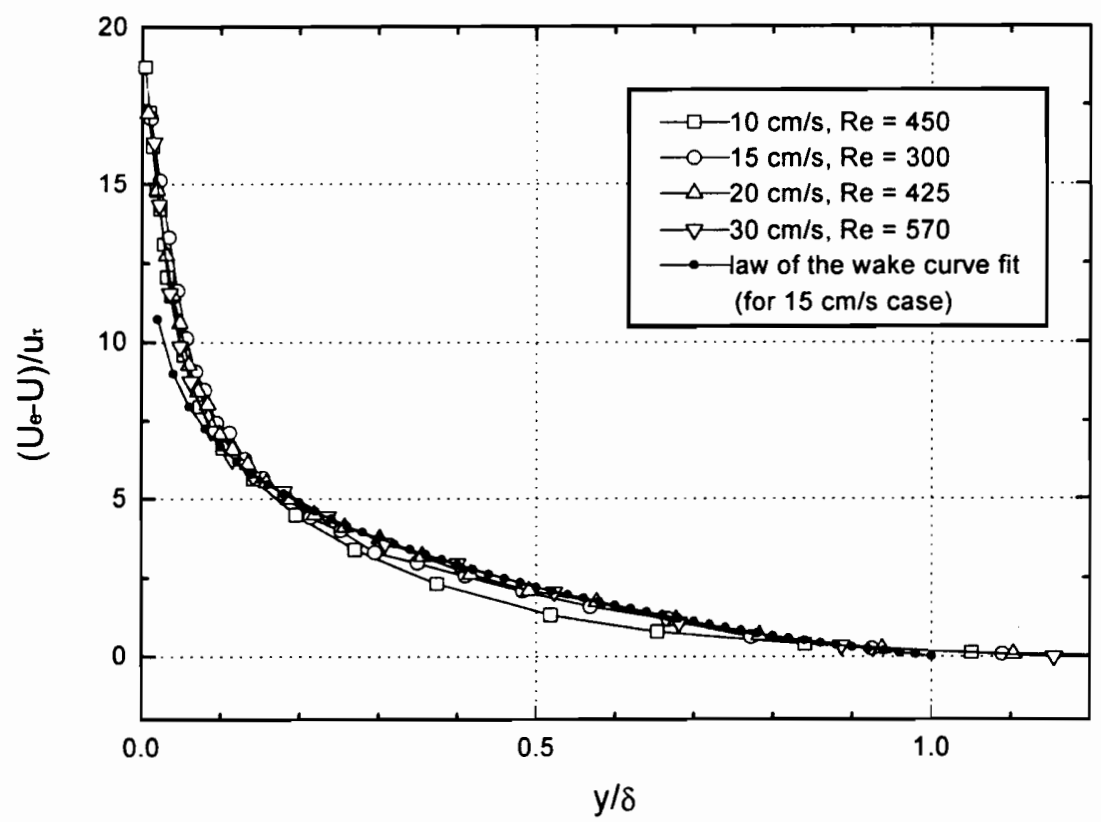

Fig. 3.7 The 2-D velocity defect profile results, $\left(U_{e}-U\right) / u_{\tau}$ vs. $y / \delta$. The law of the wake curve fit is shown for the $U_{e}=15 \mathrm{~cm} / \mathrm{s}$ case, using Coles' constants $(\kappa=0.40, C=$ $5.1)$. 

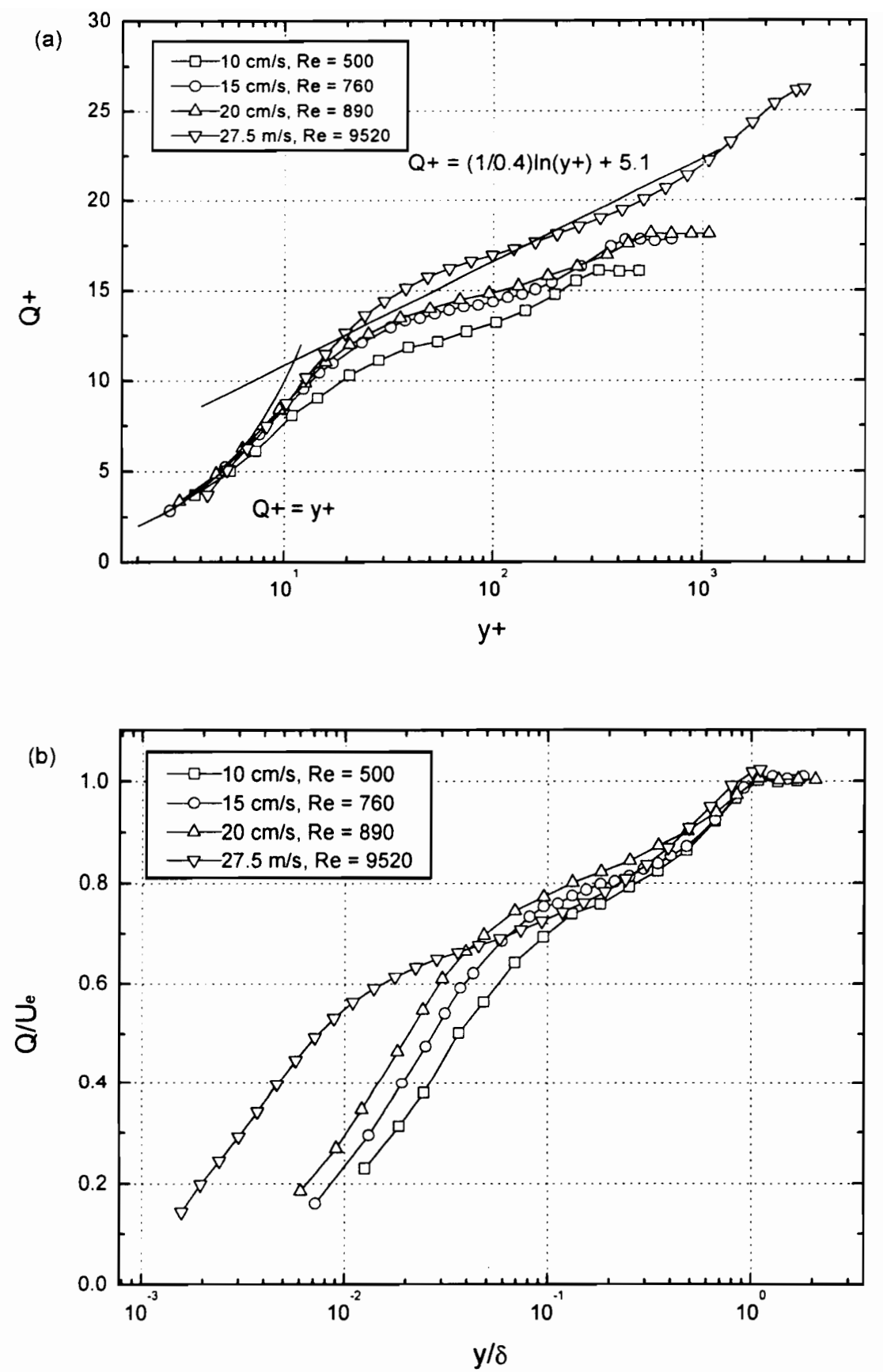

Fig. 3.8 3-D boundary layer profiles of $Q\left(Q=\bar{U}_{\mathrm{FS}} / \cos \left(\beta_{w}\right)_{\mathrm{FS}}\right)$ (from Johnston, 1960). (a) is non-dimensionalized using viscous units, with the inner and log region curve fits shown. Johnston wall law used with Coles' constants. (b) shows $Q$ nondimensionalized using outer scaling. Note: All $\operatorname{Re}_{\theta}=9520$ data are from Ölçmen and Simpson (1995). 

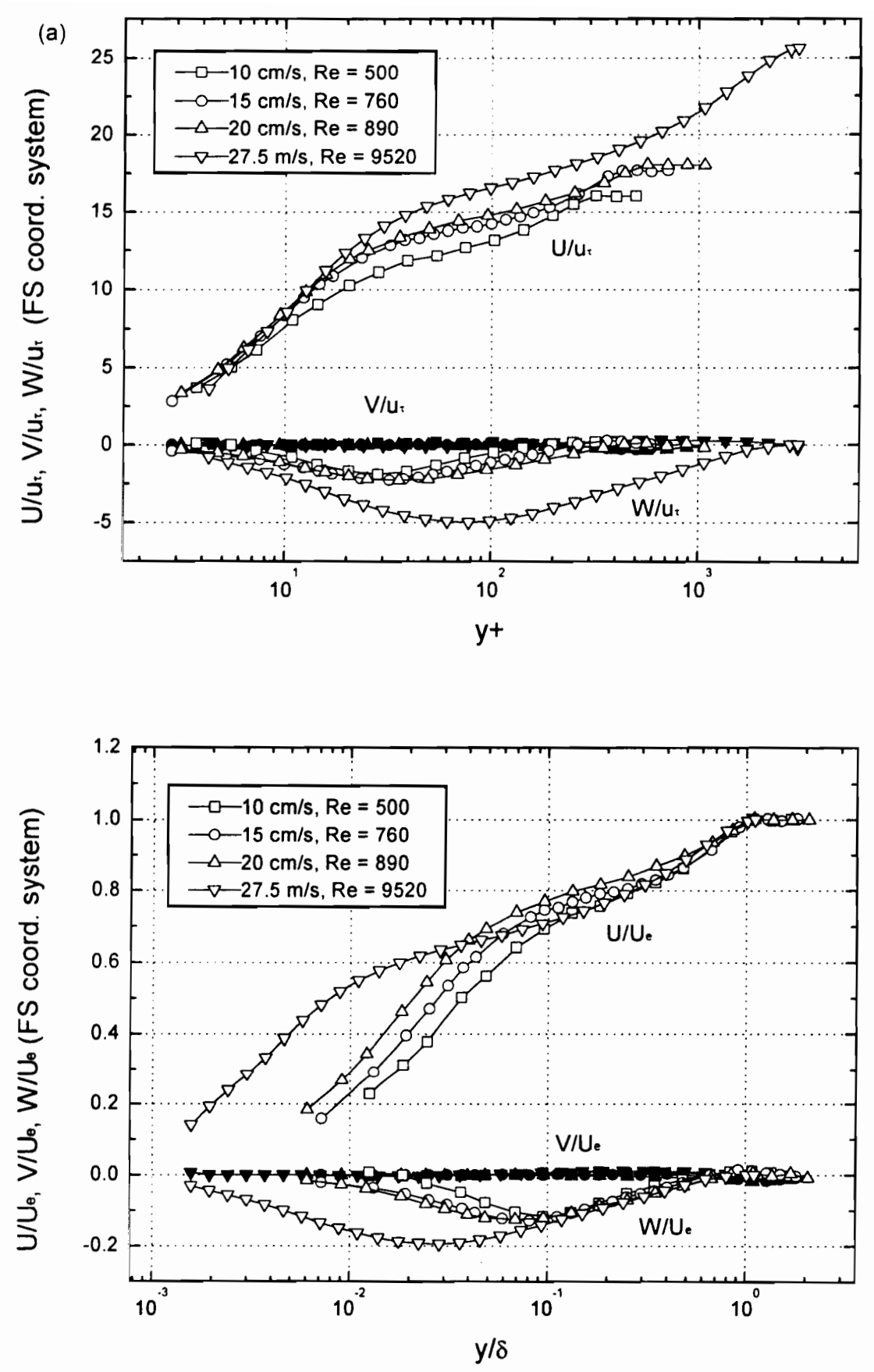

Fig. 3.9 3-D mean velocity boundary layer profiles, presented using the freestream coordinate system. Profiles in (a) are non-dimensionalized using inner scaling. (b) shows the $U, V$, and $W$ results non-dimensionalized using outer scaling. Profile data are labeled on the plots. 

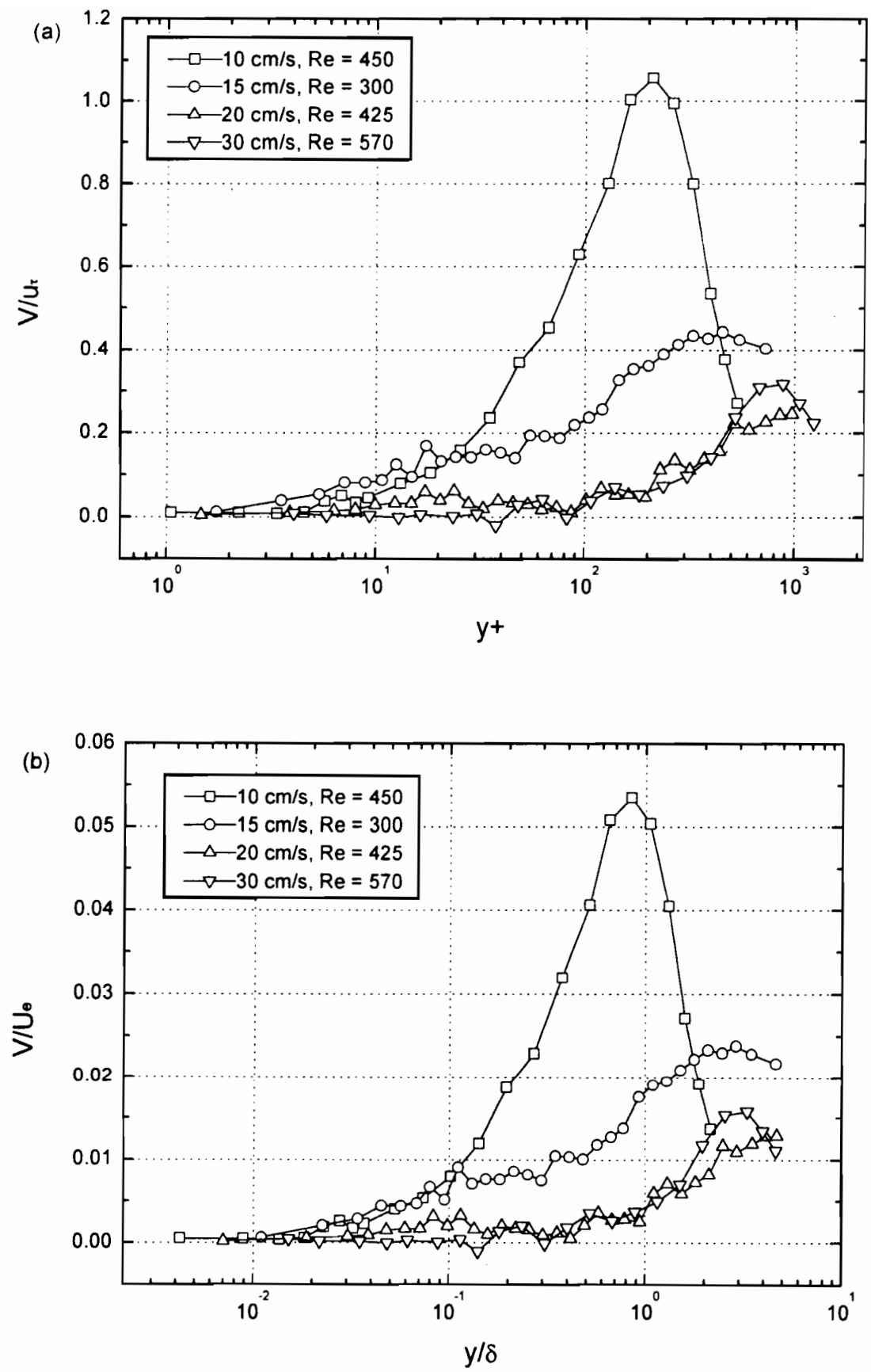

Fig. 3.10 Detail of the 2-D $V$ results. 

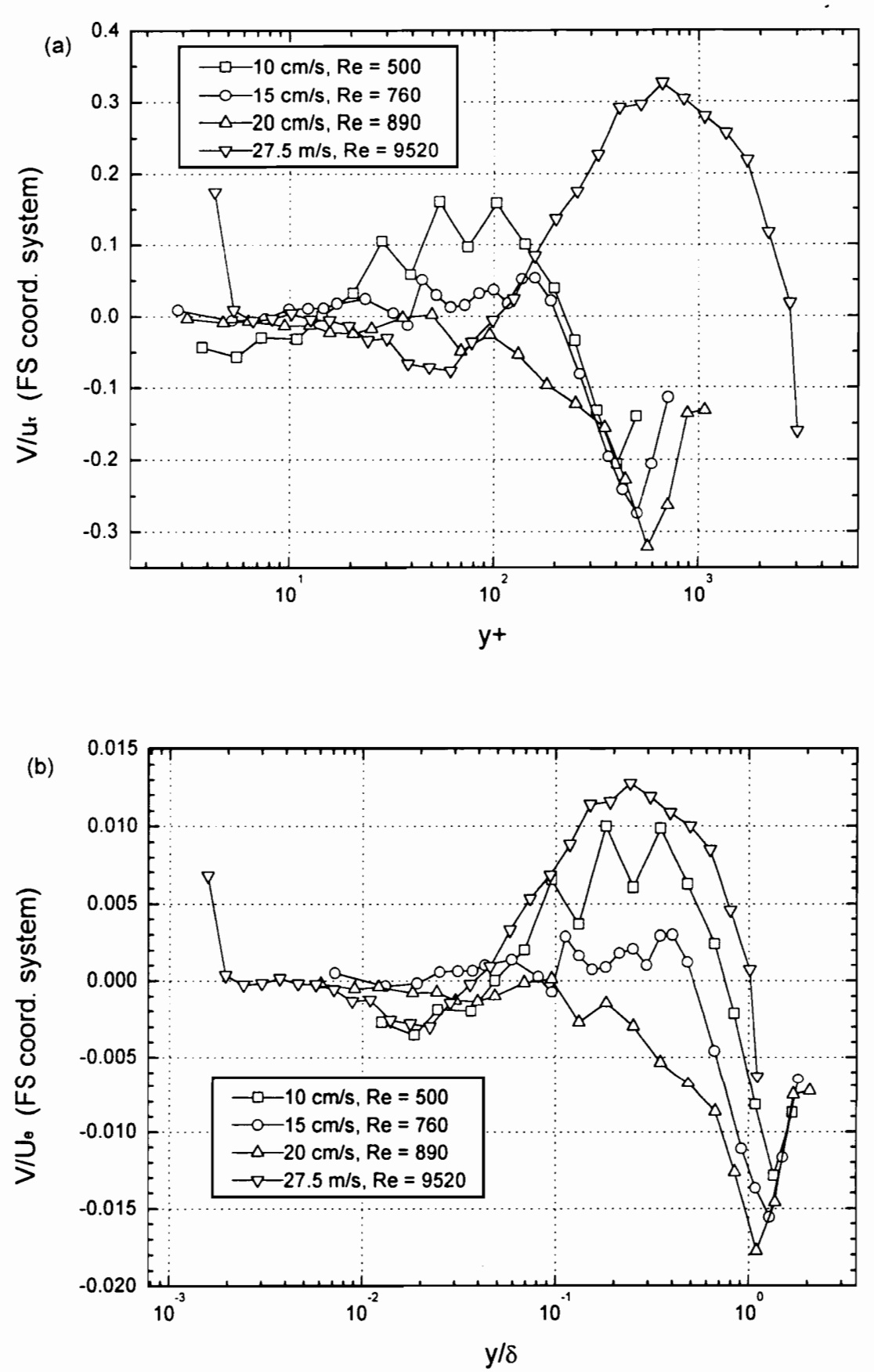

Fig. 3.11 Detail of the 3-D $V$ results. 


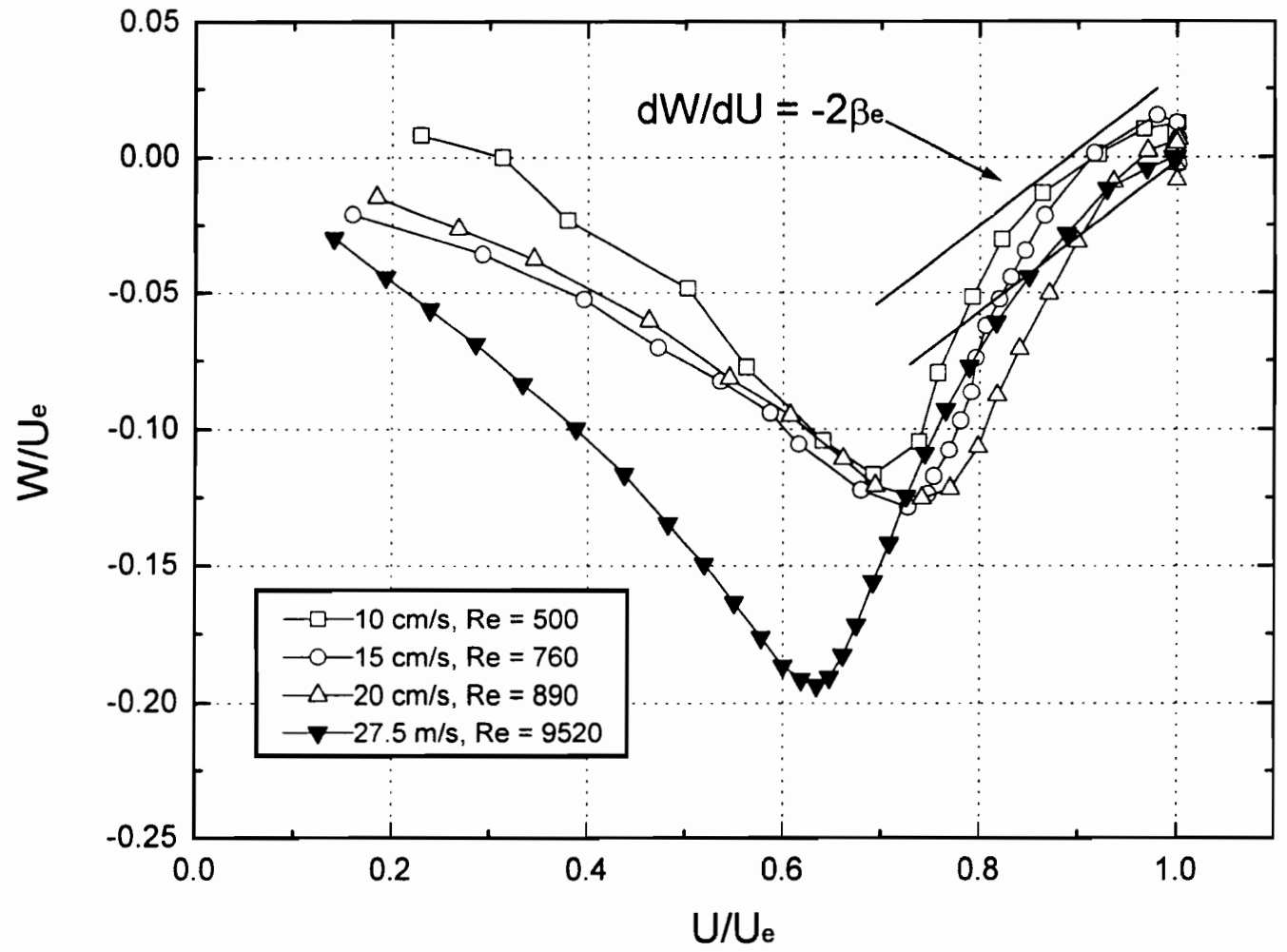

Fig. 3.12 Hodograph plot (Johnston, 1960), $U$ and $W$ scaled using $U_{\boldsymbol{e}}$. The predicted slope of the outer region is indicated as shown. The average freestream turning angle for the four flow cases was $-8.1^{\circ}$, which was used for the approximation to the curve slopes shown on the plot. 

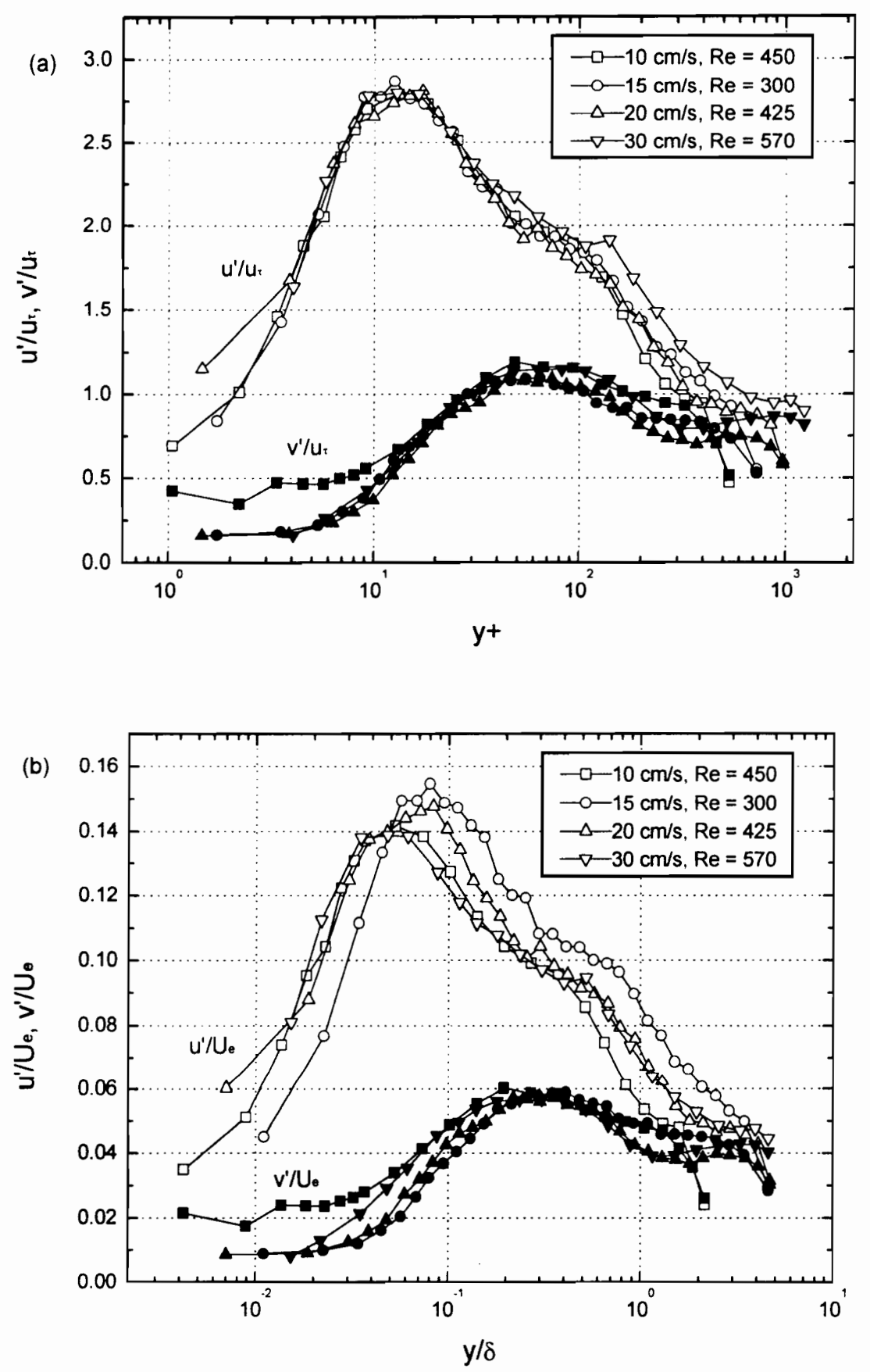

Fig. 3.13 2-D normal stress results (a) non-dimensionalized using inner scaling, and (b) nondimensionalized using outer scaling. The open symbols are the $u^{\prime}$ results, and the closed symbols are the $v^{\prime}$ results. 


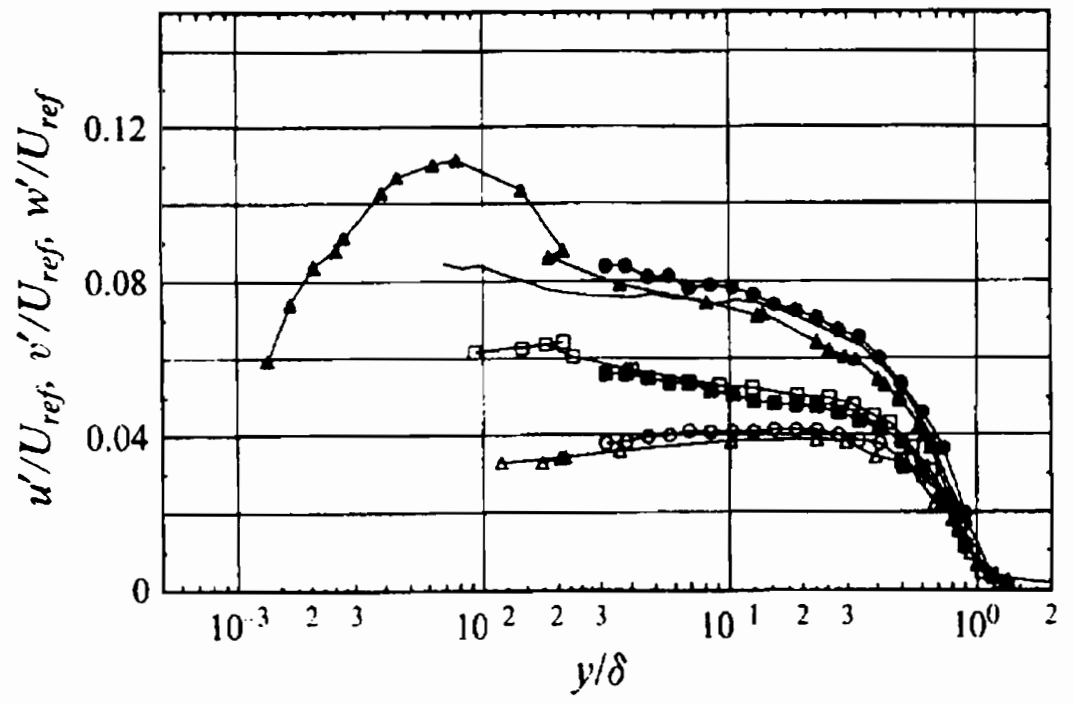

Fig. 3.14 2-D normal stress results from Klebanoff (1955) (solid lines) and Ölçmen and Simpson, station 0 data (1995). Figure reprinted from Ölçmen and Simpson. $\operatorname{Re}_{\theta}$ for the Ölçmen and Simpson data at station 0 was approximately 7800 . 

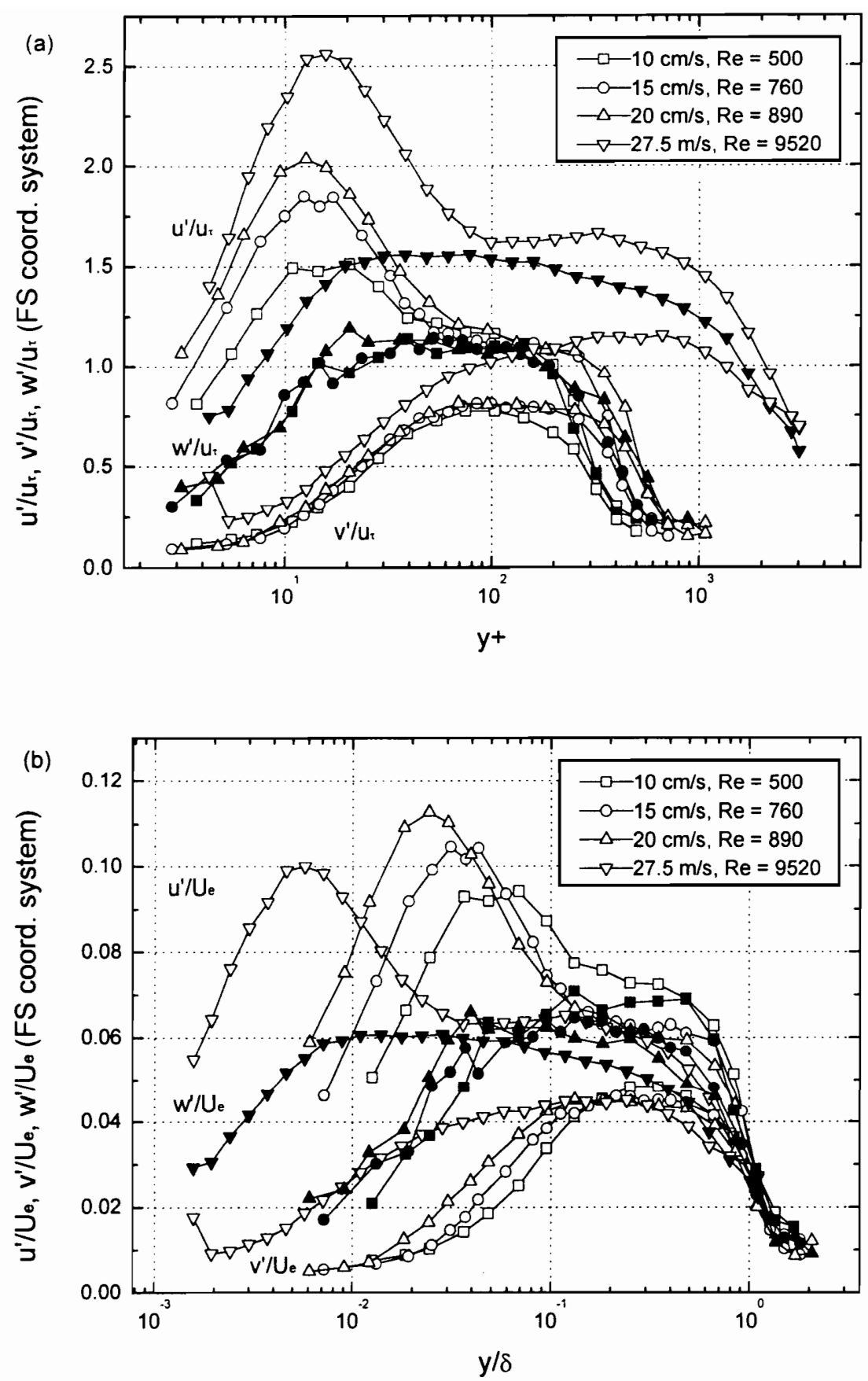

Fig. 3.15 3-D normal stress results presented using the freestream coordinate system. (a) non-dimensionalized using inner scaling, and (b) non-dimensionalized using outer scaling. Profile results are labeled on each plot. 

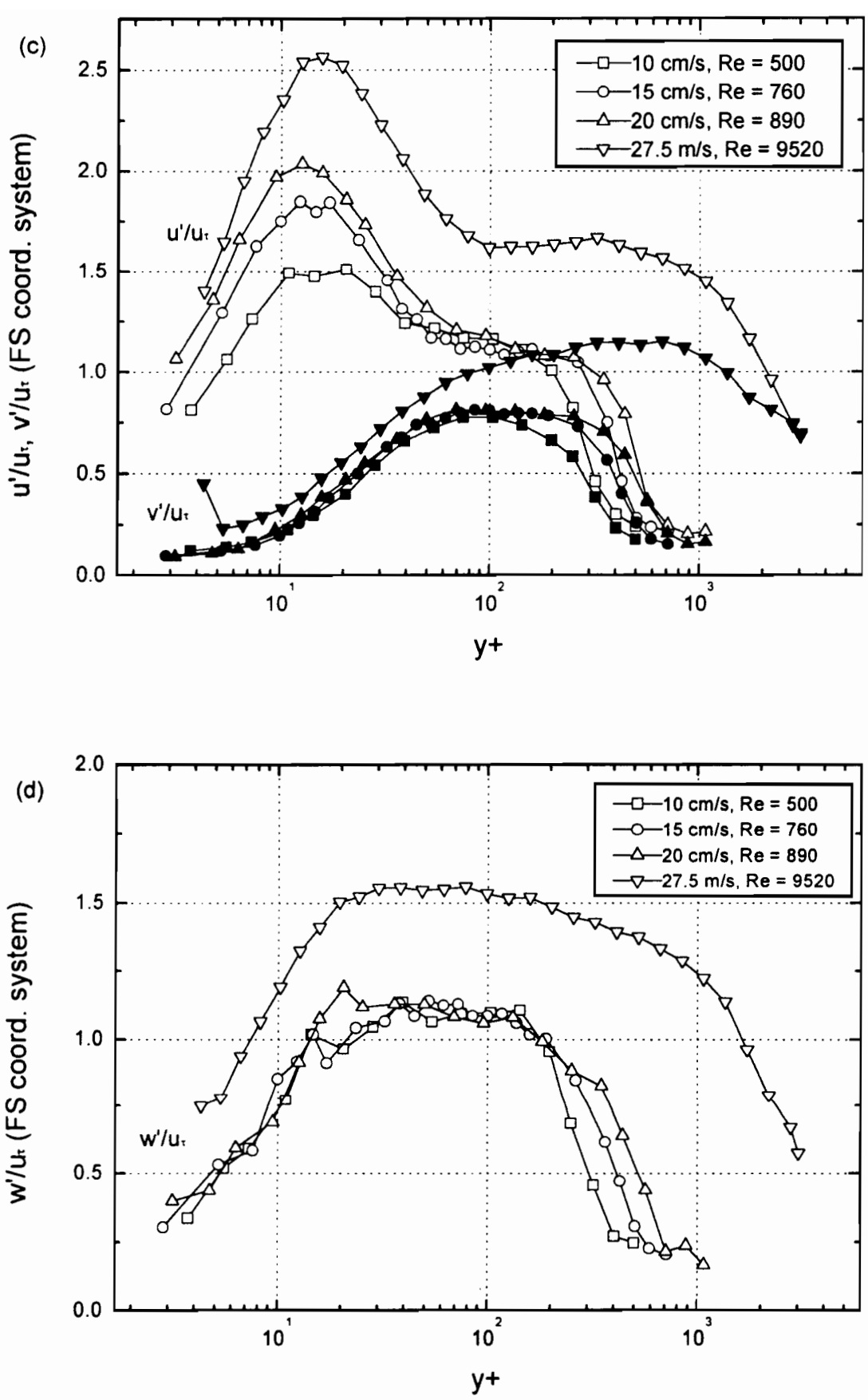

Fig. 3.15 (cont.) 3-D normal stress results presented using the freestream coordinate system, plotted separately to show data details. (c) $u^{\prime}$ (open symbols) and $v^{\prime}$ (solid symbols) results, and (d) $w^{\prime}$ results. All data non-dimensionalized using inner scaling. 

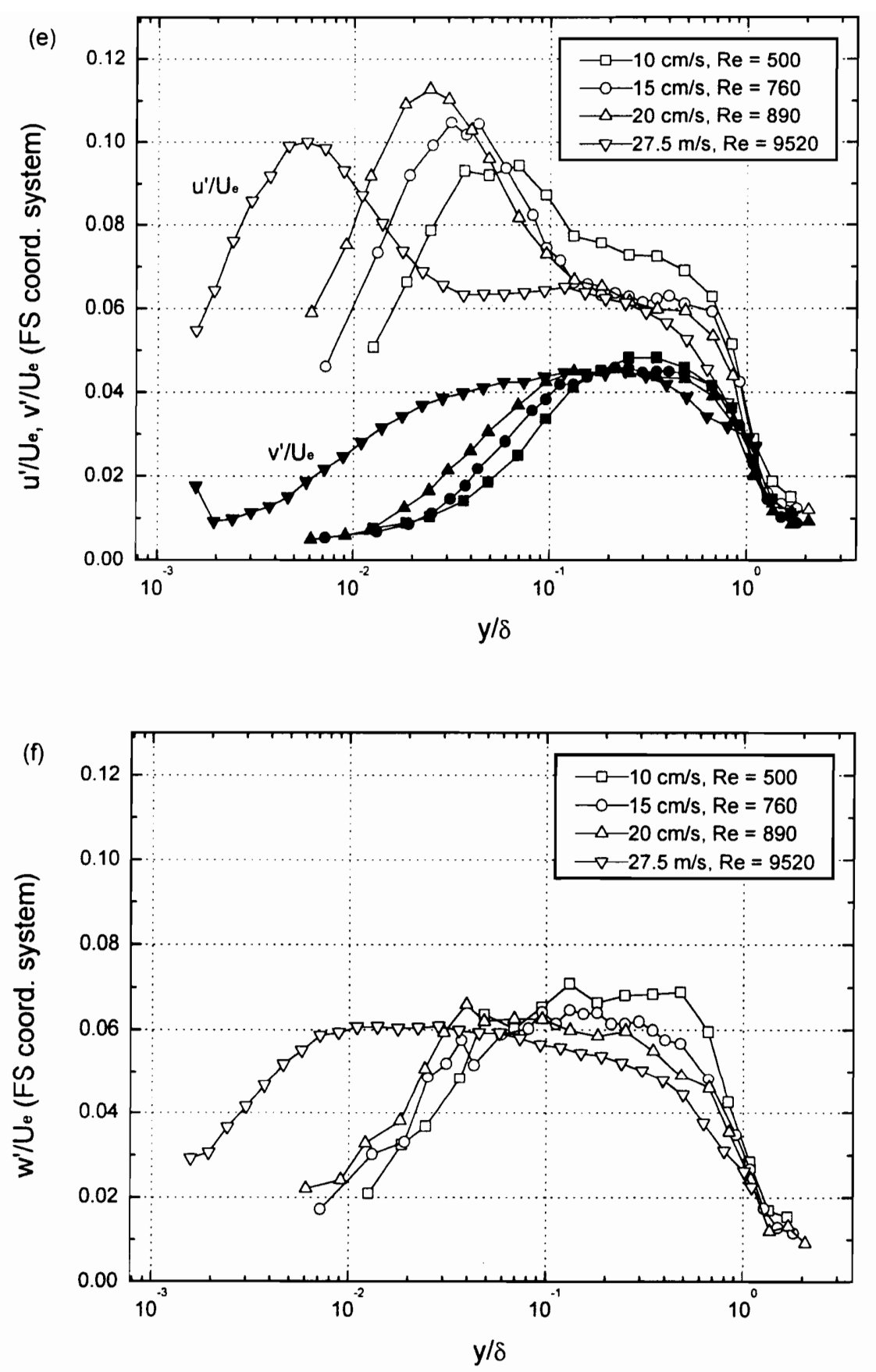

Fig. 3.15 (cont.) 3-D normal stress results presented using the freestream coordinate system, plotted separately to show data details. (e) $u^{\prime}$ (open symbols) and $v^{\prime}$ (solid symbols) results, and (f) $w^{\prime}$ results. All data non-dimensionalized using outer scaling. 


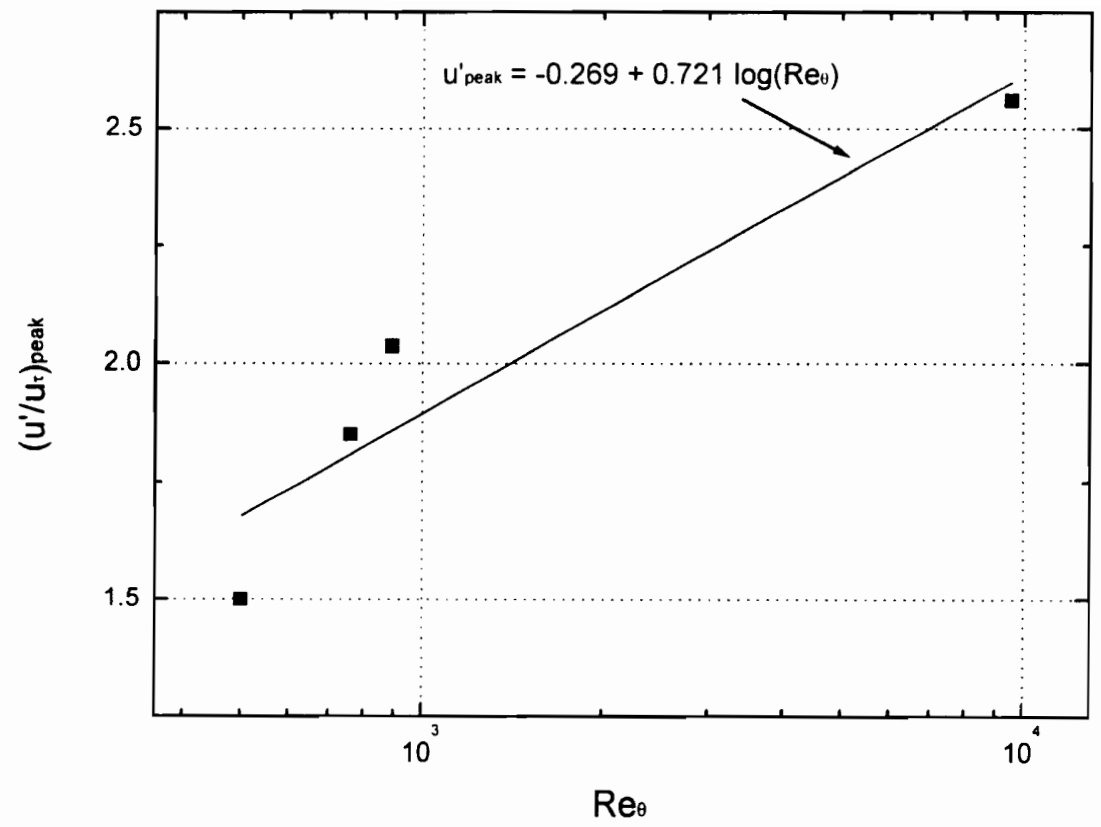

Fig. 3.16 Fit results for the peak $u^{\prime}$ normal stress for the 3-D boundary layer cases, freestream coordinate system. The correlation coefficient for the curve fit was 0.94 . 

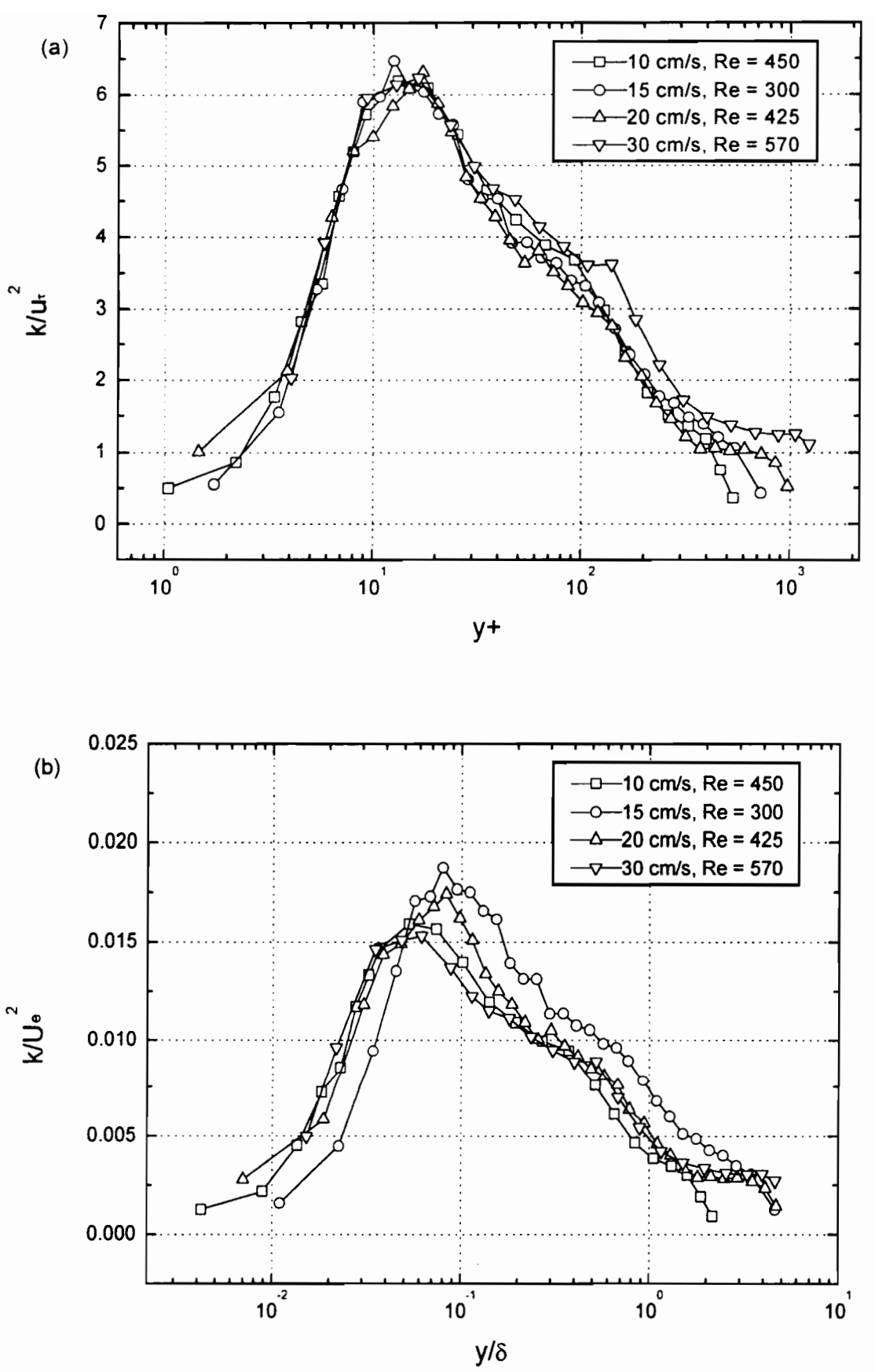

Fig. 3.17 2-D results for the turbulent kinetic energy, $k$. $w^{\prime}$ was not measured for the 2-D case, but was estimated for the calculation of $k$ using $w^{\prime}=0.5\left(u^{\prime}+v^{\prime}\right)$ (Tummers et. al., 1992). 

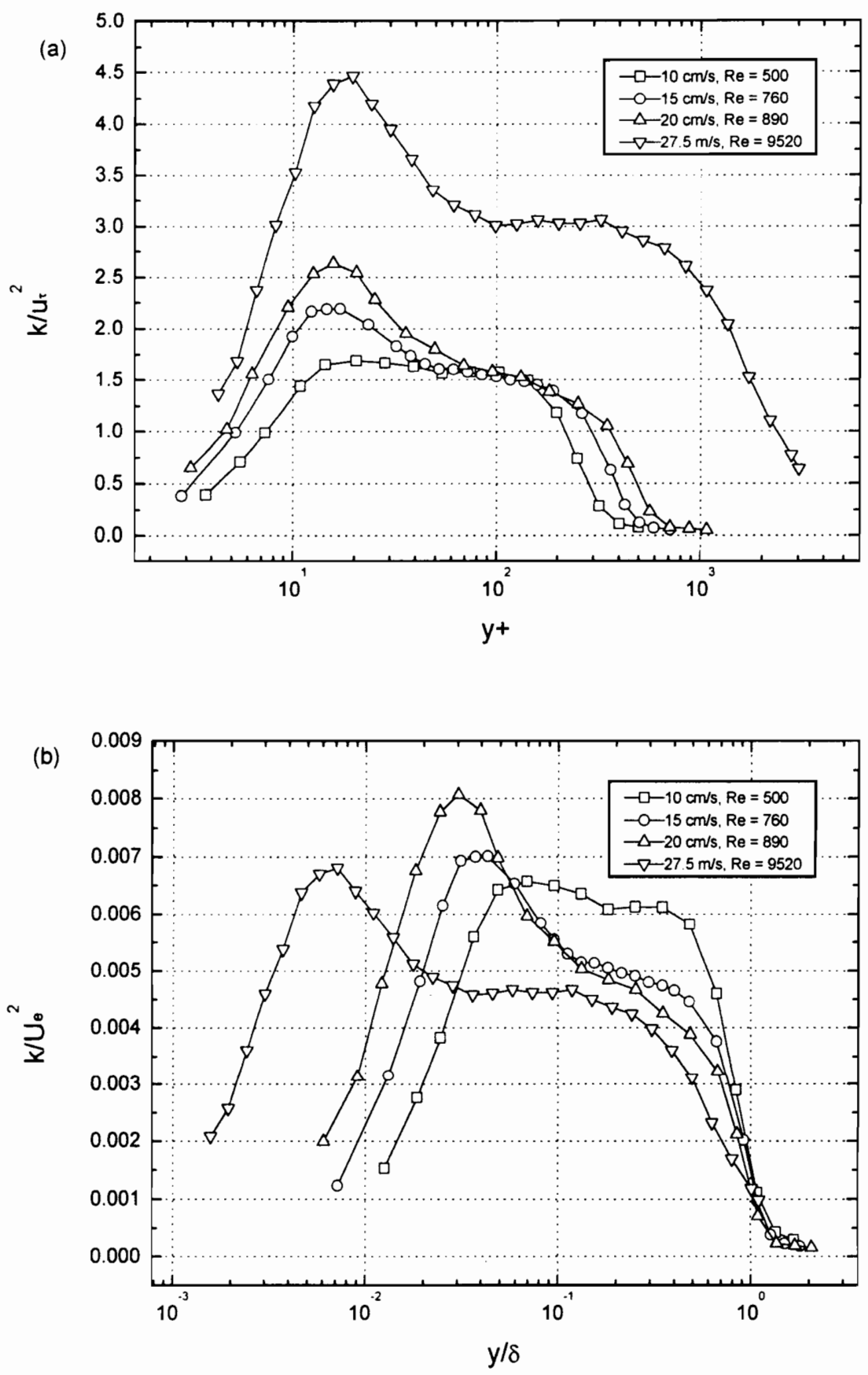

Fig. 3.18 3-D results for the turbulent kinetic energy, $k$. (a) non-dimensionalized using inner scaling, (b) non-dimensionalized using outer scaling. 


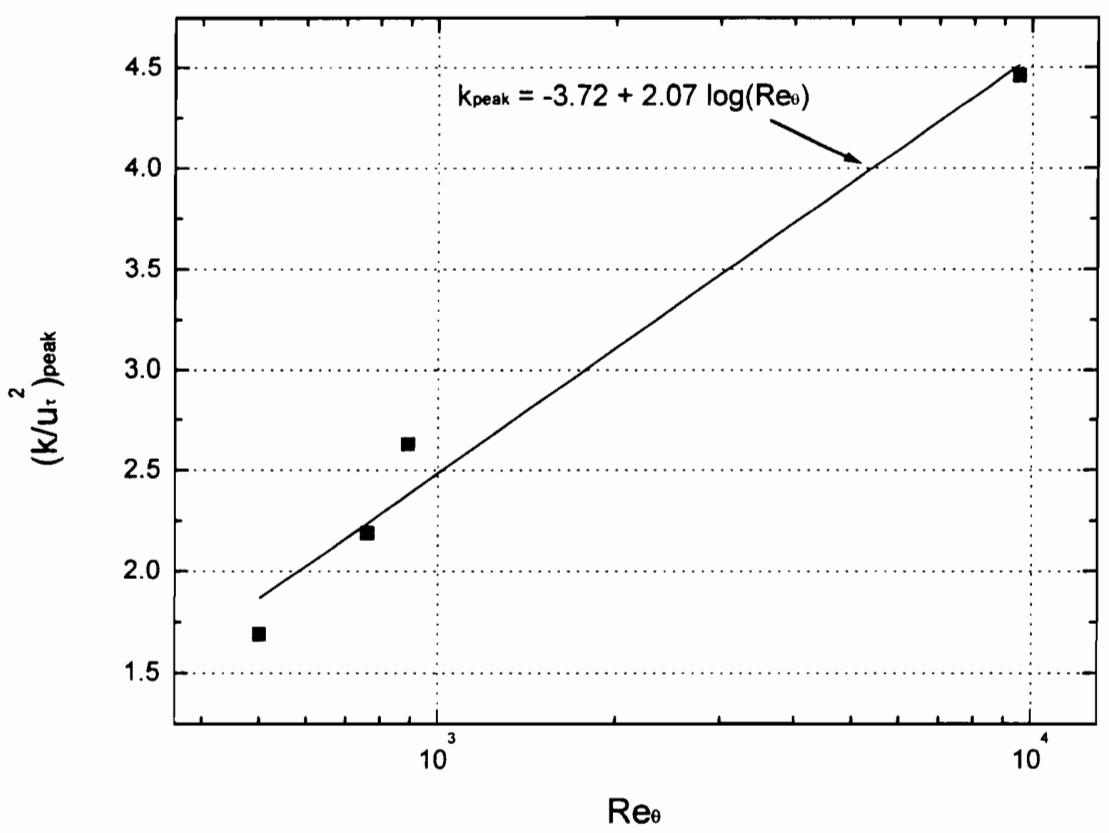

Fig. 3.19 Fit results for the peak turbulent kinetic energy levels for the 3-D boundary layer cases, results are coordinate system independent. The correlation coefficient for the curve fit was 0.989 . 

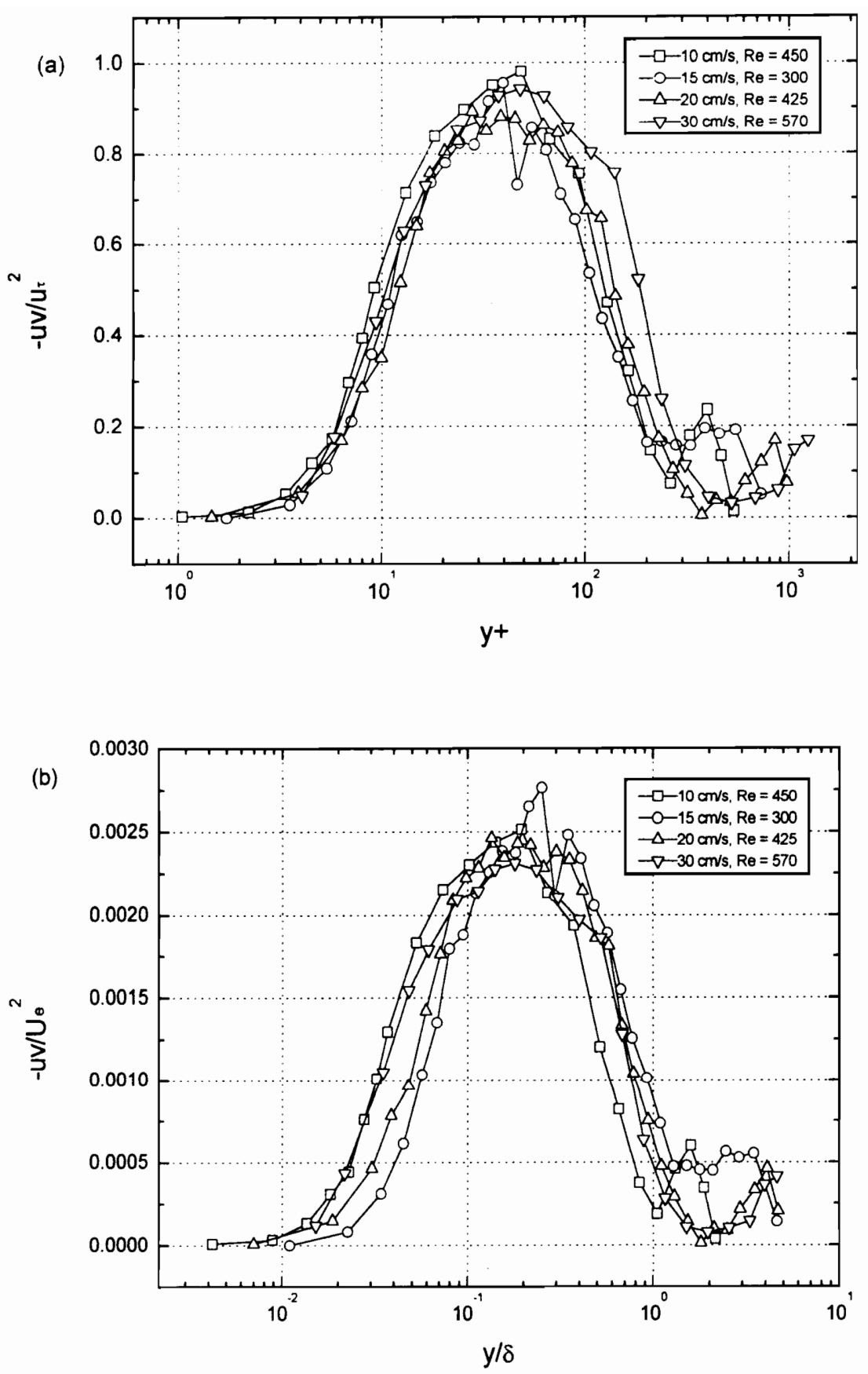

Fig. 3.20 2-D mean Reynolds shear stress results, non-dimensionalized using (a) inner scaling, and (b) outer scaling. For a $2-\mathrm{D}$ flow, $-\overline{v w}$ and $-\overline{u w}$ should be zero. 

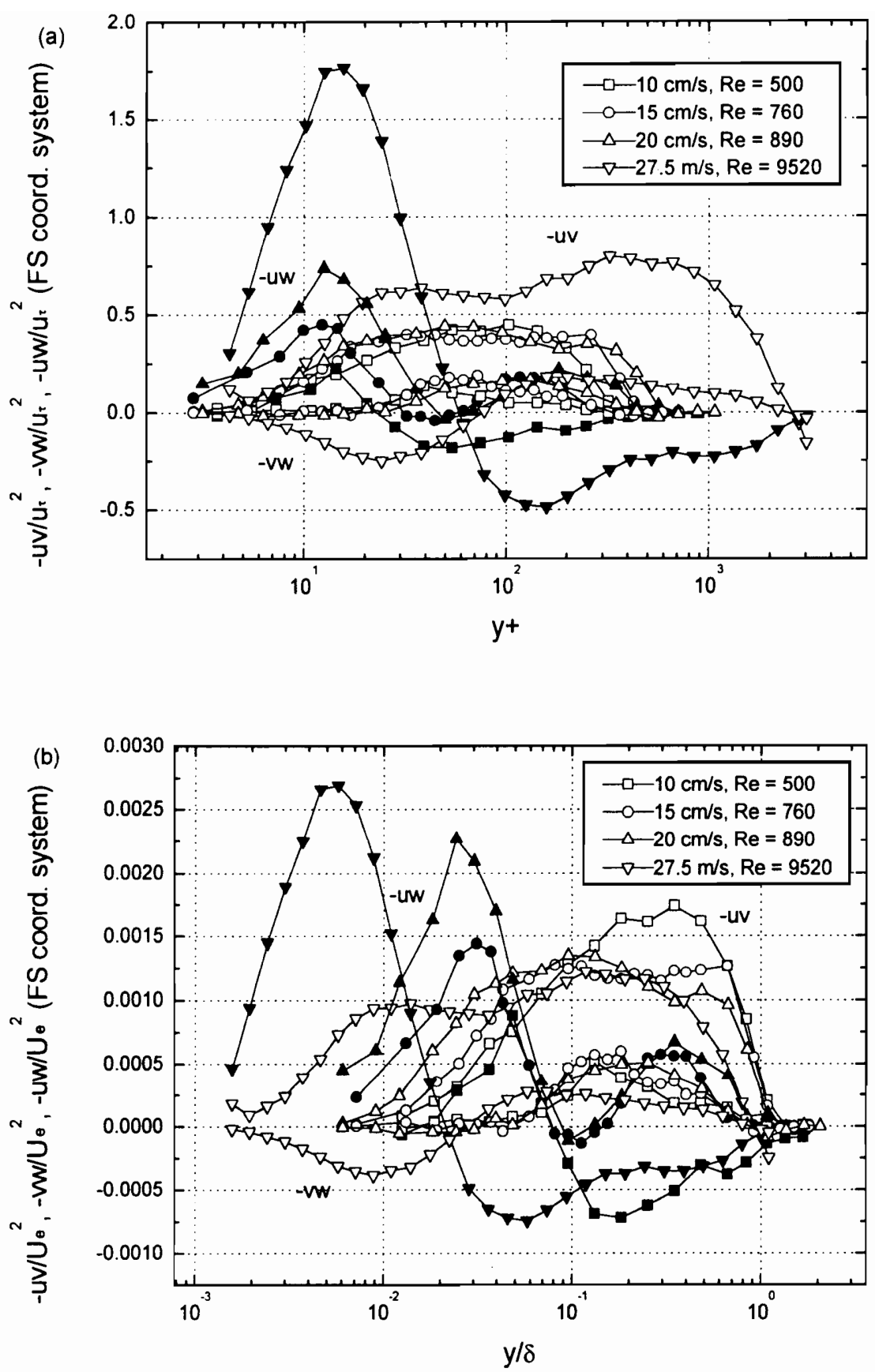

Fig. 3.21 3-D mean Reynolds shear stress results, non-dimensionalized using (a) inner scaling, and (b) outer scaling. Solid symbols are the $-\overline{u w}$ results. All data are presented in freestream coordinates. $\operatorname{Re}_{\theta}=9520$ data are from Ölçmen and Simpson (1995). 

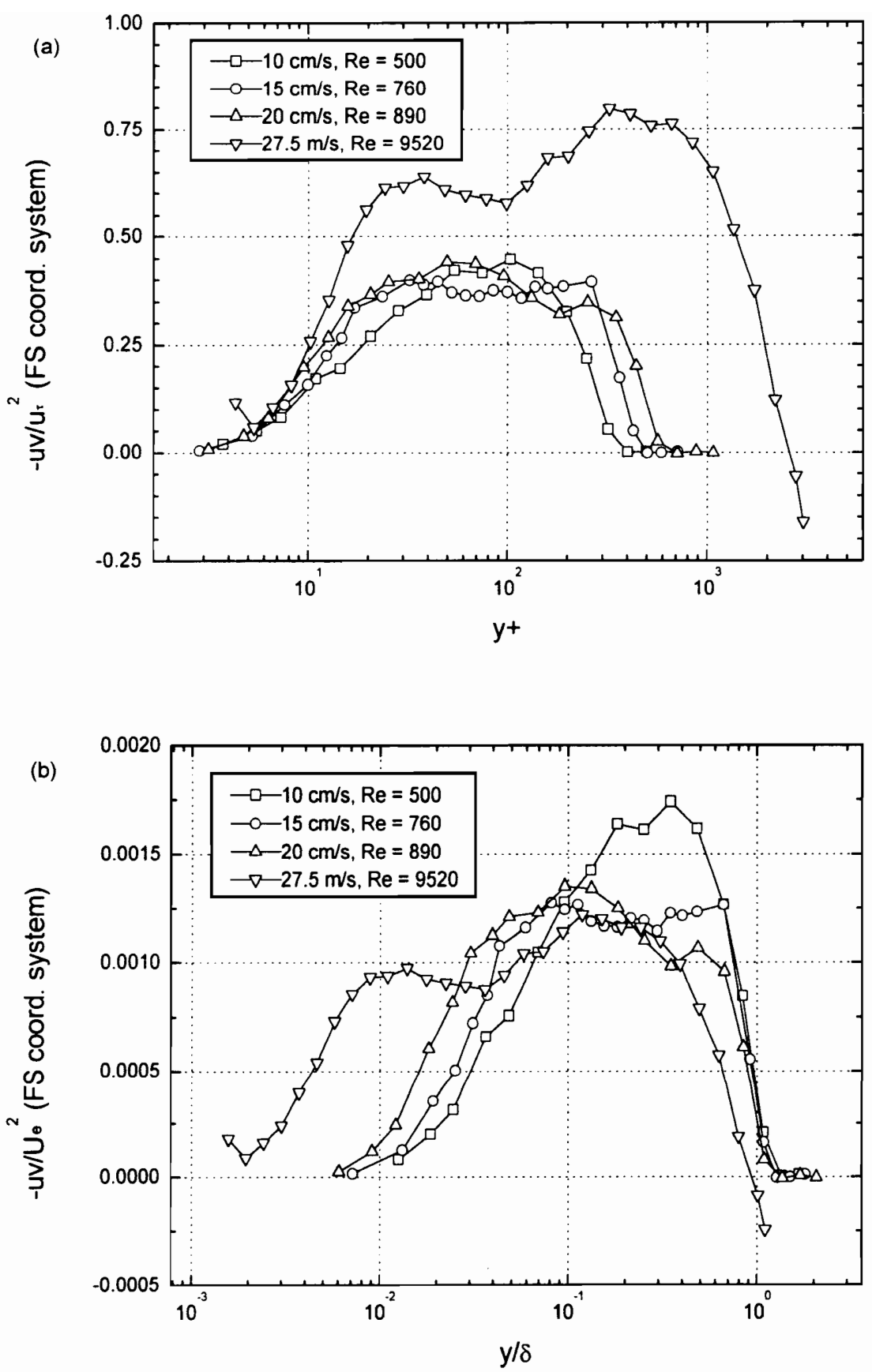

Fig. 3.22 3-D mean Reynolds shear stress $-\overline{u v}$ results (detail from Fig. 3.21) nondimensionalized using (a) inner scaling, and (b) outer scaling. 

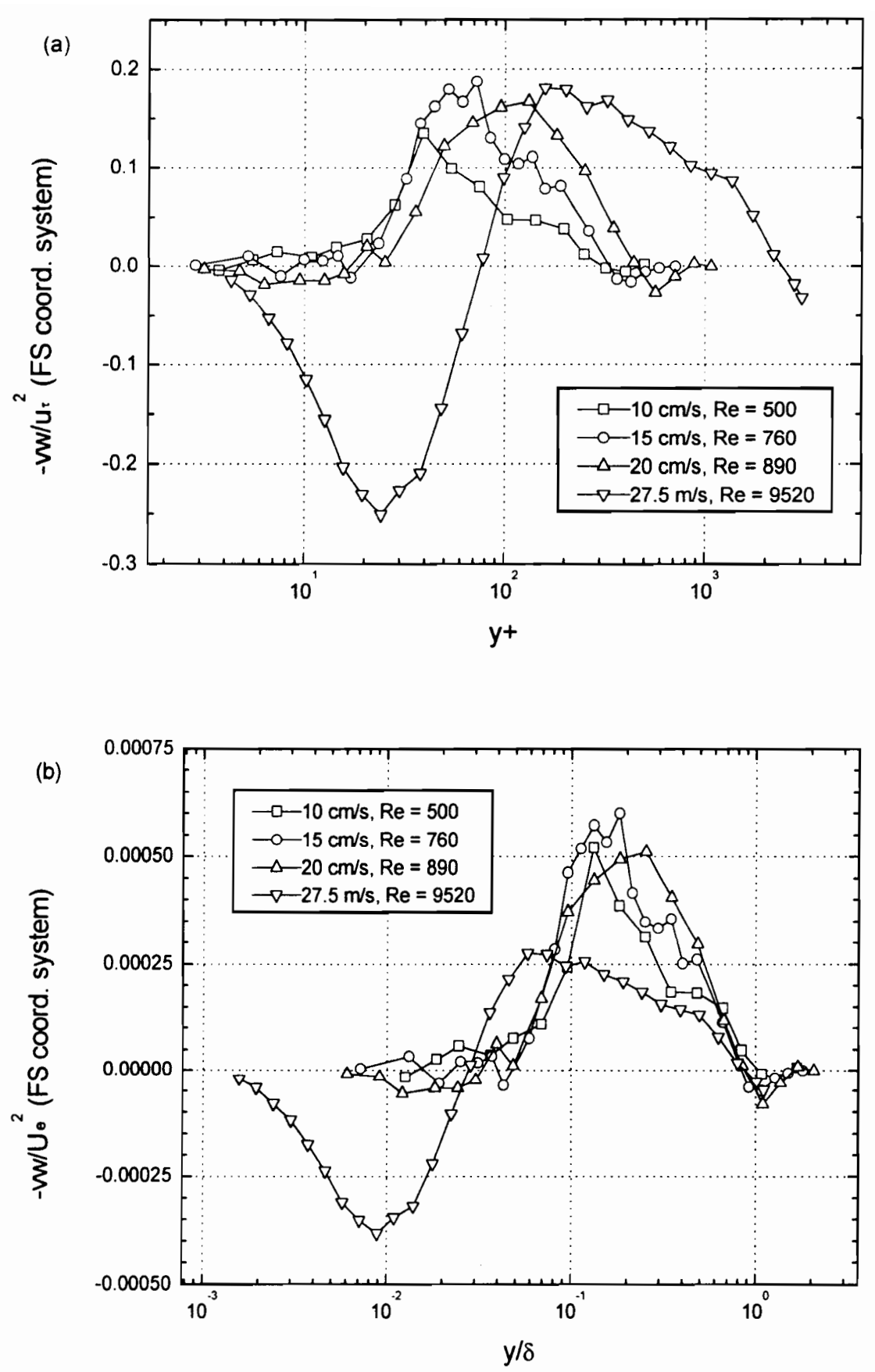

Fig. 3.23 3-D mean Reynolds shear stress $-\overline{v w}$ results (detail from Fig. 3.21) nondimensionalized using (a) inner scaling, and (b) outer scaling. 

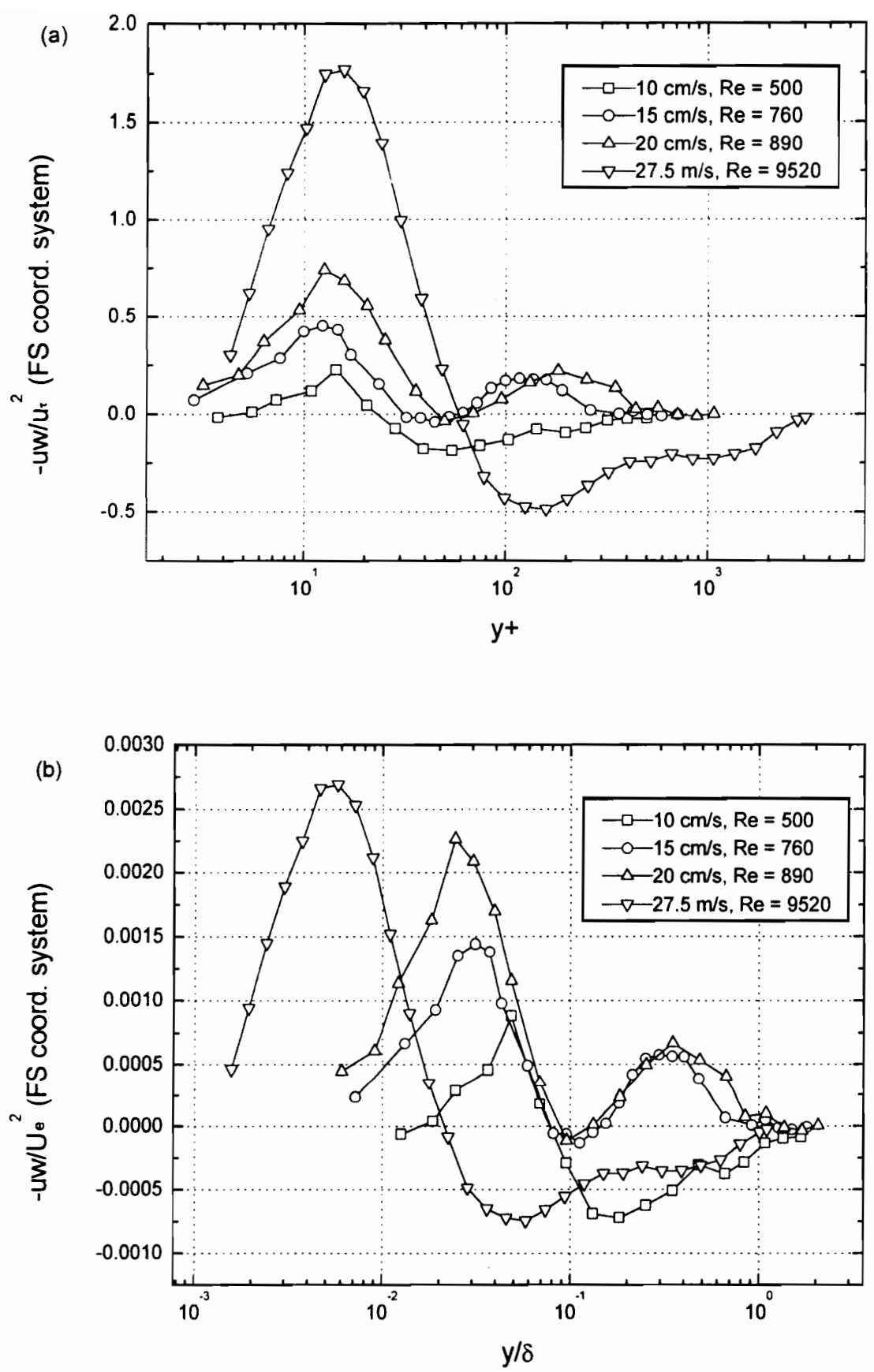

Fig. 3.24 3-D mean Reynolds shear stress $-\overline{u w}$ results (detail from Fig. 3.21) nondimensionalized using (a) inner scaling, and (b) outer scaling. 

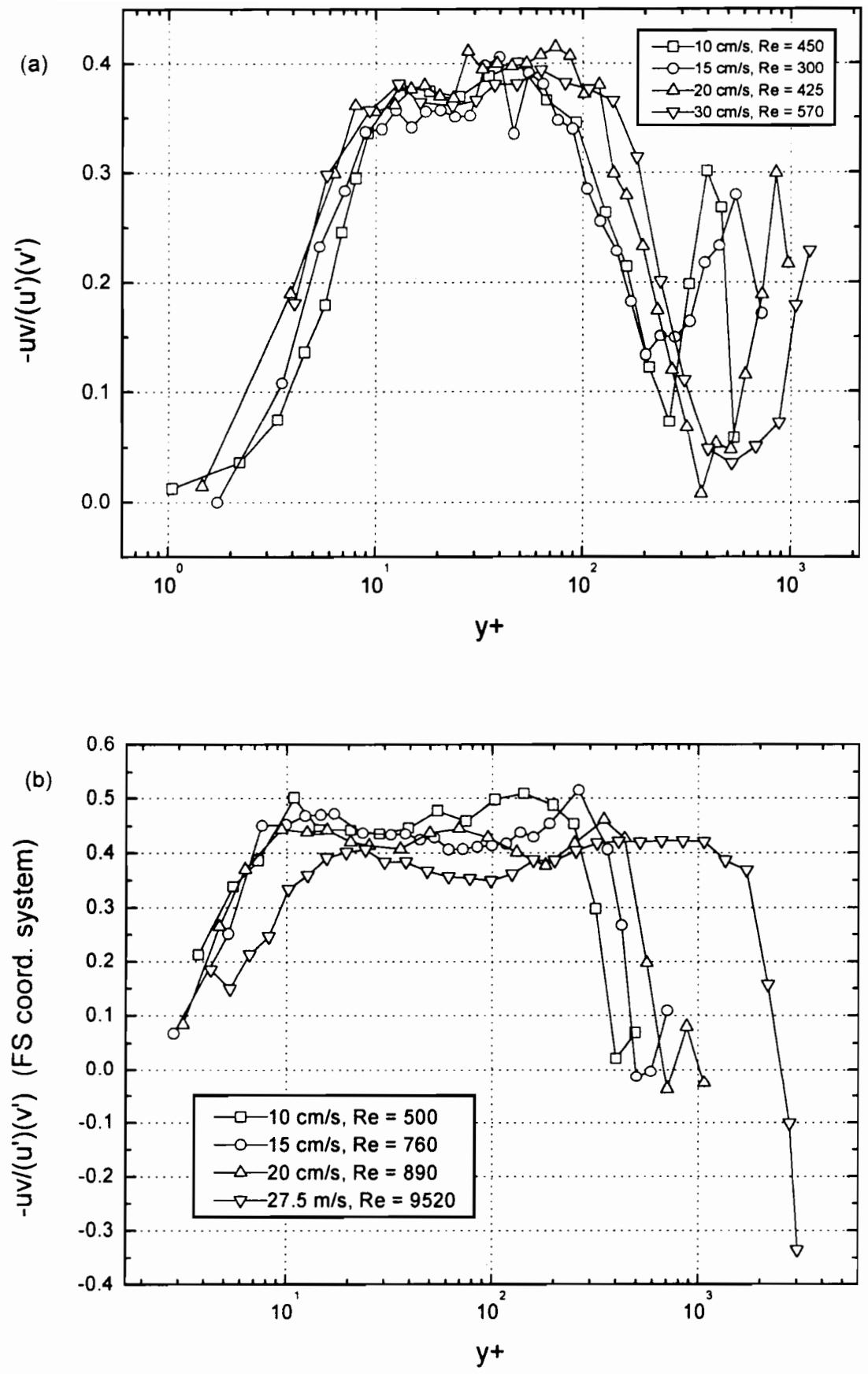

Fig. 3.25 Mean Reynolds shear stress correlation results, plotted vs. $y^{+}$for (a) the 2-D flow cases and (b) the 3-D flow cases. 3-D data presented in freestream coordinates. 


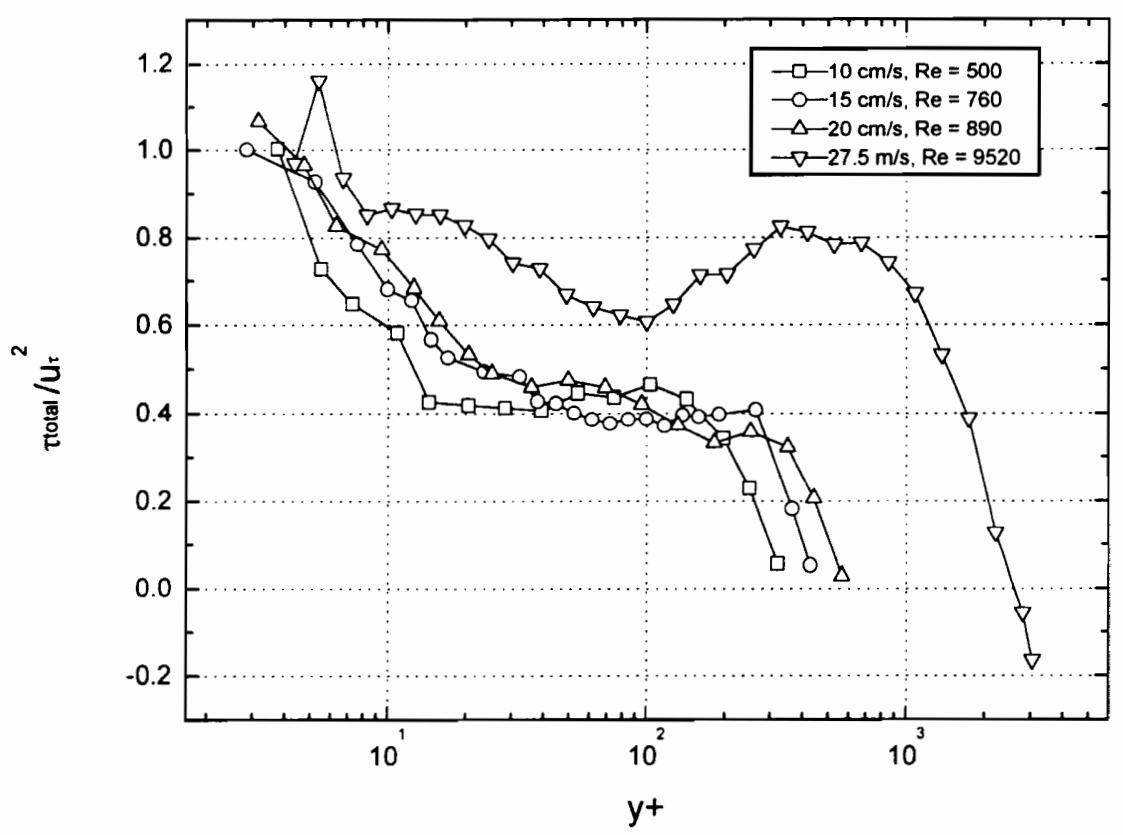

Fig. 3.26 Profiles of the total shear stress magnitude for the 3-D boundary layers. These results are the sum of the laminar and turbulent shear stresses at each point. 


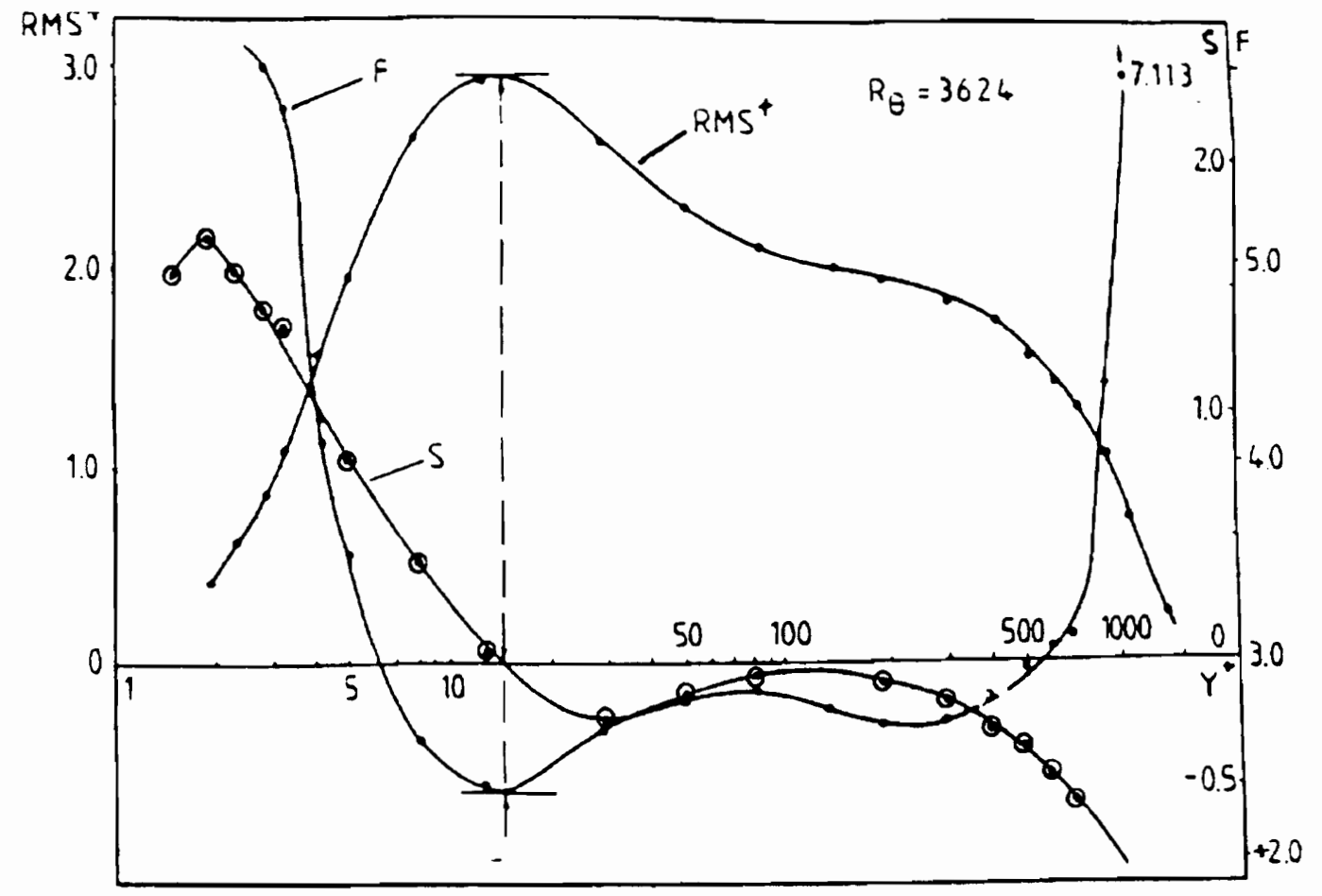

Fig. 3.27 Typical streamwise velocity rms, skewness, and flatness results for a 2-D turbulent boundary layer. Note the different scales used for each quantity (from Durst et al., 1992). 

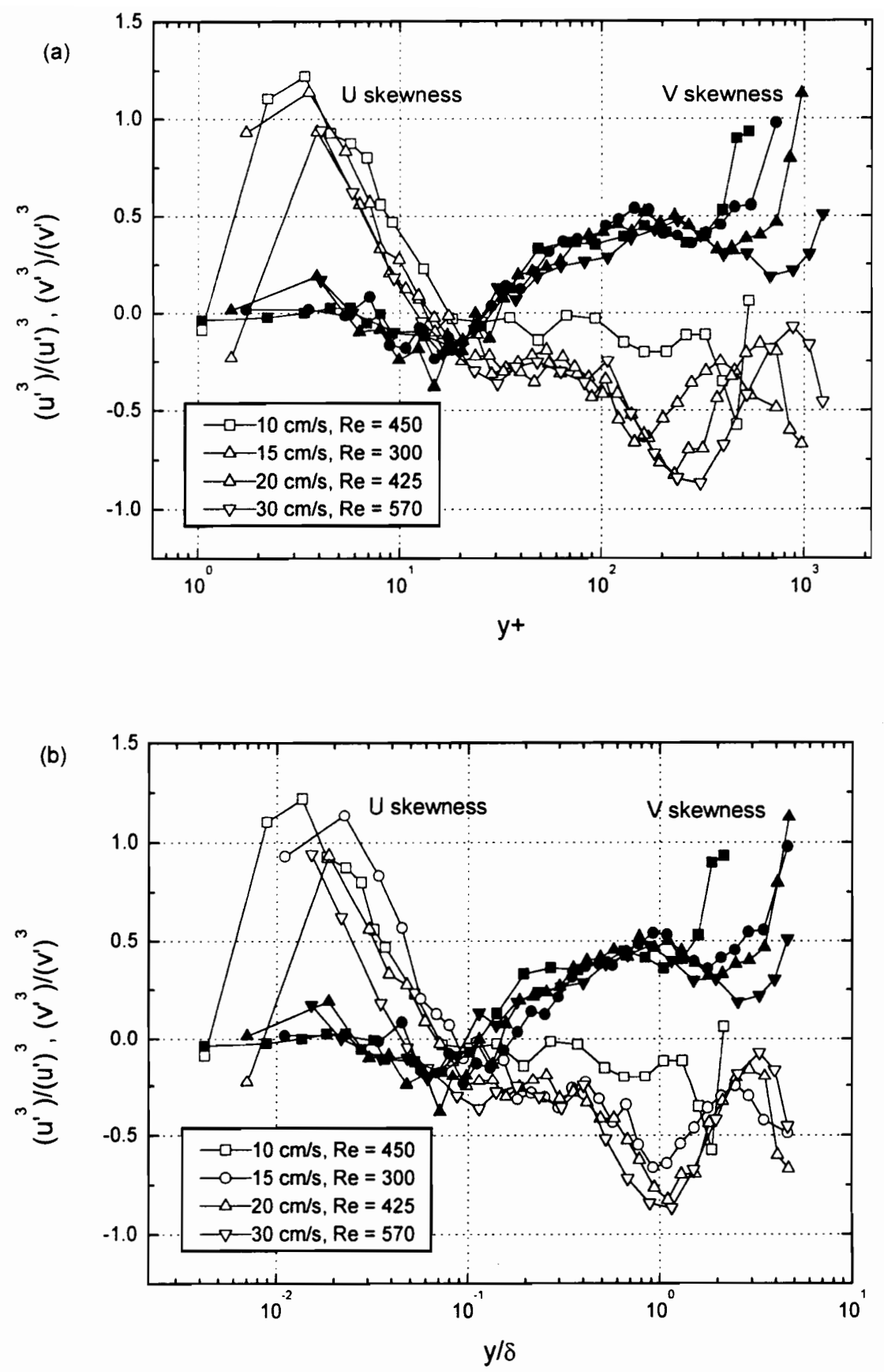

Fig. 3.28 2-D skewness results for $u$ (open symbols) and $v$ (solid symbols), plotted vs. (a) $y^{+}$. and (b) $y / \delta$. 

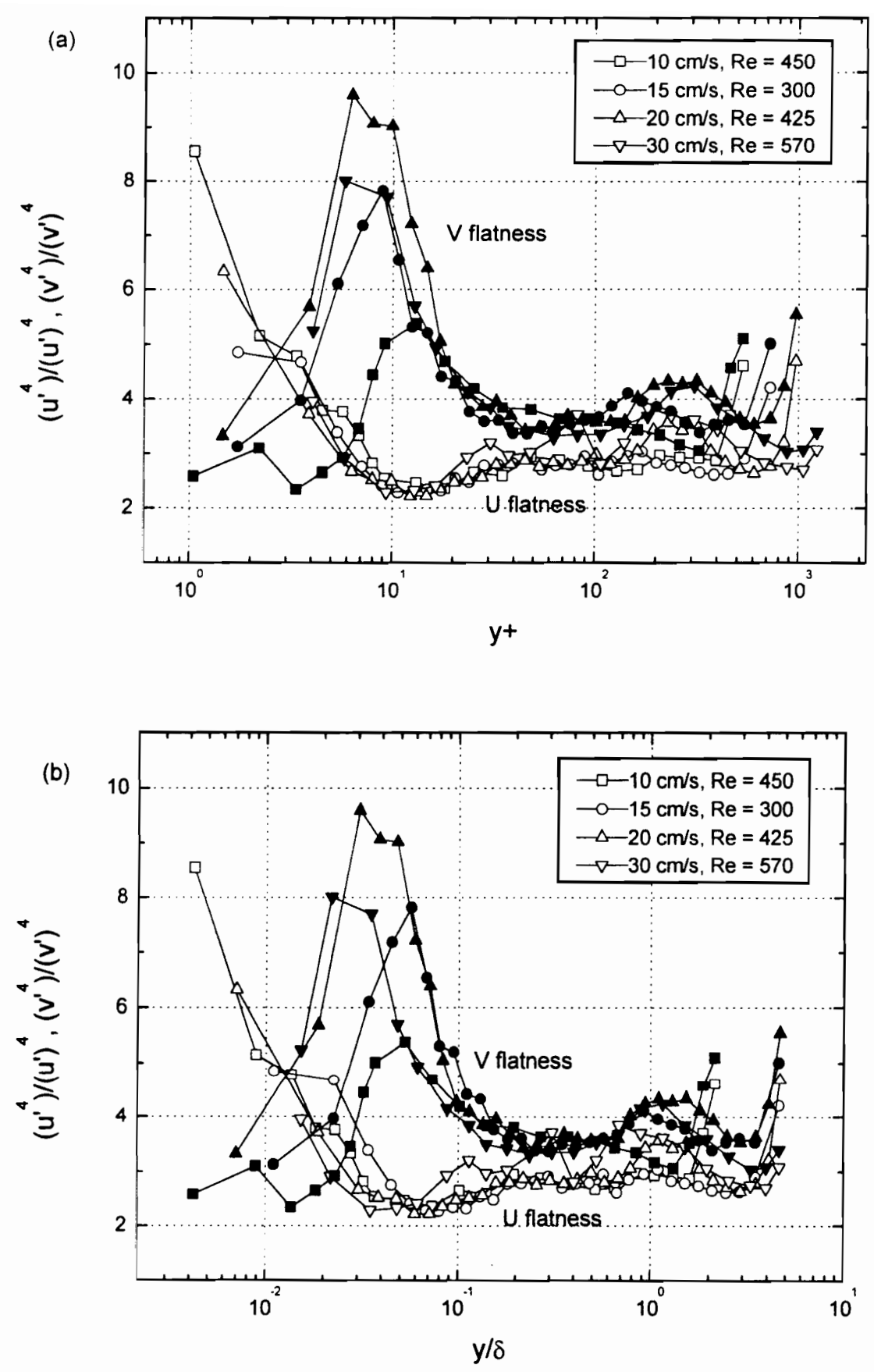

Fig. 3.29 2-D flatness results for $u$ (open symbols) and $v$ (solid symbols), plotted vs. (a) $y^{+}$, and $(\mathrm{b}) y / \delta$ 

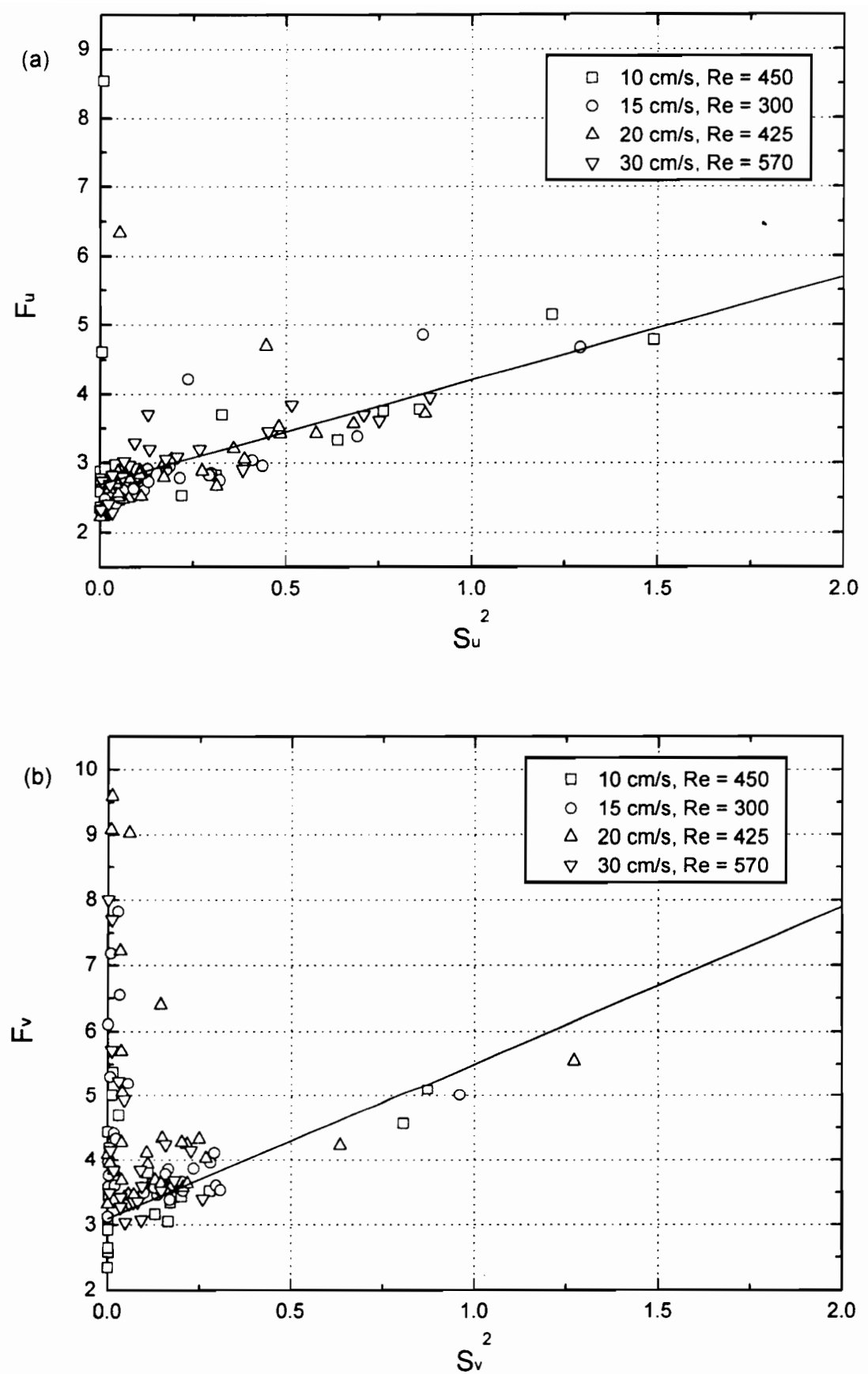

Fig. 3.30 2-D flatness vs. skewness ${ }^{2}$ results for $u$ and $v$. (a) $F_{u}$ vs. $S_{u}^{2}$, the curve equation is $F_{u}=1.5 S_{u}^{2}+2.7$. (b) $F_{v}$ vs. $S_{v}^{2}$, the curve equation is $F_{v}=2.4 S_{u}^{2}+3.1$. (both curve equations from Durst et al.., 1991). 

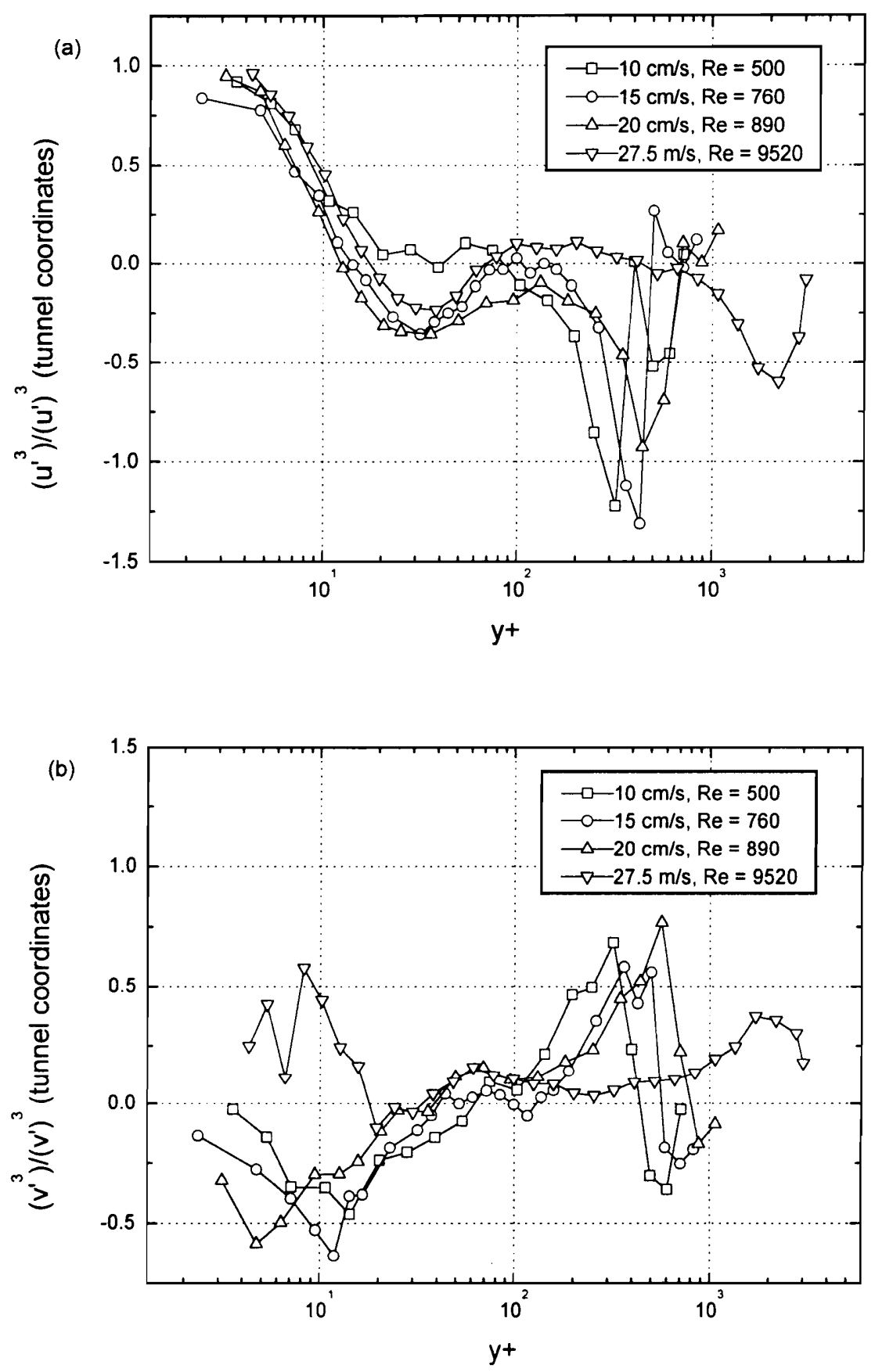

Fig. 3.31 Skewness results for the 3-D boundary layers, including Ölçmen's high Reynolds number data. Results for $u^{\prime 3} /\left(u^{\prime}\right)^{3}$ (a) and $v^{\prime 3} /\left(v^{\prime}\right)^{3}$ (b) are plotted using $y^{+}$. These results are presented in the tunnel coordinate system. 

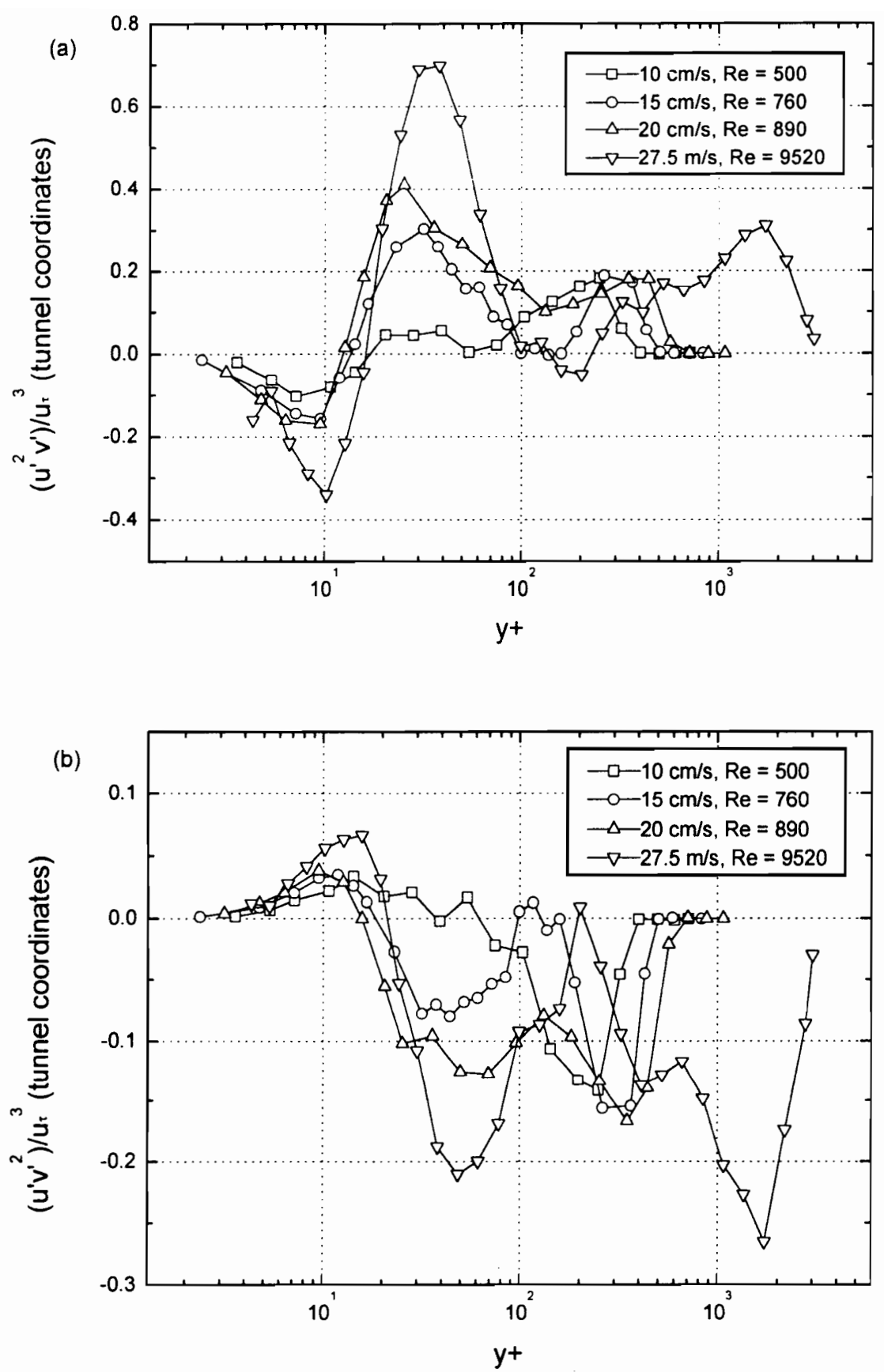

Fig. 3.32 Triple products results for the 3-D boundary layers, including Ölçmen's high Reynolds number data. Results for $u^{\prime 2} v^{\prime}(\mathrm{a})$ and $u^{\prime} v^{\prime 2}$ (b) are plotted using inner scaling. These results are presented in the tunnel coordinate system. 

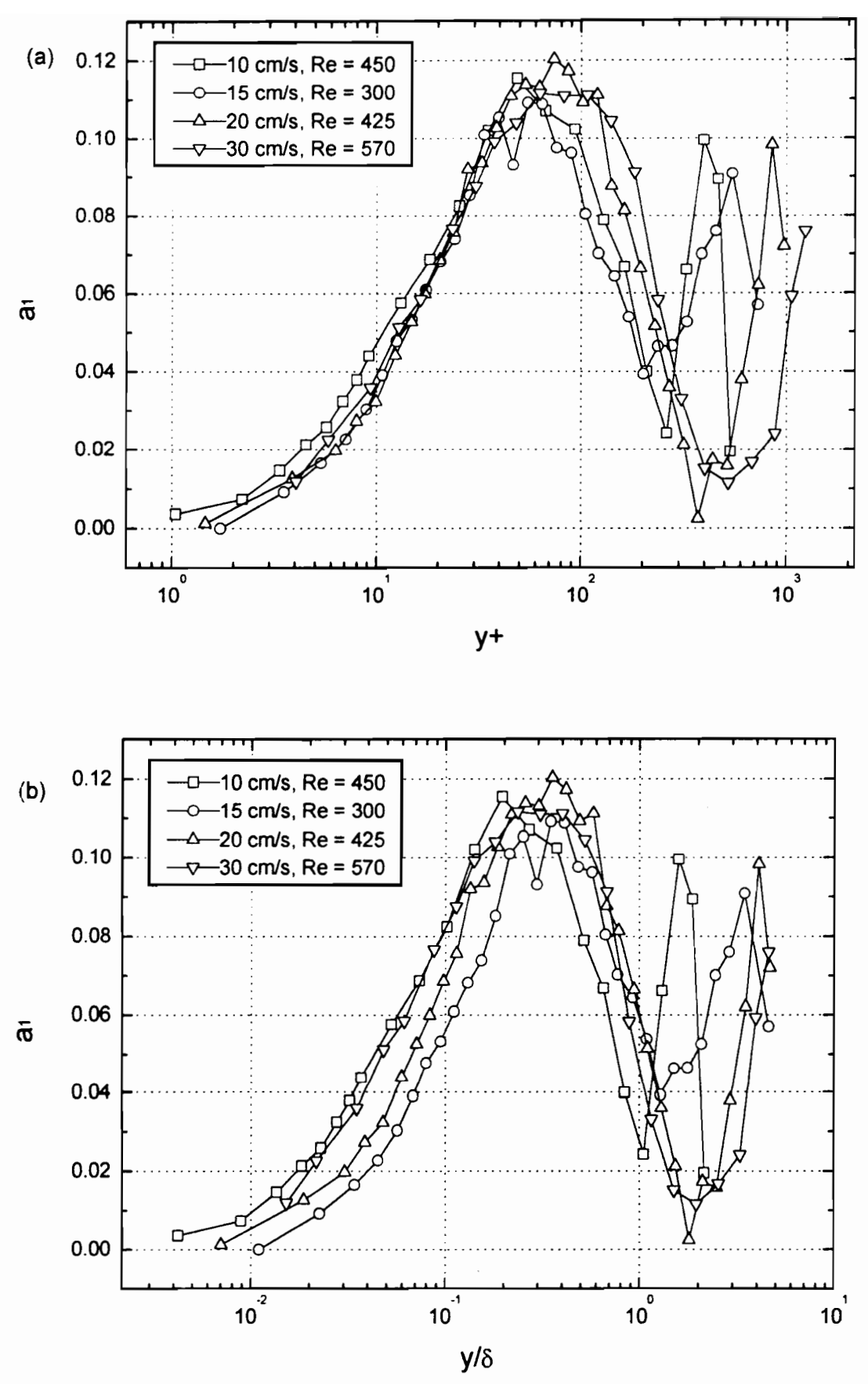

Fig. 3.33 2-D Townsend structural parameter results, plotted vs. (a) $y^{+}$, and (b) $y / \delta$. See Fig. 3.17 for the 2-D turbulent kinetic energy estimation method and results. 

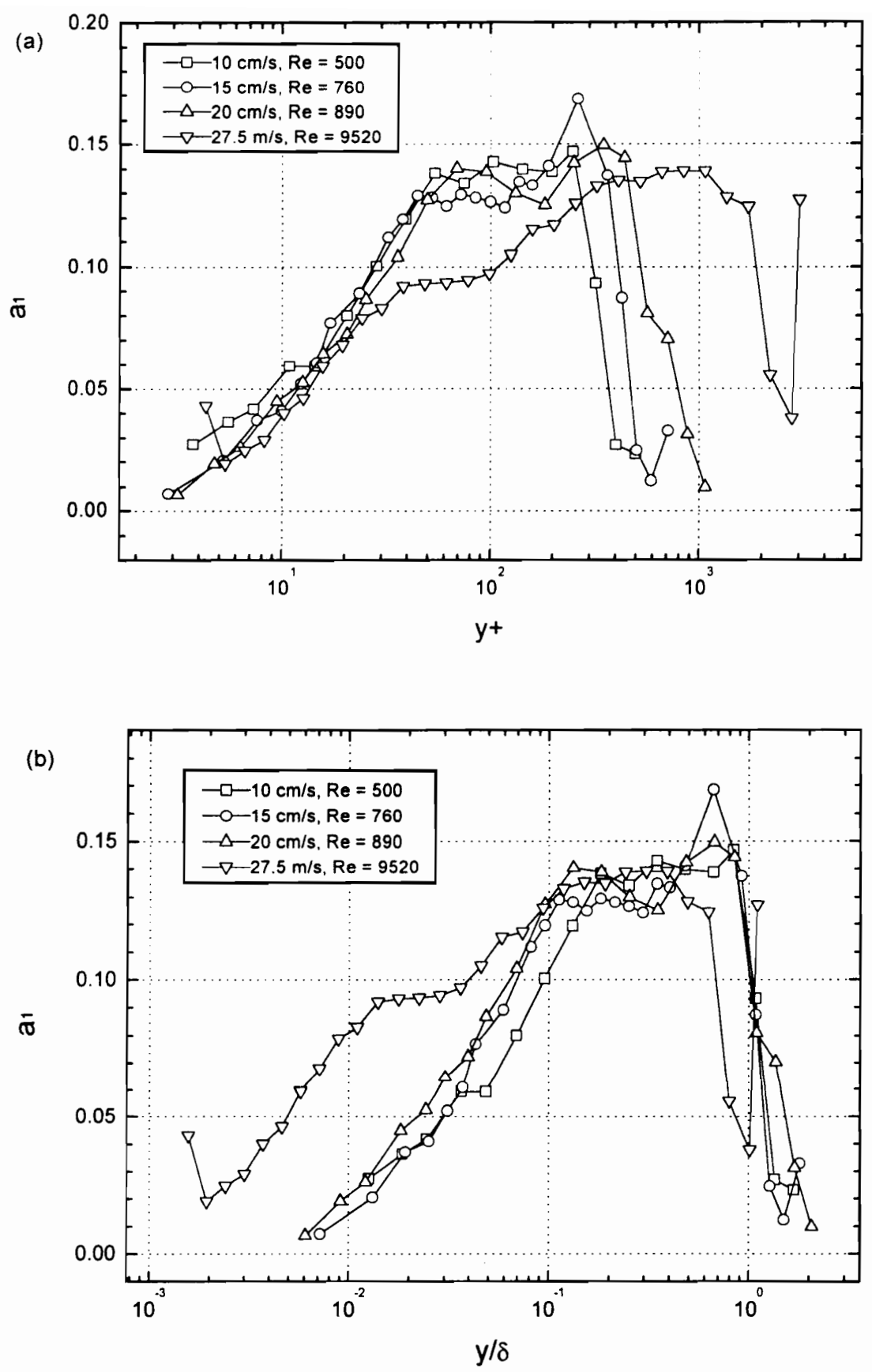

Fig. 3.34 3-D Townsend structural parameter results, plotted vs. (a) $y^{+}$, and (b) $y / \delta$. 

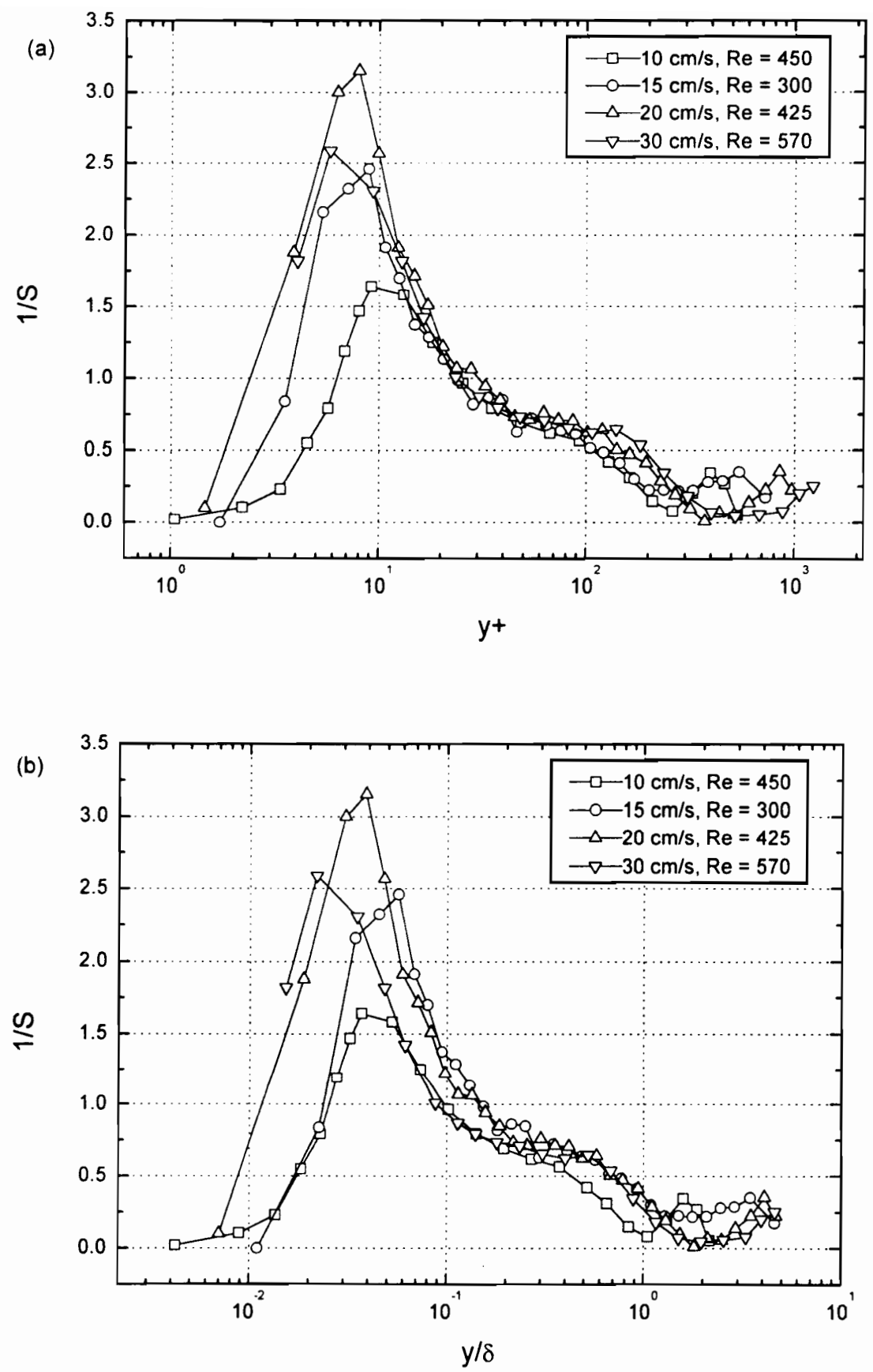

Fig. 3.35 2-D shear stress parameter, $S$, results. $1 / S$ is plotted vs. (a) $y^{+}$, and (b) $y / \delta$. 

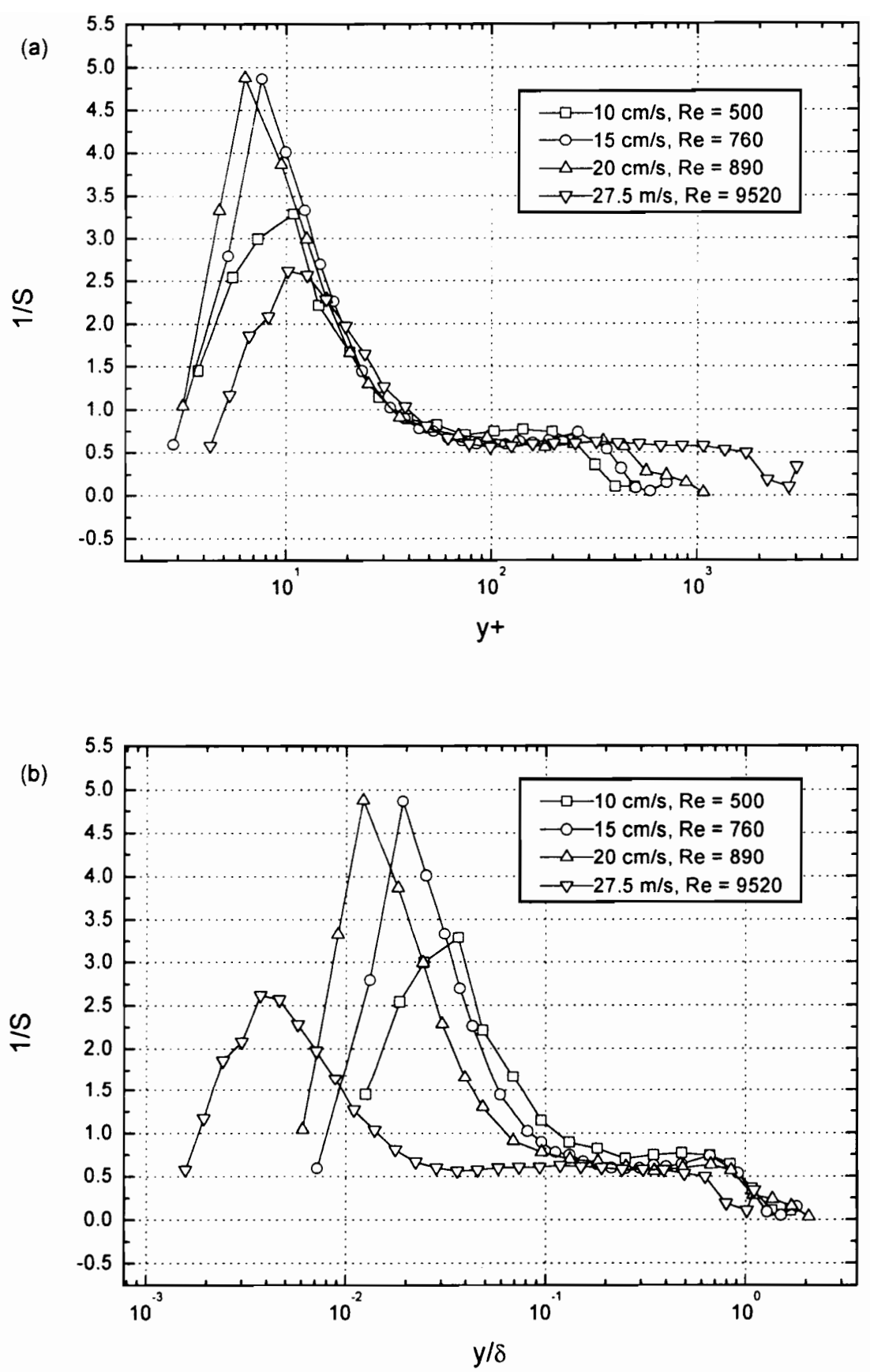

Fig. 3.36 3 -D shear stress parameter, $S$, results. $1 / S$ is plotted vs. (a) $y^{+}$, and (b) $y / \delta$. Note: $S$ is invariant to coordinate system rotations about the $y$ axis. 

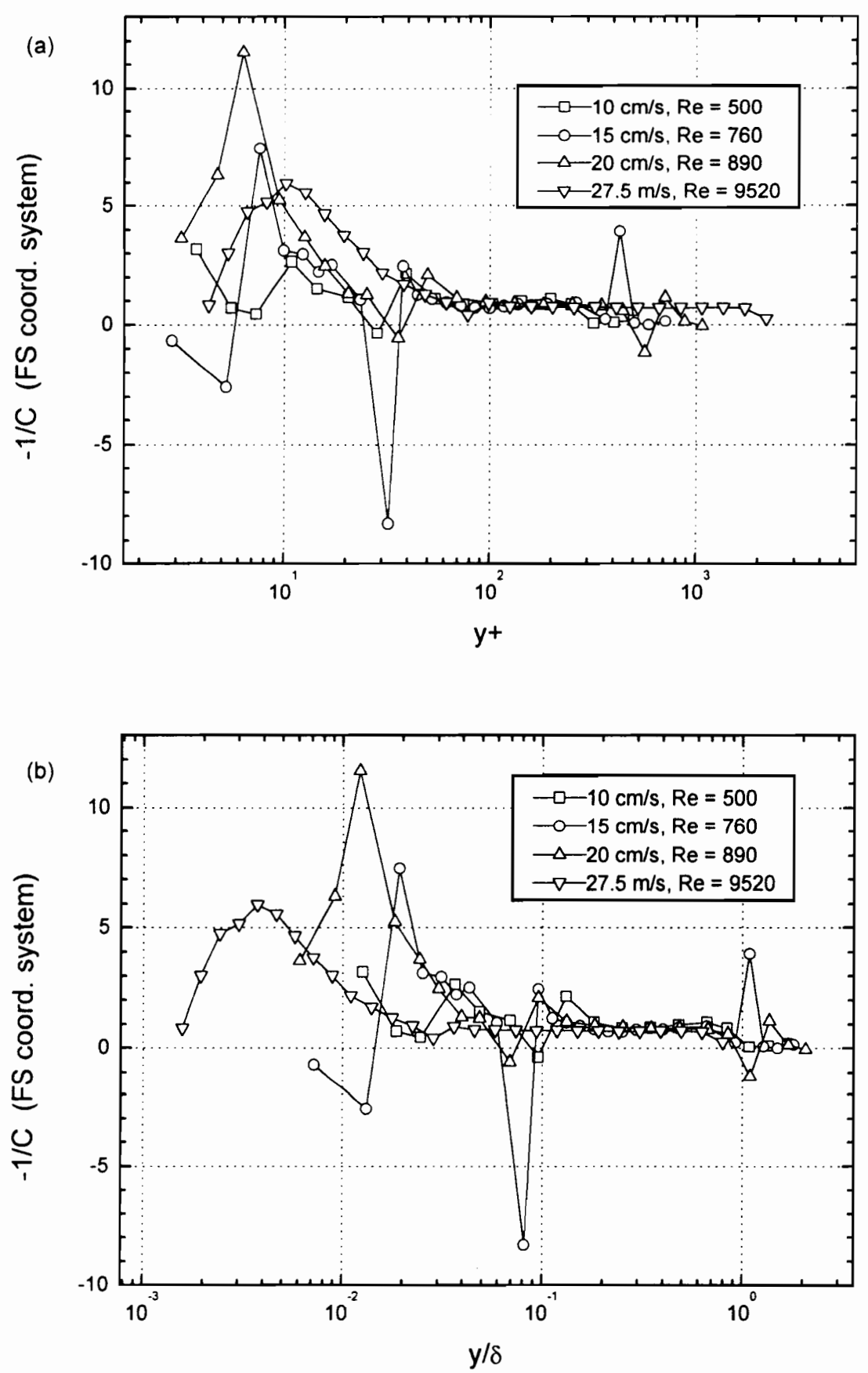

Fig. 3.37 $-1 / C$ results for the $3-\mathrm{D}$ flow. $1 / C$ is the ratio of $-\overline{u w}_{\text {production }}$ to $-\bar{v}_{\text {production }}$. Results calculated using the freestream coordinate system. The constant level in the outer regions of the boundary layers is approximately 0.7 . 

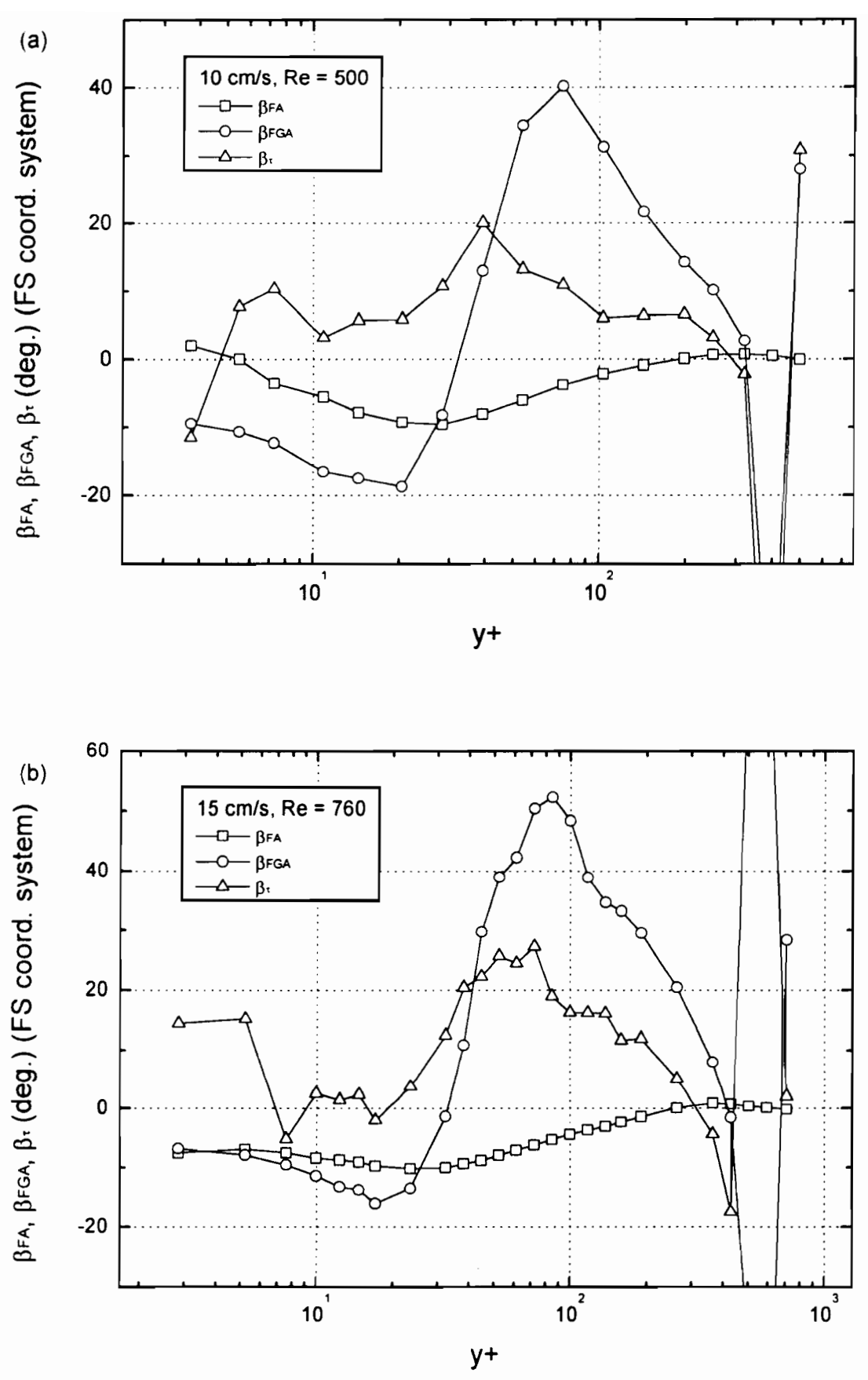

Fig. 3.38 Freestream coordinate system results for $\beta_{\mathrm{FA}}, \beta_{\mathrm{FGA}}$, and $\beta_{\tau}$ at various Reynolds numbers for station \#5. (a) $\operatorname{Re}_{\theta}=500$, (b) $\operatorname{Re}_{\theta}=760$, (c) $\operatorname{Re}_{\theta}=890$, and (d) $\operatorname{Re}_{\theta}=9520$. (c) and (d) are continued on the following page. 

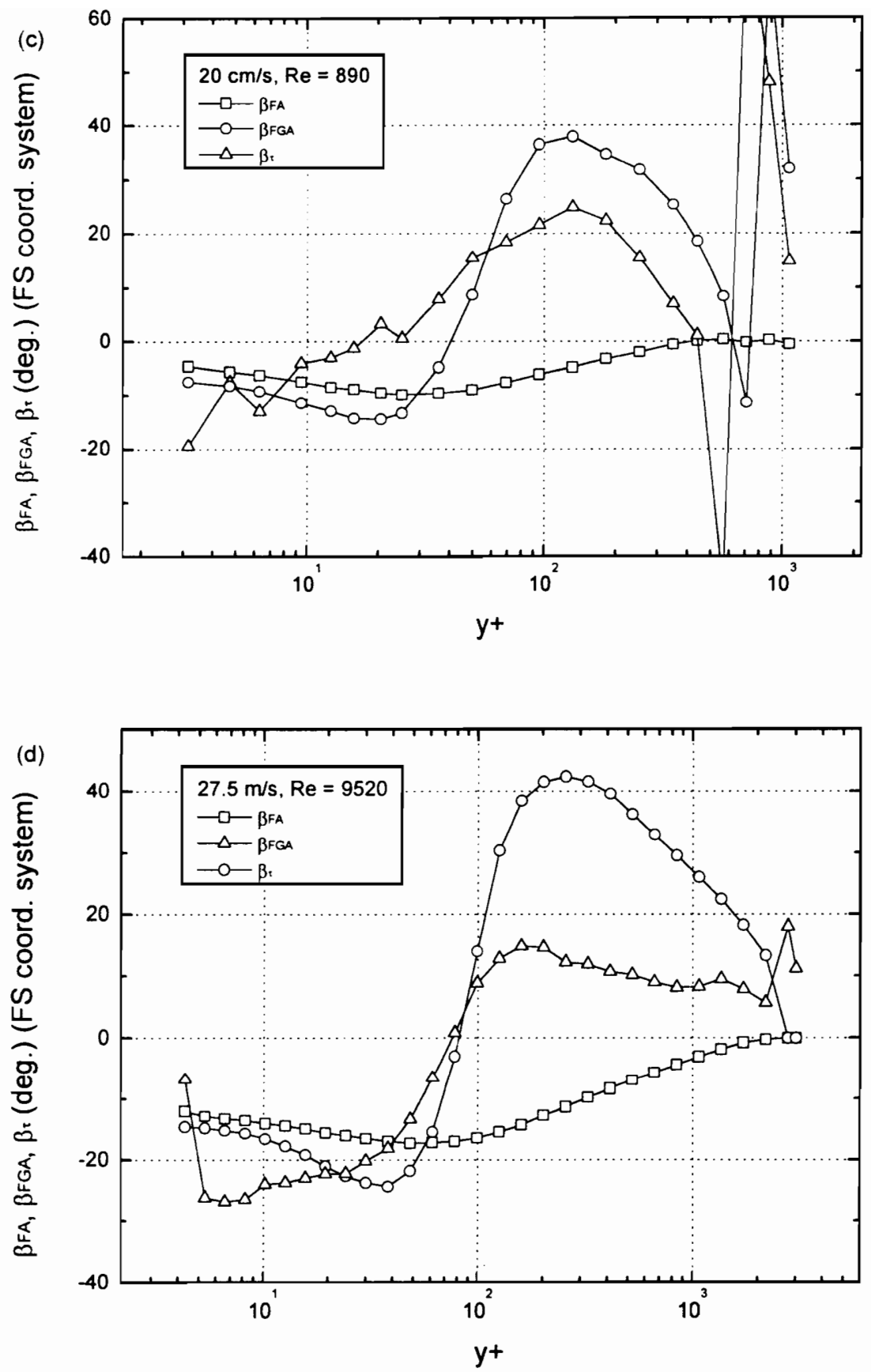

Fig. 3.38 (cont.) See previous page for caption. 

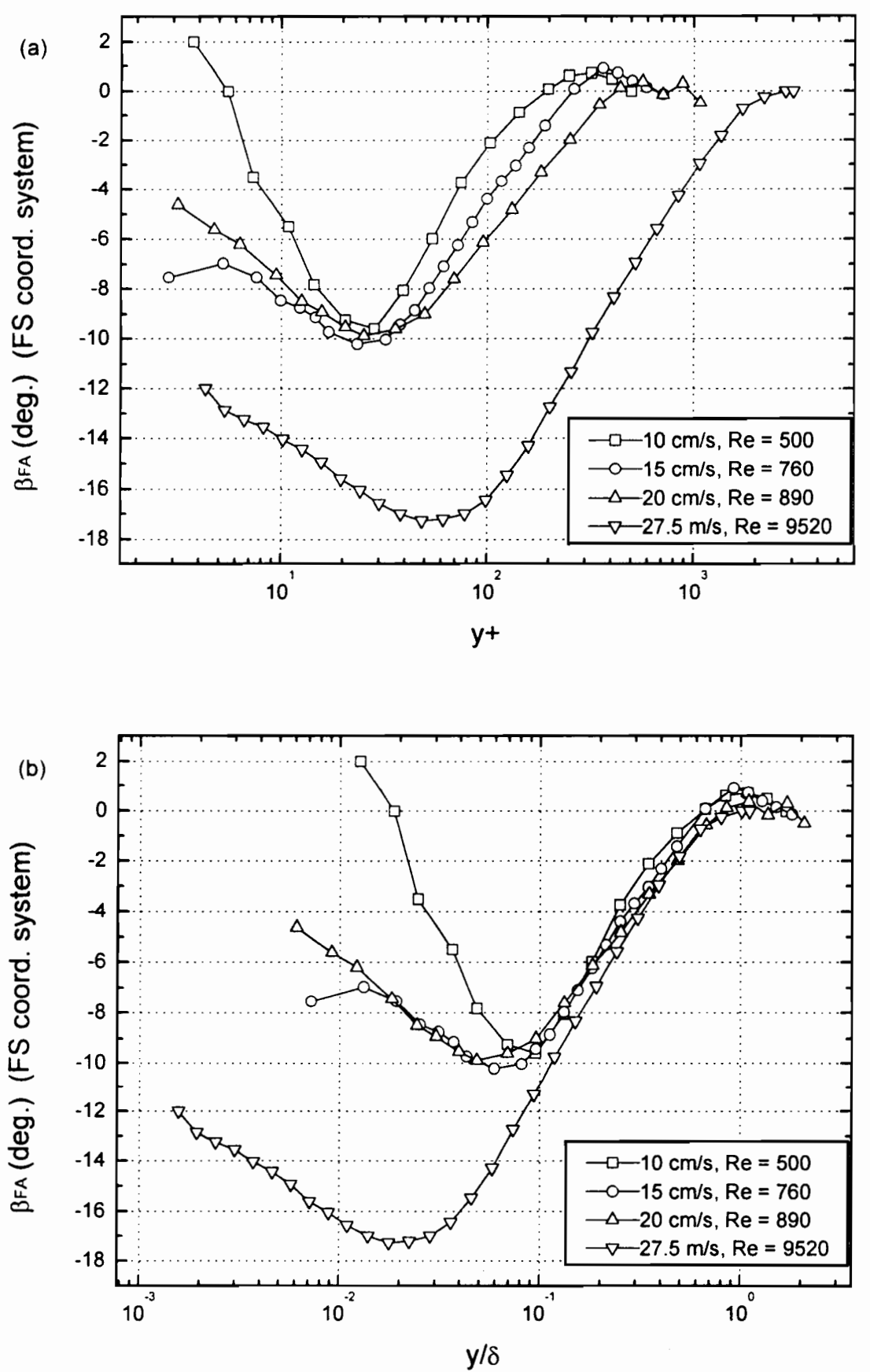

Fig. 3.39 $\beta_{\mathrm{FA}}$ freestream results at station \#5 for all Reynolds numbers, plotted vs. (a) $y^{+}$, and (b) $y / \delta$. 

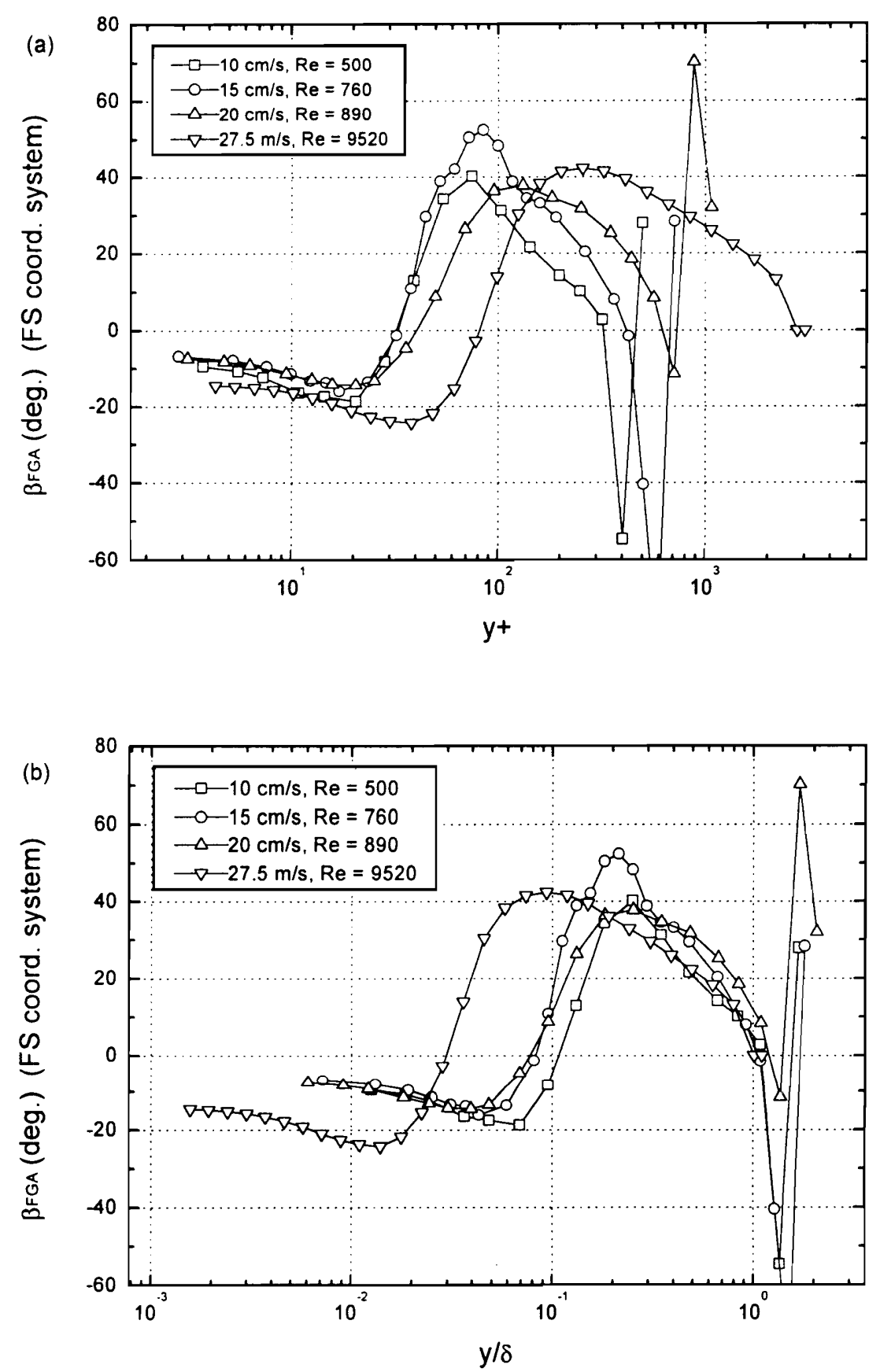

Fig. 3.40 $\beta_{\mathrm{FGA}}$ freestream results at station \#5 for all Reynolds numbers, plotted vs. (a) $y^{+}$, and (b) $y / \delta$. 

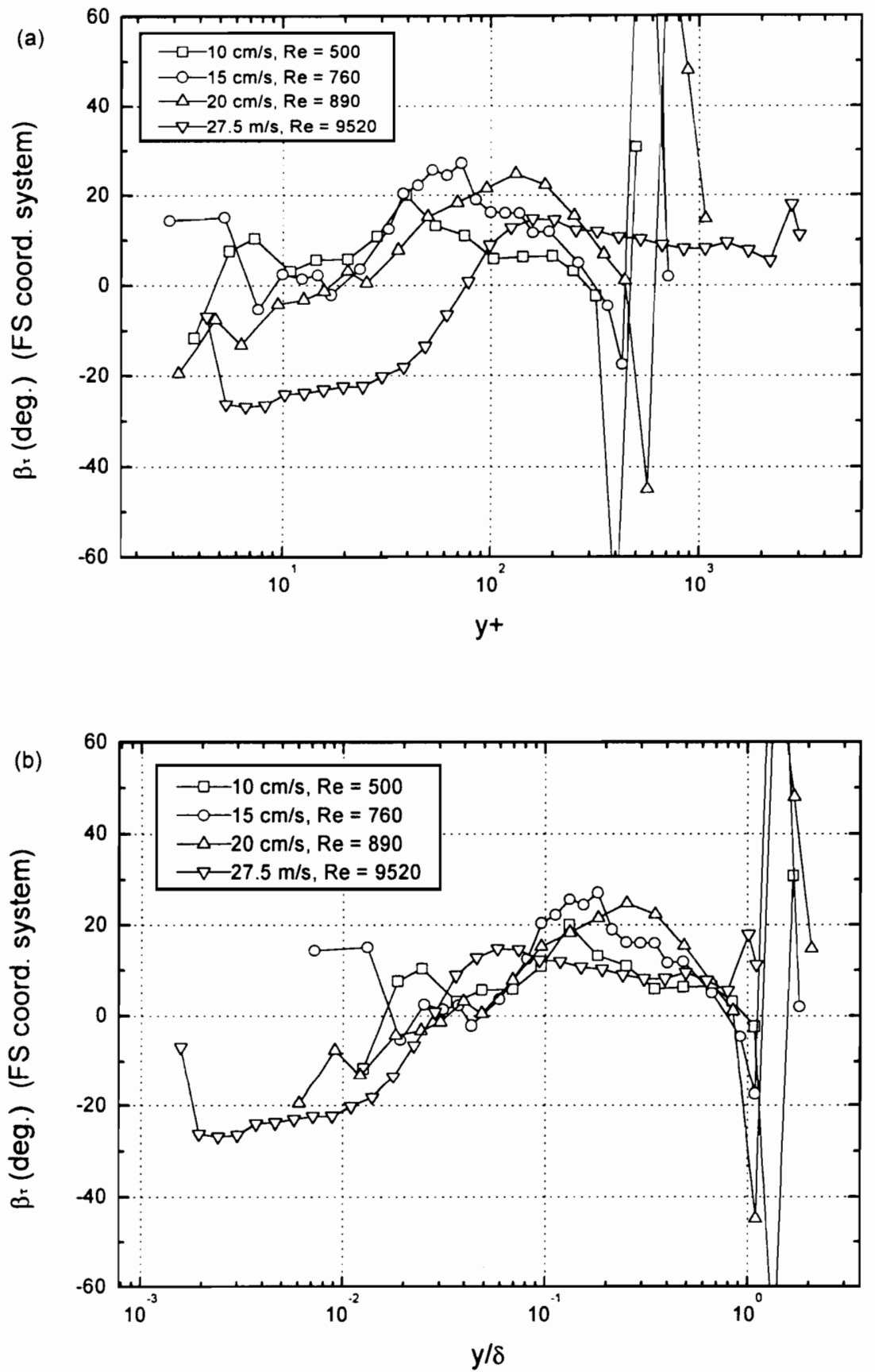

Fig. 3.41 $\beta_{\tau}$ freestream results at station \#5 for all Reynolds numbers, plotted vs. (a) $y^{+}$, and (b) $y / \delta$. 

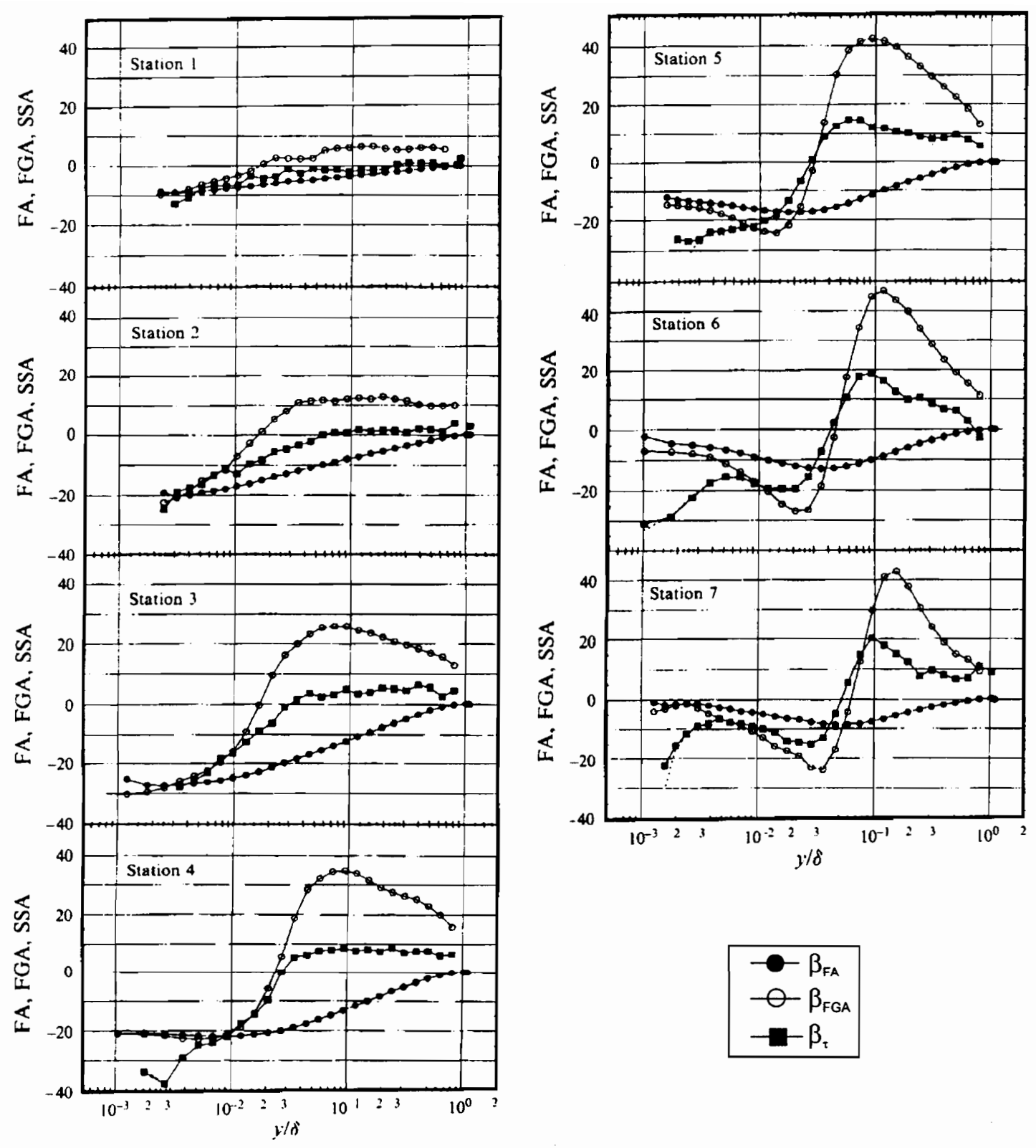

Fig. 3.42 Freestream coordinate system results for $\beta_{\mathrm{FA}}(\mathrm{FA}), \beta_{\mathrm{FGA}}(\mathrm{FGA})$, and $\beta_{\tau}$ (SSA) at $U_{\text {ref }}=27.5 \mathrm{~m} / \mathrm{s}$, approach flow $\operatorname{Re}_{\theta}=7000$ (reprinted from Ölçmen and Simpson, 1995). Where visible, dashed lines indicate gradient-broadening-corrected shear stress data. These data show the development of this 3-D flow up to and past station 5 . 

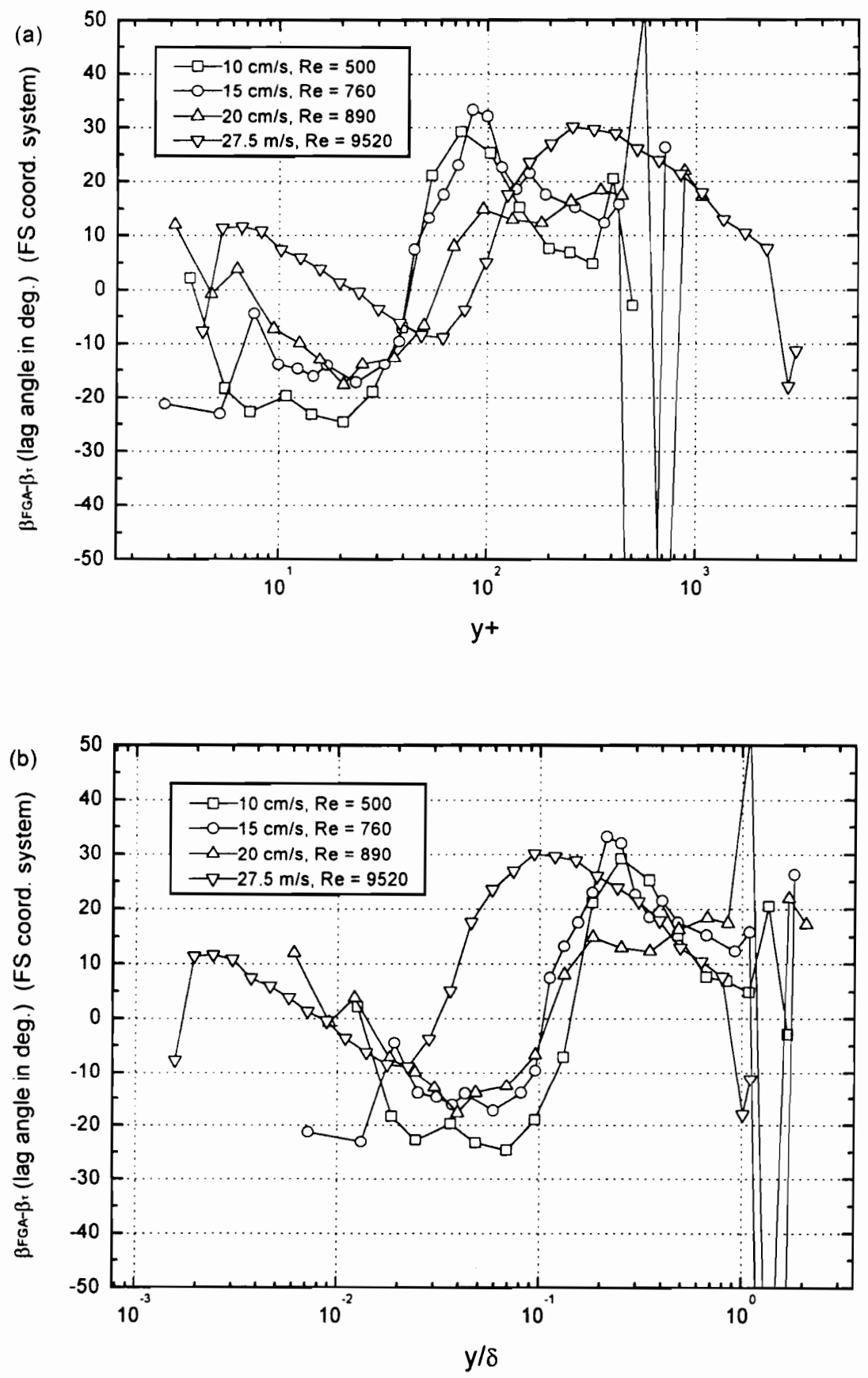

Fig. 3.43 Shear stress lag angle $\left(\beta_{\text {lag }}\right)$ freestream results at station \#5 for all Reynolds numbers, plotted vs. (a) $y^{+}$, and (b) $y / \delta$. 

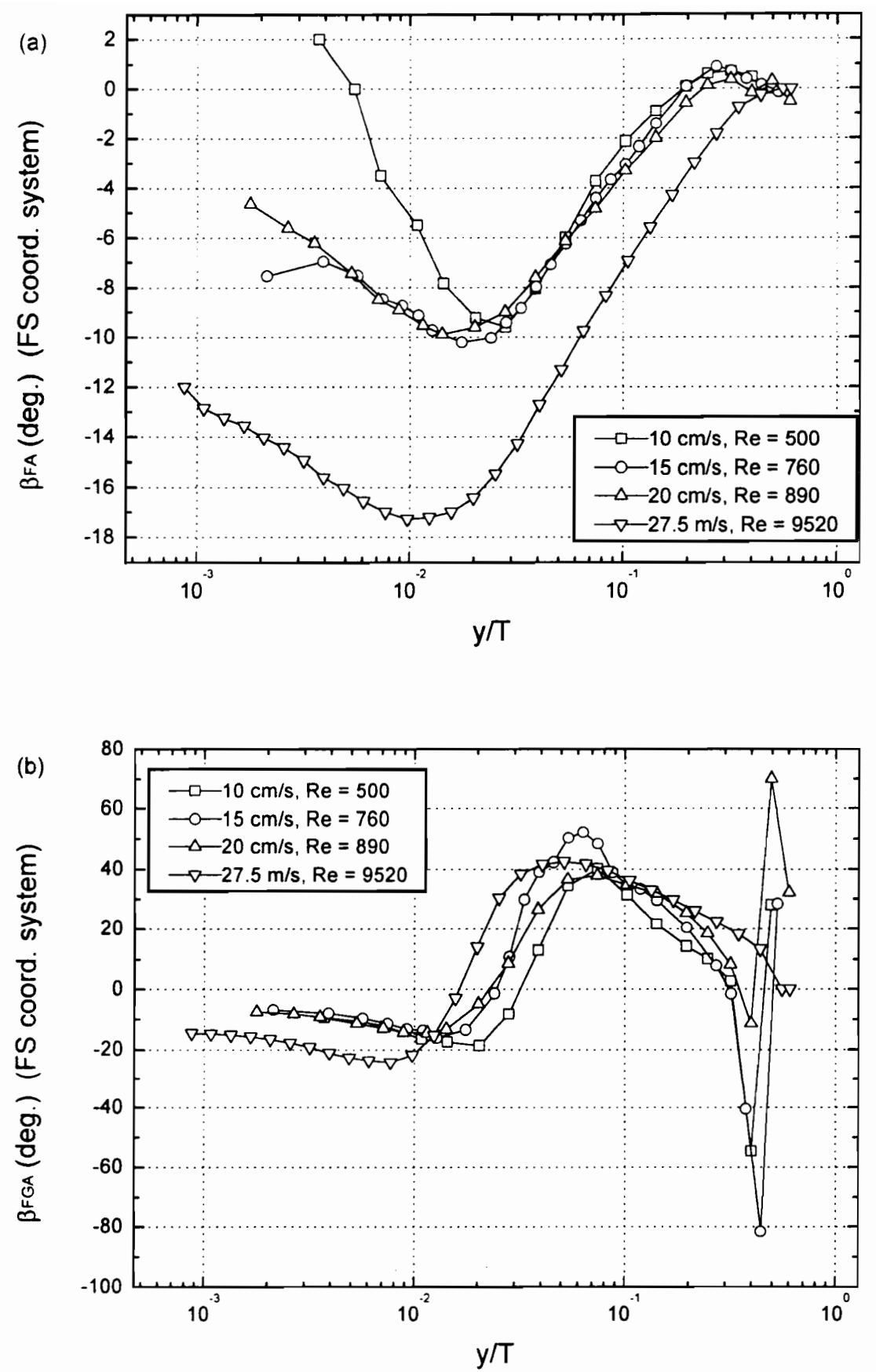

Fig. 3.44 Freestream results for (a) $\beta_{\mathrm{FA}}$, (b) $\beta_{\mathrm{FGA}}$, (c) $\beta_{\tau}$, and (d) $\beta_{\mathrm{lag}}$ at various Reynolds numbers for station $\# 5$, plotted vs. $y / T$. (c) and (d) are continued on the next page. 

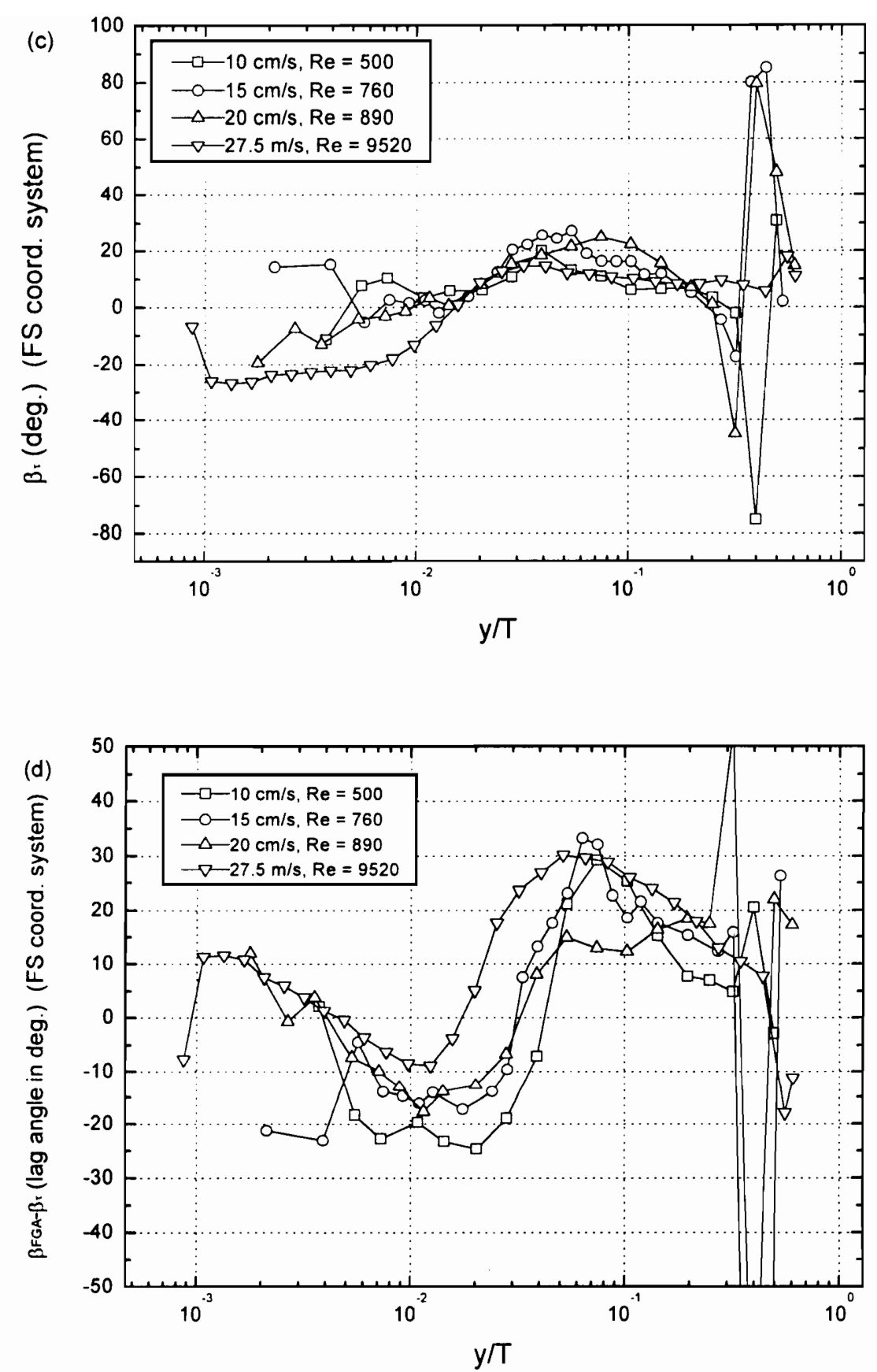

Fig. 3.44 (cont.) See previous page for caption. 

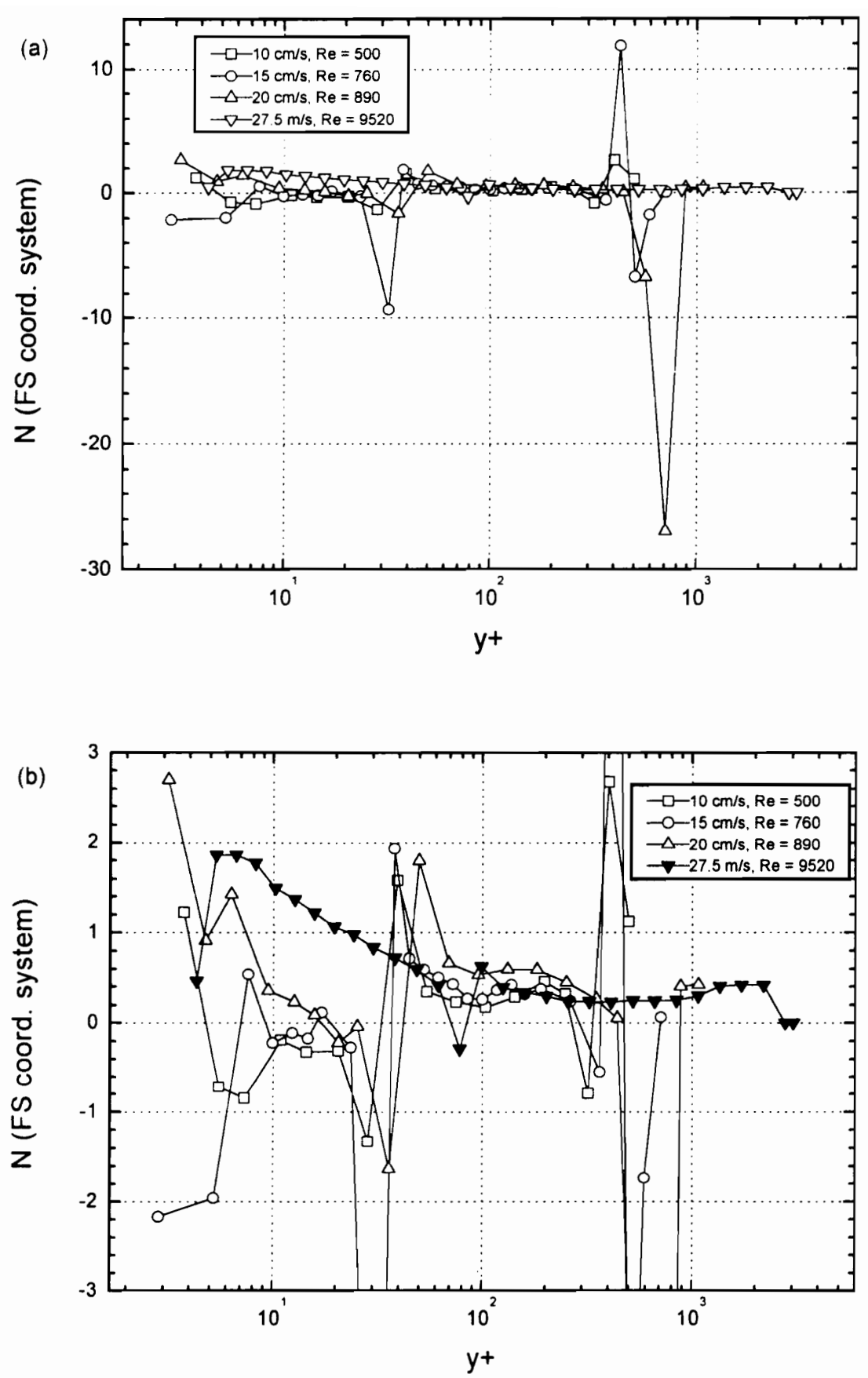

Fig. 3.45 Freestream results for the anisotropy factor, $N$, various Reynolds numbers at Station \#5. (b) is a detail view of (a), with Ölçmen and Simpson's data (1995) highlighted. 

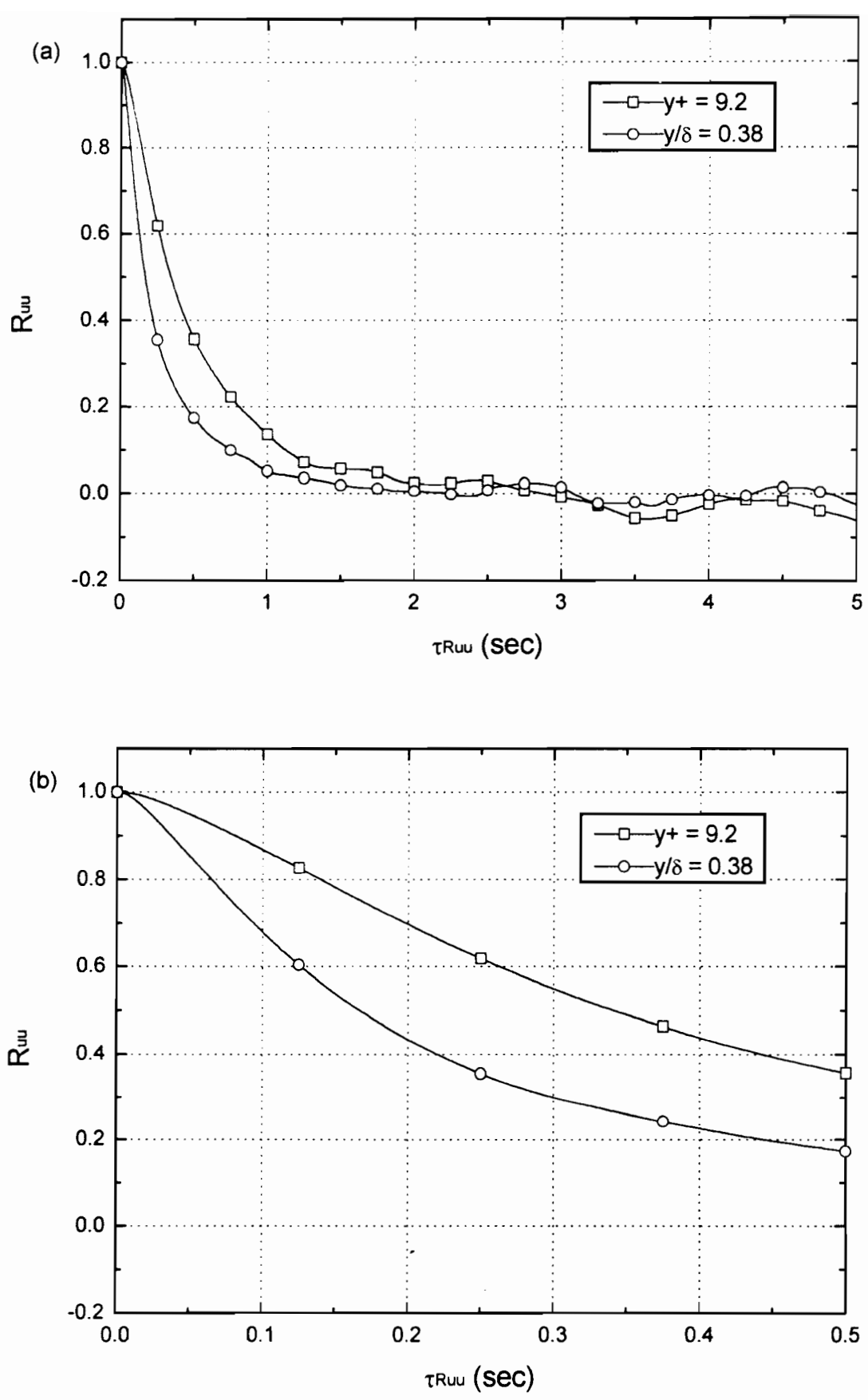

Fig. $3.462-\mathrm{D} U$ autocorrelation results, $U_{r e f}=10 \mathrm{~cm} / \mathrm{s}, \operatorname{Re}_{\theta}=450$. (a) shows the results from $\tau_{R u u}=0$ to 5 seconds. Details of the results from $\tau_{R u u}=0.0$ to 0.5 seconds are shown in (b). Note that not all data points are marked with a symbol. 

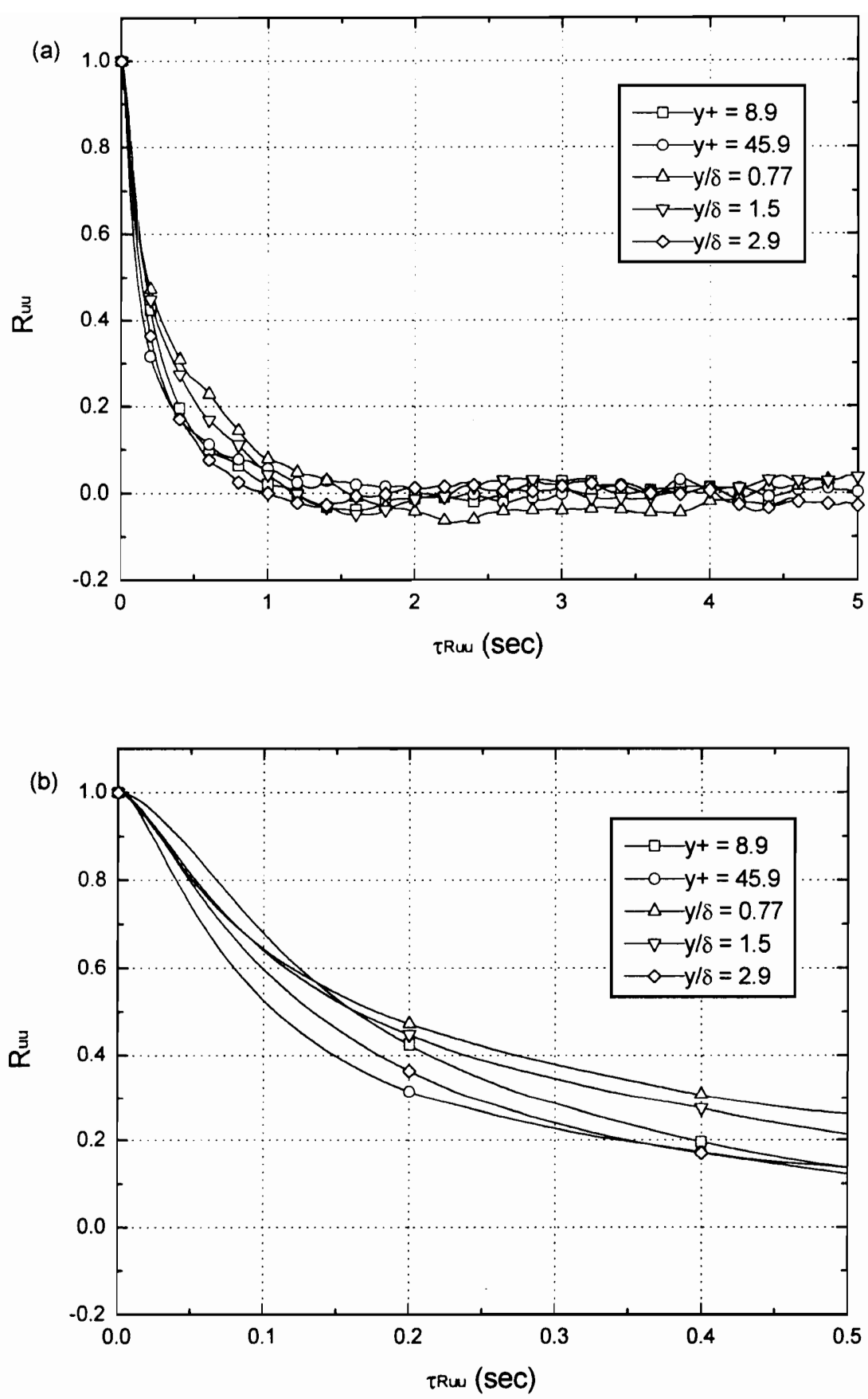

Fig. 3.47 2-D $U$ autocorrelation results, $U_{\text {ref }}=15 \mathrm{~cm} / \mathrm{s}, \operatorname{Re}_{\theta}=300$. (a) shows the results from $\tau_{R u u}=0$ to 5 seconds. Details of the results from $\tau_{R u u}=0.0$ to 0.5 seconds are shown in (b). Note that not all data points are marked with a symbol. 

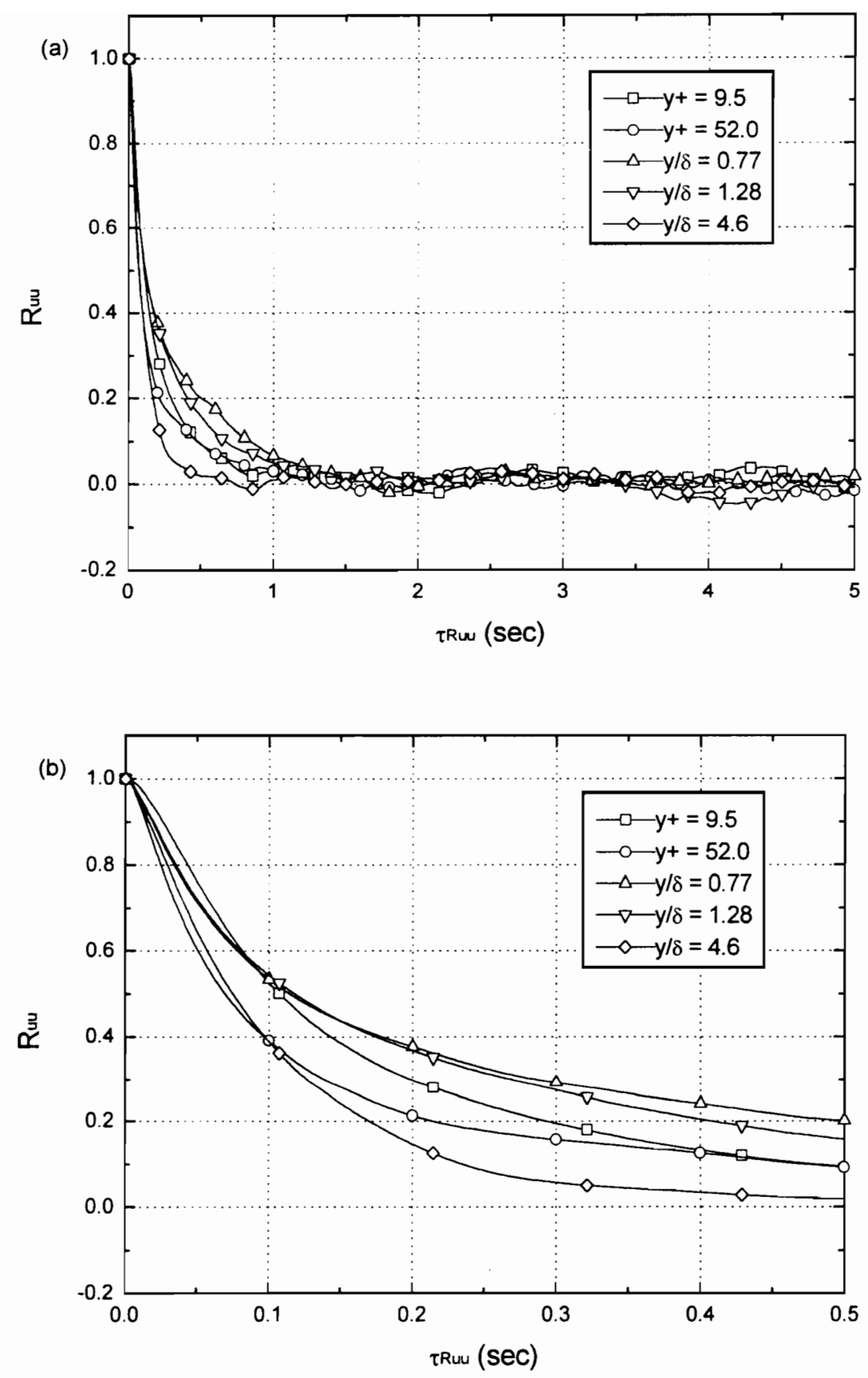

Fig. $3.482-\mathrm{D} U$ autocorrelation results, $U_{\text {ref }}=20 \mathrm{~cm} / \mathrm{s}, \operatorname{Re}_{\theta}=425$. (a) shows the results from $\tau_{R u u}=0$ to 5 seconds. Details of the results from $\tau_{R u u}=0.0$ to 0.5 seconds are shown in (b). Note that not all data points are marked with a symbol. 

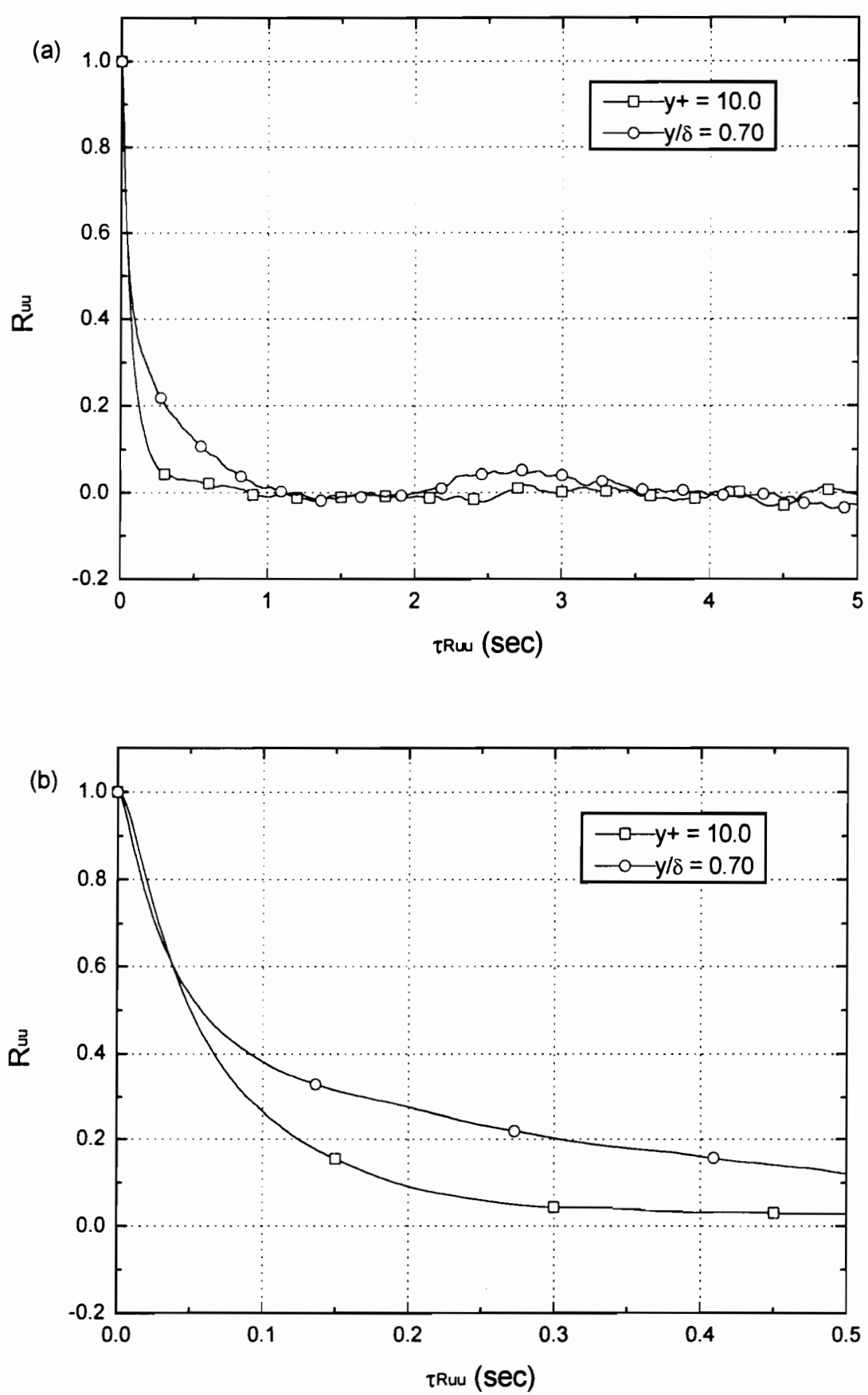

Fig. $3.492-\mathrm{D} U$ autocorrelation results, $U_{r e f}=30 \mathrm{~cm} / \mathrm{s}, \operatorname{Re}_{\theta}=570$. (a) shows the results from $\tau_{R u u}=0$ to 5 seconds. Details of the results from $\tau_{R u u}=0.0$ to 0.5 seconds are shown in (b). Note that not all data points are marked with a symbol. 

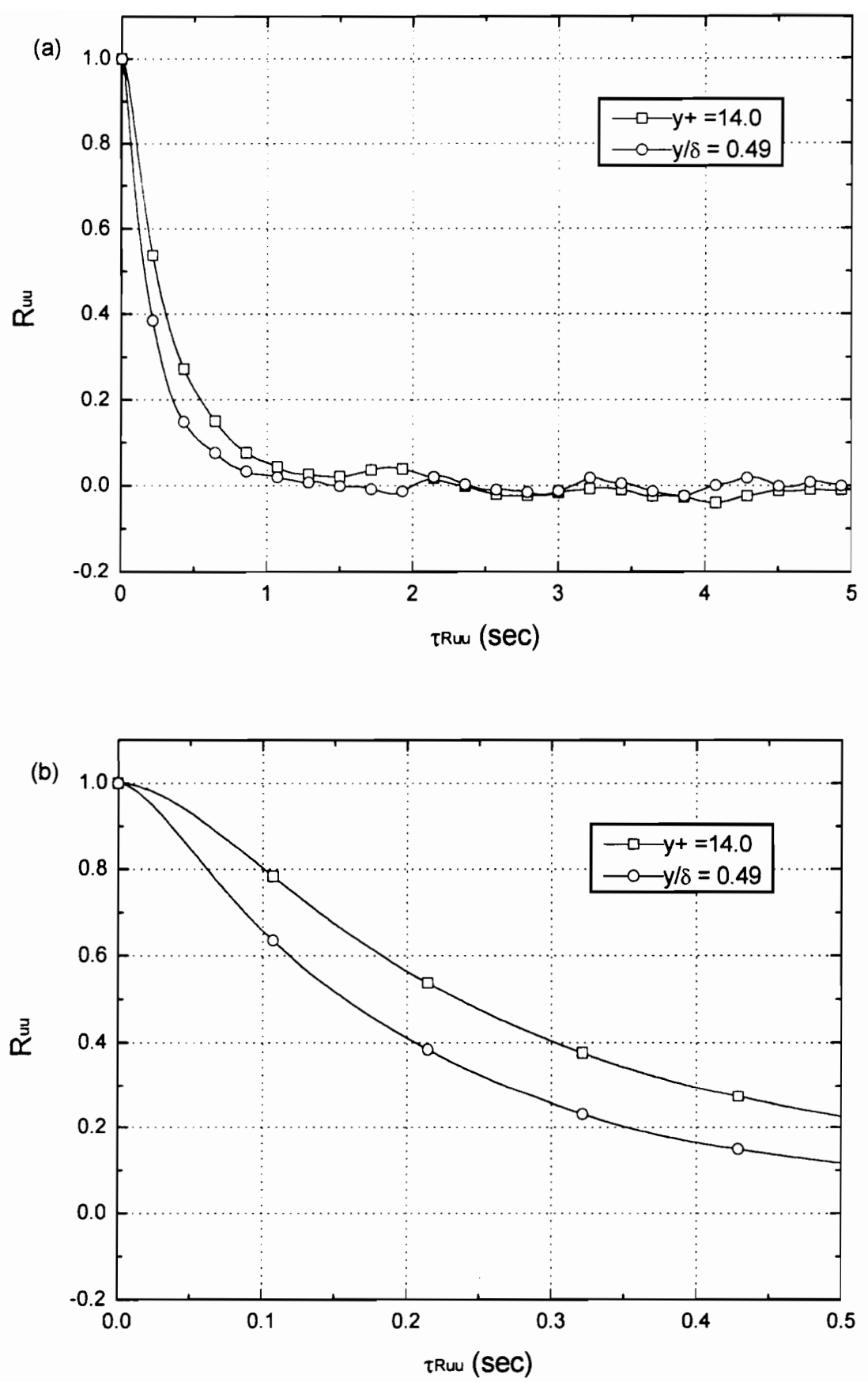

Fig. 3.50 3-D $U$ autocorrelation results, $U_{\text {ref }}=10 \mathrm{~cm} / \mathrm{s}, \operatorname{Re}_{\theta}=500$. These are tunnel coordinate system results. (a) shows the results from $\tau_{R u u}=0$ to 5 seconds. Details of the results from $\tau_{R u u}=0.0$ to 0.5 seconds are shown in (b). Note that not all data points are marked with a symbol. 

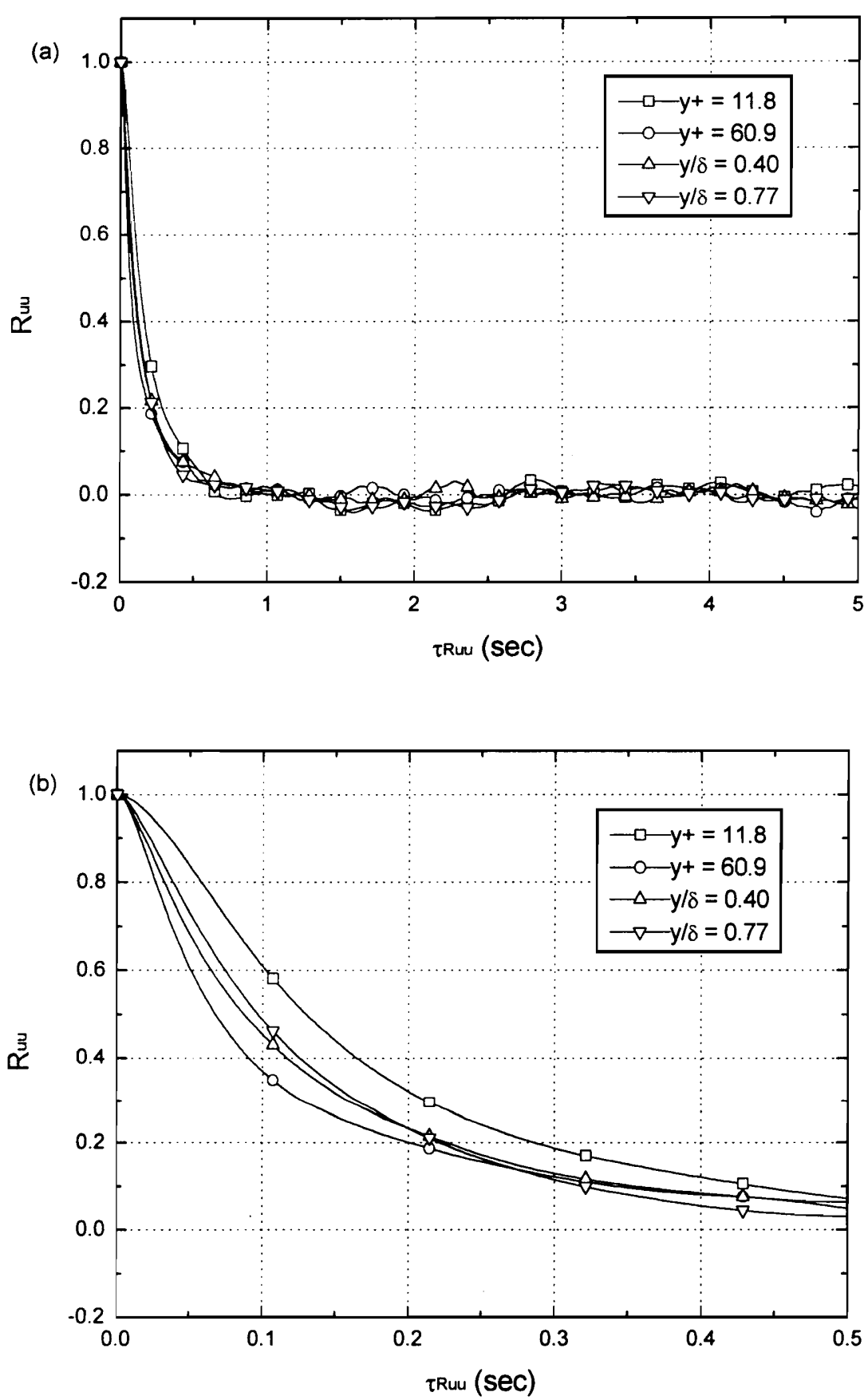

Fig. 3.51 3-D $U$ autocorrelation results, $U_{r e f}=15 \mathrm{~cm} / \mathrm{s}, \operatorname{Re}_{\theta}=760$. These are tunnel coordinate system results. (a) shows the results from $\tau_{R u u}=0$ to 5 seconds. Details of the results from $\tau_{R u u}=0.0$ to 0.5 seconds are shown in (b). Note that not all data points are marked with a symbol. 

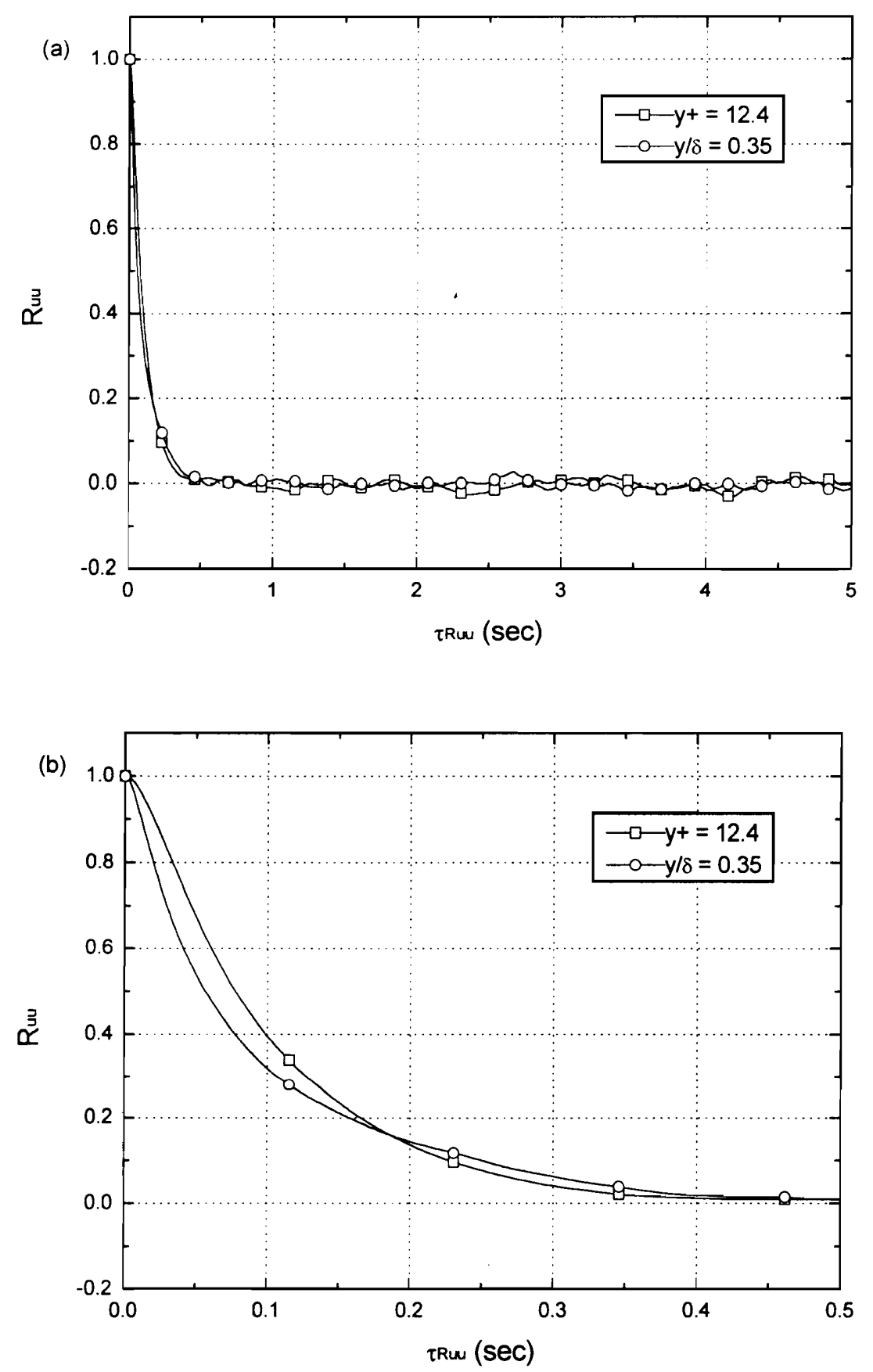

Fig. 3.52 3-D $U$ autocorrelation results, $U_{r e f}=20 \mathrm{~cm} / \mathrm{s}, \operatorname{Re}_{\theta}=890$. These are tunnel coordinate system results. (a) shows the results from $\tau_{R u u}=0$ to 5 seconds. Details of the results from $\tau_{R u u}=0.0$ to 0.5 seconds are shown in (b). Note that not all data points are marked with a symbol. 

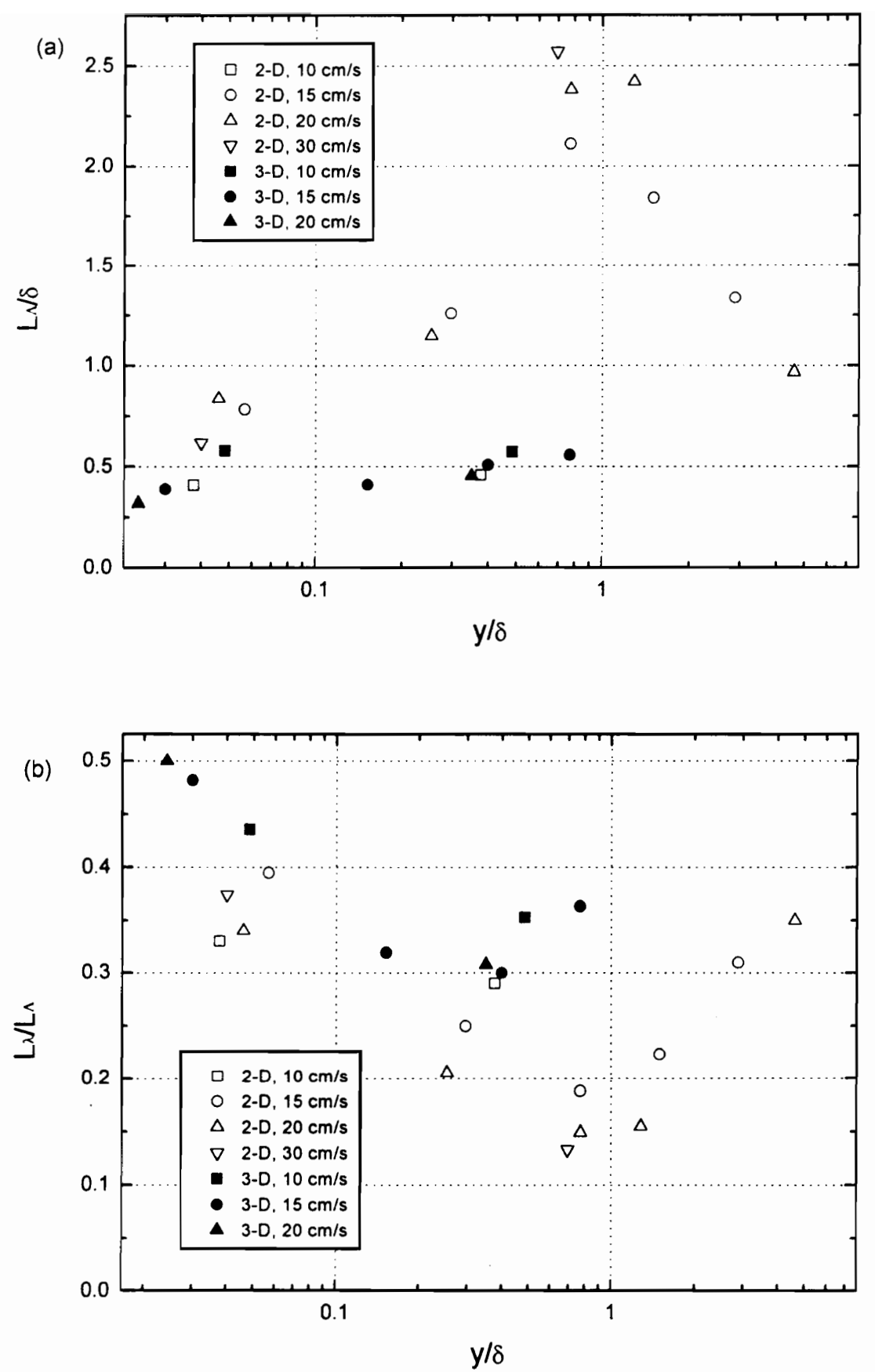

Fig. 3.53 Comparison of the autocorrelation length scale results for the 2-D and 3-D data. (a) $L_{\Lambda}$ vs. $y / \delta$, and (b) $L_{\lambda} / L_{\Lambda}$ vs. $y / \delta$. 

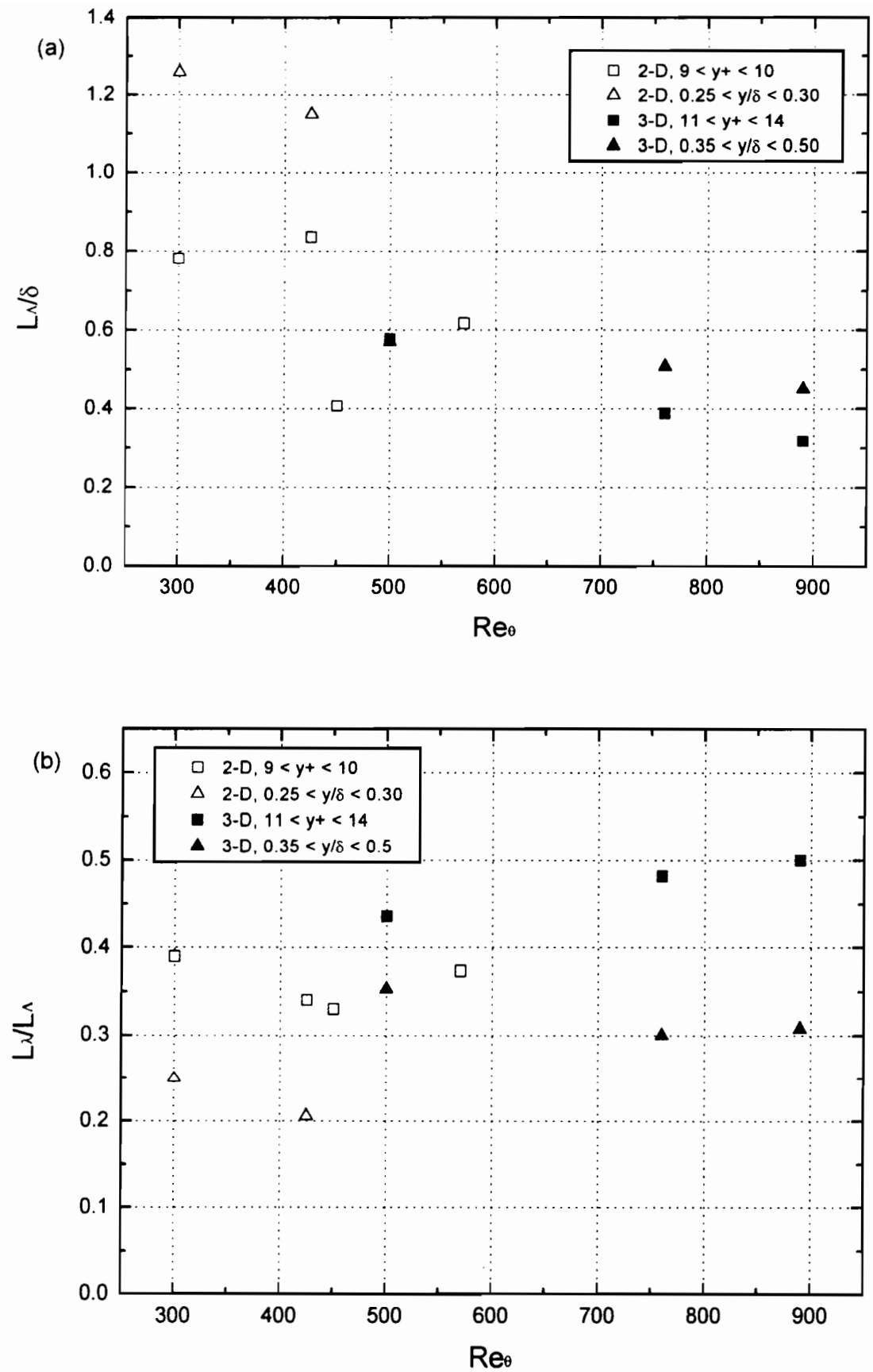

Fig. 3.54 Autocorrelation length scale results vs. $\operatorname{Re}_{\theta}$ for 2-D and 3-D data. 3-D data are tunnel coordinate system results. (a) $L_{\Lambda} / \delta$ vs. $\operatorname{Re}_{\theta}$, and (b) $L_{\Lambda} / L_{\lambda}$ vs. $\operatorname{Re}_{\theta}$. Data are grouped for different values of $y$. 

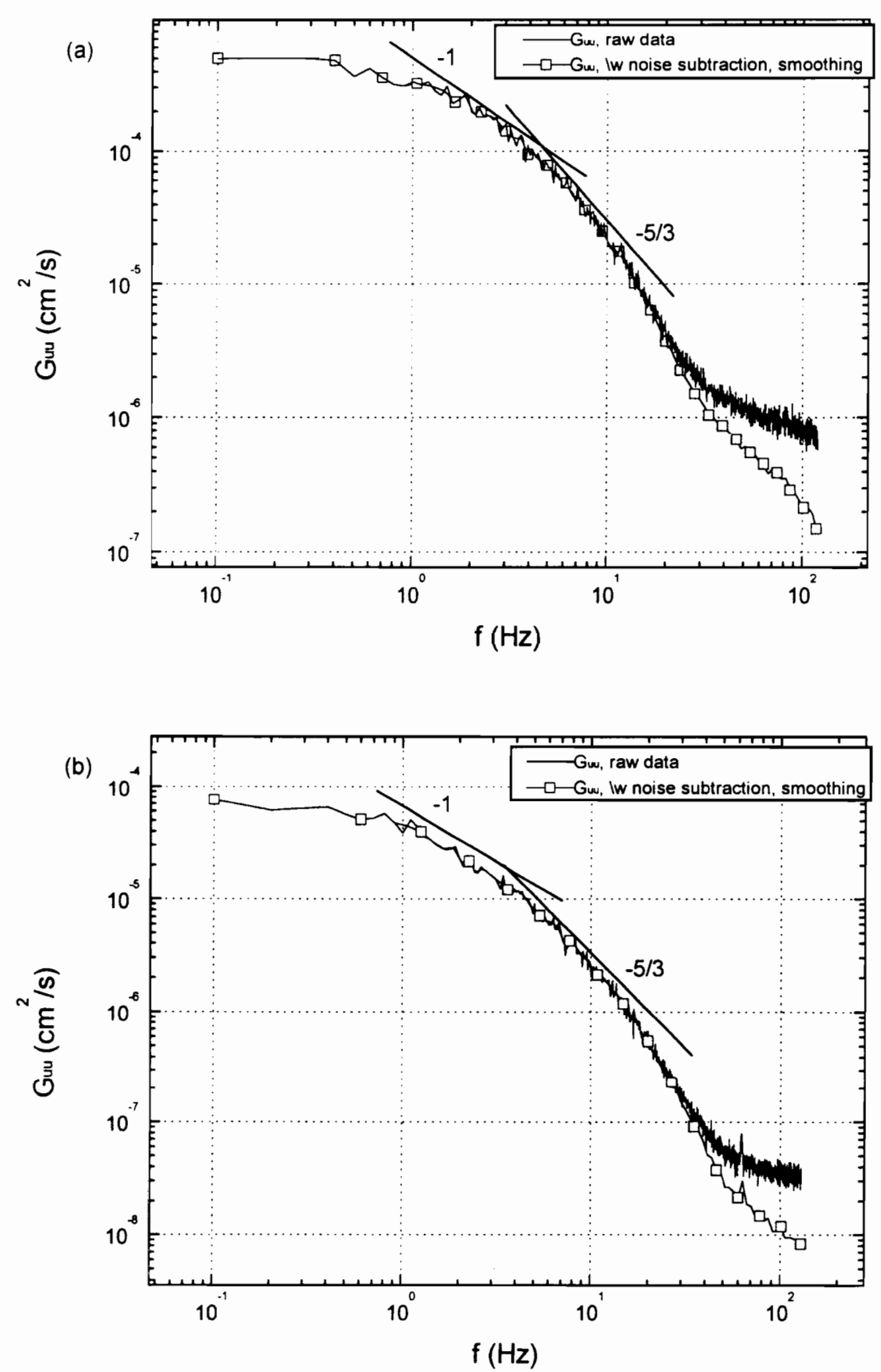

Fig. 3.55 Noise subtraction and smoothing results for typical LDV raw spectral data. $G_{u u}$ is the dimensional energy per hertz. (a) $2-\mathrm{D}, U_{\text {ref }}=30 \mathrm{~cm} / \mathrm{s}, y^{+}=10$ results. (b) 3-D, $U_{r e f}=20 \mathrm{~cm} / \mathrm{s}, y / \delta=0.35$ results. 

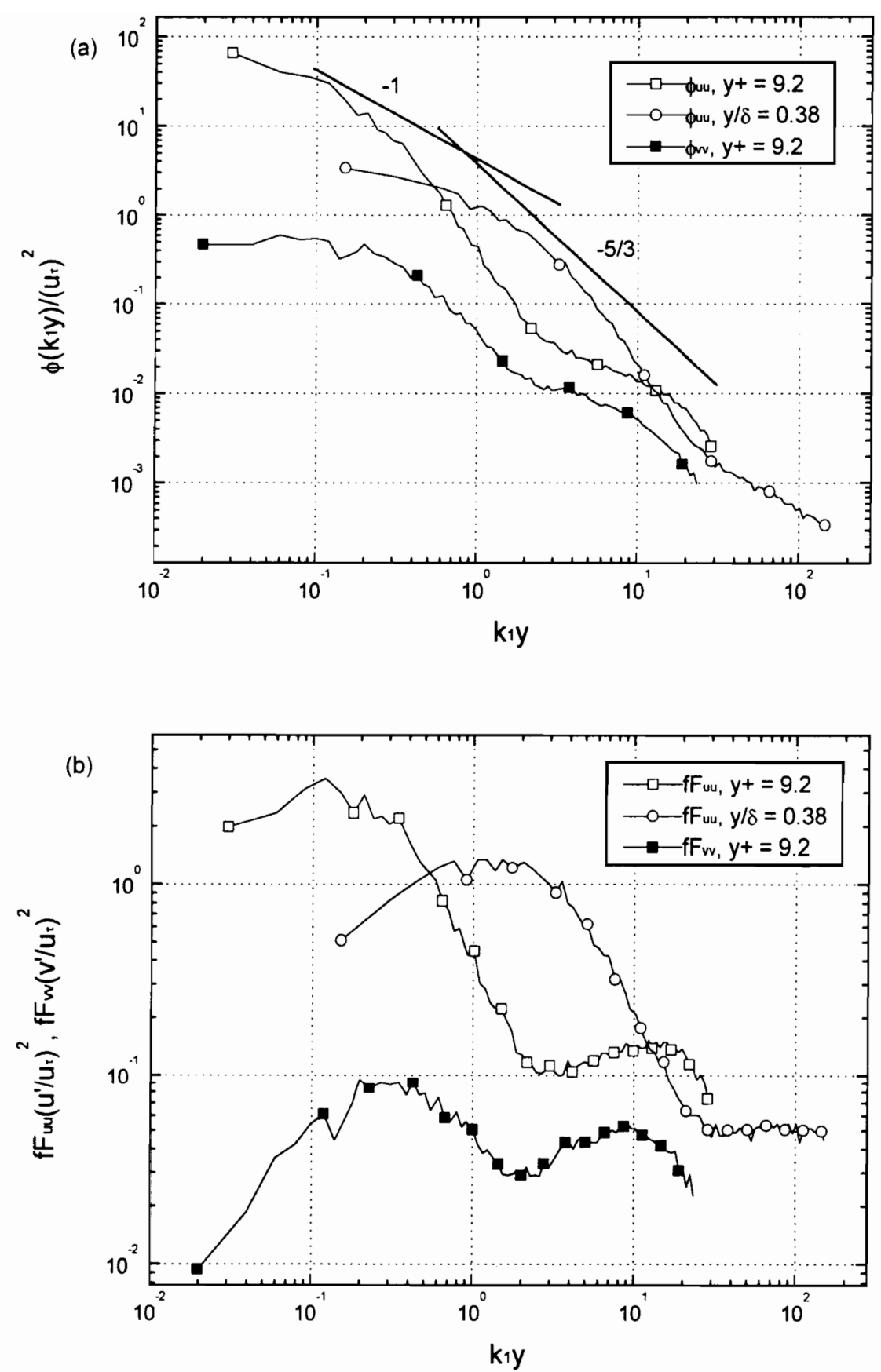

Fig. $3.562-\mathrm{D} U$ and $V$ power spectral results, $U_{r e f}=10 \mathrm{~cm} / \mathrm{s}, \operatorname{Re}_{\theta}=450$. (a) Spectra non-dimensionalized using inner scaling, and (b) non-dimensional first moment of spectra. Note that not all data points are marked with a symbol. 

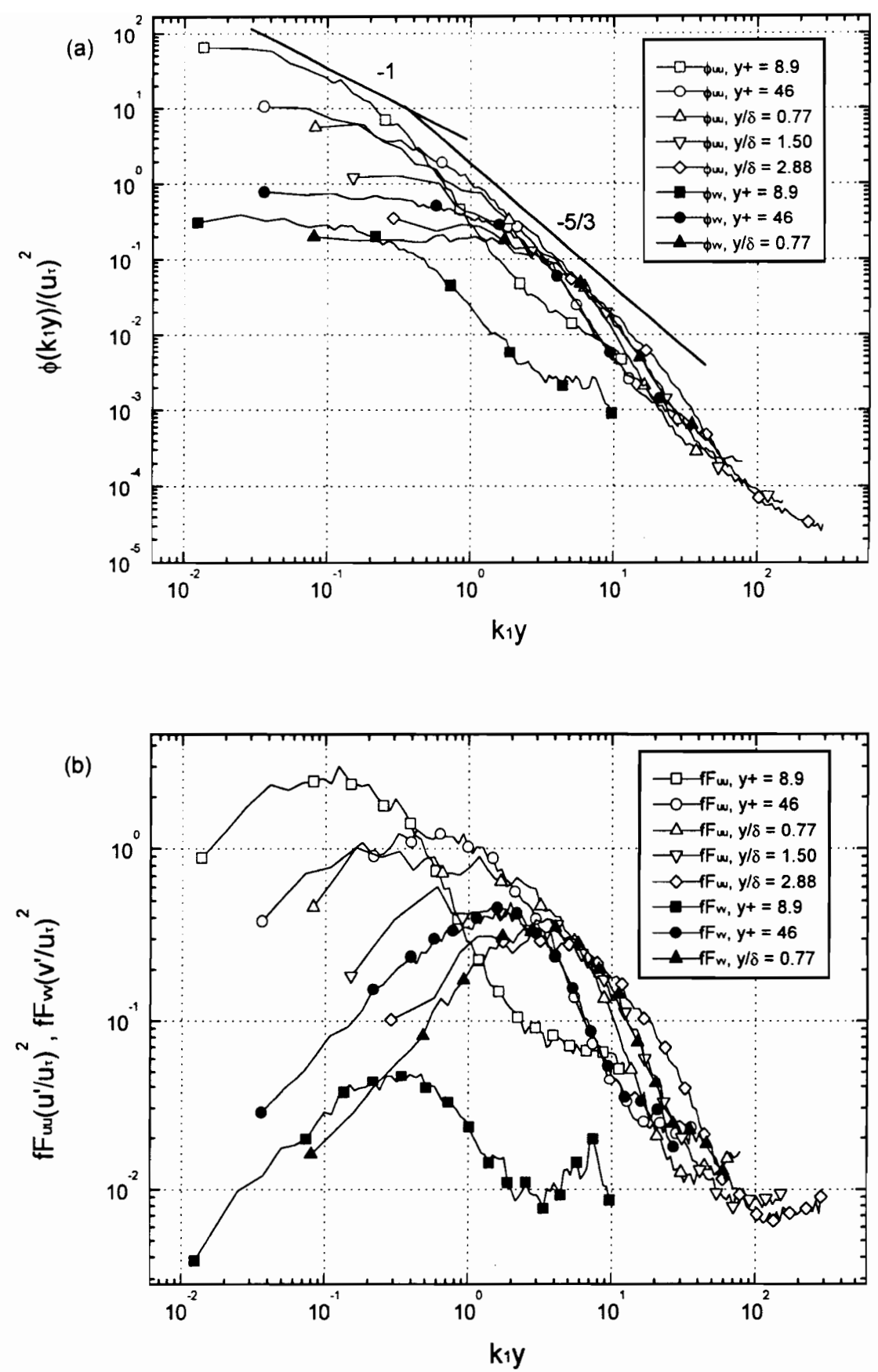

Fig. 3.57 2-D $U$ and $V$ power spectral results, $U_{\text {ref }}=15 \mathrm{~cm} / \mathrm{s}, \operatorname{Re}_{\theta}=300$. (a) Spectra non-dimensionalized using inner scaling, and (b) non-dimensional first moment of spectra. Note that not all data points are marked with a symbol. 

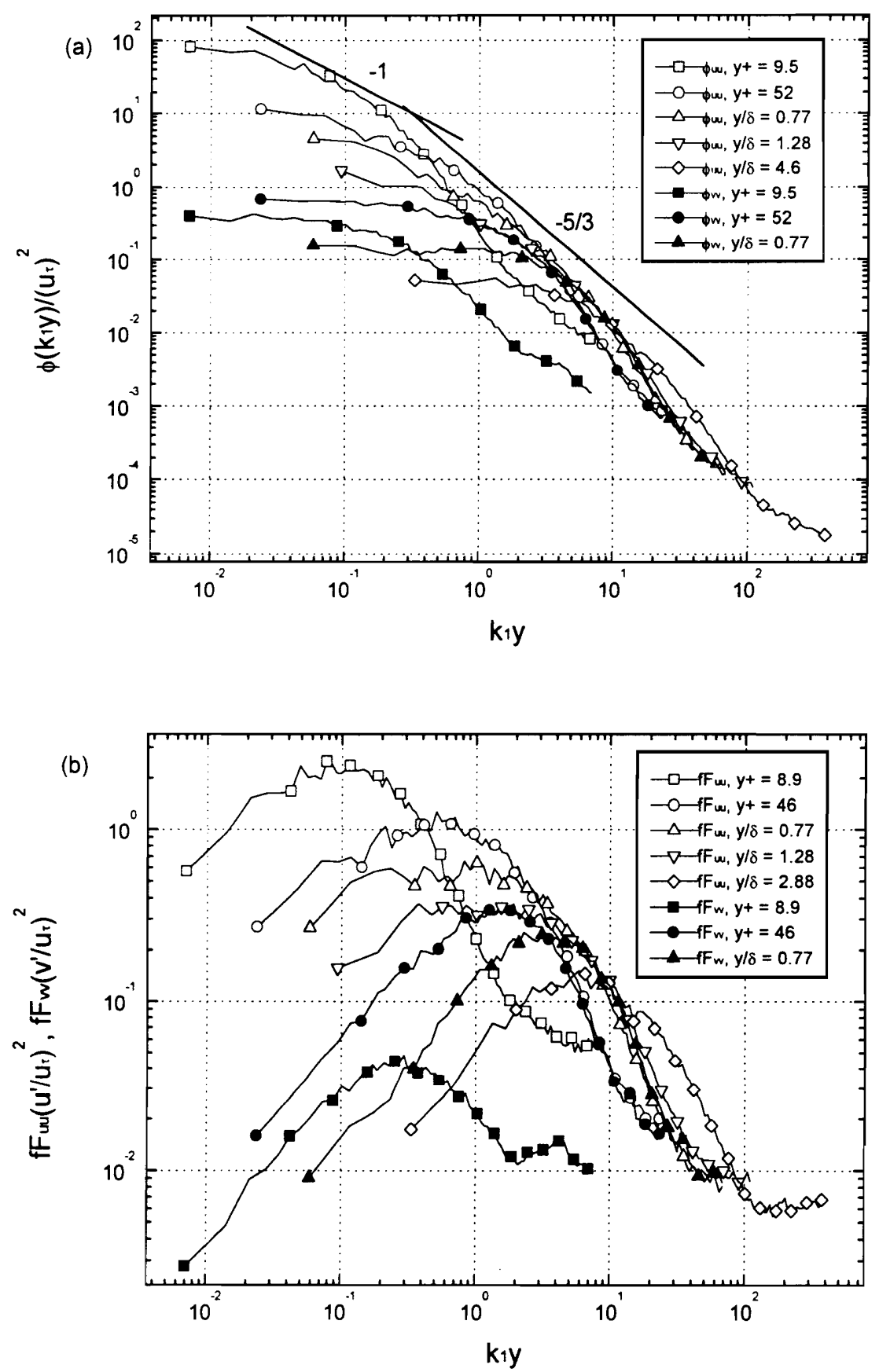

Fig. 3.58 2-D $U$ and $V$ power spectral results, $U_{\text {ref }}=20 \mathrm{~cm} / \mathrm{s}, \operatorname{Re}_{\theta}=425$. (a) Spectra non-dimensionalized using inner scaling, and (b) non-dimensional first moment of spectra. Note that not all data points are marked with a symbol. 

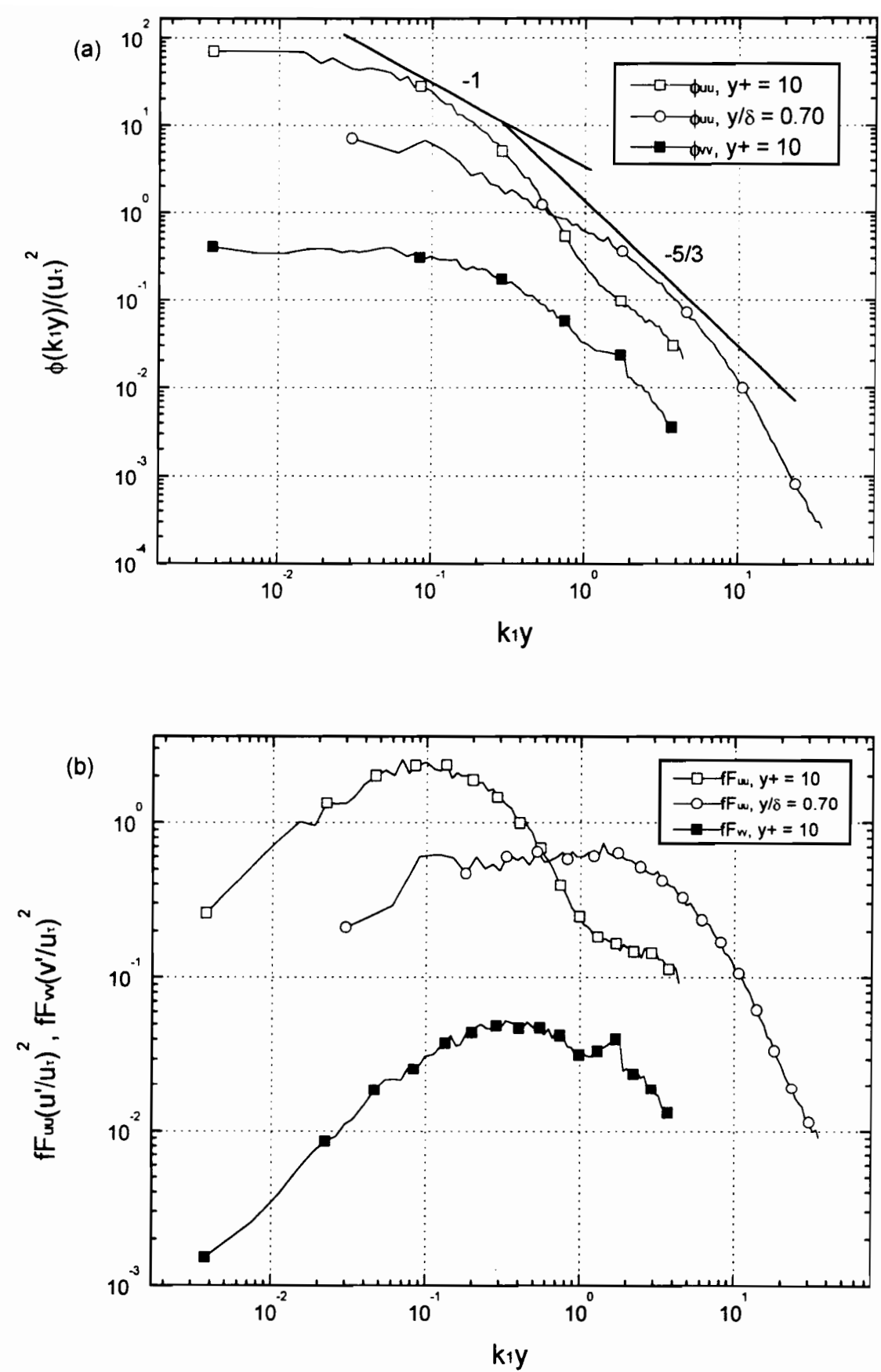

Fig. 3.59 2-D $U$ and $V$ power spectral results, $U_{r e f}=30 \mathrm{~cm} / \mathrm{s}, \operatorname{Re}_{\theta}=570$. (a) Spectra non-dimensionalized using inner scaling, and (b) non-dimensional first moment of spectra. Note that not all data points are marked with a symbol. 


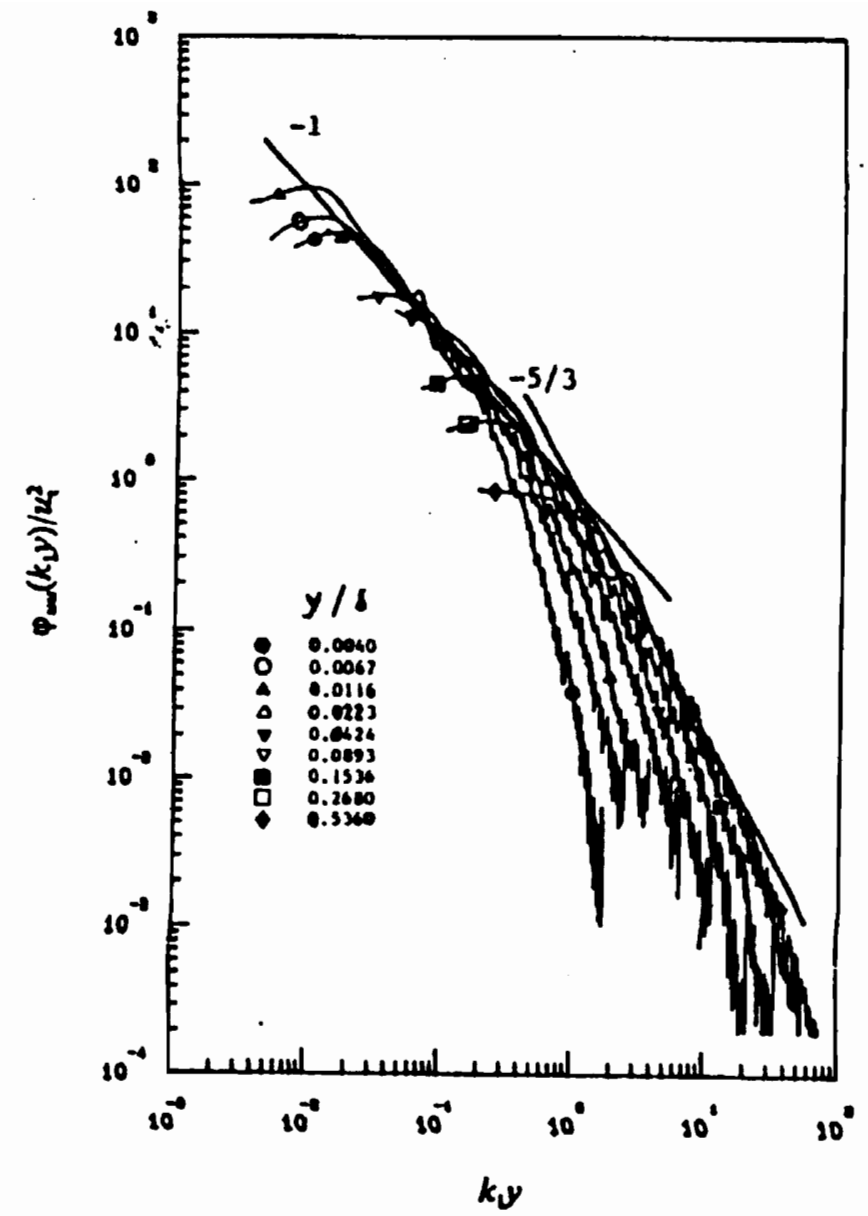

Fig. 3.60 2-D $U$ power spectra from Ahn and Simpson's (1987) constant temperature hotwire measurements. Results are normalized using inner scaling, $\operatorname{Re}_{\theta}=9000$. 


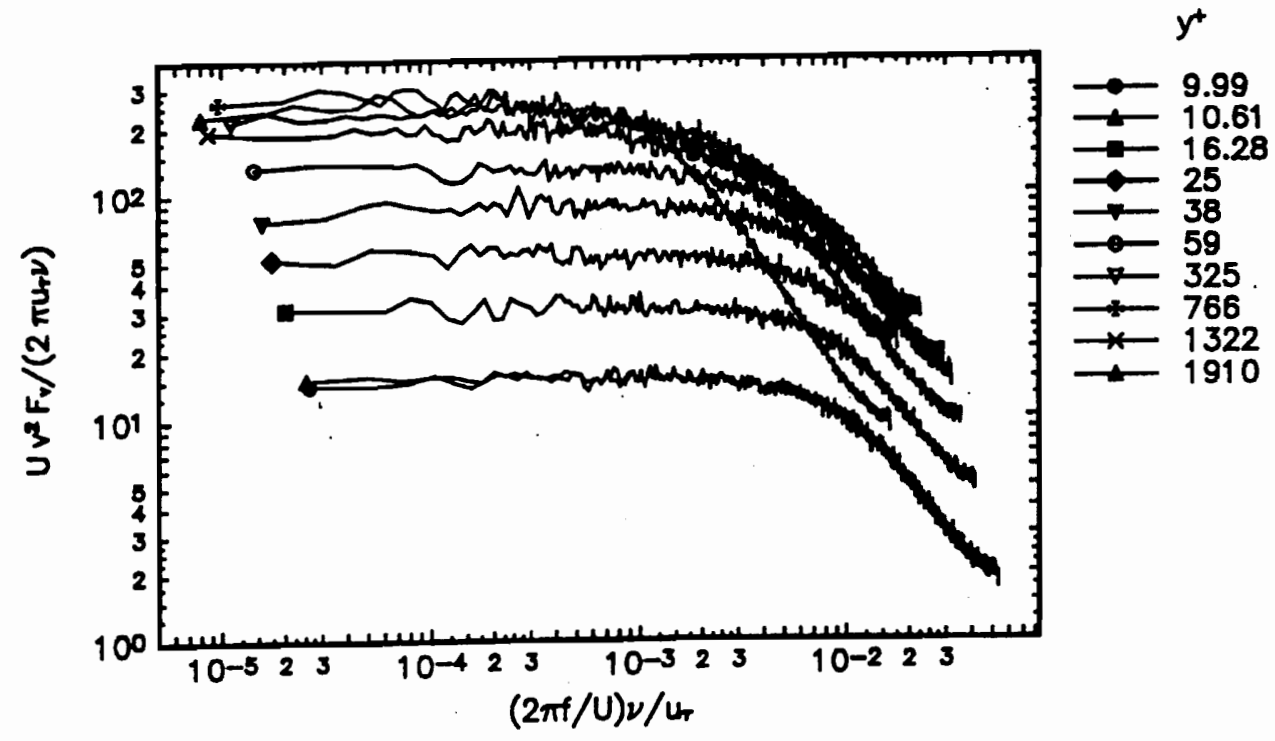

Fig. 3.61 2-D $V$ power spectra from Ölçmen (1997), non-dimensionalized using innerscaling, $\operatorname{Re}_{\theta}=5700$. 

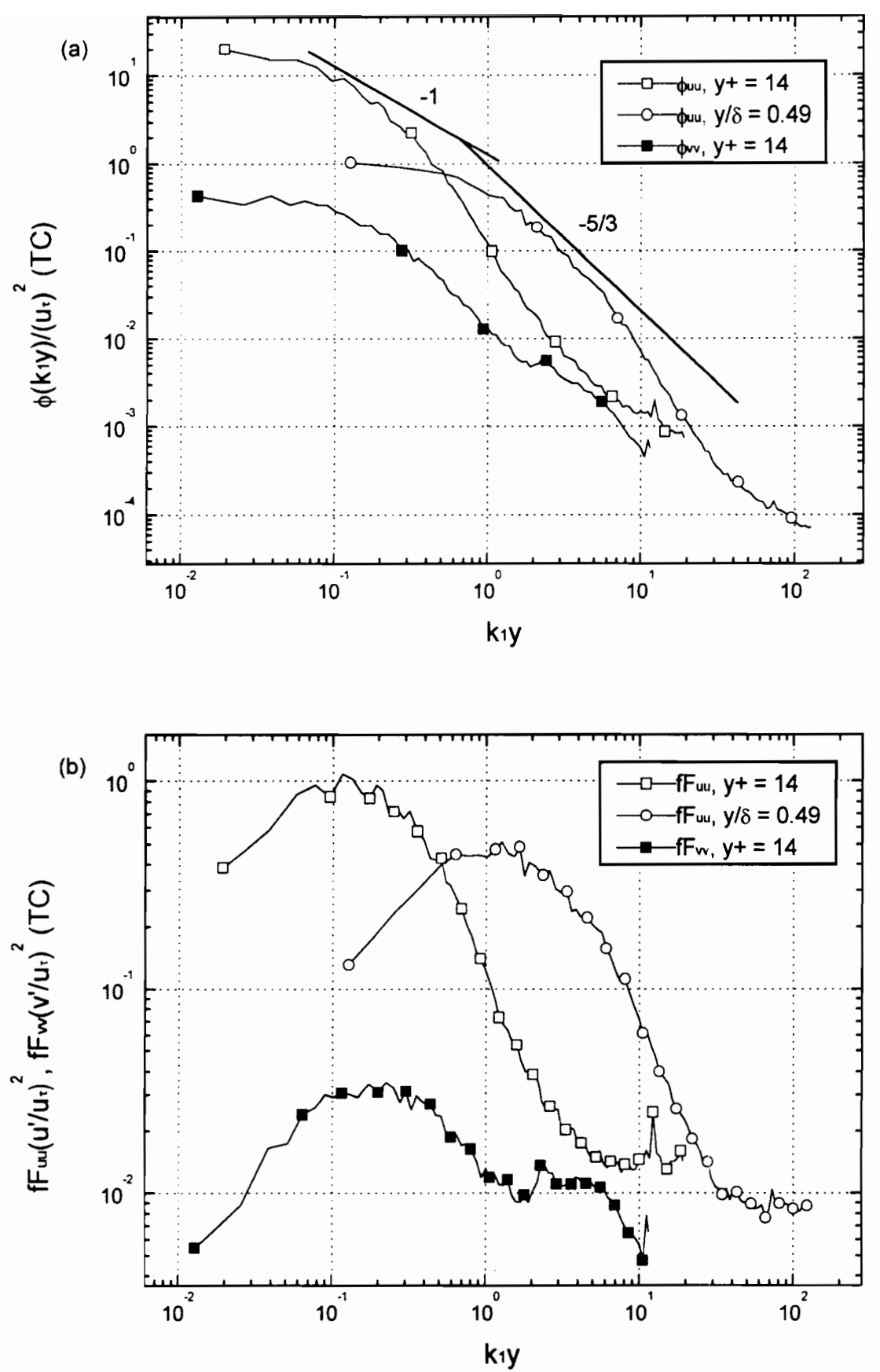

Fig. 3.62 3-D $U$ and $V$ power spectral results, $U_{r e f}=10 \mathrm{~cm} / \mathrm{s}, \operatorname{Re}_{\theta}=500$, tunnel coordinate system data. (a) Spectra non-dimensionalized using inner scaling, and (b) nondimensional first moment of spectra. Note that not all data points are marked with a symbol. 

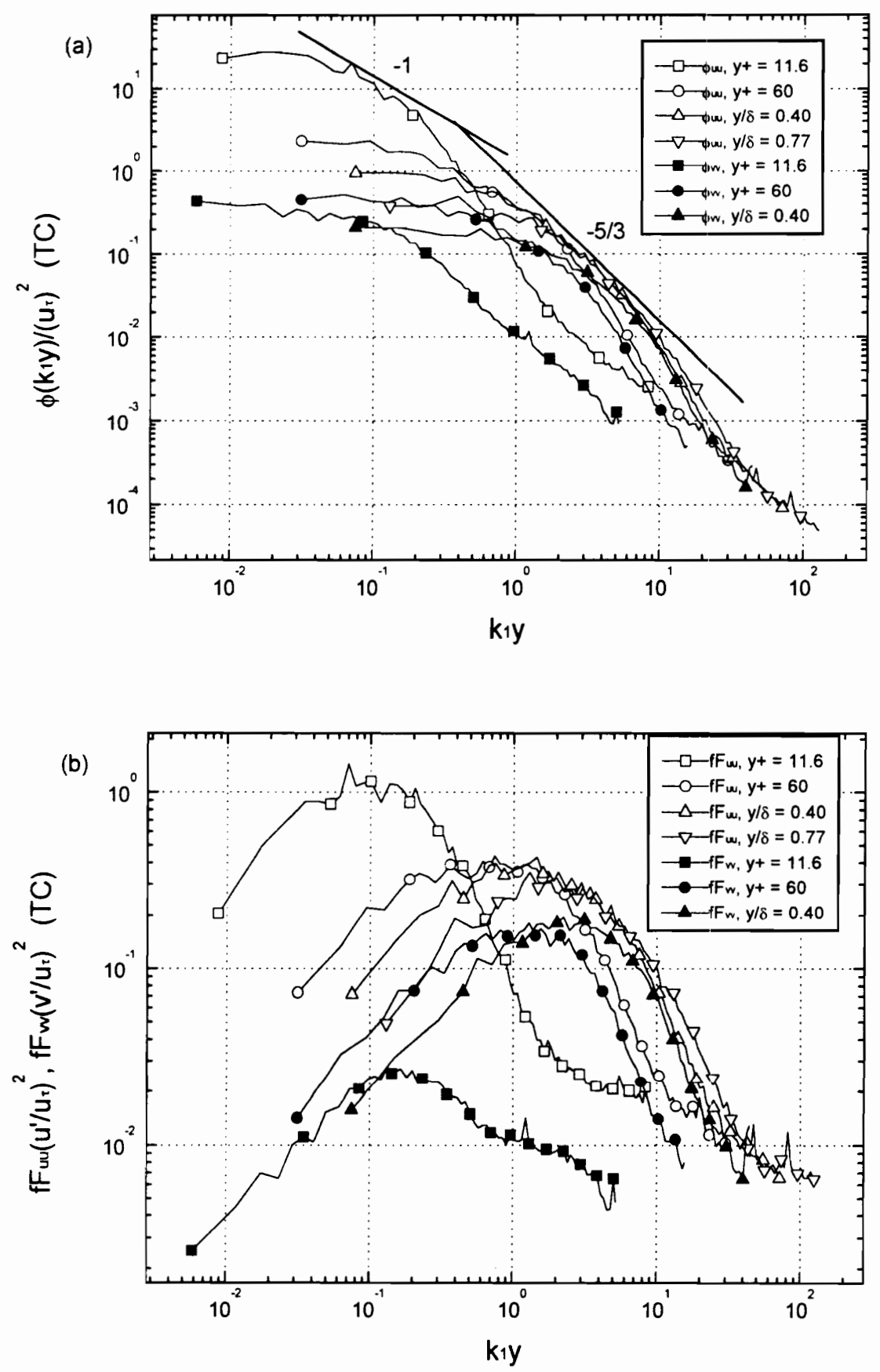

Fig. 3.63 3-D $U$ and $V$ power spectral results, $U_{r e f}=15 \mathrm{~cm} / \mathrm{s}, \operatorname{Re}_{\theta}=760$, tunnel coordinate system data. (a) Spectra non-dimensionalized using inner scaling, and (b) nondimensional first moment of spectra results. Note that not all data points are marked with a symbol. 

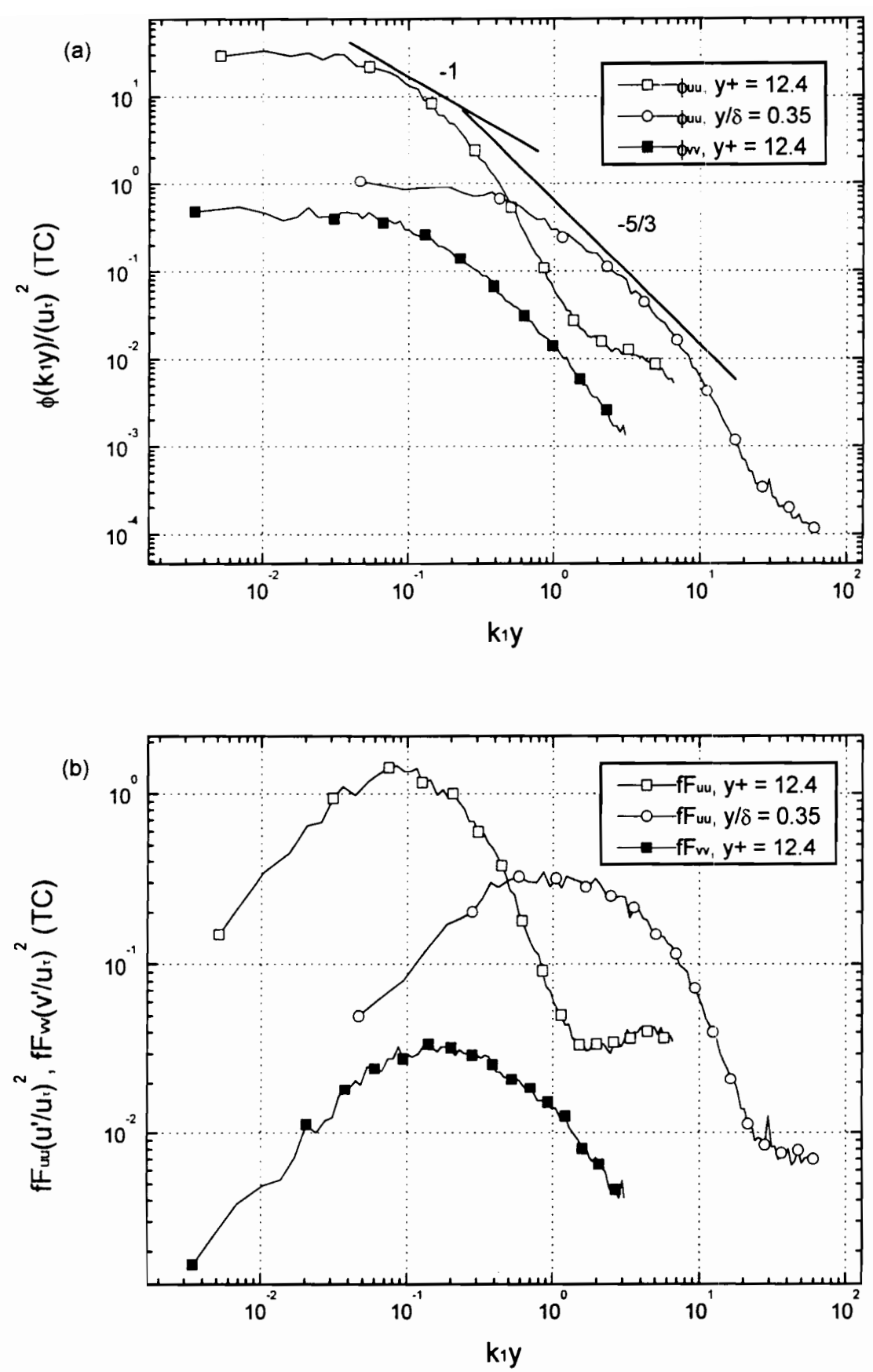

Fig. 3.64 3-D $U$ and $V$ power spectral results, $U_{r e f}=20 \mathrm{~cm} / \mathrm{s}, \operatorname{Re}_{\theta}=890$, tunnel coordinate system data. (a) Spectra non-dimensionalized using inner scaling, and (b) nondimensional first moment of spectra. Note that not all data points are marked with a symbol. 


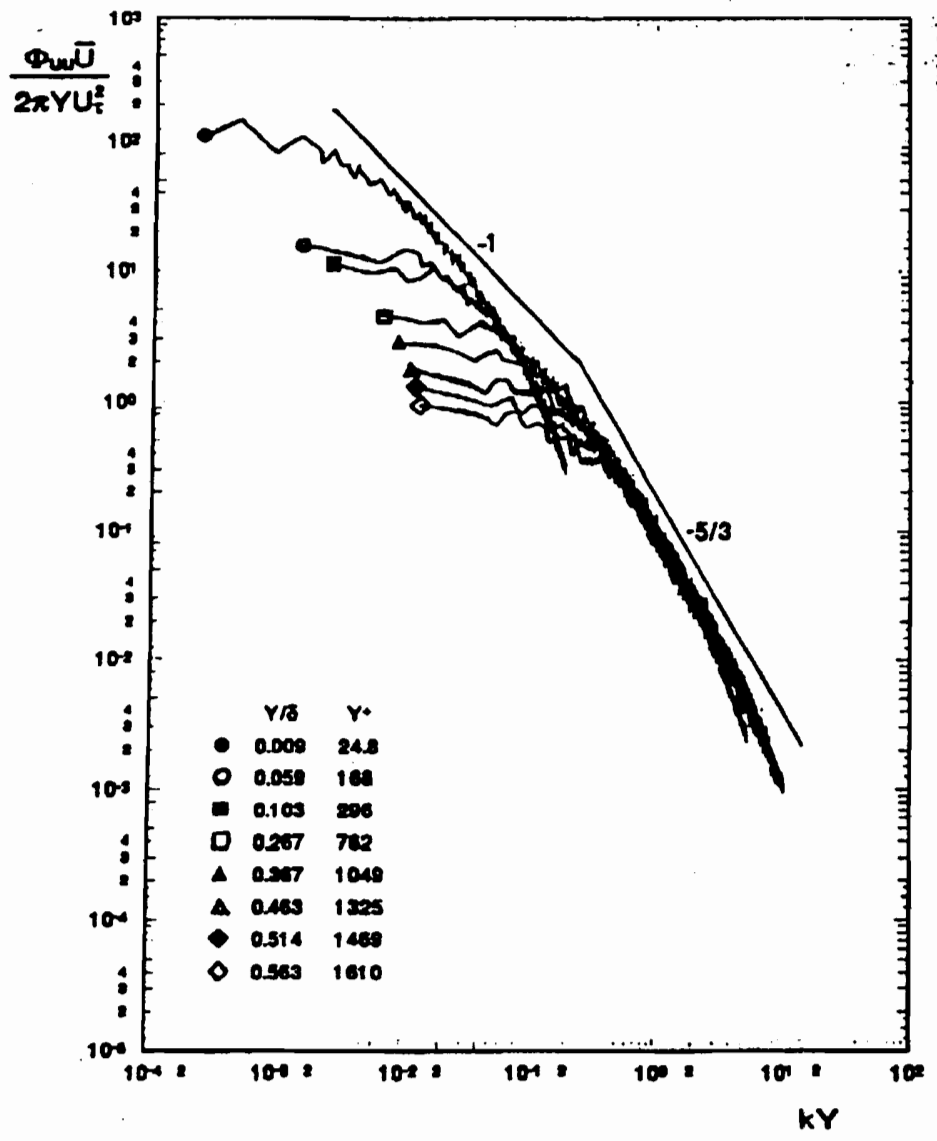

Fig. 3.65 3-D $U$ power spectra from Ha and Simpson's (1993b) hot-wire measurements. Results are presented using inner scaling. These data were obtained using the same flow geometry as the author's, and are for Ölçmen's station 5. Flow conditions are the same as Ölçmen and Simpson's (1995) test case (local $\left.\operatorname{Re}_{\theta} \approx 9500\right)$. The local freestream coordinate system was used to obtain these results. 

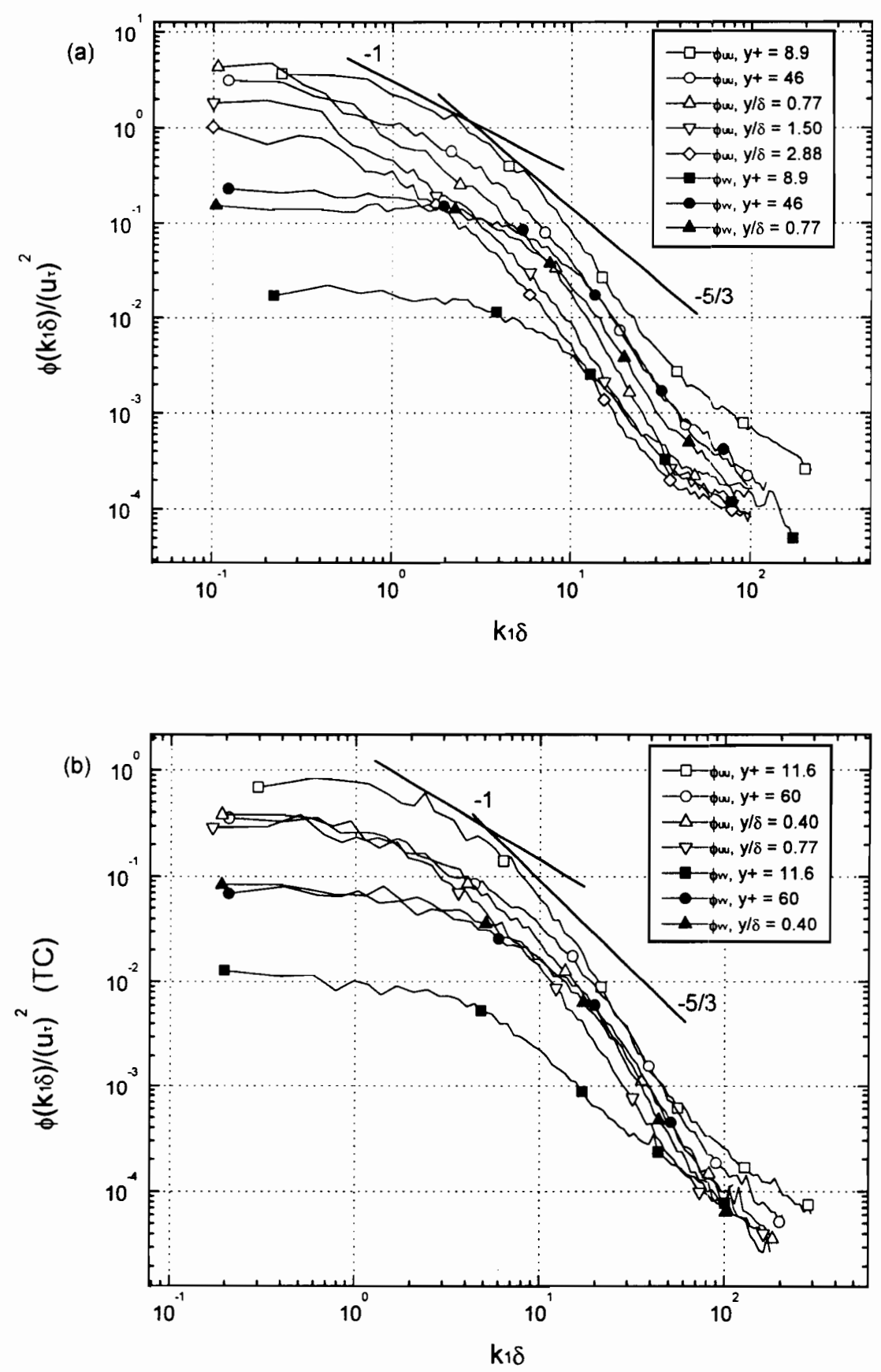

Fig. 3.66 $U$ and $V$ power spectral results, $U_{r e f}=15 \mathrm{~cm} / \mathrm{s}$, non-dimensionalized using outer scaling $(\delta)$. (a) $2-\mathrm{D}, \mathrm{Re}_{\theta}=300$, and (b) $3-\mathrm{D}, \mathrm{Re}_{\theta}=760$. 3-D data are tunnel coordinate system data. 

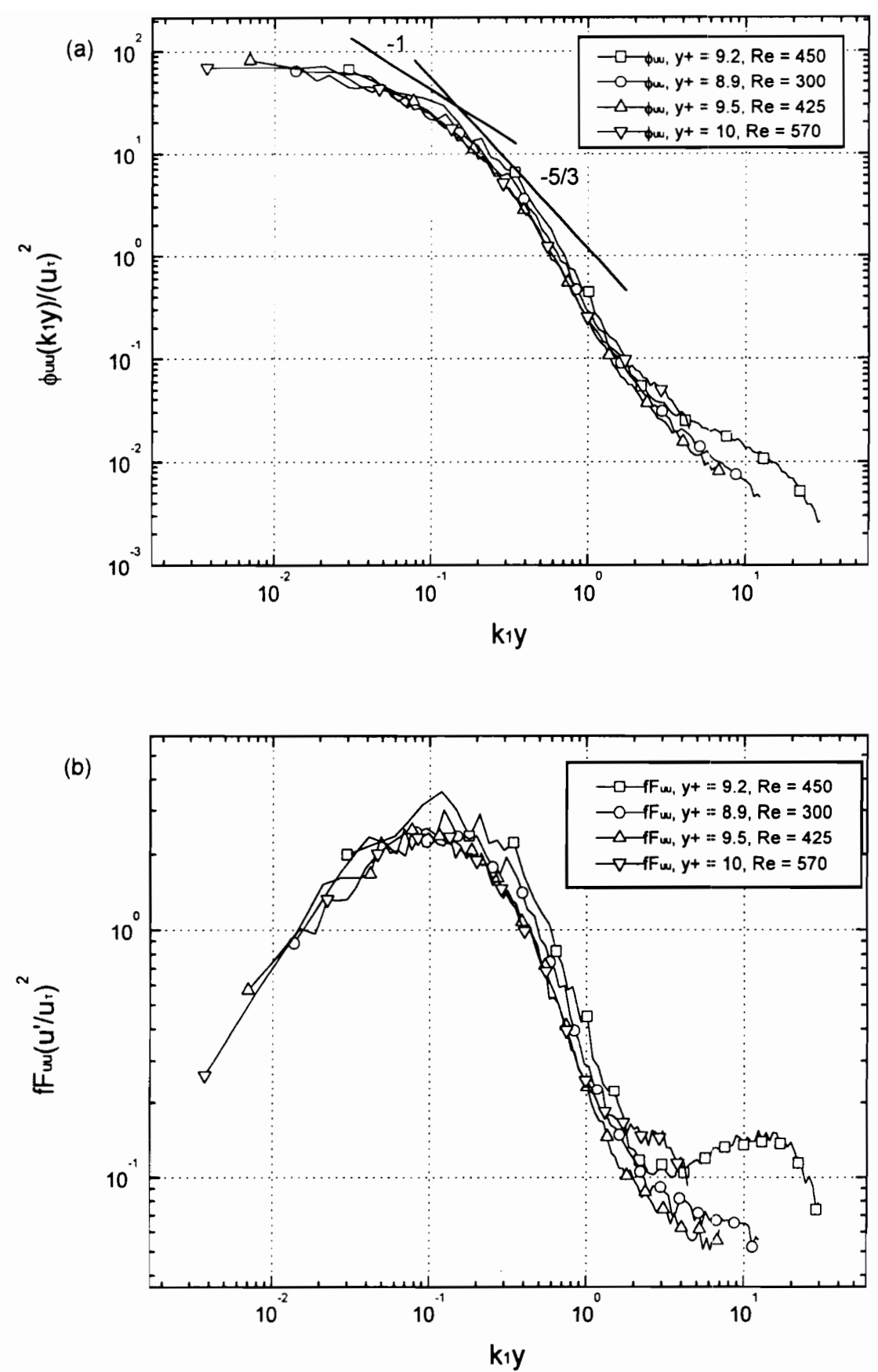

Fig. 3.67 2-D $U$ spectral results for a range of Reynolds numbers, $8.9<y^{+}<10$. (a) Spectra non-dimensionalized using inner scaling, and (b) non-dimensional first moment of spectra. 

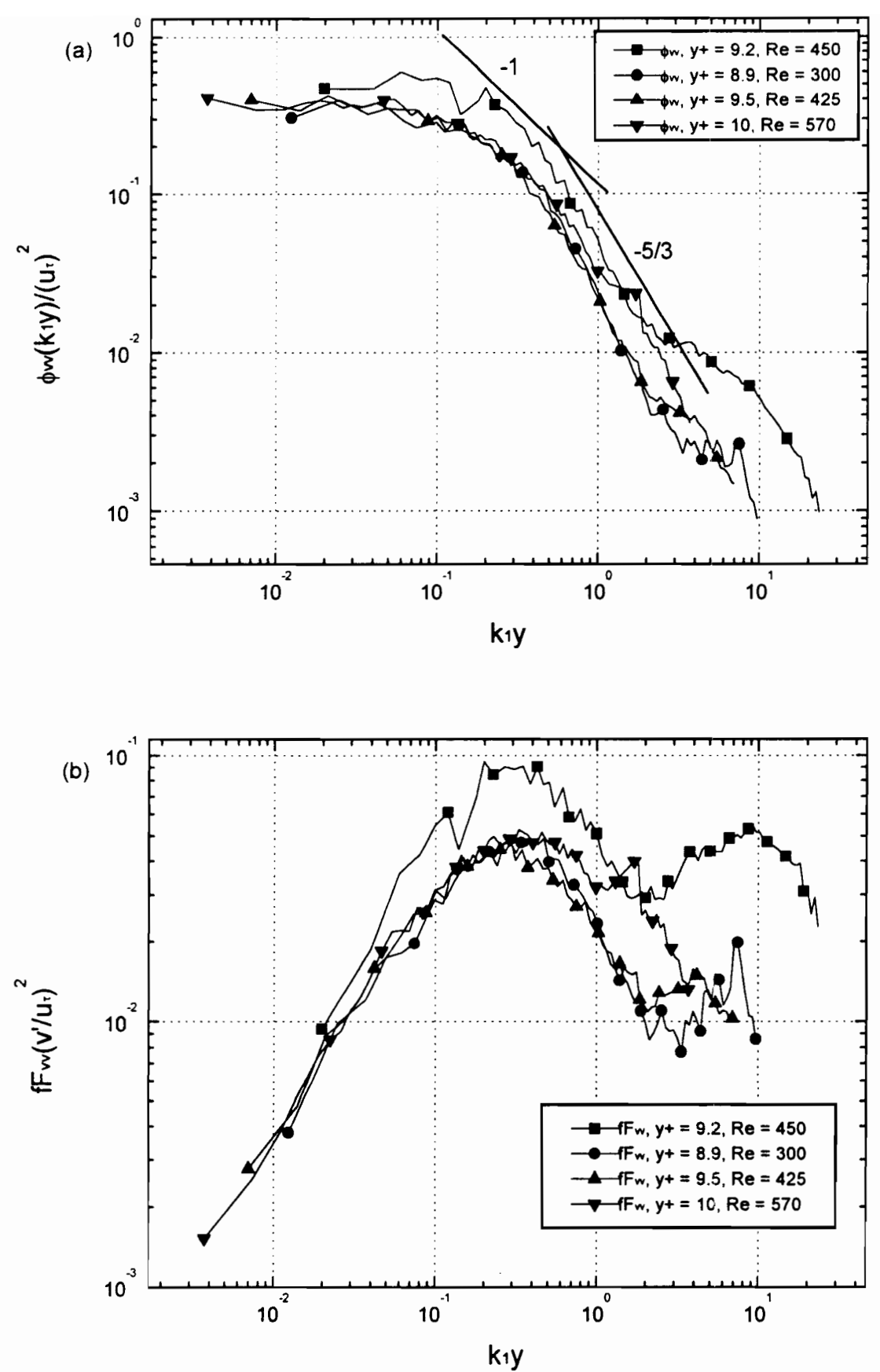

Fig. 3.68 2-D $V$ spectral results for a range of Reynolds numbers, $8.9<y^{+}<10$. (a) Spectra non-dimensionalized using inner scaling, and (b) non-dimensional first moment of spectra. 

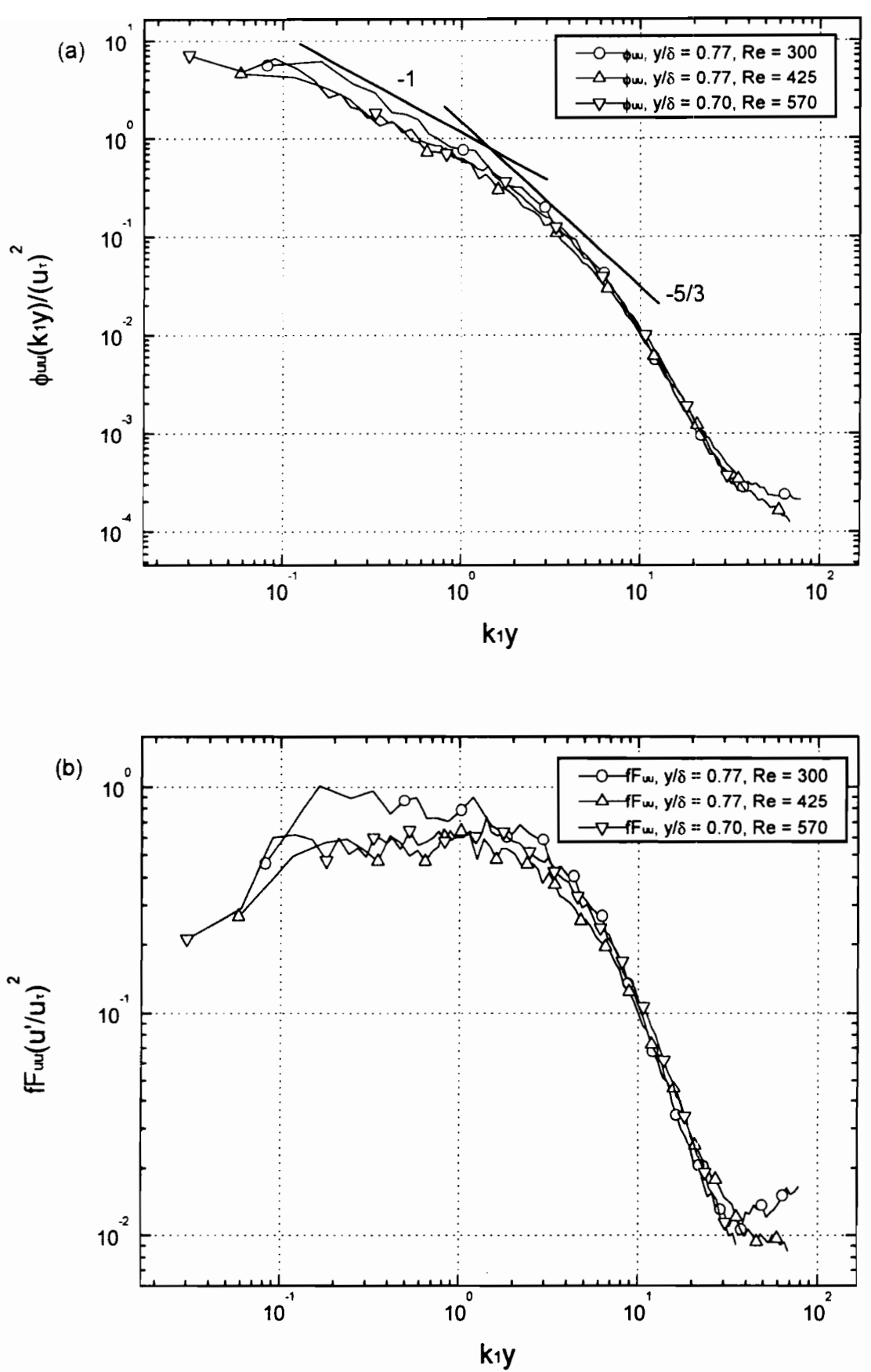

Fig. 3.69 2-D $U$ spectral results for a range of Reynolds numbers, $0.70<y / \delta<0.77$. (a) Spectra non-dimensionalized using inner scaling, and (b) non-dimensional first moment of spectra. 

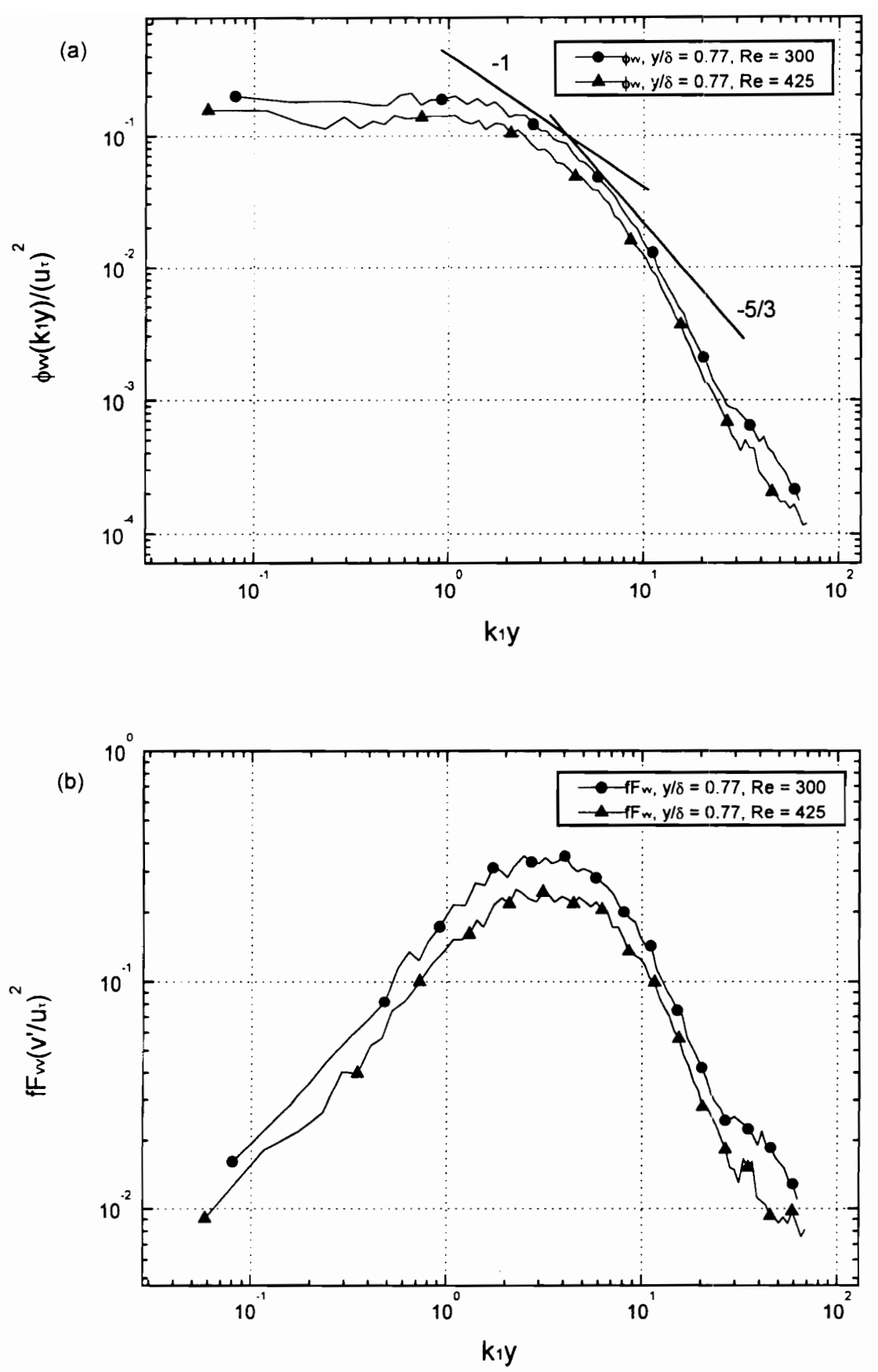

Fig. 3.70 2-D $V$ spectral results for a range of Reynolds numbers, $y / \delta=0.77$. (a) Spectra non-dimensionalized using inner scaling, and (b) non-dimensional first moment of spectra. 

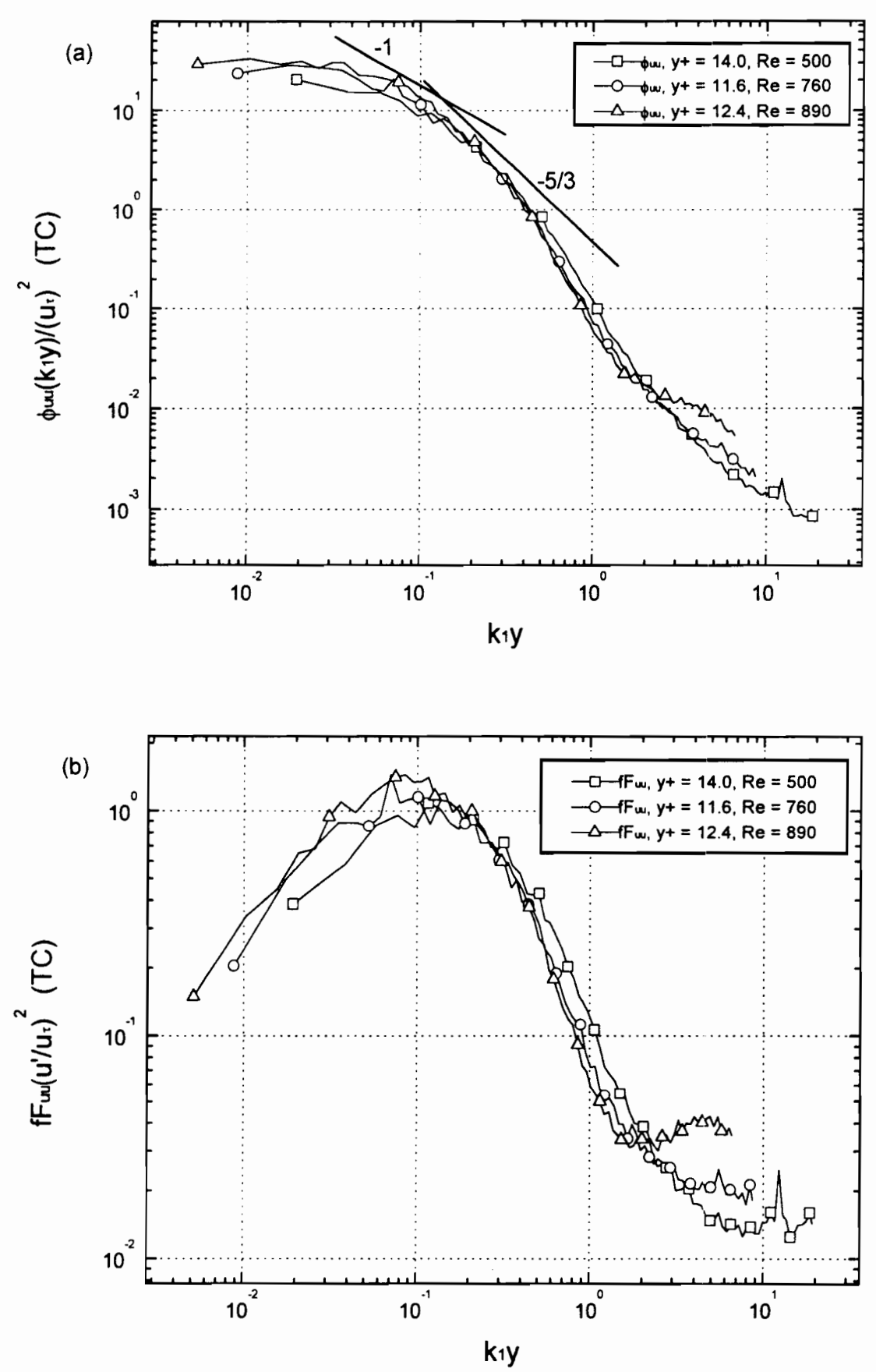

Fig. 3.71 3-D $U$ spectral results for a range of Reynolds numbers, $11.6<y^{+}<14.0$, tunnel coordinate system data. (a) Spectra non-dimensionalized using inner scaling, and (b) non-dimensional first moment of spectra. 

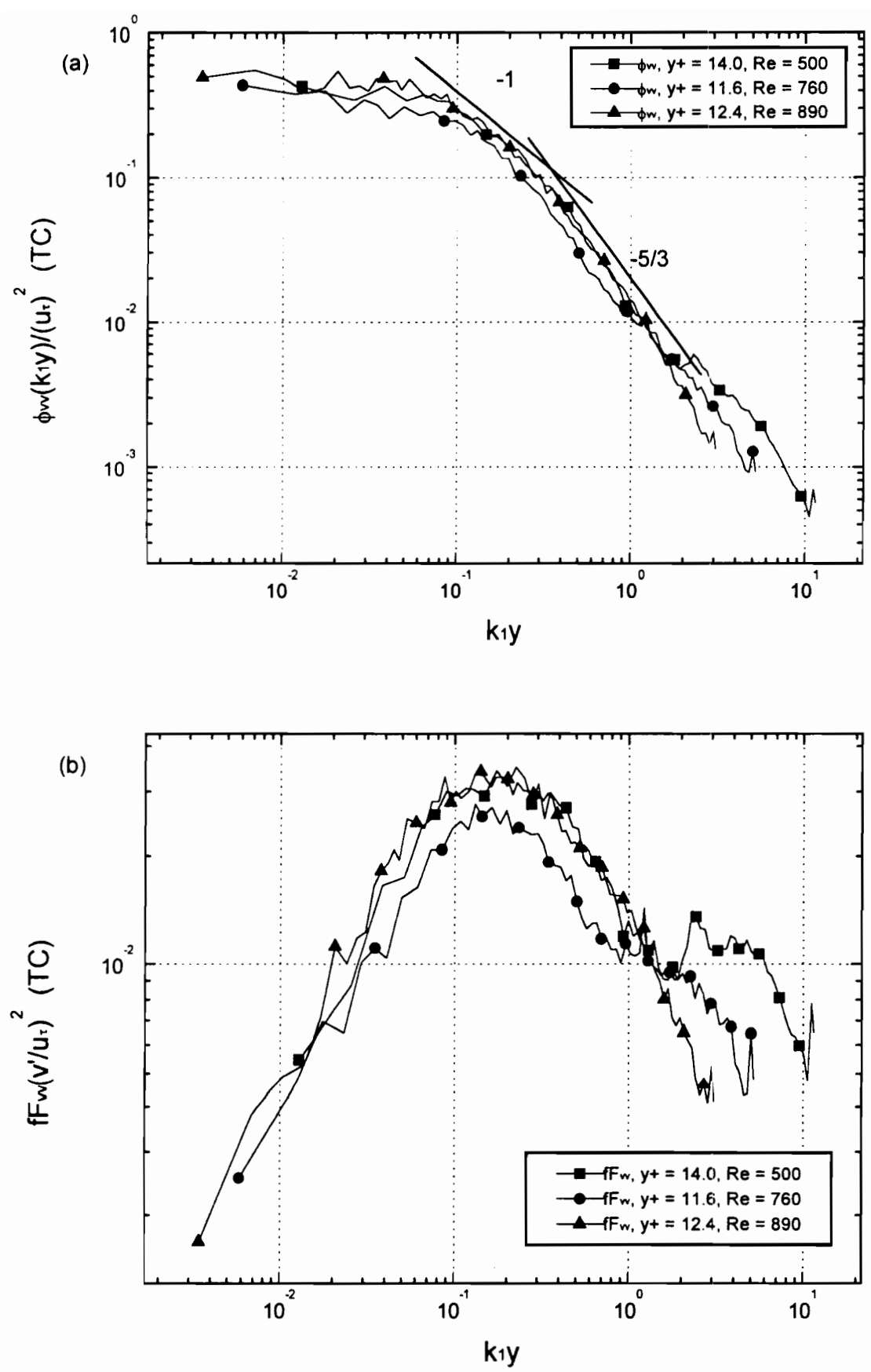

Fig. 3.72 3-D $V$ spectral results for a range of Reynolds numbers, $11.6<y^{+}<14.0$, tunnel coordinate system data. (a) Spectra non-dimensionalized using inner scaling, and (b) non-dimensional first moment of spectra. 

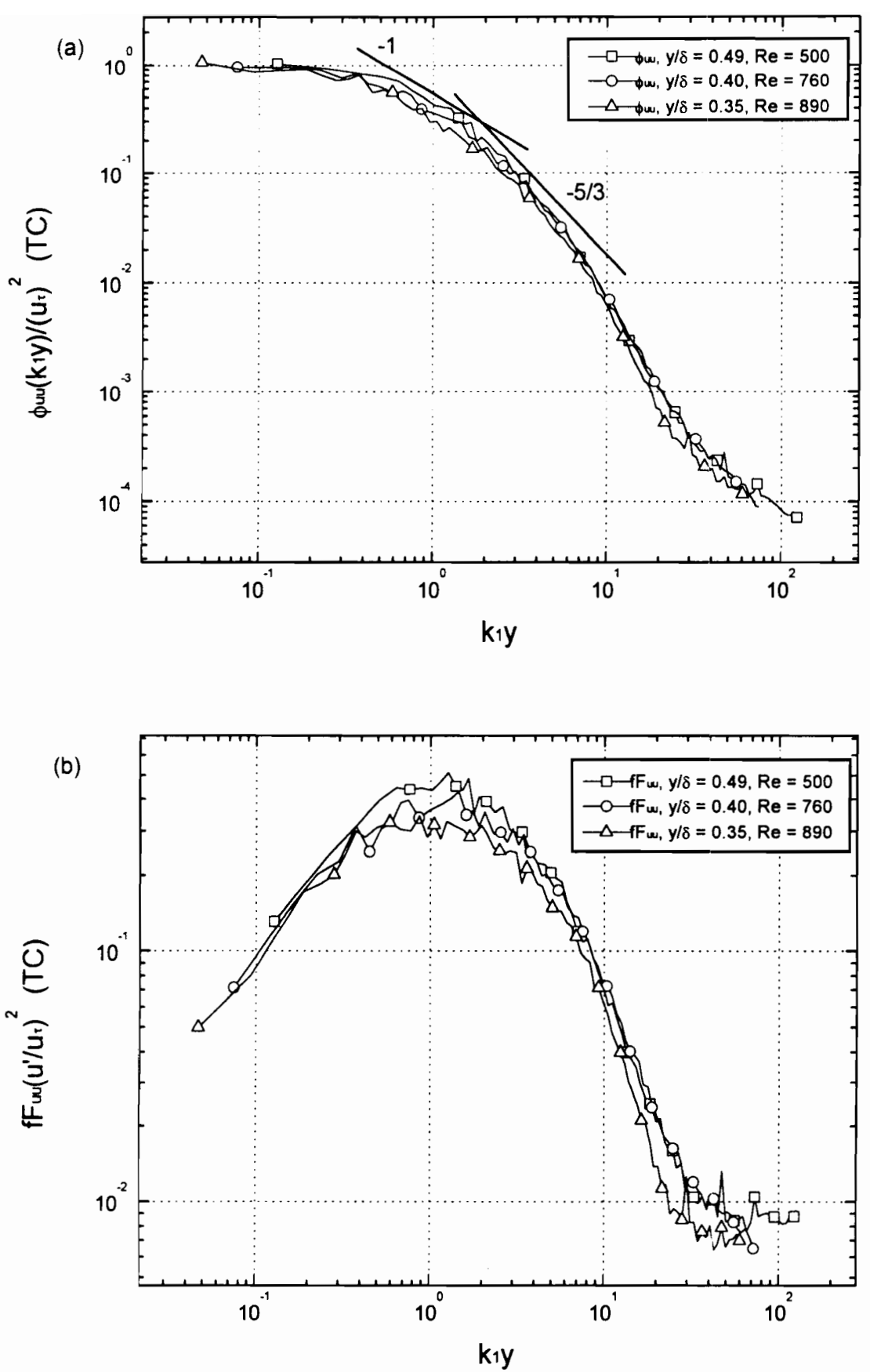

Fig. 3.73 3-D $U$ spectral results for a range of Reynolds numbers, $0.35<y / \delta<0.49$, tunnel coordinate system data. (a) Spectra non-dimensionalized using inner scaling, and (b) non-dimensional first moment of spectra. 

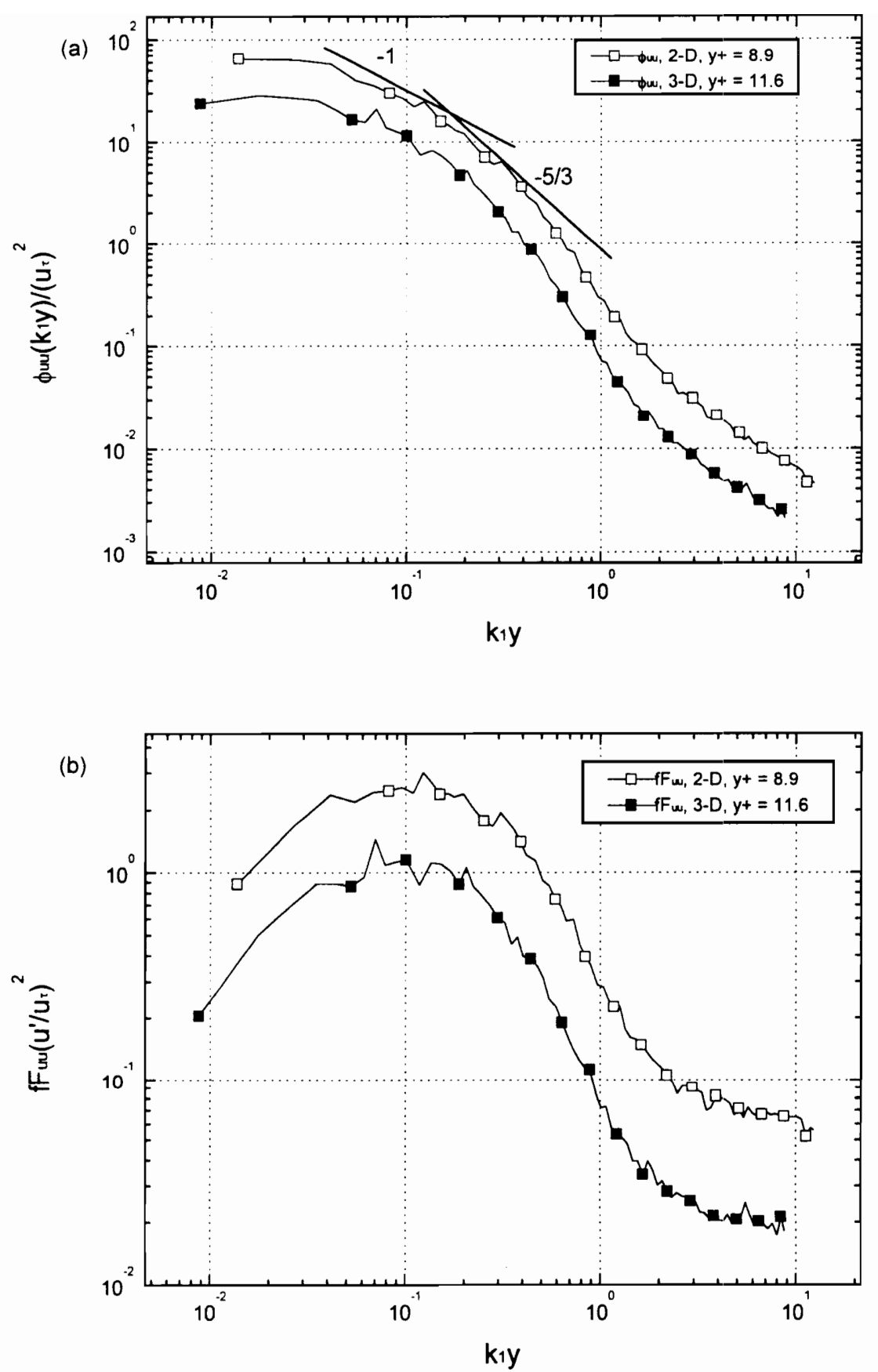

Fig. 3.74 3-D effects on the $U$ power spectral results, $y^{+} \approx 10, U_{\text {ref }}=15 \mathrm{~cm} / \mathrm{s}$. (a) Spectra non-dimensionalized using inner scaling, and (b) non-dimensional first moment of spectra. 

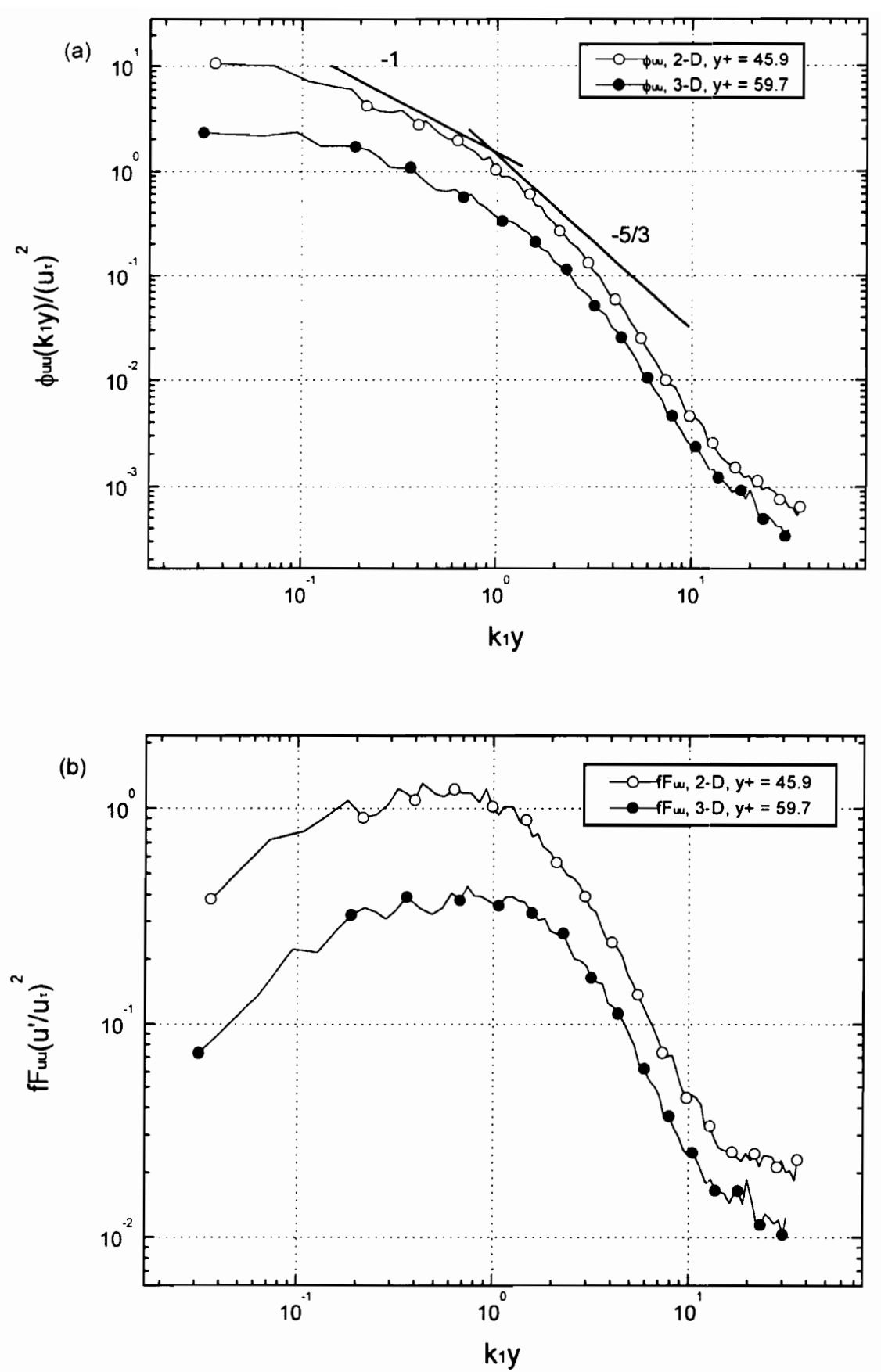

Fig. 3.75 3-D effects on the $U$ power spectral results, $y^{+} \approx 50, U_{\text {ref }}=15 \mathrm{~cm} / \mathrm{s}$. (a) Spectra non-dimensionalized using inner scaling, and (b) non-dimensional first moment of spectra. 

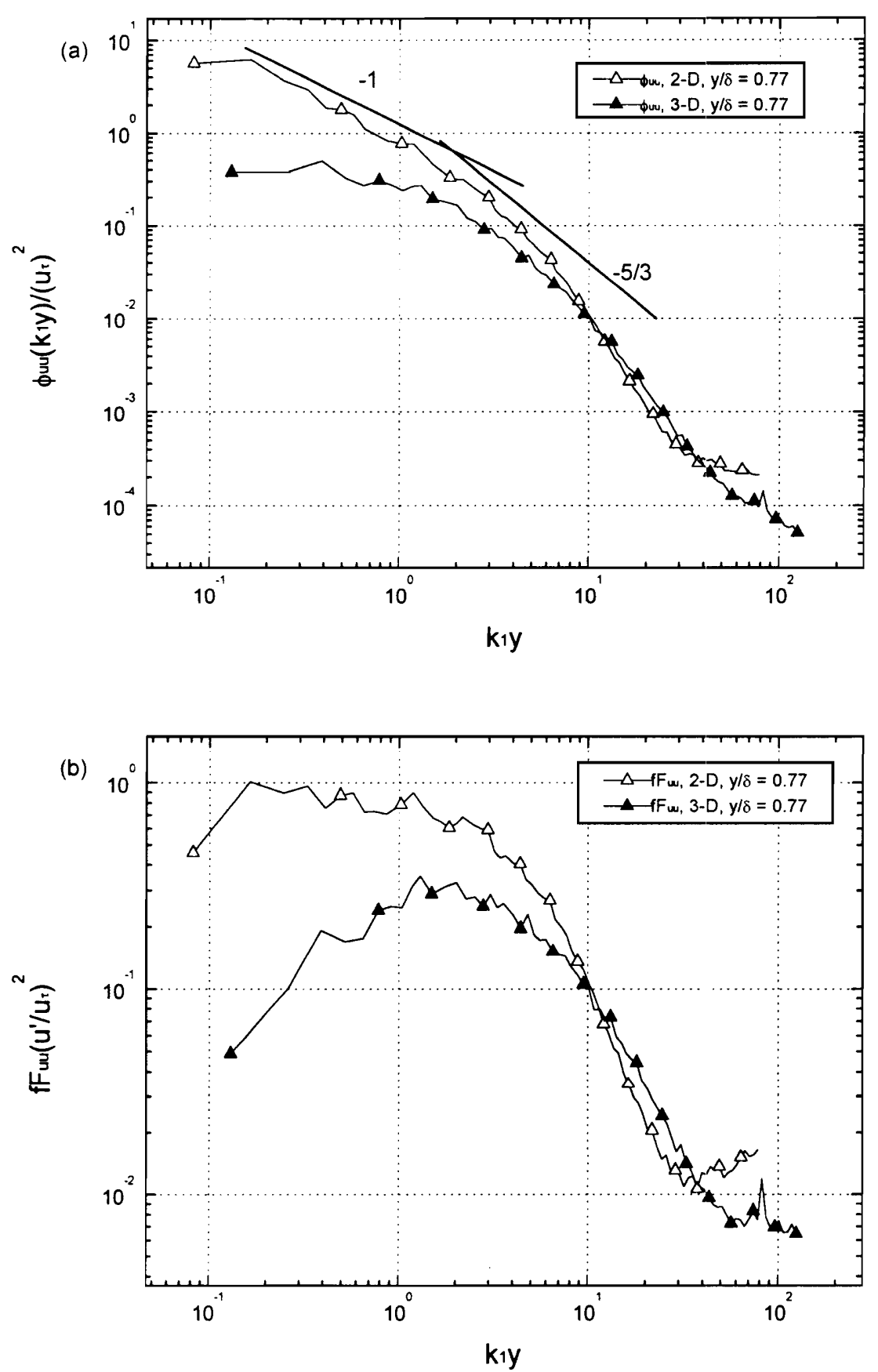

Fig. 3.76 $3-\mathrm{D}$ effects on the $U$ power spectral results, $y^{/} \delta=0.77, U_{\text {ref }}=15 \mathrm{~cm} / \mathrm{s}$. (a) Spectra non-dimensionalized using inner scaling, and (b) non-dimensional first moment of spectra. 

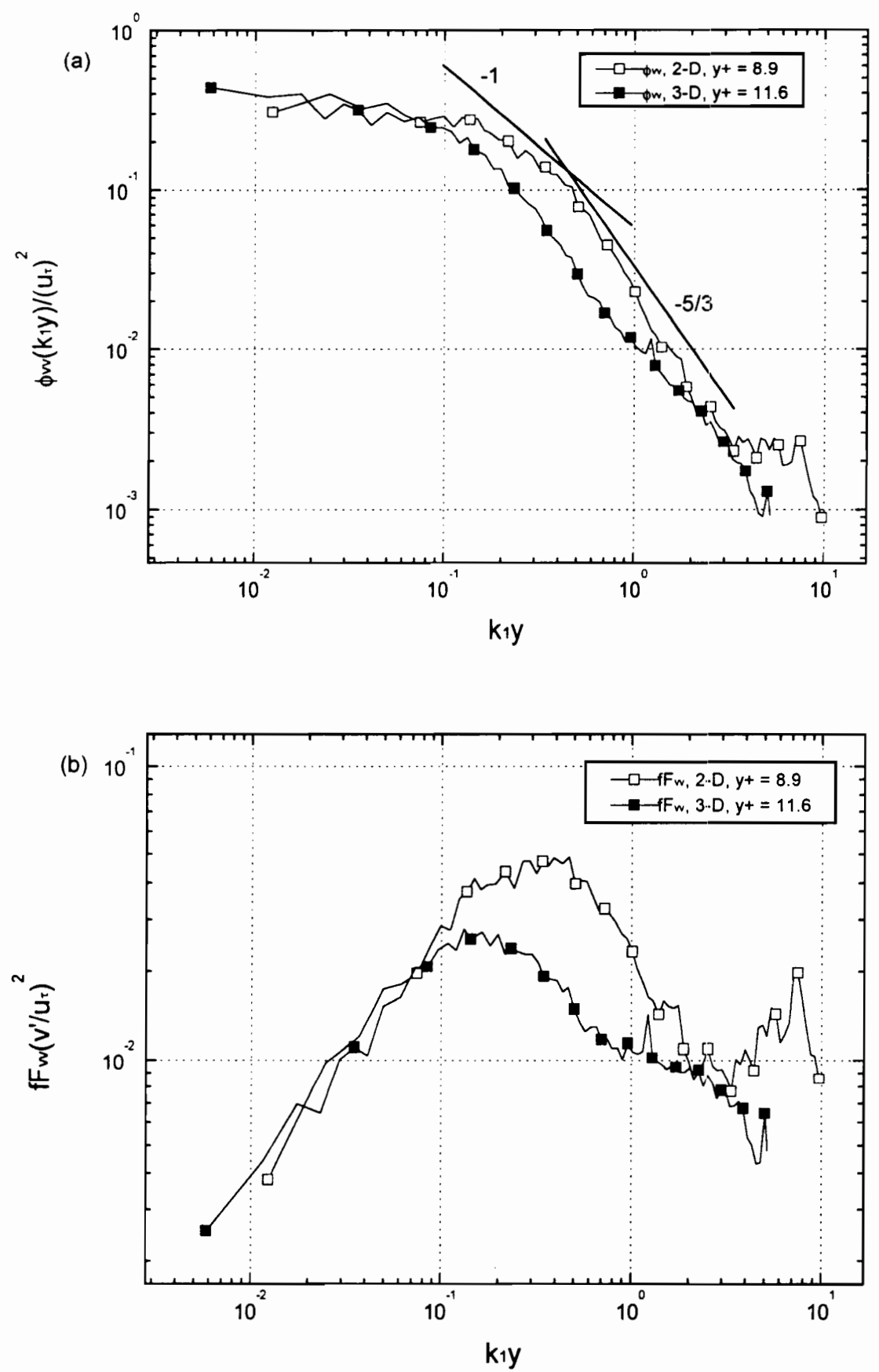

Fig. 3.77 3-D effects on the $V$ power spectral results, $y^{+} \approx 10, U_{r e f}=15 \mathrm{~cm} / \mathrm{s}$. (a) Spectra non-dimensionalized using inner scaling, and (b) non-dimensional first moment of spectra. 

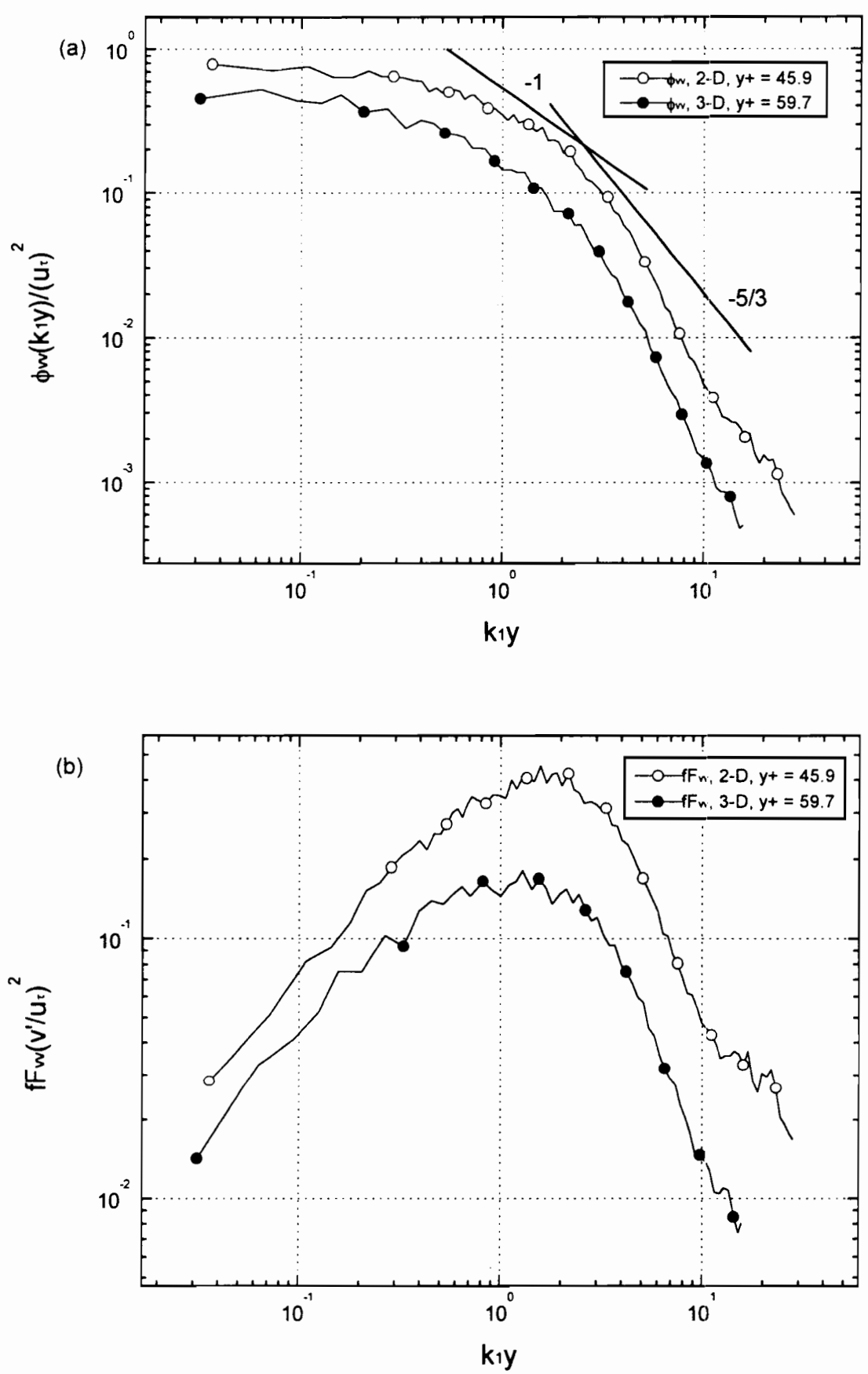

Fig. $3.783-\mathrm{D}$ effects on the $V$ power spectral results, $y^{+} \approx 50, U_{r e f}=15 \mathrm{~cm} / \mathrm{s}$. (a) Spectra non-dimensionalized using inner scaling, and (b) non-dimensional first moment of spectra. 

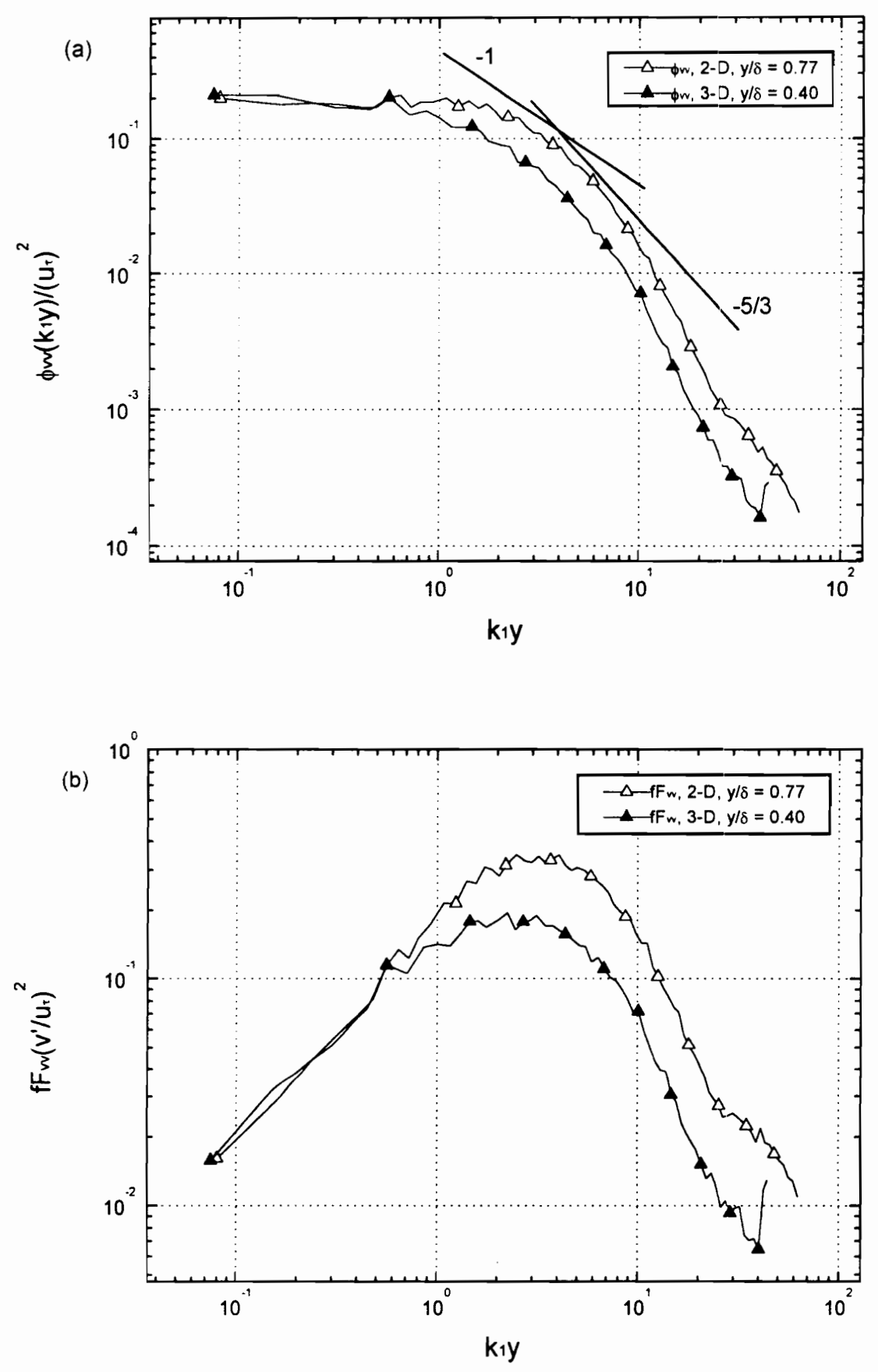

Fig. 3.79 3-D effects on the $V$ power spectral results, $y / \delta=0.77$ and $0.40, U_{\text {ref }}=15 \mathrm{~cm} / \mathrm{s}$. (a) Spectra non-dimensionalized using inner scaling, and (b) non-dimensional first moment of spectra. 


\section{Flow Direction}
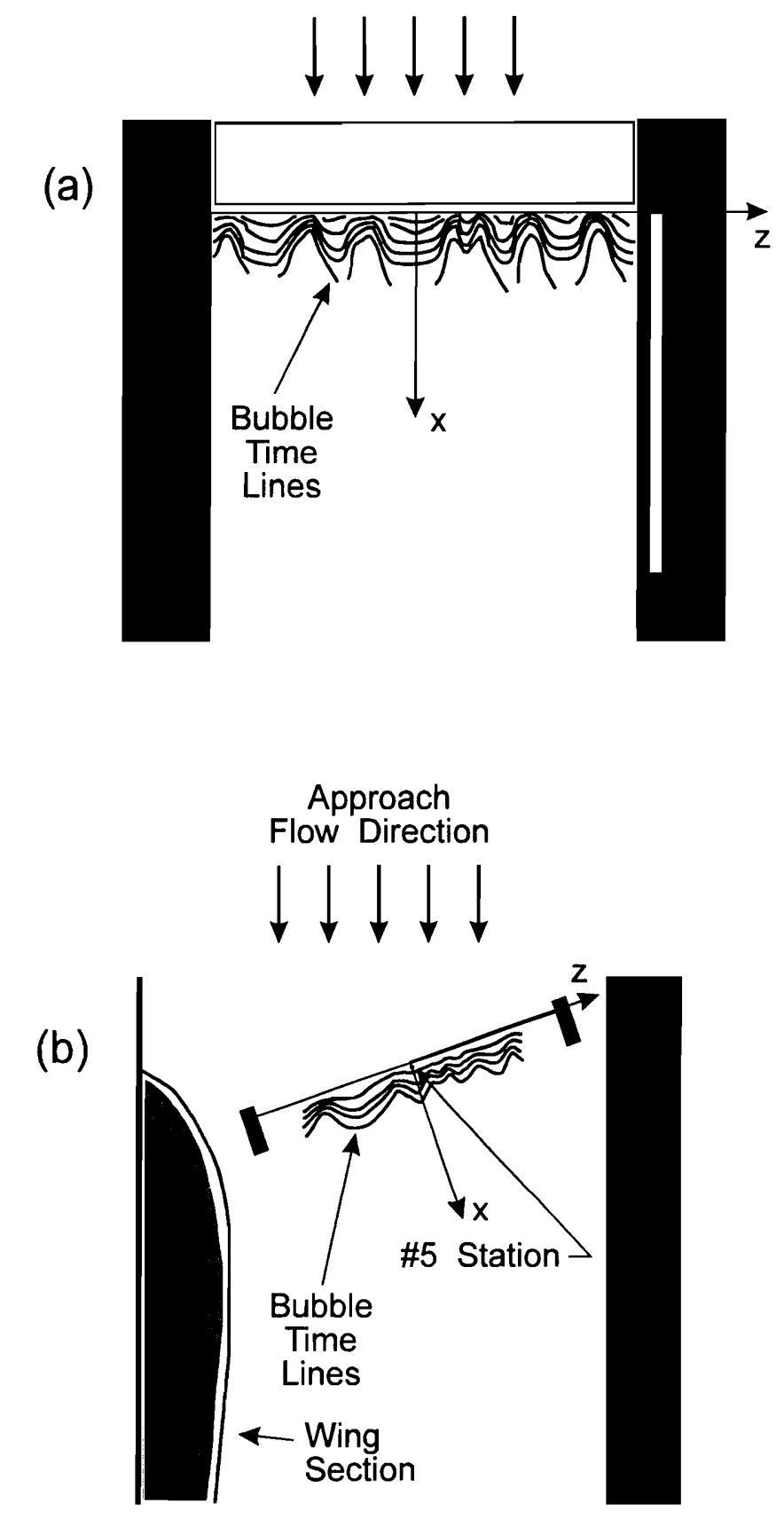

Fig. 4.1 Fig. 2.31 with wire coordinate systems drawn. The point of reference is from underneath test section floor, with the positive $y$ axis is going into the page. The 3 - $\mathrm{D}$ bubble wire is rotated $20^{\circ}$ in sketch (b). 


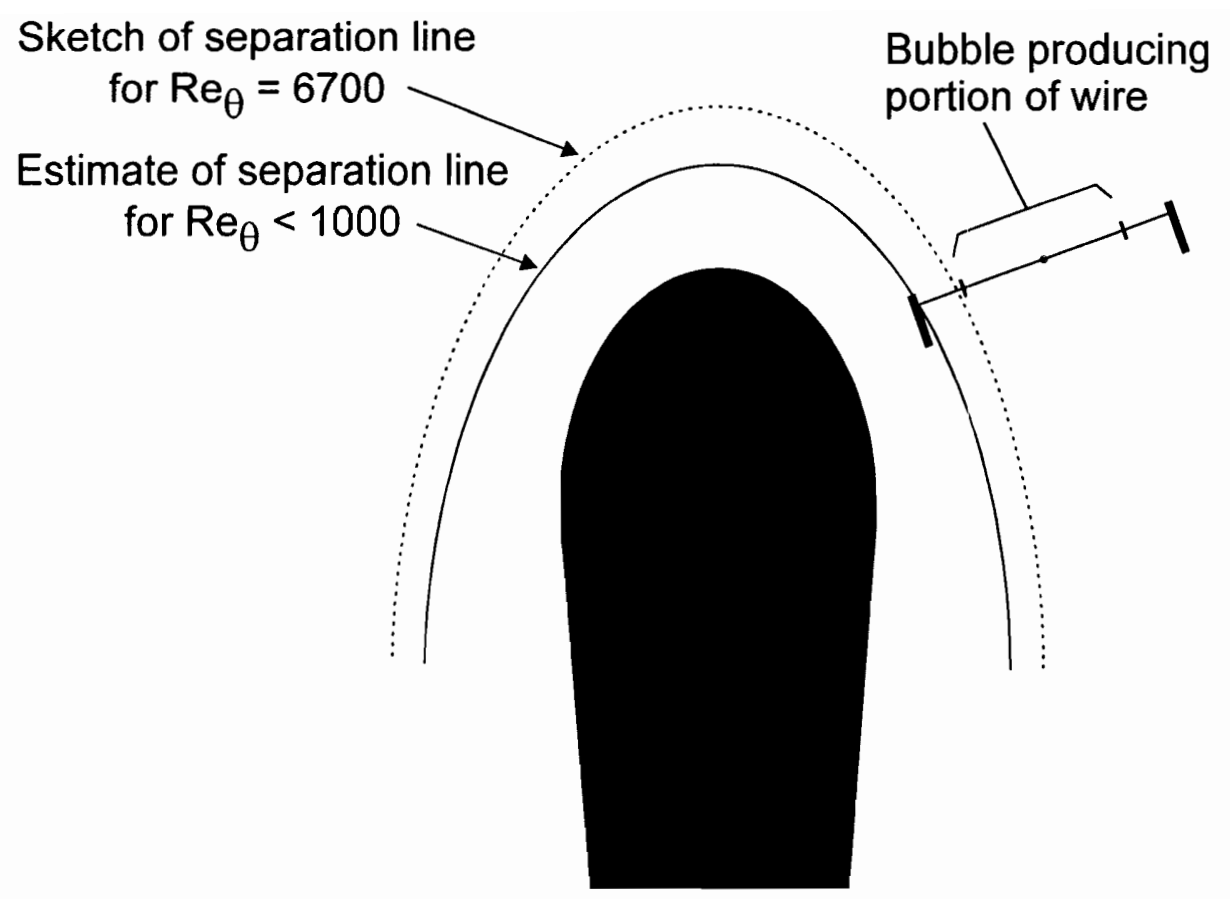

Fig. 4.2 Sketch of the estimated separation line location relative to the bubble-producing portion of the hydrogen-bubble wire for the author's low Reynolds number 3-D flow cases. High Reynolds number data are from Ölçmen and Simpson (1995). 


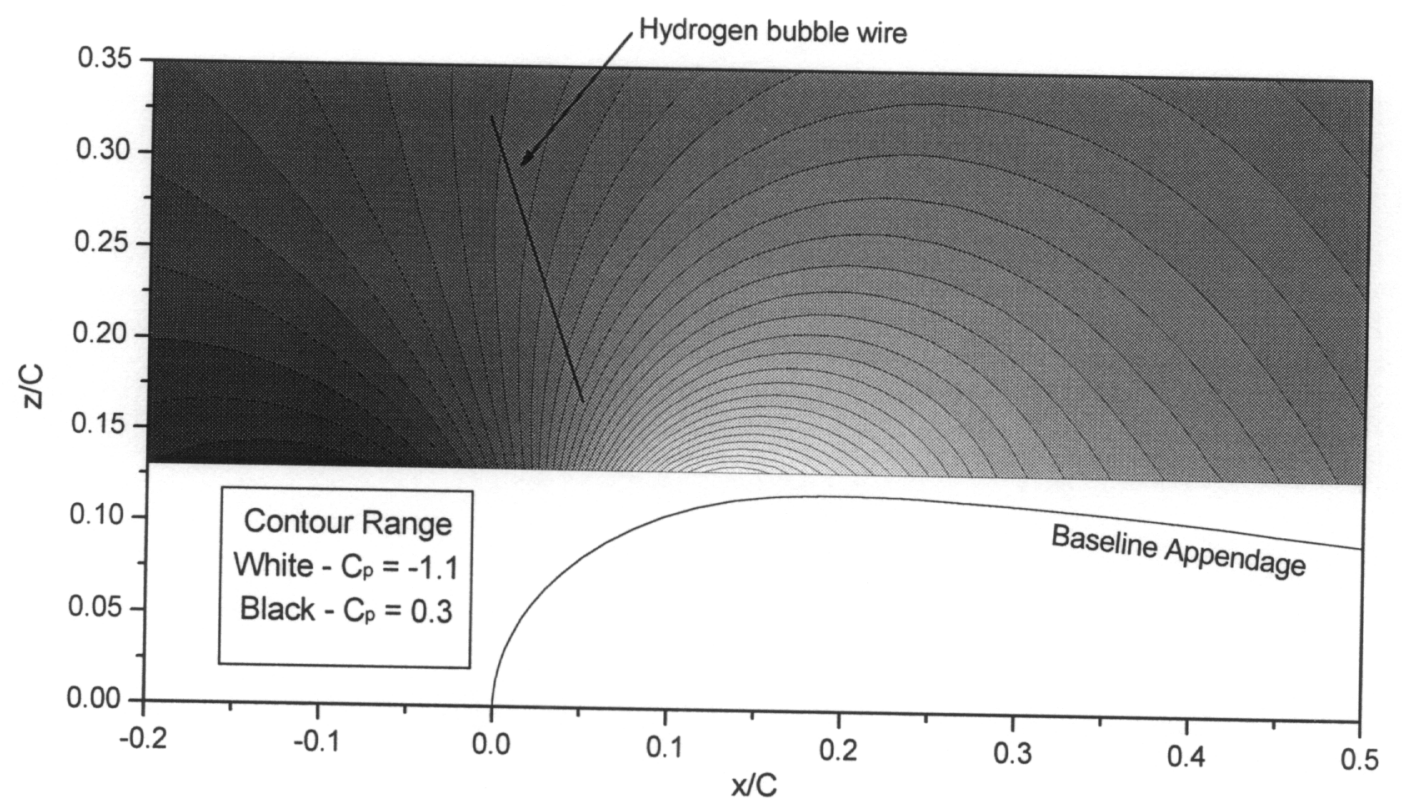

Fig. 4.3 Computed freestream pressure contours for the baseline appendage geometry. $\Delta C_{p} \approx 0.04$ between adjacent contours levels. Only the bubble-producing portion of the 3-D bubble wire is indicated. 


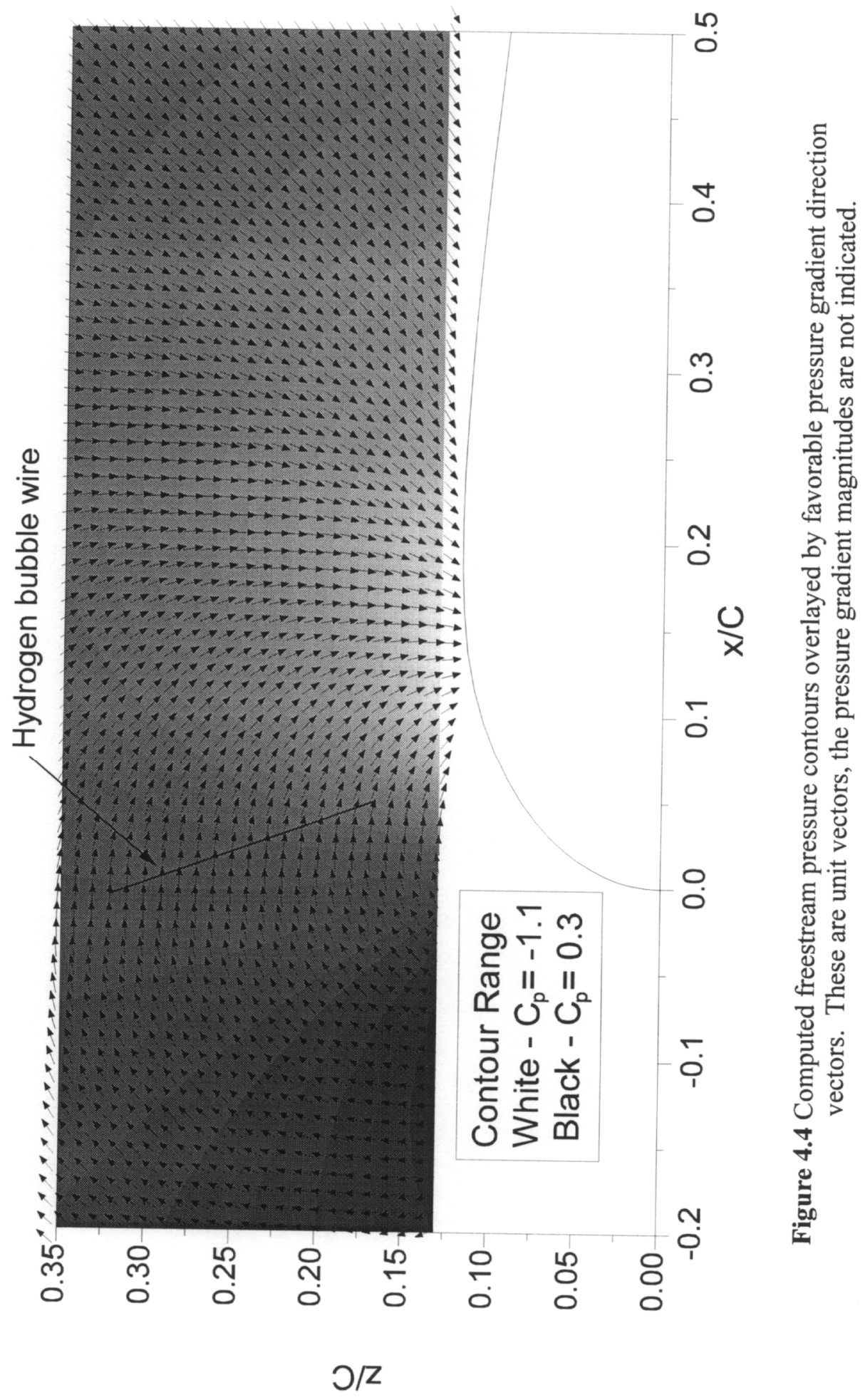




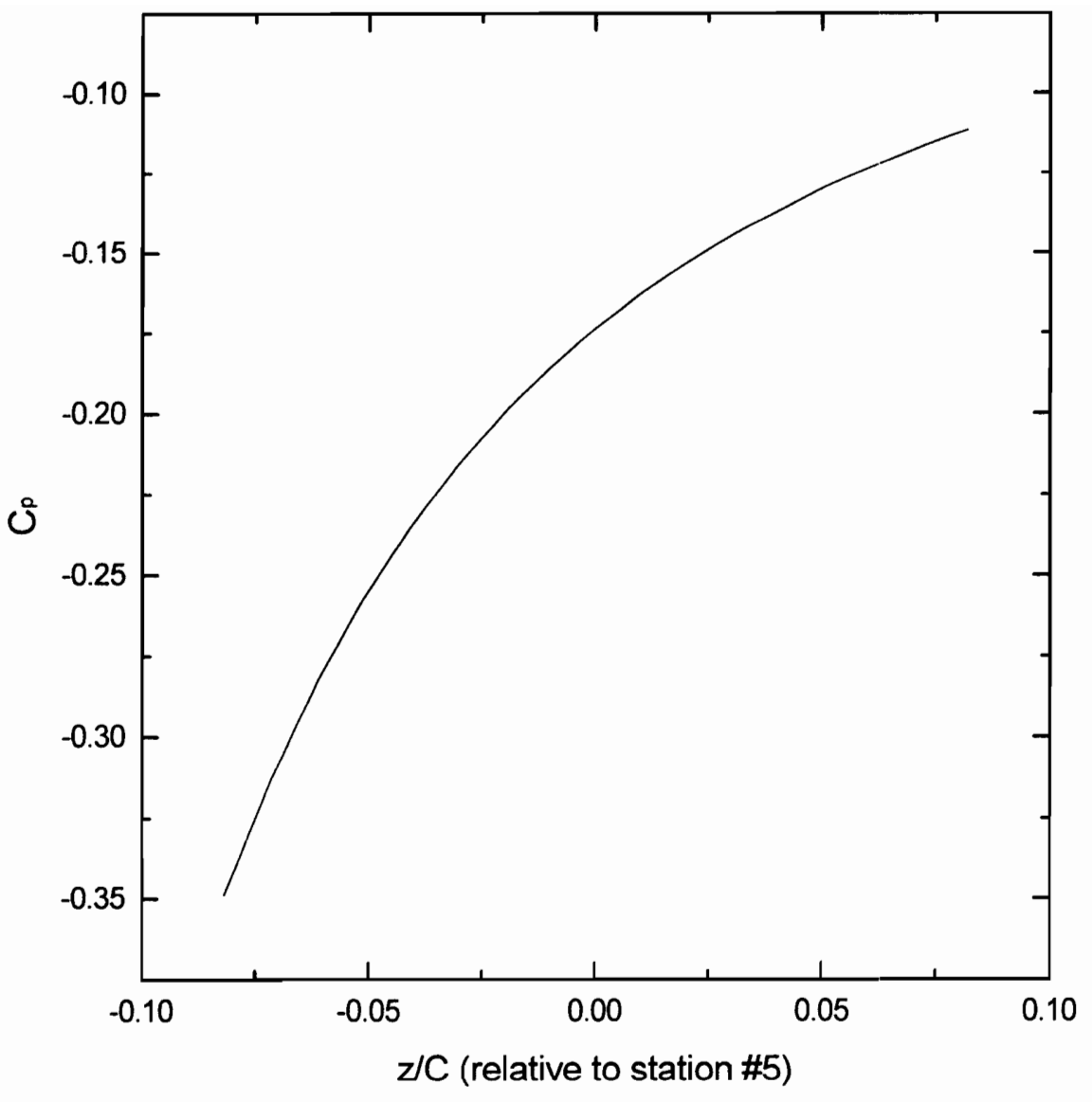

Fig. 4.5 Detail of the computed freestream pressure coefficient along the 3-D bubble wire. Station \#5 is located at $z / C=0.0$. 


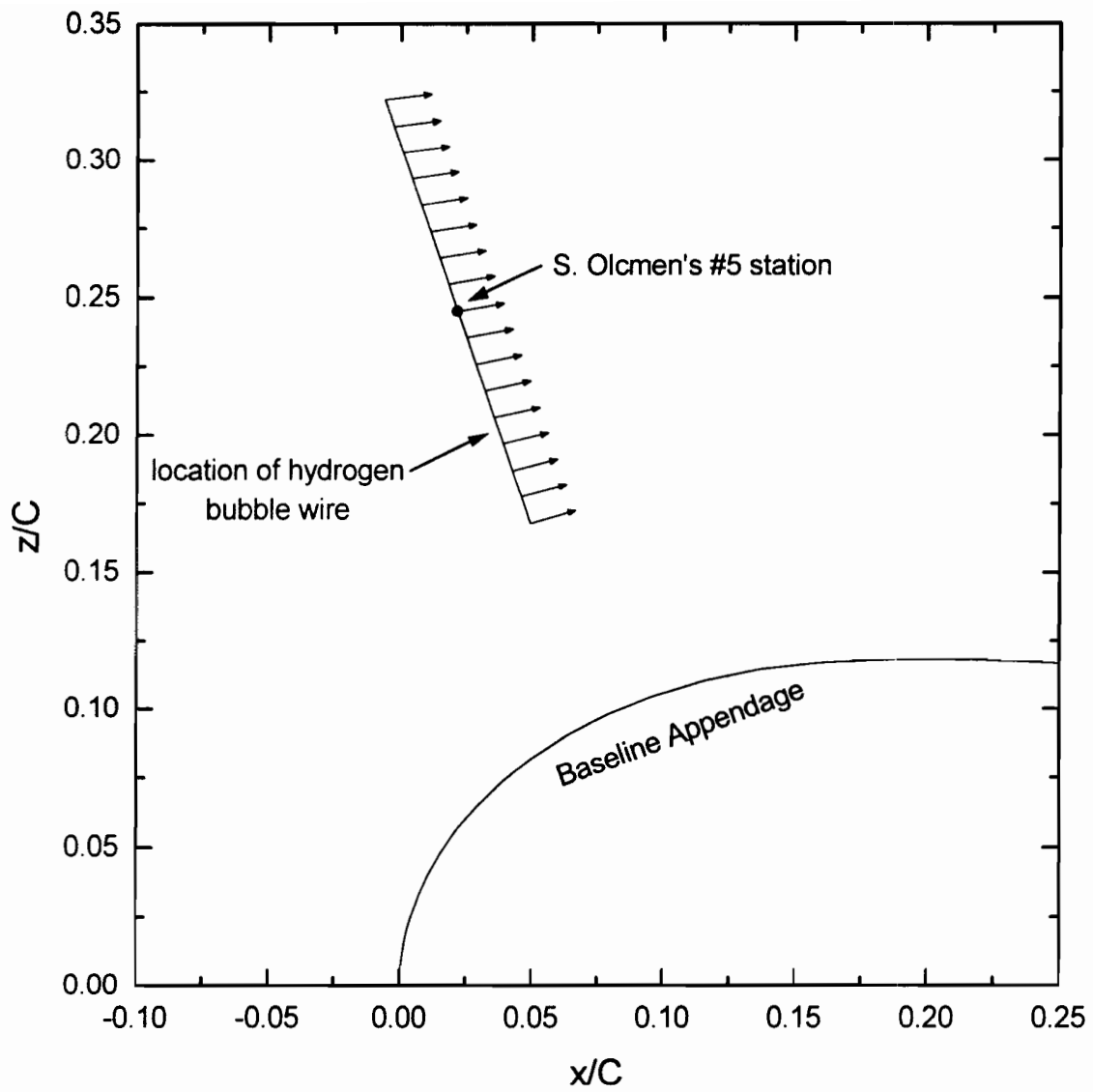

Fig. 4.6 Computed freestream flow direction along 3-D bubble wire. $x / C$ and $z / C$ are with respect to the tunnel coordinate system is shown. 


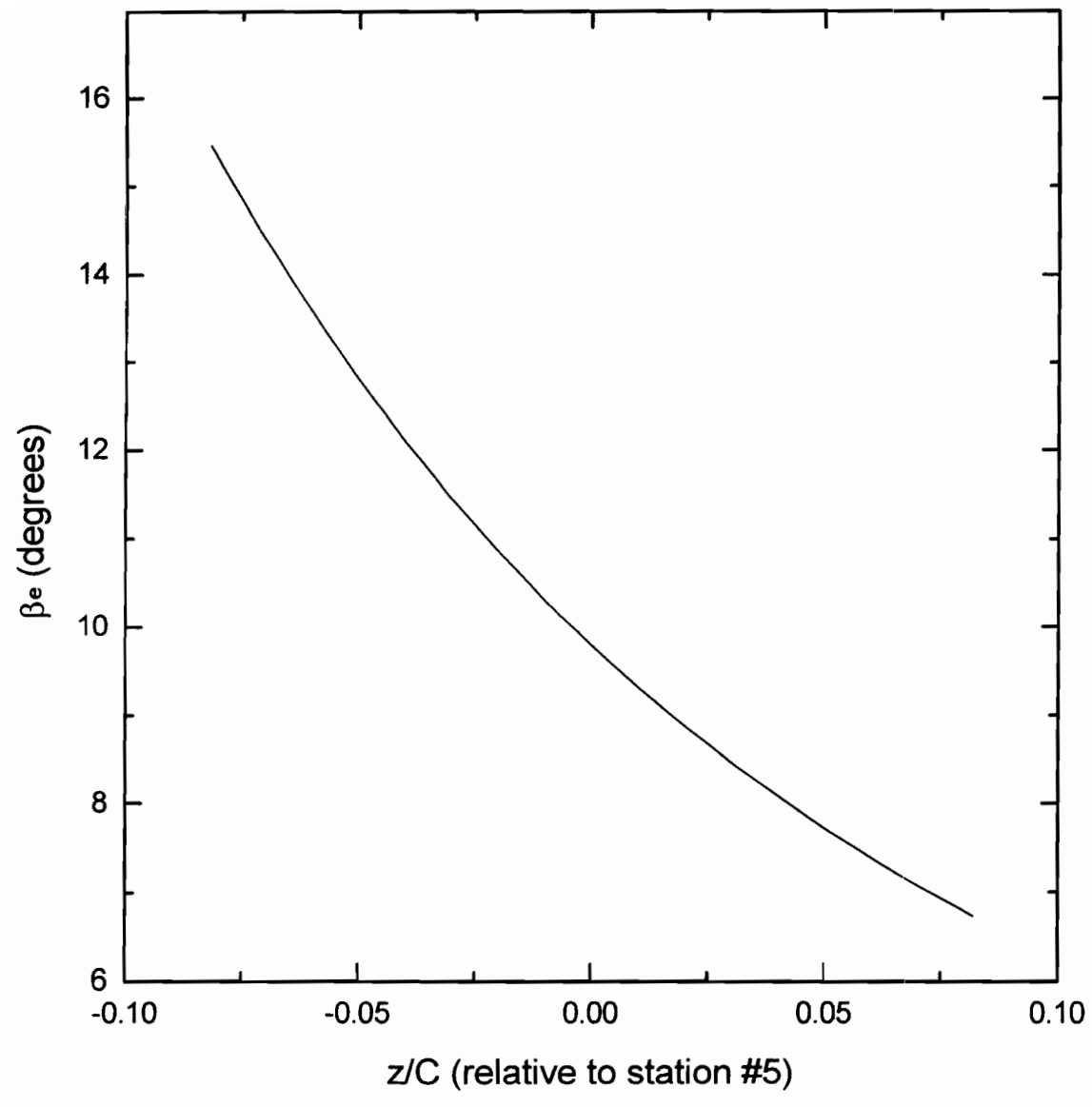

Fig. 4.7 Detail of computed freestream flow directions along 3-D bubble wire. Station \#5 is at $z / C=0.0$. 

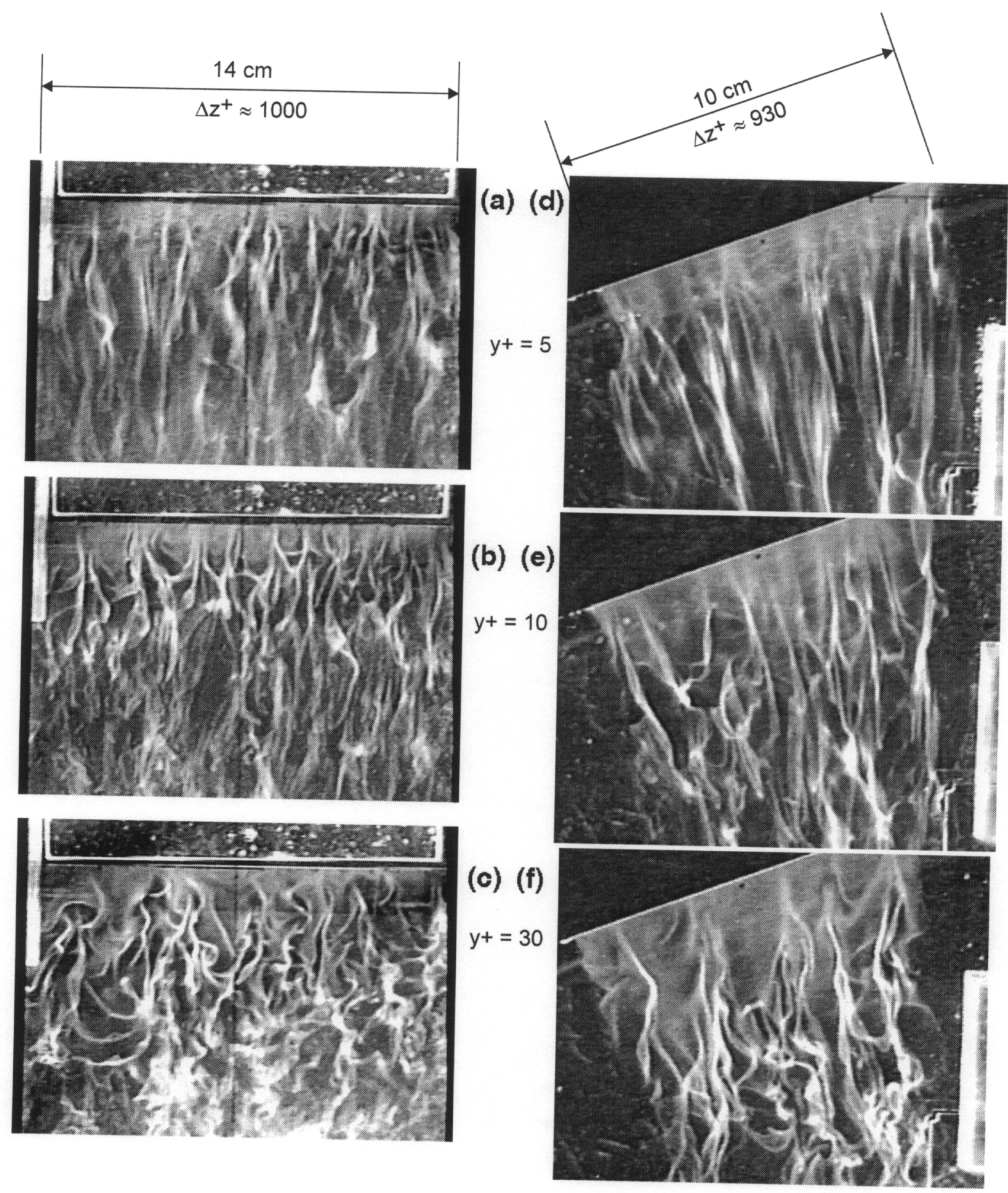

Fig. 4.8 Constant-sheet hydrogen-bubble images for a nominal $U_{e}$ of $15 \mathrm{~cm} / \mathrm{s}$. (a) 2-D, $y^{+}=5$, (b) $2-\mathrm{D}, y^{+}=10$, (c) $2-\mathrm{D}, y^{+}=30$, (d) $3-\mathrm{D}, y^{+}=5$, (e) $3-\mathrm{D}, y^{+}=10$, (f) $2-\mathrm{D}, y^{+}=30$. The 3 -D flow visualization is with the wire inclined $20^{\circ}$ to the flow. Ölçmen's station \#5 is the small dot near the middle of the wire in the 3-D images. 

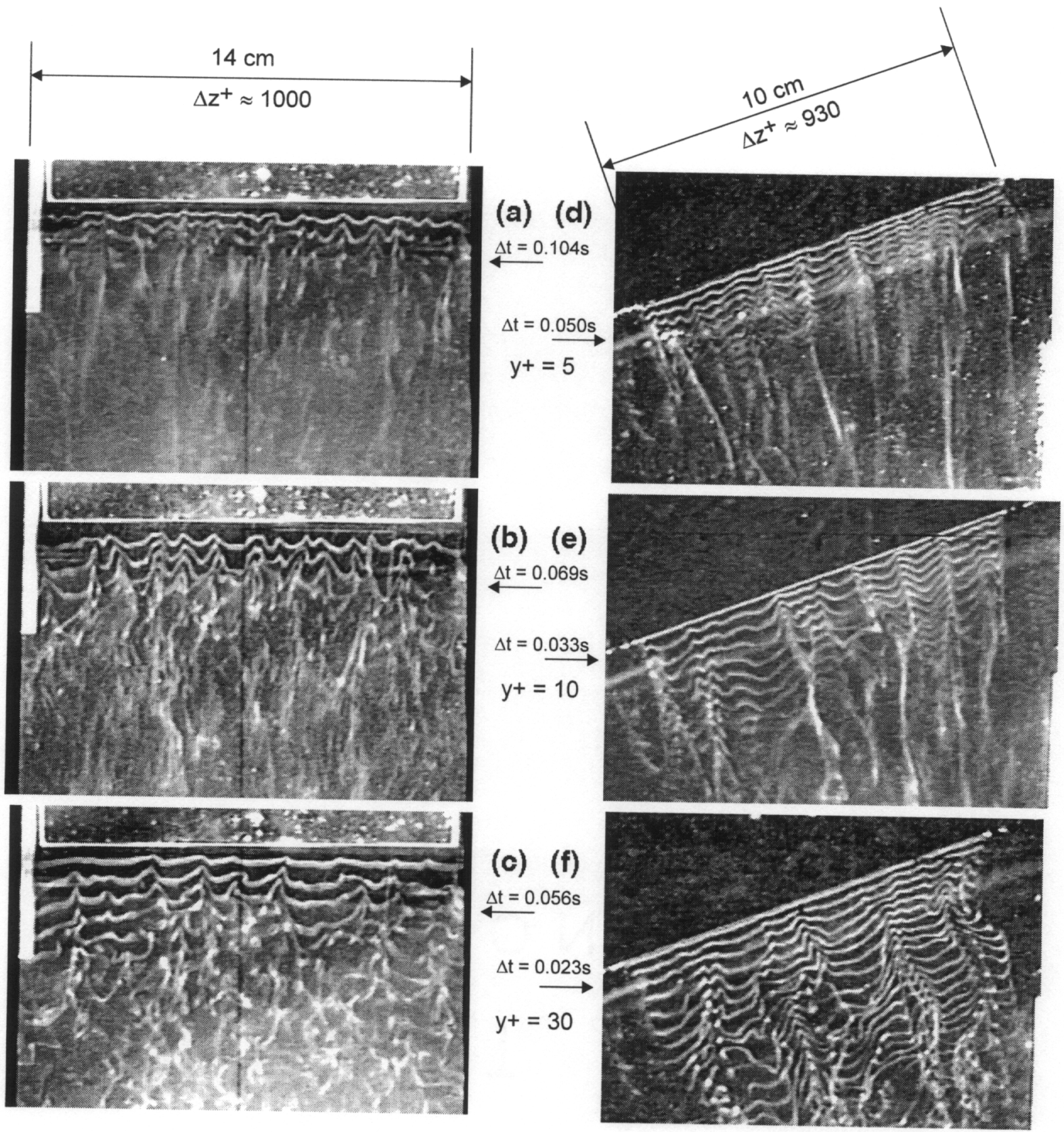

Fig. 4.9 Time-line hydrogen-bubble images for a nominal $U_{e}$ of $15 \mathrm{~cm} / \mathrm{s}$. (a) $2-\mathrm{D}, y^{+}=$ 5 , (b) $2-\mathrm{D}, y^{+}=10$, (c) $2-\mathrm{D}, y^{+}=30$, (d) $3-\mathrm{D}, y^{+}=5$, (e) $3-\mathrm{D}, y^{+}=10$, (f) $2-\mathrm{D}, y^{+}=30$. The wire pulsing rates are indicated for each frame. The 3 -D flow visualization is with the wire inclined $20^{\circ}$ to the flow. Ölçmen's station \#5 is the small dot near the middle of the wire in the 3-D images. 

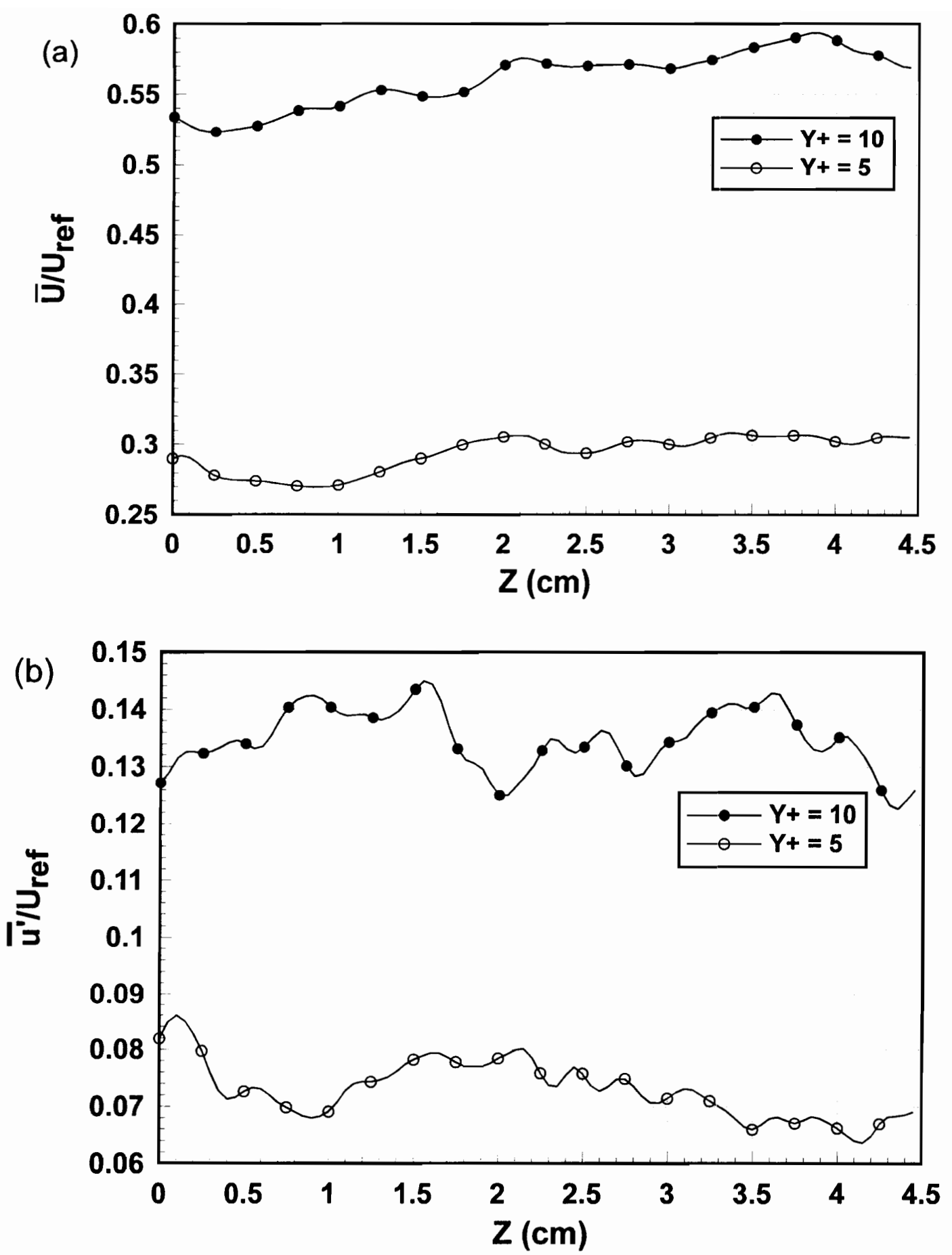

Fig. 4.10 2-D quantitative flow-visualization results for $U$ and $u^{\prime}, U_{e}=15 \mathrm{~cm} / \mathrm{s}, \operatorname{Re}_{\theta}=$ 300 . The $z$ axis is parallel to the bubble wire. The region used for gathering flow structure statistics for the $2-\mathrm{D}$ case was $0.0<z<4.5$. 

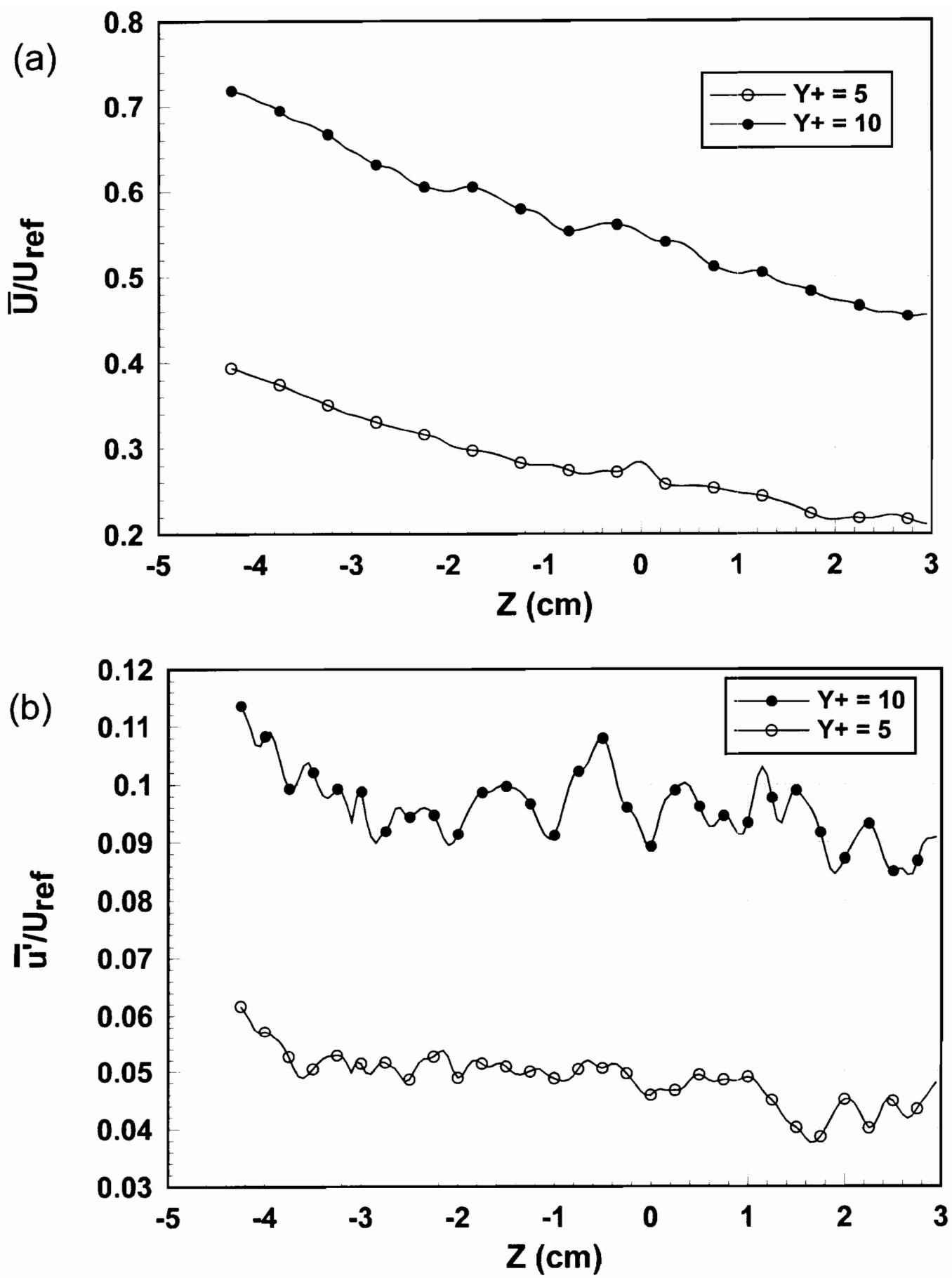

Fig. 4.11 3-D quantitative flow-visualization results for $U$ and $u^{\prime}$, bubble wire at $20^{\circ}, U_{e}=$ $15 \mathrm{~cm} / \mathrm{s}, \operatorname{Re}_{\theta}=760$. The $z$ axis is parallel to the bubble wire, with station \#5 at $z=0.0$ for this figure. The region used for gathering flow structure statistics for the 3 -D case was $-1.5<z<3.0$. 


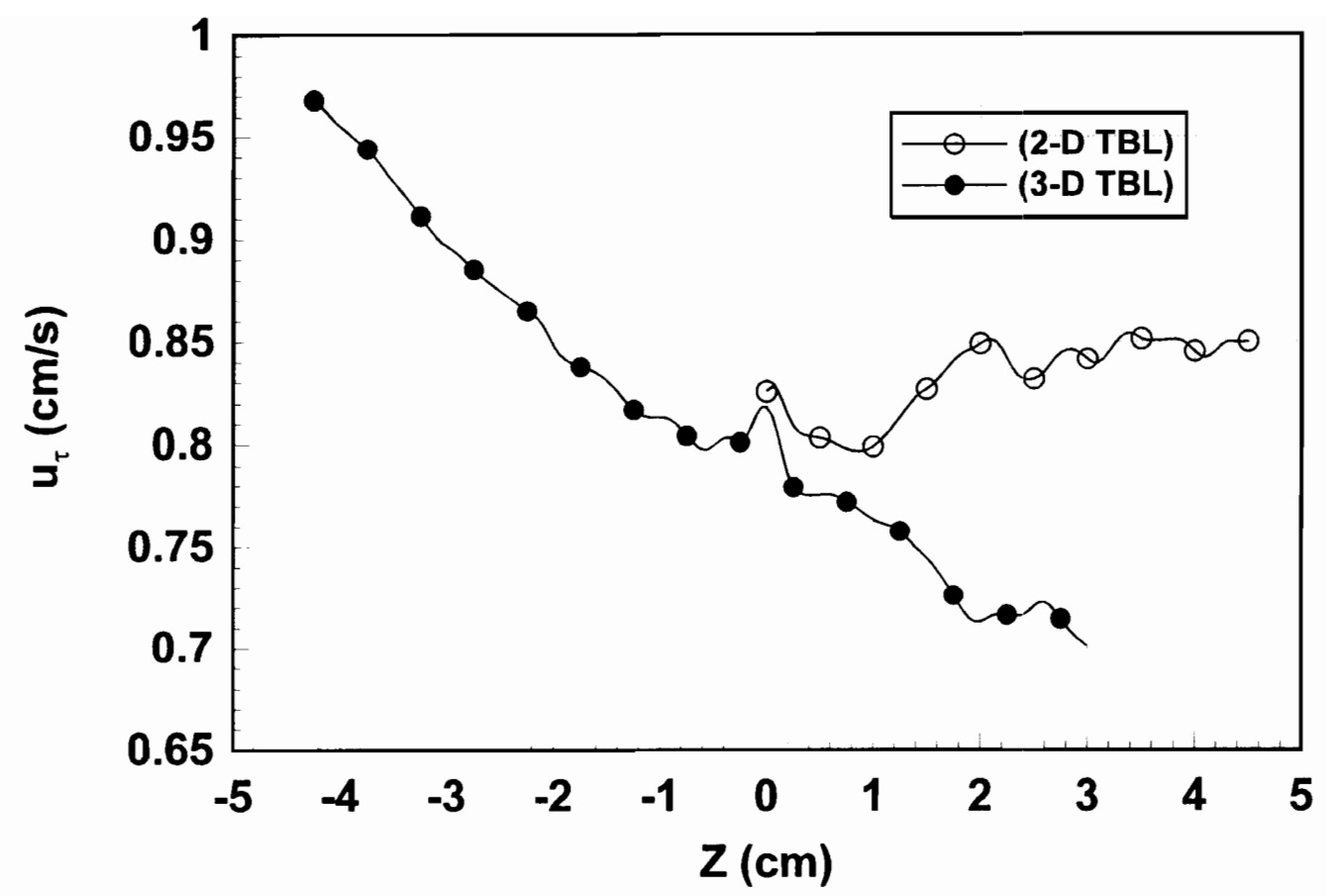

Fig. 4.12 Skin friction results determined for the $U_{e}=15 \mathrm{~cm} / \mathrm{s}$ cases. Results determined from $y^{+}=5$ mean velocity data. 


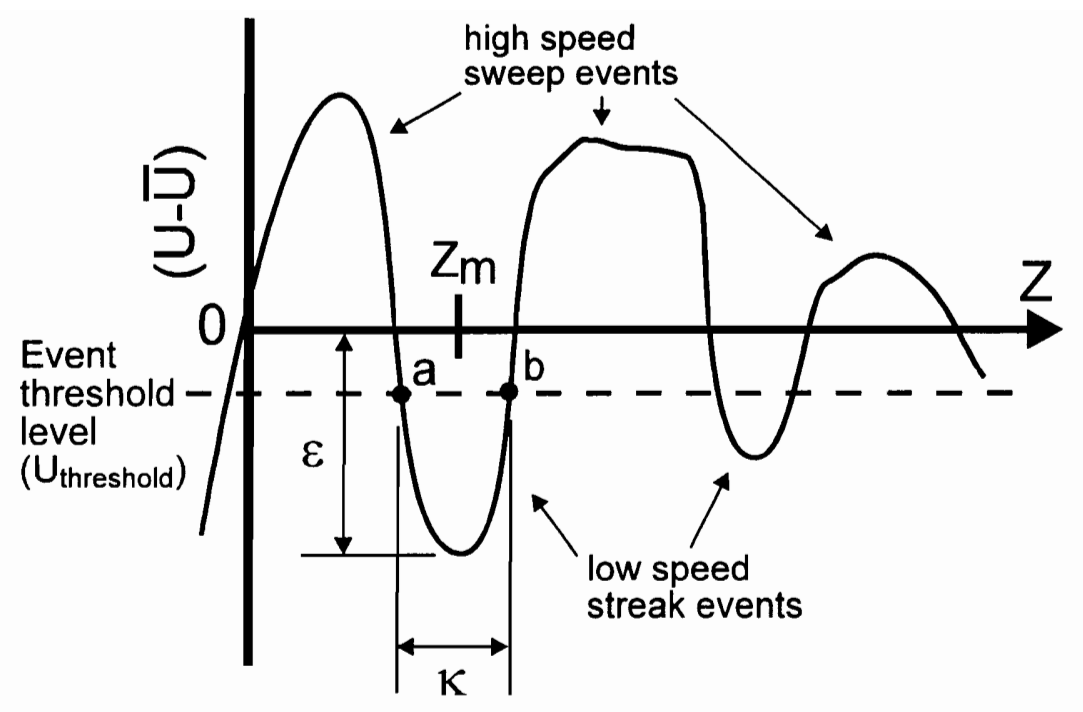

Fig. 4.13 Sketch defining event geometry parameters used to calculate quantitative structural quantities $(\lambda, \kappa, E$, and $\Omega$ ). These parameters can refer to low or high speed events. Note that $z_{m}$ indicates the maximum velocity fluctuation location for each event. These parameters can be easily applied to 3-D flow cases by allowing the mean velocity distribution to vary with $z$. 

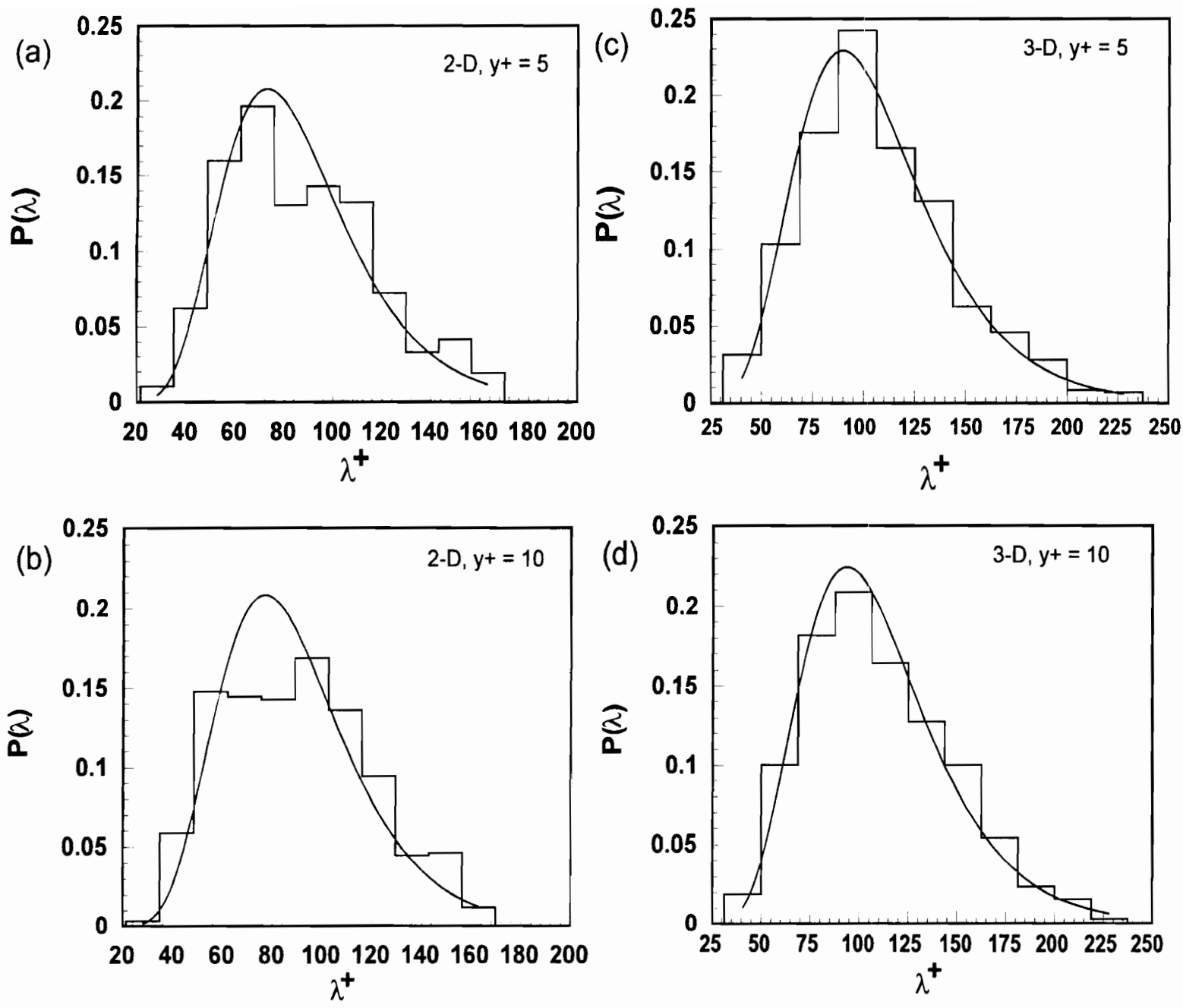

Fig. 4.14 $\lambda^{+}$probability distributions for (a) 2-D, $y^{+} \approx 5$; 581 events (b) 2-D, $y^{+} \approx 10$; 581 events $(\mathrm{c}) 3-\mathrm{D}, y^{+} \approx 5 ; 610$ events $(\mathrm{d}) 3-\mathrm{D}, y^{+} \approx 10 ; 627$ events The superimposed smooth curves are the log-normal distribution curve fits. 


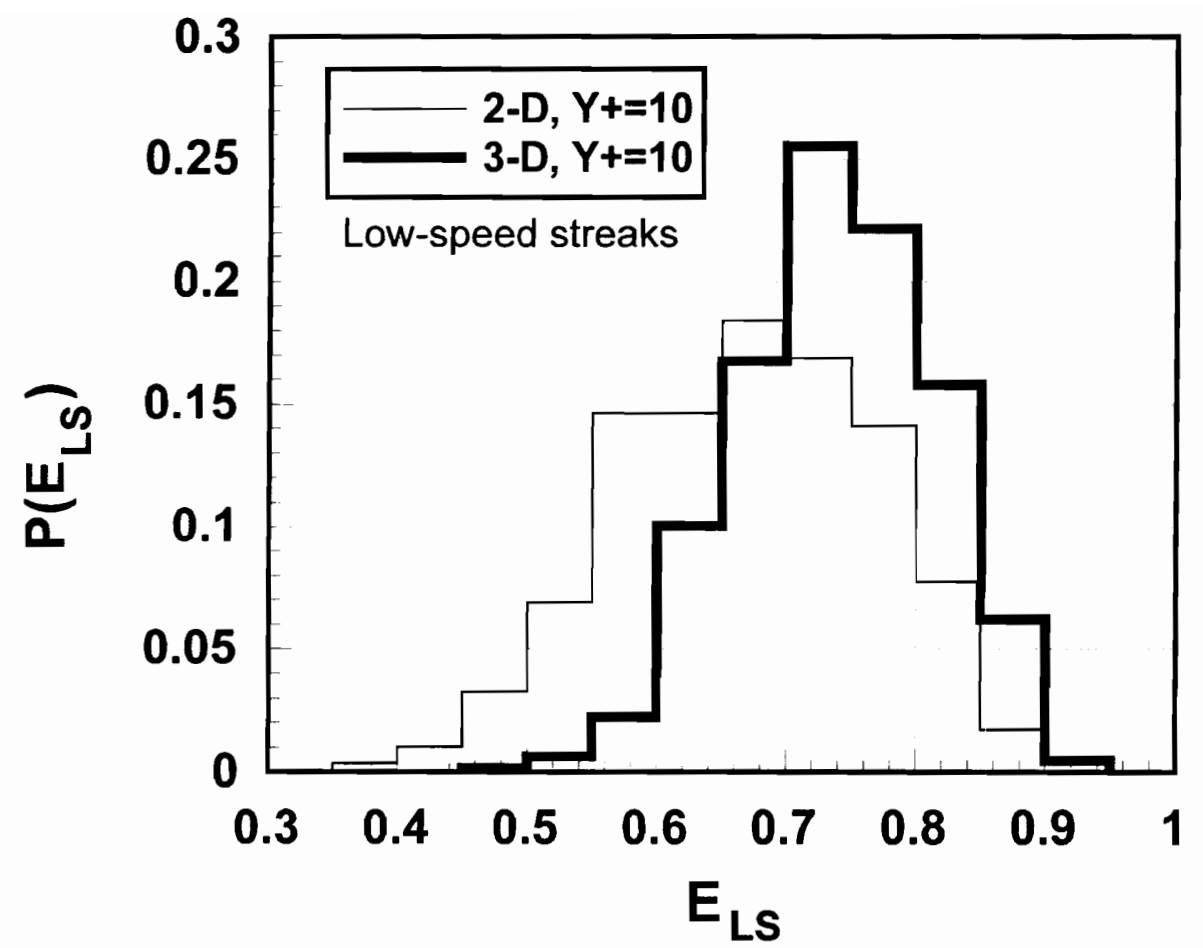

Fig. 4.15 The low-speed event momentum flux ratio $\left(E_{\mathrm{LS}}\right)$ probability distributions for 2-D and 3-D turbulent boundary layers at $y^{+} \approx 10, U_{e}=15 \mathrm{~cm} / \mathrm{s}$. 

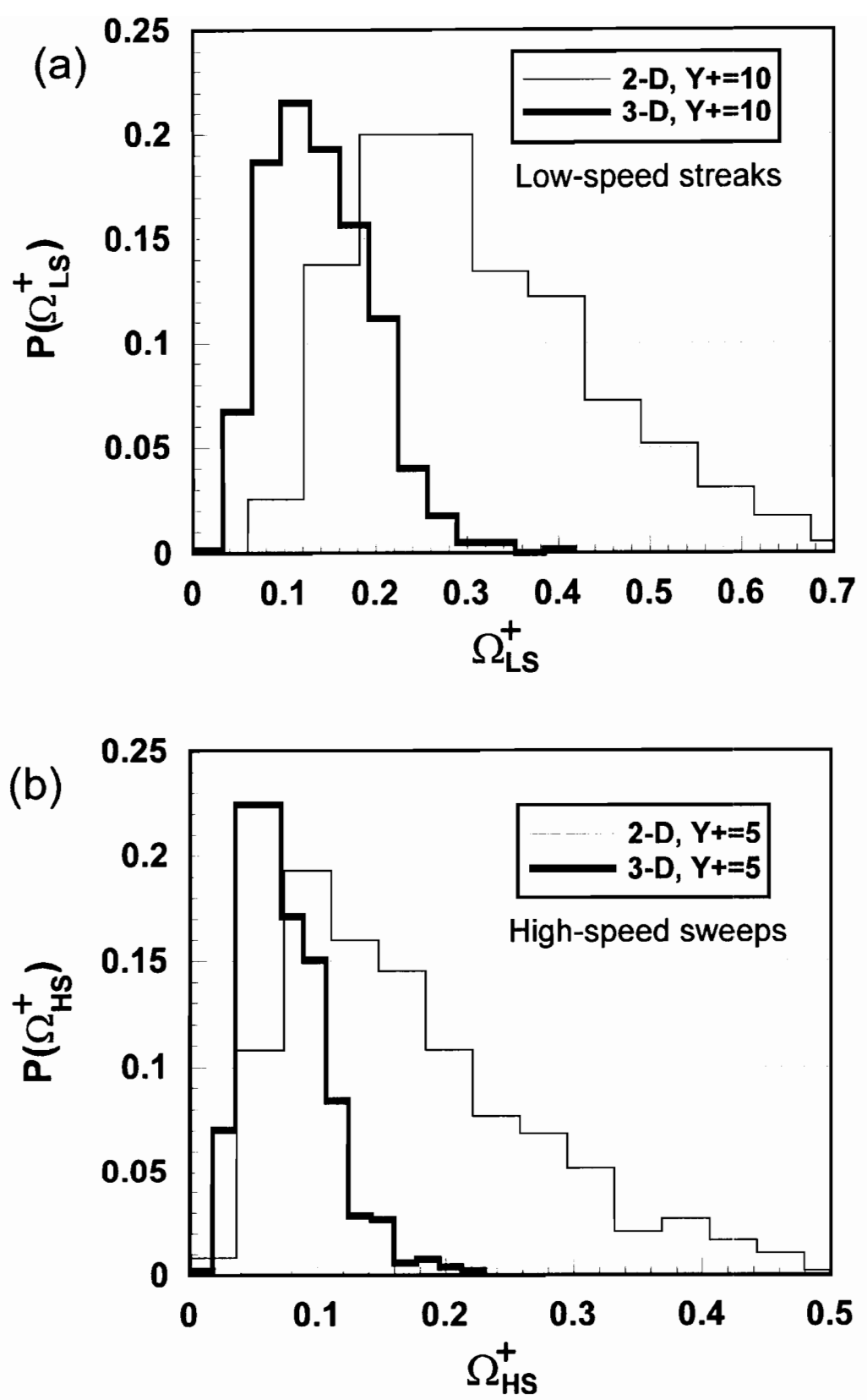

Fig. 4.16 Spatially-averaged vorticity $\left(\Omega^{+}\right)$probability distributions for the 2-D and 3-D turbulent boundary layers for the $U_{e}=15 \mathrm{~cm} / \mathrm{s}$ cases. (a) $\Omega_{\mathrm{LS}}, y^{+} \approx 10$, (b) $\Omega_{\mathrm{HS}}$, $y^{+} \approx 5$. 


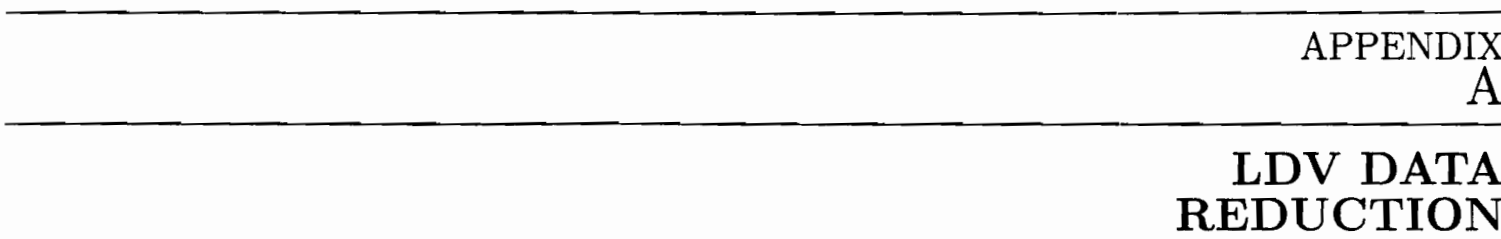

The major LDV data reduction equations are given in this appendix. This overview outlines how the data were taken from frequency vs. time information in the different optical coordinate systems to the tunnel coordinate system for the 2-D data and freestream coordinate system for the 3-D data. Only the mean and Reynolds stress results are discussed. Note that overbars are used in this appendix to distinguish mean values from instantaneous velocity realizations.

\section{$2-D$ data reduction}

One profile was measured for the 2-D data, so only $U$ and $V$ velocity components were obtained. The $U$ measurement volume fringe normal was aligned with $x_{\mathrm{TC}}$, so

$$
U_{T C}=U_{m}=\left(d_{f s}\right)_{\text {blue }}\left(f-f_{s}\right)_{\text {blue }}
$$

where $U_{m}$ is the shorthand version for the instantaneous $U$ velocity component measured by the blue laser beam pair. The calculation of all terms involving only $U_{\mathrm{TC}}$ is very straightforward for the 2-D case.

The $V$ measurement volume fringe normal was tilted $\phi=5.6^{\circ}$ from the floor normal ( $y_{\mathrm{TC}}$ axis, see Fig. 2.22), and was perpendicular to $x_{\mathrm{TC}}$. Because of this tilt, the $V$ results must be corrected to minimize contamination from non-zero terms involving $W$. The equation for $V_{m}$ (the instantaneous velocity component measured by the green beams) is

$$
V_{T C} \cos \phi+W_{T C} \sin \phi=V_{m}=\left(d_{f s}\right)_{\text {green }}\left(f-f_{s}\right)_{\text {green }}
$$

where $\phi$ is the tilt angle shown in Fig. 2.22. By solving for $V_{\mathrm{TC}}$, we obtain

$$
V_{T C}=\frac{V_{m}}{\cos \phi}-W_{T C} \tan \phi
$$

When the time average of equation A.3 is taken, we are left with

$$
\bar{V}_{T C}=\frac{\bar{V}_{m}}{\cos \phi}
$$


since $\bar{W}_{T C}=0$. The $V$ component normal stress equation becomes

$$
{\overline{v^{\prime 2}}}_{T C}=\overline{\left(\frac{V_{m}}{\cos \phi}\right)^{2}}-\left(\frac{\bar{V}_{m}}{\cos \phi}\right)^{2}+{\overline{w^{\prime 2}}}_{T C} \tan \phi
$$

The last term in equation A.5 can be estimated by taking the average of $\overline{u^{\prime 2}}$ and $\overline{v^{\prime 2}}$ as recommended by Nakayama (1983). For the author's 2-D LDV configuration and test flow, $\overline{w^{\prime 2}} \tan \phi$ contributes a $15 \%$ to $20 \%$ relative error to $\overline{v^{\prime 2}} T C$. This error estimate can be subtracted out of equation A.5, but the overall uncertainty of $\overline{v^{\prime 2}} T C$ does increase. The equation for $-\overline{u v}_{\mathrm{TC}}$ contains a term involving $W_{\mathrm{TC}}$, but this term goes to zero when the time average is taken.

Note that when calculating the time averages for these 2-D results (and the following 3 -D results), it is understood that the velocity bias correction discussed in section 2.2 .5 is being applied. The details of the third and fourth order fluctuating quantities are not listed here. These terms are much more complex, and contain $W$ terms similar to equation A.5. However, estimates of these contaminating terms can be subtracted from these results, such as done for equation A.5.

\section{3-D data reduction}

The 3-D data reduction was more complex compared to the 2-D data, but no approximations of "contaminating" terms were necessary. The 3 orientations of the LDV system for taking the 3-D data are shown in Fig. 2.22. These orientations are labeled "A," "B," and "C."

Three orthogonal coordinate systems were used to obtain results in the tunnel coordinate system. Coordinate system 1 was oriented using cases $\mathrm{B}$ and $\mathrm{C}$. The $x_{1}$ axis was aligned with case $\mathrm{B}$, and the $z_{1}$ axis was aligned with case $\mathrm{C}$. The actual LDV angles for cases $\mathrm{B}$ and $\mathrm{C}$ were aligned within $\pm 0.2^{\circ}$ of $x_{1}$ and $z_{1}$ respectively. Note that coordinate systems 1 and 2 are both contained in a plane inclined to the tunnel floor.

Coordinate system 2 is defined by a $45^{\circ}$ rotation from coordinate system 1 about the $y_{1}$ axis. This rotation is in a clockwise direction if one is looking along the $-y_{1}$ axis (the top view of the coordinate systems in Fig. 2.22). The LDV orientation used for case A was aligned with coordinate system 2 .

Coordinate system 3 is defined by a rotation about the $x_{2}$ axis through the angle $\phi$ to match the tunnel coordinate system orientation. Note that the tilt angle $\phi$ was $5.6^{\circ}$ for case $A$, and $5.2^{\circ}$ for cases $\mathrm{B}$ and $\mathrm{C}$. This small change in $\phi$ between cases was neglected for the $3-\mathrm{D}$ data reduction. A tilt angle $5.6^{\circ}$ was used for the final transformation to coordinate system 3 . 
The 3-D reduction equations were obtained thru the application of coordinate transformations. This section does not present a detailed derivation of the 3 - $D$ reduction equations. Only the final equations used to reduce the data are listed. Subscripts are used to designate intermediate mean or fluctuating quantities.

In coordinate system 1 , all mean and normal stress quantities are known $\left(\bar{U}_{B}=\right.$ $\bar{U}_{1}, \bar{U}_{C}=\bar{W}_{1}, \bar{V}_{B}=\bar{V}_{C}=\bar{V}_{1}$, etc.) Measurements of $V$ were redundant, since the vertical axis of orientations $\mathrm{A}, \mathrm{B}$, and $\mathrm{C}$ were (nearly) aligned. Two of the shear stress quantites are known for coordinate system $1\left(\overline{u^{\prime} v^{\prime}}{ }_{1}\right.$ and $\left.\overline{v^{\prime} w^{\prime}}{ }_{1}\right)$, but the "coupled" shear stress $\overline{u^{\prime} w^{\prime}}{ }_{1}$ has not been measured and is unknown. Since $\overline{u^{\prime 2}}{ }_{2}$ is already known from direct measurements from LDV case $\mathrm{A}, \overline{u^{\prime} w^{\prime}}{ }_{1}$ can be solved for:

$$
{\overline{u^{\prime} w^{\prime}}}_{1}=\frac{1}{2(\sin \alpha)(\cos \alpha)}\left({\overline{u^{\prime 2}}}_{1} \cos ^{2} \alpha+\overline{w^{\prime 2}}{ }_{1} \sin ^{2} \alpha-\overline{u^{\prime 2}}{ }_{2}\right)
$$

In equation A.6 and the following equations, $\alpha$ is the angle between $x_{1}$ and $x_{2}$. For the author's case this angle is $45^{\circ}$. Now all quantities are known in coordinate system 1.

In coordinate system 2 , the following terms were measured directly from LDV case A: $\bar{U}_{2}, \bar{V}_{2}, \bar{u}_{2}{ }_{2},{\overline{v^{\prime 2}}}_{2}$, and ${\overline{u^{\prime} v^{\prime}}}_{2}$. The equations used to get the terms involving $W$ are:

$$
\begin{gathered}
\bar{W}_{2}=\bar{U}_{1} \sin \alpha+\bar{W}_{1} \cos \alpha \\
{\overline{w^{\prime 2}}}_{2}=\bar{u}^{\prime 2}{ }_{1} \sin ^{2} \alpha+2{\overline{u^{\prime} w^{\prime}}}_{1}(\sin \alpha)(\cos \alpha)+{\overline{w^{\prime 2}}}_{1} \cos ^{2} \alpha \\
\overline{v^{\prime} w^{\prime}}{ }_{2}={\overline{u^{\prime} v^{\prime}}}_{1} \sin \alpha+\overline{v^{\prime} w^{\prime}}{ }_{1} \cos \alpha \\
\overline{u^{\prime} w^{\prime}}{ }_{2}=\left({\overline{u^{\prime 2}}}_{1}-\overline{w^{\prime 2}}{ }_{1}\right)(\cos \alpha)(\sin \alpha)+\left(\cos ^{2} \alpha-\sin ^{2} \alpha\right) \overline{u^{\prime} w^{\prime}}{ }_{1}
\end{gathered}
$$

The second term of equation A.10 is zero if $\alpha=45^{\circ}$. Now all terms in coordinate system 2 are known.

The third and final transformation on our journey to coordinate system 3 is a small rotation thru the tilt angle $\phi$ about the $x_{2}$ axis. Recall that this coordinate system is aligned with the tunnel coordinate system. The equations for all mean and fluctuating quantities for coordinate system 3 are:

$$
\begin{gathered}
\bar{U}_{3}=\bar{U}_{2} \\
\bar{V}_{3}=\bar{W}_{2} \sin \phi+\bar{V}_{2} \cos \phi \\
\bar{W}_{3}=\bar{W}_{2} \cos \phi-\bar{V}_{2} \sin \phi \\
{\overline{u^{\prime 2}}}_{3}={\overline{u^{\prime 2}}}_{2}
\end{gathered}
$$




$$
\begin{gathered}
\overline{v^{\prime 2}}{ }_{3}=\overline{w^{\prime 2}}{ }_{2} \sin ^{2} \phi+\overline{v^{\prime 2}}{ }_{2} \cos ^{2} \phi+2 \overline{v^{\prime} w^{\prime}}{ }_{2}(\cos \phi)(\sin \phi) \\
\overline{w^{\prime 2}}{ }_{3}=\overline{w^{\prime 2}}{ }_{2} \cos ^{2} \phi+\overline{v^{\prime 2}}{ }_{2} \sin ^{2} \phi-2 \overline{v^{\prime} w^{\prime}}{ }_{2}(\cos \phi)(\sin \phi) \\
\overline{u^{\prime} v^{\prime}}{ }_{3}=\overline{u^{\prime} w^{\prime}}{ }_{2} \sin \phi+{\overline{u^{\prime} v^{\prime}}}_{2} \cos \phi \\
\overline{u^{\prime} w^{\prime}}{ }_{3}=\overline{u^{\prime} w^{\prime}}{ }_{2} \cos \phi-{\overline{u^{\prime} v^{\prime}}}_{2} \sin \phi \\
\overline{v^{\prime} \bar{w}_{3}}{ }_{3}=\left({\overline{w^{\prime 2}}}_{2}-{\overline{v^{\prime 2}}}_{2}\right)(\cos \phi)(\sin \phi)+\left(\cos ^{2} \phi-\sin ^{2} \phi\right) \overline{v^{\prime} w^{\prime}}{ }_{2}
\end{gathered}
$$

Now all mean. normal stress, and shear stress quantities are known in the tunnel coordinate system orientation. Obtaining the data in freestream coordinates, wall coordinates, etc. required additional transformations about the $y_{3}$ axis using the appropriate angles.

$\mathrm{By}$ using redundant results for the 3-D measurements, small differences in flow speeds and LDV measurement volume $y$ positioning could be determined and corrected. The following redundant relationships are written using "A," "B," and "C" subscripts to signify the different LDV configurations. The first relationship was mentioned previously in this appendix.

$$
\begin{gathered}
\bar{V}_{A}=\bar{V}_{B}=\bar{V}_{C} \\
\bar{U}_{A}=\bar{U}_{B} \cos \alpha+\bar{U}_{C} \sin \alpha \\
{\overline{v^{\prime 2}}}_{A}={\overline{v^{\prime 2}}}_{B}={\overline{v^{\prime 2}}}_{C} \\
{\overline{u^{\prime} v^{\prime}}}_{A}={\overline{u^{\prime} v^{\prime}}}_{B} \cos \alpha+{\overline{u^{\prime} v^{\prime}}}_{C} \sin \alpha
\end{gathered}
$$

These equations are strictly true only if cases $\mathrm{A}, \mathrm{B}$, and $\mathrm{C}$ all share a common $y$ axis. As previously mentioned, the small change in tilt angle $\left(0.4^{\circ}\right)$ between case $A$ and cases $B$ and $\mathrm{C}$ was neglected. A unique algorithm was developed to reduce the $3-\mathrm{D}$ data taking these redundant relationships into account.

Because of the way the 3-D data were taken, the only higher-order results that were calculated were the "raw" results for case A $\left(x_{A}\right.$ aligned with $x_{T C}, y_{A}$ tilted at a $5.6^{\circ}$ to $\left.y_{T C}\right)$. These results were the same as could be obtained for the 2-D LDV measurements. 


\section{DATA REDUCTION PROGRAMS}

Many custom data reduction routines were programmed by the author during the course of this work. All programs were written using Borland's $\mathrm{C} / \mathrm{C}++$ development package and compiled to run under DOS on PC-compatible computers. This appendix lists the major programs written, the approximate number of lines in the source code, and a brief description of the program's function.

Program Name

REDLDV ......... (1,675 code lines, for LDV data) Initial LDV data reduction program, plots histograms on screen with interactive user input used to reject data points, saves data using various weighting schemes, saves histograms, saves "quadrant" data for all fluctuating quantities.

2DRP10B.......... (420 code lines, for LDV data) Reduces 2-D data into their final form, calculates shape factors, friction velocities.

3DRP10 ......... (1,275 code lines, for LDV data) Reduces 3-D data into their final form, transforms data to different coordinate systems, calculates shape factors and friction velocities, flow angles, correlation coefficients, etc.

MKBLK14......... (856 code lines, for LDV data) Transformed long time record LDV data into evenly spaced data blocks for spectral and autocorrelation calculations.

FBLK10 .......... (361 code lines, for LDV data) Digital filter for data blocks used for autocorrelation calculations.

DFTLDV20....... (420 code lines, for LDV data) Routine for calculating Discreet Fourier Transforms and ensemble averaging over many data blocks.

ACLDV20 ........ (380 code lines, for LDV data) Routine for calculating autocorrelation functions and ensemble averaging over many data blocks.

REDFV32 ........ (2,555 code lines, for flow-visualization data) Program to quantitatively analyze hydrogen-bubble time line images. Saves raw $U(z)$ data vs. time from a series of images in binary format. This program would read, enhance, display, and analyze each image. The analysis was approximately $90 \%$ automated, with user interaction required to initially align the image with the master calibration and to verify that the data were acceptable. Program parameters could be changed interactively during a program run. Examples shown in Luse (1993) were used for some of the image data handling routines used by this program. 
RDATA30 ....... (2,516 code lines, for flow-visualization data) Program to reduce raw image data results from "REDFV32." Output included final mean $U(z)$ and $u^{\prime}(z)$ distributions, or could output the time history of $U$ at a selected $z$ location. Program also could output spatial correlation data and contour data. These last two data reduction schemes were not very informative, so the program "STATS10" was developed.

STATS10 ........ (408 code lines, for flow-visualization data) Program to quantify near wall structures using data from "RDATA30." Output included streak and sweep spacing and histogram data, event widths and magnitudes, and event vorticity averages.

With some additional developmental effort and further documentation, the author believes that the image analysis program "REDFV32" and its associated image data reduction programs would benefit other researchers performing similar flow-visualization studies. It is a quite flexible program, adaptable to other time line geometries. Interested parties can contact the author by sending email to "fleming@apollo.aoe.vt.edu." 
The author was born April 16, 1966, and grew up on a commercial beef cattle farm in southwest Virginia. He graduated from Rural Retreat High School in 1984, and recieved his B. S. degree in Aerospace Engineering from VPI\&SU in May, 1988. He began his graduate studies at Virginia Tech in the fall of 1988, and successfully defended his Master's thesis, entitled "An Experimental Study of a Turbulent Wing-Body Junction and Wake Flow," in July, 1991. His immediate plans are to continue working at Virginia Tech in the Aerospace Engineering Department's post-doctoral research program.

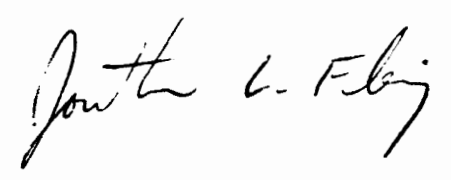

Jonathan L. Fleming 